\title{
Gene Expression Responses in a Cellular Model of Parkinson's Disease
}

Louis Beverly Brill II

Manassas, Virginia

B.A., Johns Hopkins University, 1995

A Dissertation presented to the Graduate Faculty of the University of Virginia in Candidacy for the Degree of Doctor of Philosophy

\section{Department of Cell Biology \\ University of Virginia \\ May, 2004}

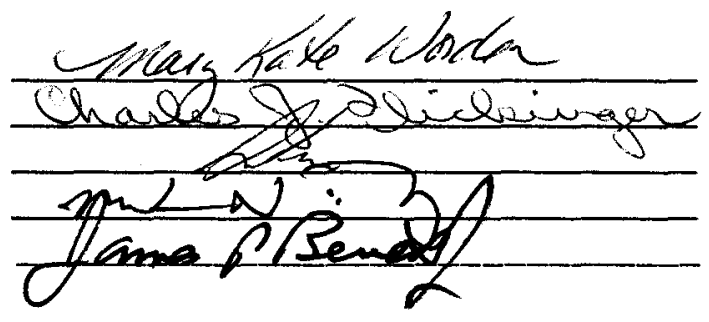


Table of Contents

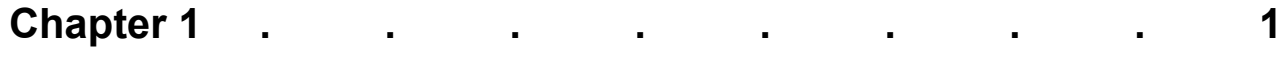

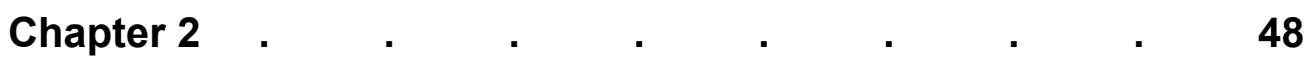

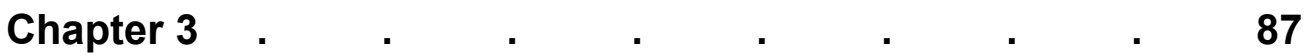

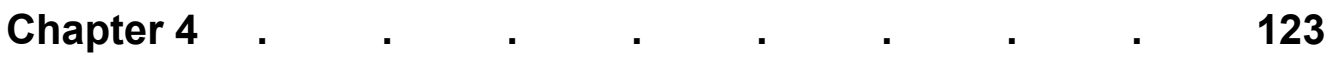

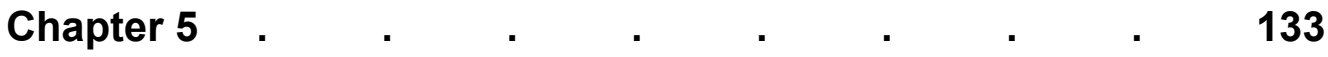

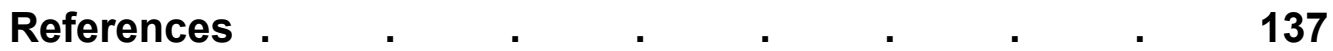

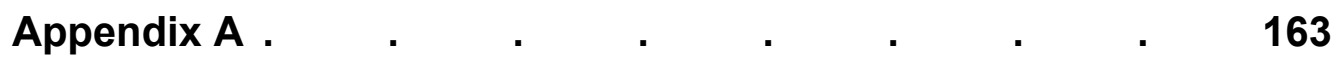

Appendix B . $\quad$. $\quad$. $\quad$. $\quad$. $\quad$. $\quad$. $\quad$. 209

Appendix C . $\quad . \quad$. $\quad . \quad$. $\quad . \quad . \quad . \quad . \quad 216$

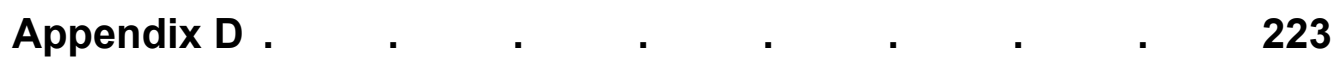

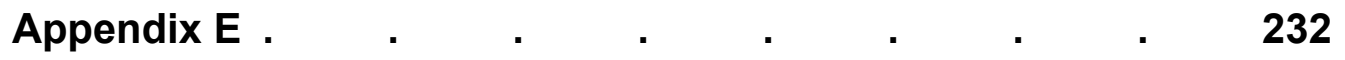

Appendix F . $\quad . \quad$. $\quad . \quad$. $\quad . \quad$. $\quad . \quad . \quad 234$

Appendix G . $\quad$. $\quad$. $\quad$. $\quad . \quad$. $\quad . \quad$.

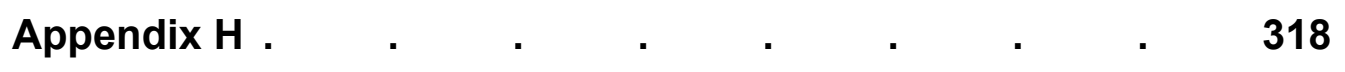

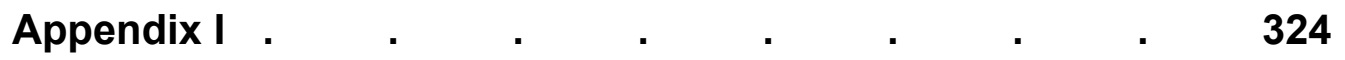




\section{Abstract}

This research represents initial steps towards understanding the relation between changes in gene expression, mitochondrial function and cell death in cell-based models of Parkinson's disease. The main hypothesis is that rapid gene expression changes in cells exposed to parkinsonian neurotoxins occur, are dependent on mitochondrial status, and directly impact intracellular signaling pathways that determine whether a cell lives or dies. Our cellular model is comprised of SH-SY5Y neuroblastoma cells exposed to the parkinsonian neurotoxin methylpyridinium ion. Transcriptomic changes are evaluated with nylon and glass-based cDNA microarray technology. Cardinal symptoms of Parkinson's disease, characteristic pathological changes, therapeutic modalities, and current theories on the etiology of the disorder are discussed. Our results verify the existence of mitochondrial-nuclear signaling in the context of electron transport chain deficits, as well as suggesting the vital roles played in this process by previously described intracellular signaling pathways. These results will serve to direct future investigations into gene expression changes relevant to the processes of cell death and cell survival in our cellular model of Parkinson's disease, and may provide important insights into the pathophysiology of the in vivo disease process. 


\section{Chapter 1}

My research in the Center for the Study of Neurodegenerative Disease has explored the relation between changes in gene expression, mitochondrial function and cell death in cell-based models of Parkinson's disease. The main hypothesis behind my work is that rapid gene expression changes in cells exposed to parkinsonian neurotoxins occur, are dependent on mitochondrial status, and directly impact intracellular signaling pathways that determine whether a cell lives or dies. I have attempted to bring two novel experimental techniques to bear upon this problem: cDNA microarrays and RNA interference (RNAi). This introductory chapter will serve as a review of the background information necessary to place the dissertation research in its proper context. The cardinal symptoms and pathology of Parkinson's disease will be discussed, as will the primary therapeutic modalities, and major hypotheses regarding the etiology of the disorder. The most recent developments in cDNA microarrays and RNA interference (RNAi) will be reviewed. Subsequent chapters will document my experimental techniques and results, along with my suggestions for future directions in cellular and molecular Parkinson's disease research. 
Background Information

\section{Parkinson's Disease}

Parkinson's disease is a progressive neurodegenerative disorder that can cause profound motor impairment in its victims. It is among the most prevalent neurological disorders, with approximately 50,000 new cases diagnosed each year in the United States alone. The four cardinal symptoms of PD are bradykinesia, tremor at rest, postural instability, and rigidity. Tremor is the symptom that is often first to prompt a patient to seek medical attention; the tremor of PD is described as a "pill-rolling" tremor, referring to the stereotyped motion of the fingers and hands. It is of low frequency (about 4-5 cycles per second) and occurs at rest. The characteristic Parkinsonian tremor tends to reduce in severity or even disappear when the patient initiates a voluntary movement.

Bradykinesia, a slowing of movements in general, is often present early in the clinical course of PD and can become highly debilitating. Inability to execute sequential motor tasks as a result of bradykinesia frequently has severe impacts on the patient's ability to perform activities of daily living. Rigidity is generally manifested in movements of the extremities (cogwheel rigidity) and, in moderate to severe cases of PD, episodes of "freezing," or the inability to initiate any voluntary movements. In addition to the obvious reduction in ability to perform activities of daily living, such episodes often provoke intense feelings of fear and helplessness, with negative impacts on the patient's mental and emotional well- 
being. Some reports have also detailed the phenomenon of akinesia paradoxica, referring to temporary periods of normal or near normal motor activity under stressful situations.

Postural abnormalities manifest in the PD patient with unusual degrees of flexion in the limbs. The trunk is often stooped or hunched forward, and when combined with flexion of the knees and elbows, the patient's center of gravity is frequently displaced forward to a sufficient degree to cause the characteristic shuffling gait of PD. As the disease progresses, cerebellar reflexes required to maintain balance are lost, with the frequent onset of episodes of falling. Falls are a major cause of morbidity and mortality in PD patients, as for the elderly population at large.

Other non-motor symptoms frequently associated with the disease process, particularly in its early stages, include a generalized loss of manual dexterity, manifesting as difficulty in performing simple motor tasks, mild depression, a reduction of facial expressions (mask facies) and micrographia, a reduction in size of handwriting. On the order of forty percent of PD patients present with non-specific sensory complaints, including numbness, tingling, aching or burning sensations, particularly in the extremities. A high percentage of patients present with anosmia, loss of the sense of smell. Anosmia may occur years before onset of the cardinal motor symptoms of PD. As the olfactory tissues are directly exposed to the environment, this phenomenon has been invoked by some researchers as an endorsement of the environmental hypothesis of PD, i.e., that a neurotoxic agent responsible for PD present in the 
environment either gains entry to the brain via the olfactory nerves, or that the olfactory receptors alternatively represent a highly vulnerable neuronal population whose demise is linked to chronic exposure to a Parkinsonian neurotoxin.

Dementia is frequently reported in PD, but there exists considerable variability in reports of its rate of occurrence (10-40\%). Some investigators have reported a higher prevalence of dementia in PD in males, and in those patients of more advanced age. Likewise, depression is reported in a variable but high portion of the PD population (37-90\%). As depression often occurs early in the clinical course, It is unclear whether the majority of depressive symptoms are related to the etiology of $\mathrm{PD}$, or rather a reaction to symptoms on the part of the patient.

Historical Context

Parkinson's Disease (PD) is generally referred to as the archetype of the hypokinetic movement disorders. The symptoms of this condition were first described in the 1817 "Essay on the Shaking Palsy" by James Parkinson, a general practitioner in the suburbs of London (Parkinson 1817). The impetus for the essay was Parkinson's keen observations of Londoners with characteristic gait abnormalties, tremors and muscle weakness that tended to increase in severity with advancing age: 
"The patient can [rarely] form any recollection of the precise period of its commencement. The first symptoms perceived are, a slight sense of weakness, with a proneness to trembling in some particular part; sometimes in the head, but most commonly in one of the hands and arms.... The propensity to lean forward becomes invincible.... As the debility increases and the influence of the will over the muscles fades away, the tremulous agitation becomes more vehement."

Parkinson and his contemporaries were hamstrung by the lack of a standardized system of neurological examination, a problem that continues in part to the present day. PD remains a diagnosis that is based primarily on clinical observation by the physician, with autopsy examinations of brain tissue and quantitative assessments of symptoms playing secondary roles. It was not until half a century following the publication of the Essay that Parkinson's name became permanently associated with the condition, formerly referred to as paralysis agitans, following acceptance by preeminent neuroscientists of the time such as Charcot and Gowers (Charcot 1861; Gowers 1893).

Following the devastating epidemics of influenza in 1918-1919, a population of patients exhibiting peculiar parkinsonian symptoms began to present at neurological clinics throughout Europe, Australia and the Americas. In addition to parkinsonian motor symptoms, cranial nerve palsies, oculogyric crisis and severe somnolence were common components of this disease. It was initially described as a form of "chronic encephalitis," and was only later to be described as postencephalitic parkinsonism, or encephalitis lethargica. One of the eminent neurologists of the period, Dr. Constantin von Economo, presented 
his description of the condition to the Vienna Psychiatric Society in April of 1917. As early as 1916, he described the typical syndromes in a monograph: "It seems strange when sleep appears as a symptom of an illness. 'Sleeping sickness' were the phenomenon of people fallingasleep while eating or working was first described in two cases in our clinic in 1916. Usually headache, nausea and fever were followed, often the next day, by sleepeing, frequently in a most uncomfortable position. One can wake them, but in severe cases, coma can rapidly lead to death. Malfunction of eye muscles, especially oculomotor dysfunction, and ptosis, was common." Von Economo was quick to obtain autopsy data from severe cases, noting inflammation in the tegmental gray matter. He classified the disorder into three primary forms: somnolentopthalmoplegic, hyperkinetic, and amyostatic-akinetic, which bore the hallmarks of parkinsonism. This amyostatic form was "characterized by a rigidity, without a real palsy and without symptoms arising from the pyramidal tract. This form of encephalitis lethargica is particularly common in the chronic cases, dominating the clinical picture as Parkinsonism. I reserve the name 'Parkinsonism,' though symptomatically identical with the amyostatic-akinetic form, rather for the chronic cases. To look at these patients one would suppose them to be in a state of profound secondary dementia. Emotions are scarcely noticeable in the face, but they are mentally intact." (von Economo, 1931).

Some efforts have been made to link instances of encephalitis lethargica, both historical and contemporary, with infection by a viral agent, as it was widely assumed that the influenza epidemic of 1918 was related to most of the cases of 
encephalitis presenting in neurological clinics at the time. As of this writing, no traces of influenza infection have been discovered in pathological specimens from patients who died of encephalitis lethargica in the 1920's through RT-PCR based testing, and its causative agent remains a mystery. The large number of patients that were brought to clinical attention with these symptoms did serve to push "Parkinsonism" into the consciousness of physicians of the time, and helped to solidify its standing as a clinicopathologic entity.

PD Therapy

A considerable period of time elapsed following the publication of Parkinson's essay before any effective therapy for PD was ascertained. Nevertheless, any rational discussion of PD as a modern clinical entity must be centered on the remarkable evolution of PD therapies over the last century. An examination of neurology textbooks of the late nineteenth century provides a pharmacologic laundry list of preparations administered to PD patients: opium, ergot, strychnine, datura, cannabis, hyoscyamine and belladonna among many others(Peterson 1890). It was around this time that anecdotal evidence began to accumulate suggesting some efficacy of belladonna extract in providing a minimal, yet consistent level of relief of symptoms. Following the large increase 
in clinic populations during the early twentieth century of patients suffering from postencephalitic parkinsonism, a large variety of compounds were again administered on an experimental basis, with the best results again obtained through the use of belladonna extract. Patients with postencephalitic parkinsonism seemed to enjoy a greater therapeutic response to belladonna than did those with classical PD, though the doses used were very high compared with past treatment regimens. The Bulgarian physician Raeff produced a wine extract of belladonna root in the early 1920's, which attained widespread popularity in the treatment of postencephalitic parkinsonism as the "Bulgarian treatment." Variations of this extract were marketed in Europe and America into the 1940's. As with earlier extracts of this type, central and peripheral anticholinergic side effects were quite common, particularly at high dosages.

The usage of natural belladonna extract as a treatment of choice for PD was eroded by the production in the 1950's of a series of manmade anticholinergic compounds derived from atropine. The first of these compounds to be employed in PD therapy was caramiphen in 1946; it is no longer produced. One of the most potent to be synthesized, benztropine, is still in limited clinical use for PD. The compound that gained the greatest clinical usage in the 1950's was the piperidine compound trihexyphenidyl. It is still in use as an adjuvant to L-dopa therapy. Some limited therapeutic effects were discovered for the common antihistamine diphenhydramine (Benadryl) and its analogous compounds, but these were never widely exploited for PD therapy. Early compounds based on phenothiazine, later the substrate for the development of 
the revolutionary tricyclic drugs such as chlorpromazine, also saw usage in PD. At least two of these, diethazine and ethopropazine, were marketed specifically as antiparkinsonian agents. Ethopropazine is still occasionally prescribed as an adjuvant to L-dopa therapy.

One of the major extrapyramidal side effects of the use of high dosages of chlorpromazine and the related tricyclic compounds was a form of parkinsonism. This observation fueled enthusiasm in the PD research community that elucidation of the biochemistry of the disease was a possibility (Merritt 1956). Following the initial investigations of the functions of acetylcholine in the CNS, Feldberg proposed in 1945 that the therapeutic value of scopolamine and atropine in PD was due to "central atropine-acetylcholine antagonism." Studies showed that the amount of antiacetylcholine activity of these compounds as measured in rodent systems correlated with their level of antiparkinsonian activity in humans. This notion was lent additional experimental support by the finding that physostigmine administration worsened parkinsonian symptoms, and could counteract the beneficial effects of benztropine in a dose-dependent manner. These experiments were key to a primary hypothesis of the biochemical nature of PD, namely that loss of activity of inhibitory dopaminergic inputs to the striatum resulted in a relative disinhibition of cholinergic neurotransmission.

The use of amantadine in PD therapy was initiated after a report (Schwab et al, 1969) detailing the improvement of symptoms in a patient initially given the compound as a prophylaxis against influenza. Amantadine was approved by the FDA for PD therapy in 1971. It has been shown to exert an indirect 
anticholinergic effect by reducing conductance of the neuronal acetylcholine receptor (Moresco et al, 2002).

The late 1950's saw a series of revolutionary developments in the pharmacological treatment of PD. Dopamine was first discovered in mammalian brain homogenates by Montagu among others in 1957 (Montagu 1957).

Carlsson, who confirmed the presence of dopamine in rodent brain homogenates in 1958, also demonstrated that administration of levodopa, a naturally occurring compound found in very high abundance in fava beans, was able to reverse the parkinsonian catalepsy induced by reserpine administration (Carlsson et al, 1958). In 1959 Bertler and Rosengren detailed the striatum-specific localization of dopamine in dogs, and Sano's group confirmed a similar localization in humans (Bertler and Rosengren, 1959; Sano et al, 1959). Ehringer and Hornykiewicz in 1960 were the first to describe a profound depletion in striatal dopamine in PD patients (Ehringer and Hornykiewicz, 1960). The stage was now set for the proposals set forth by Hornykiewicz and Carlsson to treat PD patients with levodopa, the blood-brain barrier permeant precursor to dopamine. In 1961, several patients at the Municipal Home for the Aged in Vienna were injected with 50 to $150 \mathrm{mg}$ of levodopa, and "abolition or substantial relief of akinesia" was the result (Birkmayer and Hornykiewicz 1961). This initial success was followed by five years of administration of different $D$ and L-dopa formulations, at dosages generally in the range of $50-300 \mathrm{mg}$, to PD patients worldwide. The heterogeneity of responses to these regimens considerably decreased the enthusiasm of most neurologists for dopa therapy, as evidenced by a 1965 
review in which Duvoisin wrote, "Despite enthusiastic claims of therapeutic benefit, no evidence has been presented that the DOPA effect is in any way specific or that it differs from the effect of other sympathomimetic amines" (Duvoisin 1965).

This pessimistic attitude was to change in relatively short order, with the publication detailing the results of administration of high oral doses (3-16 $\mathrm{g}$ daily) of racemic D,L-dopa to 16 patients (Cotzias et al, 1967). Eight patients experienced "complete, sustained disappearance or marked amelioration of their individual manifestations of Parkinsonism." Among the other patients, two exhibited improvement but not abolishment of motor symptoms. A gradual increase in the daily dose, $0.2 \mathrm{mg} /$ day as opposed to a larger increment of 0.5 $\mathrm{mg} / \mathrm{day}$, substantially reduced the nausea, vomiting and disorientation previously observed with high dose dopa administration. Over the next several years, multiple studies confirmed these results, with most reports describing $75-80 \%$ of patients as having significant improvement of PD symptoms. Despite the side effects that were found to occur, such as hyperkinetic dyskinesias, these new regimens represented a quantum leap in the management of PD. Levodopa remains the most widely used pharmacologic treatment of PD to the present day.

The other major class of pharmaceutical compounds used in treating PD is dopamine agonists. These compounds act through association with D1 or D2 dopamine receptors which are linked to activation or inhibition of adenylate cyclase, respectively, and opening of potassium channels in the case of D2 receptors. The first generation of dopamine agonists were derivatives of ergot 
(bromocriptine, lisuride and pergolide) and have been generally replaced by two synthetic agonists pramipexole and ropinirole.

With regards to initial therapy for a newly diagnosed case of PD, the primary choice to be made concerns use of a dopamine agonist versus L-dopa based therapy. Owing to the tendency of the L-dopa side effect profile to increase and therapeutic efficacy at a given dose to decrease with ongoing administration, with noticeable deterioration of response in two to five years, many practictioners seek to delay beginning this therapy while symptomatic relief can be obtained through the use of dopamine agonists, such as pramipexole and ropinirole. Several clinical trials randomly assigning patients to L-dopa or dopamine agonist therapy have demonstrated that the agonists produce significantly less incidence of dyskinesias, while L-dopa treated patients tend to have better overall motor function. This likely represents a therapeutic tradeoff, and the likely trend in the near-term for initial treatment of PD is likely to favor the dopamine agonists, especially for early-stage patients without significant motor symptoms.

There have been surgical attempts to address PD symptoms of rigidity and tremor through the years, ranging from wholesale extirpation of the motor cortex in the early twentieth century (producing paralysis, but definitively abolishing tremor), evolving into thalamotomies and pallidotomies, designed to interrupt the prokinetic cortex-striatum-pallidum-thalamus-cortex neuronal loop and generally are highly effective in abolishing severe medication-resistant tremor but are overall less effective at treating other PD symptoms. More 
recently, the use of deep brain stimulation techniques, in which a high-frequency electrical device is surgically implanted in either the globus pallidus or subthalamic nucleus, have produced favorable short term results (Olanow, 2002).

Another recent development in the PD therapeutic armamentarium has been the implanation of fetal neurons into the nigra, with the hope that environmental cues would induce the fetal cells to differentiate and act as replacements for dopamine neurons lost. In a recent report by Freed et al, forty patients were randomly assigned to fetal cell implantation or sham procedure groups, with the sham group being offered the fetal cells at the conclusion of the twelve month study period (Freed et al, 2001). The results of the trial were disappointing, with no significant differences reported in patient-assessed severity of symptoms between the experimental and control groups. Despite this lack of symptomatic improvement, they observed a $40 \%$ increase in fluorodopa uptake in the nigra of the fetal cell group as compared to a slight decrease in uptake in the control group, as assessed by positron emission tomography. Additionally, severe dystonias and dyskinesias were associated with a significant number of the transplanted patients (5/33). The very recently reported results of the other major US trial of fetal brain transplantation showed similarly negative outcomes in terms of improving Parkinson's symptoms (Olanow, et al, 2003). These results have made fetal cell implantation a less attractive therapeutic option for now, especially in light of the ethical concerns being raised regarding the use of cells of fetal origins in all venues of biomedical research. 
PD Pathology

Pathologically, PD is characterized by loss of the pigmented dopaminergic neurons of the substantia nigra (SN), and formation of Lewy bodies, eosinophilic cytoplasmic inclusions comprised of misfolded aggregates of alpha synuclein, neurofilaments, tubulin and ubiquitin. Lewy bodies are usually most prominent in the substantia nigra pars compacta and locus coeruleus of PD patients. The loss of neurons in the $\mathrm{SN}$ follows a characteristic pattern, with most loss occurring laterally in the ventral part of the SN pars compacta (Hirsch 1988). The loss of dopaminergic neurons results in a depletion of dopamine within the basal ganglia (Ehringer and Hornykiewicz, 1960), and it is this depletion that is responsible for production of the motor symptoms of the disease (Lee et al, 1994).

Neurodegeneration occurring in locations outside the SN, including the ventral tegmental area, locus coeruleus and the basal nucleus of Meynert are thought to contribute to the cognitive Parkinsonian symptoms, such as dementia, that occur in around 30 percent of PD cases (Agid et al, 1990; Greenfield and Bosanquet, 1953; Candy et al, 1983; Aarsland et al, 1996). PD symptoms do not manifest themselves clinically until loss of 60 to 80 percent of $\mathrm{SN}$ dopaminergic neurons has occurred, generating widespread acceptance for a long presymptomatic period during which neuronal death is occurring but gross motor symptoms are not manifested. During this preclinical phase of the disease process, compensatory mechanisms such as increased expression/sensitivity of 
dopamine receptors on remaining neurons are thought to prevent gross motor deficits (Agid et al, 1990). It is this preclinical phase and the compensatory capacity of the basal ganglia that it implies that causes some investigators to believe that a PD "therapeutic window" exists, during which it would be possible to slow or stop the neuronal loss and thus prevent most or all of the debilitation associated with the disease.

A major issue that frequently complicates discussions of PD nomenclature is the teleological distinction that must be made between PD and parkinsonism, which refers to the individual cardinal motor symptoms of the disease. Classic idiopathic PD, referring to the cardinal symptoms in the presence of autopsyverified pathology of the substantia nigra with the presence of Lewy bodies, is distinct from the symptoms of parkinsonism, which can be individually produced by known insults to specific areas of the CNS. Parkinsonism can result from exposure to infectious agents (as in the epidemics of encephalitis lethargica mentioned above), environmental toxins (MPTP, manganese, $\mathrm{CO}$, methanol, cyanide compounds), medications (neuroleptics, reserpine, lithium), organic disturbances of the CNS (stroke, trauma, increased intracranial pressure, tumor, subdural hematoma), genetic syndromes (Hallervorden—Spatz disease, Pick's disease, Wilson's disease, Huntington's disease) and even metabolic disturbances (hypoparathyroidism, hepatic coma) (Di Monte et al, 2002; Poewe and Wenning, 2002). 
Genetic and other risk factors

Although the characteristic neuropathology of PD has been well known for decades, intensive research efforts have yet to elucidate the cause of death of SN dopaminergic neurons in PD. It has long been suspected that genetic predispositions to the disease may play a role, and investigations into nuclear and mitochondrial DNA mutations are ongoing. The most comprehensive survey of genetic contribution to idiopathic PD to date was carried out by Tanner et al, and comprised an analysis of members of the National Academy of Sciences/National Research Council World War II Veteran Twins Registry (Tanner et al, 1999). Out of 19,482 white male twins screened, 268 suspected cases of PD were identified. A total of 161 twin pairs in which 1 or 2 twins had PD were found. The overall concordance rate was similar for monozygotic versus dizygotic twins (15.5\% and $11.1 \%$ respectively) in cases of PD diagnosed after 50 years of age. In cases diagnosed prior to 50 , all examined monozygotic twin pairs (4 of them) were concordant for PD, versus 2 of 12 dizygotic twin pairs. These results were interpreted by the authors to suggest that genetics do not play a major role in PD with a typical age of onset, but may play a significant role in PD cases with a younger age of onset. Conversely, a study by Sveinbjörnsdottir and colleagues using an extensive Icelandic genealogic database found a possible effect for typical age of onset PD (Sveinbjörnsdottir et al, 1999). Briefly, those persons determined to have PD had a higher degree of interrelatedness than those unaffected. One must consider, however, that the 
Icelandic population has a fairly low degree of genetic diversity overall, which may bias the results somewhat.

Mutations in genes such as Parkin, an E3 ubiqutin ligase, have been characterized and found to play a significant role in juvenile-onset forms of the disease, but such rare genotypes do not account for the vast majority of PD, the non-familial or sporadic forms of the disease. Lucking and colleagues screened 73 families with PD with atypical age of onset for mutations in the parkin gene. Parkin mutations were found in $49 \%$ of the families screened. The individual mutations varied widely with 19 homozygous and heterozygous exon rearrangements being found, as well as 16 different point mutations (Lucking et al, 2000). This study suggests that parkin mutations may play a prominent role in PD with early age of onset. In 1997, a study demonstrated linkage with the $\alpha-$ synuclein gene, located on chromosome 4q21-23, in an Italian-Greek family with early onset PD (Polymeropoulos et al, 1997). Despite this, a-synuclein mutations have not been found to be common in early onset PD, and have not been documented in any case of idiopathic PD. It is possible that certain alleles of these genes in combination with others may contribute to idiopathic PD by enhancing vulnerability to environmental or toxic factors.

There exists a considerable body of literature regarding the effect that lifestyle and environment may play in the development of PD. Historically, increased risks of developing PD have been associated with pesticide exposure, rural versus urban residence, consumption of well water and not smoking tobacco products. One recent study in humans described the reduction in risk of 
PD in over eight thousand Japanese-American males associated with increased caffeine consumption (Ross et al, 2000). The relative risk of developing PD for those that did not consume coffee was 2.2 times that of the coffee drinkers, with an increase of 5 times for nonconsumers verus those that consumed seven or more cups of coffee (or equivalent amounts of caffeine) per day.

Other hypotheses proposed for the etiology of PD include an acceleration of the normal processes of aging of CNS neurons, exposure to endogenous or exogenous toxins (the environmental hypothesis), and alterations of protein handling leading to cytotoxic misaggregation. An intriguing alternative school of thought holds that PD is rather multifactorial, with genetic predispositions to neuronal death combining with circumstances of environment or lifestyle to produce the neuropathology and symptoms of the disease. As the extreme heterogeneity of the disease has been extensively illustrated, and studies attempting to evaluate concordance of PD in monozygotic twins have not proven conclusive, we may begin to articulate the idea of PD as a range within the spectrum of neurodegenerative disease, a pathology that represents a common neuronal response to many different circumstances. If we wish to move forward in our investigations under the assumption that there is likely to be no single cause for the majority of cases of PD, we must modify our experimental strategies accordingly. Just as the therapy of AIDS has been revolutionized by the usage of "cocktails" of multiple drugs to prevent HIV replication, greater success than has heretofore been attained in neuroprotective trials for PD drugs may be obtained by using them in combination rather than singularly. In the 
same spirit, molecular investigations of the causes of the selective cell death in the substantia nigra should incorporate the testing of the contributions multiple gene products in parallel rather than singular genes or proteins in isolation. While this mode of thinking may at face value seem to flout the traditional imperative of reductionism in modern biology, this is not the case; the objective of any such multifactorial investigation, regardless of the number of molecular entities involved or the volume of data generated, must be the elucidation of the common pathways and cascades that can synthesize such diverse biological inputs into the final output of selective dopaminergic neuronal loss. A starting point for our investigations has been a common paradigm of many of the leading hypotheses of the etiology of PD: oxidative stress.

\section{Oxidative Stress in $P D$}

Oxidative stress is defined as an overabundance of reactive oxygen species (ROS) relative to the cell's antioxidant capabilities, leading to ROS attack on proteins, lipids and nucleic acids. Multiple sources of intracellular ROS have been described, the most important of which by far are the mitochondria. Mitochondria are well known to every student of biology as the primary source of ATP in eukaryotic cells through the action of the electron transport chain (ETC), and account for 85 to 90 percent of the oxygen used by a typical cell under physiological conditions (Chance et al, 1979). Less well articulated in many 
courses of study is the concomitant production of ROS as a byproduct of this oxygen use. While mitochondria have been shown to have antioxidant mechanisms in place to deal with some of their own ROS production (and that the presence of these mechanisms are essential to cell survival; Melov et al, 1998), it has also been demonstrated that a typical mitochondrion produces ROS at a rate that exceeds the ameliorative capacity of these enzymes. This results in the incomplete usage of 1 to 3 percent of oxygen consumed by the cell (Boveris and Chance, 1973).

These altered stochiometries of electron flow between the protein complexes of the mitochondrial electron transport chain are thought to be responsible for generation of such compounds as hydrogen peroxide, superoxide radicals and hydroxyl radicals. Although the former two species are not extremely deleterious to the cell, with specialized enzyme defenses designed to catalyze their degradation, they are capable of reacting with other elements commonly found within the cell to form significantly more dangerous compounds.

A body of experimental evidence documenting the increased appearance of markers of oxidative damage in PD postmortem brain samples as compared to controls has accumulated over the last 10-15 years. Most of these studies have focused on immunohistochemical methods of detection of ROS-mediated damage to cellular lipids, proteins and/or nucleic acids. Alam et al have described increased levels of protein carbonyl groups (a marker for ROS attack on histidine, arginine, lysine and proline residues) in $\mathrm{SN}$, caudate and putamen of PD postmortem brains compared to controls (Alam et al, 1997). Good et al have 
reported the presence of nitrotyrosine residues, produced by peroxynitrite radical attacks upon tyrosine amino acid residues, within the cores of Lewy bodies in PD brain samples (Good et al, 1998). This indicates that peroxynitrite acts within the vulnerable/dying neruons selectively affected by PD. Increased levels of 8hydroxy-2'-deoxyguanosine (8-OHdG), a product of nucleic acid oxidation, have been found in SN of PD patients (Alam et al, 1997; Zhang et al, 1999).

Additionally, Kikuchi et al found increased levels of 8-hydroxy-2'-deoxyguanosine (8-OHdG) and 8-hydroxyguanosine (8-OHG), products of reactive oxidization of nucleic acids in serum and cerebrospinal fluid of PD patients compared to controls (Kikuchi et al, 2002), indicating that this finding was not an exclusively postmortem phenomenon. This finding was recently independently confirmed (Abe et al, 2003). The levels of polyunsaturated fatty acids, the substrate for ROS peroxidation of cellular lipids, are decreased in several brain regions of PD postmortem brains relative to controls; the same study confirmed that levels of malondialdehyde, an intermediate compound in lipid peroxidation, are increased in the same PD postmortem brains (Dexter et al, 1989). Taken together, this likely indicates that lipid peroxidation by ROS proceeds at an elevated rate in PD. Additionally, Yoritaka et al have documented the greatly increased presence of 4hydroxy-nonenal (HNE) protein adducts in PD nigral neurons compared to controls. HNE is an unsaturated aldehyde species that is one of the major products of membrane lipid peroxidation and is reported to inhibit nucleic acid and protein synthesis and interfere with heat shock protein and other enzyme induction (Yoritaka et al, 1996). 
Ubisemiquinone produced by the normal processes of the ETC is the primary electron donor responsible for the generation of superoxide radicals in mitochondria (Turrens et al, 1985). The superoxide radical attacks enzymes that contain iron sulfur centers, such as aconitase, succinate dehydrogenase and (in the mitochondria) NADH-ubiquinone oxidoreductase (Pryor, 1986; Gardner et al, 1995). Due to the threat posed to operation of the ETC by its presence, mitochondrial superoxide is rapidly converted to hydrogen peroxide by the action of mitochondrial manganese superoxide dismutase (SOD2). Two other superoxide dismutase enzymes are present in mammalian cells, SOD1, a copper-zince superoxide dismutase present in the cytosol, and SOD3, another copper-zinc dismutase that is expressed extracellularly (Turrens et al, 1985). In SOD2 knockout mice, mitochondrial superoxide radical is not converted into hydrogen peroxide and causes extensive damage to complex I of the ETC, $\mathrm{NADH}$-ubiquinone oxidoreductase, succinate dehydrogenase and aconitase, all of which have iron-sulfur moieties. This ETC damage leads to a reduction in ATP production and increased lactic acid production. The mice develop lactic acidosis, cardiomyopathy and degeneration of the basal ganglia, closely mimicking symptoms seen in humans with inherited ETC mutations. Mice that lack the copper-zinc superoxide dismutases are not affected at birth, but develop axonal neurodegeneration later in life (Li et al, 1995; Lebovitz et al, 1996).

While hydrogen peroxide, chemically, is not a true oxygen radical (it lacks unpaired electrons), it is significant in the ROS picture due to its ability to be enzymatically transformed into many different derivatives, and its ability to diffuse 
across several cell radii. Hydrogen peroxide in combination with ferrous iron or other metals can create hydroxyl radicals by the Fenton reaction (Raha et al, 2000). Hydroxyl radicals are extremely reactive, with a very short half life. They cause peroxidation of lipids, proteins and nucleic acids. The primary means of limiting hydroxyl radical toxicity is to limit the production of hydrogen peroxide and the availability of free transition metals (i.e., substrates for the Fenton reaction). As the production of hydroxyl radicals is highly hazardous to the cell, most hydrogen peroxide generated in the mitochondria is processed by glutathione peroxidase (GPX) into water (Beyer et al, 1991).

The other major ROS produced in the mitochondria is peroxynitrite. It is formed by the reaction of superoxide radical with nitric oxide (NO). Recent evidence demonstrates that mitochondria possess their own nitric oxide synthase (mtNOS), a sub-isoform of neuronal nitric oxide synthase. At physiological levels, NO inhibits the opening of the mitochondrial permeability transition pore (MTP), while higher levels promote its opening (Brookes et al, 2000). NO is also capable of influencing the ETC by reversible inhibition of cytochrome c oxidase. It is a highly diffusible compound that freely crosses lipid membranes, therefore if a cell produces abnormally high levels of $\mathrm{NO}$ it is theoretically capable of inhibiting respiration within neighboring cells. This property may explain varying degrees of damage to neuronal ETCs that develops when astrocytes in combined neuron-astrocyte cultures produce excess NO (Bolanos et al, 1995). In the astrocytes, glutathione (GSH) is preferentially depleted (Ju et al, 2000). 
Peroxynitrite radical, while not as damaging as hydroxy radical, is nevertheless highly reactive and dangerous. It modifies proteins by nitrosylation of tyrosine residues and by oxidizing tryptophan and cysteine residues (Ischiropoulos and al-Mehdi, 1995). Within mitochondria, it is particularly damaging to complexes I, II, IV and V of the ETC, as well as aconitase, SOD2 and creatine kinase. It can also react with mitochondrial lipid membranes and mitochondrial DNA, which has no protective histone scaffolding (as nuclear DNA does) to shield it from such attack (Brown and Borutaite, 1999). Damage to these molecules can induce mitochondrial swelling, calcium release and opening of the MTP. Mitochondrial calcium uptake has been shown to induce mtNOS, leading to a concomitant increase in peroxynitrite radical and release of calcium; this is hypothesized to consitute a feedback loop preventing excessive accumulation of mitochondrial calcium. These studies demonstrate that excessive production of ROS by mitochondria can cause extensive damage to the brain among other organs.

It is currently a matter of active debate as to whether manifestations of oxidative stress represent a causative factor for dopaminergic cell death or simply a byproduct of such neuronal loss. This distinction is critical; the merits of continued study of this question are clear, as even if induction of ROS is a downstream event from initial dopaminergic cell losses, it may promote a reactive environment that weakens the remaining neurons and contribute to their eventual demise. 
It has been suggested that $\mathrm{SN}$ dopaminergic neurons exhibit a set of predisposing factors that make them particularly vulnerable to increases in ROS. While the normal catabolism of dopamine itself through the action of monoamine oxidase leads to hydrogen peroxide formation, nonenzymatic breakdown of dopamine within the neuron produces neuromelanin pigment, which can in turn promote the production of hydroxyl radicals when exposed to free iron (Fahn and Cohen, 1992; Jellinger et al, 1992). Hirsch, et al. showed that indeed, the SN neurons most susceptible to neurodegeneration were those with relatively higher levels of neuromelanin (Hirsch et al, 1988). Some researchers have inferred that the subcellular localization of the neuromelanin within the phagolysosomic membrane compartments represent an attempt by the cell to protect itself from its own neurotransmitter metabolism, with the lipofuscin serving as a "sponge" for excess oxygen radicals or other endogenous toxins (Brunk and Terman, 2002).

Another primary factor underlying neuronal viability to ROS is the relative scarcity of antioxidant enzymes (AOE) in the brain relative to other tissues. Potential relevance to PD was described when a study reported reduction in the reduced form of glutathione in the SN of PD postmortem brain samples (Perry et al, 1982), later independently confirmed (Sofic et al, 1992; Pearce et al, 1997). The reduction of glutathione levels is apparently most prominent in glial cells, perhaps reflective of transport of the glutathione from glia to the more highly "threatened" dopaminergic neurons. This notion is supported by the finding of an increase in glial gamma-glutamyl transpeptidase, an enzyme involved in the production of active glutathione. Additonally, a global decrease in glutathione 
levels as opposed to a selective depletion of the reduced (GSH) form, would be expected to manifest itself in an unchanged reduced:nonreduced glutathione ratio; in SN of PD patients, GSH is reduced without significant changes in the nonreduced form, implying the presence of increased oxidative stress (Kish et al, 1985). These drops in GSH are often accompanied by reductions in catalase and GSH peroxidase expression (Ambani et al, 1975; Kish et al, 1985), which further predispose the cells to oxidative damage.

Another potential source for ROS in the SN comes from the activation of microglial cells. In response to increased levels of ROS in the extracellular milieu, these cells are induced to produce cytokines and nitric oxide. Expression of the inducible nitric oxide synthase (iNOS) has been found to be upregulated in PD brain generally (Hunot et al, 1996). This in turn leads to impairment of ETC and enhanced generation of peroxynitrite species by the methods previously described.

Increased levels of iron have been reported in SN pars compacta of PD patients and in neuromelanin-containing vesicles of dopamine neurons (Dexter et al, 1989; Hirsch et al, 1991). Although the cause of the increased levels are unclear, a decrease in cellular ferritin and/or an increase in lactoferrin receptor expression may be partly to blame (Riederer et al, 1989; Dexter et al, 1990; Leveugle et al, 1996). This increase in iron levels could theoretically contribute to increased ROS in these neurons, via increased availability of substrates for combination with hydrogen peroxide in Fenton reactions. 


\section{Parkinsonian neurotoxins}

Perhaps no event revolutionized the oxidative stress hypothesis of Parkinson's disease more than the discovery of 1-methyl-4-phenyl-1, 2, 3, 6tetrahydropyridine (MPTP). MPTP is a widely used chemical feedstock that was accidentally produced as a byproduct in the synthesis of an analogue of meperidine (Demerol). The MPTP-contaminated drug was sold illicitly in northern California in the early 1980's. The drug abusers that injected this compound, generally in their early 20 's, began to exhibit acute and chronic symptoms typical of long standing PD in an aged patient. The patients that came to medical attention generally reported burning at the injection site, followed by a metallic taste in the mouth, jerky motions of the extremities and a variety of sensory abnormalities including "dreamy or spacey feelings," hallucinations and loss of vision. These symptoms generally disappeared within one to two hours following injection, depending on the dose administered. Those that used the compound repeatedly developed a series of chronic symptoms, including stiffness of the muscles, slowness of movements (bradykinesia) and resting tremor. Physical examination of the subjects revealed "virtually all of the typical features of PD" (Langston et al, 1983). Although the subjects did not suffer from the dementia that often accompanies advanced PD, Langston et al did note minor abnormalities of mental functioning consistent with nondemented patients with PD of long standing. The similarities of the group to idiopathic PD patients 
extended to their response to therapy: the motor symptoms they experienced could be alleviated with L-dopa therapy, with the accompanying "on-off" effects and dyskinesias seen in PD patients nearing the end of the period of effective Ldopa therapy. As these patients were relatively young and otherwise healthy, the pathologic lesions responsible for their symptoms were not confirmed by autopsy. Tragically, this data did become available when a student at the University of Maryland began to synthesize and self-administer an opiate analog. He also developed parkinsonian symptoms, following a failed synthesis (Davis et al, 1979). This troubled person eventually committed suicide, and upon autopsy substantial loss of cells in the substantia nigra was observed, virtually identical to the cellular pathology observed in advanced PD.

One of the most fundamental differences between MPTP-induced Parkinsonism and idiopathic PD is that the former is an acute syndrome, manifesting within hours-days-weeks, whereas the latter is a chronic process that requires decades to produce the characteristic clinical syndrome. Despite this major difference, MPTP remains one of the best-studied animal models of PD.

The presence of the lipophilic phenyl group aids MPTP in crossing the blood-brain barrier. Once inside the CNS, MPTP is taken up by glial cells, where formation of the toxic metabolite methylpyridinium ion (MPP+) is catalyzed by the enzyme monoamine oxidase B (Heikkila et al, 1984). MPP+ is then selectively accumulated by $\mathrm{SN}$ dopaminergic neurons by virtue of its affinity for the dopamine transporter (Javitch et al, 1985). Once inside the neuron, MPP+ is accumulated by the mitochondria, where it potently inhibits complex I of the 
electron transport chain (Hasegawa et al, 1990). It is hypothesized that alteration of the stochiometry of electron transport through complex I interference is responsible for an increase in ROS formation by the neuron in question, leading to oxidative intracellular damage eventually culminating in the observed cell death. Przedborkski and colleagues reported that transgenic mice engineered to overexpress the superoxide dismutase SOD1 were more resistant to MPTP intoxication than wild-type mice, bolstering the notion of MPTP's induction of ROS (Przedborski et al, 1992). As noted, SOD1 acts primarily to catalyze the dismutation of the superoxide anion into hydrogen peroxide. As the superoxide anion is not highly toxic in vivo, attention must be paid to a highly toxic product of superoxide's reaction with nitric oxide: peroxynitrite. This idea has been lent support primarily by the group of Flint Beal, who have noted severally that specific inhibitors of the neuronal nitric oxide synthase (nNOS) are protective against MPTP intoxication and nigral cell death in rodents, and that knockout mice lacking nNOS are also protected against MPTP's effects relative to wildtype mice (Grunewald and Beal, 1999; Matthews et al, 1997; Przedborski et al, 1996; Schulz et al, 1995). A reactive gliosis of the substantia nigra and striatum is a prominent feature of MPTP intoxication in mice (Dehmer et al, 2000), as was noted by Langston in humans(Langston et al, 1999). Apart from this proliferation, the glial cells in the vicinity begin to express inducible nitric oxid synthase (iNOS). Similar to the situation with nNOS, neurons die at a much reduced rate in the substantia nigra of iNOS knockout mice, but dopaminergic fibers are observed to atrophy and dopamine stores are depleted (Dehmer et al, 
2000; Itzhak et al, 1999; Liberatore et al, 1999). NOS inhibitors have been observed to block the neurotoxic effects of MPTP in nonhuman primate models (Hantraye et al, 1996). Finally, cellular markers of the deleterious effects of NObased reactions, such as nitrosylation of proteins, as well as iNOS upregulation, have been observed in the substantia nigra of PD patients (Good et al, 1998).

When taken together, this evidence argues strongly in favor of the involvement of NO in the neurotoxic effects of MPTP and its derivatives, and for this reason we decided to study the effects of nitric oxide modulation in our cellular model of MPP+ intoxication with studies involving application of the NO-scavenging agent PTIO.

Cell Death in PD

A controversial area of PD research deals with the manner in which neurons of the substantia nigra die. The paradigm of apoptosis, or programmed cell death, has dominated most of the research into this area of PD. Mochizuki reported TUNEL staining (indicative of DNA fragmentation) of nuclei in PD patients in 1996(Mochizuki et al, 1996). Ultrastructural analyses by Anglade et al reported typical apoptotic membrane blebbing and nuclear condensation in substantia nigra, with portions of pigment-containing neurons endocytosed by surrounding glial cells (Anglade et al, 1997). The most widely studied forms of apoptotic cell death involve the activation of a complex intracellular signaling cascade culminating in the activation of caspases, or cysteine-aspartate-serine 
proteases. Activation of caspase 3, one of the principal downstream effectors of the process, has been reported by Oo et al in isolated dopamine neurons of the substantia nigra, and by Hartmann et al in PD dopaminergic neurons(Oo et al, 2002; Hartmann et al, 2000). The tumor necrosis factor cell death cascade culminates in the activation of caspase 8 , which was also reported by Hartmann to occur to a greater extent in dopaminergic neurons from PD patients than in those of control subjects.

Evidence from several cell culture models, including SH-SY5Y neuroblastomas, indicates that dopamine itself is capable of inducing apoptosis (Ziv et al, 1994; Massenaro et al, 1996; Gabby et al, 1996; Junn and Mouradian, 2001). This effect is counteracted by overexpression of the antiapoptotic protein Bcl-2(Offen et al, 1997; Ziv et al, 1997). As the breakdown of dopamine has been noted to produce excess ROS burdens in the cell, it is presumed that this is contributory to the apoptotic death seen in these models. Studies by Barzilai et al detail the involvement of collapsin, a protein involved in axonal guidance, and TCP-1, a heat shock protein family member, in dopamine-mediated apoptosis in sympathetic neurons (Barzilai et al, 2000). The use of antibodies against collapsin and of antisense DNA for the TCP isoform delta have attenuated apoptotic death in these neurons. Dopamine is also capable of apoptotic induction in SY5Y cells, by the criteria of caspase 3 and 9 activation and nuclear condensation (Junn and Mouradian 2001). P38 MAP kinase activation and mitochondrial cytochrome $\mathrm{c}$ release were found to mediate this process in SY5Ys. MPP+ treatment has been demonstrated by Hartmann and others to 
cause apoptosis in dopaminergic PC12 cells and in primary cultures of midbrain neurons (Hartmann et al, 2000; Viswanath et al, 2001). Previous work in our laboratory has demonstrated $\mathrm{MPP}^{+}$induced apoptosis in SY5Y cells (Fall and Bennett, 1999). This study further demonstrated that this apoptosis was directly related to excess production of reactive oxygen species through an inhibition of complex I of the mitochondrial electron transport chain; Rho ${ }^{0}$ SY5Y cells, which lack mitochondrial DNA and do not carry out oxidative phosphorylation, do not undergo apoptosis when exposed to MPP ${ }^{+}$. Work by Cassarino et al carried out at the University of Virginia Center for the Study of Neurodegenerative Diseases (CSND) has further demonstrated that such $\mathrm{MPP}^{+}$treatment leads to activation (through phosphorylation) of JNK kinase, JNK and c-jun, which are in turn responsible for the activation of the caspases that carry out apoptosis (Cassarino et al, 2000). Concurrent work by Halvorsen (Halvorsen et al, 2002) detailed the activation of nuclear factor kappa-beta and protein kinase B (Akt) early in the time course of exposure of SY5Y cells to MPP ${ }^{+}$. The signaling cascades controlled by these molecules are generally considered to be anti-apoptotic; these results indicated that the response to $\mathrm{MPP}^{+}$in SY5Y cells may involve factors outside the realm of electron transport inhibition, and served to fuel my interest in determining what other signaling cascades might be operative in this situation.

Previous data have demonstrated that expression of $p 53$, crucial to the apoptotic process, is upregulated in the striatum and midbrain of PD patients (Alves da Costa et al, 2002). Previous work in the CSND (Halvorsen et al, 2002; 
Dennis and Bennett, 2003) has described the concurrent activation of both proapoptotic and anti-apoptotic signaling pathways in cellular models of PD.

Alternatively, work by Jellinger (Jellinger and Stadelmann, 2000) and Banati (Banati et al, 1998) failed to note any significant morphological signs of apoptosis in PD brain compared to control. Some of the heterogeneity of results obtained by these studies must be due to variations in the techniques and standards used to define apoptotic versus nonapoptotic cells; the preponderance of biochemically based studies measuring pro and anti-apoptotic protein and mRNA levels in cell and animal models and in PD brain samples seems to favor the notion of apoptotic cell death as occurring in PD. This has served to generate interest in determining whether the rate of dopaminergic cell death can be reduced through administration of anti-apoptotic compounds. It is uncertain whether inhibition of the apoptotic cascades in nigral cells would be sufficient to prevent their death. Encouraging data on the apotosis-preventive properties of the compound CGP3466B, thought to act via inhibition of expression of the proapoptotic protein $\mathrm{Bcl}-2$, has been obtained by different groups (Andringa et al, 2000; Waldmeier et al, 2000). 


\section{Technical approaches}

A major technological advance that has greatly increased our ability to rapidly examine many different gene expression responses simultaneously is the cDNA microarray. In its most basic form, the array consists of an ordered grouping of DNA that is adsorbed to a non-permeant surface material. When these fixed DNAs are exposed to homologous DNAs in solution, hybridization between the DNA species occurs. Generally, the DNAs that are washed over the array are labeled to allow detection by fluorescence, excited particle emission, etc. With this experimental paradigm, it is possible to examine the relative levels of particular DNA molecules within a sample in a highly parallel manner (limited by the number of genes in the fixed array). The first type of cDNA microarray to become commercially available involved 300 to 400 base pair cDNAs, selected for lack of secondary structure and uniqueness of primary sequence, covalently bonded to a nylon membrane. Following isolation of total RNA or mRNA from an experimental sample, a pool of cDNAs representative of the sample would be generated by a reverse transcription reaction using the mRNA as substrate.

Generally, a radioactive nucleotide, usually ${ }^{32} \mathrm{P}$ or ${ }^{33} \mathrm{P}$, would be incorporated into these so-called "first strand" cDNAs. These radioactive probe cDNAs would then be applied in solution to the nylon membrane bearing the target cDNAs, and hybridization at controlled temperature would occur. Following a series of washes, hybridization of the probe to target would be visualized by a 
standardized method, most often storage phosphor imaging but sometimes x-ray film exposure. This method had the advantage of being highly replicable and straightforward to learn due to its commonalities with Southern and northern blot techniques. However, it suffers from a relatively small dynamic range and from the difficulties associated with the usage of radioactive materials. Its use has been largely supplanted by arrays spotted onto glass microscope slides, with fluorescent based detection methods replacing radioactivity.

One of the first methods for producing DNA arrays on glass slides was developed by Affymetrix Corporation, as a variation of the laser photolithography methods used to produce computer chips. In brief, synthetic linker molecules with photolabile attachment sites are attached covalently to the glass slide, then a mask is placed over the slide prior to exposure to laser light. This treatment makes the attachment sites so exposed available for reaction with bi-functional deoxynucleotides, thus beginning the process of building an oligonucleotide chain at a series of discrete spots on the array. The process of masking, laser exposure and addition of deoxynucleotides is repeated cyclically until an oligonucleotide of the desired length and sequence has been constructed. The most common physical standards for Affymetrix arrays are $1.28 \times 1.28 \mathrm{~cm}$, containing approximately $5 \times 10^{5}$ oligonucleotides, 25 base pairs in length. Approximately 15 oligonucleotides are constructed to study a given gene product, with oligonucleotide sequences overlapping one another within the target gene. Oligonucleotides are synthesized with $100 \%$ homology to the target sequence (perfect matches) or with single base pair substitutions (mismatches). 
Following hybridization with fluorescently labeled probes, the signals observed in perfect match and mismatch oligonucleotides are compared; the signal from the mismatch oligos is intended to serve as a control for background noise and/or crosshybridization from other probes. This array technology has several significant advantages, including the ability to examine in excess of 30,000 genes on a single array, a (relatively) long period of use by the research community and a strong manufacturing base. However, they also have negative aspects; studies by Kuo and colleagues at the National Cancer Institute compared signals obtained from identical RNA samples derived from various cancer cell lines on cDNA type and Affymetrix type arrays, with very poor correlational results (Kuo et al, 2002). Additionally, the arrays are extremely costly, and this factor coupled with the difficulties in data analysis and comparison that are brought about by use of the perfect match/mismatch system has caused our laboratory to consider them as less than ideal for our intended work.

Our laboratory's interest in microarray technology has arisen from a continuum of work that has taken place over the last five to seven years, in which we have investigated the contributions of mitochondria, cell signaling pathways, and gene expression responses to apoptotic death in a cellular model of Parkinson's disease: SY5Y neuroblastoma cells exposed to the mitochondrial neurotoxin MPP+. The recent availability of high quality microarrays at a reasonable cost has made the investigation of the responses of thousands of genes to experimental perturbations in this system scientifically and economically feasible; we hope that the results that we obtain from such "gene discovery" 
studies will provide us with the initial building blocks to construct an integrated theory for the dependence of cell death/survival on gene expression in such models, and in so doing, to begin to add quantitative methods to the validation of such models as they apply to Parkinson's disease in vivo.

Recent developments in gene array analysis of Parkinson's disease

Several groups have published results of cDNA microarray analyses of animal models of PD. Moussa Youdim's group at the Technion in Haifa, Israel administered MPTP to mice chronically and found significant up or downregulation of over 50 genes related to oxidative stress, inflammation, nitric oxide and glutamate metabolism, and neurotrophic factors in extracts from midbrain (Mandel et al, 2002). Most of these gene expression changes were prevented by administration of the D1-D2 dopamine receptor antagonist Rapomorphine, which also has ROS-scavenging properities. Administration of the enantiomer, L-apomorphine, which shares the ROS scavenging properties of the R-enantiomer but lacks its activity at dopamine receptors, had a virtually identical effect. This study demonstrated that $\mathrm{MPP}^{+}$administration can produce gene expression changes in a rodent model, and more importantly, that some portion of these gene expression changes are dependent on the status of ROS within the cell. 
Brown and coworkers employed a new data analysis technique, that they call voxelation, in their study of whole brain gene expression in mice chronically administered methamphetamine. Briefly, ten coronal mouse brain slices were divided into four quadrants each, giving a total of 40 volume elements (voxels). 55 genes with highly correlated temporal expression patterns were differentially expressed between methamphetamine-treated and control mice, and the expression of each gene was determined for each voxel, providing spatial expression information (Brown et al, 2002). These genes had mainly neuronal morphology, intracellular signaling and apoptosis-related functions associated with them. This study was the first to suggest that a portion of the neuronal response to a parkinsonian neurotoxic insult might involve changes in morphology and cell-cell contact status.

Napolitano and colleagues recently published data concerning the gene expression response changes observed in the striatum of rats following nigral injection of the highly neurotoxic dopamine adduct 6-hydroxydopamine (6-OHDA) over a two month period (Napolitano et al, 2002). Significant alterations of expression of genes involved in transcriptional regulation and cell cycle regulation were observed. Specifically, the activity of the dopamine-PKA-Cdk5 cascade was severely downregulated, resulting in dephosphorylation of the DARPP-32 phosphoprotein that is thought to be a primary mediator of dopamine signaling. Additionally, genes involved in glutamate transmission of striatal neurons were significantly affected. This study, owing to its extended period of 6-OHDA administration, was the first to address what gene expression changes 
might be involved in alterations of nigral neurotransmission in response to a parkinsonian neurotoxic insult.

Yoo and colleagues provoked oxidative stress in the dopaminergic neural cell line SN4741 by exposure to hydrogen peroxide or MPP+. 36 genes were identified that showed significant changes in response to the oxidative stimulus, including nuclear components of complex I of the mitochondrial electron transport chain, genes involved in membrane trafficking, oxidative stress markers and oxidoreductases (Yoo et al, 2003). Specifically, components B8 and B17 of mitochondrial complex I were downregulated in response to oxidative stress, while syntaxin 8 and heme-oxygenase 1 were upregulated. Heme oxygenase 1 has been previously demonstrated to be a major constituent of Lewy bodies, one of the pathognomic findings associated with idiopathic PD. In addition to performing their studies in a cell model of PD, this study was significant in that several of the genes showed changes in their temporal regulation over the period of following induction of oxidative stress (4-16 hours).

While a considerable corpus of experimental evidence for oxidative damage in the postmortem brains of PD patients exists, the relationship between this pathology, mitochondrial and other sources of oxidative stress and the neurodegeneration leading to the sporadic PD phenotype is highly complex and as yet incompletely understood. These relationships are nevertheless important and highly worthy of exhaustive investigation, as interventions against the processes that lead to PD may also have a place in the therapy of other common neurodegenerative conditions (Alzheimer's disease, amyotrophic lateral 
sclerosis, etc.). Primary importance should be placed on the development of cell and animal models that more closely approximate sporadic PD than those currently available, and detailed investigation of genomic, transcriptomic, proteomic and intracellular signaling changes that are inherent to the pathology and symptomatology. Despite the recent completion of the Human Genome Project, fundamental gaps in our knowledge remain regarding the nature of the regulation of gene expression, sufficient to make a comprehensive evaluation of all expression events in a given cell or tissue impractical at this time.

I would like for us then to consider the experiments that I detail in the following chapters to be initial steps toward a deeper understanding of PD, moving beyond the clinicopathologic definitions of the disease to elucidation of the molecular mechanisms that are responsible for the death of dopaminergic neurons, the sine qua non of the disorder. These experiments could not and should not be construed as the end point of such endeavors, but hopefully as a guideline for future experiments that may examine the roles of many more gene products and their modulation in the context of parkinsonian insult, with our overarching objective as always being the discovery of new targets for therapeutic intervention in the disease process. The results that we have obtained represent a novel paradigm in the multiplex analysis of gene function in a widely used cellular model of Parkinson's disease. 
RNA Interference

We began to seek out methods with which we could directly apply data gleaned from our array work to parametric evaluation of our cellular models of PD. One such logical avenue is the experimental perturbation of specific RNA signals previously observed in cells. Within the last three years, a significant body of publications has documented the newfound importance of RNA interference (RNAi), a form of post-transcriptional gene silencing. The effect was first observed by Jorgensen et al in their attempts to alter coloration in petunias by introducing a pigment producing gene under control of a constitutive promoter (Jorgensen et al, 1996). Rather than producing uniform deepening of pigmentation, a variety of striped patterns and even loss of endogenous pigment was observed. They named this phenomenon "cosuppression" due to the curious disruption of expression of both the introduced gene and the homologous endogenous gene. Since the original findings of Jorgensen were published, similar effects have been observed in many other species of plants as well as in fungi such as Neurospora, where Cogoni et al reported that the silencing effect could be transferred between nuclei in heterokaryotic strains (Cogoni et al, 1996). This particular result served to demonstrate that a trans-acting agent was involved. In some species of plants, the mechanism of silencing induced by transgenes appeared to be mediated by methylation of the homologous nuclear genes in question. In other species reduction of expression occurred at a post- 
transcriptional stage, as the homologous transcript was produced but rapidly degraded.

The first evidence that dsRNA could lead to gene silencing came from work in the nematode Caenorhabditis elegans. Eight years ago, researchers Guo and Kemphues were attempting to use antisense RNA to shut down expression of the par-1 gene in order to assess its function (Guo and Kemphues, 1995). As expected, injection of the antisense RNA disrupted expression of par-1, but quizzically, injection of the sense-strand control did too. This result was a puzzle until three years later. It was then that Fire and colleagues first injected dsRNA, a mixture of both sense and antisense strands, into $C$. elegans. This injection resulted in much more efficient silencing than injection of either the sense or the antisense strands alone. Injection of just a few molecules of dsRNA per cell was sufficient to completely silence the homologous gene's expression (Fire et al, 1998). Furthermore, injection of dsRNA into the gut of the worm caused gene silencing not only throughout the worm, but also in its first generation offspring. The potency of RNAi inspired Fire and Timmons to try feeding nematodes bacteria that had been engineered to express dsRNA homologous to the $C$. elegans unc-22 gene (Timmons et al, 2001). Surprisingly, these worms developed an unc-22 null-like phenotype. Further work showed that soaking worms in dsRNA was also able to induce silencing. These strategies, whereby large numbers of nematodes are exposed to dsRNA, have enabled large-scale screens to select for RNAi-defective C. elegans mutants and have led to large numbers of gene knockout studies within this organism. Additionally, the effect 
was found to be exquisitely sequence-specific; discrepancy of even a few base pairs between the dsRNA and the target mRNA virtually abolished the silencing. RNAi has been used experimentally in these non-mammalian systems to generate transient silencing of specific genes of interest, especially those which are not amenable to more traditional gene knockout methods (e.g., those that produce embryonic lethality and thus cannot be studied in the adult animal).

RNAi has also been observed in Drosophila. Although a strategy in which yeast were engineered to produce dsRNA and then fed to fruit flies failed to work, microinjecting Drosophila embryos with dsRNA does induce silencing. Silencing can also be induced by biolistic techniques in which dsRNA is "shot" into Drosophila embryos, or by engineering flies to carry DNA containing an inverted repeat of the gene to be silenced. Over the last few years, these RNAi strategies have been used as reverse genetics tools in Drosophila organisms, embryo lysates, and cells to characterize various loss-of-function phenotypes. Zamore's group found that dsRNA added to Drosophila embryo lysates was processed to 21-23 nucleotide species. They also found that the homologous endogenous mRNA was cleaved only in the region corresponding to the introduced dsRNA and that cleavage occurred at 21-23 nucleotide intervals (Haley et al, 2003). Current models of RNAi divide the process into broad "initiation" and "effector" stages. In the initiation step, input dsRNA is digested into 21-23 nucleotide small interfering RNAs (siRNAs), which have also been called "guide RNAs." Evidence indicates that siRNAs are produced when the enzyme Dicer, a member of the RNase III family of dsRNA-specific ribonucleases, processively cleaves dsRNA in 
an ATP-dependent, processive manner. Successive cleavage events degrade the RNA to 19-21 bp duplexes (siRNAs), each with 2-nucleotide 3' overhangs. In the effector step, the siRNA duplexes bind to a nuclease complex to form what is known as the RNA-induced silencing complex, or RISC. An ATPdepending unwinding of the siRNA duplex is required for activation of the RISC. The active RISC then targets the homologous transcript by base pairing interactions and cleaves the mRNA $\sim 12$ nucleotides from the 3 ' terminus of the siRNA. Although the mechanism of cleavage is at this date unclear, research indicates that each RISC contains a single siRNA and an RNase that appears to be distinct from Dicer. Because of the remarkable potency of RNAi in some organisms, an amplification step within the RNAi pathway has also been proposed. Amplification could occur by copying of the input dsRNAs, which would generate more siRNAs, or by replication of the siRNAs themselves. Alternatively or in addition, amplification could be effected by multiple turnover events of the RISC.

The presence of RNAi in mammalian cells was only recently established (Elbashir et al, 2001). Transfection of dsRNA in excess of 30 base pairs usually results in a non-specific "pan-suppression" of RNA transcripts. Two pathways contributing to this effect have been known for some time. In the first, the presence of the long dsRNAs activate the PKR protein kinase, which serves to phosphorylate and inactivate elF2a, translation initiation factor (Manche et al, 1992). This has the effect of limiting the translation of most RNA transcripts in the cell. In the second pathway, introduction of long dsRNA catalyzes activation 
of RNAse L, which degrades intracellular RNAs nonspecifically (Minks et al, 1979).

dsRNAs less than 30 base pairs in length, including siRNAs, do not appear to activate the PKR nonspecific response pathway in mature mammalian cells. Utilizing the knowledge that siRNAs can induce RNAi in Drosophila embryos, Elbashir and colleagues transiently transfected different mammalian cell cultures (Elbashir et al, 2001) and achieved as much as $90 \%$ reductions in target RNA levels. As in Drosophila and C. elegans, the effect was extremely sequence specific, with one base pair changes frequently being sufficient to abrogate silencing entirely. The design of siRNAs for use in mammalian cells is still a largely empirical process; there are apparently differential characteristics within genes, as yet unknown, that modulate the effectiveness of silencing by siRNAs localized to specific intragenic regions.

Most of the initial RNAi experiments in mammalian cells were carried out by tranfecting the cells (using a lipophilic carrier molecule such as Oligofectamine ${ }^{\mathrm{TM}}$ ) with chemically synthesized siRNAs. While this method has the advantage of initiating RNAi very quickly (usually within 18 hours), chemical synthesis of RNA is considerably more costly than DNA synthesis, and the induced RNAi lasts for a relatively short time. More recent approaches have included the transfection of RNA produced in T7 in vitro transcription reactions, which has the advantage of greatly reduced production costs but still has a relatively short half-life, and transfection of RNAi expression vectors, which can be stably integrated into the genome of the host cell and are theoretically capable 
of providing transcriptional silencing of indefinite duration. Ambion Inc. has been at the forefront of efforts to produce and market such expression vectors. Their latest such products incorporate selectable markers into the vectors, allowing selection of successfully tranfected cells. This approach offers significant signal to noise ratio advantages when compared to other methods that result in production of a mixed population of cells, some successfully transfected and others not.

What is the role of RNA interference within a cell? Why has this function been so evolutionarily conserved through many different phyla? Phillip Sharp of MIT has been one of the leading proponents of the anti-viral hypothesis, which holds that RNAi is an ancient mechanism used by primitive eukaryotes to combat invading double stranded RNA viruses. Other theories, less prominent in the literature, cite RNAi as a possible defense against transposons or "jumping genes" and the deleterious effects they are capable of producing. In the attempts to identify the gene products required for RNAi in organisms as diverse as Arabidopsis, Neurospora and C. elegans, many researchers noted that such disruptions often resulted in organisms with severe errors in development, lending suppor to the idea that RNAi may also have a role to play in some aspects of development. 
In summary, the history of the study of Parkinson's disease spans a relatively long period of time, with a dramatic increase in our knowledge of the disorder within only the last twenty years. The results that we outline in the following chapters verify the existence of mitochondrial-nuclear signaling in the context of electron transport chain deficits, as well as suggesting the vital roles played in this process by previously described intracellular signaling pathways. These results will serve to direct future investigations into gene expression changes relevant to the processes of cell death and cell survival in our cellular model of Parkinson's disease, and may provide important insights into the pathophysiology of the in vivo disease process. 


\section{Chapter 2}

The first work that I undertook in the Center for the Study of Neurodegenerative Diseases involved the application of nylon membrane-based cDNA microarray technology to the issue of gene expression responses in a cellular model of Parkinson's disease, and concurrently investigated how these changes were dependent upon the energetic and genetic state of mitochondria and upon cell signaling pathways previously determined to modulate the cell death response under these conditions. The experiments were the basis for the publication "Dependence on electron transport chain function and intracellular signaling of genomic responses in SH-SY5Y cells to the mitochondrial neurotoxin MPP $(+)$ ", published in the journal Experimental Neurology (Brill and Bennett, 2003).

\section{Summary}

SH-SY5Y neuroblastoma cells exposed to the complex I inhibitor/parkinsonian neurotoxin methylpyridinium ion $\left(\mathrm{MPP}^{+}\right)$activate both survival and death-promoting signaling pathways and undergo MEK/ERKdependent, phosphatidylinositol-3 kinase-dependent and c-Jun kinasedependent cell death. Because genomic responses to $\mathrm{MPP}^{+}$are not extensively characterized, we used nylon cDNA arrays to measured gene expression following exposure to an apoptosis-producing $\left[\mathrm{MPP}^{+}\right]$. Many changes occurred within 5 minutes, and all gene expression changes appeared before biochemical 
and morphological markers of apoptosis. Selective ablation of the mitochondrial genome of SY5Y cells through long-term exposure to low concentrations of ethidium bromide gives rise to a metabolically altered cell known as a $\rho^{0}$ (Cassarino et al., 1997; Cassarino et al., 2000; Swerdlow et al., 1996). These cells have no detectable mitochondrial DNA, and do not carry out oxidative phosphorylation. The majority of gene expression changes in SY5Y were not found in $\rho^{0}$ cells, indicating dependence of these changes on intact electron transport activity. $\rho^{0}$ cells exposed to MPP ${ }^{+}$produced different expression profiles, indicating the potential for responses independent of complex I inhibition. MPP ${ }^{+}$-induced gene expression patterns in normal SY5Y cells were sensitive to inhibitors of MEK/ERK (U0126) or phosphatidylinositol-3 kinase (LY 294002), demonstrating regulation of gene expression by these survivalpromoting signaling pathways. The primary signaling molecules mediating these $\mathrm{MPP}^{+}$-induced gene expression changes are unknown but ultimately utilize MEK/ERK and phosphatidylinositol-3 kinase signaling. Genes suppressed by U0126 or LY294002 during MPP ${ }^{+}$exposure may mediate cell survival; those expressed in the presence of U0126 or LY294002 may mediate cell death in this in vitro model of Parkinson's disease.

$\underline{\text { Introduction }}$

Among adults, Parkinson's disease (PD) is the most common neurodegenerative movement disorder and second most common 
neurodegenerative brain disease. Motor deficits of PD arise from progressive loss of dopamine neurons in midbrain substantia nigra zona compacta, appear when $\sim 60-75 \%$ of these neurons have died and progress towards severe disability as the remaining nigral dopamine neurons are lost at a rate of $\sim 10 \%$ per year. While effective dopamine-replacement symptomatic treatments exist, none has been shown to change death rate of nigral neurons and alter disease outcome. Understanding how these dopamine neurons die may lead to neuroprotective therapies that will arrest progression of PD symptoms.

While the causes of neuronal cell death in PD remain elusive, significant insight arose in the mid 1980's when the mechanism of illness was defined in opiate addicts who had accidentally injected the pro-neurotoxin N-methyl-4phenyl-1,2,3,6-tetrahydropyridine (MPTP) (Ballard et al. 1985; Davis et al. 1979; Langston et al. 1983). These individuals developed rapidly progressive and severe parkinsonism responsive to dopamine replacement therapy. Animal studies showed that nigral dopamine neurons died following accumulation through the dopamine transporter of methylpyridinium ion (MPP ${ }^{+}$), the twoelectron monoamine oxidase oxidation product of MPTP and a potent inhibitor of complex I of the electron transport chain (Burns et al. 1983; Chiba et al. 1984; Jenner et al. 1984; Langston et al.1984; Javitch et al. 1985; Nicklas et al. 1985; Snyder and D'Amato 1986).

Because multiple PD tissues including platelets (Parker et al., 1989), muscle (Bindoff et al., 1991), fibroblasts (Mytilineau et al., 1994), and both nigral and nonigral brain (Mann et al., 1992; Mizuno et al., 1989) have reduced activity 
of complex I, it has been suggested that PD represents a systemic complex I deficiency state that results in selective death primarily of nigral dopamine neurons. Such a concept was significantly strengthened by experiments with chronic systemic infusion into rats of the potent complex I inhibitor rotenone (Betarbet, et al, 2000). These animals developed a parkinsonian clinical condition and marked, selective loss of nigral dopamine neurons.

MPTP experiments in transgenic mice have demonstrated involvement of several gene products in neurotoxicity to dopamine neurons. Knockout of neuronal (Przedborski et al. 1996) or inducible (Dehmer et al. 2000) nitric oxide synthase, poly (ADP-ribose) polymerase (PARP, Mandir et al. 1999) or the growth regulatory gene p53 (Trimmer et al. 1996) reduces MPTP-induced loss of nigral dopamine neurons. These nigral dopamine neurons are protected against MPTP toxicity if the anti-apoptotic protein bcl-2 is overexpressed (Offen et al. 1998; Yang et al. 1998), or if the pro-apoptotic protein bax is knocked out (Vila et al. 2001).

Exposure of SH-SY5Y human neuroblastoma cells to $\mathrm{MPP}^{+}$induces apoptotic death (Fall and Bennett, 1999) and brings about many responses that likely regulate this process. These responses include an increase in oxidative stress (Cassarino et al., 1997), activation of pro- and anti-apoptotic signaling pathways (Cassarino et al., 2000; Halvorsen et al., 2002), and increasing levels of the anti-apoptotic proteins bcl-2 and bcl- $\mathrm{X}_{\mathrm{L}}$ (Veech et al., 2000; Dennis and Bennett, 2002) and pro-apoptotic protein Bax in a nitric oxide-dependent manner (Dennis and Bennett, 2002). We have previously shown that $\mathrm{MPP}^{+}$induced 
typical oligosomal DNA laddering in SY5Y cells, brought about DNA fragmentation measured with flow cytometry and increased reactive oxygen species (ROS) production and anaerobic metabolism, estimated by lactate production (Fall and Bennett, 1999).

Selective ablation of the mitochondrial genome of SY5Y cells through long-term exposure to low concentrations of ethidium bromide gives rise to a metabolically altered cell known as a $\rho^{0}$ (Cassarino et al., 1997; Cassarino et al., 2000; Swerdlow et al., 1996). Exposure of $\rho^{0}$ cells to $5 \mathrm{mM} \mathrm{MPP}^{+}$does not induce apoptotic cell death. We also found that $\rho^{0}$ cells exposed to $5 \mathrm{mM} \mathrm{MPP}^{+}$ did not show increases in ROS or lactate production, demonstrating the necessity of intact electron transport chain function for $\mathrm{MPP}^{+}$-induced increases in oxidative stress and anaerobic metabolism (Fall and Bennett, 1999).

We have examined the time course of cytochrome $\mathrm{C}$ release and caspase 3-dependent DNA fragmentation after exposure of SY5Y cells to MPP ${ }^{+}$; cytochrome $C$ appears in the cytoplasm within 2-4 hours, and caspasedependent DNA degradation occurs between 12 and 24 hours (Dennis and Bennett, 2002). These findings suggest to us that acute complex I inhibition with $\mathrm{MPP}^{+}$may result in alteration of expression of multiple genes related to cell survival through signals as yet undetermined.

To explore this possibility, to determine if $\mathrm{MPP}^{+}$-induced changes in gene expression depend on electron transport activity, and to explore what signaling pathways or molecules mediate these expression changes, we began this gene 
expression study in SH-SY5Y cells exposed to MPP ${ }^{+}$. We utilized cDNA arrays that displayed well-characterized genes related to cell death/survival and neuronal function. To achieve maximum sensitivity, we selected nylon-based cDNA arrays to which we hybridized ${ }^{32} \mathrm{P}$-labeled probes. We wished to compare any changes in expression of this gene set to changes observed in $\mathrm{SH}-\mathrm{SY} 5 \mathrm{Y}$ cells devoid of mitochondrial DNA and electron transport activity ( $\rho^{0}$ cells), cells treated with inhibitors of signaling pathways, other neurotoxins or neuroprotective drugs, and ultimately to postmortem PD brain samples.

Our results indicate that $\mathrm{MPP}^{+}$induces changes in expression of many different genes in SH-SY5Y cells. These gene expression changes occur hours before the appearance of biochemical and morphological markers of apoptosis. The majority of these changes are dependent on the presence of an intact mitochondrial genome and/or a functional electron transport chain (i.e., they are ablated in $\rho^{0}$ cells), and in addition are sensitive to inhibition of MEK or phosphatidylinositol-3-kinase (PI3K) signaling pathways. These data suggest that $\mathrm{MPP}^{+}$exerts significant effects on cells that factor in their commitment to apoptosis beyond inhibition of mitochondrial electron transport. Further, our results imply the existence of an efficient communication network between mitochondria and the nuclear genome, potentially permitting mitochondria to have more extensive control over cell functioning than previously thought. 


\section{Materials and Methods}

Cell Culture and Treatment.

SH-SY5Y cells were obtained from the American Tissue Culture Collection (ATCC; Manassas, VA) and maintained in culture as previously described (Cassarino et al., 1997; Cassarino et al., 2000; Swerdlow et al., 1996). $\rho^{0}$ cells were generated by chronic incubation with ethidium bromide and maintained as previously described (Cassarino et al., 1997; Cassarino et al., 2000; Swerdlow et al., 1996). They had no mtDNA detectable by polymerase chain reaction and no electron transport chain activity based on assays of complex I and complex IV. 3 x $10^{6}$ control or $\rho^{0}$ cells were placed onto $10 \mathrm{~cm}$ tissue culture plates (Greiner Bio-One; Longwood, $\mathrm{FL}$ ) and maintained at $37^{\circ} \mathrm{C}$ for 24 hours prior to exposure to $5 \mathrm{mM} \mathrm{MPP}^{+}$(Sigma-Aldrich; St. Louis, MO) for multiple periods up to 3 hours in the presence or absence of $20 \mu \mathrm{M}$ LY 294002 or $50 \mu \mathrm{M}$ UO 126 for gene array hybridization studies. SH-SY5Y cells were exposed to $5 \mathrm{mM} \mathrm{MPP}^{+}$for multiple periods up to 24 hours for cell death studies.

\section{RNA Isolation.}

Total RNA was isolated from cell cultures immediately following completion of any MPP ${ }^{+}$exposure using the RNEasy Mini system (Qiagen; Valencia, CA). RNA samples were archived at $-80^{\circ} \mathrm{C}$. 
cDNA Arrays.

Radioactively labeled cDNA probes were prepared from each total RNA samples and hybridized to Atlas nylon "Apoptosis" and "Neurobiology" arrays (Clontech Laboratories; Palo Alto, CA) as per kit protocol. $\alpha{ }^{32}$ P-dATP was obtained from Amersham Pharmacia Biotech, Piscataway, NJ. Array hybridization images were recorded in .TIF format using a Cyclone storage phosphor system (Packard Instrument Company; Meriden, CT). Exposure times were between 24 hours and 72 hours.

\section{$\underline{\text { RT-PCR. }}$}

cDNA was prepared from experimental total RNA samples using the Advantage RT-for-PCR kit (Clontech Laboratories) using oligo(dT)primers according to the manufacturers directions. PCR was carried out using the Advantage cDNA PCR kit (Clontech Laboratories), with the indicated Atlas PCR primers according to manufacturers directions. Samples were held at $94^{\circ} \mathrm{C}$ for 1 minute, followed by 33 cycles of $\left[94^{\circ} \mathrm{C}-30\right.$ seconds, $68^{\circ} \mathrm{C}-2$ minutes], followed in turn by a final $68^{\circ} \mathrm{C}$ for 5 minutes. PCR products were separated on $2 \%$ agarose gels and visualized by ethidium bromide incorporation. Caspase 7 and NIK were selected for RT-PCR verification of both statistically significant and nonsignificant gene expression changes. 


\section{Statistical Analysis.}

Three independent cell culture, RNA extraction and array hybridization experiments were performed for each period of exposure to MPP ${ }^{+}$. Optical density and background levels for each gene were extracted from TIFF files using ImaGene software (BioDiscovery; Los Angeles, CA). Optical densities for individual genes were normalized to the mean optical density calculated from hybridization signals for 405 ribosomal protein, 605 ribosomal protein and cytoplasmic $\beta$-actin "housekeeping genes" in each array experiment, allowing direct comparisons across arrays. When normalization was performed on the basis of combined densities for all genes present on each array, signals were found to be no greater than $1 \%$ different from those obtained through housekeeping gene normalization. Normalized gene hybridization signals of at least 0.1 relative intensity were analyzed by cluster analysis using Cluster software and results visualized using TreeView software (both from M. Eisen, Lawrence Berkeley National Laboratory, http://rana.lbl.gov). One-way repeated measures ANOVA was used to evaluate differences in gene hybridization signals across time of $\mathrm{MPP}^{+}$exposure. Two-way repeated measures ANOVA was used to compare gene hybridization signals across time of $\mathrm{MPP}^{+}$exposure and cell type when comparing SY5Y to $\rho^{0}$. P values less than 0.05 were considered significant. 


\section{$\underline{\text { Results }}$}

\section{SY5Y Response to MPP}

Our approach to this gene expression study was to use the most sensitive array detection technology ( ${ }^{32} \mathrm{P}$-labeled cDNA oligonucleotide probes) and multiple, independent $(n=3)$ experiments for each condition to control for biological and methodological variations. Cluster analysis provides a nonweighted, grouping paradigm that allows deliniation of gene groups whose relative expression levels change with similar patterns. Output of the Cluster software program is presented in Figure 1; a branching dendrogram indicating the grouping of the genes and a corresponding graphical representation of each gene expression profile is shown. Analysis of the four second-order nodes identified by the program indicated marked differences in temporal gene expression patterns among these clusters. Cluster 1, the smallest of the four, identified genes $(n=18)$ with a punctuated decrease in expression at the 15 minute timepoint, and little significant regulation at other timepoints. Cluster 2 identified a large group of genes $(n=171)$ characterized by an even earlier decrease in expression, at the 5 minute timepoint. Some genes in this cluster had little other significant regulation, whereas others were consistently downregulated throughout the time course; others still are notable for slight upregulation of expression at the 90 minute timepoint. Cluster 3 represents genes $(n=83)$ whose expression was upregulated early in the time course, the majority of which have their peaks of expression at the 30 minute timepoint. The 
fourth and final cluster contains genes $(n=106)$ that are upregulated at the later (90 minute plus) points in the timecourse, with considerable heterogeneity of expression at the earlier timepoints. The complete listing of genes for each mathematical cluster is provided in the Supplemental Data section.

We used parametric ANOVA testing to search for any significant variation in expression of individual genes. 36 individual genes were found to be significantly regulated during $\mathrm{MPP}^{+}$exposure. A listing of these genes is presented in Table 1, part A.

\section{SY5Y $\rho^{0}$ Response to MPP ${ }^{+}$}

In order to investigate the contribution of electron transport chain activity to the cellular genomic response to $\mathrm{MPP}^{+}$, we collected expression data from SY5Y $\rho^{0}$ cultures exposed in triplicate to $5 \mathrm{mM} \mathrm{MPP}^{+}$for $0,15,60$ and 120 minutes. and compared these to expression profiles of control SY5Y exposed to $\mathrm{MPP}^{+}$for identical time periods. Expression profiles of genes detected in both SY5Y and SY5Y $\rho^{0}$ cells are compared by cell type in Figure 2 . Of the 378 genes detected in SY5Y cells, 261 were also detected in SY5Y $\rho^{0}$ cells. There were 20 genes detected in SY $5 Y \rho^{0}$ cells that were not detected in control SY5Ys. For the 261 in-common genes, data from the time points measured in $\rho^{0}$ cells were compared to equivalent data from control cells by 2-way ANOVA and found to be significantly different $(P<0.05)$. Furthermore, of the 261 in-common 
genes, 51 were found to be regulated in opposite directions at all timepoints measured in control and $\rho^{0} \mathrm{SY} 5 \mathrm{Y}$ cells.

17 individual genes were found to be significantly regulated in $\rho^{0}$ cells during $\mathrm{MPP}^{+}$exposure. A listing of these genes is presented in Table 1, part B. Note that only 2 out of 770 genes detected across both cell types, furin and GADD153, were found to be significantly regulated in both cell types.

\section{SY5Y Response to MPP ${ }^{+}$in the presence of LY 294002 and UO 126}

Previous results (Halvorsen, et al, 2002) indicated that inhibition of PI3kinase or ERK signaling pathways accelerated $\mathrm{MPP}^{+}$induced cell death in $\mathrm{SH}-$ SY5Y cells. We therefore analyzed gene expression in SH-SY5Y cells exposed to LY 294002 (LY) and UO 126 (UO), pharmacological inhibitors of PI3-kinase and ERK signaling, respectively, to determine what changes in gene expression might be involved in commitment to cell death. LY 294002 is a reversible, specific inhibitor of PI3-kinase that competes for ATP binding on PI3-kinase; its IC50 for class I PI3-kinases is $1 \mu \mathrm{M}$ and for class II PI3-kinases is $19 \mu \mathrm{M}$ (Vlahos, et al, 1994). UO 126 is a specific inhibitor of MEK1 and MEK2; it inhibits phosphorylation-activated as well of constitutively active forms of both MEK1 and MEK2, with $\mathrm{IC}_{50}$ values of $10 \mu \mathrm{M}$ and $<0.1 \mu \mathrm{M}$, respectively (Favata, et al 1998). We found distinct groups of genes that were significantly regulated during exposure to these compounds; a total of 22 individual genes modulated by LY treatment are summarized in Table 2 part A. 51 genes modulated by UO 
treatment are summarized in Table 2 part B. 7 genes out of 76 significantly regulated by either $\mathrm{LY}$ or $\mathrm{UO}$ plus $\mathrm{MPP}^{+}$were also significantly regulated by $\mathrm{MPP}^{+}$alone. 4 genes are significantly regulated by both drugs in the presence of $\mathrm{MPP}^{+}$

To estimate the effect of LY and UO on baseline gene expression, we compared expression data from control SH-SY5Y cells and SY5Ys exposed to either LY or UO alone. Results are presented in Figure 3. Baseline expression was highly similar for both LY and UO when compared to control, with a slight overall tendency toward reduced expression (note relative positions of regression lines in both scatterplots to equivalence lines).

\section{$\underline{\text { RT-PCR }}$}

We sought to validate the gene array data in terms of gene expressions that change and those that don't change. PCR primers specific for arrayed genes caspase 7 and NIK were obtained from Clontech laboratories. RT-PCR was optimized and performed using RNA from control SY5Y cells and SY5Ys treated with $5 \mathrm{mM} \mathrm{MPP}^{+}$for 90 minutes. We examined the caspase 7 gene because it codes for an executioner caspase and had a low level of increase after MPP ${ }^{+}$ (1.5-fold, NS). RT-PCR showed an increase in caspase-7 mRNA of 1.3-fold in the same samples. The NIK gene exhibited one of the largest increases after $\mathrm{MPP}^{+}$(see Table 1A). At 90 minutes of $\mathrm{MPP}^{+}$treatment the gene arrays showed an increase in NIK expression of 5.8-fold. RT-PCR assay of the same samples showed an increase of 6.0-fold. Thus, RT-PCR analysis agreed with array data 
for genes with both a low, non-significant level of increase, and a high level of increase.

\section{Discussion}

MPTP treatment of animals and $\mathrm{MPP}^{+}$exposure of neural cells have been extensively utilized as models of neuronal death for Parkinson's disease. Prior characterizations of the effects of the parkinsonism-producing protoxin MPTP and its toxic metabolite $\mathrm{MPP}^{+}$at the cellular level have focused on the role of $\mathrm{MPP}^{+}$as an inhibitor of complex I of the mitochondrial electron transport chain (ETC). The increased production of ROS resulting from shunting of electrons away from their normal acceptor molecules as a result of this ETC inhibition has been suggested as a possible mediator of the damage to cellular components and eventual progression to apoptotic cell death that follows exposure to MPP ${ }^{+}$. Our experiments utilized a focused genomics approach to address interrelated questions about $\mathrm{MPP}^{+}$toxicity. First, we wished to determine the changes in gene expression that occur in cells in response to $\mathrm{MPP}^{+}$exposure prior to apoptosis. Second, by utilizing genomic responses to $\mathrm{MPP}^{+}$in $\rho^{0}$ cells, we explored the involvement of the ETC in mediating observed expression changes in native SH-SY5Y neuroblastoma cells. Finally, we utilized genomic response screening by array in combination with pharmacological manipulation of signaling pathways known to be contributory to $\mathrm{MPP}^{+}$induced cell death, with an objective of determining which pathways may be responsible for initiating or preventing cell death. 
The statistical analysis of microarray data is still far from standardized, and the relative merits of multiple methods are matters of active discourse. Further, it remains unknown if there are any correlations among mRNA changes and alterations in protein levels in this or many other cell models. With this in mind, we adopted a conservative, parametric analysis strategy that relied upon experimental replication and well-characterized statistical tests. We have listed all gene changes that achieved statistical significance and have not assigned an arbitrary minimum value of "biological significance." Having proceeded in this manner, we believe that the results presented here are a minimal listing of genomic responses, and the reader should not assume that additional, significant genomic responses do not or cannot occur under similar experimental conditions.

The very rapid appearance of gene expression changes observed in SY5Y cells exposed to MPP ${ }^{+}$was surprising. As our studies of cell death markers in the SY5Y-MPP ${ }^{+}$model had found that the earliest markers of apoptosis appeared in the 2-4 hour time range, it was interesting to note that significant alterations in gene expression occurred within 5-15 minutes of MPP exposure. While this does not mean that any of these alterations in expression are required for apoptosis to occur, it is unlikely that all the observed changes are unrelated to the process of apoptosis. Given that the criteria deciding commitment to apoptosis are incompletely defined, it is possible that the results presented here will eventually lead to a more robust depiction of the process of programmed cell death that might allow for earlier identification of cells fated for apoptosis, perhaps by observing changes in genomic expression. 
These studies were undertaken with an initial objective of monitoring the changes in expression of genes known to be involved in apoptosis, e.g. caspases, bcl family proteins, etc., as well as those important to broader mitochondrial functions, e.g. porin, nitric oxide synthase, etc. The diversity of gene responses induced by MPP ${ }^{+}$treatment is difficult to overstate, ranging from cyclin-dependent kinases required for mitosis to the workaday enzymes of basic cellular metabolism. As such, the little-understood effects of $\mathrm{MPP}^{+}$on cellular components other than mitochondria have assumed a new importance in our considerations for future experiments.

We found that the expression level of 378 genes could be reliably detected across the period of $\mathrm{MPP}^{+}$exposure in control and $\rho^{0} \mathrm{SY} 5 \mathrm{Y}$ cells combined. Of these genes, expression levels for 261 were present in $\rho^{0}$ samples. $\rho^{0}$ cells have no detectable mtDNA and no measurable ETC activity, therefore compounds that inhibit ETC activity are unlikely to produce ROS through conventional mitochondrial mechanisms in $\rho^{0}$ cells. In previous work we found that SH-SY5Y $\rho^{0}$ cells neither undergo apoptosis after extended exposure to $5 \mathrm{mM} \mathrm{MPP}^{+}$, nor produce detectable increases in ROS or increases in glycolytic metabolism after MPP ${ }^{+}$exposure (Fall and Bennett, 1999). Thus, the changes in gene expression observed in $\rho^{0}$ cells treated with $\mathrm{MPP}^{+}$are unlikely to be mediated by increases in oxidative stress or intracellular acidification. Given that $\mathrm{MPP}^{+}$is regarded primarily as an inhibitor of the ETC, it was quite remarkable to note that greater than two-thirds of gene expression changes that 
occur following treatment with $\mathrm{MPP}^{+}$are likely to be completely unrelated to this aspect of its toxicity; the logical corollary to this idea is that those gene changes observed in SY5Y but not $\rho^{0}$ cells following $\mathrm{MPP}^{+}$treatment are in some way dependent on ETC function.

We found that the expression of two genes, furin and GADD153 were significantly regulated in both SY5Y and $\rho^{0}$ cells. Furin is a Ca(2+)-dependent serine endoprotease that belongs to the subtilisin-like proprotein convertase (SPC) family. Furin has been shown to be expressed in all tissues and cell lines examined and to be mainly localized in the trans-Golgi network, although some proportion of the furin molecules cycle between this compartment and the cell surface. This endoprotease is capable of cleaving precursors of a wide variety of proteins, including growth factors, serum proteins, including proteases of the blood-clotting and complement systems, matrix metalloproteinases, receptors, viral-envelope glycoproteins and bacterial exotoxins (Nakayama, 1997). Furin has recently been implicated in the extracellular cleavage of proneurotrophins, including proNGF. This regulatory control is particularly interesting in the light of evidence that proneurotrophins stimulate apoptosis in target cells, whereas the proteolyzed, mature forms promote nerve cell survival (Lee, et al, 2001).

Growth arrest- and DNA damage-inducible gene 153 (GADD153), a leucine-zipper transcription factor which is part of the CCAAT/enhancer-binding

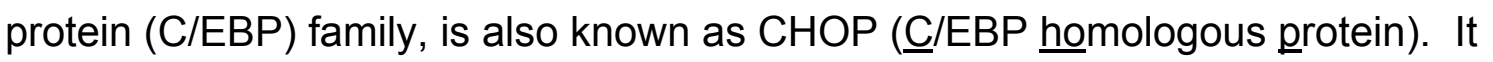
is not expressed at detectable levels in growing mammalian cells but is strongly upregulated in response to various cellular insults (Fawcett, et al, 1996; Luethy 
and Holbrook, 1992). Recent experiments have shown a functional link between GADD153 expression and cellular alterations including growth arrest, apoptosis and even tumorigenesis. Injection of GADD153 protein into NIH3T3 cells caused G1/S arrest of the cell cycle (Barone, et al, 1994). In contrast, overexpression of GADD153 in M1 myeloblastic leukemia cells induced apoptosis, which can be modified by the anti-apoptotic protein, Bcl-2 (Matsumoto, et al, 1996). In addition, compared to the wild type, mouse embryonic fibroblasts derived from gadd $153^{-/-}$ knockout animals undergo significantly less apoptosis in response to endoplasmic reticulum (ER) stress, a powerful upregulator of GADD153 expression (Zinszner, et al, 1998). GADD153 induction has been shown to be required for apoptosis in a human colon cancer cell line induced by treatment with bile acids (Qiao, et al, 2002). Recently, Conn ,et al (2002) reported that treatment of SY5Y cells with $1 \mathrm{mM} \mathrm{MPP}^{+}$differentially altered expression of 313 genes after 24 hours, and that GADD153 expression increased the most. They also found a modest increase in GADD153 protein by Western blot at 24 hours, with protein levels declining after 24 hours in spite of continued increase in mRNA.

Our findings may provide an example of the interplay of pro- and antiapoptotic elements within cells exposed to MPP+; as furin expression was reduced and GADD153 was increased in normal SY5Y cells in response to $\mathrm{MPP}^{+}$, this may indicate anti-apoptotic and pro-apoptotic roles for these gene products, respectively, with $\mathrm{MPP}^{+}$exposure directing the balance toward cell death. The changes observed in $\rho^{0}$ cells are similar to those in control SY5Y 
cells (furin down, GADD153 up); taken together, these results may suggest that these expression changes are necessary but not sufficient for apoptosis, as $\rho^{0}$ cells do not undergo apoptosis in response to $5 \mathrm{mM} \mathrm{MPP}^{+}$(Fall and Bennett, 1999).

How might MPP+ bring about gene expression changes in $\rho^{0}$ cells devoid of ETC function? An additional non-ETC action for $\mathrm{MPP}^{+}$is its recently described ability to open a traditional mitochondrial transition pore (Cassarino, et al, 1999). This transition pore opening was observed in liver mitochondria to occur synergistically with nitric oxide and was incompletely inhibited by ROS scavenging enzymes, suggesting a possible direct interaction of $\mathrm{MPP}^{+}$with transition pore components. This concept is supported by our observation that $\rho^{0}$ cells can be induced to undergo caspase 3-mediated chromatin cleavage into DNA-histone complexes, if metabolic substrate for ATP production (pyruvate) is supplied (Dennis and Bennett, under review). An additional consideration is that $\mathrm{MPP}^{+}$-induced genomic alterations we observed in $\rho^{0}$ cells may wholly or partly derive from non-mitochondrial actions of $\mathrm{MPP}^{+}$. Whatever the final explanations are, $\mathrm{MPP}^{+}$is clearly capable of inducing multiple cellular genomic responses beyond the purview of its known ETC inhibitory actions. This observation raises the problem of how $\mathrm{MPP}^{+}$effects in cells and brain are to be interpreted. Assuming that biochemical changes or cell death in MPTP/MPP ${ }^{+}$experiments derive exclusively from ETC inhibition is no longer viable. 
When compared to the number of genes detected in SY5Y and $\rho^{0}$ cells, only a small portion, $\sim 9 \%(35 / 378)$, were found to change significantly over the time course of $\mathrm{MPP}^{+}$exposure. When this set of genes is compared to similar sets derived from $\rho^{0}$ cells or from SY5Y cells exposed to inhibitors of the MEK and/or PI3K pathways, we note a very small number of genes in common. While it is not surprising that significant differences exist between the datasets, particularly between $\rho^{0}$ cells and SY5Ys, the degree of dissimilarity is striking; only 7 genes out of 76 significantly regulated by either LY or UO plus MPP ${ }^{+}$were also significantly regulated by $\mathrm{MPP}^{+}$alone (Table 4 parts $\mathrm{A}$ and $\mathrm{B}$ ). This high degree of dissimilarity between the sets of genes regulated by LY or UO plus $\mathrm{MPP}^{+}$and those regulated by $\mathrm{MPP}^{+}$alone compels us to consider the possibility that cellular responses, mitochondrial and otherwise, to $\mathrm{MPP}^{+}$are in some way dependent upon ERK and/or PI3K activity. Furthermore, there are only 4 genes ( 2 of which encode lysosomal proteins) that are significantly regulated by both drugs in the presence of $\mathrm{MPP}^{+}$(Table 4 part C).

In only one gene, LIMK-1, was expression increased by MPP+ alone and decreased by MPP+ in combination with an inhibitor (UO 126). While this nonconcordance of response is consistent with LIMK-1 performing a necessary cell survival function in response to MPP+ insult, its connection with known apoptosis/survival mechanisms is unclear. It is a serine threonine protein kinase remarkable for its $2 \mathrm{~N}$-terminal LIM domains that are highly conserved cysteinerich structures containing 2 zinc fingers thought to be responsible for the 
specificity of its protein-protein interactions. Its best characterized cellular function is the transduction of phosphorylation changes in Rho protein to changes in the state of the actin cytoskeleton via phosphorylation of cofilin (CFL1) (Maekawa, et al, 1999). Additionally, LIMK-1 hemizygosity has been associated with Williams syndrome, a developmental disorder in which patients suffer from poor visuospatial constructive cognition (Frangiskakis, et al, 1996). As previous work in our laboratory has documented the ability of inhibition of MEK and PI3K to accelerate progression to cell death in the presence of MPP ${ }^{+}$, these data suggest the possibility that these 4 genes or related gene products may be involved in mediating cell death induced by $\mathrm{MPP}^{+}$. These findings also serve to provide direction for our future inquiries, particularly the question of whether the sets of genes observed to change significantly in the presence of either inhibitor in addition to $\mathrm{MPP}^{+}$represent bona fide independent pathways leading to apoptosis, or act under control of other unidentified cell signaling components.

A potential limitation of our study is that we utilized $\mathrm{SH}-\mathrm{SY} 5 \mathrm{Y}$ neuroblastoma cells exposed to a high [MPP $\left.{ }^{+}\right]$. SH-SY5Y cells expressing the dopamine transporter (DAT) and exposed to $10 \mu \mathrm{M}[\mathrm{MPP}+]$ showed acute mitochondrial impairment and delayed (2-3 days) apoptotic death (Stephans, et al, 2002). This finding suggests that it is the low level of DAT expression in native SH-SY5Y that requires high $\left[\mathrm{MPP}^{+}\right]$for induction of apoptosis in our paradigm. We chose to use this paradigm in order to be able to compare gene expression profiles with our earlier studies in SH-SY5Y cells on the rate and characteristics 
of apoptotic cell death (Fall and Bennett, 1999), activation of intracellular signaling (Cassarino, et al, 2000; Halvorsen, et al, 2002) and NF-kB transcription factor (Cassarino, et al, 2000), release of cytochrome $C$ and activation of caspases (Abramova, et al, 2002) and changes in mRNA and protein levels for apoptosis-regulating bcl-family proteins (Veech, et al, 2000; Dennis and Bennett, 2002). However, we note that because the nature of cell death in Parkinson's disease remains controversial (Graeber and Moran, 2002), our use of acute, $\mathrm{MPP}^{+}$-induced apoptosis in undifferentiated, dividing neuroblastoma cells may be of limited relevance to neurodegeneration in this clinical disease.

Finally, our findings demonstrate an extensive and rapidly acting signaling system between mitochondrial "distress" and nuclear gene expression. We do not yet know which molecules or signaling pathways mediate these expression changes, but it is likely that such signaling is necessary for cell survival in the face of mitochondrial insults or failure. Because increasing evidence points to mitochondrial impairments as a leading cause of neurodegeneration (Cassarino and Bennett, 1999), elucidating the signaling mechanisms between mitochondria and the nuclear genetic machinery of their host cells could point to additional strategies to increase neuronal survival in these chronic, progressive diseases. 
Chapter 2 Table 1.

A. Genes significantly regulated in $\mathrm{SH}-\mathrm{SY} 5 \mathrm{Y}$ exposed to $\mathrm{MPP}^{+}(\mathrm{n}=36)$

\begin{tabular}{|c|c|c|c|c|}
\hline Gene & $\begin{array}{l}\text { Chromosomal } \\
\text { Location }\end{array}$ & $\begin{array}{l}\text { Genbank } \\
\#\end{array}$ & $\begin{array}{l}\text { Max fold } \\
\text { change } \\
\text { from } \\
\text { baseline }\end{array}$ & $\begin{array}{l}p- \\
\text { value }\end{array}$ \\
\hline $\begin{array}{l}\text { 94-kDa glucose-regulated } \\
\text { protein (GRP94) }\end{array}$ & $\begin{array}{l}12 \mathrm{q} 24.2- \\
12 \mathrm{q} 24.3\end{array}$ & X15187 & -0.83 & 0.020 \\
\hline $\begin{array}{l}\text { phosphatidylinositol 4-kinase } \\
\text { alpha (PI4-kinase; PTDINS-4- } \\
\text { kinase; PI4K-alpha) }\end{array}$ & $22 q 11.21$ & L36151 & -0.82 & 0.007 \\
\hline $\begin{array}{l}\text { fur; furin precursor; paired basic } \\
\text { amino acid residue cleaving } \\
\text { enzyme (PACE); dibasic } \\
\text { processing enzyme* }\end{array}$ & $15 q 25-15 q 26$ & $\mathrm{X} 17094$ & -0.81 & 0.005 \\
\hline $\begin{array}{l}\text { p21-activated kinase alpha } \\
\text { (PAK-alpha; PAK1) }\end{array}$ & 11q13-11q14 & U24152 & -0.81 & 0.034 \\
\hline $\begin{array}{l}\text { lumican precursor (LUM); } \\
\text { keratan sulfate proteoglycan; } \\
\text { LDC }\end{array}$ & $\begin{array}{l}12 q 21.3- \\
12 q 22\end{array}$ & U18728 & -0.79 & 0.021 \\
\hline $\begin{array}{l}\text { protein-tyrosine phosphatase } \\
\text { 2C (PTP-2C); SH-PTP2 }\end{array}$ & $12 q 24.1$ & L08807 & -0.74 & 0.028 \\
\hline $\begin{array}{l}\text { cAMP-dependent protein kinase } \\
\text { alpha-catalytic subunit (PKA C- } \\
\text { alpha) }\end{array}$ & 19p13.1 & X07767 & -0.70 & 0.010 \\
\hline $\begin{array}{l}\text { phosphatidylethanolamine- } \\
\text { binding protein (PBP); } \\
\text { neuropolypeptide H3 }\end{array}$ & $12 q 24.23$ & D16111 & -0.71 & 0.013 \\
\hline Ras-related protein RAB-5B & $12 q 13$ & X54871 & -0.69 & 0.033 \\
\hline $\begin{array}{l}\text { mitochondrial ATP synthase } \\
\text { alpha chain precursor; ATP5A1 }\end{array}$ & $18 q 12-q 21$ & D14710 & -0.68 & 0.019 \\
\hline $\begin{array}{l}\text { casein kinase I alpha isoform } \\
\text { (CKI-alpha); CK1; CSNK1A }\end{array}$ & $13 q 13$ & X80693 & -0.66 & 0.046 \\
\hline Ras-related protein RAB-32 & $6 q 24.3$ & U71127 & -0.65 & 0.048 \\
\hline $\begin{array}{l}\text { ephrin type-A receptor } 1 \\
\text { precursor; tyrosine-protein } \\
\text { kinase receptor eph }\end{array}$ & $17 q 32-17 q 36$ & M18391 & -0.65 & 0.025 \\
\hline $\begin{array}{l}\text { outer mitochondrial membrane } \\
\text { protein porin; porin } 31 \mathrm{HL} \text {; porin } \\
\text { 31HM }\end{array}$ & $5 q 31$ & L06132 & -0.64 & 0.045 \\
\hline $\begin{array}{l}\text { beta-adrenergic receptor kinase } \\
2 \text { (beta-ARK2) }\end{array}$ & $22 q 11$ & X69117 & -0.63 & 0.006 \\
\hline laminin beta 1 subunit precursor & $7 q 31.1-7 q 31.3$ & M61916 & -0.61 & 0.044 \\
\hline
\end{tabular}




\begin{tabular}{|c|c|c|c|c|}
\hline (laminin B1; LAMB1) & & & & \\
\hline $\begin{array}{l}\text { casein kinase I delta isoform } \\
\text { (CKI-delta); CSNK1D }\end{array}$ & $17 q 25$ & U29171 & -0.61 & 0.016 \\
\hline $\begin{array}{l}\text { insulin-like growth factor II } \\
\text { receptor (IGFR II) }\end{array}$ & $11 \mathrm{p} 15.5$ & Y00285 & -0.61 & 0.006 \\
\hline $\begin{array}{l}\text { metabotropic glutamate } \\
\text { receptor } 7 \text { precursor (GRM7; } \\
\text { MGLUR7) }\end{array}$ & 3p26.1-p25.1 & X94552 & -0.60 & 0.038 \\
\hline $\begin{array}{l}\text { cholinergic receptor nicotinic } \\
\text { alpha polipeptide } 3 \text { (CHRNA3) }\end{array}$ & $2 q 24-2 q 32$ & M37981 & -0.59 & 0.012 \\
\hline $\begin{array}{l}\text { Ras-related protein RAB-11A; } \\
\text { YPT3 }\end{array}$ & $\begin{array}{l}15 \mathrm{q} 21.3- \\
\mathrm{q} 22.31\end{array}$ & X53143 & -0.59 & 0.037 \\
\hline $\begin{array}{l}\text { sodium-dependent } \\
\text { noradrenaline transporter; } \\
\text { norepinephrine transporter } \\
\text { (NET) }\end{array}$ & $16 q 12.2$ & M65105 & -0.59 & 0.030 \\
\hline $\begin{array}{l}\text { protein-tyrosine phosphatase } \\
\text { mu precursor (R-PTP-mu) }\end{array}$ & $18 p 11.2$ & X58288 & -0.57 & 0.025 \\
\hline $\begin{array}{l}\text { lysosomal acid phosphatase } \\
\text { precursor (LAP); ACP2 }\end{array}$ & $11 \mathrm{p} 11.2$ & $\mathrm{X} 12548$ & -0.56 & 0.035 \\
\hline $\begin{array}{l}\text { MAP kinase-activated protein } \\
\text { kinase } 2 \text { (MAPKAP kinase } 2 \text {; } \\
\text { MAPKAPK-2) }\end{array}$ & $1 q 32$ & U12779 & -0.56 & 0.024 \\
\hline $\begin{array}{l}\text { vesicular acetylcholine } \\
\text { transporter (VAChT) }\end{array}$ & $10 q 11.2$ & U10554 & -0.51 & 0.041 \\
\hline LIM domain kinase 1 (LIMK-1) & $7 q 11.23$ & D26309 & 0.68 & $<0.001$ \\
\hline $\begin{array}{l}\text { protein kinase C inhibitor } \\
\text { protein-1 (KCIP-1) }\end{array}$ & $20 q 13.1$ & X57346 & 0.79 & 0.031 \\
\hline Ras-related protein RAB5A & $3 p 24-p 22$ & M28215 & 0.97 & 0.023 \\
\hline $\begin{array}{l}\text { RBQ1 retinoplastoma binding } \\
\text { protein }\end{array}$ & 16p12-p11.2 & X85133 & 0.98 & 0.035 \\
\hline $\begin{array}{l}\text { serine/threonine protein } \\
\text { phosphatase PP1-gamma } 1 \\
\text { catalytic subunit (PP-1G) }\end{array}$ & $12 q 24.1-q 24.2$ & X74008 & 1.0 & 0.025 \\
\hline $\begin{array}{l}\text { transforming protein rhoA H12 } \\
\text { (RHO12; ARH12; ARHA) }\end{array}$ & $3 p 21.3$ & L25080 & 1.2 & 0.045 \\
\hline $\begin{array}{l}\text { growth arrest \& DNA-damage- } \\
\text { inducible protein } 153 \\
(\text { GADD153)* }\end{array}$ & $\begin{array}{l}12 q 13.1- \\
12 q 13.2\end{array}$ & S40706 & 1.3 & 0.028 \\
\hline $\begin{array}{l}\text { G1/S-specific cyclin D1 } \\
\text { (CCND1); cyclin PRAD1; bcl-1 } \\
\text { oncogene }\end{array}$ & $11 q 13$ & X59798 & 1.5 & 0.024 \\
\hline $\begin{array}{l}\text { NIK serine/threonine protein } \\
\text { kinase }\end{array}$ & $17 q 21$ & Y10256 & 9.4 & 0.039 \\
\hline
\end{tabular}




\begin{tabular}{|l|l|l|l|l|}
\hline cell division protein kinase 3 & $17 q 22$ & X66357 & 11. & 0.027 \\
\hline
\end{tabular}

B. Genes significantly regulated in $\mathrm{SH}-\mathrm{SY} 5 \mathrm{Y} \rho^{0}$ exposed to $\mathrm{MPP}^{+}(n=17)$

\begin{tabular}{|l|l|l|l|l|}
\hline Gene & $\begin{array}{l}\text { Chromosomal } \\
\text { Location }\end{array}$ & $\begin{array}{l}\text { Genbank } \\
\#\end{array}$ & $\begin{array}{l}\text { Max fold } \\
\text { change } \\
\text { from } \\
\text { baseline }\end{array}$ & $\begin{array}{l}\text { p- } \\
\text { value }\end{array}$ \\
\hline $\begin{array}{l}\text { endothelial nitric oxide } \\
\text { synthase (EC-NOS; ENOS); } \\
\text { type III NOS }\end{array}$ & $7 q 36$ & $M 93718$ & -11 & 0.011 \\
\hline $\begin{array}{l}\text { adenosine A1 receptor } \\
\text { (ADORA1) }\end{array}$ & 1932.1 & $S 56143$ & -7.4 & 0.005 \\
\hline $\begin{array}{l}\text { apolipoprotein E precursor } \\
\text { (APOE) }\end{array}$ & $19 q 13.2$ & $M 12529$ & -2.6 & 0.018 \\
\hline $\begin{array}{l}\text { corticotropin releasing factor } \\
\text { receptor 1 precursor (CRF-R; } \\
\text { CRF1) }\end{array}$ & $17 q 12-q 22$ & $X 72304$ & -1.9 & $<0.001$ \\
\hline $\begin{array}{l}\text { Fur; furin precursor; paired } \\
\text { basic amino acid residue } \\
\text { cleaving enzyme (PACE); } \\
\text { dibasic processing enzyme* }\end{array}$ & $15 q 25-15 q 26$ & $X 17094$ & -1.9 & 0.014 \\
\hline $\begin{array}{l}\text { ER-Golgi intermediate } \\
\text { compartment 53-kDa protein } \\
\text { (ERGIC-53); GP58; MR60; } \\
\text { mannose-binding lectin } \\
\text { (LMAN1) }\end{array}$ & $18 q 21.3-q 22$ & $X 71661$ & -1.5 & 0.006 \\
\hline $\begin{array}{l}\text { superior cervical ganglion-10 } \\
\text { protein (SCG10); neuron- } \\
\text { specific growth-associated } \\
\text { protein; stathmin homolog }\end{array}$ & $8 q 21.12$ & $S 82024$ & -1.2 & 0.004 \\
\hline $\begin{array}{l}\text { annexin II (ANX2); lipocortin } \\
\text { Il; calpactin I heavy subunit; } \\
\text { chromobindin 8; protein I; } \\
\text { placental anticoagulant } \\
\text { protein IV (PAPIV) }\end{array}$ & $15 q 21-q 22$ & $D 00017$ & -1.1 & 0.028 \\
\hline $\begin{array}{l}\text { protein kinase C alpha } \\
\text { polypeptide (PKC-alpha; } \\
\text { PKCA) }\end{array}$ & $17 q 22-q 23.2$ & $M 22199$ & -1.1 & \\
\hline
\end{tabular}




\begin{tabular}{|l|l|l|l|l|}
\hline Calmodulin & $19 q 13.2-q 13.3$ & $J 04046$ & -1.1 & 0.022 \\
\hline $\begin{array}{l}\text { beta-D-galactosidase } \\
\text { precursor; lactase; acid beta- } \\
\text { galactosidase; GLB1 }\end{array}$ & $3 p 21.33$ & $M 27507$ & 0.50 & 0.013 \\
\hline drebrin E & $5 q 35.3$ & $U 00802$ & 0.53 & 0.047 \\
\hline $\begin{array}{l}\text { serine/threonine protein } \\
\text { phosphatase PP2A-beta } \\
\text { catalytic subunit }\end{array}$ & $8 p 12-p 11.2$ & $X 12656$ & 0.55 & 0.002 \\
\hline $\begin{array}{l}\text { induced myeloid leukemia cell } \\
\text { differentiation protein MCL-1 }\end{array}$ & $1 q 21$ & $L 08246$ & 0.58 & 0.019 \\
\hline $\begin{array}{l}\text { c-jun proto-oncogene; } \\
\text { transcription factor AP-1 }\end{array}$ & $1 p 32-p 31$ & $J 04111$ & 0.64 & 0.018 \\
\hline $\begin{array}{l}\text { dopamine beta-hydroxylase } \\
\text { (DBH); dopamine-beta- } \\
\text { monooxygenase precursor }\end{array}$ & $9 q 34$ & $X 13255$ & 0.68 & 0.015 \\
\hline $\begin{array}{l}\text { growth arrest \& DNA- } \\
\text { damage-inducible protein 153 } \\
\text { (GADD153)* }\end{array}$ & $12 q 13.2$ & $S 40706$ & 0.83 & 0.007 \\
\hline
\end{tabular}

\section{$\underline{\text { Table } 1 \text { Legend }}$}

${ }^{*}$ denotes genes significantly regulated in both cell types.

"Max fold change from baseline" refers to the maximum change in signal observed for a given gene over all time points examined as compared to average (mean) signal observed in control cells. The time point of maximal change in expression varies from gene to gene. 
Chapter 2 Table 2.

A. Genes significantly regulated in SH-SY5Y exposed to LY 294002 and MPP ${ }^{+}$ $\underline{(n=22)}$

\begin{tabular}{|c|c|c|c|c|}
\hline Gene & Location & $\begin{array}{l}\text { Genbank } \\
\#\end{array}$ & $\begin{array}{l}\text { Max fold } \\
\text { change } \\
\text { from } \\
\text { baseline }\end{array}$ & $\begin{array}{l}p- \\
\text { value }\end{array}$ \\
\hline $\begin{array}{l}\text { cyclin-dependent kinase } 4 \text { inhibitor } \\
\text { (CDK4I; CDKN2); p16-INK4; } \\
\text { multiple tumor suppressor } 1 \text { (MTS1) }\end{array}$ & $9 p 21$ & L27211 & -0.59 & 0.045 \\
\hline ras-related protein $\mathrm{RAB}-32^{*}$ & $6 q 24.3$ & U71127 & -0.34 & 0.009 \\
\hline $\begin{array}{l}\text { protein phosphatase PP2A 55-kDa } \\
\text { regulatory subunit neuronal isoform; } \\
\text { protein phosphatase PP2A B } \\
\text { subunit beta; beta-PR55† }\end{array}$ & $\begin{array}{l}5 q 31- \\
5 q 33\end{array}$ & M64930 & -0.25 & 0.004 \\
\hline $\begin{array}{l}\text { cyclophilin } 3 \text { protein (CYP3); } \\
\text { mitochondrial peptidyl-prolyl cis- } \\
\text { trans isomerase precursor } \\
\text { (PPIASE); rotamase }\end{array}$ & $\begin{array}{l}10 q 22- \\
q 23\end{array}$ & M80254 & -0.23 & 0.037 \\
\hline $\begin{array}{l}\text { serine/threonine-protein kinase } \\
\text { PCTAIRE } 1 \text { (PCTK1) }\end{array}$ & $\begin{array}{l}\text { Xp11.3- } \\
\text { p11.23 }\end{array}$ & X66363 & -0.22 & 0.032 \\
\hline $\begin{array}{l}\text { cyclin-dependent kinase } 4 \text { inhibitor } \\
\text { D (CDKN2D); p19-INK4D }\end{array}$ & $19 p 13$ & U40343 & -0.22 & 0.025 \\
\hline $\begin{array}{l}\text { cation-dependent mannose-6- } \\
\text { phosphate receptor precursor (CD } \\
\text { man-6-P receptor; CD-MPR); MPR } \\
46\end{array}$ & $12 p 13$ & M16985 & -0.19 & 0.005 \\
\hline syntaxin 7 (STX7) & $6 q 23.1$ & U77942 & -0.17 & 0.030 \\
\hline c-myc binding protein MM-1 & $12 q 13.13$ & D89667 & -0.14 & 0.022 \\
\hline $\begin{array}{l}\text { dystroglycan precursor; dystrophin- } \\
\text { associated glycoprotein } 1 \text { (DAG1) }\end{array}$ & $3 p 21$ & L19711 & -0.07 & 0.029 \\
\hline $\begin{array}{l}\text { MAP kinase-activated protein } \\
\text { kinase } 2 \text { (MAPKAP kinase } 2 ; \\
\text { MAPKAPK-2)* }\end{array}$ & $1 q 32$ & U12779 & -0.02 & 0.015 \\
\hline $\begin{array}{l}\text { protein-tyrosine phosphatase 1B } \\
\text { (PTP-1B) }\end{array}$ & $\begin{array}{l}20 q 13.1- \\
q 13.2\end{array}$ & M31724 & 0.13 & 0.008 \\
\hline $\begin{array}{l}\text { activin receptor type IIB (ACTRIIB; } \\
\text { ACVR2) }\end{array}$ & $3 p 22$ & X77533 & 0.20 & 0.022 \\
\hline $\begin{array}{l}\text { protein SEC23 homolog isoform A } \\
\text { (SEC23A) }\end{array}$ & $14 q 13.2$ & X97064 & 0.24 & 0.005 \\
\hline MAPK/ERK kinase kinase 3 (MEK & $17 q 11.2$ & L36719 & 0.26 & 0.018 \\
\hline
\end{tabular}




\begin{tabular}{|c|c|c|c|c|}
\hline kinase 3; MEKK3) & & & & \\
\hline $\begin{array}{l}\text { alpha-soluble NSF attachment } \\
\text { protein (SNAP-alpha) }\end{array}$ & $19 q 13.33$ & U39412 & 0.31 & 0.010 \\
\hline $\begin{array}{l}\text { Rac-alpha serine/threonine kinase } \\
\text { (rac-PK-alpha); protein kinase B } \\
\text { (PKB); c-akt; akt1 }\end{array}$ & $14 q 32.32$ & M63167 & 0.43 & 0.027 \\
\hline $\begin{array}{l}\text { cytosolic dynein heavy chain } \\
\text { (DYHC) }\end{array}$ & $14 q 32.3$ & L23958 & 0.44 & 0.012 \\
\hline $\begin{array}{l}\text { lysosomal acid lipase/cholesteryl } \\
\text { ester hydrolase precursor (LAL); } \\
\text { acid cholesteryl ester hydrolase; } \\
\text { sterol esterase; lipase A (LIPA); } \\
\text { cholesteryl esterase† }\end{array}$ & $\begin{array}{l}\text { 10q23.2- } \\
\text { q23.3 }\end{array}$ & M74775 & 0.50 & 0.015 \\
\hline $\begin{array}{l}\text { lysosome membrane protein II } \\
\text { (LIMP II); 85-kDa lysosomal } \\
\text { membrane sialoglycoprotein } \\
\text { (LGP85); CD36 antigen-like } 2 \\
\text { (CD36L2)† }\end{array}$ & $4 q 21.21$ & D12676 & 0.53 & 0.012 \\
\hline $\begin{array}{l}\text { insulin-like growth factor-binding } \\
\text { protein } 3 \text { precursor (IGF-binding } \\
\text { protein 3; IGFBP3; IBP3)† }\end{array}$ & 7p13-p12 & M31159 & 0.66 & 0.015 \\
\hline $\begin{array}{l}\text { neuron-derived orphan receptor } 1 \\
\text { (NOR1); mitogen-induced nuclear } \\
\text { orphan receptor (MINOR); CHN }\end{array}$ & $9 q 22$ & D78579 & 0.80 & 0.025 \\
\hline
\end{tabular}


B. Genes significantly regulated in SH-SY5Y exposed to $\mathrm{MPP}^{+}$and $\mathrm{UO} 126$ $\underline{(n=51)}$

\begin{tabular}{|c|c|c|c|c|}
\hline Gene & Location & $\begin{array}{l}\text { Genbank } \\
\text { \# }\end{array}$ & $\begin{array}{l}\text { Max fold } \\
\text { change } \\
\text { from } \\
\text { baseline }\end{array}$ & $\begin{array}{l}p- \\
\text { value }\end{array}$ \\
\hline $\begin{array}{l}\text { chromaffin granule amine } \\
\text { transporter; vesicular amine } \\
\text { transporter } 1 \text { (VAT1) }\end{array}$ & 8p21.3 & U39905 & -0.98 & 0.005 \\
\hline $\begin{array}{l}\text { ER lumen protein retaining receptor } \\
1 ; K D E L \text { receptor } 1 ; E R D 21\end{array}$ & $19 q 13.3$ & X55885 & -0.91 & 0.023 \\
\hline $\begin{array}{l}\text { mitochondrial 10-kDa heat shock } \\
\text { protein (HSP10); 10-kDa chaperonin } \\
\text { (CPN10); HSPE1 }\end{array}$ & $2 q 33.1$ & U07550 & -0.84 & 0.018 \\
\hline $\begin{array}{l}\text { protein phosphatase PP2A 55-kDa } \\
\text { regulatory subunit alpha isoform; } \\
\text { protein phosphatase PP2A B subunit } \\
\text { alpha isoform; alpha-PR55† }\end{array}$ & $8 p 21.1$ & M64929 & -0.79 & 0.042 \\
\hline $\begin{array}{l}\text { neural-cadherin precursor }(\mathrm{N}- \\
\text { cadherin; NCAD); cadherin } 2(\mathrm{CDH} 2)\end{array}$ & $18 q 11.2$ & M34064 & -0.76 & 0.001 \\
\hline $\begin{array}{l}\text { lumican precursor (LUM); keratan } \\
\text { sulfate proteoglycan; LDC }{ }^{*}\end{array}$ & $\begin{array}{l}12 \mathrm{q} 21.3- \\
\mathrm{q} 22\end{array}$ & U18728 & -0.75 & 0.041 \\
\hline LIM domain kinase 1 (LIMK-1) ${ }^{\star}$ & $7 q 11.23$ & D26309 & -0.74 & 0.032 \\
\hline $\begin{array}{l}\text { serine/threonine protein } \\
\text { phosphatase PP1-gamma } 1 \text { catalytic } \\
\text { subunit (PP-1G) }\end{array}$ & $\begin{array}{l}12 q 24.1- \\
q 24.2\end{array}$ & X74008 & -0.72 & 0.036 \\
\hline $\begin{array}{l}\text { paraneoplastic encephalomyelitis } \\
\text { antigen HUD; HU-antigen D }\end{array}$ & $1 \mathrm{p} 34$ & M62843 & -0.71 & 0.039 \\
\hline $\begin{array}{l}\text { epidermal growth factor receptor } \\
\text { kinase substrate EPS } 8\end{array}$ & $\begin{array}{l}12 q 23- \\
q 24\end{array}$ & U12535 & -0.68 & 0.038 \\
\hline $\begin{array}{l}\text { dihydropyridine-sensitive L-type } \\
\text { calcium channel beta-3 subunit } \\
\text { (CAB3A/CAB3B); CACNLB3 }\end{array}$ & $12 q 13$ & U07139 & -0.65 & 0.040 \\
\hline $\begin{array}{l}\text { lung group IB phospholipase A2 } \\
\text { precursor (PLA2); } \\
\text { phosphatidylcholine 2-acylhydrolase }\end{array}$ & $\begin{array}{l}\text { 12q23- } \\
\text { q24.1 }\end{array}$ & M21054 & -0.65 & 0.034 \\
\hline $\begin{array}{l}\text { mitochondrial stress-70 protein } \\
\text { precursor; } 75-k D a \text { glucose-regulated } \\
\text { protein (GRP75); peptide-binding } \\
\text { protein } 74 \text { (PBP74); mortalin (MOT); } \\
\text { HSPA9B }\end{array}$ & $5 q 31.1$ & L15189 & -0.63 & 0.022 \\
\hline flavoprotein subunit of complex II; & $5 p 15$ & D30648 & -0.62 & 0.049 \\
\hline
\end{tabular}




\begin{tabular}{|c|c|c|c|c|}
\hline $\begin{array}{l}\text { succinate-ubiquinone } \\
\text { dehydrogenase flavoprotein subunit } \\
\text { precursor (SDHA; SDH2) }\end{array}$ & & & & \\
\hline $\begin{array}{l}\text { coatomer alpha subunit; alpha-coat } \\
\text { protein; alpha-COP; HEP-COP }\end{array}$ & 1q23-q25 & U24105 & -0.62 & 0.021 \\
\hline $\begin{array}{l}\text { 94-kDa glucose-regulated protein } \\
\text { (GRP94) }^{*}\end{array}$ & $\begin{array}{l}12 \mathrm{q} 24.2- \\
12 \mathrm{q} 24.3\end{array}$ & X15187 & -0.60 & 0.002 \\
\hline $\begin{array}{l}\text { casein kinase II alpha' subunit (CK } \\
\text { II); CSNK2A2 }\end{array}$ & $\begin{array}{l}\text { 16p13.3- } \\
\text { p13.2 }\end{array}$ & M55268 & -0.58 & 0.043 \\
\hline Golgi SNARE; GS27 & $17 q 21$ & AF007548 & -0.55 & 0.014 \\
\hline $\begin{array}{l}\text { lysosome membrane protein II (LIMP } \\
\text { II); 85-kDa lysosomal membrane } \\
\text { sialoglycoprotein (LGP85); CD36 } \\
\text { antigen-like } 2 \text { (CD36L2)† }\end{array}$ & $4 q 21.21$ & D12676 & -0.53 & 0.022 \\
\hline $\begin{array}{l}\text { ras-related protein R-ras2; ras-like } \\
\text { protein TC21; teratocarcinoma } \\
\text { oncogene }\end{array}$ & $11 \mathrm{p} 15.3$ & M31468 & -0.51 & 0.019 \\
\hline $\begin{array}{l}\text { vesicle-membrane fusion protein } \\
\text { SNAP23A }\end{array}$ & $15 q 13.3$ & Y09567 & -0.49 & 0.021 \\
\hline $\begin{array}{l}\text { cholinephosphate } \\
\text { cytidylyltransferase; } \\
\text { phosphorylcholine transferase; CTP }\end{array}$ & $3 q 29$ & L28957 & -0.48 & 0.023 \\
\hline $\begin{array}{l}\text { protein-tyrosine phosphatase MEG2 } \\
\text { (PTPASE-MEG2) }\end{array}$ & $15 q 22.9$ & M83738 & -0.46 & 0.047 \\
\hline $\begin{array}{l}\text { laminin gamma } 1 \text { subunit precursor } \\
\text { (LAMC1); laminin B2 subunit } \\
\text { (LAMB2) }\end{array}$ & $1 q 31$ & J03202 & -0.45 & 0.032 \\
\hline $\begin{array}{l}\text { lysosomal acid lipase/cholesteryl } \\
\text { ester hydrolase precursor (LAL); acid } \\
\text { cholesteryl ester hydrolase; sterol } \\
\text { esterase; lipase A (LIPA); cholesteryl } \\
\text { esterase† }\end{array}$ & $\begin{array}{l}\text { 10q23.2- } \\
\text { q23.3 }\end{array}$ & M74775 & -0.43 & 0.049 \\
\hline $\begin{array}{l}\text { ER-Golgi intermediate compartment } \\
\text { 53-kDa protein (ERGIC-53); GP58; } \\
\text { MR60; mannose-binding lectin } \\
\text { (LMAN1) }\end{array}$ & $\begin{array}{l}18 q 21.3- \\
q 22\end{array}$ & X71661 & -0.42 & 0.017 \\
\hline $\begin{array}{l}\text { guanine nucleotide-binding protein } \\
\text { beta subunit-like protein 12; receptor } \\
\text { of activated protein kinase C } 1 \\
\text { (RACK1) }\end{array}$ & $5 q 35.3$ & M24194 & -0.40 & 0.017 \\
\hline p53 cellular tumor antigen & $17 \mathrm{p} 13.1$ & M14694 & -0.39 & 0.019 \\
\hline $\begin{array}{l}\text { casein kinase I alpha isoform (CKI- } \\
\text { alpha); CK1; CSNK1A* }\end{array}$ & $13 q 13$ & X80693 & -0.33 & 0.047 \\
\hline membrane-bound \& soluble & $22 q 11.21$ & M65212 & -0.29 & 0.017 \\
\hline
\end{tabular}




\begin{tabular}{|c|c|c|c|c|}
\hline $\begin{array}{l}\text { catechol-O-methyltransferase } \\
\text { (COMT) }\end{array}$ & & & & \\
\hline $\begin{array}{l}\text { protein-tyrosine phosphatase D1 } \\
\text { (PTP-D1) }\end{array}$ & $14 q 31.3$ & X79510 & -0.26 & 0.024 \\
\hline $\begin{array}{l}\text { cell division protein kinase } 6 \text { (CDK6); } \\
\text { serine/threonine protein kinase } \\
\text { PLSTIRE }\end{array}$ & $7 q 21-q 22$ & X66365 & 0.43 & 0.046 \\
\hline $\begin{array}{l}\text { E2F dimerization partner 1; DRTF1- } \\
\text { polypeptide } 1 \text { (DP1) }\end{array}$ & $13 q 34$ & L23959 & 0.44 & 0.047 \\
\hline $\begin{array}{l}\text { p73 (monoallelically expressed p53- } \\
\text { related protein) }\end{array}$ & $1 \mathrm{p} 36.3$ & Y11416 & 0.45 & 0.014 \\
\hline $\begin{array}{l}\text { cell division cycle protein 25A } \\
\text { (CDC25A); M-phase inducer } \\
\text { phosphatase } 1\end{array}$ & $3 p 21$ & M81933 & 0.88 & 0.034 \\
\hline CDC27HS protein & $\begin{array}{l}17 q 12- \\
17 q 23.2\end{array}$ & U00001 & 0.88 & 0.034 \\
\hline $\begin{array}{l}\text { p35 cyclin-like CAK1-associated } \\
\text { protein }\end{array}$ & $14 q 23$ & X92669 & 0.92 & 0.046 \\
\hline $\begin{array}{l}\text { RBQ1 retinoplastoma binding } \\
\text { protein* }^{*}\end{array}$ & $\begin{array}{l}\text { 16p12- } \\
\text { p11.2 }\end{array}$ & $\mathrm{X} 85133$ & 1.0 & 0.042 \\
\hline RBQ-3 & $1 q 32$ & X85134 & 1.0 & 0.015 \\
\hline $\begin{array}{l}\text { insulin-like growth factor-binding } \\
\text { protein } 3 \text { precursor (IGF-binding } \\
\text { protein 3; IGFBP3; IBP3)† }\end{array}$ & 7p13-p12 & M31159 & 1.2 & 0.033 \\
\hline $\begin{array}{l}\text { Peptidyl-prolyl cis-transisomerase } \\
\text { nima-interacting } 1 \text { (PIN1) }\end{array}$ & $19 \mathrm{p} 13$ & U49070 & 1.3 & 0.018 \\
\hline $\begin{array}{l}\text { NEDD5 protein homolog; DIFF6; } \\
\text { KIAA0158 }\end{array}$ & $2 q 37$ & D63878 & 1.3 & 0.026 \\
\hline $\begin{array}{l}\text { glutathione S-transferase mu1 } \\
\text { (GSTM1; GST1); HB subunit 4; } \\
\text { GTH4 }\end{array}$ & 1p13.3 & $\mathrm{X} 08020$ & 1.4 & 0.032 \\
\hline PDCD2 & $6 q 27$ & S78085 & 1.4 & 0.027 \\
\hline $\begin{array}{l}\text { Ubiquitin-conjugating enzyme E2 32- } \\
\text { kDa complementing protein; } \\
\text { ubiquitin-protein ligase; ubiquitin } \\
\text { carrier protein; CDC34 }\end{array}$ & $19 p 13.3$ & L22005 & 1.7 & 0.047 \\
\hline $\begin{array}{l}\text { glutathione S-transferase A1 (GTH1; } \\
\text { GSTA1); HA subunit 1; GST-epsilon }\end{array}$ & 6p12.2 & M25627 & 1.9 & 0.011 \\
\hline $\begin{array}{l}\text { Wee1Hu CDK tyrosine 15-kinase; } \\
\text { wee-1-like protein kinase }\end{array}$ & $\begin{array}{l}11 \mathrm{p} 15.3- \\
\mathrm{p} 15.1\end{array}$ & U10564 & 2.0 & 0.039 \\
\hline CDC10 protein homolog & $\begin{array}{l}\text { 7p14.3- } \\
\text { p14.1 }\end{array}$ & S72008 & 2.3 & 0.030 \\
\hline $\begin{array}{l}\text { TNF-alpha converting enzyme } \\
\text { (TACEA); transmembrane }\end{array}$ & $2 \mathrm{p} 25$ & U69611 & 2.7 & 0.015 \\
\hline
\end{tabular}




\begin{tabular}{|l|l|l|l|l|}
\hline $\begin{array}{l}\text { metalloproteinase/disintegrin; } \\
\text { adamalysin }\end{array}$ & & & & \\
\hline c-raf proto-oncogene & $3 p 25$ & X03484 & 3.3 & 0.032 \\
\hline $\begin{array}{l}\text { G2/mitotic-specific cyclin B1 } \\
\text { (CCNB1) }\end{array}$ & $5 q 12$ & M25753 & 3.6 & 0.037 \\
\hline
\end{tabular}

Table 2 Legend

* denotes genes also significantly regulated in SY5Y exposed to MPP ${ }^{+}$alone (see Table 1 part A).

† denotes genes significantly regulated during exposure to both LY 294002 and UO 126.

"Max fold change from baseline" refers to the maximum change in signal observed for a given gene over all time points examined as compared to average (mean) signal observed in control cells. 
Chapter 2 Table 3

A. Genes regulated by MPP+ and by MPP+/LY 294002 in combination

\begin{tabular}{|l|l|}
\hline Gene & Function \\
\hline ras-related protein RAB-32 & Vesicular trafficking; GTPase \\
\hline MAP kinase-activated protein kinase 2 & Intracellular signal transduction \\
(MAPKAP kinase 2; MAPKAPK-2) & \\
\hline
\end{tabular}

B. Genes regulated by MPP+ and by MPP+/UO126 in combination

\begin{tabular}{|l|l|}
\hline Gene & Function \\
\hline sulfate proteoglycan; LDC & extracellular matrix (ECM) component \\
\hline LIM domain kinase 1 (LIMK-1) & $\begin{array}{l}\text { mediates Rho/actin cytoskeleton } \\
\text { signaling via phosphorylation of cofilin }\end{array}$ \\
\hline $\begin{array}{l}\text { 94-kDa glucose-regulated protein } \\
\text { (GRP94) }\end{array}$ & $\begin{array}{l}\text { adenotin, cell-surface 96 kDa } \\
\text { glycoprotein; binds adenosine; similar } \\
\text { to various stress-induced proteins }\end{array}$ \\
\hline Casein kinase I alpha isoform (CKI- & serine/threonine protein kinase, has \\
RBQ1 retinoblastoma binding protein & broad specificity \\
\hline
\end{tabular}


C. Genes regulated by LY 294002 and UO 126 in combination

\begin{tabular}{|l|l|}
\hline Gene & Function \\
\hline protein phosphatase PP2A 55-kDa & Regulatory subunit of protein phosphatase \\
regulatory subunit neuronal isoform; & 2 \\
protein phosphatase PP2A B & \\
subunit beta; beta-PR55 & \\
\hline $\begin{array}{l}\text { Iysosomal acid lipase/cholesteryl } \\
\text { ester hydrolase precursor (LAL); } \\
\text { acid cholesteryl ester hydrolase; } \\
\text { sterol esterase; lipase A (LIPA); } \\
\text { cholesteryl esterase† }\end{array}$ & ester core lipids \\
\hline $\begin{array}{l}\text { Iysosome membrane protein II } \\
\text { (LIMP II); 85-kDa lysosomal } \\
\text { membrane sialoglycoprotein } \\
\text { (LGP85); CD36 antigen-like 2 } \\
\text { (CD36L2)† }\end{array}$ & significant homology with collagen type I \\
\hline $\begin{array}{l}\text { insulin-like growth factor-binding } \\
\text { protein 3 precursor (IGF-binding } \\
\text { protein 3; IGFBP3; IBP3)† }\end{array}$ & may bind to and modulate insulin-like \\
growth factor activity; induces early \\
apoptosis and has potential tumor \\
\hline
\end{tabular}




\section{Chapter 2 Figure Legends}

Figure 1. (Left) Gene expression changes in SY5Y cells over the time course (5180

minutes) of MPP+ exposure, as represented by Cluster analysis ( $\mathrm{N}=378$ genes).

Black indicates no change in expression relative to control (no MPP+); increasing intensities of green and red indicate greater depression (maximum observed=8.7 fold) and elevation (maximum observed=8.6 fold) of expression, respectively. The locations of the four mathematical clusters are indicated by the color-coded vertical bars to the right. (Right) Plots of mean expression ratios (experimental/control) for genes in each mathematical cluster at each time point of MPP+ exposure. Cluster 1, N=18 genes; cluster 2, N=171; cluster 3, N=83 genes; cluster 4, $\mathrm{N}=106$ genes.

Figure 2. Gene expression changes in control SY5Y compared to mtDNA-free $\rho^{0}$ cells at selected time points of MPP+ exposure $(15,60,120$ minutes), as represented by Cluster analysis. Color code is same as for Figure 1, with the addition of gray, which indicates that the gene is not represented in the $\rho^{0}$ group.

Figure 3. $(A, B)$ Scatterplots comparing the normalized basal gene expression levels in SY5Y control cells ( $\mathrm{X}$-axis) compared to expression levels in SY5Y cells pretreated with (A) U0126, an inhibitor of MEK/ERK; or (B) LY294002, an inhibitor of PI3-kinase. (C) Survival curves of SY5Y cells incubated for 24 hours 
with U0126 or LY294002 either in the absence of or presence of MPP+. Data adapted from Halvorsen, et al, 2002. 


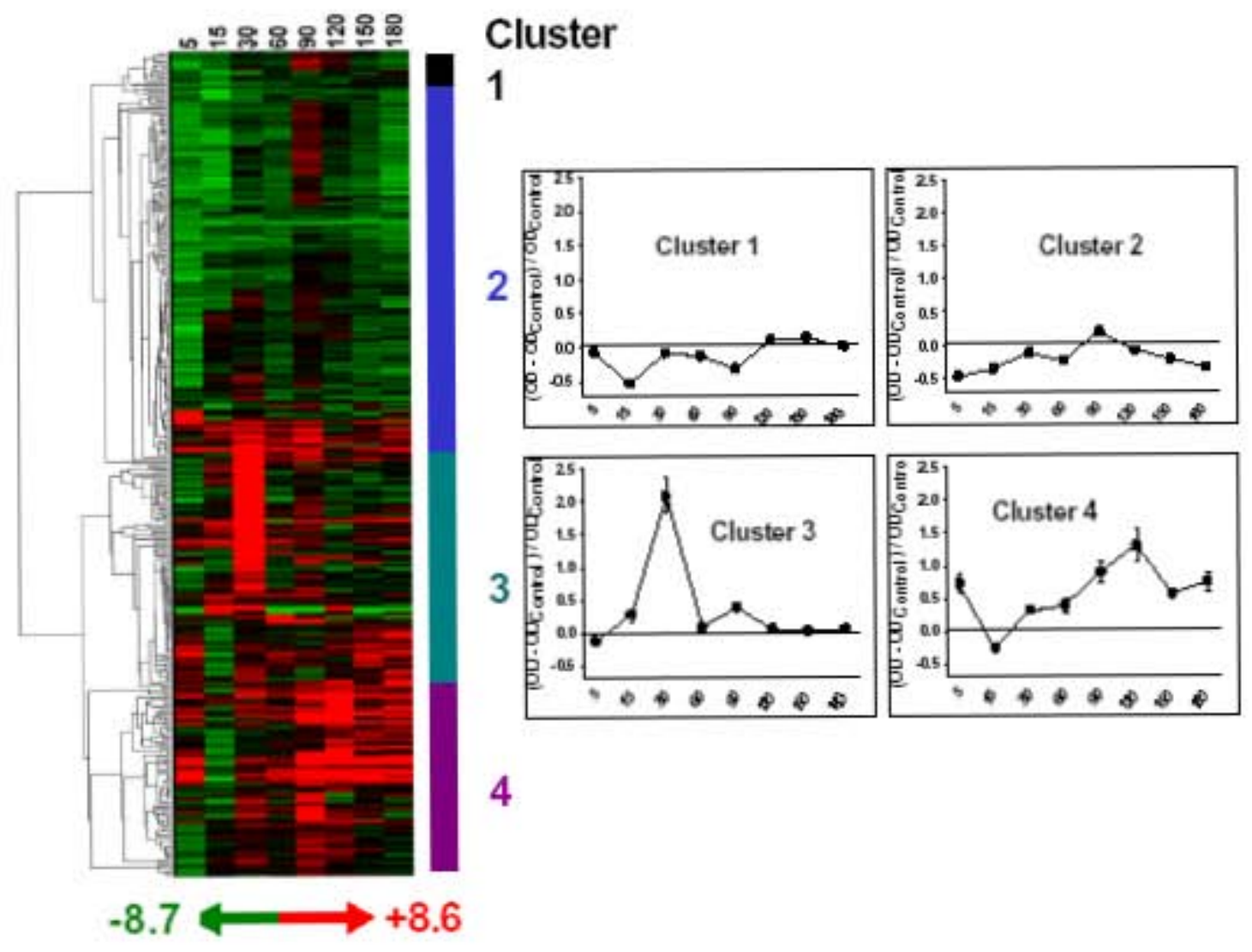

Chapter 2 Figure 1. 
15601201560120

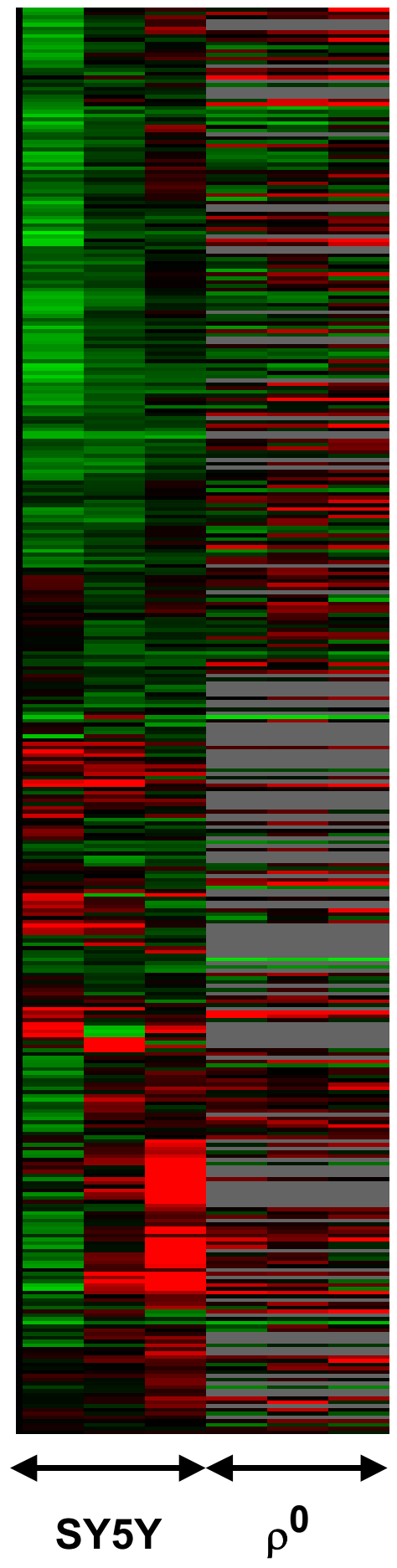

Chapter 2 Figure 2. 

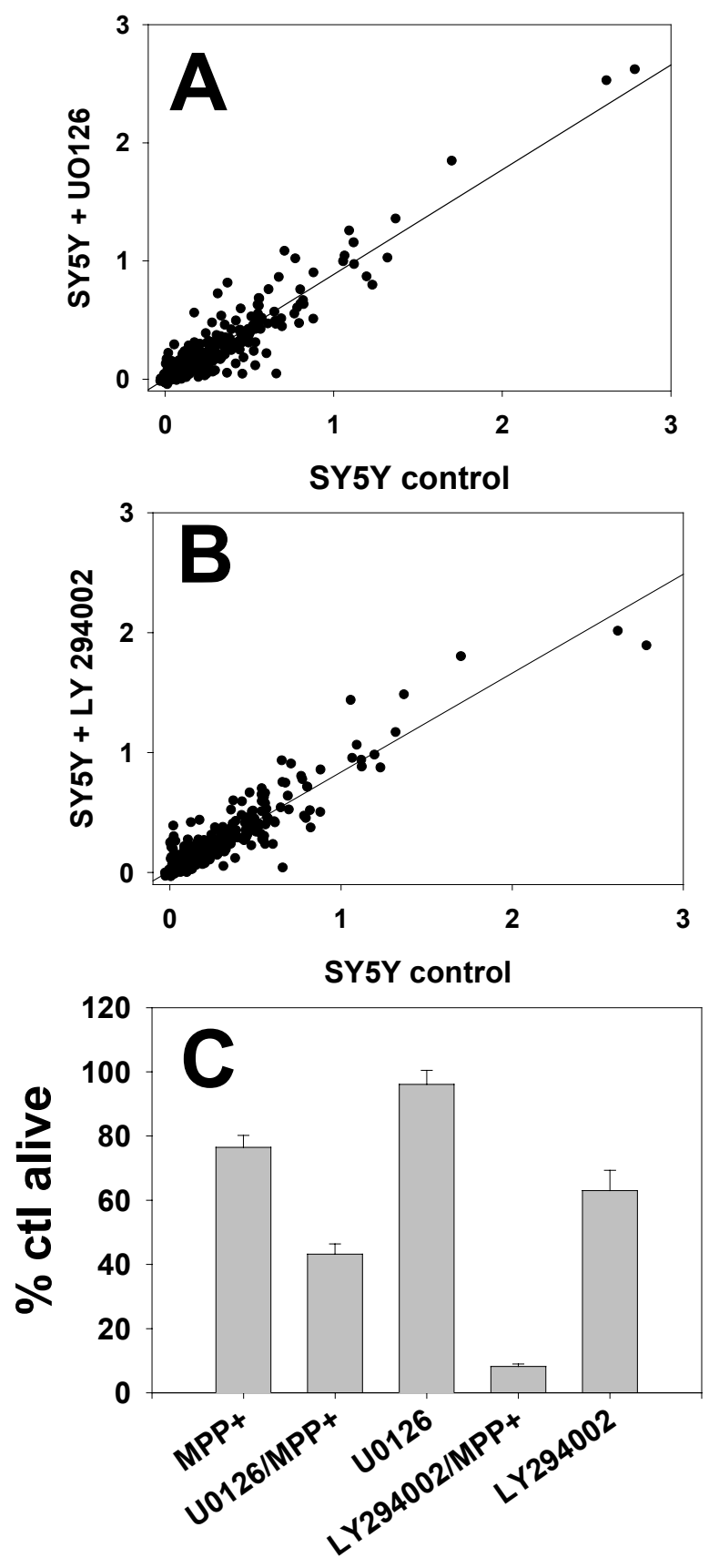

Chapter 2 Figure 3. 


\section{Chapter 3}

\section{Applications of novel molecular technologies in the study of gene expression response to MPP+.}

\section{Introduction}

Following our analysis of the dependence of nuclear gene expression response on mitochondrial DNA integrity, we were left with a number of intriguing potential directions for follow-up studies. The rapidity and robustness of gene expression change in response to MPP+ in our limited gene set made us very interested in how these results would or would not generalize to a larger set of genes. The profound changes in the gene expression responses that were effected by chemical modulators of cell signaling reinforced our conviction that such responses are complex, multifactorial events that would be difficult, at best to dissect through conventional molecular and biochemical techniques. As compared to our nylon array study, we observed that larger numbers of genes were altered in their expression as a result of MPP+ exposure; this is not surprising considering the expansion of the number of genes assayed per array. Given the dependence in MPP+-exposed cells of molecular events and cell signaling upon both nitric oxide and NFkB activation that has been demonstrated by previous work in our group, we tested the hypothesis that transcriptosome changes following MPP+ exposure might have similar dependencies. We were intrigued to find that $\sim 90 \%$ of a large number of genes that were regulated by MPP+ appeared to be dependent on NO or NFkB translocation to the nucleus. 
Not only were these findings consistent with previous observations, but they suggested that mitochondria might use endogenously produced $\mathrm{NO}$ as a signaling molecule to communicate ETC impairment to the nuclear transcriptosomal machinery, and that NFkB transcription factor played a significant upstream regulatory role in this process. We also found that the magnitude of gene response was much greater at the $15 \mathrm{~m}$ timepoint than that at $90 \mathrm{~m}$ of $\mathrm{MPP}+/$ drug exposure. This datum reinforces the notion, first articulated following our nylon array work, that gene expression changes in response to MPP+ are very rapid, and can change relatively quickly (or at least more quickly than a process that might rely on turnover and synthesis of proteins de novo). One potential weakness of our protocol is that we continue to make use of the MPP+ SY5Y model; as noted previously the acute nature of cell death in this model does not follow a similar timecourse as the prolonged attrition of cells that occurs in the PD process. While acknowledging this fact, the ease of availability and reproducibility that we have observed with RNA samples from SY5Ys made them the best available choice for this initial study of the relationship between acute mitochondrial bioenergetic impairment and changes in the cell transcriptosome.

While a comprehensive evaluation of every gene expression level measured is clearly beyond the scope of our work, we discuss several genes identified in the microarray screens that may play as yet unidentified roles in MPP+ neurotoxicity. Finally, we describe our efforts to alter the course of MPP+ induced cell death through several RNA interference methods, in addition to 
presenting a preliminary analysis of the ontological categorizations of transcriptionally regulated genes in our experimental system using a publicly available software package, GoMiner.

\section{Materials and Methods}

Cell Culture and Treatment.

SH-SY5Y cells were obtained from the American Tissue Culture Collection (ATCC; Manassas, VA) and maintained in culture as previously described (Cassarino et al., 1997; Cassarino et al., 2000; Swerdlow et al., 1996). $3 \times 10^{6}$ SY5Y cells were placed onto $10 \mathrm{~cm}$ tissue culture plates (Greiner Bio-One; Longwood, $\mathrm{FL}$ ) and maintained at $37^{\circ} \mathrm{C}$ for 24 hours prior to exposure to $5 \mathrm{mM}$ $\mathrm{MPP}^{+}$(Sigma-Aldrich; St. Louis, MO) for $15 \mathrm{~m}$ or $90 \mathrm{~m}$ in the presence or absence of $15 \mathrm{~m}$ of pretreatment with either the nitric oxide scavenger 2-Phenyl-4,4,5,5tetramethylimidazoline-3-oxide-1-oxyl (PTIO) (Akaike et al, 1993) at $1 \mathrm{mM}$ or the nuclear factor kappa B nuclear translocation inhibitor peptide SN50 (Lin et al, 1995) at $18 \mu \mathrm{M}$ (both obtained from Calbiochem). Cultures were also exposed to carrier (phosphate buffered saline) alone, and to SN50 or PTIO in the absence of $\mathrm{MPP}+$

RNA Isolation.

Total RNA was isolated from cell cultures immediately following completion of any $\mathrm{MPP}^{+} / \mathrm{SN} 50 / \mathrm{PTIO}$ exposure using the RNEasy Mini system 
(Qiagen; Valencia, CA), according to manufacturer's protocol. RNA samples were archived at $-80^{\circ} \mathrm{C}$.

\section{cDNA Arrays.}

The general experimental plan for our microarray protocol is diagrammed in Figure 1. cDNA probes were prepared from each total RNA sample and hybridized to "human 19k3" cDNA microarrays (University of Toronto Microarray Centre, Toronto, Ontario) using the Perkin Elmer MicroMax kit according to manufacturer's protocol. Briefly, each RNA isolate is reverse transcribed into cDNA that incorporates either fluorescein or biotin moieties on cytosine residues via provision of deoxycytosine so labeled. In our experiments, cDNA from control SY5Y samples always bears fluorescein, and experimental samples are labeled with biotin. These labeled cDNA populations are mixed and hybridized to the microarrays overnight under constant temperature and humidity. Following a series of washes, the arrays are exposed to an anti-fluorescein antibodyhorseradish peroxidase conjugate. This localizes HRP to the regions of fluorescein-cDNA hybridization. Application of a Cyanine 3-conjugated tyramide compound catalyzes the rapid deposition Cyanine 3 tyramide immediately adjacent to the immobilized HRP. In this manner, many fluorescent molecules are deposited at the site of hybridization, resulting in signal amplification relative to using cDNAs that are synthesized to directly incorporate fluorescent moieties. Following Cy3 deposition, residual HRP is inactivated and the array is exposed to streptavidin HRP, which binds to the biotinylated nucleotides. In a similar 
reaction to the Cy3 process described, the conjugated HRP activity is used to catalyze deposition of Cy5 tyramide. In this way, the signals from the 2 RNA samples are amplified with separate fluorophores on the same array. Two hybridizations were carried out in which both Cy3 and Cy5 fluorophores were associated with control (untreated) SY5Y cDNA to determine the prevalence of fluorophore specific alterations ("dye-swap" effects) on gene expression signals. Array hybridization fluorescence digital images were acquired and saved in .TIF format using ScanArray software package with a ScanArray 4000 microarray scanner (Packard BioChip Technologies).

\section{Statistical Analysis.}

A major limitation of many published microarray studies is limited biological and technical replication, with complex statistical approaches used to extract significance in the absence of replication. Our approach to avoid this limitation was to perform for each experimental condition three independent biological replications (cell culture, drug exposure, RNA isolation) and duplicate hybridizations for each independent biological sample. Each hybridization experiment comprised the hybridization of cDNA samples derived from experimental and control (untreated) SY5Y cells, labeled with cyanine 5 or cyanine 3 fluors respectively, to cDNA microarray slides. Fluorescence intensity and background levels for each gene hybridization were extracted from TIFF files using the QuantArray software package. Net hybridization intensities for individual genes were obtained through background subtraction followed by 
normalization to the mean hybridization signals for each individual microarray in toto, allowing direct comparisons across experimental conditions. Following normalization, gene hybridization intensities greater than $2 x$ the signal obtained from a standardized region of the array with no adsorbed DNA were used in downstream analyses. For experiments in which SY5Y cells were treated with $\mathrm{MPP}^{+}, \mathrm{PTIO}$ or SN50 alone, genes that exhibited a \pm 2-fold change were considered to have altered expression. For experiments involving comparison of $\mathrm{MPP}^{+}$alone to $\mathrm{MPP}^{+}$in combination with $\mathrm{PTIO}$ or SN50, Student's t-tests were used to compare ratios of hybridization intensities of experimental and control samples for each individual gene spot in each treatment condition. P values were calculated for each gene, ranked from least to greatest and compared to their corresponding critical $p$ values obtained with the Benjamini-Hochberg calculation $\left(p_{B H}\right)$ with significance set at $p \leq 0.05$. $P$ values less than $P_{B H}$ were considered significant.

The control-control hybridizations to check for dye-swap effects were processed in the same way as described for the other hybridization experiments above, and array-normalized, background subtracted intensity ratios Cy5:Cy3 were compared. The results are presented in Figure 2.

\section{Gene Ontology Analysis}

We attempted to define biological meaning to the transcript changes by looking for correlations among gene ontology groups in our array datasets with "GoMiner" (Zeeberg, et al, 2003; http://genomebiology/2003/4/4/R28), an open- 
source program that organizes gene lists in the context of Gene Ontology categories (Ashburner, et al, 2000). GENBANK ID's of our microarray clones were annotated to gene symbols using DAVID (Database for Annotation, Visualization and Integrated Discovery; Dennis, et al, 2003; http://genomebiology/2003/4/9/R60). The gene symbols from DAVID were then annotated with a $(+1)$ or $(-1)$ to indicate increased or decreased expression, respectively, and analyzed with the GoMiner engine. The output from GoMiner includes both grouping of experimental genes into Gene Ontology groups and a "relative enrichment" (RE) calculation for each Gene Ontology group $(\mathrm{RE}=\{$ (changed genes in category/total genes in category)/(changed genes in chip/total genes in chip)\}). We used the GoMiner RE values to test the hypothesis that two different experimental manipulations altered Gene Ontology groups in similar ways by constructing correlation plots of the RE values for genes increased or decreased in expression in the different experimental conditions. We used RE values for the second order nodes in the GoMiner output for the initial correlations. For second order nodes where $n>1000$ genes, we also analyzed correlations for the third order nodes in the GoMiner output.

\section{RT-PCR.}

cDNA was prepared from experimental total RNA samples using the Advantage RT-for-PCR kit (Clontech Laboratories) using oligo(dT)primers according to the manufacturers directions. PCR was carried out using the Advantage cDNA PCR kit (Clontech Laboratories), with the indicated PCR 
primers according to manufacturer's directions. Samples were held at $94^{\circ} \mathrm{C}$ for 1 minute, followed by 33 cycles of $\left[94^{\circ} \mathrm{C}-30\right.$ seconds, $68^{\circ} \mathrm{C}-2$ minutes], followed in turn by a final $68^{\circ} \mathrm{C}$ for 5 minutes. PCR products were separated on $2 \%$ agarose gels and visualized by ethidium bromide incorporation. SYN2 and GA3PDH were selected for RT-PCR verification of both statistically significant and nonsignificant gene expression changes.

SN50 effects on MPP+ induced cell death.

Cultures of $10^{4}$ SY5Y cells were plated in individual wells of Corning 96 well clear bottom, black plates with DMEM and 10\% FBS. Cells were treated with either MPP+ $(5 \mathrm{mM})$, SN50 peptide $(18 \mu \mathrm{M})$ or a combination of the two for time periods ranging from 2 to $24 \mathrm{~h}$. Cell viability was measured using a 3-(4,5dimethylthiazol-2-yl)-2,5-diphenyl tetrazolium bromide-based spectrophotometric assay. The results are depicted in Figure 3. Data compiled by Dr. Jameel Dennis indicate that PTIO at the concentrations used in the present experiments has no measurable effect on MPP+ induced cell death through $24 \mathrm{~h}$ of concurrent exposure (Dennis and Bennett, 2003).

RNA interference.

As described in chapter 1, RNA interference refers to the catalyzed destruction of specific RNA sequences via the introduction of short double stranded RNA species of approximately $10-20$ base pairs, so-called short interfering RNAs (siRNAs). The general sequence of events in this process is 
depicted in Figure 4. This technology was appealing to us as it presented a seemingly direct route to modulation of the gene expression signals we observed in our microarray work, and evaluation of their effects on cell death in our model system. Several methods were used to generate siRNA directed against SYN2, DDIT3, FGD1 and RET gene products. Methods employed included chemical synthesis of siRNA, in vitro transcription of complementary dsRNAs that were then subjected to RNAse III digestion to produce siRNAs, and transfection of SY5Y cells with vectors designed to drive constitutive expression of siRNA hairpin species under the control of RNA polymerase II.

To date, we have had siRNAs chemically synthesized for SYN2, DDIT3 and FGD1, though none have proven effective in decreasing their respective RNA signals as measured by RT-PCR assay. Figure 5 depicts typical RT-PCR results following transfection of SY5Y cells with our chemically synthesized siRNAs. In part $A$, lane $L$ is a 100 bp DNA ladder, lane 1 is a positive control PCR product, lanes 2 and 3 are FGD1 RT-PCR products from SY5Y cells transfected with control and FGD1 siRNA, respectively, and lanes 4 and 5 are RT-PCR products from SY5Y cels transfected with control and DDIT3 siRNA, respectively. In part B of Figure 5, lane 1 is a positive control PCR product, while lanes 2 and 3 contain SYN2 PCR products from SY5Y cells transfected with control and SYN2-directed siRNAs, respectively. Lanes 4 and 5 and lanes 6 and 7 duplicate lanes 2 and 3 . The lack of attenuation of signal in any of the gene product-directed siRNA transfected samples suggests that the siRNAs are ineffective at silencing their targets. 
For in vitro transcription, T7 promoters were appended to FGD1 and SYN2 PCR products and used to generate an approximately 200 bp double stranded RNA corresponding to a C-terminal region of the transcript, which was subjected to RNAse III digestion. The products of this digestion were transfected into SY5Y cells, and they were not found to be efficacious in lowering FGD1 expression by RT-PCR; in Figure 6, Lane "L" contains a 100 bp DNA ladder, lane 1 is a positive control PCR product, lanes 2 and 3 are SYN2 PCR products from SY5Y cells transfected with control siRNA or FGD1 siRNA respectively, and lanes 4 and 5 duplicate lanes 2 and 3 . The presence of PCR products in both control and directed siRNA transfected samples suggests that the silencing protocol is ineffective.

Vectors encoding hairpin siRNAs directed against C-terminal regions of SYN2, RET, FGD1 and DDIT3 gene products were cloned into $p$ Silencer expression vectors (Ambion), and transfected into SY5Y cells, but unexpectedly high cellular toxicity was observed following hygromycin selection of transfected cells, despite high estimates of transfection efficiency and multiple successful transfection experiments utilizing the same cell line with different plasmid expression vectors in the past. No positive transformants were recovered following hygromycin selection with any of the pSilencer constructs. A diagram of the $p$ Silencer vector used is presented in Figure 7. 


\section{$\underline{\text { Results }}$}

cDNA Microarrays

Following background subtraction, normalization, log transformation and comparison to control RNA hybridization, a total of 533 genes of 19,200 total $(2.7 \%)$ were found to have increased or decreased 2 -fold in response to 15 minutes of $5 \mathrm{mM}$ MPP+ exposure. These genes are listed, along with available LocusLink identifers and Gene Ontology annotations, if available, in Appendix A. Of these, were 409 were upregulated and 124 were downregulated. Of these $533,65(11.7 \%)$ were found to be significantly altered in their expression in the presence of PTIO, and $93(16.7 \%)$ were found to be significantly altered in their expression in the presence of SN50 peptide. These genes are listed, along with available LocusLink and Gene Ontology information in Appendices B and C. The relative sizes of these gene groups are presented in Figure 8, part A.

At 90 minutes of MPP+ treatment, 143 genes $(0.7 \%)$ were found to have increased or decreased 2-fold or more as compared to control. These genes and available annotations for them are listed in Appendix D. Of these 143 genes, 136 were upregulated and 7 were downregulated. Of these 143, 2 (cellular retinoic acid-binding protein 2:CRABP2 and integrin alpha 1:ITGA1) were found to be significantly altered in their expression in the presence of PTIO with MPP+, and none changed significantly in the presence of SN50 peptide with MPP+.

Forty genes were found to have changed $+/-2$ at both $15 \mathrm{~m}$ and $90 \mathrm{~m}$ MPP+ timepoints. These genes and available annotations are listed in Appendix 
E. The relative representation of these genes in relation to the total number of genes assayed by cDNA microarray is presented in Figure 8, part B.

A gene by gene comparison of between the $15 \mathrm{~m} \mathrm{MPP}+$ and $15 \mathrm{~m} \mathrm{MPP}+$ with PTIO conditions revealed 706 genes differentially regulated. This response, while similar in magnitude to that observed in the comparison of $15 \mathrm{~m} \mathrm{MPP}+$ to control in numbers of genes, comprises an almost completely distinct set of genes $(65 / 706$, or greater than $90 \%$ dissimilar). These genes and any available annotative information are listed in Appendix F. When a similar comparison between the $15 \mathrm{~m} \mathrm{MPP+}$ and $15 \mathrm{~m} \mathrm{MPP+}$ with SN50 condition is carried out, 492 genes are found to be differentially regulated. These genes are listed in Appendix G. At $90 \mathrm{~m}$ of MPP+ treatment, the presence of PTIO induces differential expression of 74 genes listed in Appendix H. Only one gene, ADAR3, is differentially expressed at $90 \mathrm{~m}$ of MPP+ treatment in the presence of SN50 peptide. 73 genes were noted to be significantly changed between the $15 \mathrm{~m}$ $\mathrm{MPP}+$ condition and 15m MPP+ with either PTIO or SN50. These genes and any available annotative information are presented in Appendix I.

\section{Gene Ontology Correlations}

The correlation plots for GoMiner RE values revealed limited correlations between Gene Ontology families in the experiments where NO was scavenged with PTIO during MPP+ exposure (Figure 9) or NFkappaB translocation to the nucleus was blocked by SN50 peptide during MPP+ exposure (Figure 10). The limited correlations were more prominent in the plots of third order node RE 
values and were seen for genes both downregulated (green) and upregulated (red). These Gene Ontology findings are consistent with the analysis of transcriptosome responses on a gene-by-gene basis in these experiments, in which the majority of genes altered by MPP+ alone were not altered when PTIO or SN50 were included with the MPP+.

We then compared the Gene Ontology families that were sensitive to the presence of the NfkappaB antagonist SN50 to those that were sensitive to the presence of the NO scavenger PTIO when SH-SY5Y cells were exposed to $\mathrm{MPP}+$. In contrast to the previously described limited RE correlations between transcriptosome responses of PTIO+MPP+ or SN50+MPP+ with those of MPP+ alone, we observed very strong correlations between the RE values of SN50sensitive genes and PTIO-sensitive genes (Figure 11).

\section{Discussion}

As in our previous nylon-based array experiments, we noted a rapid and robust nuclear gene expression response to the application of the mitochondrial neurotoxin MPP+ to SH-SY5Y cells. Upon examination of our data, it becomes apparent that there are major differences in gene expression during the time course of MPP+ exposure. The magnitude of expression changes is considerably higher at $15 \mathrm{~m}$ than at $90 \mathrm{~m}$, which is in accord with a key finding of our nylon array work, that expression change is greatest early in the timecourse of MPP+ exposure. The fact that only 40 genes are changing $+/-2$ fold at both 
$15 \mathrm{~m}$ and $90 \mathrm{~m}$ would argue strongly for the presence of distinct "early" and "late" effects of MPP+ on gene expression. Functional categorizations available for genes that change at the $15 \mathrm{~m}$ timepoint are presented in Table 1 and those for the $90 \mathrm{~m}$ timepoint in Table 2. It is evident that the numbers of genes listed in each of these tables is considerably smaller than the total number of genes identified to change $+/-2$ fold for each condition; this illustrates a major issue confronting those working with all types of genomic datasets, that of curation. The ontological categorizations that are attached to a given gene ID are subjective; that is to say, they are susceptible to errors of classification and omission due to the fact that they must be assigned by one or at most a small group of persons acting together, relying in large part only on publicly accessible data of widely varying quality. This makes it untenable, in the majority of cases of microarray studies of mammalian samples in particular, to derive large scale conclusions about interconnections of various metabolic and cell signaling pathways in the absence of a higher order (e.g., ontological) analysis. With this limitation in mind, we will note that the largest ontological categories represented at the $15 \mathrm{~m}$ timepoint, when the majority of changes appear to occur, are DNA binding and modifying enzymes, and protein kinases. This is in accordance with our earlier finding that the majority of early gene expression changes induced in the SY5Y cells by MPP+ are related to intracellular signalling. We also note that the finding of such rapid gene expression response is consistent with rapid mitochondrial-nuclear communication, as no experimental evidence has as yet demonstrated MPP+ to exert direct effects on nuclear gene expression, and as 
we have previously demonstrated that the nuclear gene expression response induced by MPP+ is dependent on mitochondrial status.

The most common gene ontology categories represented in the genes that are differentially expressed at $15 \mathrm{~m}$ of MPP+ treatment in the presence of PTIO are listed in Table 3, and those of genes that vary significantly in the presence of SN50 peptide at $15 \mathrm{~m}$ of concurrent MPP+ treatment are listed in Table 4. The most common categorizations in both cases are gene products that bind nucleic acids, and protein kinases, consistent with an intracellular signalling response regulating transcription of different genes.

Perhaps one of the most interesting findings in this dataset are the startling effects seen with PTIO and SN50 treatment concurrent with $15 \mathrm{~m}$ of MPP+ exposure. The number of genes differentially expressed in the presence of PTIO is roughly equivalent to the number that vary $+/-2$ fold or greater with $15 \mathrm{~m}$ MPP+ exposure as compared to control, yet the groups of genes are almost totally dissimilar. A similar effect is seen with SN50, with about $80 \%$ dissimilarity noted. These results strongly imply that the nuclear gene expression response induced by this mitochondrial neurotoxin is dependent upon nitric oxide and/or NFkB signaling. The finding that the vast majority of gene expression changes documented at both $15 \mathrm{~m}$ and $90 \mathrm{~m}$ of MPP+ exposure do not occur in the presence of inhibitors of NFkB nuclear translocation or of a nitric oxide scavenging compound suggests that these expression responses are mediated through NFkB and/or nitric oxide. As mitochondria possess intraorganellar nitric oxide synthase, these data are consistent with nitric oxide being responsible for 
signal transduction to the nucleus. NFkB's role in translocation to the nucleus and upregulation of gene transcription there is well known, and our data are consistent with it being involved in the mitochondrial-nuclear signaling that we have observed.

One important aspect of high-density microarray studies that has been neglected by many researchers is the issue of multiple hypothesis testing. When parametric statistical analyses are applied to datasets involving thousands of individual comparisons, the chance of introduction of type I (false positive) errors grows dramatically (for an excellent review, see Westfall and Young, 1993). A familiar method of compensating for this effect is the Bonferroni correction, in which the $p$ value associated with a significant result for a given test $(\alpha)$ is altered by the formula $\alpha / n$, where $\mathrm{n}$ is the number of simultaneous tests. For example, if $\mathrm{t}$ tests were used to evaluate microarray results for significance at $\alpha=0.05$, and the array comprised a total of 1000 gene spots, the $p$ value associated with statistical significance would be $0.05 / 1000$, or $5 \times 10^{-5}$. This transformation, while undeniably effective at reducing type I errrors, is generally considered to sacrifice too much statistical power in the process. Indeed, when the Bonferroni correction is applied to the $\mathrm{t}$ statistics that we calculated to compare gene expression changes with MPP+ in the presence of PTIO or SN50, a p value of $2.6 \times 10-6$ is required to achieve significance ( $\alpha$ of 0.05 divided by 19,200 gene features/array). With regards to our microarray datasets, the BenjaminiHochberg transformation, while among the least restrictive of the multiple testing 
correction algorithms available, strikes a reasonable balance between elimination of false positives and inclusiveness of potentially "real" differences in gene expression based on the number of genes that meet significance criteria. This method entails the calculation of $p$ values followed by their ranking from lowest to highest, 1 through $\mathrm{n}$. For type I error control at level $\alpha$, a critical $p$ value is calculated for each ranked position in the list, by mulitplying the rank by the desired $\alpha$, and dividing by $\mathrm{n}$, the total number of rankings. Initial $\mathrm{p}$ values for each test are then compared to the $p$ critical value for the test's ranking. $\mathrm{P}$ values less than or equal to $p$ critical are considered to be significant, with the chance of false discovery error less than or equal to $\alpha$. For our experiments, $\alpha$ is always set equal to 0.05 .

Several lab groups have recently described methods using Bayesian networks for the statistical analysis of microarray data. These methods have the advantage of being able to assign unambiguous confidence intervals for differences between unlimited numbers of sets of gene expression data, and are particularly useful when the number of samples is low and/or some expression values are missing, but suffer from the same potential for false-discovery errors as more conventional parametric analyses do when making thousands of comparisons simultaneously. We have preliminarily evaluated one publicly available software package (Bayesian Analysis of Gene Expression Levels, BAGEL, Townsend and Hartl, 2002) which performs such analyses, and at this 
time, in light of the quality of our data sets, do not feel that it offers us significant improvements over the parametric methods that we have presented here.

Multiple laboratories have reported significant "dye-swap" effects, in which gene expression levels recorded for particular genes in microarray analyses vary depending on the fluorophore associated with the cDNA hybridized to the array. The tyramide-signal amplification protocol that we employed avoids the majority of these issues, as the fluorophore deposition is in great excess to the number of labeled nucleotides in an individual hybridized cDNA, but it was heartening to note that these effects only occur in a very small number of cases, less than $5 \%$ of all gene spots, as shown by our Figure 2 findings. From this, we can move forward with future array experiments with the tyramide system knowing that dyeswapping is not necessary in this context, saving time and expense.

It is clear from our data that major changes in regulation occur between the time points that we are studying. We began to study the generated lists for genes that displayed interesting regulatory characteristics, and that also might have relevance to neurodegenerative processes in general and PD in particular.

The first such gene that we have identified is synapsin 2 (SYN2). SYN2 is a member of the synapsin family of genes which encode neuronal phosphoproteins associated with the cytoplasmic surface of synaptic vesicles. Family members are implicated in synaptogenesis and the modulation of neurotransmitter release, suggesting a potential role in several psychiatric and neurodegenerative diseases. This member of the synapsin family encodes a neuron-specific phosphoprotein that selectively binds to small synaptic vesicles 
in the presynaptic nerve terminal. SYN2 is upregulated approximately 4 fold in response to $15 \mathrm{~m}$ of MPP+ treatment.

The second gene that we have singled out for downstream analysis is faciogenital dysplasia 1 (FGD1). FGD1 can bind specifically to the Rho family GTPase Cdc42Hs and stimulate the GDP-GTP exchange of the isoprenylated form of $\mathrm{Cdc} 42 \mathrm{Hs}$. It also stimulates the mitogen activated protein kinase cascade leading to c-Jun kinase SAPK/JNK1 activation. FGD1 has an essential role in embryonic development, and FGD1 gene mutations result in the human developmental disorder, Aarskog-Scott syndrome. We were especially interested in FGD1 in light of the key roles played by the MAP kinase cascade in $\mathrm{MPP}+$ signalling described by previous work in the CSND. FGD1 is upregulated approximately 3 fold in response to $15 \mathrm{~m}$ of MPP+ treatment.

The third gene to be considered is DNA-damage-inducible transcript 3 (DDIT3). It is a nuclear transcription factor shown to be upregulated in a variety of situations where genomic damage is induced by oxidative stress and other means. This makes DDIT3 a potential transducer of oxidative damage signals from mitochondria to the nucleus, a process that we have shown is dependent on mitochondrial DNA status. DDIT3 is downregulated to approximately $33 \%$ of control levels in response to $15 \mathrm{~m}$ of MPP+ treatment.

The final gene that we identified is the ret proto-oncogene (RET). This gene is a member of the cadherin superfamily, and encodes a receptor tyrosine kinase, which are cell-surface molecules that generally transduce signals for cell growth and differentiation. RET plays a crucial role in neural crest development, 
and it can undergo oncogenic rearrangement both in vivo and in vitro. Mutations in this gene are associated with the disorders multiple endocrine neoplasia, type IIA, multiple endocrine neoplasia, type IIB, Hirschsprung disease, and medullary thyroid carcinoma. Alternative splicing has been described, with at least 4 transcript variants. Most importantly for PD considerations, RET comprises one half of the cell surface receptor complex for glial-derived neurotrophic factor (GDNF), which was identified in 1993 as a key survival factor for dopaminergic neurons in the striatum. Several clinical trials are underway in which GDNF is infused into the putamens of PD patients in hopes of slowing the neuronal cell death processes ongoing. If mitochondrial neurotoxicity is a key component of the apoptotic process, RET may be one of the molecules that is under its regulation. In our dataset, RET is found to be decreased to approximately $29 \%$ of control expression after 15m of MPP+ treatment.

The fact that we have encountered great difficulties in implementing our RNAi strategy is disheartening, but we still feel that it provides the most logical method for directly and specifically mediating gene expression in a wide variety of experimental systems, and hope to pursue it in the future in this and other disease models. Initial publications were highly enthusiastic regarding the ease of use and efficacy of these technologies, but subsequent experience in our laboratory and others, is proving contrary.

Finally, our intial experiences with the GoMiner package have proven extremely valuable. The fact that groups of genes may be highly functionally correlated, while being highly dissimilar at the individual gene identity level, 
encourages us to pursue this type of analysis further. We believe that directed analysis of curated gene families provides the best possible system to date for attempting to assign biological significance to the growing amount of publicly available microarray data. With regards to our data, the major finding made possible by GoMiner, that both nitric oxide and NFkB seem to regulate the same biological response to MPP+ in SY5Y cells represents a novel investigative tack that may well prove valuable in the design of future experiments. 
Chapter 3 Table 1. Ontological categorization of genes that change $+/-2$ fold after 15m MPP+ exposure.

\begin{tabular}{|l|c|}
\hline \multicolumn{1}{|c|}{ CATEGORY } & NUMBER \\
\hline purine nucleotide binding & 36 \\
\hline DNA binding & 29 \\
\hline $\begin{array}{l}\text { hydrolase activity, acting } \\
\text { on acid anhydrides }\end{array}$ & 16 \\
\hline $\begin{array}{l}\text { transferase activity, } \\
\text { transferring phosphorus- } \\
\text { containing groups }\end{array}$ & 16 \\
\hline protein kinase activity & 14 \\
\hline $\begin{array}{l}\text { hydrolase activity, acting } \\
\text { on ester bonds }\end{array}$ & 13 \\
\hline RNA binding & 11 \\
\hline peptidase activity & 11 \\
\hline $\begin{array}{l}\text { transmembrane receptor } \\
\text { activity }\end{array}$ & 11 \\
\hline calcium ion binding & 10 \\
\hline \begin{tabular}{l} 
cytoskeletal protein \\
binding \\
\hline $\begin{array}{l}\text { transition metal ion } \\
\text { binding }\end{array}$
\end{tabular} & 10 \\
\hline
\end{tabular}


Chapter 3 Table 2. Ontological categorization of genes that change $+/-2$ fold after 90m MPP+ exposure.

\begin{tabular}{|l|r|}
\hline CATEGORY & NUMBER \\
\hline DNA binding & 9 \\
\hline calcium ion binding & 5 \\
\hline purine nucleotide binding & 4 \\
\hline $\begin{array}{l}\text { transition metal ion } \\
\text { binding }\end{array}$ \\
\hline $\begin{array}{l}\text { hydrolase activity, acting } \\
\text { on acid anhydrides }\end{array}$ \\
\hline $\begin{array}{l}\text { hydrolase activity, acting } \\
\text { on ester bonds }\end{array}$ \\
\hline protein kinase activity & 3 \\
\hline $\begin{array}{l}\text { transferase activity, } \\
\text { transferring phosphorus- } \\
\text { containing groups }\end{array}$ & 3 \\
\hline lamin binding \\
\hline magnesium ion binding
\end{tabular}


Chapter 3 Table 3 . Ontological categorization of genes significantly different between 15m MPP+ exposure and 15m MPP+ with PTIO.

\begin{tabular}{|c|c|}
\hline CATEGORY & NUMBER \\
\hline nucleotide binding & 32 \\
\hline DNA binding & 26 \\
\hline $\begin{array}{l}\text { transferase activity, transferring } \\
\text { phosphorus-containing groups }\end{array}$ & 22 \\
\hline protein kinase activity & 18 \\
\hline $\begin{array}{l}\text { hydrolase activity, acting on acid } \\
\text { anhydrides }\end{array}$ & 14 \\
\hline RNA binding & 13 \\
\hline peptidase activity & 12 \\
\hline transition metal ion binding & 12 \\
\hline calcium ion binding & 9 \\
\hline $\begin{array}{l}\text { hydrolase activity, acting on ester } \\
\text { bonds }\end{array}$ & 9 \\
\hline primary active transporter activity & 7 \\
\hline $\begin{array}{l}\text { small GTPase regulatory/interacting } \\
\text { protein activity }\end{array}$ & 7 \\
\hline transmembrane receptor activity & 7 \\
\hline cytoskeletal protein binding & 6 \\
\hline alpha-type channel activity & 5 \\
\hline cytokine activity & 5 \\
\hline transcription factor binding & 5 \\
\hline Unclassified & 571 \\
\hline
\end{tabular}


Chapter 3 Table 4 . Ontological categorization of genes significantly different between 15m MPP+ exposure and 15m MPP+ with SN50

\begin{tabular}{|l|c|}
\hline CATEGORY & NUMBER \\
\hline purine nucleotide binding & 18 \\
\hline DNA binding & 16 \\
\hline $\begin{array}{l}\text { transferase activity, transferring } \\
\text { phosphorus-containing groups }\end{array}$ & 15 \\
\hline calcium ion binding & 13 \\
\hline protein kinase activity & 11 \\
\hline RNA binding & 10 \\
\hline cation transporter activity & 9 \\
\hline transition metal ion binding & 9 \\
\hline transmembrane receptor activity & 9 \\
\hline $\begin{array}{l}\text { hydrolase activity, acting on acid } \\
\text { anhydrides }\end{array}$ & 8 \\
\hline peptidase activity & 8 \\
\hline $\begin{array}{l}\text { hydrolase activity, acting on ester } \\
\text { bonds }\end{array}$ & 7 \\
\hline primary active transporter activity & 6 \\
\hline unclassified & 399 \\
\hline
\end{tabular}


Chapter 3 Figure 1. Outline of generalized microarray experimental procedure.

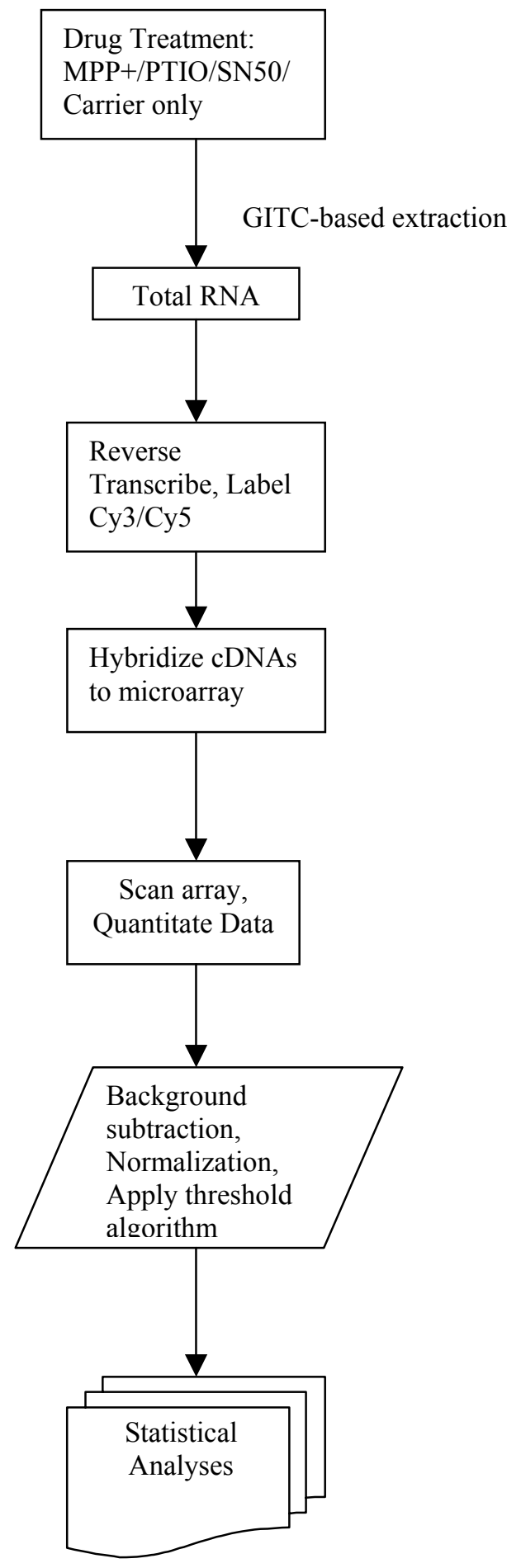


Chapter 3 Figure 2. Analysis of "dye-swap" microarray replicates.

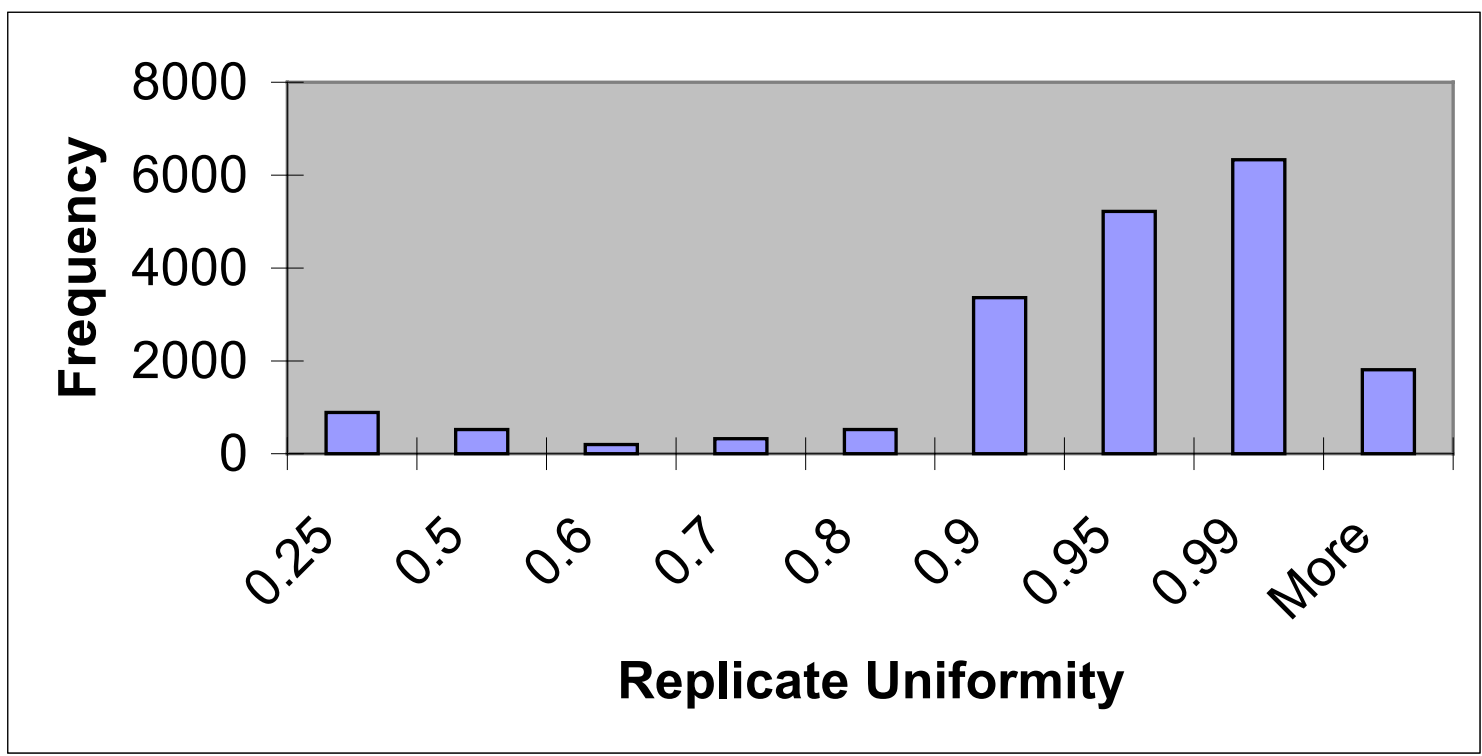

Hybridization 1: Histogram of Gene-Spot Cy5:Cy3 Uniformity Frequencies

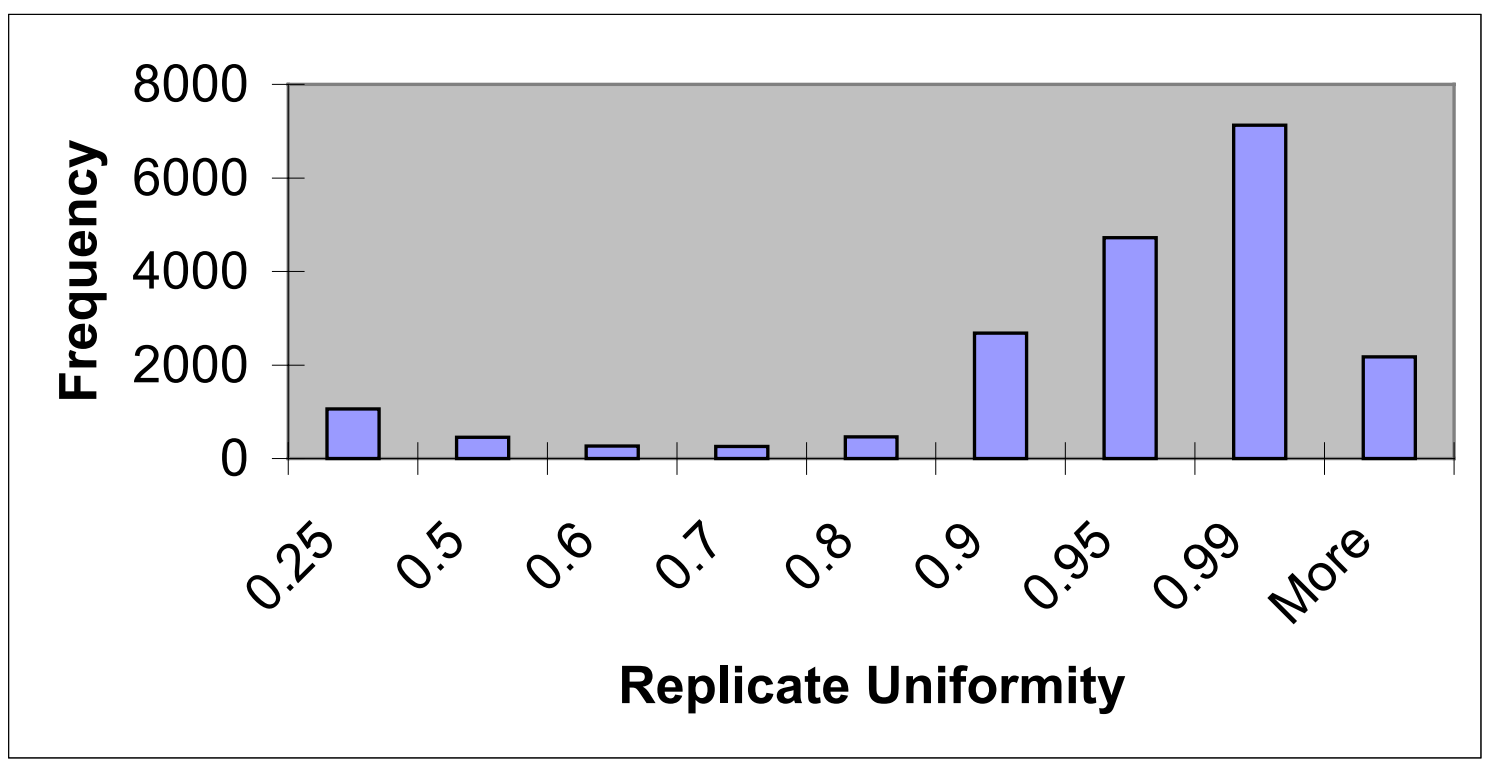

Hybridization 2: Histogram of Gene-Spot Cy5:Cy3 Uniformity Frequencies 
Chapter 3 Figure 3. SN50 peptide does not affect MPP+ induced cell death in SY5Y neuroblastoma.

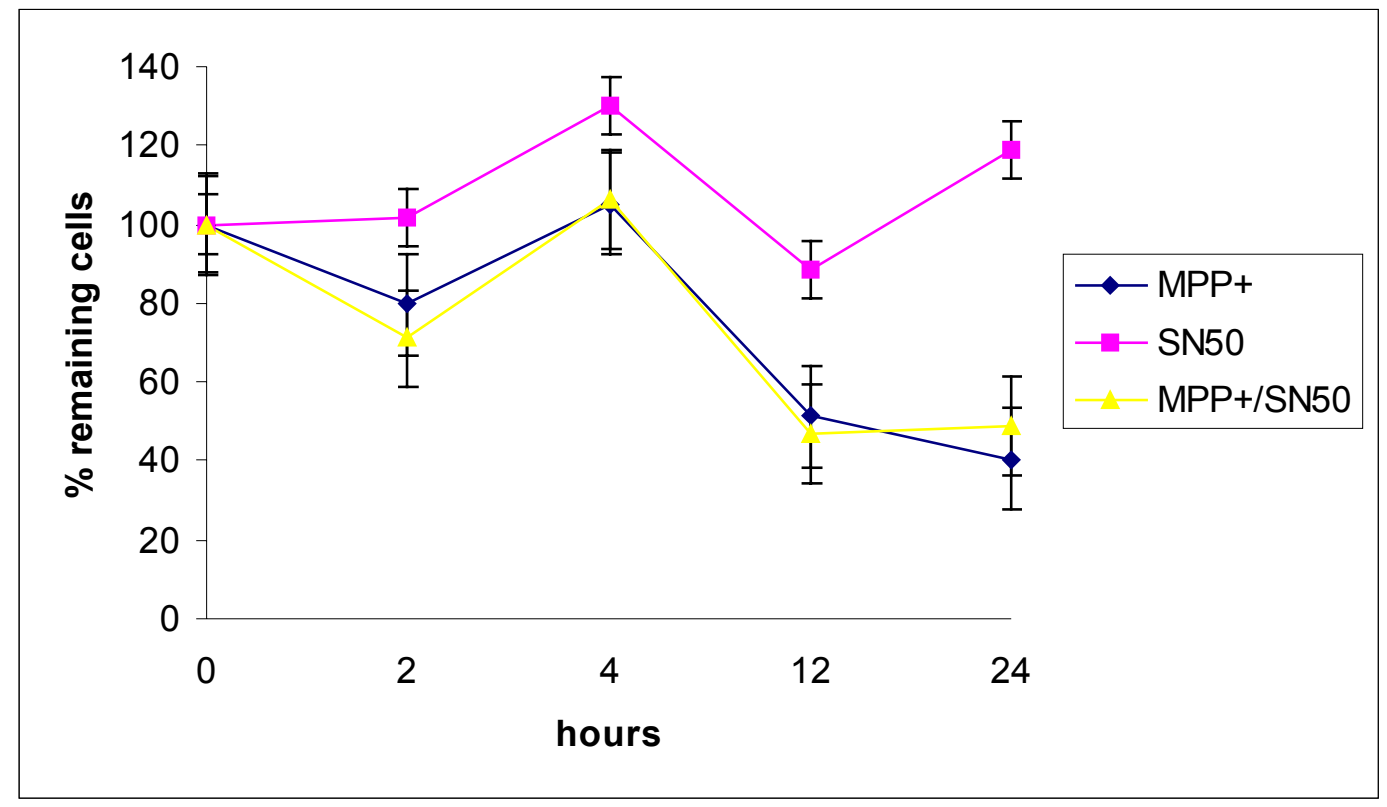


Chapter 3 Figure 4. General Mechanism of RNA Interference via dsRNA.

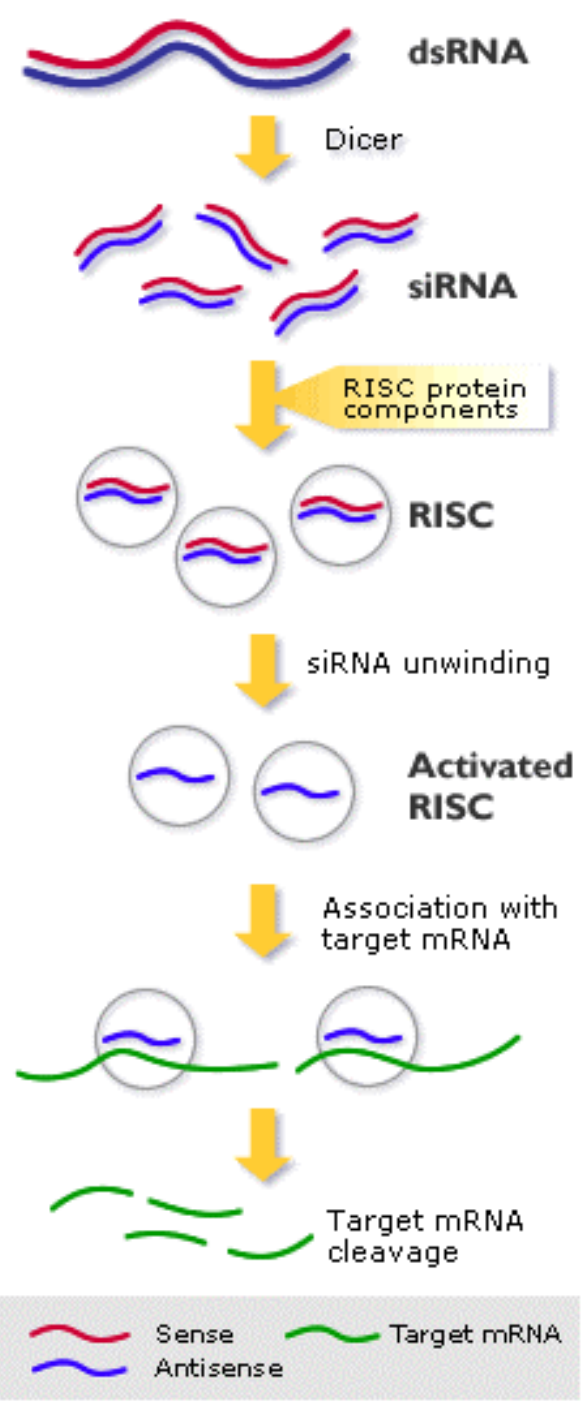

Artwork @Ambion, Inc. 
Chapter 3 Figure 5. Transfection of Chemically Synthesized siRNAs.

\section{A. FGD1, DDIT3}

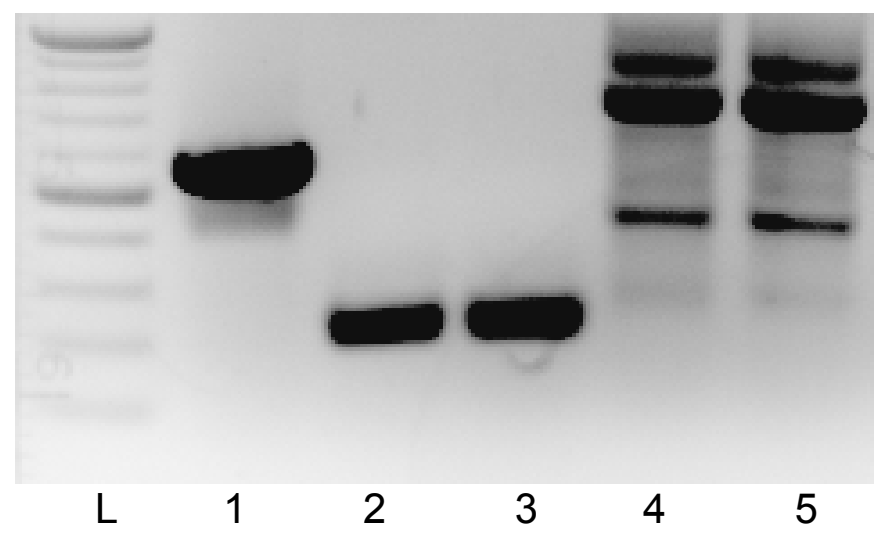

Assay for chemically synthesized siRNA efficacy: (L) 100 bp DNA ladder. (1) positive control PCR product. (2-3) FGD1 PCR products indicating no silencing. (4-5) DDIT3 PCR products indicating no silencing. See Methods for details.

B. SYN2

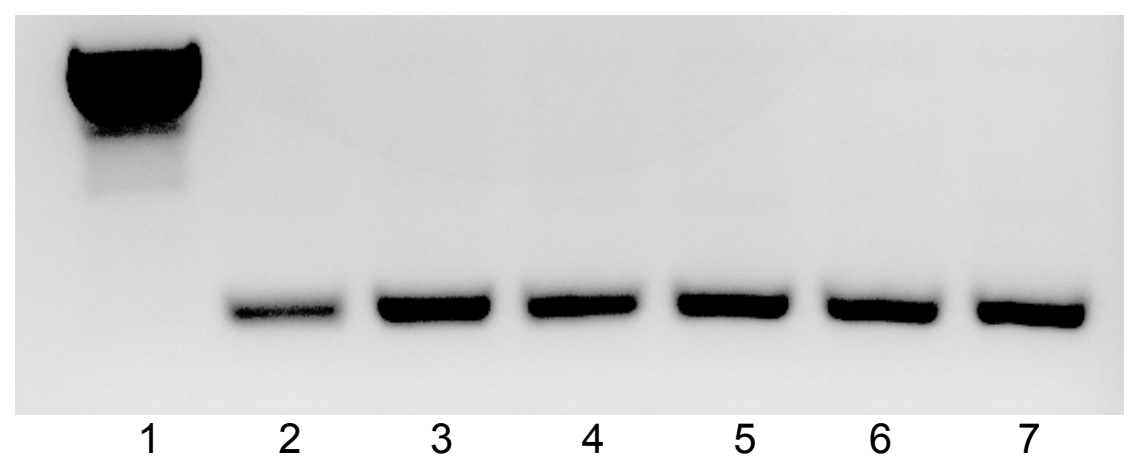

Assay for chemically synthesized siRNA efficacy: (1) positive control PCR product. (2-3) SYN2 PCR products indicating no silencing in SYN2 and control siRNA transfected cells, respectively. $(4-5,6-7)$ duplicates of lanes 2 and 3 . See Methods for details. 
Chapter 3 Figure 6

A. Experimental design for In vitro transcription/RNAse III siRNA production for use in mammalian gene silencing.
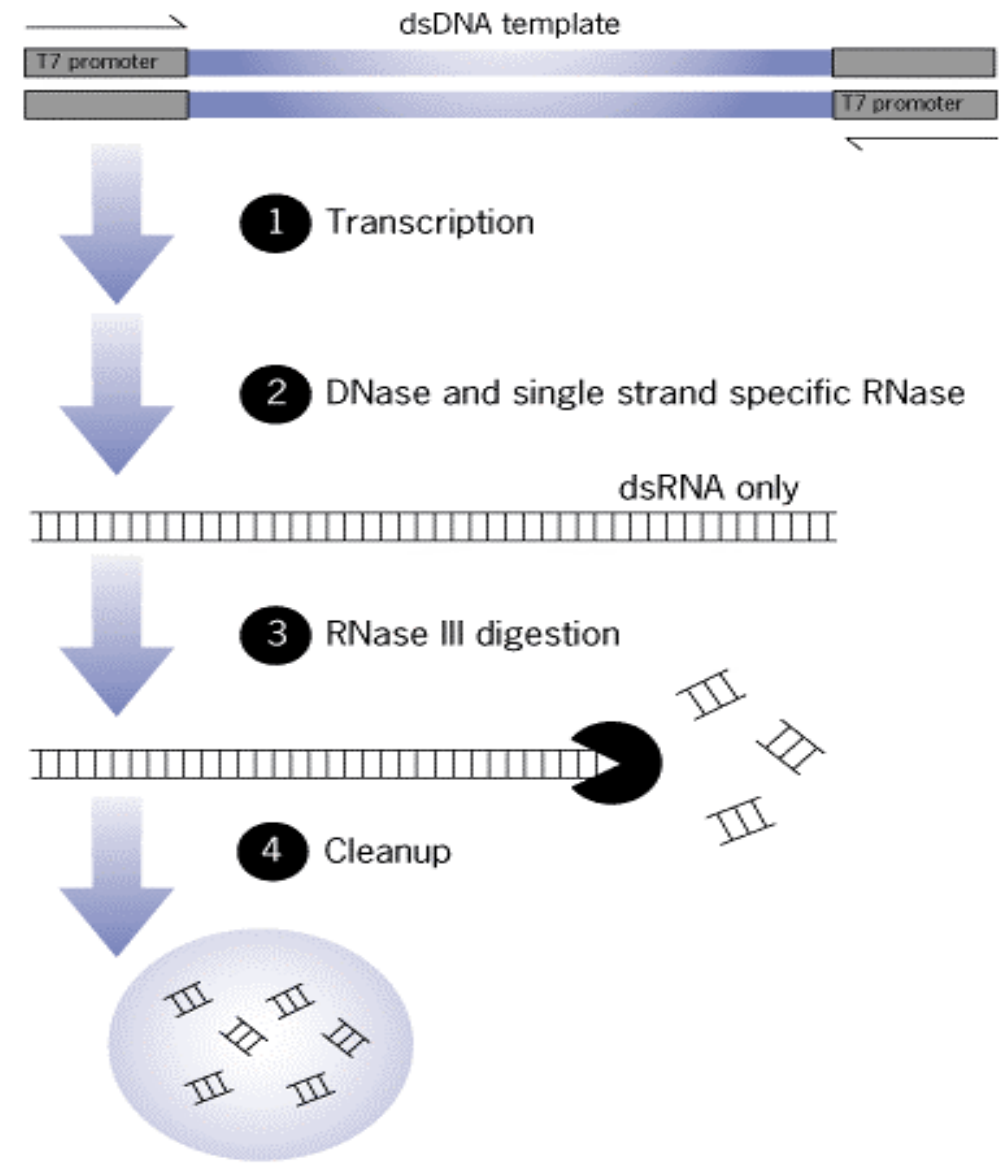

siRNAs ready for transfection

Artwork (Ambion, Inc.

B.

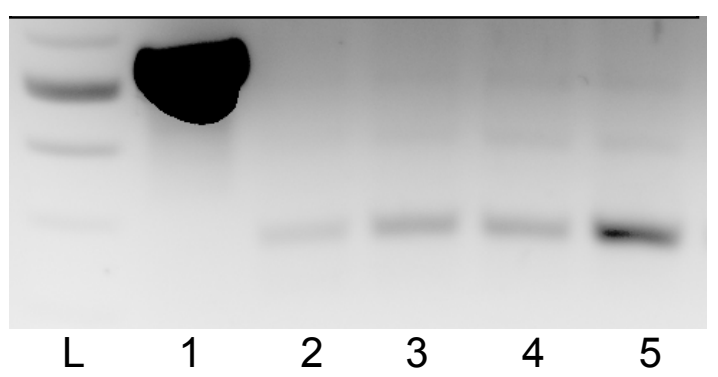

PCR Assay for efficacy of in vitro transcribed siRNA. (L) 100 bp DNA ladder. (1) Positive control PCR. $(2,4)$ SYN2 PCR products indicating no silencing in siRNA transfected cells. $(3,5)$ SYN2 PCR products in control siRNA transfected cells. 
Chapter 3 Figure 7 . Example of a plasmid expression vector for siRNA induction in mammalian cells.

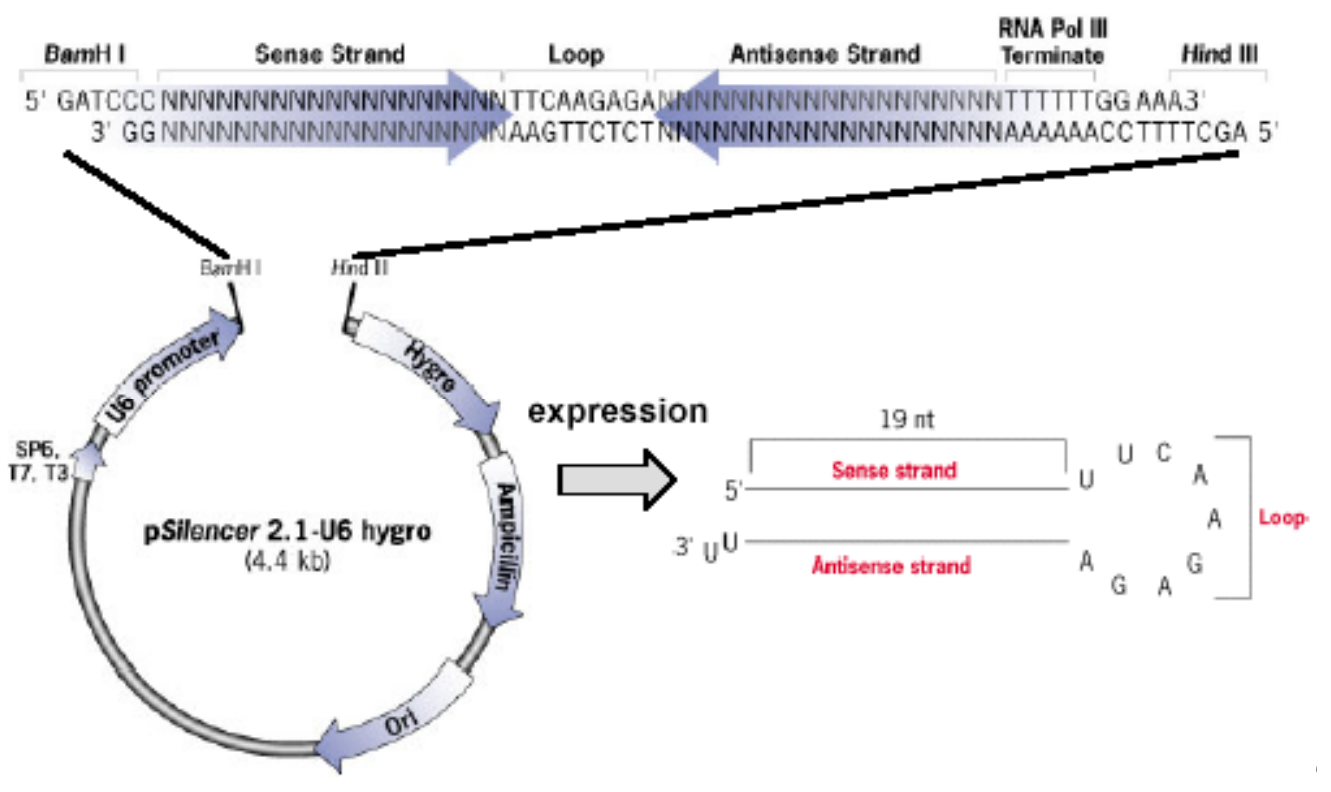

Artwork @Ambion, Inc. 
A.

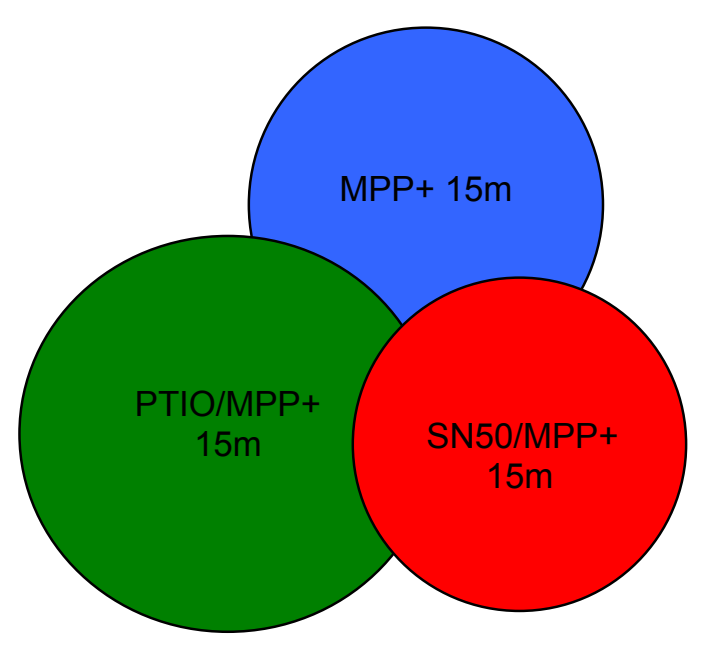

B.

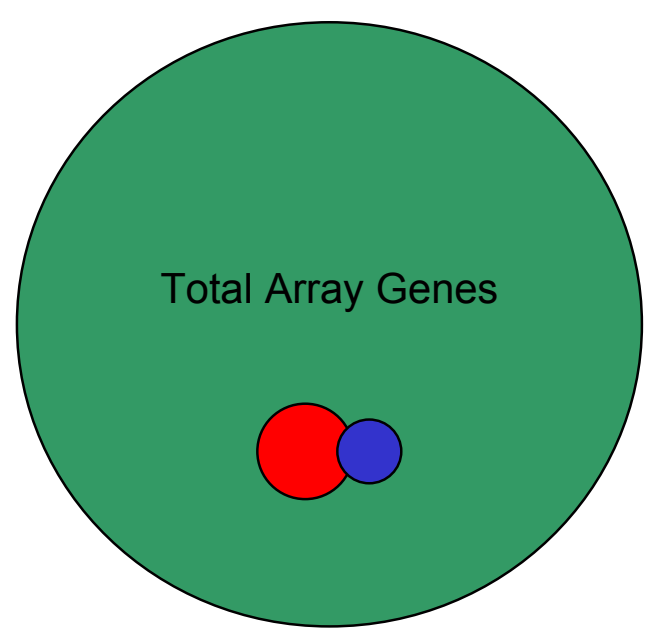

Chapter 3 Figure 8. (A) Area-proportional Venn diagram representing commonalities between genes found to be significantly regulated in SY5Y cells with exposure to $15 \mathrm{~m}$ MPP+ alone or in combination with PTIO or SN50. (B) Area proportional Venn diagram representing the number of genes significantly regulated at $15 \mathrm{~m}$ (red) and 90m (blue) of MPP+ exposure in SY5Y cells in relation to the total number of genes assayed by cDNA microarray. 

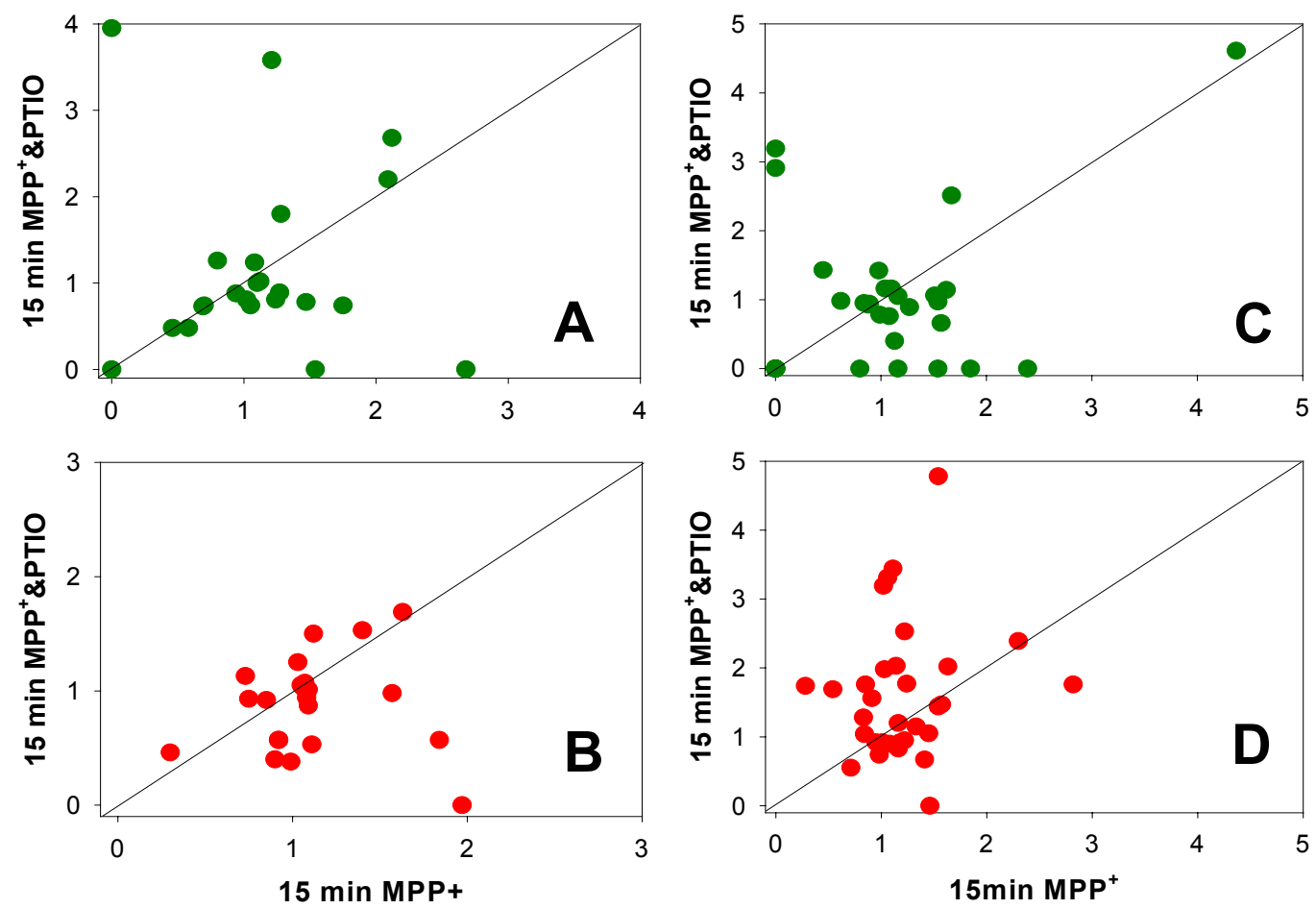

Chapter 3 Figure 9. Correlations between RE values of transcripts grouped into Gene Ontology families by GoMiner software from experiments comparing transcriptosome changes in response to $\mathrm{MPP}^{+}$alone to those in response to $\mathrm{MPP}^{+}$in presence of the NO scavenger PTIO. RE values for genes downregulated (green) and upregulated (red) are plotted for the second order GoMiner nodes $(A, B)$ and third order nodes $(C, D)$. See Methods for details. 

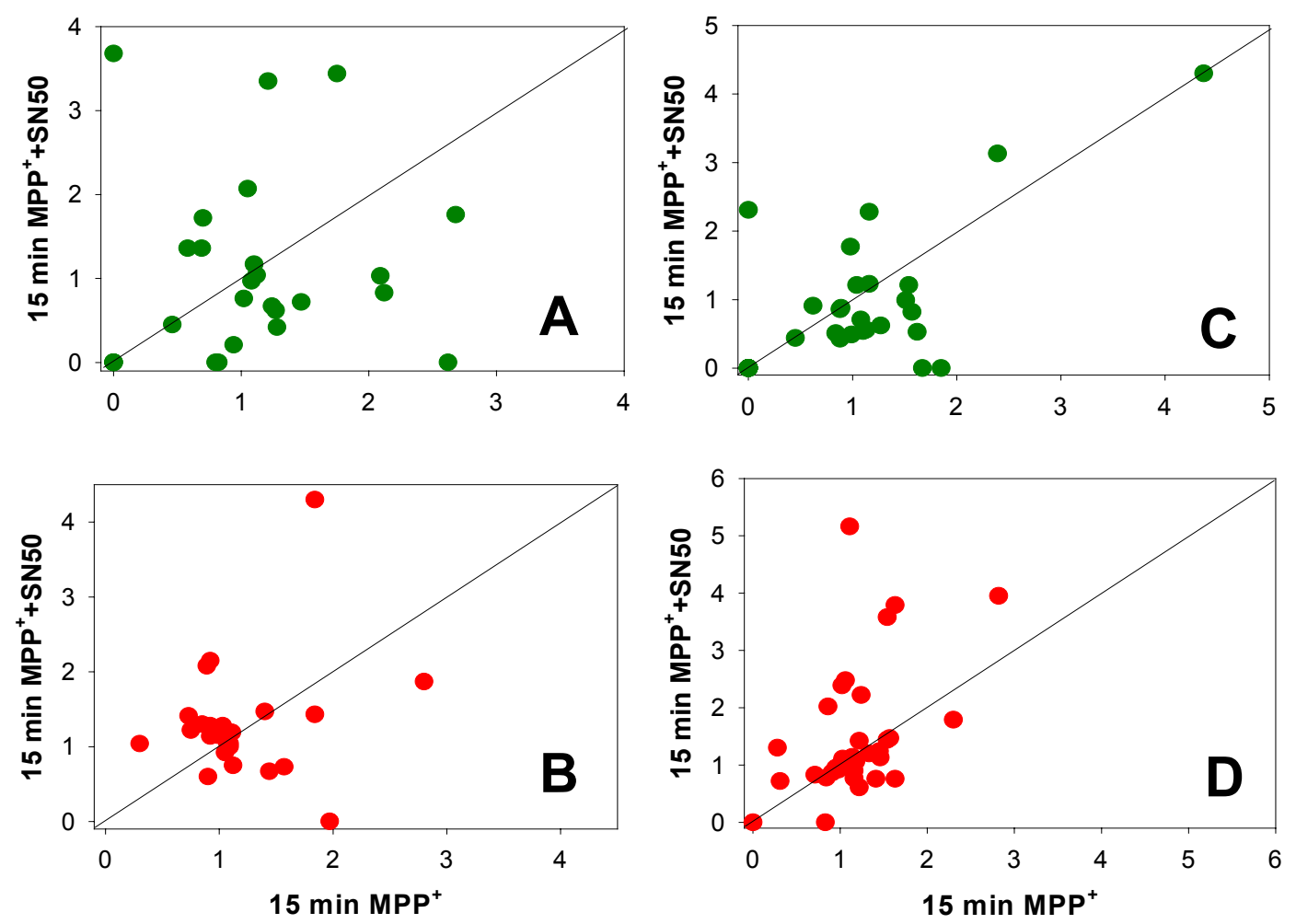

Chapter 3 Figure 10 . Correlations between RE values of transcripts grouped into Gene Ontology families by GoMiner software from experiments comparing transcriptosome changes in response to MPP+ alone to those in response to $\mathrm{MPP}+$ in presence of the NfkappaB antagonist SN50. RE values for genes downregulated (green) and upregulated (red) are plotted for the second order GoMiner nodes $(A, B)$ and third order nodes $(C, D)$. See Methods for details. 

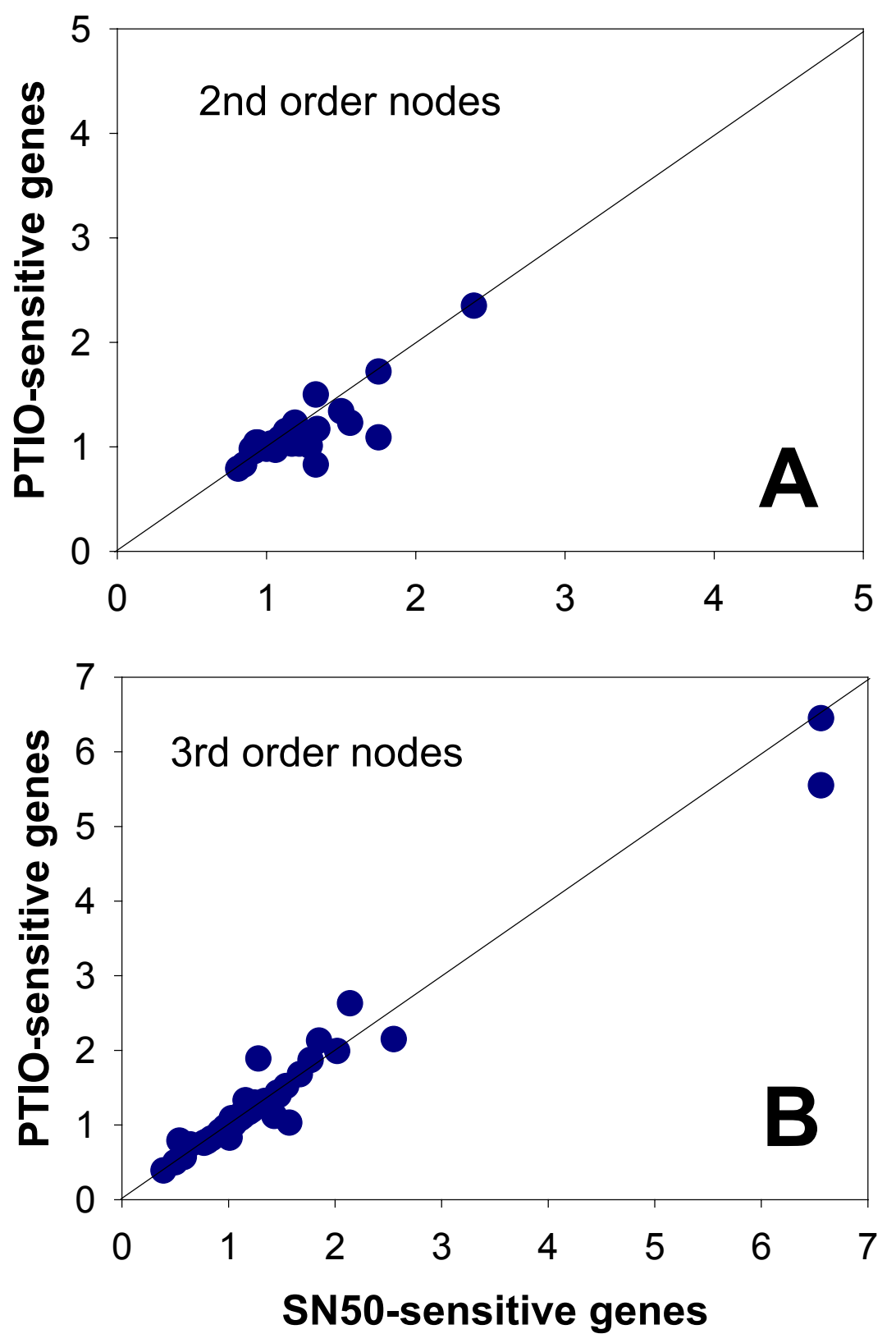

Chapter 3 Figure 11. Correlations between RE values of transcripts grouped into Gene Ontology families by GoMiner software from experiments comparing transcriptosome changes for MPP+ altered genes sensitive to blockade by SN50 compared to those sensitive to blockade by PTIO. 


\section{Chapter 4}

\section{Analysis of brain tissue with varying post mortem intervals: methodological considerations.}

One important aspect of the evolution of our work with microarrays is the extension of the experimental paradigm into other model systems. Following our work with nylon arrays utilizing samples derived from our cell model of PD, we were interested in the applicability of our methods to samples that might be more directly indicative of the processes occurring in the disease state. An example of such a sample would be post mortem brain tissue samples from PD and agematched control patients. Several facilities maintain brain banks comprising anywhere from dozens to thousands of samples from a broad spectrum of neurodegenerative disease patients. A critical issue facing such facilities is the quality of the samples they keep, which is dependent upon multiple factors which certainly include the robustness of the physical plant, quality of curation and the like, but is most directly affected by the method and speed of collection of the tissues at the time of harvest. Post mortem interval (PMI) is a term that refers to the time elapsed between death of the tissue donor and the placement of the tissue under storage conditions. Samples range widely in initial quality, as time elapsed from death to autopsy, and indeed the "agonal period," or period of energetic/nutritional/trophic compromise to the tissue prior to death. 
In isolation of RNA from frozen brain tissue samples archived in the UVA CSND brain bank and in others obtained from a bank curated by Dr. Rajput of the University of Saskatchewan, the quality and yield varied widely. The RNA yield did not appear to depend directly on the PMI of a given sample. In cooperation with Stacey Trotter, a MSTP student performing a summer research rotation in the Bennett lab, we conducted experiments that investigated RNA yield from brain samples from rats after varying PMI. ST performed the animal work and hybridization experiments; LB and JB were jointly responsible for the experimental planning and data analysis, These results were published as a short communication in the journal Brain Research in June of 2002.

Postmortem brain tissue is increasingly being used for studying gene expression in neuropsychiatric diseases. RT-PCR, Northern blots, and in situ hybridization all require stable mRNA of high quality (Augood et al, 1999; Benisty et al, 1998; Chen et al, 1999; Dwivedi et al, 2001; Growdon et al, 1999; Osterlund et al, 1999; Marcinkiewicz and Seidah, 2000; Schramm et al, 1999). More recently, gene array technology, allowing the examination of hundreds or thousands of mRNA levels simultaneously, is being used for human postmortem brain studies (Hakak et al, 2001; Lewohl et al, 2000). However, discrepancies in postmortem conditions represent a potential source of significant variation among samples. 
Previous studies have focused on testing the quality of mRNA in postmortem brain tissue using different postmortem conditions (Bahn et al, 2001; Barton et al, 1993; Castensson et al, 1993; Gilmore et al, 1993; Harrison et al, 1995; Kingsbury et al, 1995; Leonard et al, 1993; Mathern et al, 1997; Pardue et al, 1994). These studies determined that postmortem interval (time between death and freezing) had little effect upon stability. Some found instead that agonal status, reflected in a decreased brain $\mathrm{pH}$, correlated strongly with a decrease in mRNA stability (Harrison et al, 1995; Kingsbury et al, 1995). Thus far, studies using postmortem brain mRNA are based on the examination of housekeeping genes as well as selected genes of interest. With the advent of gene array technology, it is necessary to revisit the issue of postmortem mRNA stability from a population-wide perspective.

In this study, we examined the effect of postmortem interval (PMI) on mRNA used in gene arrays. Mice were subjected to scenarios that mimic circumstances of postmortem collection of human brains. Our gene arrays indicate that mRNA shows no consistent decline in stability or quality after a PMI of 4 hours at room temperature, followed by overnight refrigeration of the corpse before brain removal. After that, there is an increasing variation in the amount of hybridization of cDNA probe to the gene array membrane, indicating a slight decrease in the stability of isolated mRNA. These findings will be useful for selection of postmortem brain tissue used in experiments involving gene array technology. 
Hilltop Balb/C male mice, 25-30 grams were killed using $\mathrm{CO}_{2}$ asphyxiation. Seven groups of mice with 3 mice per group were created based on postmortem interval:

\begin{tabular}{|l|c|c|c|}
\hline Group Name & $\begin{array}{l}\text { Time spent at } \\
\text { room } \\
\text { temperature } \\
\text { (hours) }\end{array}$ & $\begin{array}{l}\text { Time spent at } \\
4^{\circ} \mathrm{C} \text { (hours) }\end{array}$ & $\begin{array}{l}\text { Total } \\
\text { Postmortem } \\
\text { Interval (hours) }\end{array}$ \\
\hline Immediate & 0 & 0 & 0 \\
\hline Morgue overnight & 0 & 18 & 18 \\
\hline RT 4 hours & 4 & 18 & 22 \\
\hline RT 8 hours & 8 & 18 & 26 \\
\hline RT 12 hours & 12 & 18 & 30 \\
\hline RT 24 hours & 24 & 18 & 42 \\
\hline
\end{tabular}

Once the interval was ended, the brains were dissected, the cerebellum removed, then the forebrain was hemisected. Both halves were placed directly on a block of dry ice. RNA was purified from one half using the RNeasy mini kit (Qiagen). The concentration of RNA was assessed by spectrophotometry. All RNA used had 260/280 ratios of $>1.5$. 
2ug total RNA were used for probe synthesis and hybridization to nylon Atlas Mouse cDNA Expression Arrays according to the manufacturer's protocol (Clontech). cDNA probes were synthesized using $\left[\alpha-{ }^{32} \mathrm{P}\right]$ dATP $(3,000 \mathrm{Ci} / \mathrm{mmol})$. After overnight hybridization and washing, the arrays were exposed to phosphoimager screens. Images were analyzed with Imagene 4.1 software (Biodiscovery).

3 independent brain samples from mice at each postmortem interval were subject to RNA extraction, cDNA labeling and hybridization to genes spotted in duplicate on the membranes. Local background was subtracted from each spot, duplicates were averaged and then normalized first to the mean OD of two standard housekeeping genes (glyceraldehyde-3-phosphate dehydrogenase and cytoplasmic beta-actin). A threshold of at least $10 \%$ of the mean housekeeping gene OD was used for establishing detection of a given gene. By this criteria, 365 of the 588 genes arrayed were detected at baseline. Each detected gene was then normalized to the total array OD for all the detected genes on a given array. The three independent values for array-normalized expression of each detected gene were then averaged for comparison across post-mortem conditions.

Postmortem interval conditions were repeated and the entire brain was dissected and the cerebellum removed. The brain was weighed and homogenized in 10 volumes of water. The $\mathrm{pH}$ was measured at room temperature using a temperature-compensating $\mathrm{pH}$ meter. 
Figure 1 shows correlation plots for array-normalized gene expression levels from brains harvested at each postmortem condition, compared to expression levels from brains harvested and frozen immediately. The postmortem conditions of overnight refrigeration ("MG_ON") as well as four hours at room temperature followed by overnight refrigeration ("RT_4HRS") yielded gene expression that correlated highly with that from brains with zero hours PMI and varied with slopes close to unity. More prolonged postmortem conditions caused the correlation to lessen and slopes to drop consistently below unity. Under these conditions more genes were observed to have expression levels both above and below the consensus line.

Figure $2 \mathrm{~A}$ shows the relationship between baseline level of normalized gene expression and the degree of change found in brains removed after 24 hours at RT/18 hours at 4 degrees. The majority of the 365 detected genes were expressed at relatively low levels. There was no clear relationship among basal levels of expression and degree of change found at the longest postmortem interval examined. Figure 2B shows the population distribution of gene expression indexed to baseline levels. Overall the population distributions were similar, with the longer postmortem intervals showing larger percentages of distribution towards the extremes. However, for all postmortem conditions 90$95 \%$ of genes fell within $+/-40 \%$ of equivalency. The small number of genes outside of this range was almost all from the later postmortem interval groups. 
Brain $\mathrm{pH}$ was examined for each of the PMI groups and ranged between 6.62 and 7.00 without correlation to any group.

We used low density nylon mouse gene arrays and ${ }^{32} \mathrm{P}$-labeled cDNA probes to examine postmortem stability of brain mRNAs isolated from mice subjected to varying postmortem conditions. Nylon arrays offer the greatest sensitivity of all available techniques and low cost, but are limited by low gene density as compared to most glass microarrays. Thus, our results must be interpreted within the confines of a limited genomic survey.

We tried to mimic common scenarios experienced by humans whose brains are harvested for experimental purposes, such as gene array studies. We found that the best correlations with immediate brain harvesting and freezing were in situations of immediate refrigeration or short postmortem time (4 hours) at room temperature. Longer postmortem time at room temperature introduced greater variability in gene expression, but the variation from immediate brain harvesting for most genes was within $+/-40 \%$. This figure is under the 1.5-1.7 fold threshold that many accept for "biologically significant" gene expression changes, although it must be emphasized that any such threshold is arbitrary.

Our preliminary results appear to justify the greater expense associated with carrying out broader genomic surveys of brain tissues harvested under varying postmortem conditions. Because of the trend we observed of increasing postmortem intervals leading to larger populations of genes at the extremes of the distribution curves, we feel such studies should be carried out to determine 
the effect of postmortem interval on larger gene population distributions. This type of information would seem essential to interpreting gene array studies of human brain diseases that utilize postmortem brains and should be validated for the arrays used in any particular study. 

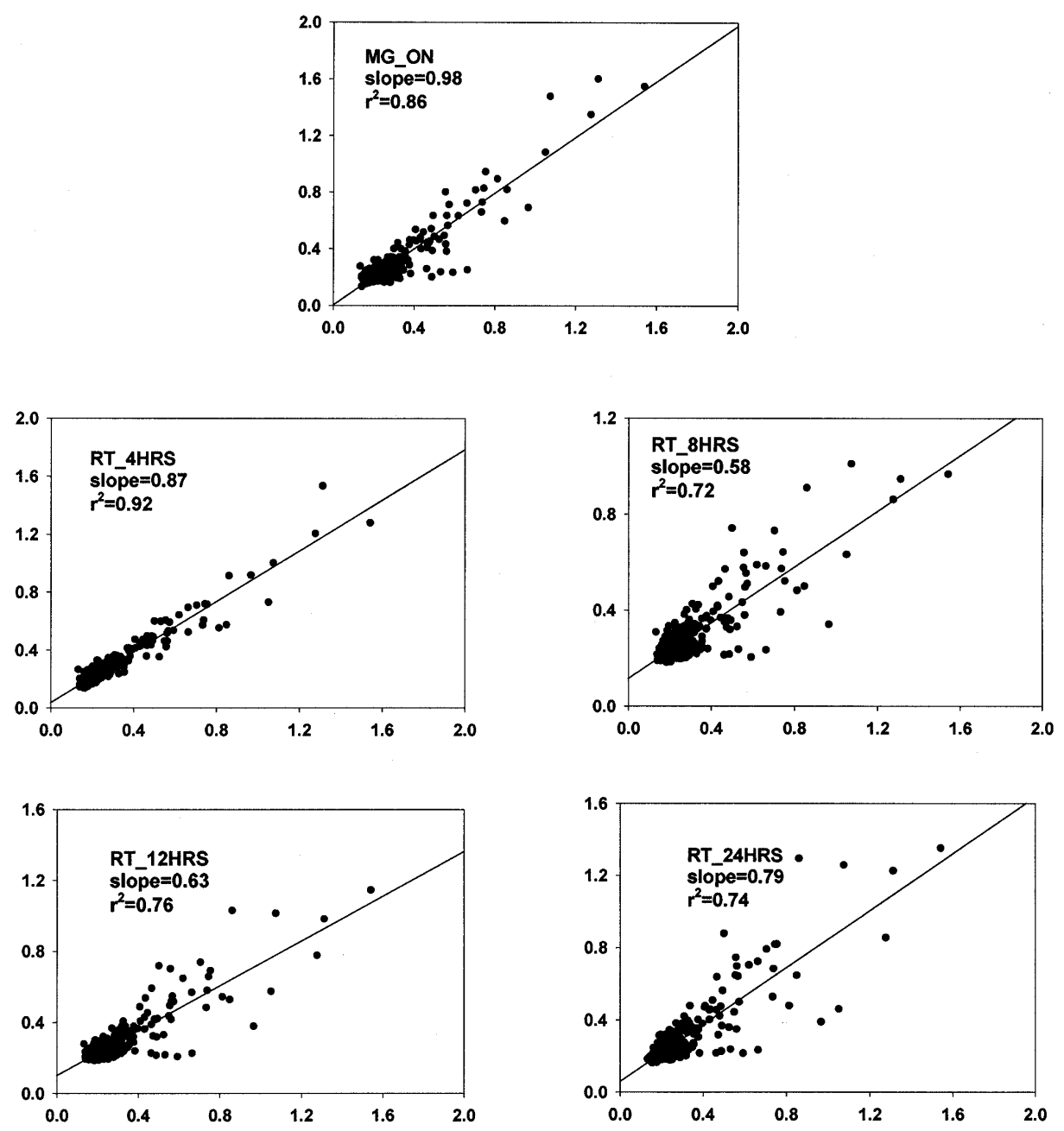

Chapter 4 Figure 1. Correlations among normalized gene expression from brains removed and frozen immediately (on X axes) and brains removed after different postmortem conditions (on $\mathrm{Y}$ axes). 


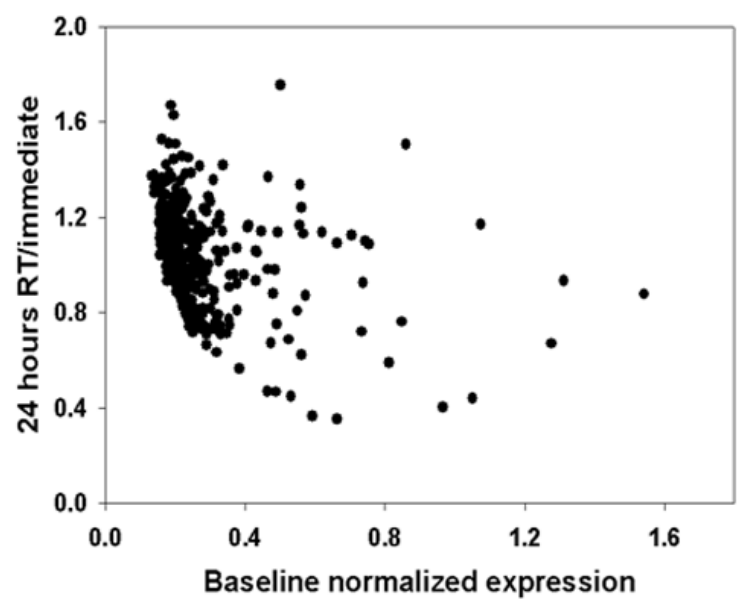

A

Normalized Mouse Brain Gene Distribution

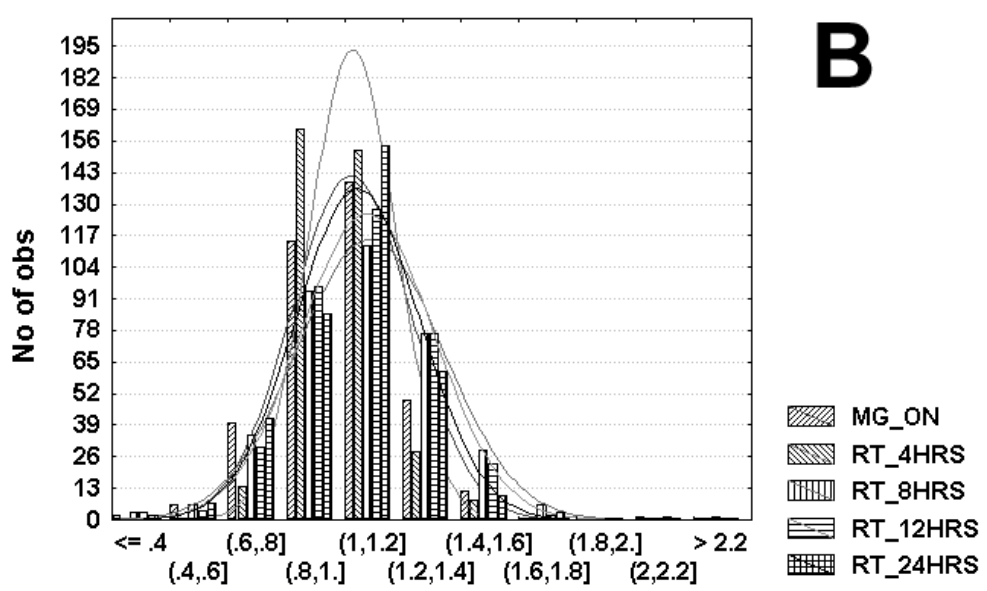

Chapter 4 Figure 2. A. Plot for the 365 detected genes of initial normalized expression level vs. ratio of expression in brains harvested at 24 hours RT/those harvested immediately. B. Histogram plots of population distributions of gene expressions from brains removed after different postmortem conditions and normalized to that of brains removed immediately. A value of 1.0 would indicate the same level of gene expression for a given gene as that found in brains removed immediately after death. 


\section{$\underline{\text { Chapter } 5}$}

Conclusions and Future Directions.

My work in the CSND has been a time of great technological and scientific innovation, and the difficulties that I have encountered- technical, statistical, and otherwise, may well be a harbinger of the types of changes that much of biomedical science will be undergoing over the next ten to twenty years. The single greatest innovation since the start of the molecular biology revolution is incontrovertibly the sequencing of the human genome. We are only now taking the first steps in applying this great volume of (unfortunately, largely unannotated) knowledge to the practical purpose of treating human disease.

Biologists, as a culture of scientists, are not used to dealing with large, noisy, quantitative data sets such as the type produced by microarray analyses. Certainly the development of enhanced data mining techniques and greater numbers and speed of analyses performed will improve the amount of data collected, but will largely fail to address the issue of quality. As such, I would recommend a substantial investment in the research community to provide a consistent framework, along the lines of a LocusLink-style system, for discussing changes in genes and in proteins in a common language. The need for this is as basic as can be, and will unfortunately require huge numbers of man-hours to accomplish, and therefore large amounts of money. Nevertheless, without this "genetic curacy," the majority of the knowledge accumulated in large scale 
studies of genes and proteins may be disjointed and proprietary in its format to the extent that it is unusable by the scientific community at large. This must be avoided at all costs.

With regards to my particular work, I feel that the repeated observation of large nuclear gene expression changes in the face of mitochondrial toxicity represents a new paradigm for approaching the study of mitochondrial pathology as it relates to the greater status of the cell. The data I have presented demonstrates that rapid mitochondrial-nuclear signaling occurs in this cell model, and that it is modulated by different effectors of intracellular signaling. Since mitochondria are known to possess endogenous nitric oxide synthase, and since nitric oxide has recently been demonstrated to impact gene expression through an NFkB dependent mechanism, our data are consistent with nitric oxide being the primary signal indicating mitochondrial distress to the nucleus, and NFkB being the effector molecule that is responsible for the gene expression response that is induced there. Given this notion, it is somewhat surprising to note that treatment with either the NFkB translocation-inhibiting peptide SN50 or the nitric oxide scavenging compound PTIO have no discernible effects upon the course of MPP+ induced cell death. By far the most likely explanation for this phenomenon is that there are multiple pathways in use by the cell to implement such responses, and if one is inhibited, another may be upregulated to cause the same net effect. While this may be somewhat depressing for those of a more reductionistic mindset, I would rather prefer to take it as an endorsement of the efforts that are currently underway to make a more comprehensive catalog of the 
interconnections between cellular systems widely available to the scientific community. The preliminary GoMiner data analysis that I have presented in Chapter 3 represents an initial effort in this vein. At present, it is the most comprehensive analysis package of its type available at no cost to the research community at large, and will undoubtedly be improved and refined even as the curation of the genome progresses.

The relevance of these findings to idiopathic Parkinson's disease process in vivo is as yet uncertain given our current profound lack of understanding of the interconnections between the various intra and extracellular signaling pathways, but the data here will hopefully provide preliminary clues for which pathways deserve the earliest and closest scrutiny. The RNAi strategies we describe above were my attempt to begin this process; the failure of these experiments was undoubtedly the most disheartening aspect of my time in the CSND, yet I am confident that this and similar approaches represent the best ways to provide direct modulation of the gene expression signals measured by microarray analysis, and that the technical problems that I have encountered are not by any means insurmountable. In addition to RNAi, the question of protein expression/modification changes must be addressed in future experiments. Despite the power of microarrays to measure thousands of RNAs simultaneously, we must be cognizant of the fact that RNA does not exist in a vacuum; many would argue that our efforts would be better served to accelerate completion of a human proteome project, to get to the level of the actuators of molecular activity in the cell. Protein array technology is at least five years 
behind DNA array technology today; the collections of antibodies and other binding proteins that are being applied to glass slides for microarray-style analyses are currently few in number, but commercial entities are rapidly improving their offerings in the hope of being first to market. Given resources, I would recommend that any lab group interested in the multiplexed study of gene expression also give serious consideration to construction of protein arrays specific for the model systems they are employing: in the case of the CSND, for example, an array comprising the proteome of dopaminergic nigrostriatal neurons would be highly useful in determining which gene expression responses observed in response to chemical or other perturbations are relevant to the overarching cellular processes of growth, differentiation and death. A cell line with mitochondrial nitric oxide synthase under the control of an inducible repressor would also prove highly useful in beginning to address the question of the contribution of mitochondrial nitric oxide to the cell death process. In this case, as with many research projects, there are indeed many more avenues worthy of investigation than we have the hands, time, and money to pursue as yet. 


\section{References}

Aarsland D, Tandberg E, Larsen JP, Cummings JL. (1996) Frequency of dementia in Parkinson disease. Arch Neurol 53:538-42.

Abe T, Isobe C, Murata T, Sato C, Tohgi H. (2003) Alteration of 8hydroxyguanosine concentrations in the cerebrospinal fluid and serum from patients with Parkinson's disease. Neurosci Lett 336:105-108.

Abramova N. A., Cassarino D. S., Khan S. M., Painter T. W. and Bennett J. P., Jr. (2002) Inhibition by $\mathrm{R}(+)$ or $\mathrm{S}(-)$ pramipexole of caspase activation and cell death induced by methylpyridinium ion or beta amyloid peptide in SH-SY5Y neuroblastoma. J. Neurosci. Res. 67, 494-500.

Agid Y, Graybiel AM, Ruberg M, Hirsch E, Blin J, Dubois B, Javoy-Agid F. (1990) The efficacy of levodopa treatment declines in the course of Parkinson's disease: do nondopaminergic lesions play a role? Adv Neurol 53:83-100.

Akaike T, Yoshida M, Miyamoto Y, Sato K, Kohno M, Sasamoto K, Miyazaki K, Ueda S, Maeda H. (1993) Antagonistic action of imidazolineoxyl Noxides against endothelium-derived relaxing factor/.NO through a radical reaction. Biochemistry 32:827-32.

Alam ZI, Daniel SE, Lees AJ, Marsden DC, Jenner P, Halliwell B. (1997) A generalised increase in protein carbonyls in the brain in Parkinson's but not incidental Lewy body disease. J Neurochem 69:1326-1329.

Alam ZI, Jenner A, Daniel SE, Lees AJ, Cairns N, Marsden CD, Jenner P, Halliwell B. (1997) Oxidative DNA damage in the parkinsonian brain: an apparent 
selective increase in 8-hydroxyguanine levels in substantia nigra. J Neurochem 69:1196-1203.

Alves Da Costa C, Paitel E, Vincent B, Checler F. (2002) Alpha-synuclein lowers p53-dependent apoptotic response of neuronal cells. Abolishment by 6hydroxydopamine and implication for Parkinson's disease. J Biol Chem 277:50980-50984.

Ambani LM, Van Woert MH, Murphy S. (1975) Brain peroxidase and catalase in Parkinson disease. Arch Neurol 32:114-118.

Andringa G, van Oosten RV, Unger W, Hafmans TG, Veening J, Stoof JC, Cools AR. (2000) Systemic administration of the propargylamine CGP 3466B prevents behavioural and morphological deficits in rats with 6-hydroxydopamineinduced lesions in the substantia nigra. Eur J Neurosci 12:3033-3043.

Anglade P, Vyas S, Javoy-Agid F, Herrero MT, Michel PP, Marquez J, Mouatt-Prigent A, Ruberg M, Hirsch EC, Agid Y. (1997) Apoptosis and autophagy in nigral neurons of patients with Parkinson's disease. Histol Histopathol 12:25-31.

Augood SJ, Waldvogel HJ, Munkle MC, Faull RL, Emson PC, (1999) Localization of calcium-binding proteins and GABA transporter (GAT-1) messenger RNA in the human subthalamic nucleus, Neuroscience 88:521-34.

Bahn S, Augood SJ, Ryan M, Standaert DG, Starkey M, Emson PC. (2001) Gene expression profiling in the post-mortem human brain- no cause for dismay. J Chem Neuroanat 22:79-94. 
Ballard P. A., Tetrud J. W. and Langston J. W. (1985) Permanent human parkinsonism due to 1-methyl-4-phenyl-1,2,3,6-tetrahydropyridine (MPTP): seven cases. Neurology 35, 949-956.

Ballif B. A. and Blenis J. (2001) Molecular mechanisms mediating mammalian mitogen-activated protein kinase (MAPK) kinase (MEK)-MAPK cell survival signals. Cell Growth Differ. 12, 397-408.

Banati RB, Daniel SE, Blunt SB. (1998) Glial pathology but absence of apoptotic nigral neurons in long-standing Parkinson's disease. Mov Disord 13:221-227.

Barone M. V., Crozat A., Tabaee A., Philipson L. and Ron D. (1994) CHOP (GADD153) and its oncogenic variant, TLS-CHOP, have opposing effects on the induction of G1/S arrest. Genes Dev. 8:453-464.

Barton AJ, Pearson RC, Najlerahim A, Harrison PJ. (1993) Pre- and postmortem influences on brain RNA. J Neurochem 61:1-11.

Barzilai A, Zilkha-Falb R, Daily D, Stern N, Offen D, Ziv I, Melamed E, Shirvan A. (2000) The molecular mechanism of dopamine-induced apoptosis: identification and characterization of genes that mediate dopamine toxicity. $J$ Neural Transm Suppl 60:59-76.

Benisty S, Boissiere F, Faucheux B, Agid Y, Hirsch EC. (1998) trkB messenger RNA expression in normal human brain and in the substantia nigra of parkinsonian patients: an in situ hybridization study. J Neurosci 86:813-26.

Bertler A, Rosengren E. (1959) Occurrence and distribution of dopamine in brain and other tissues. Experientia 15:10-11. 
Betarbet R, Sherer TB, MacKenzie G, Garcia-Osuna M, Panov AV, Greenamyre JT (2000) Chronic systemic pesticide exposure reproduces features of Parkinson's disease. Nat Neurosci 3:1301-1306.

Beyer W, Imlay J, Fridovich I. (1991) Superoxide dismutases. Prog Nucleic Acid Res Mol Biol 40:221-253.

Bindoff LA, Birch-Machin M, Cartilidge NE, Parker WD, Jr, Turnbull DM (1991) Respiratory chain abnormalities in skeletal muscle from patients with Parkinson's disease. Jour Neurol Sci 104:203-208.

Birkmayer W, Hornykiewicz O. (1961) Der L-3,4-Dioxyphenylalanin(= DOPA)—Effekt bei der Parkinson-Akinese. Wien Klin Wochenschr 3:787-788.

Bolanos JP, Heales SJ, Land JM, Clark JB. (1995) Effect of peroxynitrite on the mitochondrial respiratory chain: differential susceptibility of neurones and astrocytes in primary culture. J Neurochem 64:1965-1972.

Boveris A, Chance B. (1973) The mitochondrial generation of hydrogen peroxide. General properties and effect of hyperbaric oxygen. Biochem $J$ 134:707-716.

Brill LB $2^{\text {nd }}$, Bennett JP. (2003) Dependence on electron transport chain function and intracellular signaling of genomic responses in SH-SY5Y cells to the mitochondrial neurotoxin MPP+. Exp Neurol 181:25-38.

Brookes PS, Salinas EP, Darley-Usmar K, Eiserich JP, Freeman BA, Darley-Usmar VM, Anderson PG. (2000) Concentration-dependent effects of nitric oxide on mitochondrial permeability transition and cytochrome $\mathrm{c}$ release. $J$ Biol Chem 275:20474-20479. 
Brown GC, Borutaite V. (1999) Nitric oxide, cytochrome c and mitochondria. Biochem Soc Symp 66:17-25.

Brown VM, Ossadtchi A, Khan AH, Yee S, Lacan G, Melega WP, Cherry SR, Leahy RM, Smith DJ. (2002) Multiplex three-dimensional brain gene expression mapping in a mouse model of Parkinson's disease. Genome Res 12:868-884.

Brunk UT, Terman A. (2002) Lipofuscin: mechanisms of age-related accumulation and influence on cell function. Free Radic Biol Med 33:611-619.

Burns R. S., Chiueh C. C., Markey S. P., Ebert M. H., Jacobowitz D. M. and Kopin I. J. (1983) A primate model of parkinsonism: selective destruction of dopaminergic neurons in the pars compacta of the substantia nigra by $\mathrm{N}$-methyl4-phenyl-1,2,3,6-tetrahydropyridine. Proc Natl Acad Sci U S A 80:4546-4550.

Candy JM, Perry RH, Perry EK, Irving D, Blessed G, Fairbairn AF, Tomlinson BE. (1983) Pathological changes in the nucleus of Meynert in Alzheimer's and Parkinson's diseases. J Neurol Sci 59:277-89.

Carlsson A, Lindqvist M, Magnusson T, Waldeck B. (1958) On the presence of 3-hydroxytyramine in brain. Science 127:471.

Cassarino DS, Bennett JP Jr. (1999) An evaluation of the role of mitochondria in neurodegenerative diseases: mitochondrial mutations and oxidative pathology, protective nuclear responses, and cell death in neurodegeneration. Brain Res Brain Res Rev 1, 1-25.

Cassarino DS, Fall CP, Swerdlow RH, Smith TS, Halvorsen EM, Miller SW, Parks JP, Parker WD Jr, and Bennett JP Jr. (1997) Elevated reactive 
oxygen species and antioxidant enzyme activities in animal and cellular models of Parkinson's disease. Biochim. Biophys Acta 1362, 77-86.

Cassarino DS, Halvorsen EM, Swerdlow RH, Abramova NN, Parker WD Jr, Sturgill TW, Bennett JP Jr. (2000) Interaction among mitochondria, mitogenactivated protein kinases, and nuclear factor-kappaB in cellular models of Parkinson's disease. J Neurochem 74:1384-1392.

Castensson A, Emilsson L, Preece P, Jazin E. (2000) High-resolution quantification of specific mRNA levels in human brain autopsies and biopsies. Genome Res 10:1219-1229.

Chance B, Schoener B, Oshino R, Itshak F, Nakase Y. (1979) Oxidationreduction ratio studies of mitochondria in freeze-trapped samples. $\mathrm{NADH}$ and flavoprotein fluorescence signals. J Biol Chem 254:4764-4771.

Charcot JM, Vulpian A. (1861) De la paralysie agitante. Gaz Hebdom Med Chir 8:765-767.

Chen L, Segal DM, Mash DC. (1999) Semi-quantitative reversetranscriptase polymerase chain reaction: an approach for the measurement of target gene expression in human brain. Brain Res Brain Res Prot 4:132-139.

Chiba K, Trevor A, Castagnoli N. (1984) Metabolism of the neurotoxic tertiary amine, MPTP, by brain monoamine oxidase. Biochem Biophys Res Commun $120,547-578$.

Cogoni C, Irelan JT, Schumacher M, Schmidhauser TJ, Selker EU, Macino G. (1996) Transgene silencing of the al-1 gene in vegetative cells of 
Neurospora is mediated by a cytoplasmic effector and does not depend on DNADNA interactions or DNA methylation. EMBO J 15:3153-3163.

Conn KJ, Gao WW, Ullman MD, McKeon-O'Malley C, Eisenhauer PB, Fine RE, Wells JM. (2002) Specific up-regulation of GADD153/CHOP in 1methyl-4-phenyl-pyridinium-treated SH-SY5Y cells. J Neurosci Res 68:755-760.

Cotzias GC, Papavasiliou PS, Gellene R. (1967) Modification of Parkinson's disease—Chronic treatment with L-dopa. N Engl J Med 280:337345.

Davis GC, Williams AC, Markey SP, Ebert MH, Caine ED, Reichert CM, Kopin IJ. (1979) Chronic Parkinsonism secondary to intravenous injection of meperidine analogues. Psychiatry Res 1(3:249-254.

Dehmer T, Lindenau J, Haid S, Dichgans J, Schulz JB. (2000) Deficiency of inducible nitric oxide synthase protects against MPTP toxicity in vivo. $J$ Neurochem 74:2213-2216.

Dennis J, Bennett JP Jr. (2003) Interactions among nitric oxide and Bclfamily proteins after MPP+ exposure of SH-SY5Y neural cells II: exogenous NO replicates MPP+ actions. J Neurosci Res 72:89-97.

Dexter DT, Carayon A, Vidailhet M, Ruberg M, Agid F, Agid Y, Lees AJ, Wells FR, Jenner P, Marsden CD. (1990) Decreased ferritin levels in brain in Parkinson's disease. J Neurochem 55:16-20.

Dexter DT, Carter CJ, Wells FR, Javoy-Agid F, Agid Y, Lees A, Jenner P, Marsden CD. (1989) Basal lipid peroxidation in substantia nigra is increased in Parkinson's disease. J Neurochem 52:381-389. 
Dexter DT, Wells FR, Lees AJ, Agid F, Agid Y, Jenner P, Marsden CD. (1989) Increased nigral iron content and alterations in other metal ions occurring in brain in Parkinson's disease. J Neurochem 52:1830-1836.

Di Monte DA, Lavasani M, Manning-Bog AB. (2002) Environmental factors in Parkinson's disease. Neurotoxicology 23:487-502.

Duvoisin RC. (1965) A review of drug therapy in parkinsonism. Bull NY Acad Med 41:898-910.

Dwivedi Y, Rizavi HS, Roberts RC, Conley RC, Tamminga CA, Pandey GN. (2001) Reduced activation and expression of ERK1/2 MAP kinase in the post-mortem brain of depressed suicide patients, J Neurochem 77:916-928.

Ehringer H, Hornykiewicz O. (1960) Veteilung von Noradrenalin und Dopamin (3-Hydroxytyramin) im Gehirn des Menschen und ihr Verhalten bei Erkrankungen des extrapyramidalen Systems. Klin Wochenschr 38:1236-1239.

Elbashir SM, Harborth J, Lendeckel W, Yalcin A, Weber K, Tuschl T. (2001) Duplexes of 21-nucleotide RNAs mediate RNA interference in cultured mammalian cells. Nature 411:494-498.

Fall CP, Bennett JP Jr. (1999) Characterization and time course of MPP+ induced apoptosis in human SH-SY5Y neuroblastoma cells. J Neurosci Res $55: 620-628$.

Fahn S, Cohen G. (1992) The oxidant stress hypothesis in Parkinson's disease: evidence supporting it. Ann Neurol 32:804-812.

Favata MF, Horiuchi KY, Manos EJ, Daulerio AJ, Stradley DA, Feeser WS, Van Dyk DE, Pitts WJ, Earl RA, Hobbs F, Copeland RA, Magolda RL, 
Scherle PA, Trzaskos JM. (1998) Identification of a novel inhibitor of mitogenactivated protein kinase kinase. J Biol Chem 273, 18623-18632.

Fawcett TW, Eastman HB, Martindale JL, Holbrook NJ. (1996) Physical and functional association between GADD153 and CCAAT/enhancer-binding protein beta during cellular stress. J Biol Chem 271, 14285-14289.

Fire A, Xu S, Montgomery MK, Kostas SA, Driver SE, Mello CC. (1998) Potent and specific genetic interference by double-stranded RNA in Caenorhabditis elegans. Nature 391:806-811.

Frangiskakis JM, Ewart AK, Morris CA, Mervis CB, Bertrand J, Robinson BF, Klein BP, Ensing GJ, Everett LA, Green ED, Proschel C, Gutowski NJ, Noble M, Atkinson DL, Odelberg SJ, Keating MT. (1996) LIM-kinase1 hemizygosity implicated in impaired visuospatial constructive cognition. Cell 86:59-69.

Freed CR, Greene PE, Breeze RE, Tsai WY, DuMouchel W, Kao R, Dillon S, Winfield H, Culver S, Trojanowski JQ, Eidelberg D, Fahn S. (2001) Transplantation of embryonic dopamine neurons for severe Parkinson's disease. N Engl J Med 344(10):710-719.

Gabby M, Tauber M, Porat S, Simantov R. (1996) Selective role of glutathione in protecting human neuronal cells from dopamine-induced apoptosis. Neuropharmacology 35:571-578.

Gardner PR, Raineri I, Epstein LB, White CW. (1995) Superoxide radical and iron modulate aconitase activity in mammalian cells. J Biol Chem 270:13399-13405. 
Gilmore JH, Lawler CP, Eaton AM, Mailman RB. (1993) Postmortem stability of dopamine D1 receptor mRNA and D1 receptors. Brain Res Molec Brain Res 18:290-296.

Good PF, Hsu A, Werner P, Perl DP, Olanow CW. (1998) Protein nitration in Parkinson's disease. J Neuropathol Exp Neurol 57:338-342.

Gowers WR. (1893) A manual of diseases of the nervous system, vol. 2: Philadelphia, Blakiston. 636-657.

Graeber MB, Moran LB. (2002) Mechanisms of cell death in neurodegenerative diseases: fashion, fiction, and facts. Brain Pathol 12:385390.

Greenfield JG, Bosanquet FD. (1953) The brain-stem lesions in Parkinsonism. J Neurochem 16:213-26.

Growdon WB, Cheung BS, Hyman BT, Rebeck GW. (1999) Lack of allelic imbalance in APOE epsilon3/4 brain mRNA expression in Alzheimer's disease, Neurosci Lett 272:83-86.

Grunewald T, Beal MF. (1999) NOS knockouts and neuroprotection. Nat Med 5:1354-1355.

Guo S, Kemphues KJ. (1995) par-1, a gene required for establishing polarity in C. elegans embryos, encodes a putative Ser/Thr kinase that is asymmetrically distributed. Cell 81:611-620.

Hakak Y, Walker JR, Li C, Wong WH, Davis KL, Buxbaum JD, Haroutunian V, Fienberg AA. (2001) Genome-wide expression analysis reveals 
dysregulation of myelination-related genes in chronic schizophrenia. Proc Nat Acad Sci U S A 98:4746-4751.

Haley B, Tang G, Zamore PD. (2003) In vitro analysis of RNA interference in Drosophila melanogaster. Methods 30:330-336.

Halvorsen EM, Dennis J, Keeney P, Sturgill TW, Tuttle JB, Bennett JB Jr. (2002) Methylpyridinium (MPP(+))- and nerve growth factor-induced changes in pro- and anti-apoptotic signaling pathways in SH-SY5Y neuroblastoma cells. Brain Res 952:98-110.

Hantraye P, Brouillet E, Ferrante R, Palfi S, Dolan R, Matthews RT, Beal MF. (1996) Inhibition of neuronal nitric oxide synthase prevents MPTP-induced parkinsonism in baboons. Nat Med 2:1017-1021.

Harrison PJ, Heath PR, Eastwood SL, Burnet PW, McDonald B, Pearson RC. (1995) The relative importance of premortem acidosis and postmortem interval for human brain gene expression studies: selective mRNA vulnerability and comparison with their encoded proteins. Neurosci Lett 200:151-154.

Hartmann A, Hunot S, Michel PP, Muriel MP, Vyas S, Faucheux BA, Mouatt-Prigent A, Turmel H, Srinivasan A, Ruberg M, Evan GI, Agid Y, Hirsch EC. (2000) Caspase-3: A vulnerability factor and final effector in apoptotic death of dopaminergic neurons in Parkinson's disease. Proc Natl Acad Sci U S A 97:2875-2880.

Hasegawa E, Takeshige K, Oishi T, Murai Y, Minakami S. (1990) 1Methyl-4-phenylpyridinium (MPP+) induces NADH-dependent superoxide 
formation and enhances NADH-dependent lipid peroxidation in bovine heart submitochondrial particles. Biochem Biophys Res Commun 170:1049-1055.

Heikkila RE, Manzino L, Cabbat FS, Duvoisin RC. (1984) Protection against the dopaminergic neurotoxicity of 1-methyl-4-phenyl-1,2,5,6tetrahydropyridine by monoamine oxidase inhibitors. Nature 311:467-469.

Hirsch E, Graybiel AM, Agid YA. (1988) Melanized dopaminergic neurons are differentially susceptible to degeneration in Parkinson's disease. Nature 6180:345-8.

Hirsch EC, Brandel JP, Galle P, Javoy-Agid F, Agid Y. (1991) Iron and aluminum increase in the substantia nigra of patients with Parkinson's disease: an X-ray microanalysis. J Neurochem 56:446-451.

Hunot S, Boissiere F, Faucheux B, Brugg B, Mouatt-Prigent A, Agid Y, Hirsch EC. (1996) Nitric oxide synthase and neuronal vulnerability in Parkinson's disease. Neuroscience 72:355-363.

Ischiropoulos H, al-Mehdi AB. (1995) Peroxynitrite-mediated oxidative protein modifications. FEBS Lett 364:279-282.

Itzhak Y, Martin JL, Ali SF. (1999) Methamphetamine- and 1-methyl-4phenyl- 1,2,3, 6-tetrahydropyridine-induced dopaminergic neurotoxicity in inducible nitric oxide synthase-deficient mice. Synapse 34:305-312.

Javitch JA, D'Amato RJ, Strittmatter SM, Snyder SH. (1985) Parkinsonism-inducing neurotoxin, N-methyl-4-phenyl-1,2,3,6 tetrahydropyridine: uptake of the metabolite $\mathrm{N}$-methyl-4-phenylpyridine by 
dopamine neurons explains selective toxicity. Proc Natl Acad Sci U S A 82:2173-2177.

Jellinger K, Kienzl E, Rumpelmair G, Riederer P, Stachelberger H, BenShachar D, Youdim MB. (1992) Iron-melanin complex in substantia nigra of parkinsonian brains: an x-ray microanalysis. J Neurochem 59:1168-1171. Jellinger KA, Stadelmann C. (2000) Mechanisms of cell death in neurodegenerative disorders. J Neural Transm Suppl 59:95-114. Jenner P, Rupniak NM, Rose S, Kelly E, Kilpatrick G, Lees A, Marsden CD. (1984) 1-Methyl-4-phenyl-1,2,3,6-tetrahydropyridine-induced parkinsonism in the common marmoset. Neurosci Lett 50:85-90.

Jorgensen RA, Cluster PD, English J, Que Q, Napoli CA. (1996) Chalcone synthase cosuppression phenotypes in petunia flowers: comparison of sense vs. antisense constructs and single-copy vs. complex T-DNA sequences. Plant Mol Biol 31:957-973.

Ju C, Yoon KN, Oh YK, Kim HC, Shin CY, Ryu JR, Ko KH, Kim WK. (2000) Synergistic depletion of astrocytic glutathione by glucose deprivation and peroxynitrite: correlation with mitochondrial dysfunction and subsequent cell death. J Neurochem 74:1989-1998.

Junn E, Mouradian MM. (2001) Apoptotic signaling in dopamine-induced cell death: the role of oxidative stress, p38 mitogen-activated protein kinase, cytochrome c and caspases. J Neurochem 78:374-383.

Kikuchi A, Takeda A, Onodera H, Kimpara T, Hisanaga K, Sato N, Nunomura A, Castellani RJ, Perry G, Smith MA, Itoyama Y. (2002) Systemic 
increase of oxidative nucleic acid damage in Parkinson's disease and multiple system atrophy. Neurobiol Dis 9:244-248.

Kingsbury AE, Foster OJ, Nisbet AP, Cairns N, Bray L, Eve DJ, Lees AJ, Marsden CD. (1995) Tissue $\mathrm{pH}$ as an indicator of mRNA preservation in human post-mortem brain. Brain Res Molec Brain Res 28:311-318.

Kish SJ, Morito C, Hornykiewicz O. (1985) Glutathione peroxidase activity in Parkinson's disease brain. Neurosci Lett 58:343-346.

Kuo WP, Jenssen TK, Butte AJ, Ohno-Machado L, Kohane IS. (2002) Analysis of matched mRNA measurements from two different microarray technologies. Bioinformatics 18:405-412.

Langston JW, Ballard P, Tetrud JW, Irwin I. (1983) Chronic Parkinsonism in humans due to a product of meperidine-analog synthesis. Science 219:979980.

Langston JW, Forno LS, Tetrud J, Reeves AG, Kaplan JA, Karluk D. (1999) Evidence of active nerve cell degeneration in the substantia nigra of humans years after 1-methyl-4-phenyl-1,2,3,6-tetrahydropyridine exposure. Ann Neurol 46:598-605.

Langston JW, Langston EB, Irwin I. (1984) MPTP-induced parkinsonism in human and non-human primates--clinical and experimental aspects. Acta Neurol Scand Suppl 100, 49-54.

Lebovitz RM, Zhang H, Vogel H, Cartwright J Jr, Dionne L, Lu N, Huang S, Matzuk MM. (1996) Neurodegeneration, myocardial injury, and perinatal death 
in mitochondrial superoxide dismutase-deficient mice. Proc Natl Acad Sci U S A 93:9782-9787.

Lee EH, Yang YL, Chia LG, Kuo JS. (1994) Interactions among deprenyl, clorgyline and 1-methyl-4-phenyl-1,2,3,6-tetrahydropyridine on catecholamines in mice. Neurosci Lett 165:149-52.

Lee R, Kermani P, Teng KK, Hempstead BL. (2001) Regulation of cell survival by secreted proneurotrophins. Science 294:1945-1948.

Leonard S, Logel J, Luthman D, Casanova M, Kirch D, Freedman R. (1993) Biological stability of mRNA isolated from human postmortem brain collections. Biol Psychiatry 33:456-466.

Leveugle B, Faucheux BA, Bouras C, Nillesse N, Spik G, Hirsch EC, Agid Y, Hof PR. (1996) Cellular distribution of the iron-binding protein lactotransferrin in the mesencephalon of Parkinson's disease cases. Acta Neuropathol (Berl) 91:566-572.

Lewohl JM, Wang L, Miles MF, Zhang L, Dodd PR, Harris RA. (2000) Gene expression in human alcoholism: microarray analysis of frontal cortex. Alcoholism Clin Exp Res 24:1873-1882.

Li Y, Huang TT, Carlson EJ, Melov S, Ursell PC, Olson JL, Noble LJ, Yoshimura MP, Berger C, Chan PH, et al. (1995) Dilated cardiomyopathy and neonatal lethality in mutant mice lacking manganese superoxide dismutase. Nat Genet 11:376-381.

Liberatore GT, Jackson-Lewis V, Vukosavic S, Mandir AS, Vila M, McAuliffe WG, Dawson VL, Dawson TM, Przedborski S. (1999) Inducible nitric 
oxide synthase stimulates dopaminergic neurodegeneration in the MPTP model of Parkinson disease. Nat Med 5:1403-1409.

Lin YZ, Yao SY, Veach RA, Torgerson TR, Hawiger J. (1995) Inhibition of nuclear translocation of transcription factor NF-kappa B by a synthetic peptide containing a cell membrane-permeable motif and nuclear localization sequence. J Biol Chem 270:14255-8.

Lucking CB, Durr A, Bonifati V, Vaughan J, De Michele G, Gasser T, Harhangi BS, Meco G, Denefle P, Wood NW, Agid Y, Brice A. (2000) Association between early-onset Parkinson's disease and mutations in the parkin gene. French Parkinson's Disease Genetics Study Group. N Engl J Med 342:1560-7. Luethy JD, Holbrook NJ. (1992) Activation of the gadd153 promoter by genotoxic agents: a rapid and specific response to DNA damage. Cancer Res $52: 5-10$.

Maekawa M, Ishizaki T, Boku S, Watanabe N, Fujita A, Iwamatsu A, Obinata T, Ohashi K, Mizuno K, Narumiya S. (1999) Signaling from Rho to the actin cytoskeleton through protein kinases ROCK and LIM-kinase. Science 285:895-898.

Manche L, Green SR, Schmedt C, Mathews MB. (1992) Interactions between double-stranded RNA regulators and the protein kinase DAI. Mol Cell Biol 12:5238-5248.

Mandel S, Grunblatt E, Maor G, Youdim MB. (2002) Early and late gene changes in MPTP mice model of Parkinson's disease employing cDNA microarray. Neurochem Res 27:1231-1243. 
Mandir AS, Przedborski S, Jackson-Lewis V, Wang ZQ, SimbulanRosenthal CM, Smulson ME, Hoffman BE, Guastella DB, Dawson VL, Dawson TM. (1999) Poly(ADP-ribose) polymerase activation mediates 1-methyl-4-phenyl1, 2,3,6-tetrahydropyridine (MPTP)-induced parkinsonism. Proc Natl Acad Sci U S A 96:5774-5779.

Mann VM, Cooper JM, Kriige D, Daniel SE, Schapira AHV, Marsden D (1992) Brain, skeletal muscle, and platelet homogenate mitochondrial functions in Parkinson's disease. Brain 115:333-342.

Marcinkiewicz M, Seidah NG. (2000) Coordinated expression of betaamyloid precursor protein and the putative beta-secretase BACE and alphasecretase ADAM10 in mouse and human brain. J Neurochem 75:2133-2143.

Masserano JM, Gong L, Kulaga H, Baker I, Wyatt RJ. (1996) Dopamine induces apoptotic cell death of a catecholaminergic cell line derived from the central nervous system. Mol Pharmacol 50:1309-1315.

Mathern GW, Pretorius JK, Kornblum HI, Mendoza D, Lozada A, Leite JP, Chimelli MC, Fried I, Sakamoto AC, Assirati JA, Levesque MF, Adelson PD, Peacock WJ. (1997) Human hippocampal AMPA and NMDA mRNA levels in temporal lobe epilepsy patients. Brain 120:1937-1959.

Matsumoto M, Minami M, Takeda K, Sakao Y, Akira S. (1996) Ectopic expression of CHOP (GADD153) induces apoptosis in M1 myeloblastic leukemia cells. FEBS Lett 395:143-147. 
Matthews RT, Yang L, Beal MF. (1997) S-Methylthiocitrulline, a neuronal nitric oxide synthase inhibitor, protects against malonate and MPTP neurotoxicity. Exp Neurol 143:282-286.

Melov S, Schneider JA, Day BJ, Hinerfeld D, Coskun P, Mirra SS, Crapo JD, Wallace DC. (1998) A novel neurological phenotype in mice lacking mitochondrial manganese superoxide dismutase. Nat Genet 18:159-163.

Merritt HH (1956) Paralysis agitans [editorial]. J Chron Dis 6:654-657.

Minks MA, Benvin S, Maroney PA, Baglioni C. (1979) Metabolic stability of 2' 5'oligo (A) and activity of 2' 5'oligo (A)-dependent endonuclease in extracts of interferon-treated and control HeLa cells. Nucleic Acids Res 6:767-780.

Mizuno Y, Ohta S, Tanaka M, Takamiya S, Suzuki K, Sato T, Oya H, Ozawa T, and Kagawa Y (1989) Deficiencies of the respiratory chain in Parkinson's disease. Biochem Biophys Res Comm 163:1450-1455.

Mochizuki H, Goto K, Mori H, Mizuno Y. (1996) Histochemical detection of apoptosis in Parkinson's disease. J Neurol Sci 137:120-123.

Montagu KA. (1957) Catechol compounds in rat tissues and in brains of different animals. Nature 180:244-245.

Moresco RM, Volonte MA, Messa C, Gobbo C, Galli L, Carpinelli A, Rizzo G, Panzacchi A, Franceschi M, Fazio F. (2002) New perspectives on neurochemical effects of amantadine in the brain of parkinsonian patients: a PET - [(11)C]raclopride study. J Neural Transm 109:1265-74. 
Mytilineau C, Werner P, Molinari S, DiRocco A, Cohen G, Yahr MD (1994) Impaired oxidative decarboxylation of pyruvate in fibroblasts from patients with Parkinson's disease. J Neural Transm-Park Dis Dement Sect 8: 223-228.

Nakayama, K. (1997) Furin: a mammalian subtilisin/Kex2p-like endoprotease involved in processing of a wide variety of precursor proteins. Biochem J :625-635.

Napolitano M, Centonze D, Calce A, Picconi B, Spiezia S, Gulino A, Bernardi G, Calabresi P. (2002) Experimental parkinsonism modulates multiple genes involved in the transduction of dopaminergic signals in the striatum. Neurobiol Dis 10:387-395.

Nicklas WJ, Vyas I, Heikkila RE. (1985) Inhibition of NADH-linked oxidation in brain mitochondria by 1-methyl-4-phenyl-pyridine, a metabolite of the neurotoxin, 1-methyl-4-phenyl-1,2,5,6-tetrahydropyridine. Life Sci 8:223-228.

Offen D, Beart PM, Cheung NS, Pascoe CJ, Hochman A, Gorodin S, Melamed E, Bernard R, Bernard O. (1998) Transgenic mice expressing human $\mathrm{Bcl}-2$ in their neurons are resistant to 6-hydroxydopamine and 1-methyl-4-phenyl1,2,3,6- tetrahydropyridine neurotoxicity. Proc Natl Acad Sci U S A 95:57895794.

Offen D, Ziv I, Panet H, Wasserman L, Stein R, Melamed E, Barzilai A. (1997) Dopamine-induced apoptosis is inhibited in PC12 cells expressing Bcl-2. Cell Mol Neurobiol 17:289-304.

Olanow CW. (2002) Surgical therapy for Parkinson's disease. Eur J Neurol 3:31-39. 
Olanow CW, Goetz CG, Kordower JH, Stoessl AJ, Sossi V, Brin MF, Shannon KM, Nauert GM, Perl DP, Godbold J, Freeman TB. (2003) A doubleblind controlled trial of bilateral fetal nigral transplantation in Parkinson's disease. Ann Neurol 54:403-14.

Oo TF, Siman R, Burke RE. (2002) Distinct nuclear and cytoplasmic localization of caspase cleavage products in two models of induced apoptotic death in dopamine neurons of the substantia nigra. Exp Neurol 175:1-9.

Osterlund MK, Keller E, Hurd YL. (2000) The human forebrain has discrete estrogen receptor alpha messenger RNA expression: high levels in the amygdaloid complex. Neuroscience 95:333-342.

Pardue S, Zimmerman AL, Morrison-Bogorad M. (1994) Selective postmortem degradation of inducible heat shock protein 70 (hsp70) mRNAs in rat brain. Cell Mol Neurobio 14:341-357.

Parker WD Jr, Boyson SJ, Parks JK (1989) Abnormalities of the electron transport chain in idiopathic Parkinson's disease. Ann Neurol 26:719-723.

Parkinson J. (1817) An essay on the shaking palsy. London, Whittingham and Rowland for Sherwood, Neely, Jones.

Pearce RK, Owen A, Daniel S, Jenner P, Marsden CD. (1997) Alterations in the distribution of glutathione in the substantia nigra in Parkinson's disease. $J$ Neural Transm 104:661-677.

Perry TL, Godin DV, Hansen S. (1982) Parkinson's disease: a disorder due to nigral glutathione deficiency? Neurosci Lett 33:305-310. 
Peterson F. (1890) Paralysis agitans. In: Starr MA, ed., Familiar Forms of Nervous Disease. New York, Columbia University Press.

Poewe W, Wenning G. (2002) The differential diagnosis of Parkinson's disease. Eur J Neurol 9 Suppl 3:23-30.

Polymeropoulos MH, Lavedan C, Leroy E, Ide SE, Dehejia A, Dutra A, Pike B, Root H, Rubenstein J, Boyer R, Stenroos ES, Chandrasekharappa S, Athanassiadou A, Papapetropoulos T, Johnson WG, Lazzarini AM, Duvoisin RC, Di lorio G, Golbe LI, Nussbaum RL. (1997) Mutation in the alpha-synuclein gene identified in families with Parkinson's disease. Science 276:2045-2047.

Pryor WA. (1986) Oxy-radicals and related species: their formation, lifetimes, and reactions. Ann Rev Physiol 48:657-667.

Przedborski S, Jackson-Lewis V, Yokoyama R, Shibata T, Dawson VL, Dawson TM. (1996) Role of neuronal nitric oxide in 1-methyl-4-phenyl-1,2,3,6tetrahydropyridine (MPTP)-induced dopaminergic neurotoxicity. Proc Natl Acad Sci U S A 93:4565-4571.

Przedborski S, Kostic V, Jackson-Lewis V, Naini AB, Simonetti S, Fahn S, Carlson E, Epstein CJ, Cadet JL. (1992) Transgenic mice with increased Cu/Znsuperoxide dismutase activity are resistant to N-methyl-4-phenyl-1,2,3,6tetrahydropyridine-induced neurotoxicity. J Neurosci 12:1658-1667.

Qiao D, Im E, Qi W, Martinez JD. (2002) Activator protein-1 and CCAAT/enhancer-binding protein mediated GADD153 expression is involved in deoxycholic acid-induced apoptosis. Biochim Biophys Acta 1583:108-16. 
Raha S, McEachern GE, Myint AT, Robinson BH. (2000) Superoxides from mitochondrial complex III: the role of manganese superoxide dismutase. Free Radic Biol Med 29:170-180.

Riederer P, Sofic E, Rausch WD, Schmidt B, Reynolds GP, Jellinger K, Youdim MB. (1989) Transition metals, ferritin, glutathione, and ascorbic acid in parkinsonian brains. J Neurochem 52:515-520.

Ross GW, Abbott RD, Petrovitch H, Morens DM, Grandinetti A, Tung KH, Tanner CM, Masaki KH, Blanchette PL, Curb JD, Popper JS, White LR. (2000) Association of coffee and caffeine intake with the risk of Parkinson disease. JAMA 283:2674-2679.

Sano I, Gamo T, Kakimoto Y, Taniguchi K, Takesada M, Nishinuma K. (1959) Distribution of catechol compounds in human brain. Biochem Biophys Acta 32:586-587.

Schramm M, Falkai P, Tepest R, Schneider-Axmann T, Przkora R, Waha A, Pietsch T, Bonte W, Bayer TA. (1999) Stability of RNA transcripts in postmortem psychiatric brains. J Neur Tran 106:329-335.

Schulz JB, Matthews RT, Muqit MM, Browne SE, Beal MF. (1995) Inhibition of neuronal nitric oxide synthase by 7-nitroindazole protects against MPTP-induced neurotoxicity in mice. J Neurochem 64:936-939.

Schwab RS, England AC Jr., Poskanzer DC, Young RR. (1969) Amantadine in the treatment of Parkinson's disease. JAMA 208:1168-1170. 
Snyder SH, D'Amato RJ. (1986) MPTP: a neurotoxin relevant to the pathophysiology of Parkinson's disease. The 1985 George C. Cotzias lecture. Neurology 36:250-258.

Sofic E, Lange KW, Jellinger K, Riederer P. (1992) Reduced and oxidized glutathione in the substantia nigra of patients with Parkinson's disease. Neurosci Lett 142:128-130.

Stephans SE, Miller GW, Levey Al, Greenamyre JT (2002) Acute mitochondrial and chronic toxicological effects of 1-methyl-4-phenylpyridinium in human neuroblastoma cells. Neurotoxicol 23:569-580.

Sveinbjornsdottir S, Hicks AA, Jonsson T, Petursson H, Gugmundsson G, Frigge ML, Kong A, Gulcher JR, Stefansson K. (2000) Familial aggregation of Parkinson's disease in Iceland. N Engl J Med 343:1765-70.

Swerdlow RH, Parks JK, Miller SW, Tuttle JB, Trimmer PA, Sheehan JP, Bennett JP Jr, Davis RE, Parker WD Jr. (1996) Origin and functional consequences of the complex I defect in Parkinson's disease. Ann Neurol 40:663-671.

Tanner CM, Ottman R, Goldman SM, Ellenberg J, Chan P, Mayeux R, Langston JW. Parkinson disease in twins: an etiologic study. (1999) JAMA 281:341-6.

Timmons L, Court DL, Fire A. (2001) Ingestion of bacterially expressed dsRNAs can produce specific and potent genetic interference in Caenorhabditis elegans. Gene 263:103-112. 
Trimmer PA, Smith TS, Jung AB, Bennett JP Jr. (1996) Dopamine neurons from transgenic mice with a knockout of the p53 gene resist MPTP neurotoxicity. Neurodegeneration 5:233-239.

Turrens JF, Alexandre A, Lehninger AL. (1985) Ubisemiquinone is the electron donor for superoxide formation by complex III of heart mitochondria. Arch Biochem Biophys 237:408-414.

Veech GA, Dennis J, Keeney PM, Fall CP, Swerdlow RH, Parker WD Jr, Bennett JP Jr. (2000) Disrupted mitochondrial electron transport function increases expression of anti-apoptotic bcl-2 and bcl-X(L) proteins in SH-SY5Y neuroblastoma and in Parkinson disease cybrid cells through oxidative stress. $J$ Neurosci Res 61:693-700.

Vila M, Jackson-Lewis VV, Vukosavic S, Djaldetti R, Liberatore G, Offen D, Korsmeyer SJ, Przedborski S. (2001) Bax ablation prevents dopaminergic neurodegeneration in the 1-methyl- 4-phenyl-1,2,3,6-tetrahydropyridine mouse model of Parkinson's disease. Proc Natl Acad Sci U S A 98:2837-2842.

Viswanath V, Wu Y, Boonplueang R, Chen S, Stevenson FF, Yantiri F, Yang L, Beal MF, Andersen JK. (2001) Caspase-9 activation results in downstream caspase-8 activation and bid cleavage in 1-methyl-4-phenyl-1,2,3,6tetrahydropyridine-induced Parkinson's disease. J Neurosci 21:9519-9528.

Vlahos CJ, Matter WF, Hui KY, Brown RF. (1994) A specific inhibitor of phosphatidylinositol 3-kinase, 2-(4-morpholinyl)-8-phenyl-4H-1-benzopyran-4one (LY294002). J Biol Chem 269:5241-5248. 
Von Economo, C. (1931) The sequelae of encephalitis lethargica. In: Newman K., ed., Encephalitis lethargica its sequelae and treatment: 105-156.

Waldmeier PC, Spooren WP, Hengerer B. (2000) CGP 3466 protects dopaminergic neurons in lesion models of Parkinson's disease. Naunyn Schmiedebergs Arch Pharmacol 362:526-537.

Yang L, Matthews RT, Schulz JB, Klockgether T, Liao AW, Martinou JC, Penney JB Jr, Hyman BT, Beal MF. (1998) 1-Methyl-4-phenyl-1,2,3,6tetrahydropyride neurotoxicity is attenuated in mice overexpressing Bcl-2. $J$ Neurosci 18:8145-8152.

Yoo MS, Chun HS, Son JJ, DeGiorgio LA, Kim DJ, Peng C, Son JH. (2003) Oxidative stress regulated genes in nigral dopaminergic neuronal cells: correlation with the known pathology in Parkinson's disease. Brain Res Mol Brain Res 110:76-84.

Yoritaka A, Hattori N, Uchida K, Tanaka M, Stadtman ER, Mizuno Y. (1996) Immunohistochemical detection of 4-hydroxynonenal protein adducts in Parkinson disease. Proc Natl Acad Sci U S A 93:2696-2701.

Zeeberg BR, Feng W, Wang G, Wang MD, Fojo AT, Sunshine M, Narasimhan S, Kane DW, Reinhold WC, Lababidi S, Bussey KJ, Riss J, Barrett JC, Weinstein JN. (2003) GoMiner: a resource for biological interpretation of genomic and proteomic data. Genome Biol 4:R28.

Zhang J, Perry G, Smith MA, Robertson D, Olson SJ, Graham DG, Montine TJ. (1999) Parkinson's disease is associated with oxidative damage to 
cytoplasmic DNA and RNA in substantia nigra neurons. Am J Pathol 154:14231429.

Zinszner H, Kuroda M, Wang X, Batchvarova N, Lightfoot RT, Remotti H, Stevens J L, Ron D. (1998) CHOP is implicated in programmed cell death in response to impaired function of the endoplasmic reticulum. Genes Dev 12:982-995.

Ziv I, Melamed E, Nardi N, Luria D, Achiron A, Offen D, Barzilai A. (1994) Dopamine induces apoptosis-like cell death in cultured chick sympathetic neurons--a possible novel pathogenetic mechanism in Parkinson's disease. Neurosci Lett 170:136-140.

Ziv I, Offen D, Barzilai A, Haviv R, Stein R, Zilkha-Falb R, Shirvan A, Melamed E. (1997) Modulation of control mechanisms of dopamine-induced apoptosis--a future approach to the treatment of Parkinson's disease? J Neural Transm Suppl 49:195-202. 
Appendix A. Genes that change $+/-2$ fold in response to $15 \mathrm{~m} \mathrm{MPP}+$.

\begin{tabular}{|c|c|c|c|c|c|}
\hline GENBANK & $\begin{array}{l}+/- \\
F O L D\end{array}$ & SYMBOL & LOCUSLINK & GENENAME & GENE ONTOLOGY \\
\hline H47026 & 2.10507 & MGAT3 & 4248 & $\begin{array}{l}\text { mannosyl (beta-1,4-)- } \\
\text { glycoprotein beta-1,4- } \\
\mathrm{N}- \\
\text { acetylglucosaminyltran } \\
\text { sferase }\end{array}$ & $\begin{array}{l}\text { Golgi apparatus; N- } \\
\text { linked glycosylation; } \\
\text { beta-1,4- } \\
\text { mannosylglycoprotein } \\
\text { beta-1,4-N- } \\
\text { acetylglucosaminyltran } \\
\text { sferase activity; } \\
\text { integral to membrane; } \\
\text { transferase activity, } \\
\text { transferring glycosyl } \\
\text { groups }\end{array}$ \\
\hline R19118 & 2.10065 & SDCBP & $\underline{6386}$ & $\begin{array}{l}\text { syndecan binding } \\
\text { protein (syntenin) }\end{array}$ & $\begin{array}{l}\text { actin modulating } \\
\text { activity; adherens } \\
\text { junction; cytoskeletal } \\
\text { adaptor activity; } \\
\text { cytoskeleton; } \\
\text { endoplasmic } \\
\text { reticulum; interleukin-5 } \\
\text { receptor binding; } \\
\text { interleukin-5 receptor } \\
\text { complex; intracellular } \\
\text { signaling cascade; } \\
\text { membrane; neurexin } \\
\text { binding; nucleus; } \\
\text { protein-membrane } \\
\text { targeting; regulation of } \\
\text { synapse; substrate- } \\
\text { bound cell migration, } \\
\text { cell extension; } \\
\text { syndecan binding }\end{array}$ \\
\hline $\mathrm{H} 26760$ & 2.02783 & $\frac{\mathrm{KIAA037}}{5}$ & $\underline{9853}$ & $\begin{array}{l}\text { KIAA0375 gene } \\
\text { product }\end{array}$ & \\
\hline T77387 & 1.99795 & & & & \\
\hline $\mathrm{H} 67530$ & 1.88371 & MYH11 & $\underline{4629}$ & \begin{tabular}{|l} 
myosin, heavy \\
polypeptide 11, smooth \\
muscle
\end{tabular} & $\begin{array}{l}\text { ATP binding; actin } \\
\text { binding; calmodulin } \\
\text { binding; cell growth } \\
\text { and/or maintenance; } \\
\text { kinesin complex; } \\
\text { motor activity; muscle } \\
\text { development; muscle } \\
\text { myosin; myosin; } \\
\text { striated muscle } \\
\text { contraction; striated } \\
\text { muscle thick filament }\end{array}$ \\
\hline
\end{tabular}




\begin{tabular}{|c|c|c|c|c|c|}
\hline R17538 & 1.84664 & $\underline{\text { SYN2 }}$ & $\underline{6854}$ & Synapsin 2 & $\begin{array}{l}\text { Neuronal } \\
\text { phosphoproteins; } \\
\text { synaptic proteins; } \\
\text { neurotransmission; } \\
\text { nucleus }\end{array}$ \\
\hline N93442 & 1.81151 & TTC11 & $\underline{51024}$ & \multicolumn{2}{|c|}{ tetratricopeptide repeat domain 11} \\
\hline R44837 & 1.76474 & & 339479 & \multicolumn{2}{|c|}{ similar to RIKEN cDNA B830045N13 } \\
\hline $\mathrm{H} 47146$ & 1.70916 & ERCC1 & $\underline{2067}$ & $\begin{array}{l}\text { excision repair cross- } \\
\text { complementing rodent } \\
\text { repair deficiency, } \\
\text { complementation } \\
\text { group } 1 \text { (includes } \\
\text { overlapping antisense } \\
\text { sequence) }\end{array}$ & $\begin{array}{l}\text { DNA repair; } \\
\text { embryogenesis and } \\
\text { morphogenesis; } \\
\text { endodeoxyribonucleas } \\
\text { e activity; nucleotide- } \\
\text { excision repair; } \\
\text { nucleus }\end{array}$ \\
\hline H83405 & 1.69136 & FGD1 & $\underline{2245}$ & $\begin{array}{l}\text { faciogenital dysplasia } \\
\text { (Aarskog-Scott } \\
\text { syndrome) }\end{array}$ & $\begin{array}{l}\text { development; guanyl- } \\
\text { nucleotide exchange } \\
\text { factor activity; } \\
\text { histogenesis and } \\
\text { organogenesis; signal } \\
\text { transduction; zinc ion } \\
\text { binding }\end{array}$ \\
\hline AA043061 & 1.68845 & $\frac{\text { DKFZP7 }}{27 \mathrm{G} 051}$ & $\underline{26147}$ & $\begin{array}{l}\text { DKFZP727G051 } \\
\text { protein }\end{array}$ & $\begin{array}{l}\text { DNA binding; } \\
\text { regulation of } \\
\text { transcription, DNA- } \\
\text { dependent } \\
\end{array}$ \\
\hline AA004997 & 1.6817 & TRAP100 & 9862 & $\begin{array}{l}\text { thyroid hormone } \\
\text { receptor-associated } \\
\text { protein }(100 \mathrm{kDa})\end{array}$ & $\begin{array}{l}\text { ATP binding; mediator } \\
\text { complex; } \\
\text { molecular function } \\
\text { unknown; nucleus; } \\
\text { regulation of } \\
\text { transcription, DNA- } \\
\text { dependent }\end{array}$ \\
\hline H62557 & 1.66864 & HSPA8 & 3312 & $\begin{array}{l}\text { heat shock } 70 \mathrm{kDa} \\
\text { protein } 8\end{array}$ & $\begin{array}{l}\text { ATP binding; heat } \\
\text { shock protein activity; } \\
\text { intracellular; non- } \\
\text { chaperonin molecular } \\
\text { chaperone ATPase } \\
\text { activity; protein folding }\end{array}$ \\
\hline N90938 & 1.6458 & $\begin{array}{l}\text { HNRPA2 } \\
\text { B1 }\end{array}$ & 3181 & $\begin{array}{l}\text { heterogeneous nuclear } \\
\text { ribonucleoprotein } \\
\text { A2/B1 }\end{array}$ & $\begin{array}{l}\text { RNA binding; RNA } \\
\text { processing; } \\
\text { heterogeneous } \\
\text { nuclear } \\
\text { ribonucleoprotein; } \\
\text { nucleus }\end{array}$ \\
\hline
\end{tabular}




\begin{tabular}{|c|c|c|c|c|c|}
\hline N32327 & 1.62862 & CPSF5 & $\underline{11051}$ & $\begin{array}{l}\text { cleavage and } \\
\text { polyadenylation } \\
\text { specific factor 5, } 25 \\
\text { kDa }\end{array}$ & $\begin{array}{l}\text { RNA binding; mRNA } \\
\text { processing; nucleus }\end{array}$ \\
\hline R19119 & 1.62086 & & & & \\
\hline N90527 & 1.58095 & PIM1 & $\underline{5292}$ & pim-1 oncogene & $\begin{array}{l}\text { ATP binding; cAMP- } \\
\text { dependent protein } \\
\text { kinase activity; cell } \\
\text { growth and/or } \\
\text { maintenance; } \\
\text { cytoplasm; } \\
\text { development; protein } \\
\text { amino acid } \\
\text { phosphorylation; } \\
\text { protein kinase CK2 } \\
\text { activity; protein } \\
\text { serine/threonine } \\
\text { kinase activity; } \\
\text { transferase activity } \\
\end{array}$ \\
\hline N20098 & 1.57982 & CD151 & $\underline{977}$ & CD151 antigen & $\begin{array}{l}\text { cell adhesion; integral } \\
\text { to plasma membrane; } \\
\text { membrane fraction }\end{array}$ \\
\hline W60305 & 1.56134 & & & & \\
\hline R84700 & 1.54625 & PKM2 & $\underline{5315}$ & $\begin{array}{l}\text { pyruvate kinase, } \\
\text { muscle }\end{array}$ & \\
\hline $\mathrm{H} 25578$ & 1.54297 & & & & \\
\hline R54918 & 1.53857 & $\begin{array}{l}\underline{F L J 1391} \\
\underline{2}\end{array}$ & $\underline{64785}$ & \multicolumn{2}{|c|}{ hypothetical protein FLJ13912 } \\
\hline AA137073 & 1.51737 & & & & \\
\hline \begin{tabular}{|l|} 
N70221 \\
\end{tabular} & 1.51581 & $\begin{array}{l}\text { KIAA050 } \\
\underline{0}\end{array}$ & $\underline{57237}$ & KIAA0500 protein & \\
\hline H45355 & 1.51462 & & & & \\
\hline H64900 & 1.51382 & & & & \\
\hline H79188 & 1.50945 & ERCC2 & $\underline{2068}$ & $\begin{array}{l}\text { excision repair cross- } \\
\text { complementing rodent } \\
\text { repair deficiency, } \\
\text { complementation } \\
\text { group } 2 \text { (xeroderma } \\
\text { pigmentosum D) }\end{array}$ & $\begin{array}{l}\text { ATP binding; ATP } \\
\text { dependent DNA } \\
\text { helicase activity; DNA } \\
\text { binding; hearing; } \\
\text { hydrolase activity; } \\
\text { magnesium ion } \\
\text { binding; nucleotide- } \\
\text { excision repair; } \\
\text { nucleus; regulation of } \\
\text { transcription, DNA- } \\
\text { dependent }\end{array}$ \\
\hline AA047172 & 1.50434 & WDR1 & $\underline{9948}$ & WD repeat domain 1 & $\begin{array}{l}\text { actin binding; } \\
\text { cytoskeleton; hearing; } \\
\text { protein binding }\end{array}$ \\
\hline AA004845 & 1.50065 & $\begin{array}{l}\text { KIAA152 } \\
\end{array}$ & $\underline{57653}$ & KIAA1529 protein & \\
\hline
\end{tabular}




\begin{tabular}{|c|c|c|c|c|c|}
\hline AA098865 & 1.49567 & BCL2L10 & 10017 & $\begin{array}{l}\text { BCL2-like } 10 \\
\text { (apoptosis facilitator) }\end{array}$ & $\begin{array}{l}\text { anti-apoptosis; } \\
\text { apoptosis inhibitor } \\
\text { activity; caspase } \\
\text { activation; integral to } \\
\text { membrane; membrane } \\
\text { fraction; } \\
\text { mitochondrion; } \\
\text { oogenesis; protein } \\
\text { binding; } \\
\text { spermatogenesis }\end{array}$ \\
\hline R84451 & 1.49119 & & & & \\
\hline N23806 & 1.48899 & $\frac{\mathrm{LOC} 1133}{86}$ & 113386 & $\begin{array}{l}\text { similar to envelope } \\
\text { protein }\end{array}$ & \\
\hline W85877 & 1.48488 & & & & \\
\hline W68333 & 1.47652 & & 346452 & LOC346452 & \\
\hline R87352 & 1.47143 & BCKDHA & $\underline{593}$ & $\begin{array}{l}\text { branched chain keto } \\
\text { acid dehydrogenase } \\
\text { E1, alpha polypeptide } \\
\text { (maple syrup urine } \\
\text { disease) }\end{array}$ & $\begin{array}{l}\text { 3-methyl-2- } \\
\text { oxobutanoate } \\
\text { dehydrogenase } \\
\text { (lipoamide) activity; 3- } \\
\text { methyl-2- } \\
\text { oxobutanoate } \\
\text { dehydrogenase } \\
\text { (lipoamide) complex; } \\
\text { alpha-ketoacid } \\
\text { dehydrogenase } \\
\text { activity; alpha- } \\
\text { ketoglutarate } \\
\text { dehydrogenase } \\
\text { complex (sensu } \\
\text { Eukarya); metabolism; } \\
\text { mitochondrion }\end{array}$ \\
\hline H69656 & 1.4693 & NARF & 26502 & $\begin{array}{l}\text { nuclear prelamin } \mathrm{A} \\
\text { recognition factor }\end{array}$ & $\begin{array}{l}\text { lamin binding; nuclear } \\
\text { lamina }\end{array}$ \\
\hline N56656 & 1.46477 & \begin{tabular}{|l|} 
D13S106 \\
E
\end{tabular} & 10208 & highly charged protein & $\begin{array}{l}\text { cysteine-type } \\
\text { endopeptidase } \\
\text { activity; ubiquitin C- } \\
\text { terminal hydrolase } \\
\text { activity; ubiquitin- } \\
\text { dependent protein } \\
\text { catabolism }\end{array}$ \\
\hline AA047135 & 1.46205 & RNH & $\underline{6050}$ & $\begin{array}{l}\text { ribonuclease/angiogeni } \\
n \text { inhibitor }\end{array}$ & $\begin{array}{l}\text { RNA catabolism; } \\
\text { ribonuclease inhibitor } \\
\text { activity }\end{array}$ \\
\hline AA101859 & 1.45684 & ENSA & 2029 & endosulfine alpha & $\begin{array}{l}\text { ion channel inhibitor } \\
\text { activity; receptor } \\
\text { binding; response to } \\
\text { nutrients; transport }\end{array}$ \\
\hline
\end{tabular}




\begin{tabular}{|c|c|c|c|c|c|}
\hline N71628 & 1.45316 & SPIB & $\underline{6689}$ & $\begin{array}{l}\text { Spi-B transcription } \\
\text { factor (Spi-1/PU.1 } \\
\text { related) }\end{array}$ & \begin{tabular}{|l} 
RNA polymerase II \\
transcription factor \\
activity; \\
biological_process \\
unknown; cytoplasm; \\
molecular_function \\
unknown; nucleus; \\
regulation of \\
transcription from Pol \\
II promoter; \\
transcription factor \\
activity
\end{tabular} \\
\hline AA131782 & 1.44189 & & & & \\
\hline H83025 & 1.43173 & & & & \\
\hline W45719 & 1.42601 & PAPA-1 & 83444 & PAP-1 binding protein & \\
\hline $\mathrm{H} 18298$ & 1.42171 & & & & \\
\hline N39391 & 1.41993 & $\begin{array}{l}\text { MGC147 } \\
\underline{99}\end{array}$ & $\underline{84296}$ & \multicolumn{2}{|c|}{ hypothetical protein MGC14799 } \\
\hline H18495 & 1.41884 & & & & \\
\hline AA194880 & 1.41282 & DC-UbP & $\underline{92181}$ & \multicolumn{2}{|c|}{ dendritic cell-derived ubiquitin-like protein } \\
\hline N24337 & 1.41241 & CD44 & $\underline{960}$ & $\begin{array}{l}\text { CD44 antigen (homing } \\
\text { function and Indian } \\
\text { blood group system) }\end{array}$ & $\begin{array}{l}\text { cell adhesion receptor } \\
\text { activity; cell-cell } \\
\text { adhesion; cell-matrix } \\
\text { adhesion; collagen } \\
\text { binding; hyaluronic } \\
\text { acid binding; integral } \\
\text { to plasma membrane; } \\
\text { receptor activity }\end{array}$ \\
\hline AA148568 & 1.4095 & SNRPB & $\underline{6628}$ & $\begin{array}{l}\text { small nuclear } \\
\text { ribonucleoprotein } \\
\text { polypeptides B and B1 }\end{array}$ & $\begin{array}{l}\text { mRNA splicing; small } \\
\text { nuclear } \\
\text { ribonucleoprotein; } \\
\text { spliceosome complex }\end{array}$ \\
\hline AA054271 & 1.40667 & GAPD & $\underline{2597}$ & $\begin{array}{l}\text { glyceraldehyde-3- } \\
\text { phosphate } \\
\text { dehydrogenase }\end{array}$ & $\begin{array}{l}\text { cytoplasm; } \\
\text { glyceraldehyde 3- } \\
\text { phosphate } \\
\text { dehydrogenase } \\
\text { (phosphorylating) } \\
\text { activity; glycolysis; } \\
\text { oxidoreductase activity }\end{array}$ \\
\hline H19297 & 1.40394 & EDIL3 & $\underline{10085}$ & $\begin{array}{l}\text { EGF-like repeats and } \\
\text { discoidin I-like domains } \\
3\end{array}$ & $\begin{array}{l}\text { calcium ion binding; } \\
\text { cell adhesion; cell } \\
\text { adhesion molecule } \\
\text { activity; development; } \\
\text { integrin binding }\end{array}$ \\
\hline W94117 & 1.40301 & & & & \\
\hline $\mathrm{H} 20520$ & 1.39971 & & & & \\
\hline
\end{tabular}




\begin{tabular}{|c|c|c|c|c|c|}
\hline $\mathrm{R} 85150$ & 1.39948 & EPHB6 & 2051 & EphB6 & $\begin{array}{l}\text { ATP binding; ephrin } \\
\text { receptor activity; } \\
\text { integral to membrane; } \\
\text { protein amino acid } \\
\text { phosphorylation; } \\
\text { protein tyrosine kinase } \\
\text { activity; receptor } \\
\text { activity; } \\
\text { transmembrane } \\
\text { receptor protein } \\
\text { tyrosine kinase } \\
\text { signaling pathway }\end{array}$ \\
\hline AA203242 & 1.39794 & ASB13 & $\underline{79754}$ & $\begin{array}{l}\text { ankyrin repeat and } \\
\text { SOCS box-containing } \\
13\end{array}$ & $\begin{array}{l}\text { intracellular signaling } \\
\text { cascade }\end{array}$ \\
\hline H93330 & 1.39314 & $\frac{\text { SLC9A3 }}{\text { R1 }}$ & 9368 & $\begin{array}{l}\text { solute carrier family } 9 \\
\text { (sodium/hydrogen } \\
\text { exchanger), isoform } 3 \\
\text { regulatory factor } 1\end{array}$ & $\begin{array}{l}\text { actin cytoskeleton; } \\
\text { intracellular signaling } \\
\text { cascade; protein } \\
\text { complex assembly }\end{array}$ \\
\hline W85843 & 1.39028 & $\begin{array}{l}\text { LOC2537 } \\
82\end{array}$ & $\underline{253782}$ & \multicolumn{2}{|c|}{ hypothetical protein LOC253782 } \\
\hline N43796 & 1.39023 & $\frac{\text { ATP6V0 }}{\text { D1 }}$ & $\underline{9114}$ & $\begin{array}{l}\text { ATPase, } \mathrm{H+} \\
\text { transporting, lysosomal } \\
38 \mathrm{kDa}, \mathrm{V} 0 \text { subunit d } \\
\text { isoform } 1\end{array}$ & $\begin{array}{l}\text { ATP biosynthesis; } \\
\text { hydrogen ion } \\
\text { transporter activity; } \\
\text { hydrogen- } \\
\text { translocating V-type } \\
\text { ATPase complex; } \\
\text { hydrogen-transporting } \\
\text { two-sector ATPase } \\
\text { activity; hydrolase } \\
\text { activity; } \\
\text { molecular_function } \\
\text { unknown; proton } \\
\text { transport }\end{array}$ \\
\hline AA057300 & 1.38962 & QDPR & $\underline{5860}$ & \begin{tabular}{|l} 
quinoid \\
dihydropteridine \\
reductase
\end{tabular} & $\begin{array}{l}\text { amino acid } \\
\text { metabolism; } \\
\text { dihydrobiopterin } \\
\text { reduction; } \\
\text { dihydropteridine } \\
\text { reductase activity; } \\
\text { electron transporter } \\
\text { activity; metabolism; } \\
\text { oxidoreductase } \\
\text { activity; phenylalanine } \\
\text { catabolism; } \\
\text { tetrahydrobiopterin } \\
\text { biosynthesis }\end{array}$ \\
\hline
\end{tabular}




\begin{tabular}{|c|c|c|c|c|c|}
\hline $\mathrm{H} 18900$ & 1.38528 & $\underline{\mathrm{TPI}}$ & $\underline{7167}$ & $\begin{array}{l}\text { triosephosphate } \\
\text { isomerase } 1\end{array}$ & $\begin{array}{l}\text { fatty acid biosynthesis; } \\
\text { gluconeogenesis; } \\
\text { glycolysis; isomerase } \\
\text { activity; metabolism; } \\
\text { pentose-phosphate } \\
\text { shunt; triose- } \\
\text { phosphate isomerase } \\
\text { activity }\end{array}$ \\
\hline H83698 & 1.38185 & $\frac{\text { MGC108 }}{20}$ & 84734 & $\begin{array}{l}\text { hypothetical protein } \\
\text { MGC10820 }\end{array}$ & kinesin complex \\
\hline W89099 & 1.36991 & CYP4F12 & $\underline{66002}$ & $\begin{array}{l}\text { cytochrome P450, } \\
\text { family } 4 \text {, subfamily F, } \\
\text { polypeptide } 12\end{array}$ & $\begin{array}{l}\text { electron transport; } \\
\text { endoplasmic } \\
\text { reticulum; membrane; } \\
\text { microsome; } \\
\text { monooxygenase } \\
\text { activity }\end{array}$ \\
\hline $\mathrm{H} 69898$ & 1.36373 & & $\underline{285465}$ & \multicolumn{2}{|c|}{ hypothetical gene supported by AK096576 } \\
\hline H69898 & 1.36373 & $\frac{F L J 2184}{1}$ & 79662 & \multicolumn{2}{|c|}{ hypothetical protein FLJ21841 } \\
\hline H82992 & 1.36359 & PIGT & $\underline{51604}$ & \multicolumn{2}{|c|}{ phosphatidyl inositol glycan class $T$} \\
\hline AA127687 & 1.36203 & \begin{tabular}{|l} 
MGC319 \\
$\underline{6}$ \\
\end{tabular} & $\underline{79064}$ & \multicolumn{2}{|c|}{ hypothetical protein MGC3196 } \\
\hline AA150384 & 1.35985 & NICE-4 & $\underline{9898}$ & NICE-4 protein & \\
\hline H43455 & 1.35791 & PP2447 & $\underline{80305}$ & \multicolumn{2}{|c|}{ hypothetical protein PP2447 } \\
\hline N45640 & 1.35736 & $\underline{\mathrm{CH}} 25 \mathrm{H}$ & $\underline{9023}$ & $\begin{array}{l}\text { cholesterol 25- } \\
\text { hydroxylase }\end{array}$ & $\begin{array}{l}\text { catalytic activity; lipid } \\
\text { metabolism; } \\
\text { membrane fraction; } \\
\text { steroid hydroxylase } \\
\text { activity } \\
\end{array}$ \\
\hline N53883 & 1.35661 & $\frac{\mathrm{KIAA027}}{6}$ & 23142 & KIAA0276 protein & \\
\hline H67193 & 1.35305 & EIF2S3 & 1968 & $\begin{array}{l}\text { eukaryotic translation } \\
\text { initiation factor } 2 \\
\text { subunit } 3 \text { gamma, } \\
52 \mathrm{kDa}\end{array}$ & $\begin{array}{l}\text { GTPase activity; } \\
\text { cytosolic small } \\
\text { ribosomal subunit } \\
\text { (sensu Eukarya); } \\
\text { eukaryotic translation } \\
\text { initiation factor } 2 \\
\text { complex; translation } \\
\text { elongation factor } \\
\text { activity; translation } \\
\text { initiation factor activity; } \\
\text { translational } \\
\text { elongation }\end{array}$ \\
\hline \begin{tabular}{|l|} 
N47284 \\
\end{tabular} & 1.3508 & & & & \\
\hline R23351 & 1.34692 & & & & \\
\hline AA059148 & 1.34334 & $\frac{\mathrm{KIAA} 119}{9}$ & $\underline{57214}$ & KIAA1199 protein & \\
\hline W46155 & 1.34043 & & & & \\
\hline
\end{tabular}




\begin{tabular}{|c|c|c|c|c|c|}
\hline W88726 & 1.3382 & MTX1 & 4580 & metaxin 1 & integral to membrane \\
\hline AA213450 & 1.33654 & & & & \\
\hline W58177 & 1.33626 & $\begin{array}{l}\mathrm{HIST} 2 \mathrm{H} 2 \\
\mathrm{AA}\end{array}$ & 8337 & histone 2, H2aa & $\begin{array}{l}\text { DNA binding; } \\
\text { chromosome; } \\
\text { chromosome } \\
\text { organization and } \\
\text { biogenesis (sensu } \\
\text { Eukarya); } \\
\text { nucleosome; } \\
\text { nucleosome } \\
\text { assembly; nucleus }\end{array}$ \\
\hline N42484 & 1.33301 & & & & \\
\hline AA019138 & 1.33299 & SLC2A5 & $\underline{6518}$ & $\begin{array}{l}\text { solute carrier family } 2 \\
\text { (facilitated } \\
\text { glucose/fructose } \\
\text { transporter), member } 5\end{array}$ & $\begin{array}{l}\text { carbohydrate } \\
\text { metabolism; } \\
\text { carbohydrate } \\
\text { transport; fructose } \\
\text { transport; fructose } \\
\text { transporter activity; } \\
\text { glucose transport; } \\
\text { glucose transporter } \\
\text { activity; integral to } \\
\text { membrane; plasma } \\
\text { membrane; sugar } \\
\text { porter activity; } \\
\text { transporter activity } \\
\end{array}$ \\
\hline AA213887 & 1.32852 & $\frac{F L J 2190}{8}$ & 79657 & \multicolumn{2}{|c|}{ hypothetical protein FLJ21908 } \\
\hline $\mathrm{H} 40607$ & 1.32381 & & & & \\
\hline N95545 & 1.3227 & IL11 & $\underline{3589}$ & interleukin 11 & $\begin{array}{l}\text { B-cell differentiation; } \\
\text { adipocyte } \\
\text { differentiation; cell } \\
\text { proliferation; cell-cell } \\
\text { signaling; cytokine } \\
\text { activity; extracellular; } \\
\text { interleukin-11 receptor } \\
\text { binding; } \\
\text { megakaryocyte } \\
\text { differentiation; platelet } \\
\text { activation; positive } \\
\text { regulation of cell } \\
\text { proliferation }\end{array}$ \\
\hline AA044803 & 1.32088 & \begin{tabular}{|l} 
FLJ2004 \\
$\underline{0}$
\end{tabular} & $\underline{54442}$ & $\begin{array}{l}\text { hypothetical protein } \\
\text { FLJ20040 }\end{array}$ & $\begin{array}{l}\text { membrane; potassium } \\
\text { ion transport; protein } \\
\text { binding; voltage-gated } \\
\text { potassium channel } \\
\text { activity; voltage-gated } \\
\text { potassium channel } \\
\text { complex }\end{array}$ \\
\hline
\end{tabular}




\begin{tabular}{|c|c|c|c|c|c|}
\hline AA031859 & 1.31813 & TIMM13 & $\underline{26517}$ & $\begin{array}{l}\text { translocase of inner } \\
\text { mitochondrial } \\
\text { membrane } 13 \text { homolog } \\
\text { (yeast) }\end{array}$ & $\begin{array}{l}\text { hearing; mitochondrial } \\
\text { inner membrane pre- } \\
\text { sequence translocase } \\
\text { complex; } \\
\text { mitochondrial } \\
\text { translocation; } \\
\text { mitochondrion; protein } \\
\text { targeting; protein } \\
\text { translocase activity; } \\
\text { zinc ion binding }\end{array}$ \\
\hline AA142881 & 1.31641 & & 284354 & similar to BC282485 1 & \\
\hline AA210768 & 1.31532 & & & & \\
\hline H19488 & 1.31527 & & & & \\
\hline AA203110 & 1.31081 & AIBZIP & 148327 & $\begin{array}{l}\text { androgen-induced } \\
\text { basic leucine zipper }\end{array}$ & $\begin{array}{l}\text { DNA binding; nucleus; } \\
\text { regulation of } \\
\text { transcription, DNA- } \\
\text { dependent }\end{array}$ \\
\hline $\mathrm{H} 62766$ & 1.30716 & & & & \\
\hline AA002135 & 1.30424 & C2 & $\underline{717}$ & $\begin{array}{l}\text { complement } \\
\text { component } 2\end{array}$ & $\begin{array}{l}\text { chymotrypsin activity; } \\
\text { classical-complement } \\
\text { pathway C3/C5 } \\
\text { convertase activity; } \\
\text { complement } \\
\text { activation, classical } \\
\text { pathway; complement } \\
\text { component C2 } \\
\text { complex; hydrolase } \\
\text { activity; proteolysis } \\
\text { and peptidolysis; } \\
\text { trypsin activity }\end{array}$ \\
\hline H87311 & 1.30351 & ERCC1 & $\underline{2067}$ & $\begin{array}{l}\text { excision repair cross- } \\
\text { complementing rodent } \\
\text { repair deficiency, } \\
\text { complementation } \\
\text { group } 1 \text { (includes } \\
\text { overlapping antisense } \\
\text { sequence) }\end{array}$ & $\begin{array}{l}\text { DNA repair; } \\
\text { embryogenesis and } \\
\text { morphogenesis; } \\
\text { endodeoxyribonucleas } \\
\text { e activity; nucleotide- } \\
\text { excision repair; } \\
\text { nucleus }\end{array}$ \\
\hline H84257 & 1.29776 & & & & \\
\hline H45746 & 1.29746 & & & & \\
\hline AA127214 & 1.29622 & IGFBP5 & 3488 & $\begin{array}{l}\text { insulin-like growth } \\
\text { factor binding protein } 5\end{array}$ & $\begin{array}{l}\text { extracellular space; } \\
\text { insulin-like growth } \\
\text { factor binding; } \\
\text { regulation of cell } \\
\text { growth; signal } \\
\text { transduction } \\
\end{array}$ \\
\hline T86338 & 1.29606 & & & & \\
\hline $\mathrm{H} 26580$ & 1.29372 & IGKC & $\underline{3514}$ & $\begin{array}{l}\text { immunoglobulin kappa } \\
\text { constant }\end{array}$ & $\begin{array}{l}\text { antigen binding; } \\
\text { immune response }\end{array}$ \\
\hline
\end{tabular}




\begin{tabular}{|c|c|c|c|c|c|}
\hline $\mathrm{H} 27908$ & 1.29153 & TUBB4 & 10381 & tubulin, beta, 4 & $\begin{array}{l}\text { GTP binding; } \\
\text { cytoskeleton; } \\
\text { microtubule; } \\
\text { microtubule-based } \\
\text { movement; structural } \\
\text { constituent of } \\
\text { cytoskeleton } \\
\end{array}$ \\
\hline $\mathrm{H} 26870$ & 1.28945 & & & & \\
\hline $\mathrm{H} 27400$ & 1.28938 & & 286202 & LOC286202 & \\
\hline $\mathrm{H} 70974$ & 1.28906 & & & & \\
\hline W47153 & 1.28838 & PTRF & $\underline{284119}$ & \multicolumn{2}{|c|}{ polymerase I and transcript release factor } \\
\hline N32700 & 1.28764 & $\underline{\text { RPS3 }}$ & $\underline{6188}$ & ribosomal protein S3 & $\begin{array}{l}\text { RNA binding; cytosolic } \\
\text { small ribosomal } \\
\text { subunit (sensu } \\
\text { Eukarya); intracellular; } \\
\text { protein biosynthesis; } \\
\text { structural constituent } \\
\text { of ribosome }\end{array}$ \\
\hline AA131933 & 1.28741 & ABP1 & $\underline{26}$ & $\begin{array}{l}\text { amiloride binding } \\
\text { protein } 1 \text { (amine } \\
\text { oxidase (copper- } \\
\text { containing)) }\end{array}$ & $\begin{array}{l}\text { amine oxidase } \\
\text { (copper-containing) } \\
\text { activity; copper ion } \\
\text { binding; drug binding; } \\
\text { heparin binding; } \\
\text { metabolism; } \\
\text { oxidoreductase } \\
\text { activity; peroxisome } \\
\end{array}$ \\
\hline AA046245 & 1.28676 & OSF-2 & $\underline{10631}$ & $\begin{array}{l}\text { osteoblast specific } \\
\text { factor } 2 \text { (fasciclin I-like) }\end{array}$ & $\begin{array}{l}\text { cell adhesion; cell } \\
\text { adhesion molecule } \\
\text { activity; extracellular } \\
\text { matrix; skeletal } \\
\text { development }\end{array}$ \\
\hline R48060 & 1.28409 & $\begin{array}{l}\text { DKFZP5 } \\
\underline{640243} \\
\end{array}$ & $\underline{25864}$ & $\begin{array}{l}\text { DKFZP564O243 } \\
\text { protein }\end{array}$ & \\
\hline W45695 & 1.28288 & H2AFZ & $\underline{3015}$ & $\begin{array}{l}\mathrm{H} 2 \mathrm{~A} \text { histone family, } \\
\text { member } \mathrm{Z}\end{array}$ & $\begin{array}{l}\text { DNA binding; } \\
\text { chromosome; } \\
\text { chromosome } \\
\text { organization and } \\
\text { biogenesis (sensu } \\
\text { Eukarya); } \\
\text { nucleosome; } \\
\text { nucleosome } \\
\text { assembly; nucleus }\end{array}$ \\
\hline N48735 & 1.27935 & & & & \\
\hline N24096 & 1.27886 & AKAP12 & $\underline{9590}$ & $\begin{array}{l}\text { A kinase (PRKA) } \\
\text { anchor protein (gravin) } \\
12\end{array}$ & $\begin{array}{l}\text { G-protein coupled } \\
\text { receptor protein } \\
\text { signaling pathway; } \\
\text { cytoplasm; protein } \\
\text { kinase A anchoring } \\
\text { activity; protein } \\
\text { targeting; protein } \\
\text { transporter activity }\end{array}$ \\
\hline
\end{tabular}




\begin{tabular}{|c|c|c|c|c|c|}
\hline H68587 & 1.27795 & & 340833 & LOC340833 & \\
\hline R49189 & 1.27581 & SLC30A6 & $\underline{55676}$ & \multicolumn{2}{|c|}{$\begin{array}{l}\text { solute carrier family } 30 \text { (zinc transporter), } \\
\text { member } 6\end{array}$} \\
\hline AA057286 & 1.27551 & $\frac{\text { TA- }}{\text { WDRP }}$ & 134430 & $\begin{array}{l}\text { T-cell activation WD } \\
\text { repeat protein }\end{array}$ & $\begin{array}{l}\text { catalytic activity; } \\
\text { metabolism }\end{array}$ \\
\hline R49895 & 1.2755 & & 350854 & similar to SNAG1 & \\
\hline W02372 & 1.27341 & & $\overline{\underline{284752}}$ & LOC284752 & \\
\hline W33064 & 1.27308 & TUBA1 & $\underline{7277}$ & $\begin{array}{l}\text { tubulin, alpha } 1 \text { (testis } \\
\text { specific) }\end{array}$ & $\begin{array}{l}\text { microtubule; structural } \\
\text { constituent of } \\
\text { cytoskeleton }\end{array}$ \\
\hline R88435 & 1.27112 & DPP6 & 1804 & dipeptidylpeptidase 6 & $\begin{array}{l}\text { catalytic activity; } \\
\text { dipeptidyl-peptidase IV } \\
\text { activity; dipeptidyl- } \\
\text { peptidase activity; } \\
\text { integral to membrane; } \\
\text { proteolysis and } \\
\text { peptidolysis }\end{array}$ \\
\hline AA039791 & 1.27003 & ICA1 & $\underline{3382}$ & $\begin{array}{l}\text { islet cell autoantigen } 1, \\
69 \mathrm{kDa}\end{array}$ & cytoplasm \\
\hline W90601 & 1.26828 & HADHA & $\underline{3030}$ & \begin{tabular}{|l|} 
hydroxyacyl- \\
Coenzyme A \\
dehydrogenase/3- \\
ketoacyl-Coenzyme A \\
thiolase/enoyl- \\
Coenzyme A \\
hydratase (trifunctional \\
protein), alpha subunit
\end{tabular} & $\begin{array}{l}\text { 3-hydroxyacyl-CoA } \\
\text { dehydrogenase } \\
\text { activity; acetyl-CoA C- } \\
\text { acetyltransferase } \\
\text { activity; fatty acid } \\
\text { metabolism; long- } \\
\text { chain enoyl-CoA } \\
\text { hydratase activity; } \\
\text { lyase activity; } \\
\text { metabolism; } \\
\text { mitochondrion; } \\
\text { oxidoreductase } \\
\text { activity; short-chain } \\
\text { enoyl-CoA hydratase } \\
\text { activity }\end{array}$ \\
\hline H50204 & 1.26813 & PKM2 & $\underline{5315}$ & $\begin{array}{l}\text { pyruvate kinase, } \\
\text { muscle }\end{array}$ & \\
\hline AA136856 & 1.26671 & TEM7R & 84898 & \multicolumn{2}{|c|}{ tumor endothelial marker 7-related precursor } \\
\hline \begin{tabular}{|l|l} 
AA044889 \\
\end{tabular} & 1.26413 & & & & \\
\hline AA054468 & 1.26402 & MYH11 & $\underline{4629}$ & $\begin{array}{l}\text { myosin, heavy } \\
\text { polypeptide } 11 \text {, smooth } \\
\text { muscle }\end{array}$ & $\begin{array}{l}\text { ATP binding; actin } \\
\text { binding; calmodulin } \\
\text { binding; cell growth } \\
\text { and/or maintenance; } \\
\text { kinesin complex; } \\
\text { motor activity; muscle } \\
\text { development; muscle } \\
\text { myosin; myosin; } \\
\text { striated muscle } \\
\text { contraction; striated } \\
\text { muscle thick filament }\end{array}$ \\
\hline
\end{tabular}




\begin{tabular}{|c|c|c|c|c|c|}
\hline R94499 & 1.26393 & GNB5 & 10681 & \multicolumn{2}{|c|}{$\begin{array}{l}\text { guanine nucleotide binding protein (G protein), } \\
\text { beta } 5\end{array}$} \\
\hline $\mathrm{H} 21137$ & 1.26251 & & & & \\
\hline H52741 & 1.26191 & & & & \\
\hline W31285 & 1.26095 & TCF3 & $\underline{6929}$ & $\begin{array}{l}\text { transcription factor } 3 \\
\text { (E2A immunoglobulin } \\
\text { enhancer binding } \\
\text { factors E12/E47) }\end{array}$ & $\begin{array}{l}\text { cell growth and/or } \\
\text { maintenance; nucleus; } \\
\text { regulation of } \\
\text { transcription, DNA- } \\
\text { dependent; } \\
\text { transcription factor } \\
\text { activity }\end{array}$ \\
\hline N24815 & 1.25981 & UBA52 & 7311 & $\begin{array}{l}\text { ubiquitin A-52 residue } \\
\text { ribosomal protein } \\
\text { fusion product } 1\end{array}$ & $\begin{array}{l}\text { nucleus; protein } \\
\text { biosynthesis; protein } \\
\text { modification; } \\
\text { ribosome; structural } \\
\text { constituent of } \\
\text { ribosome } \\
\end{array}$ \\
\hline AA059335 & 1.25971 & & & & \\
\hline H70759 & 1.259 & & & & \\
\hline H86672 & 1.25743 & & & & \\
\hline R95136 & 1.25654 & & & & \\
\hline \begin{tabular}{|l|} 
N47105 \\
\end{tabular} & 1.25626 & CSPG2 & 1462 & $\begin{array}{l}\text { chondroitin sulfate } \\
\text { proteoglycan } 2 \\
\text { (versican) }\end{array}$ & $\begin{array}{l}\text { calcium ion binding; } \\
\text { cell recognition; } \\
\text { development; } \\
\text { extracellular matrix; } \\
\text { heterophilic cell } \\
\text { adhesion; hyaluronic } \\
\text { acid binding; sugar } \\
\text { binding }\end{array}$ \\
\hline H61040 & 1.25569 & & & & \\
\hline \begin{tabular}{|l|}
$H 27334$ \\
\end{tabular} & 1.25455 & DDR1 & $\underline{780}$ & $\begin{array}{l}\text { discoidin domain } \\
\text { receptor family, } \\
\text { member } 1\end{array}$ & $\begin{array}{l}\text { ATP binding; cell } \\
\text { adhesion; integral to } \\
\text { plasma membrane; } \\
\text { protein amino acid } \\
\text { phosphorylation; } \\
\text { receptor activity; } \\
\text { transferase activity; } \\
\text { transmembrane } \\
\text { receptor protein } \\
\text { tyrosine kinase } \\
\text { activity; } \\
\text { transmembrane } \\
\text { receptor protein } \\
\text { tyrosine kinase } \\
\text { signaling pathway }\end{array}$ \\
\hline N31846 & 1.2541 & $\mathrm{ACP} 2$ & $\underline{53}$ & $\begin{array}{l}\text { acid phosphatase } 2 \\
\text { lysosomal }\end{array}$ & $\begin{array}{l}\text { acid phosphatase } \\
\text { activity; hydrolase } \\
\text { activity; integral to } \\
\text { membrane; lysosomal } \\
\text { membrane }\end{array}$ \\
\hline
\end{tabular}




\begin{tabular}{|c|c|c|c|c|c|}
\hline N72582 & 1.25336 & & & & \\
\hline R85191 & 1.25308 & $\frac{\text { FLJ3136 }}{4}$ & 146956 & homolog of yeast EME1 & 1 endonuclease \\
\hline $\mathrm{H} 45010$ & 1.25288 & ICAP-1A & $\underline{9270}$ & $\begin{array}{l}\text { integrin cytoplasmic } \\
\text { domain-associated } \\
\text { protein } 1\end{array}$ & $\begin{array}{l}\text { cell adhesion receptor } \\
\text { activity; cell-matrix } \\
\text { adhesion; membrane; } \\
\text { protein C-terminus } \\
\text { binding; protein kinase } \\
\text { cascade }\end{array}$ \\
\hline N64846 & 1.25133 & $\frac{\text { KIAA141 }}{6}$ & $\underline{55636}$ & KIAA1416 protein & \\
\hline AA131302 & 1.24851 & & & & \\
\hline R88818 & 1.24836 & GSTM1 & $\underline{2944}$ & $\begin{array}{l}\text { glutathione S- } \\
\text { transferase M1 }\end{array}$ & $\begin{array}{l}\text { cytoplasm; glutathione } \\
\text { transferase activity; } \\
\text { tumor suppressor }\end{array}$ \\
\hline W52537 & 1.24787 & PSMA2 & $\underline{5683}$ & $\begin{array}{l}\text { proteasome (prosome, } \\
\text { macropain) subunit, } \\
\text { alpha type, } 2\end{array}$ & $\begin{array}{l}\text { 26S proteasome; } \\
\text { cytosol; } \\
\text { endopeptidase } \\
\text { activity; proteasome } \\
\text { core complex (sensu } \\
\text { Eukarya); proteasome } \\
\text { endopeptidase } \\
\text { activity; ubiquitin- } \\
\text { dependent protein } \\
\text { catabolism }\end{array}$ \\
\hline R90757 & 1.24601 & RPH3A & $\underline{22895}$ & $\begin{array}{l}\text { likely ortholog of } \\
\text { mouse rabphilin } 3 \mathrm{~A}\end{array}$ & $\begin{array}{l}\text { intracellular protein } \\
\text { transport; membrane; } \\
\text { protein transporter } \\
\text { activity; synaptic } \\
\text { junction; synaptic } \\
\text { vesicle; zinc ion } \\
\text { binding }\end{array}$ \\
\hline AA026475 & 1.24268 & & & & \\
\hline N39088 & 1.24252 & & & & \\
\hline N67453 & 1.24188 & CDKN1A & 1026 & $\begin{array}{l}\text { cyclin-dependent } \\
\text { kinase inhibitor 1A } \\
\text { (p21, Cip1) }\end{array}$ & $\begin{array}{l}\text { cell cycle arrest; cell } \\
\text { cycle regulator; cyclin- } \\
\text { dependent protein } \\
\text { kinase inhibitor } \\
\text { activity; induction of } \\
\text { apoptosis by } \\
\text { intracellular signals; } \\
\text { negative regulation of } \\
\text { cell proliferation; } \\
\text { nucleus; oncogenesis; } \\
\text { protein kinase activity; } \\
\text { regulation of CDK } \\
\text { activity; regulation of } \\
\text { cell cycle; tumor } \\
\text { suppressor }\end{array}$ \\
\hline N67453 & 1.24188 & & 286039 & \multicolumn{2}{|c|}{ similar to hypothetical protein } \\
\hline
\end{tabular}




\begin{tabular}{|c|c|c|c|c|c|}
\hline H94541 & 1.24167 & C20orf64 & 112858 & $\begin{array}{l}\text { chromosome } 20 \text { open } \\
\text { reading frame } 64\end{array}$ & $\begin{array}{l}\text { ATP binding; nucleus; } \\
\text { protein amino acid } \\
\text { phosphorylation; } \\
\text { protein binding; } \\
\text { protein } \\
\text { serine/threonine } \\
\text { kinase activity; } \\
\text { transferase activity } \\
\end{array}$ \\
\hline T39206 & 1.24141 & & & & \\
\hline H26552 & 1.23804 & $\frac{\text { MGC539 }}{5}$ & $\underline{79026}$ & $\begin{array}{l}\text { hypothetical protein } \\
\text { MGC5395 }\end{array}$ & $\begin{array}{l}\text { intracellular signaling } \\
\text { cascade }\end{array}$ \\
\hline N50057 & 1.23646 & ORMDL2 & 29095 & \multicolumn{2}{|c|}{ ORM1-like 2 (S. cerevisiae) } \\
\hline N78414 & 1.23531 & \begin{tabular}{|l} 
LOC1449 \\
97 \\
\end{tabular} & 144997 & \multicolumn{2}{|c|}{ hypothetical protein LOC144997 } \\
\hline $\mathrm{H} 69440$ & 1.23469 & $\frac{\mathrm{ANKRD} 1}{3}$ & $\underline{88455}$ & $\begin{array}{l}\text { ankyrin repeat domain } \\
13\end{array}$ & \\
\hline AA043685 & 1.23316 & & & & \\
\hline R28329 & 1.23314 & $\frac{\text { MGC160 }}{63}$ & 114129 & \multicolumn{2}{|c|}{ hypothetical protein MGC16063 } \\
\hline AA054115 & 1.23251 & & & & \\
\hline AA043227 & 1.23068 & CNN3 & $\underline{1266}$ & calponin 3, acidic & $\begin{array}{l}\text { actin binding; } \\
\text { calmodulin binding; } \\
\text { cellular_component } \\
\text { unknown; smooth } \\
\text { muscle contraction; } \\
\text { tropomyosin binding; } \\
\text { troponin C binding }\end{array}$ \\
\hline AA152194 & 1.23037 & $\frac{\text { PTP9Q2 }}{2}$ & 138639 & $\begin{array}{l}\text { protein tyrosine } \\
\text { phosphatase } \\
\text { PTP9Q22 }\end{array}$ & $\begin{array}{l}\text { protein amino acid } \\
\text { dephosphorylation; } \\
\text { protein tyrosine } \\
\text { phosphatase activity; } \\
\text { protein } \\
\text { tyrosine/serine/threoni } \\
\text { ne phosphatase } \\
\text { activity }\end{array}$ \\
\hline
\end{tabular}




\begin{tabular}{|c|c|c|c|c|c|}
\hline $\mathrm{H} 71213$ & 1.22872 & $\underline{F 2}$ & $\underline{2147}$ & $\begin{array}{l}\text { coagulation factor II } \\
\text { (thrombin) }\end{array}$ & $\begin{array}{l}\text { STAT protein nuclear } \\
\text { translocation; acute- } \\
\text { phase response; } \\
\text { apoptosis; blood } \\
\text { coagulation; calcium } \\
\text { ion binding; caspase } \\
\text { activation; } \\
\text { chymotrypsin activity; } \\
\text { development; } \\
\text { extracellular space; } \\
\text { hydrolase activity; } \\
\text { proteolysis and } \\
\text { peptidolysis; } \\
\text { regulation of cell cycle; } \\
\text { response to wounding; } \\
\text { soluble fraction; } \\
\text { thrombin activity; } \\
\text { trypsin activity; } \\
\text { tyrosine } \\
\text { phosphorylation of } \\
\text { STAT protein }\end{array}$ \\
\hline H29730 & 1.22773 & & & & \\
\hline N91039 & 1.22672 & DDX5 & $\underline{1655}$ & $\begin{array}{l}\text { DEAD (Asp-Glu-Ala- } \\
\text { Asp) box polypeptide } 5\end{array}$ & $\begin{array}{l}\text { ATP binding; ATP } \\
\text { dependent helicase } \\
\text { activity; RNA helicase } \\
\text { activity; cell growth; } \\
\text { nucleus }\end{array}$ \\
\hline H68805 & 1.22625 & & & & \\
\hline H93450 & 1.22599 & ZNF347 & $\underline{84671}$ & zinc finger protein 347 & $\begin{array}{l}\text { DNA binding; nucleus; } \\
\text { regulation of } \\
\text { transcription, DNA- } \\
\text { dependent }\end{array}$ \\
\hline H84293 & 1.22425 & SLC12A5 & $\underline{57468}$ & $\begin{array}{l}\text { solute carrier family 12, } \\
\text { (potassium-chloride } \\
\text { transporter) member } 5\end{array}$ & $\begin{array}{l}\text { amino acid transport; } \\
\text { amino acid-polyamine } \\
\text { transporter activity; } \\
\text { cell ion homeostasis; } \\
\text { chloride transport; } \\
\text { integral to membrane; } \\
\text { ion transport; } \\
\text { potassium ion } \\
\text { transport; } \\
\text { potassium:chloride } \\
\text { symporter activity; } \\
\text { sodium ion transport; } \\
\text { symporter activity; } \\
\text { transporter activity } \\
\end{array}$ \\
\hline T95863 & 1.22308 & & & & \\
\hline AA115496 & 1.22285 & TRAP25 & 90390 & \multicolumn{2}{|c|}{ TRAP/Mediator complex component } \\
\hline AA058632 & 1.22273 & KIF1B & 23095 & $\begin{array}{l}\text { kinesin family member } \\
1 \mathrm{~B}\end{array}$ & \\
\hline
\end{tabular}




\begin{tabular}{|c|c|c|c|c|c|}
\hline H51834 & 1.22179 & TTC1 & $\underline{7265}$ & $\begin{array}{l}\text { tetratricopeptide repeat } \\
\text { domain } 1\end{array}$ & $\begin{array}{l}\text { chaperone activity; } \\
\text { protein binding; } \\
\text { protein folding }\end{array}$ \\
\hline T77398 & 1.22123 & AOC3 & $\underline{8639}$ & $\begin{array}{l}\text { amine oxidase, copper } \\
\text { containing } 3 \text { (vascular } \\
\text { adhesion protein 1) }\end{array}$ & $\begin{array}{l}\text { amine metabolism; } \\
\text { amine oxidase } \\
\text { (copper-containing) } \\
\text { activity; cell adhesion; } \\
\text { cell adhesion molecule } \\
\text { activity; copper ion } \\
\text { binding; electron } \\
\text { transporter activity; } \\
\text { inflammatory } \\
\text { response; integral to } \\
\text { membrane; } \\
\text { oxidoreductase } \\
\text { activity; plasma } \\
\text { membrane }\end{array}$ \\
\hline AA151577 & 1.22066 & HPRT1 & $\underline{3251}$ & $\begin{array}{l}\text { hypoxanthine } \\
\text { phosphoribosyltransfer } \\
\text { ase } 1 \text { (Lesch-Nyhan } \\
\text { syndrome) }\end{array}$ & $\begin{array}{l}\text { behavior; cytoplasm; } \\
\text { hypoxanthine } \\
\text { phosphoribosyltransfer } \\
\text { ase activity; } \\
\text { magnesium ion } \\
\text { binding; nucleoside } \\
\text { metabolism; purine } \\
\text { salvage; transferase } \\
\text { activity, transferring } \\
\text { glycosyl groups }\end{array}$ \\
\hline $\mathrm{H} 45972$ & 1.21983 & & & & \\
\hline AA099685 & 1.2175 & PIBF1 & 10464 & \multicolumn{2}{|c|}{ progesterone-induced blocking factor 1} \\
\hline N48160 & 1.21254 & LCMR1 & 219541 & \multicolumn{2}{|c|}{ lung cancer metastasis-related protein 1} \\
\hline AA028111 & 1.21238 & CXorf9 & $\underline{54440}$ & \multicolumn{2}{|c|}{ chromosome X open reading frame 9} \\
\hline $\mathrm{H} 20790$ & 1.21043 & & 348024 & \multicolumn{2}{|c|}{ similar to TPIP alpha lipid phosphatase } \\
\hline W33065 & 1.20973 & $\underline{\underline{F L}}$ & 339175 & \multicolumn{2}{|c|}{ hypothetical protein FLJ12760 } \\
\hline R98517 & 1.20701 & $\frac{\mathrm{HIST} 1 \mathrm{H} 1}{\underline{\mathrm{C}}}$ & $\underline{3006}$ & histone 1, H1c & $\begin{array}{l}\text { DNA binding; } \\
\text { chromosome; } \\
\text { chromosome } \\
\text { organization and } \\
\text { biogenesis (sensu } \\
\text { Eukarya); } \\
\text { nucleosome; } \\
\text { nucleosome } \\
\text { assembly; nucleus }\end{array}$ \\
\hline $\mathrm{H} 45472$ & 1.20642 & & & & \\
\hline AA152297 & 1.20615 & PNPO & $\underline{55163}$ & $\begin{array}{l}\text { pyridoxine-5'- } \\
\text { phosphate oxidase }\end{array}$ & $\begin{array}{l}\text { pyridoxamine- } \\
\text { phosphate oxidase } \\
\text { activity; pyridoxine } \\
\text { biosynthesis }\end{array}$ \\
\hline
\end{tabular}




\begin{tabular}{|c|c|c|c|c|c|}
\hline $\mathrm{H} 83003$ & 1.20601 & IGSF1 & 3547 & $\begin{array}{l}\text { immunoglobulin } \\
\text { superfamily, member } 1\end{array}$ & $\begin{array}{l}\text { cell adhesion; integral } \\
\text { to plasma membrane }\end{array}$ \\
\hline $\mathrm{H} 51160$ & 1.20446 & $\begin{array}{l}\text { PPP2R1 } \\
\text { A }\end{array}$ & $\underline{5518}$ & $\begin{array}{l}\text { protein phosphatase } 2 \\
\text { (formerly } 2 A \text { ), } \\
\text { regulatory subunit A } \\
\text { (PR 65), alpha isoform }\end{array}$ & $\begin{array}{l}\text { protein phosphatase } \\
\text { type } 2 A \text { activity }\end{array}$ \\
\hline W37621 & 1.20397 & MEF2B & $\underline{4207}$ & $\begin{array}{l}\text { MADS box } \\
\text { transcription enhancer } \\
\text { factor 2, polypeptide B } \\
\text { (myocyte enhancer } \\
\text { factor 2B) }\end{array}$ & $\begin{array}{l}\text { muscle development; } \\
\text { nucleus; transcription } \\
\text { activating factor; } \\
\text { transcription co- } \\
\text { activator activity; } \\
\text { transcription factor } \\
\text { activity; transcription } \\
\text { from Pol II promoter }\end{array}$ \\
\hline N33086 & 1.20201 & & & & \\
\hline \begin{tabular}{|l} 
R89790 \\
\end{tabular} & 1.20005 & & & & \\
\hline $\mathrm{H} 00498$ & 1.19972 & $\frac{\mathrm{PPP} 2 \mathrm{R} 3}{\mathrm{~A}}$ & $\underline{5523}$ & $\begin{array}{l}\text { protein phosphatase } 2 \\
\text { (formerly } 2 A \text { ), } \\
\text { regulatory subunit B", } \\
\text { alpha }\end{array}$ & $\begin{array}{l}\text { calcium ion binding; } \\
\text { protein phosphatase } \\
\text { type } 2 A \text {, intrinsic } \\
\text { regulator activity }\end{array}$ \\
\hline W25557 & 1.19882 & $\underline{\text { TRIM28 }}$ & $\underline{10155}$ & $\begin{array}{l}\text { tripartite motif- } \\
\text { containing } 28\end{array}$ & $\begin{array}{l}\text { nucleus; regulation of } \\
\text { transcription from Pol } \\
\text { Il promoter; } \\
\text { transcription co- } \\
\text { repressor activity; } \\
\text { transcription factor } \\
\text { activity; zinc ion } \\
\text { binding }\end{array}$ \\
\hline R47938 & 1.19757 & $\frac{\text { FLJ3209 }}{6}$ & 148646 & \multicolumn{2}{|c|}{ hypothetical protein FLJ32096 } \\
\hline H64569 & 1.19614 & & & & \\
\hline $\mathrm{H} 18190$ & 1.19576 & $\underline{J A K 1}$ & $\underline{3716}$ & $\begin{array}{l}\text { Janus kinase } 1 \text { (a } \\
\text { protein tyrosine kinase) }\end{array}$ & $\begin{array}{l}\text { ATP binding; } \\
\text { cytoskeleton; } \\
\text { intracellular signaling } \\
\text { cascade; protein } \\
\text { amino acid } \\
\text { phosphorylation; } \\
\text { protein tyrosine kinase } \\
\text { activity; transferase } \\
\text { activity } \\
\end{array}$ \\
\hline AA057398 & 1.19564 & & & & \\
\hline N777703 & 1.19466 & $\underline{\underline{\mathrm{MGC216}}}$ & $\underline{93594}$ & \multicolumn{2}{|c|}{ unknown MGC21654 product } \\
\hline AA039677 & 1.19306 & $\begin{array}{l}\text { DKFZP4 } \\
34 \mathrm{~A} 0131 \\
\end{array}$ & $\underline{54441}$ & $\begin{array}{l}\text { DKFZp434A0131 } \\
\text { protein }\end{array}$ & \\
\hline N71552 & 1.19282 & $\begin{array}{l}\text { DKFZp43 } \\
\text { 4D1428 } \\
\end{array}$ & $\underline{84213}$ & \multicolumn{2}{|c|}{ hypothetical protein DKFZp434D1428 } \\
\hline R47758 & 1.19013 & LMNB2 & $\underline{84823}$ & lamin B2 & $\begin{array}{l}\text { S-specific transcription } \\
\text { in mitotic cell cycle; } \\
\text { lamin filament }\end{array}$ \\
\hline
\end{tabular}




\begin{tabular}{|c|c|c|c|c|c|}
\hline $\mathrm{H} 09945$ & 1.18823 & & & & \\
\hline $\mathrm{H} 70359$ & 1.18679 & & & & \\
\hline $\mathrm{H} 68440$ & 1.1866 & PIP5K1B & $\underline{8395}$ & \multicolumn{2}{|c|}{$\begin{array}{l}\text { phosphatidylinositol-4-phosphate 5-kinase, } \\
\text { type I, beta }\end{array}$} \\
\hline AA204701 & 1.18363 & & & & \\
\hline R82834 & 1.18305 & & & & \\
\hline R50905 & 1.18121 & TUBB & $\underline{7280}$ & $\begin{array}{l}\text { tubulin, beta } \\
\text { polypeptide }\end{array}$ & $\begin{array}{l}\text { cytoskeleton; } \\
\text { structural constituent } \\
\text { of cytoskeleton }\end{array}$ \\
\hline AA057126 & 1.17985 & $\frac{\text { KIAA141 }}{6}$ & $\underline{55636}$ & KIAA1416 protein & \\
\hline N94432 & 1.17822 & & & & \\
\hline N20665 & 1.17448 & MSI2 & 124540 & \multicolumn{2}{|c|}{ musashi homolog 2 (Drosophila) } \\
\hline AA036801 & 1.1743 & PRDX2 & $\underline{7001}$ & peroxiredoxin 2 & $\begin{array}{l}\text { antioxidant activity; } \\
\text { cytoplasm; electron } \\
\text { transporter activity; } \\
\text { oxidoreductase } \\
\text { activity; response to } \\
\text { oxidative stress; } \\
\text { thioredoxin peroxidase } \\
\text { activity }\end{array}$ \\
\hline AA129727 & 1.17119 & RAB5C & $\underline{5878}$ & $\begin{array}{l}\text { RAB5C, member RAS } \\
\text { oncogene family }\end{array}$ & $\begin{array}{l}\text { GTP binding; RAB } \\
\text { small monomeric } \\
\text { GTPase activity; } \\
\text { intracellular protein } \\
\text { transport; protein } \\
\text { transporter activity; } \\
\text { small GTPase } \\
\text { mediated signal } \\
\text { transduction }\end{array}$ \\
\hline H61812 & 1.16962 & CDK4 & $\underline{1019}$ & $\begin{array}{l}\text { cyclin-dependent } \\
\text { kinase } 4\end{array}$ & $\begin{array}{l}\text { G1/S transition of } \\
\text { mitotic cell cycle; cell } \\
\text { proliferation; cyclin- } \\
\text { dependent protein } \\
\text { kinase activity; } \\
\text { oncogenesis; } \\
\text { regulation of cell cycle }\end{array}$ \\
\hline N28915 & 1.16928 & CERK & 64781 & ceramide kinase & \\
\hline R23374 & 1.16888 & $\begin{array}{l}\text { FLJ1046 } \\
\underline{2}\end{array}$ & 55711 & \multicolumn{2}{|c|}{ hypothetical protein FLJ10462 } \\
\hline H63763 & 1.16855 & & & & \\
\hline W85995 & 1.1684 & & & & \\
\hline $\mathrm{H} 45128$ & 1.16752 & IGHG3 & $\underline{3502}$ & $\begin{array}{l}\text { immunoglobulin heavy } \\
\text { constant gamma } 3 \\
\text { (G3m marker) }\end{array}$ & $\begin{array}{l}\text { antigen binding; } \\
\text { immune response; } \\
\text { membrane fraction }\end{array}$ \\
\hline $\mathrm{H} 67696$ & 1.16713 & & & & \\
\hline R01530 & 1.16687 & & & & \\
\hline
\end{tabular}




\begin{tabular}{|c|c|c|c|c|c|}
\hline N31469 & 1.16319 & NCKAP1 & $\underline{10787}$ & $\begin{array}{l}\text { NCK-associated } \\
\text { protein } 1\end{array}$ & $\begin{array}{l}\text { apoptosis; central } \\
\text { nervous system } \\
\text { development; integral } \\
\text { to membrane }\end{array}$ \\
\hline AA031564 & 1.16281 & $\frac{\mathrm{LOC} 1134}{44}$ & 113444 & \multicolumn{2}{|c|}{ hypothetical protein BC011880 } \\
\hline N47654 & 1.16033 & $\begin{array}{l}\text { KIAA014 } \\
\underline{0}\end{array}$ & $\underline{9679}$ & $\begin{array}{l}\text { KIAA0140 gene } \\
\text { product }\end{array}$ & \\
\hline R81035 & 1.15962 & EIF5A & 1984 & \multicolumn{2}{|c|}{ eukaryotic translation initiation factor $5 \mathrm{~A}$} \\
\hline H84008 & 1.15948 & & & & \\
\hline R72577 & 1.15882 & $\frac{F L J 1175}{3}$ & $\underline{79712}$ & \multicolumn{2}{|c|}{ hypothetical protein FLJ11753 } \\
\hline AA054170 & 1.15736 & & & & \\
\hline AA059274 & 1.15565 & $\frac{\mathrm{KIAA} 159}{4}$ & $\underline{57695}$ & KIAA1594 protein & \\
\hline H93017 & 1.15546 & $\underline{\mathrm{ECH} 1}$ & $\underline{1891}$ & $\begin{array}{l}\text { enoyl Coenzyme A } \\
\text { hydratase } 1, \\
\text { peroxisomal }\end{array}$ & $\begin{array}{l}\text { energy pathways; } \\
\text { enoyl-CoA hydratase } \\
\text { activity; fatty acid } \\
\text { beta-oxidation; fatty } \\
\text { acid metabolism; } \\
\text { isomerase activity; } \\
\text { mitochondrion; } \\
\text { peroxisome }\end{array}$ \\
\hline H95467 & 1.1545 & MIDORI & $\underline{57538}$ & $\begin{array}{l}\text { likely ortholog of } \\
\text { mouse myocytic } \\
\text { induction/differentiation } \\
\text { originator }\end{array}$ & $\begin{array}{l}\text { ATP binding; kinase } \\
\text { activity; protein amino } \\
\text { acid phosphorylation; } \\
\text { protein } \\
\text { serine/threonine } \\
\text { kinase activity }\end{array}$ \\
\hline AA114905 & 1.15449 & SPAG7 & 9552 & $\begin{array}{l}\text { sperm associated } \\
\text { antigen } 7\end{array}$ & nucleic acid binding \\
\hline R21970 & 1.15446 & GTF2H2 & $\underline{2966}$ & $\begin{array}{l}\text { general transcription } \\
\text { factor IIH, polypeptide } \\
2,44 \mathrm{kDa}\end{array}$ & $\begin{array}{l}\text { DNA repair; nucleus; } \\
\text { regulation of } \\
\text { transcription, DNA- } \\
\text { dependent }\end{array}$ \\
\hline AA033685 & 1.15394 & & & & \\
\hline AA121514 & 1.1534 & ZNF197 & 10168 & zinc finger protein 197 & $\begin{array}{l}\text { transcription factor } \\
\text { activity }\end{array}$ \\
\hline R53840 & 1.15265 & $\frac{\text { RABGEF }}{1}$ & $\underline{27342}$ & $\begin{array}{l}\text { RAB guanine } \\
\text { nucleotide exchange } \\
\text { factor (GEF) } 1\end{array}$ & $\begin{array}{l}\text { DNA binding; zinc ion } \\
\text { binding }\end{array}$ \\
\hline \begin{tabular}{|l|} 
R21702 \\
\end{tabular} & 1.15133 & & & & \\
\hline
\end{tabular}




\begin{tabular}{|c|c|c|c|c|c|}
\hline H84617 & 1.15113 & PCAF & $\underline{8850}$ & $\begin{array}{l}\text { p300/CBP-associated } \\
\text { factor }\end{array}$ & $\begin{array}{l}\text { N-acetyltransferase } \\
\text { activity; cell cycle; cell } \\
\text { cycle arrest; chromatin } \\
\text { modeling; histone } \\
\text { deacetylase activity; } \\
\text { negative regulation of } \\
\text { cell proliferation; } \\
\text { nucleus; protein amino } \\
\text { acid acetylation; } \\
\text { regulation of } \\
\text { transcription, DNA- } \\
\text { dependent; } \\
\text { transcription cofactor } \\
\text { activity; transferase } \\
\text { activity } \\
\end{array}$ \\
\hline H59810 & 1.15093 & $\underline{\mathrm{CLU}}$ & 1191 & \begin{tabular}{|l|} 
clusterin (complement \\
lysis inhibitor, SP- \\
40,40, sulfated \\
glycoprotein 2, \\
testosterone-repressed \\
prostate message 2, \\
apolipoprotein J) \\
\end{tabular} & $\begin{array}{l}\text { apoptosis; cell death; } \\
\text { complement } \\
\text { activation, classical } \\
\text { pathway; fertilization } \\
\text { (sensu Animalia); lipid } \\
\text { metabolism }\end{array}$ \\
\hline N31625 & 1.14819 & $\frac{\mathrm{KIAA} 190}{9}$ & $\underline{153478}$ & KIAA1909 protein & \\
\hline AA135646 & 1.14651 & hIAN6 & 155038 & \multicolumn{2}{|c|}{ human immune associated nucleotide 6} \\
\hline N30177 & 1.14608 & $\frac{\mathrm{LOC} 2859}{58}$ & $\underline{285958}$ & \multicolumn{2}{|c|}{ hypothetical protein LOC285958 } \\
\hline T87888 & 1.14578 & $\frac{\mathrm{KIAA} 104}{6}$ & 22867 & KIAA1046 protein & \\
\hline AA045281 & 1.1453 & $\underline{P A G}$ & $\underline{55824}$ & \begin{tabular}{|l|} 
phosphoprotein \\
associated with \\
glycosphingolipid- \\
enriched microdomains
\end{tabular} & $\begin{array}{l}\text { antimicrobial humoral } \\
\text { response (sensu } \\
\text { Invertebrata); integral } \\
\text { to plasma membrane; } \\
\text { signal transduction; } \\
\text { transmembrane } \\
\text { receptor protein } \\
\text { tyrosine kinase } \\
\text { adaptor protein activity }\end{array}$ \\
\hline AA046610 & 1.14455 & & & & \\
\hline $\mathrm{H} 62770$ & 1.13654 & & & & \\
\hline N30471 & 1.13607 & \begin{tabular}{|l|} 
DKFZp58 \\
611420 \\
\end{tabular} & $\underline{222161}$ & \multicolumn{2}{|c|}{ hypothetical protein DKFZp586I1420 } \\
\hline W07300 & 1.13597 & AP1G1 & 164 & $\begin{array}{l}\text { adaptor-related protein } \\
\text { complex 1, gamma } 1 \\
\text { subunit } \\
\end{array}$ & $\begin{array}{l}\text { Golgi apparatus; } \\
\text { clathrin adaptor; } \\
\text { coated pit; } \\
\text { endocytosis; } \\
\text { intracellular protein } \\
\text { transport; protein } \\
\text { transporter activity } \\
\end{array}$ \\
\hline AA069532 & 1.13508 & & & & \\
\hline
\end{tabular}




\begin{tabular}{|c|c|c|c|c|c|}
\hline AA203751 & 1.13496 & $\begin{array}{l}\text { KIAA195 } \\
\underline{6}\end{array}$ & $\underline{147686}$ & KIAA1956 protein & $\begin{array}{l}\text { DNA binding; nucleus; } \\
\text { regulation of } \\
\text { transcription, DNA- } \\
\text { dependent }\end{array}$ \\
\hline H84844 & 1.13238 & & & & \\
\hline R85333 & 1.13207 & & & & \\
\hline R26844 & 1.131 & & & & \\
\hline R47859 & 1.13045 & NPR1 & $\underline{4881}$ & $\begin{array}{l}\text { natriuretic peptide } \\
\text { receptor A/guanylate } \\
\text { cyclase A } \\
\text { (atrionatriuretic peptide } \\
\text { receptor A) } \\
\\
\end{array}$ & $\begin{array}{l}\text { ATP binding; cGMP } \\
\text { biosynthesis; } \\
\text { guanylate cyclase } \\
\text { activity; integral to } \\
\text { membrane; } \\
\text { intracellular signaling } \\
\text { cascade; lyase } \\
\text { activity; peptide } \\
\text { receptor activity, G- } \\
\text { protein coupled; } \\
\text { protein amino acid } \\
\text { phosphorylation; } \\
\text { protein kinase activity; } \\
\text { receptor activity; } \\
\text { receptor guanylate } \\
\text { cyclase activity; } \\
\text { regulation of blood } \\
\text { pressure }\end{array}$ \\
\hline H66198 & 1.12923 & & $\underline{343171}$ & \multicolumn{2}{|c|}{ similar to seven transmembrane helix receptor } \\
\hline N92573 & 1.12567 & MVD & $\underline{4597}$ & $\begin{array}{l}\text { mevalonate } \\
\text { (diphospho) } \\
\text { decarboxylase }\end{array}$ & $\begin{array}{l}\text { cholesterol } \\
\text { biosynthesis; } \\
\text { diphosphomevalonate } \\
\text { decarboxylase activity; } \\
\text { isoprenoid } \\
\text { biosynthesis; lyase } \\
\text { activity }\end{array}$ \\
\hline H50914 & 1.12561 & & $\underline{284665}$ & \multicolumn{2}{|c|}{ hypothetical gene supported by BC023596 } \\
\hline N56889 & 1.12525 & $\underline{\text { SOS2 }}$ & $\underline{6655}$ & $\begin{array}{l}\text { son of sevenless } \\
\text { homolog } 2 \\
\text { (Drosophila) }\end{array}$ & $\begin{array}{l}\text { cellular_component } \\
\text { unknown; guanyl- } \\
\text { nucleotide exchange } \\
\text { factor activity; small } \\
\text { GTPase mediated } \\
\text { signal transduction }\end{array}$ \\
\hline AA099647 & 1.12465 & TSPAN-2 & $\underline{10100}$ & tetraspan 2 & $\begin{array}{l}\text { cell adhesion; cell } \\
\text { motility; cell } \\
\text { proliferation; integral } \\
\text { to membrane; mystery } \\
\text { cell fate differentiation } \\
\text { (sensu Drosophila) }\end{array}$ \\
\hline H60488 & 1.12462 & $\mathrm{H} 326$ & 50717 & $\mathrm{H} 326$ & \\
\hline
\end{tabular}




\begin{tabular}{|c|c|c|c|c|c|}
\hline $\mathrm{H} 27352$ & 1.12415 & $\underline{\mathrm{HRAS}}$ & $\underline{3265}$ & $\begin{array}{l}\text { v-Ha-ras Harvey rat } \\
\text { sarcoma viral } \\
\text { oncogene homolog }\end{array}$ & \begin{tabular}{|l|} 
GTPase activity; cell \\
motility; cell shape and \\
cell size control; cell \\
surface receptor linked \\
signal transduction; \\
chemotaxis; \\
cytoplasm; \\
histogenesis and \\
organogenesis; \\
peripheral plasma \\
membrane protein; \\
plasma membrane; \\
regulation of cell cycle; \\
signal transduction \\
\end{tabular} \\
\hline AA149333 & 1.1237 & ACTN1 & $\underline{87}$ & actinin, alpha 1 & $\begin{array}{l}\text { actin binding; actin } \\
\text { cytoskeleton; calcium } \\
\text { ion binding; structural } \\
\text { constituent of } \\
\text { cytoskeleton }\end{array}$ \\
\hline AA210872 & 1.12192 & $\frac{\text { DKFZp66 }}{7 \mathrm{E} 0512}$ & $\underline{202025}$ & \multicolumn{2}{|c|}{ hypothetical protein DKFZp667E0512 } \\
\hline R90824 & 1.11871 & TMEM10 & $\underline{93377}$ & \begin{tabular}{|l|} 
transmembrane protein \\
10
\end{tabular} & integral to membrane \\
\hline W52472 & 1.11848 & PCDH1 & $\underline{5097}$ & \multicolumn{2}{|c|}{ protocadherin 1 (cadherin-like 1) } \\
\hline H71358 & 1.11817 & & & & \\
\hline R41363 & 1.11746 & & & & \\
\hline R28090 & 1.11676 & $\frac{\mathrm{KIAA} 149}{5}$ & $\underline{57631}$ & KIAA1495 protein & \\
\hline N32669 & 1.11488 & RBT1 & $\underline{29946}$ & \multicolumn{2}{|c|}{ RPA-binding trans-activator } \\
\hline R71629 & 1.11417 & LST1 & $\underline{7940}$ & $\begin{array}{l}\text { leukocyte specific } \\
\text { transcript } 1\end{array}$ & $\begin{array}{l}\text { cellular defense } \\
\text { response; } \\
\text { defense/immunity } \\
\text { protein activity; } \\
\text { immune response; } \\
\text { integral to plasma } \\
\text { membrane }\end{array}$ \\
\hline AA136159 & 1.11406 & MGST1 & $\underline{4257}$ & $\begin{array}{l}\text { microsomal glutathione } \\
\text { S-transferase } 1\end{array}$ & $\begin{array}{l}\text { glutathione } \\
\text { transferase activity; } \\
\text { membrane; } \\
\text { microsome; } \\
\text { mitochondrion; } \\
\text { transferase activity } \\
\end{array}$ \\
\hline $\mathrm{H} 14332$ & 1.11314 & & & & \\
\hline H65775 & 1.11187 & & & & \\
\hline N45213 & 1.11181 & & 147808 & \multicolumn{2}{|c|}{ similar to zinc finger protein Zec } \\
\hline N22392 & 1.11168 & CLDN11 & $\underline{5010}$ & \begin{tabular}{|l} 
claudin 11 \\
(oligodendrocyte \\
transmembrane
\end{tabular} & $\begin{array}{l}\text { integral to membrane; } \\
\text { structural molecule } \\
\text { activity; tight junction }\end{array}$ \\
\hline
\end{tabular}




\begin{tabular}{|c|c|c|c|c|c|}
\hline & & & & protein) & \\
\hline AA054473 & 1.11148 & GOSR2 & $\underline{9570}$ & $\begin{array}{l}\text { golgi SNAP receptor } \\
\text { complex member } 2\end{array}$ & $\begin{array}{l}\text { ER to Golgi transport; } \\
\text { Golgi apparatus; } \\
\text { integral to membrane; } \\
\text { intracellular protein } \\
\text { transport; kinesin } \\
\text { complex; membrane } \\
\text { fusion; protein } \\
\text { transporter activity; v- } \\
\text { SNARE activity }\end{array}$ \\
\hline H30081 & 1.10991 & & & & \\
\hline AA054300 & 1.10989 & KLHL4 & 56062 & $\begin{array}{l}\text { kelch-like } 4 \\
\text { (Drosophila) }\end{array}$ & $\begin{array}{l}\text { actin binding; actin } \\
\text { cytoskeleton } \\
\text { organization and } \\
\text { biogenesis; } \\
\text { cytoskeleton; protein } \\
\text { binding }\end{array}$ \\
\hline W02842 & 1.10882 & TBX2 & $\underline{6909}$ & T-box 2 & $\begin{array}{l}\text { development; nucleus; } \\
\text { regulation of } \\
\text { transcription, DNA- } \\
\text { dependent; } \\
\text { transcription factor } \\
\text { activity }\end{array}$ \\
\hline H59454 & 1.1079 & & & & \\
\hline H46133 & 1.10788 & $\mathrm{BAI} 2$ & $\underline{576}$ & $\begin{array}{l}\text { brain-specific } \\
\text { angiogenesis inhibitor } \\
2\end{array}$ & $\begin{array}{l}\text { G-protein coupled } \\
\text { receptor activity; } \\
\text { integral to membrane; } \\
\text { neuropeptide signaling } \\
\text { pathway }\end{array}$ \\
\hline AA021582 & 1.10723 & GFAP & $\underline{2670}$ & $\begin{array}{l}\text { glial fibrillary acidic } \\
\text { protein }\end{array}$ & $\begin{array}{l}\text { intermediate filament; } \\
\text { structural constituent } \\
\text { of cytoskeleton }\end{array}$ \\
\hline H59405 & 1.10629 & $\frac{F L J 1029}{8}$ & $\underline{54682}$ & \multicolumn{2}{|c|}{ hypothetical protein FLJ10298 } \\
\hline R28033 & 1.10612 & PTPN18 & $\underline{26469}$ & $\begin{array}{l}\text { protein tyrosine } \\
\text { phosphatase, non- } \\
\text { receptor type } 18 \\
\text { (brain-derived) }\end{array}$ & $\begin{array}{l}\text { non-membrane } \\
\text { spanning protein } \\
\text { tyrosine phosphatase } \\
\text { activity; protein amino } \\
\text { acid } \\
\text { dephosphorylation }\end{array}$ \\
\hline AA142943 & 1.10577 & DOK1 & $\underline{1796}$ & $\begin{array}{l}\text { docking protein } 1, \\
62 \mathrm{kDa} \text { (downstream of } \\
\text { tyrosine kinase } 1 \text { ) }\end{array}$ & $\begin{array}{l}\text { cell surface receptor } \\
\text { linked signal } \\
\text { transduction; insulin } \\
\text { receptor binding; } \\
\text { protein binding; } \\
\text { transmembrane } \\
\text { receptor protein } \\
\text { tyrosine kinase } \\
\text { signaling pathway }\end{array}$ \\
\hline
\end{tabular}




\begin{tabular}{|c|c|c|c|c|c|}
\hline W04610 & 1.10503 & H3F3A & $\underline{3020}$ & H3 histone, family $3 \mathrm{~A}$ & \\
\hline AA045373 & 1.10484 & TCEAL1 & 9338 & $\begin{array}{l}\text { transcription elongation } \\
\text { factor A (SII)-like } 1\end{array}$ & $\begin{array}{l}\text { RNA polymerase II } \\
\text { transcription factor } \\
\text { activity; negative } \\
\text { regulation of } \\
\text { transcription from Pol } \\
\text { II promoter; nucleus; } \\
\text { regulation of } \\
\text { transcription, DNA- } \\
\text { dependent; } \\
\text { transcription factor } \\
\text { activity; translation } \\
\text { elongation factor } \\
\text { activity }\end{array}$ \\
\hline N45505 & 1.10213 & VAV1 & 7409 & vav 1 oncogene & $\begin{array}{l}\text { cell growth and/or } \\
\text { maintenance; } \\
\text { diacylglycerol binding; } \\
\text { guanyl-nucleotide } \\
\text { exchange factor } \\
\text { activity; intracellular } \\
\text { signaling cascade; } \\
\text { nucleus; transcription } \\
\text { factor activity }\end{array}$ \\
\hline N45013 & 1.10126 & & & & \\
\hline R22402 & 1.10059 & & & & \\
\hline H86198 & 1.10011 & & & & \\
\hline AA034916 & 1.09997 & $\begin{array}{l}\text { FLJ1145 } \\
\underline{7}\end{array}$ & $\underline{79809}$ & \multicolumn{2}{|c|}{ hypothetical protein FLJ11457 } \\
\hline N90061 & 1.09992 & STE & $\underline{6783}$ & $\begin{array}{l}\text { sulfotransferase, } \\
\text { estrogen-preferring }\end{array}$ & \begin{tabular}{|l} 
estrone \\
sulfotransferase \\
activity; steroid \\
binding; steroid \\
metabolism; \\
transferase activity \\
\end{tabular} \\
\hline H52939 & 1.09857 & & & & \\
\hline $\mathrm{H} 16193$ & 1.09761 & & & & \\
\hline AA035066 & 1.0976 & $\frac{\text { MGC426 }}{8}$ & $\underline{83607}$ & \multicolumn{2}{|c|}{ hypothetical protein MGC4268 } \\
\hline AA040852 & 1.09709 & $\frac{\text { KIAA132 }}{1}$ & $\underline{57532}$ & KIAA1321 protein & \\
\hline $\mathrm{H} 88577$ & 1.09513 & HNRPH1 & 3187 & $\begin{array}{l}\text { heterogeneous nuclear } \\
\text { ribonucleoprotein } \mathrm{H} 1 \\
(\mathrm{H})\end{array}$ & $\begin{array}{l}\text { RNA binding; RNA } \\
\text { processing; } \\
\text { heterogeneous } \\
\text { nuclear } \\
\text { ribonucleoprotein; } \\
\text { nucleus; poly(U) } \\
\text { binding; } \\
\text { ribonucleoprotein } \\
\text { complex }\end{array}$ \\
\hline
\end{tabular}




\begin{tabular}{|c|c|c|c|c|c|}
\hline N90836 & 1.09501 & FMR1 & $\underline{2332}$ & $\begin{array}{l}\text { fragile X mental } \\
\text { retardation } 1\end{array}$ & $\begin{array}{l}\text { mRNA binding; } \\
\text { nucleoplasm; } \\
\text { polysome; soluble } \\
\text { fraction }\end{array}$ \\
\hline AA044181 & 1.09376 & ENAH & $\underline{5740}$ & \multicolumn{2}{|c|}{ enabled homolog (Drosophila) } \\
\hline $\mathrm{H} 23933$ & 1.09286 & & & & \\
\hline AA131391 & 1.09165 & TRIM29 & $\underline{23650}$ & $\begin{array}{l}\text { tripartite motif- } \\
\text { containing } 29\end{array}$ & $\begin{array}{l}\text { transcription factor } \\
\text { activity; transcription } \\
\text { from Pol II promoter }\end{array}$ \\
\hline R85044 & 1.09161 & SMPD1 & $\underline{6009}$ & $\begin{array}{l}\text { sphingomyelin } \\
\text { phosphodiesterase 1, } \\
\text { acid lysosomal (acid } \\
\text { sphingomyelinase) }\end{array}$ & $\begin{array}{l}\text { carbohydrate } \\
\text { metabolism; hydrolase } \\
\text { activity, acting on } \\
\text { glycosyl bonds; } \\
\text { lysosome; } \\
\text { neurogenesis; signal } \\
\text { transduction; } \\
\text { sphingomyelin } \\
\text { metabolism; } \\
\text { sphingomyelin } \\
\text { phosphodiesterase } \\
\text { activity }\end{array}$ \\
\hline N22938 & 1.09154 & SAA4 & $\underline{6291}$ & $\begin{array}{l}\text { serum amyloid A4, } \\
\text { constitutive }\end{array}$ & $\begin{array}{l}\text { acute-phase } \\
\text { response; acute- } \\
\text { phase response } \\
\text { protein activity; } \\
\text { extracellular; lipid } \\
\text { transporter activity }\end{array}$ \\
\hline R88711 & 1.09131 & & & & \\
\hline R85232 & 1.09047 & ACCN2 & $\underline{41}$ & $\begin{array}{l}\text { amiloride-sensitive } \\
\text { cation channel 2, } \\
\text { neuronal }\end{array}$ & $\begin{array}{l}\text { amiloride-sensitive } \\
\text { sodium channel } \\
\text { activity; integral to } \\
\text { membrane; ion } \\
\text { channel activity; ion } \\
\text { transport; sodium ion } \\
\text { transport }\end{array}$ \\
\hline $\mathrm{H} 71112$ & 1.0887 & MCM2 & $\underline{4171}$ & $\begin{array}{l}\mathrm{MCM} 2 \\
\text { minichromosome } \\
\text { maintenance deficient } \\
2, \text { mitotin (S. } \\
\text { cerevisiae) }\end{array}$ & $\begin{array}{l}\text { ATP binding; DNA } \\
\text { binding; DNA } \\
\text { dependent ATPase } \\
\text { activity; DNA } \\
\text { replication; DNA } \\
\text { replication initiation; } \\
\text { cell cycle; chromatin; } \\
\text { nucleus; regulation of } \\
\text { transcription, DNA- } \\
\text { dependent }\end{array}$ \\
\hline
\end{tabular}




\begin{tabular}{|c|c|c|c|c|c|}
\hline T54547 & 1.08811 & DF & 1675 & $\begin{array}{l}\text { D component of } \\
\text { complement (adipsin) }\end{array}$ & $\begin{array}{l}\text { chymotrypsin activity; } \\
\text { complement } \\
\text { activation, alternative } \\
\text { pathway; complement } \\
\text { factor D activity; } \\
\text { hydrolase activity; } \\
\text { proteolysis and } \\
\text { peptidolysis; trypsin } \\
\text { activity }\end{array}$ \\
\hline W40304 & 1.08804 & API5 & $\underline{8539}$ & apoptosis inhibitor 5 & $\begin{array}{l}\text { anti-apoptosis; } \\
\text { apoptosis inhibitor } \\
\text { activity }\end{array}$ \\
\hline AA041264 & 1.08588 & ATP2B1 & $\underline{490}$ & $\begin{array}{l}\text { ATPase, Ca++ } \\
\text { transporting, plasma } \\
\text { membrane } 1\end{array}$ & $\begin{array}{l}\text { ATP binding; calcium } \\
\text { ion transport; calcium- } \\
\text { transporting ATPase } \\
\text { activity; calmodulin } \\
\text { binding; cation } \\
\text { transport; hydrolase } \\
\text { activity; integral to } \\
\text { plasma membrane; } \\
\text { magnesium ion } \\
\text { binding; metabolism; } \\
\text { transport }\end{array}$ \\
\hline H38321 & 1.08519 & $\underline{\underline{F} L J 1436}$ & $\underline{84861}$ & $\begin{array}{l}\text { hypothetical protein } \\
\text { FLJ14360 }\end{array}$ & protein binding \\
\hline N81000 & 1.08328 & & & & \\
\hline H52253 & 1.08181 & IGHG3 & 3502 & $\begin{array}{l}\text { immunoglobulin heavy } \\
\text { constant gamma } 3 \\
\text { (G3m marker) }\end{array}$ & $\begin{array}{l}\text { antigen binding; } \\
\text { immune response; } \\
\text { membrane fraction }\end{array}$ \\
\hline $\mathrm{H} 40662$ & 1.08154 & KNS2 & 3831 & kinesin 2 60/70kDa & $\begin{array}{l}\text { kinesin complex; } \\
\text { microtubule motor } \\
\text { activity; microtubule- } \\
\text { based process }\end{array}$ \\
\hline H68885 & 1.08128 & TSSC3 & 7262 & $\begin{array}{l}\text { tumor suppressing } \\
\text { subtransferable } \\
\text { candidate } 3\end{array}$ & apoptosis; imprinting \\
\hline H59563 & 1.081 & CASP10 & 843 & \multicolumn{2}{|c|}{$\begin{array}{l}\text { caspase } 10, \text { apoptosis-related cysteine } \\
\text { protease }\end{array}$} \\
\hline N74428 & 1.08013 & & & & \\
\hline R46282 & 1.07975 & & & & \\
\hline AA101839 & 1.07876 & & & & \\
\hline N93133 & 1.07856 & & & & \\
\hline N40017 & 1.07723 & MRPL24 & 79590 & $\begin{array}{l}\text { mitochondrial } \\
\text { ribosomal protein L24 }\end{array}$ & $\begin{array}{l}\text { intracellular; protein } \\
\text { biosynthesis; } \\
\text { ribosome; structural } \\
\text { constituent of } \\
\text { ribosome }\end{array}$ \\
\hline
\end{tabular}




\begin{tabular}{|c|c|c|c|c|c|}
\hline AA037284 & 1.07698 & APRT & $\underline{353}$ & $\begin{array}{l}\text { adenine } \\
\text { phosphoribosyltransfer } \\
\text { ase }\end{array}$ & $\begin{array}{l}\text { adenine } \\
\text { phosphoribosyltransfer } \\
\text { ase activity; adenine } \\
\text { salvage pathway; } \\
\text { nucleoside } \\
\text { metabolism; } \\
\text { transferase activity, } \\
\text { transferring glycosyl } \\
\text { groups }\end{array}$ \\
\hline AA011556 & 1.07686 & & & & \\
\hline T66831 & 1.07643 & & & & \\
\hline AA207094 & 1.07521 & & & & \\
\hline W37783 & 1.07349 & RRAS2 & $\underline{22800}$ & $\begin{array}{l}\text { related RAS viral (r- } \\
\text { ras) oncogene } \\
\text { homolog } 2\end{array}$ & $\begin{array}{l}\text { GTP binding; RAS } \\
\text { small monomeric } \\
\text { GTPase activity; cell } \\
\text { growth and/or } \\
\text { maintenance; } \\
\text { endoplasmic } \\
\text { reticulum; plasma } \\
\text { membrane; small } \\
\text { GTPase mediated } \\
\text { signal transduction }\end{array}$ \\
\hline $\mathrm{H} 12075$ & 1.07259 & $\frac{F L J 3795}{3}$ & 129450 & hypothetical protein FL. & J37953 \\
\hline H69011 & 1.07177 & $\underline{\mathrm{SKIL}}$ & $\underline{6498}$ & SKI-like & $\begin{array}{l}\text { cell differentiation; cell } \\
\text { growth and/or } \\
\text { maintenance; } \\
\text { molecular_function } \\
\text { unknown; nucleus }\end{array}$ \\
\hline W90661 & 1.07093 & & & & \\
\hline R96672 & 1.07087 & CYP2D6 & $\underline{1565}$ & $\begin{array}{l}\text { cytochrome P450, } \\
\text { family } 2 \text {, subfamily D, } \\
\text { polypeptide } 6\end{array}$ & $\begin{array}{l}\text { cytochrome P450 } \\
\text { activity }\end{array}$ \\
\hline R87923 & 1.07057 & RPIP8 & $\underline{10900}$ & $\begin{array}{l}\text { RaP2 interacting } \\
\text { protein } 8\end{array}$ & $\begin{array}{l}\text { small GTPase } \\
\text { mediated signal } \\
\text { transduction; small } \\
\text { GTPase } \\
\text { regulatory/interacting } \\
\text { protein activity }\end{array}$ \\
\hline N34901 & 1.06882 & GALNT7 & $\underline{117248}$ & \multicolumn{2}{|c|}{$\begin{array}{l}\text { UDP-N-acetyl-alpha-D- } \\
\text { galactosamine:polypeptide N- } \\
\text { acetylgalactosaminyltransferase } 7\end{array}$} \\
\hline
\end{tabular}




\begin{tabular}{|c|c|c|c|c|c|}
\hline AA057293 & 1.06798 & PPIC & $\underline{5480}$ & $\begin{array}{l}\text { peptidylprolyl } \\
\text { isomerase C } \\
\text { (cyclophilin C) }\end{array}$ & $\begin{array}{l}\text { FK506-sensitive } \\
\text { peptidyl-prolyl cis- } \\
\text { trans isomerase; } \\
\text { antimicrobial humoral } \\
\text { response (sensu } \\
\text { Invertebrata); } \\
\text { chaperone activity; } \\
\text { cyclophilin; cyclophilin- } \\
\text { type peptidy-prolyl cis- } \\
\text { trans isomerase } \\
\text { activity; cyclosporin A } \\
\text { binding; cytoplasm; } \\
\text { isomerase activity; } \\
\text { protein folding; signal } \\
\text { transduction }\end{array}$ \\
\hline AA147500 & 1.06795 & & $\underline{282963}$ & \multicolumn{2}{|c|}{$\begin{array}{l}\text { hypothetical gene supported by AL833529; } \\
\text { AK021993 }\end{array}$} \\
\hline $\mathrm{H} 45213$ & 1.066 & & & & \\
\hline H85193 & 1.06535 & & & & \\
\hline N41763 & 1.06085 & & & & \\
\hline AA147534 & 1.06047 & & & & \\
\hline H91962 & 1.06007 & & & & \\
\hline AA213442 & 1.05913 & & & & \\
\hline AA036758 & 1.05844 & S100A4 & $\underline{6275}$ & $\begin{array}{l}\text { S100 calcium binding } \\
\text { protein A4 (calcium } \\
\text { protein, calvasculin, } \\
\text { metastasin, murine } \\
\text { placental homolog) }\end{array}$ & $\begin{array}{l}\text { calcium ion binding; } \\
\text { invasive growth }\end{array}$ \\
\hline R88082 & 1.0574 & & & & \\
\hline W42634 & 1.05675 & FAP & $\underline{2191}$ & $\begin{array}{l}\text { fibroblast activation } \\
\text { protein, alpha }\end{array}$ & $\begin{array}{l}\text { dipeptidyl-peptidase IV } \\
\text { activity; integral to } \\
\text { membrane; prolyl } \\
\text { oligopeptidase activity; } \\
\text { proteolysis and } \\
\text { peptidolysis }\end{array}$ \\
\hline R54729 & 1.05414 & & & & \\
\hline H41751 & 1.05392 & GTF2F1 & 2962 & $\begin{array}{l}\text { general transcription } \\
\text { factor IIF, polypeptide } \\
1,74 \mathrm{kDa}\end{array}$ & $\begin{array}{l}\text { DNA binding; general } \\
\text { RNA polymerase II } \\
\text { transcription factor } \\
\text { activity; nucleus; } \\
\text { protein binding; } \\
\text { regulation of } \\
\text { transcription, DNA- } \\
\text { dependent; } \\
\text { transcription co- } \\
\text { activator activity; } \\
\text { transcription factor } \\
\text { TFIIF complex; } \\
\text { transcription initiation } \\
\text { from Pol II promoter }\end{array}$ \\
\hline
\end{tabular}




\begin{tabular}{|c|c|c|c|c|c|}
\hline AA204664 & 1.05298 & SMC1L2 & 27127 & \begin{tabular}{|l|} 
SMC1 structural \\
maintenance of \\
chromosomes 1-like 2 \\
(yeast)
\end{tabular} & $\begin{array}{l}\text { ATP binding; ATP- } \\
\text { binding cassette } \\
\text { (ABC) transporter } \\
\text { activity; cell cycle; } \\
\text { chromosome } \\
\text { segregation; kinesin } \\
\text { complex; meiosis; } \\
\text { membrane; nucleus; } \\
\text { transport }\end{array}$ \\
\hline N30528 & 1.05281 & PPARD & $\underline{5467}$ & \multicolumn{2}{|c|}{$\begin{array}{l}\text { peroxisome proliferative activated receptor, } \\
\text { delta }\end{array}$} \\
\hline N70531 & 1.05242 & STMN1 & 3925 & $\begin{array}{l}\text { stathmin 1/oncoprotein } \\
18\end{array}$ & $\begin{array}{l}\text { cell growth and/or } \\
\text { maintenance; cytosol; } \\
\text { intracellular signaling } \\
\text { cascade; kinesin } \\
\text { complex; microtubule- } \\
\text { based process; signal } \\
\text { transducer activity }\end{array}$ \\
\hline R66261 & 1.05162 & PRG2 & $\underline{5553}$ & $\begin{array}{l}\text { proteoglycan } 2, \text { bone } \\
\text { marrow (natural killer } \\
\text { cell activator, } \\
\text { eosinophil granule } \\
\text { major basic protein) }\end{array}$ & $\begin{array}{l}\text { cytoplasm; } \\
\text { extracellular; heparin } \\
\text { binding; heterophilic } \\
\text { cell adhesion; immune } \\
\text { response; } \\
\text { inflammatory } \\
\text { response; lectin; sugar } \\
\text { binding; toxin activity; } \\
\text { xenobiotic metabolism }\end{array}$ \\
\hline N78926 & 1.0507 & MYL6 & 4637 & $\begin{array}{l}\text { myosin, light } \\
\text { polypeptide } 6 \text {, alkali, } \\
\text { smooth muscle and } \\
\text { non-muscle }\end{array}$ & $\begin{array}{l}\text { muscle myosin; non- } \\
\text { muscle myosin; } \\
\text { structural constituent } \\
\text { of muscle }\end{array}$ \\
\hline R48615 & 1.04859 & C14orf21 & 161424 & $\begin{array}{l}\text { chromosome } 14 \text { open } \\
\text { reading frame } 21\end{array}$ & RNA binding \\
\hline H49225 & 1.04854 & & & & \\
\hline R51354 & 1.04815 & $\frac{\text { CNTNAP }}{2}$ & 26047 & contactin associated prc & otein-like 2 \\
\hline AA063424 & 1.04652 & C6orf80 & $\underline{25901}$ & chromosome 6 open rea & ading frame 80 \\
\hline N63012 & 1.04631 & & & & \\
\hline W49770 & 1.04582 & $\frac{\text { MORF4L }}{\underline{2}}$ & $\underline{9643}$ & mortality factor 4 like 2 & $\begin{array}{l}\text { molecular_function } \\
\text { unknown; nucleus; } \\
\text { regulation of cell } \\
\text { growth }\end{array}$ \\
\hline
\end{tabular}




\begin{tabular}{|c|c|c|c|c|c|}
\hline R53559 & 1.04558 & NME1 & $\underline{4830}$ & $\begin{array}{l}\text { non-metastatic cells 1, } \\
\text { protein (NM23A) } \\
\text { expressed in }\end{array}$ & $\begin{array}{l}\text { ATP binding; CTP } \\
\text { biosynthesis; GTP } \\
\text { biosynthesis; UTP } \\
\text { biosynthesis; kinase } \\
\text { activity; negative } \\
\text { regulation of cell cycle; } \\
\text { negative regulation of } \\
\text { cell proliferation; } \\
\text { nucleoside } \\
\text { triphosphate } \\
\text { biosynthesis; } \\
\text { nucleoside- } \\
\text { diphosphate kinase } \\
\text { activity; nucleus; } \\
\text { transferase activity } \\
\end{array}$ \\
\hline AA115689 & 1.04489 & & & & \\
\hline AA028109 & 1.04465 & RAB23 & $\underline{51715}$ & $\begin{array}{l}\text { RAB23, member RAS } \\
\text { oncogene family }\end{array}$ & $\begin{array}{l}\text { GTP binding; RAB } \\
\text { small monomeric } \\
\text { GTPase activity; } \\
\text { intracellular protein } \\
\text { transport; protein } \\
\text { transporter activity; } \\
\text { small GTPase } \\
\text { mediated signal } \\
\text { transduction }\end{array}$ \\
\hline N33745 & 1.04362 & $\frac{\text { KIAA084 }}{1}$ & $\underline{23354}$ & KIAA0841 protein & nucleic acid binding \\
\hline W04822 & 1.04293 & & & & \\
\hline $\mathrm{H} 02088$ & 1.04178 & RBAF600 & $\underline{23352}$ & \multicolumn{2}{|c|}{ retinoblastoma-associated factor 600} \\
\hline H30357 & 1.04171 & ATP4A & $\underline{495}$ & $\begin{array}{l}\text { ATPase, } \mathrm{H}+/ \mathrm{K}+ \\
\text { exchanging, alpha } \\
\text { polypeptide }\end{array}$ & $\begin{array}{l}\text { ATP binding; } \\
\text { hydrogen/potassium- } \\
\text { exchanging ATPase } \\
\text { activity; hydrolase } \\
\text { activity; integral to } \\
\text { plasma membrane; } \\
\text { magnesium ion } \\
\text { binding; metabolism; } \\
\text { potassium ion } \\
\text { transport; proton } \\
\text { transport; transport }\end{array}$ \\
\hline N39691 & 1.04084 & CFL1 & $\underline{1072}$ & cofilin 1 (non-muscle) & $\begin{array}{l}\text { Rho protein signal } \\
\text { transduction; actin } \\
\text { cytoskeleton } \\
\text { organization and } \\
\text { biogenesis; actin } \\
\text { modulating activity; } \\
\text { cytoskeleton; nucleus }\end{array}$ \\
\hline $\mathrm{H} 27034$ & 1.04005 & IGKC & $\underline{3514}$ & $\begin{array}{l}\text { immunoglobulin kappa } \\
\text { constant }\end{array}$ & $\begin{array}{l}\text { antigen binding; } \\
\text { immune response }\end{array}$ \\
\hline AA203153 & 1.03836 & & & & \\
\hline
\end{tabular}




\begin{tabular}{|c|c|c|c|c|c|}
\hline AA036635 & 1.03821 & AKAP10 & $\underline{11216}$ & $\begin{array}{l}\text { A kinase (PRKA) } \\
\text { anchor protein } 10\end{array}$ & $\begin{array}{l}\text { mitochondrion; protein } \\
\text { binding; protein } \\
\text { localization; signal } \\
\text { transducer activity; } \\
\text { signal transduction }\end{array}$ \\
\hline W52156 & 1.03787 & OXTR & $\underline{5021}$ & oxytocin receptor & $\begin{array}{l}\text { G-protein signaling, } \\
\text { coupled to IP3 second } \\
\text { messenger } \\
\text { (phospholipase C } \\
\text { activating); endosome; } \\
\text { integral to plasma } \\
\text { membrane; lactation; } \\
\text { muscle contraction; } \\
\text { oxytocin receptor } \\
\text { activity; pregnancy; } \\
\text { rhodopsin-like } \\
\text { receptor activity; } \\
\text { vasopressin receptor } \\
\text { activity }\end{array}$ \\
\hline T77422 & 1.0375 & & & & \\
\hline W01319 & 1.03748 & $\mathrm{BHC} 80$ & $\underline{51317}$ & \multicolumn{2}{|c|}{ BRAF35/HDAC2 complex (80 kDa) } \\
\hline T84788 & 1.03644 & & & & \\
\hline N55079 & 1.0356 & F10 & $\underline{2159}$ & coagulation factor $X$ & $\begin{array}{l}\text { blood coagulation; } \\
\text { blood coagulation } \\
\text { factor X activity; } \\
\text { calcium ion binding; } \\
\text { chymotrypsin activity; } \\
\text { extracellular; } \\
\text { hydrolase activity; } \\
\text { proteolysis and } \\
\text { peptidolysis; trypsin } \\
\text { activity }\end{array}$ \\
\hline AA131450 & 1.03424 & & & & \\
\hline R20373 & 1.03415 & TMP21 & $\underline{10972}$ & $\begin{array}{l}\text { transmembrane } \\
\text { trafficking protein }\end{array}$ & $\begin{array}{l}\text { ER to Golgi transport; } \\
\text { Golgi apparatus; } \\
\text { integral to plasma } \\
\text { membrane; } \\
\text { intracellular protein } \\
\text { transport; membrane } \\
\text { fraction; microsome; } \\
\text { protein carrier activity; } \\
\text { protein transporter } \\
\text { activity }\end{array}$ \\
\hline R47945 & 1.03168 & CCT5 & $\underline{22948}$ & $\begin{array}{l}\text { chaperonin containing } \\
\text { TCP1, subunit } 5 \\
\text { (epsilon) }\end{array}$ & $\begin{array}{l}\text { ATP binding; } \\
\text { chaperone activity }\end{array}$ \\
\hline
\end{tabular}




\begin{tabular}{|c|c|c|c|c|c|}
\hline $\mathrm{H} 38879$ & 1.02995 & PSPH & $\underline{5723}$ & $\begin{array}{l}\text { phosphoserine } \\
\text { phosphatase }\end{array}$ & $\begin{array}{l}\text { hydrolase activity; } \\
\text { magnesium ion } \\
\text { binding; metabolism; } \\
\text { phosphoserine } \\
\text { phosphatase activity; } \\
\text { serine biosynthesis }\end{array}$ \\
\hline R44307 & 1.02859 & $\frac{\text { PPP1R9 }}{\text { B }}$ & $\underline{84687}$ & $\begin{array}{l}\text { protein phosphatase } 1, \\
\text { regulatory subunit 9B, } \\
\text { spinophilin }\end{array}$ & $\begin{array}{l}\text { intracellular signaling } \\
\text { cascade; membrane; } \\
\text { transport; transporter } \\
\text { activity }\end{array}$ \\
\hline W87556 & 1.02814 & & & & \\
\hline N80976 & 1.02773 & \begin{tabular}{|l|}
$\underline{2} \mathrm{OC} 5125$ \\
$\underline{2}$
\end{tabular} & $\underline{51252}$ & \multicolumn{2}{|c|}{ hypothetical protein LOC51252 } \\
\hline AA059076 & 1.02713 & MTMR9 & $\underline{66036}$ & \multicolumn{2}{|c|}{ myotubularin related protein 9} \\
\hline H86858 & 1.02685 & & & & \\
\hline W89025 & 1.02603 & & & & \\
\hline N91128 & 1.02596 & SFTPB & $\underline{6439}$ & $\begin{array}{l}\text { surfactant, pulmonary- } \\
\text { associated protein B }\end{array}$ & $\begin{array}{l}\text { extracellular space; } \\
\text { histogenesis and } \\
\text { organogenesis; } \\
\text { lysosome; respiratory } \\
\text { gaseous exchange; } \\
\text { sphingolipid } \\
\text { metabolism; surfactant } \\
\text { activity }\end{array}$ \\
\hline $\mathrm{H} 04530$ & 1.02471 & ECHS1 & $\underline{1892}$ & $\begin{array}{l}\text { enoyl Coenzyme A } \\
\text { hydratase, short chain, } \\
1, \text { mitochondrial }\end{array}$ & $\begin{array}{l}\text { energy pathways; fatty } \\
\text { acid beta-oxidation; } \\
\text { fatty acid metabolism; } \\
\text { long-chain enoyl-CoA } \\
\text { hydratase activity; } \\
\text { lyase activity; } \\
\text { mitochondrion; short- } \\
\text { chain enoyl-CoA } \\
\text { hydratase activity } \\
\end{array}$ \\
\hline N73980 & 1.02454 & KRT8 & $\underline{3856}$ & keratin 8 & $\begin{array}{l}\text { cytoskeleton } \\
\text { organization and } \\
\text { biogenesis; } \\
\text { intermediate filament; } \\
\text { kinesin complex; } \\
\text { phosphorylation; } \\
\text { structural molecule } \\
\text { activity }\end{array}$ \\
\hline H58631 & 1.02371 & & & & \\
\hline W42638 & 1.02059 & STAM2 & $\underline{10254}$ & \begin{tabular}{|l|} 
signal transducing \\
adaptor molecule (SH3 \\
domain and ITAM \\
motif) 2
\end{tabular} & $\begin{array}{l}\text { intracellular protein } \\
\text { transport }\end{array}$ \\
\hline R39421 & 1.02039 & PIGM & $\underline{93183}$ & $\begin{array}{l}\text { phosphatidylinositol } \\
\text { glycan, class M }\end{array}$ & transferase activity \\
\hline
\end{tabular}




\begin{tabular}{|c|c|c|c|c|c|}
\hline R89056 & 1.02026 & LAMP1 & $\underline{3916}$ & $\begin{array}{l}\text { lysosomal-associated } \\
\text { membrane protein } 1\end{array}$ & $\begin{array}{l}\text { integral to plasma } \\
\text { membrane; lysosome; } \\
\text { membrane fraction }\end{array}$ \\
\hline H44888 & 1.01975 & & & & \\
\hline W88660 & 1.01804 & PDIP38 & $\underline{26073}$ & \multicolumn{2}{|c|}{ polymerase delta interacting protein 38} \\
\hline AA056998 & 1.01557 & & & & \\
\hline N25523 & 1.01481 & HSPE1 & 3336 & $\begin{array}{l}\text { heat shock } 10 \mathrm{kDa} \\
\text { protein } 1 \text { (chaperonin } \\
10 \text { ) }\end{array}$ & $\begin{array}{l}\text { co-chaperonin activity; } \\
\text { heat shock protein } \\
\text { activity; } \\
\text { mitochondrion; protein } \\
\text { folding }\end{array}$ \\
\hline R82691 & 1.01433 & & 348700 & \multicolumn{2}{|c|}{$\begin{array}{l}\text { similar to RAN-binding protein 2-like } 1 \text { isoform } \\
\text { 1; sperm membrane protein BS-63; RAN- } \\
\text { binding protein 2-like } 1\end{array}$} \\
\hline W24523 & 1.01417 & $\frac{M G C 202}{62}$ & 138311 & \multicolumn{2}{|c|}{ hypothetical protein MGC20262 } \\
\hline AA054948 & 1.01407 & & & & \\
\hline AA069448 & 1.01373 & & & & \\
\hline N36272 & 1.01165 & & & & \\
\hline H59595 & 1.01126 & & & & \\
\hline AA043638 & 1.00939 & & & & \\
\hline AA127098 & 1.00921 & & & & \\
\hline W19744 & 1.00892 & & & & \\
\hline N57249 & 1.00664 & PSMB5 & $\underline{5693}$ & $\begin{array}{l}\text { proteasome (prosome, } \\
\text { macropain) subunit, } \\
\text { beta type, } 5\end{array}$ & $\begin{array}{l}\text { 26S proteasome; } \\
\text { cytosol; } \\
\text { endopeptidase } \\
\text { activity; proteasome } \\
\text { core complex (sensu } \\
\text { Eukarya); proteasome } \\
\text { endopeptidase } \\
\text { activity; ubiquitin- } \\
\text { dependent protein } \\
\text { catabolism }\end{array}$ \\
\hline R08339 & 1.00641 & & & & \\
\hline R48610 & 1.00556 & TTC7 & $\underline{57217}$ & \multicolumn{2}{|c|}{ tetratricopeptide repeat domain 7} \\
\hline AA029012 & 1.00498 & SMA5 & 11042 & SMA5 & \begin{tabular}{|l} 
biological_process \\
unknown; \\
carbohydrate \\
metabolism; \\
cellular_component \\
unknown; hydrolase \\
activity, hydrolyzing O- \\
glycosyl compounds; \\
molecular_function \\
unknown
\end{tabular} \\
\hline
\end{tabular}




\begin{tabular}{|c|c|c|c|c|c|}
\hline AA036787 & 1.00455 & NCK2 & 8440 & NCK adaptor protein 2 & $\begin{array}{l}\text { intracellular signaling } \\
\text { cascade; negative } \\
\text { regulation of cell } \\
\text { proliferation; } \\
\text { regulation of EGF } \\
\text { receptor activity } \\
\end{array}$ \\
\hline R07186 & 1.00438 & & & & \\
\hline AA002041 & 1.00431 & ZNF262 & 9202 & zinc finger protein 262 & $\begin{array}{l}\text { DNA binding; } \\
\text { development; } \\
\text { extracellular; hormone } \\
\text { activity }\end{array}$ \\
\hline N78467 & 1.00392 & PWP1 & $\underline{11137}$ & $\begin{array}{l}\text { nuclear } \\
\text { phosphoprotein similar } \\
\text { to S. cerevisiae PWP1 }\end{array}$ & nucleus; transcription \\
\hline W78129 & 1.00383 & $\underline{F G G}$ & $\underline{2266}$ & $\begin{array}{l}\text { fibrinogen, gamma } \\
\text { polypeptide }\end{array}$ & $\begin{array}{l}\text { blood coagulation; } \\
\text { fibrinogen; fibrinogen } \\
\text { gamma chain; positive } \\
\text { regulation of cell } \\
\text { proliferation; } \\
\text { regulation of blood } \\
\text { pressure }\end{array}$ \\
\hline $\mathrm{H} 14840$ & 1.00372 & $\frac{M G C 266}{8}$ & $\underline{81605}$ & \multicolumn{2}{|c|}{ hypothetical protein MGC2668 } \\
\hline $\mathrm{H} 68441$ & 1.00241 & $\begin{array}{l}\text { FLJ1405 } \\
4\end{array}$ & $\underline{79614}$ & \multicolumn{2}{|c|}{ hypothetical protein FLJ14054 } \\
\hline R74161 & 1.00161 & PYGL & $\underline{5836}$ & $\begin{array}{l}\text { phosphorylase, } \\
\text { glycogen; liver (Hers } \\
\text { disease, glycogen } \\
\text { storage disease type } \\
\text { VI) }\end{array}$ & $\begin{array}{l}\text { carbohydrate } \\
\text { metabolism; glycogen } \\
\text { metabolism; glycogen } \\
\text { phosphorylase activity; } \\
\text { transferase activity, } \\
\text { transferring glycosyl } \\
\text { groups }\end{array}$ \\
\hline R77028 & 1.00093 & LTBR & $\underline{4055}$ & $\begin{array}{l}\text { lymphotoxin beta } \\
\text { receptor (TNFR } \\
\text { superfamily, member } \\
\text { 3) }\end{array}$ & $\begin{array}{l}\text { apoptosis; immune } \\
\text { response; integral to } \\
\text { membrane; signal } \\
\text { transduction; } \\
\text { transmembrane } \\
\text { receptor activity }\end{array}$ \\
\hline R50087 & 1.00091 & GREB1 & 9687 & GREB1 protein & \\
\hline AA131884 & 1.00077 & $\begin{array}{l}\mathrm{LOC5105} \\
\underline{7} \\
\end{array}$ & $\underline{51057}$ & \multicolumn{2}{|c|}{ hypothetical protein LOC51057 } \\
\hline R07617 & -1.0006 & ABCD4 & $\underline{5826}$ & $\begin{array}{l}\text { ATP-binding cassette, } \\
\text { sub-family D (ALD), } \\
\text { member } 4\end{array}$ & $\begin{array}{l}\text { ATP binding; ATP- } \\
\text { binding cassette } \\
\text { (ABC) transporter } \\
\text { activity; integral to } \\
\text { membrane; membrane } \\
\text { fraction; nucleotide } \\
\text { binding; peroxisomal } \\
\text { membrane; transport; } \\
\text { transporter activity }\end{array}$ \\
\hline
\end{tabular}




\begin{tabular}{|c|c|c|c|c|c|}
\hline T89328 & -1.0012 & $\frac{\text { PPP1R1 }}{2 \mathrm{~A}}$ & $\underline{4659}$ & $\begin{array}{l}\text { protein phosphatase 1, } \\
\text { regulatory (inhibitor) } \\
\text { subunit } 12 \mathrm{~A}\end{array}$ & $\begin{array}{l}\text { actin cytoskeleton; } \\
\text { regulation of muscle } \\
\text { contraction; signal } \\
\text { transducer activity }\end{array}$ \\
\hline W52903 & -1.0018 & $\frac{\mathrm{HSPC} 17}{7 \underline{7}}$ & $\underline{51510}$ & $\begin{array}{l}\text { hypothetical protein } \\
\text { HSPC } 177\end{array}$ & $\begin{array}{l}\text { molecular_function } \\
\text { unknown }\end{array}$ \\
\hline R00555 & -1.007 & & & & \\
\hline R80587 & -1.0082 & $\frac{\text { PPP2R2 }}{A}$ & $\underline{5520}$ & $\begin{array}{l}\text { protein phosphatase } 2 \\
\text { (formerly } 2 A) \text {, } \\
\text { regulatory subunit B } \\
(P R 52), \text { alpha isoform }\end{array}$ & $\begin{array}{l}\text { protein amino acid } \\
\text { dephosphorylation; } \\
\text { protein phosphatase } \\
\text { type } 2 \mathrm{~A} \text { complex; } \\
\text { protein phosphatase } \\
\text { type } 2 \mathrm{~A} \text {, intrinsic } \\
\text { regulator activity; } \\
\text { signal transduction }\end{array}$ \\
\hline T86348 & -1.0112 & GATM & $\underline{2628}$ & $\begin{array}{l}\text { glycine } \\
\text { amidinotransferase (L- } \\
\text { arginine:glycine } \\
\text { amidinotransferase) }\end{array}$ & $\begin{array}{l}\text { creatine biosynthesis; } \\
\text { cytosol; glycine } \\
\text { amidinotransferase } \\
\text { activity; } \\
\text { mitochondrion; } \\
\text { transferase activity }\end{array}$ \\
\hline R46353 & -1.0127 & AEBP1 & 165 & AE binding protein 1 & $\begin{array}{l}\text { carboxypeptidase A } \\
\text { activity; } \\
\text { carboxypeptidase } \\
\text { activity; cell adhesion; } \\
\text { cytoplasm; muscle } \\
\text { development; } \\
\text { proteolysis and } \\
\text { peptidolysis; skeletal } \\
\text { development; } \\
\text { transcription factor } \\
\text { activity }\end{array}$ \\
\hline R26717 & -1.0129 & \begin{tabular}{|l|}
$\underline{47}$ \\
\end{tabular} & 134147 & $\begin{array}{l}\text { hypothetical protein } \\
\text { BC001573 }\end{array}$ & hydrolase activity \\
\hline R75921 & -1.0145 & $\frac{\mathrm{SDBCAG}}{84}$ & 51614 & \multicolumn{2}{|c|}{ serologically defined breast cancer antigen 84} \\
\hline W04539 & -1.0151 & $\frac{\mathrm{KIAA029}}{5}$ & 23060 & KIAA0295 protein & \\
\hline AA129569 & -1.0162 & SLC35E2 & $\underline{9906}$ & \multicolumn{2}{|c|}{ solute carrier family 35 , member E2 } \\
\hline N69900 & -1.0163 & NIN283 & 84937 & nerve injury gene 283 & \\
\hline T81574 & -1.0203 & & & & \\
\hline N79754 & -1.0213 & ELAC1 & 55520 & $\begin{array}{l}\text { elaC homolog } 1 \text { (E. } \\
\text { coli) }\end{array}$ & \\
\hline H51151 & -1.0224 & $\frac{\text { GABARA }}{\text { PL1 }}$ & 23710 & $\begin{array}{l}\text { GABA(A) receptor- } \\
\text { associated protein like } \\
1\end{array}$ & receptor activity \\
\hline
\end{tabular}




\begin{tabular}{|c|c|c|c|c|c|}
\hline AA043506 & -1.0225 & $\underline{\text { CEBPD }}$ & 1052 & $\begin{array}{l}\text { CCAAT/enhancer } \\
\text { binding protein } \\
\text { (C/EBP), delta }\end{array}$ & $\begin{array}{l}\text { DNA binding; nucleus; } \\
\text { regulation of } \\
\text { transcription, DNA- } \\
\text { dependent; } \\
\text { transcription from Pol } \\
\text { Il promoter }\end{array}$ \\
\hline R53445 & -1.0227 & & & & \\
\hline T84038 & -1.0257 & POU2F1 & 5451 & $\begin{array}{l}\text { POU domain, class } 2, \\
\text { transcription factor } 1\end{array}$ & $\begin{array}{l}\text { nucleus; regulation of } \\
\text { transcription, DNA- } \\
\text { dependent; } \\
\text { transcription factor } \\
\text { activity }\end{array}$ \\
\hline R20409 & -1.0259 & & & & \\
\hline R20620 & -1.0272 & $\frac{\mathrm{LOC} 6417}{4}$ & 64174 & putative dipeptidase & \\
\hline AA148455 & -1.0278 & UBE2V2 & $\underline{7336}$ & $\begin{array}{l}\text { ubiquitin-conjugating } \\
\text { enzyme E2 variant } 2\end{array}$ & $\begin{array}{l}\text { cell proliferation; } \\
\text { ligase activity; protein } \\
\text { modification; protein } \\
\text { polyubiquitination; } \\
\text { regulation of DNA } \\
\text { repair; regulation of } \\
\text { cell cycle; ubiquitin } \\
\text { conjugating enzyme } \\
\text { activity; ubiquitin } \\
\text { cycle; ubiquitin-protein } \\
\text { ligase activity }\end{array}$ \\
\hline AA036809 & -1.0279 & & & & \\
\hline $\mathrm{H} 64812$ & -1.0289 & RPS28 & $\underline{6234}$ & ribosomal protein S28 & $\begin{array}{l}\text { RNA binding; cytosolic } \\
\text { small ribosomal } \\
\text { subunit (sensu } \\
\text { Eukarya); intracellular; } \\
\text { protein biosynthesis; } \\
\text { ribosome; structural } \\
\text { constituent of } \\
\text { ribosome }\end{array}$ \\
\hline R06463 & -1.029 & TRAP1 & 10131 & heat shock protein 75 & $\begin{array}{l}\text { ATP binding; } \\
\text { biological_process } \\
\text { unknown; } \\
\text { cellular_component } \\
\text { unknown; chaperone } \\
\text { activity; } \\
\text { mitochondrion; tumor } \\
\text { necrosis factor } \\
\text { receptor binding }\end{array}$ \\
\hline N74391 & -1.0328 & & & & \\
\hline N63587 & -1.0384 & RPL24 & $\underline{6152}$ & ribosomal protein L24 & $\begin{array}{l}\text { RNA binding; cytosolic } \\
\text { large ribosomal } \\
\text { subunit (sensu } \\
\text { Eukarya); intracellular; } \\
\text { protein biosynthesis; } \\
\text { ribosome; structural } \\
\text { constituent of } \\
\text { ribosome }\end{array}$ \\
\hline
\end{tabular}




\begin{tabular}{|c|c|c|c|c|c|}
\hline R12744 & -1.0392 & SPARC & $\underline{6678}$ & $\begin{array}{l}\text { secreted protein, } \\
\text { acidic, cysteine-rich } \\
\text { (osteonectin) }\end{array}$ & $\begin{array}{l}\text { basement membrane; } \\
\text { calcium ion binding; } \\
\text { collagen binding; } \\
\text { ossification }\end{array}$ \\
\hline N75422 & -1.0417 & $\frac{F L J 2266}{2}$ & 79887 & \multicolumn{2}{|c|}{ hypothetical protein FLJ22662 } \\
\hline $\mathrm{H} 06405$ & -1.0465 & PCTK1 & $\underline{5127}$ & $\begin{array}{l}\text { PCTAIRE protein } \\
\text { kinase } 1\end{array}$ & $\begin{array}{l}\text { protein amino acid } \\
\text { phosphorylation; } \\
\text { protein kinase activity; } \\
\text { protein } \\
\text { serine/threonine } \\
\text { kinase activity; } \\
\text { regulation of cell cycle }\end{array}$ \\
\hline R55277 & -1.0469 & & & & \\
\hline W40409 & -1.0485 & KARS & 3735 & lysyl-tRNA synthetase & \\
\hline T99304 & -1.0525 & ZNF297B & 23099 & $\begin{array}{l}\text { zinc finger protein } \\
297 B\end{array}$ & $\begin{array}{l}\text { DNA binding; nucleus; } \\
\text { protein binding; } \\
\text { regulation of } \\
\text { transcription, DNA- } \\
\text { dependent }\end{array}$ \\
\hline AA056130 & -1.0558 & PTK7 & $\underline{5754}$ & $\begin{array}{l}\text { PTK7 protein tyrosine } \\
\text { kinase } 7\end{array}$ & $\begin{array}{l}\text { plasma membrane; } \\
\text { proteoglycan integral } \\
\text { to plasma membrane; } \\
\text { receptor activity; } \\
\text { signal transduction; } \\
\text { transmembrane } \\
\text { receptor protein } \\
\text { tyrosine kinase activity }\end{array}$ \\
\hline R55995 & -1.0573 & & & & \\
\hline $\mathrm{H} 08370$ & -1.0653 & A2BP1 & $\underline{54715}$ & $\begin{array}{l}\text { ataxin 2-binding } \\
\text { protein } 1\end{array}$ & $\begin{array}{l}\text { Golgi apparatus; RNA } \\
\text { binding }\end{array}$ \\
\hline N39407 & -1.0671 & KIF21A & $\underline{55605}$ & kinesin family member 2 & $21 \mathrm{~A}$ \\
\hline AA101827 & -1.0723 & & & & \\
\hline W32710 & -1.0724 & & $\underline{348153}$ & $\begin{array}{l}\text { similar to nuclear pore } c \\
\text { protein }\end{array}$ & complex interacting \\
\hline W38749 & -1.0733 & MAL2 & 114569 & $\begin{array}{l}\text { mal, T-cell } \\
\text { differentiation protein } 2\end{array}$ & integral to membrane \\
\hline N73898 & -1.0798 & EIF2B1 & $\underline{1967}$ & $\begin{array}{l}\text { eukaryotic translation } \\
\text { initiation factor } 2 \mathrm{~B}, \\
\text { subunit } 1 \text { alpha, } 26 \mathrm{kDa}\end{array}$ & $\begin{array}{l}\text { GTP binding; } \\
\text { eukaryotic translation } \\
\text { initiation factor 2B } \\
\text { complex; guanyl- } \\
\text { nucleotide exchange } \\
\text { factor activity; } \\
\text { translation initiation } \\
\text { factor activity; } \\
\text { translational initiation }\end{array}$ \\
\hline AA031950 & -1.0821 & & & & \\
\hline
\end{tabular}




\begin{tabular}{|c|c|c|c|c|c|}
\hline R00507 & -1.0827 & $\frac{F L J 3308}{4}$ & 149483 & \multicolumn{2}{|c|}{\begin{tabular}{l|l}
3 & hypothetical protein FLJ33084
\end{tabular}} \\
\hline T93322 & -1.0831 & & & & \\
\hline N75518 & -1.0849 & TPR & $\underline{7175}$ & $\begin{array}{l}\text { translocated promoter } \\
\text { region (to activated } \\
\text { MET oncogene) }\end{array}$ & $\begin{array}{l}\text { cytoplasm; kinesin } \\
\text { complex; nuclear pore; } \\
\text { nucleus; protein- } \\
\text { nucleus import; } \\
\text { transport }\end{array}$ \\
\hline T69714 & -1.0878 & POLB & $\underline{5423}$ & $\begin{array}{l}\text { polymerase (DNA } \\
\text { directed), beta }\end{array}$ & $\begin{array}{l}\text { DNA binding; DNA } \\
\text { dependent DNA } \\
\text { replication; DNA } \\
\text { repair; alpha DNA } \\
\text { polymerase activity; } \\
\text { beta DNA polymerase } \\
\text { activity; delta DNA } \\
\text { polymerase activity; } \\
\text { deoxycytidyl } \\
\text { transferase activity, } \\
\text { template dependent; } \\
\text { epsilon DNA } \\
\text { polymerase activity; } \\
\text { eta DNA polymerase } \\
\text { activity; gamma DNA- } \\
\text { directed DNA } \\
\text { polymerase activity; } \\
\text { iota DNA polymerase } \\
\text { activity; kappa DNA } \\
\text { polymerase activity; } \\
\text { lambda DNA } \\
\text { polymerase activity; } \\
\text { mu DNA polymerase } \\
\text { activity; nu DNA } \\
\text { polymerase activity; } \\
\text { nucleus; sigma DNA } \\
\text { polymerase activity; } \\
\text { theta DNA polymerase } \\
\text { activity; transferase } \\
\text { activity; zeta DNA } \\
\text { polymerase activity } \\
\end{array}$ \\
\hline N66814 & -1.0891 & TRIM32 & $\underline{22954}$ & $\begin{array}{l}\text { tripartite motif- } \\
\text { containing } 32\end{array}$ & $\begin{array}{l}\text { nucleus; transcription } \\
\text { co-activator activity; } \\
\text { zinc ion binding }\end{array}$ \\
\hline AA151307 & -1.0911 & GNB2 & $\underline{2783}$ & $\begin{array}{l}\text { guanine nucleotide } \\
\text { binding protein (G } \\
\text { protein), beta } \\
\text { polypeptide } 2\end{array}$ & $\begin{array}{l}\text { G-protein coupled } \\
\text { receptor protein } \\
\text { signaling pathway; } \\
\text { heterotrimeric G- } \\
\text { protein GTPase } \\
\text { activity; heterotrimeric } \\
\text { G-protein complex; } \\
\text { signal transducer } \\
\text { activity; signal } \\
\text { transduction }\end{array}$ \\
\hline
\end{tabular}




\begin{tabular}{|c|c|c|c|c|c|}
\hline N54788 & -1.0942 & CYBRD1 & $\underline{79901}$ & $\begin{array}{l}\text { cytochrome b } \\
\text { reducatse } 1\end{array}$ & $\begin{array}{l}\text { electron transport; } \\
\text { integral to membrane }\end{array}$ \\
\hline R34626 & -1.1005 & & & & \\
\hline R75598 & -1.1008 & NBL1 & $\underline{4681}$ & $\begin{array}{l}\text { neuroblastoma, } \\
\text { suppression of } \\
\text { tumorigenicity } 1\end{array}$ & $\begin{array}{l}\text { negative regulation of } \\
\text { cell cycle }\end{array}$ \\
\hline $\mathrm{H} 00122$ & -1.103 & ADCY1 & 107 & $\begin{array}{l}\text { adenylate cyclase } 1 \\
\text { (brain) }\end{array}$ & \\
\hline H09869 & -1.1039 & GNAI2 & $\underline{2771}$ & $\begin{array}{l}\text { guanine nucleotide } \\
\text { binding protein (G } \\
\text { protein), alpha } \\
\text { inhibiting activity } \\
\text { polypeptide } 2\end{array}$ & $\begin{array}{l}\text { G-protein coupled } \\
\text { receptor protein } \\
\text { signaling pathway; } \\
\text { GTP binding; } \\
\text { heterotrimeric G- } \\
\text { protein GTPase } \\
\text { activity; negative } \\
\text { regulation of adenylate } \\
\text { cyclase activity; } \\
\text { response to nutrients; } \\
\text { signal transducer } \\
\text { activity; signal } \\
\text { transduction }\end{array}$ \\
\hline AA040090 & -1.1059 & \begin{tabular}{|l|l|} 
LOC9297 \\
$\underline{9}$
\end{tabular} & $\underline{92979}$ & hypothetical protein $\mathrm{BC}$ & 009489 \\
\hline R35596 & -1.1104 & SCHIP1 & $\underline{29970}$ & \begin{tabular}{|l} 
schwannomin \\
interacting protein 1
\end{tabular} & cytoplasm \\
\hline AA129598 & -1.112 & NCE2 & $\underline{140739}$ & $\begin{array}{l}\text { NEDD8-conjugating } \\
\text { enzyme }\end{array}$ & $\begin{array}{l}\text { ligase activity; protein } \\
\text { modification; ubiquitin } \\
\text { conjugating enzyme } \\
\text { activity; ubiquitin } \\
\text { cycle; ubiquitin-protein } \\
\text { ligase activity }\end{array}$ \\
\hline W37491 & -1.1126 & BLVRB & $\underline{645}$ & $\begin{array}{l}\text { biliverdin reductase B } \\
\text { (flavin reductase } \\
\text { (NADPH)) }\end{array}$ & $\begin{array}{l}\text { biliverdin reductase } \\
\text { activity; flavin } \\
\text { reductase activity; } \\
\text { oxidoreductase activity }\end{array}$ \\
\hline W05611 & -1.1185 & & & & \\
\hline \begin{tabular}{|l|} 
N69433 \\
\end{tabular} & -1.1188 & & & & \\
\hline H94329 & -1.1203 & DMD & $\underline{1756}$ & $\begin{array}{l}\text { dystrophin (muscular } \\
\text { dystrophy, Duchenne } \\
\text { and Becker types) }\end{array}$ & $\begin{array}{l}\text { cell shape and cell } \\
\text { size control; } \\
\text { cytoskeleton; muscle } \\
\text { contraction; muscle } \\
\text { development; } \\
\text { peripheral plasma } \\
\text { membrane protein; } \\
\text { structural constituent } \\
\text { of cytoskeleton } \\
\end{array}$ \\
\hline R85505 & -1.1218 & & & & \\
\hline R54798 & -1.1241 & $\frac{F L J 3181}{0}$ & 158038 & \multicolumn{2}{|c|}{ hypothetical protein FLJ31810 } \\
\hline
\end{tabular}




\begin{tabular}{|c|c|c|c|c|c|}
\hline AA121547 & -1.1282 & IMPDH2 & $\underline{3615}$ & \begin{tabular}{|l} 
IMP (inosine \\
monophosphate) \\
dehydrogenase 2
\end{tabular} & $\begin{array}{l}\text { GMP biosynthesis; } \\
\text { IMP dehydrogenase } \\
\text { activity; } \\
\text { oxidoreductase } \\
\text { activity; purine } \\
\text { nucleotide } \\
\text { biosynthesis } \\
\end{array}$ \\
\hline AA031958 & -1.1321 & & & & \\
\hline T98046 & -1.1324 & PFDN4 & $\underline{5203}$ & prefoldin 4 & $\begin{array}{l}\text { chaperonin-mediated } \\
\text { tubulin folding; co- } \\
\text { chaperone activity; } \\
\text { cytosol; protein } \\
\text { binding; protein } \\
\text { folding; tubulin-specific } \\
\text { chaperone activity }\end{array}$ \\
\hline $\mathrm{H} 09331$ & -1.1324 & $\frac{\text { DKFZp43 }}{4 \mathrm{D} 177}$ & 84224 & \multicolumn{2}{|c|}{ hypothetical protein DKFZp434D177 } \\
\hline N89807 & -1.1348 & PDGFC & 56034 & \multicolumn{2}{|c|}{4 platelet derived growth factor $\mathrm{C}$} \\
\hline AA001906 & -1.1363 & TRAP240 & $\underline{9969}$ & $\begin{array}{l}\text { thyroid hormone } \\
\text { receptor-associated } \\
\text { protein, } 240 \mathrm{kDa} \\
\text { subunit }\end{array}$ & $\begin{array}{l}\text { nucleus; receptor } \\
\text { activity; regulation of } \\
\text { transcription, DNA- } \\
\text { dependent }\end{array}$ \\
\hline W24393 & -1.1373 & STX10 & $\underline{8677}$ & syntaxin 10 & $\begin{array}{l}\text { Golgi membrane; } \\
\text { integral to membrane; } \\
\text { intracellular protein } \\
\text { transport; kinesin } \\
\text { complex; protein } \\
\text { transporter activity }\end{array}$ \\
\hline R28325 & -1.1527 & $\frac{\text { MGC105 }}{00}$ & 83719 & \multicolumn{2}{|c|}{ hypothetical protein MGC10500 } \\
\hline T94861 & -1.1564 & $\frac{\text { PPP1R1 }}{5 \mathrm{~B}}$ & $\underline{84919}$ & \multicolumn{2}{|c|}{$\begin{array}{l}\text { protein phosphatase 1, regulatory (inhibitor) } \\
\text { subunit } 15 \mathrm{~B}\end{array}$} \\
\hline R40485 & -1.159 & MMP16 & $\underline{4325}$ & $\begin{array}{l}\text { matrix } \\
\text { metalloproteinase } 16 \\
\text { (membrane-inserted) }\end{array}$ & $\begin{array}{l}\text { collagen catabolism; } \\
\text { enzyme activator } \\
\text { activity; extracellular } \\
\text { matrix; hydrolase } \\
\text { activity; integral to } \\
\text { plasma membrane; } \\
\text { metalloendopeptidase } \\
\text { activity; zinc ion } \\
\text { binding } \\
\end{array}$ \\
\hline T97765 & -1.1651 & & & & \\
\hline
\end{tabular}




\begin{tabular}{|c|c|c|c|c|c|}
\hline N91912 & -1.1656 & PLA2G12 & $\underline{81579}$ & $\begin{array}{l}\text { phospholipase A2, } \\
\text { group XII }\end{array}$ & $\begin{array}{l}\text { biological_process } \\
\text { unknown; calcium ion } \\
\text { binding; calcium- } \\
\text { dependent cytosolic } \\
\text { phospholipase A2 } \\
\text { activity; calcium- } \\
\text { dependent secreted } \\
\text { phospholipase A2 } \\
\text { activity; calcium- } \\
\text { independent cytosolic } \\
\text { phospholipase A2 } \\
\text { activity; } \\
\text { cellular_component } \\
\text { unknown; hydrolase } \\
\text { activity; lipid } \\
\text { catabolism }\end{array}$ \\
\hline H93058 & -1.1701 & & $\underline{285707}$ & hypothetical gene supp & orted by BC035411 \\
\hline R43193 & -1.1734 & \begin{tabular}{|l} 
FLJ1174 \\
$\underline{9}$
\end{tabular} & $\underline{79643}$ & $\begin{array}{l}\text { hypothetical protein } \\
\text { FLJ11749 }\end{array}$ & $\begin{array}{l}\text { molecular_function } \\
\text { unknown }\end{array}$ \\
\hline R41846 & -1.1771 & & & & \\
\hline $\mathrm{H} 28801$ & -1.1838 & AD158 & $\underline{84230}$ & $\begin{array}{l}\text { hypothetical protein } \\
\text { AD158 }\end{array}$ & \\
\hline R56495 & -1.1919 & IKBKG & $\underline{8517}$ & $\begin{array}{l}\text { inhibitor of kappa light } \\
\text { polypeptide gene } \\
\text { enhancer in B-cells, } \\
\text { kinase gamma }\end{array}$ & $\begin{array}{l}\text { NIK-I-kappaB/NF- } \\
\text { kappaB cascade; } \\
\text { immune response; } \\
\text { induction of apoptosis; } \\
\text { kinesin complex; } \\
\text { nucleus; regulation of } \\
\text { transcription, DNA- } \\
\text { dependent; signal } \\
\text { transducer activity }\end{array}$ \\
\hline W79682 & -1.1937 & $\frac{\mathrm{LOC5} 5103}{5}$ & $\underline{51035}$ & ORF & \\
\hline W19514 & -1.1961 & $\frac{\mathrm{LOC} 1584}{27}$ & 158427 & PP4189 & \\
\hline R80802 & -1.1987 & KDELR1 & $\underline{10945}$ & $\begin{array}{l}\text { KDEL (Lys-Asp-Glu- } \\
\text { Leu) endoplasmic } \\
\text { reticulum protein } \\
\text { retention receptor } 1\end{array}$ & $\begin{array}{l}\text { KDEL sequence } \\
\text { binding; endoplasmic } \\
\text { reticulum; integral to } \\
\text { membrane; } \\
\text { intracellular protein } \\
\text { transport; membrane } \\
\text { fraction; protein } \\
\text { transporter activity; } \\
\text { receptor activity }\end{array}$ \\
\hline \begin{tabular}{|l|} 
N71104 \\
\end{tabular} & -1.1998 & & 283520 & \multicolumn{2}{|c|}{ hypothetical gene supported by AK095358 } \\
\hline
\end{tabular}




\begin{tabular}{|c|c|c|c|c|c|}
\hline W52798 & -1.2049 & EMD & $\underline{2010}$ & $\begin{array}{l}\text { emerin (Emery- } \\
\text { Dreifuss muscular } \\
\text { dystrophy) }\end{array}$ & $\begin{array}{l}\text { integral to membrane; } \\
\text { muscle contraction; } \\
\text { muscle development; } \\
\text { nonselective vesicle } \\
\text { transport; nuclear } \\
\text { membrane }\end{array}$ \\
\hline T98139 & -1.2051 & $\underline{H L A-B}$ & $\underline{3106}$ & $\begin{array}{l}\text { major } \\
\text { histocompatibility } \\
\text { complex, class I, B }\end{array}$ & $\begin{array}{l}\text { MHC class I receptor } \\
\text { activity; antigen } \\
\text { presentation, } \\
\text { endogenous antigen; } \\
\text { antigen processing, } \\
\text { endogenous antigen } \\
\text { via MHC class I; } \\
\text { immune response; } \\
\text { integral to plasma } \\
\text { membrane }\end{array}$ \\
\hline T85060 & -1.2115 & & & & \\
\hline R40307 & -1.2133 & & & & \\
\hline AA149232 & -1.2165 & SREBF2 & $\underline{6721}$ & $\begin{array}{l}\text { sterol regulatory } \\
\text { element binding } \\
\text { transcription factor } 2\end{array}$ & $\begin{array}{l}\text { DNA binding; Golgi } \\
\text { apparatus; RNA } \\
\text { polymerase II } \\
\text { transcription factor } \\
\text { activity; cholesterol } \\
\text { metabolism; } \\
\text { endoplasmic } \\
\text { reticulum; integral to } \\
\text { membrane; lipid } \\
\text { metabolism; nucleus; } \\
\text { regulation of } \\
\text { transcription from Pol } \\
\text { II promoter }\end{array}$ \\
\hline H99444 & -1.2218 & $\begin{array}{l}\text { DKFZP5 } \\
64 \mathrm{D} 172 \\
\end{array}$ & 83989 & \multicolumn{2}{|c|}{ hypothetical protein DKFZp564D172 } \\
\hline N74415 & -1.2274 & & & & \\
\hline R75796 & -1.232 & PABPN1 & $\underline{8106}$ & \multicolumn{2}{|c|}{ poly(A) binding protein, nuclear 1} \\
\hline N71043 & -1.2357 & SRPR & $\underline{6734}$ & $\begin{array}{l}\text { signal recognition } \\
\text { particle receptor } \\
\text { ('docking protein') }\end{array}$ & $\begin{array}{l}\text { GTP binding; } \\
\text { cotranslational } \\
\text { membrane targeting; } \\
\text { integral to membrane; } \\
\text { nucleotide binding; } \\
\text { protein targeting; } \\
\text { receptor activity; } \\
\text { signal recognition } \\
\text { particle; signal } \\
\text { recognition particle } \\
\text { binding; signal } \\
\text { recognition particle } \\
\text { receptor complex }\end{array}$ \\
\hline N/A2 & -1.239 & & & & \\
\hline
\end{tabular}




\begin{tabular}{|c|c|c|c|c|c|}
\hline R34387 & -1.2463 & FAAH & $\underline{2166}$ & $\begin{array}{l}\text { fatty acid amide } \\
\text { hydrolase }\end{array}$ & $\begin{array}{l}\text { amidase activity; fatty } \\
\text { acid metabolism; } \\
\text { insoluble fraction; } \\
\text { membrane fraction; } \\
\text { receptor binding }\end{array}$ \\
\hline \begin{tabular}{|l|} 
AA195088 \\
\end{tabular} & -1.2496 & & & & \\
\hline AA010141 & -1.2497 & $\begin{array}{l}\text { SERPIN } \\
\text { H1 }\end{array}$ & $\underline{871}$ & $\begin{array}{l}\text { serine (or cysteine) } \\
\text { proteinase inhibitor, } \\
\text { clade H (heat shock } \\
\text { protein 47), member 1, } \\
\text { (collagen binding } \\
\text { protein 1) }\end{array}$ & heat shock response \\
\hline N69956 & -1.2507 & \begin{tabular}{|l|} 
LOC2839 \\
04
\end{tabular} & $\underline{283904}$ & \multicolumn{2}{|c|}{ hypothetical protein LOC283904 } \\
\hline N77925 & -1.2561 & NDUFA1 & $\underline{4694}$ & $\begin{array}{l}\text { NADH dehydrogenase } \\
\text { (ubiquinone) } 1 \text { alpha } \\
\text { subcomplex, } 1,7.5 \mathrm{kDa}\end{array}$ & $\begin{array}{l}\text { NADH dehydrogenase } \\
\text { (ubiquinone) activity; } \\
\text { NADH dehydrogenase } \\
\text { activity; energy } \\
\text { pathways; membrane } \\
\text { fraction; } \\
\text { mitochondrion; } \\
\text { oxidoreductase activity }\end{array}$ \\
\hline T94447 & -1.2644 & CTXL & $\underline{23584}$ & $\begin{array}{l}\text { cortical thymocyte } \\
\text { receptor (X. laevis } \\
\text { CTX) like }\end{array}$ & $\begin{array}{l}\text { antigen binding; } \\
\text { integral to plasma } \\
\text { membrane; membrane } \\
\text { fraction }\end{array}$ \\
\hline AA046449 & -1.2701 & ARF1 & $\underline{375}$ & $\begin{array}{l}\text { ADP-ribosylation factor } \\
1\end{array}$ & $\begin{array}{l}\text { Golgi apparatus; } \\
\text { intracellular protein } \\
\text { transport; plasma } \\
\text { membrane; protein } \\
\text { transporter activity; } \\
\text { receptor signaling } \\
\text { protein activity; small } \\
\text { GTPase mediated } \\
\text { signal transduction; } \\
\text { small monomeric } \\
\text { GTPase activity }\end{array}$ \\
\hline AA057729 & -1.2746 & $\begin{array}{l}\text { FLJ1323 } \\
\underline{6}\end{array}$ & $\underline{79962}$ & \multicolumn{2}{|c|}{ hypothetical protein FLJ13236 } \\
\hline H63653 & -1.2777 & HLA-B & $\underline{3106}$ & $\begin{array}{l}\text { major } \\
\text { histocompatibility } \\
\text { complex, class I, B }\end{array}$ & $\begin{array}{l}\text { MHC class I receptor } \\
\text { activity; antigen } \\
\text { presentation, } \\
\text { endogenous antigen; } \\
\text { antigen processing, } \\
\text { endogenous antigen } \\
\text { via MHC class I; } \\
\text { immune response; } \\
\text { integral to plasma } \\
\text { membrane }\end{array}$ \\
\hline
\end{tabular}




\begin{tabular}{|c|c|c|c|c|c|}
\hline R07336 & -1.279 & $\mathrm{KCNH} 2$ & $\underline{3757}$ & $\begin{array}{l}\text { potassium voltage- } \\
\text { gated channel, } \\
\text { subfamily H (eag- } \\
\text { related), member } 2\end{array}$ & $\begin{array}{l}\text { cation transport; } \\
\text { delayed rectifier } \\
\text { potassium channel } \\
\text { activity; hearing; } \\
\text { integral to membrane; } \\
\text { membrane fraction; } \\
\text { muscle contraction; } \\
\text { potassium ion } \\
\text { transport; regulation of } \\
\text { heart rate; two- } \\
\text { component sensor } \\
\text { molecule activity; two- } \\
\text { component signal } \\
\text { transduction system } \\
\text { (phosphorelay); } \\
\text { voltage-gated } \\
\text { potassium channel } \\
\text { complex }\end{array}$ \\
\hline N53657 & -1.3195 & & & & \\
\hline R13346 & -1.3199 & ENT4 & $\underline{222962}$ & \multicolumn{2}{|c|}{ equilibrative nucleoside transporter 4} \\
\hline AA055938 & -1.3223 & LAMA4 & $\underline{3910}$ & laminin, alpha 4 & $\begin{array}{l}\text { basal lamina; } \\
\text { extracellular matrix } \\
\text { glycoprotein }\end{array}$ \\
\hline R38827 & -1.3263 & $\begin{array}{l}\text { CACNA2 } \\
\text { D2 }\end{array}$ & $\underline{9254}$ & $\begin{array}{l}\text { calcium channel, } \\
\text { voltage-dependent, } \\
\text { alpha } 2 / \text { delta subunit } 2\end{array}$ & membrane \\
\hline AA045613 & -1.3668 & $\frac{\mathrm{HSD11B}}{1}$ & $\underline{3290}$ & $\begin{array}{l}\text { hydroxysteroid (11- } \\
\text { beta) dehydrogenase } 1\end{array}$ & $\begin{array}{l}11 \text {-beta- } \\
\text { hydroxysteroid } \\
\text { dehydrogenase } \\
\text { activity; } \\
\text { biological_process } \\
\text { unknown; metabolism; } \\
\text { microsome; } \\
\text { oxidoreductase } \\
\text { activity; steroid } \\
\text { metabolism }\end{array}$ \\
\hline AA193553 & -1.4141 & & & & \\
\hline AA023029 & -1.4229 & PPP5C & $\underline{5536}$ & \multicolumn{2}{|c|}{ protein phosphatase 5 , catalytic subunit } \\
\hline H62618 & \begin{tabular}{|c|}
-1.424 \\
\end{tabular} & & & & \\
\hline R76163 & -1.4362 & ZYX & $\underline{7791}$ & zyxin & $\begin{array}{l}\text { cell adhesion; cell } \\
\text { adhesion molecule } \\
\text { activity; cell-cell } \\
\text { signaling; focal } \\
\text { adhesion; integral to } \\
\text { plasma membrane; } \\
\text { plasma membrane; } \\
\text { signal transduction }\end{array}$ \\
\hline H01987 & -1.4431 & MLL3 & $\underline{58508}$ & \multicolumn{2}{|c|}{ myeloid/lymphoid or mixed-lineage leukemia3 } \\
\hline
\end{tabular}




\begin{tabular}{|c|c|c|c|c|c|}
\hline N29429 & -1.5061 & CGI-57 & $\underline{27013}$ & hypothetical protein CG & $1-57$ \\
\hline AA032288 & -1.6506 & & & & \\
\hline R72685 & -1.6521 & PLD3 & $\underline{23646}$ & phospholipase D3 & $\begin{array}{l}\text { catalytic activity; } \\
\text { metabolism; } \\
\text { phospholipase D } \\
\text { activity }\end{array}$ \\
\hline N65982 & -1.6535 & & & & \\
\hline W68050 & -1.6844 & LGALS1 & $\underline{3956}$ & $\begin{array}{l}\text { lectin, galactoside- } \\
\text { binding, soluble, } 1 \\
\text { (galectin 1) }\end{array}$ & $\begin{array}{l}\text { apoptosis; heterophilic } \\
\text { cell adhesion; sugar } \\
\text { binding }\end{array}$ \\
\hline H49989 & -1.7217 & BOCT & $\underline{51310}$ & $\begin{array}{l}\text { potent brain type } \\
\text { organic ion transporter }\end{array}$ & $\begin{array}{l}\text { integral to membrane; } \\
\text { transport; transporter } \\
\text { activity }\end{array}$ \\
\hline H26465 & -1.7737 & GSN & $\underline{2934}$ & $\begin{array}{l}\text { gelsolin (amyloidosis, } \\
\text { Finnish type) }\end{array}$ & $\begin{array}{l}\text { actin cytoskeleton; } \\
\text { actin filament } \\
\text { polymerization; actin } \\
\text { filament severing } \\
\text { activity; barbed-end } \\
\text { actin capping/severing } \\
\text { activity; calcium ion } \\
\text { binding; cytosol; } \\
\text { extracellular; structural } \\
\text { constituent of } \\
\text { cytoskeleton }\end{array}$ \\
\hline R13936 & -1.7787 & DHPS & $\underline{1725}$ & $\begin{array}{l}\text { deoxyhypusine } \\
\text { synthase }\end{array}$ & \begin{tabular}{|l} 
deoxyhypusine \\
synthase activity; \\
hypusine biosynthesis \\
from peptidyl-lysine; \\
positive regulation of \\
cell proliferation; \\
protein biosynthesis; \\
transferase activity
\end{tabular} \\
\hline $\mathrm{H} 24956$ & -1.844 & RET & $\underline{5979}$ & \begin{tabular}{|l|} 
ret proto-oncogene \\
(multiple endocrine \\
neoplasia and \\
medullary thyroid \\
carcinoma 1, \\
Hirschsprung disease)
\end{tabular} & $\begin{array}{l}\text { oncogenesis; posterior } \\
\text { midgut development; } \\
\text { protein amino acid } \\
\text { phosphorylation; } \\
\text { protein tyrosine kinase } \\
\text { activity; receptor } \\
\text { activity; signal } \\
\text { transduction }\end{array}$ \\
\hline W69432 & -1.8457 & DDIT3 & $\underline{1649}$ & $\begin{array}{l}\text { DNA-damage inducible } \\
\text { transcript } 3\end{array}$ & $\begin{array}{l}\text { Transcription co- } \\
\text { repressor activity; } \\
\text { transcription factor } \\
\text { activity; cell cyle } \\
\text { arrest; response to } \\
\text { DNA damage } \\
\text { stimulus; cell growth } \\
\text { and/or maintenance; } \\
\text { regulation of } \\
\text { transcription, DNA- } \\
\text { dependent; nucleus }\end{array}$ \\
\hline
\end{tabular}




\begin{tabular}{|l|l|l|l|l|l|}
\hline R00207 & -2.214 & $\underline{\text { SLC22A3 }}$ & $\underline{6581}$ & $\begin{array}{l}\text { solute carrier family 22 } \\
\text { (extraneuronal } \\
\text { monoamine } \\
\text { transporter), member 3 }\end{array}$ & $\begin{array}{l}\text { integral to plasma } \\
\text { membrane; ion } \\
\text { transport; ion } \\
\text { transporter activity; } \\
\text { membrane fraction; } \\
\text { organic cation } \\
\text { transport; organic } \\
\text { cation transporter } \\
\text { activity }\end{array}$ \\
\hline H86199 & -2.2282 & & & & \\
\hline H19719 & -2.4026 & GEMIN5 & 25929 & gem (nuclear organelle) associated protein 5 \\
\hline N52375 & -4.3698 & & & & \\
\hline
\end{tabular}


Appendix B. Genes Differentially Regulated by $15 \mathrm{~m} \mathrm{MPP+}$ and $15 \mathrm{~m}$ MPP+/PTIO in Combination.

\begin{tabular}{|c|c|c|c|c|c|}
\hline GENBANK & $P$ VALUE & SYMBOL & $\begin{array}{c}\text { LOCUS } \\
\text { LINK }\end{array}$ & GENE NAME & GENE ONTOLOGY \\
\hline H01987 & $8.26 \mathrm{E}-10$ & MLL3 & 58508 & \multicolumn{2}{|c|}{ myeloid/lymphoid or mixed-lineage leukemia3 } \\
\hline H09869 & $3.24 \mathrm{E}-07$ & GNAI2 & $\underline{2771}$ & $\begin{array}{l}\text { guanine nucleotide binding } \\
\text { protein (G protein), alpha } \\
\text { inhibiting activity polypeptide } \\
2\end{array}$ & $\begin{array}{l}\text { G-protein coupled } \\
\text { receptor protein } \\
\text { signaling pathway; } \\
\text { GTP binding; } \\
\text { heterotrimeric G- } \\
\text { protein GTPase } \\
\text { activity; negative } \\
\text { regulation of } \\
\text { adenylate cyclase } \\
\text { activity; response to } \\
\text { nutrients; signal } \\
\text { transducer activity; } \\
\text { signal transduction }\end{array}$ \\
\hline W19744 & $8.06 \mathrm{E}-07$ & & & & \\
\hline R07186 & $2.21 \mathrm{E}-06$ & & & & \\
\hline AA037284 & $2.26 \mathrm{E}-06$ & APRT & $\underline{353}$ & $\begin{array}{l}\text { adenine } \\
\text { phosphoribosyltransferase }\end{array}$ & $\begin{array}{l}\text { adenine } \\
\text { phosphoribosyltransfe } \\
\text { rase activity; adenine } \\
\text { salvage pathway; } \\
\text { nucleoside } \\
\text { metabolism; } \\
\text { transferase activity, } \\
\text { transferring glycosyl } \\
\text { groups }\end{array}$ \\
\hline H59405 & $3.27 \mathrm{E}-06$ & FLJ10298 & 54682 & $\begin{array}{l}\text { hypothetical protein } \\
\text { FLJ10298 }\end{array}$ & \\
\hline H51834 & 3.35E-06 & TTC1 & $\underline{7265}$ & $\begin{array}{l}\text { tetratricopeptide repeat } \\
\text { domain } 1\end{array}$ & $\begin{array}{l}\text { chaperone activity; } \\
\text { protein binding; } \\
\text { protein folding }\end{array}$ \\
\hline T86338 & 5.85E-06 & & & & \\
\hline R76163 & 1.15E-05 & $\underline{Z Y X}$ & 7791 & zyxin & $\begin{array}{l}\text { cell adhesion; cell } \\
\text { adhesion molecule } \\
\text { activity; cell-cell } \\
\text { signaling; focal } \\
\text { adhesion; integral to } \\
\text { plasma membrane; } \\
\text { plasma membrane; } \\
\text { signal transduction }\end{array}$ \\
\hline $\mathrm{H} 68587$ & $4.89 \mathrm{E}-05$ & & 340833 & LOC340833 & \\
\hline
\end{tabular}




\begin{tabular}{|c|c|c|c|c|c|}
\hline W52537 & $6.17 \mathrm{E}-05$ & PSMA2 & $\underline{5683}$ & $\begin{array}{l}\text { proteasome (prosome, } \\
\text { macropain) subunit, alpha } \\
\text { type, } 2\end{array}$ & $\begin{array}{l}\text { 26S proteasome; } \\
\text { cytosol; } \\
\text { endopeptidase } \\
\text { activity; proteasome } \\
\text { core complex (sensu } \\
\text { Eukarya); proteasome } \\
\text { endopeptidase } \\
\text { activity; ubiquitin- } \\
\text { dependent protein } \\
\text { catabolism }\end{array}$ \\
\hline $\mathrm{H} 26552$ & $8.54 \mathrm{E}-05$ & MGC5395 & $\underline{79026}$ & $\begin{array}{l}\text { hypothetical protein } \\
\text { MGC5395 }\end{array}$ & $\begin{array}{l}\text { intracellular signaling } \\
\text { cascade }\end{array}$ \\
\hline R20373 & $9.30 \mathrm{E}-05$ & TMP21 & $\underline{10972}$ & $\begin{array}{l}\text { transmembrane trafficking } \\
\text { protein }\end{array}$ & $\begin{array}{l}\text { ER to Golgi transport; } \\
\text { Golgi apparatus; } \\
\text { integral to plasma } \\
\text { membrane; } \\
\text { intracellular protein } \\
\text { transport; membrane } \\
\text { fraction; microsome; } \\
\text { protein carrier activity; } \\
\text { protein transporter } \\
\text { activity }\end{array}$ \\
\hline W68050 & 9.91E-05 & LGALS1 & $\underline{3956}$ & $\begin{array}{l}\text { lectin, galactoside-binding, } \\
\text { soluble, } 1 \text { (galectin 1) }\end{array}$ & $\begin{array}{l}\text { apoptosis; heterophilic } \\
\text { cell adhesion; sugar } \\
\text { binding }\end{array}$ \\
\hline H67193 & 0.000143814 & EIF2S3 & 1968 & $\begin{array}{l}\text { eukaryotic translation } \\
\text { initiation factor } 2 \text {, subunit } 3 \\
\text { gamma, } 52 \mathrm{kDa}\end{array}$ & $\begin{array}{l}\text { GTPase activity; } \\
\text { cytosolic small } \\
\text { ribosomal subunit } \\
\text { (sensu Eukarya); } \\
\text { eukaryotic translation } \\
\text { initiation factor } 2 \\
\text { complex; translation } \\
\text { elongation factor } \\
\text { activity; translation } \\
\text { initiation factor } \\
\text { activity; translational } \\
\text { elongation }\end{array}$ \\
\hline R22402 & 0.000145207 & & & & \\
\hline AA040852 & 0.000146241 & KIAA1321 & $\underline{57532}$ & KIAA1321 protein & \\
\hline R51354 & 0.000171049 & $\begin{array}{l}\text { CNTNAP } \\
\underline{2} \\
\end{array}$ & $\underline{26047}$ & $\begin{array}{l}\text { contactin associated protein- } \\
\text { like } 2\end{array}$ & \\
\hline AA069448 & 0.000177002 & & & & \\
\hline N95545 & 0.000178172 & IL11 & $\underline{3589}$ & interleukin 11 & $\begin{array}{l}\text { B-cell differentiation; } \\
\text { adipocyte } \\
\text { differentiation; cell } \\
\text { proliferation; cell-cell } \\
\text { signaling; cytokine } \\
\text { activity; extracellular; } \\
\text { interleukin-11 receptor } \\
\text { binding; } \\
\text { megakaryocyte } \\
\text { differentiation; platelet } \\
\text { activation; positive }\end{array}$ \\
\hline
\end{tabular}




\begin{tabular}{|c|c|c|c|c|c|}
\hline & & & & & $\begin{array}{l}\text { regulation of cell } \\
\text { proliferation }\end{array}$ \\
\hline R94499 & 0.000233405 & GNB5 & $\underline{10681}$ & ${ }_{5}^{\text {guanine nucleotide binding pro }}$ & otein (G protein), beta \\
\hline W01319 & 0.000249061 & $\mathrm{BHC} 80$ & $\underline{51317}$ & $\begin{array}{l}\text { BRAF35/HDAC2 complex (80 } \\
\mathrm{kDa})\end{array}$ & \\
\hline R13346 & 0.0003047 & ENT4 & 222962 & $\begin{array}{l}\text { equilibrative nucleoside } \\
\text { transporter } 4\end{array}$ & \\
\hline W47153 & 0.000339402 & PTRF & $\underline{284119}$ & $\begin{array}{l}\text { polymerase I and transcript } \\
\text { release factor }\end{array}$ & \\
\hline AA131933 & 0.000353436 & $\mathrm{ABP1}$ & $\underline{26}$ & $\begin{array}{l}\text { amiloride binding protein } 1 \\
\text { (amine oxidase (copper- } \\
\text { containing)) }\end{array}$ & $\begin{array}{l}\text { amine oxidase } \\
\text { (copper-containing) } \\
\text { activity; copper ion } \\
\text { binding; drug binding; } \\
\text { heparin binding; } \\
\text { metabolism; } \\
\text { oxidoreductase } \\
\text { activity; peroxisome }\end{array}$ \\
\hline R89790 & 0.000381932 & & & & \\
\hline W85877 & 0.000608409 & & & & \\
\hline AA101859 & 0.000631871 & ENSA & 2029 & endosulfine alpha & $\begin{array}{l}\text { ion channel inhibitor } \\
\text { activity; receptor } \\
\text { binding; response to } \\
\text { nutrients; transport } \\
\end{array}$ \\
\hline N71628 & 0.000686384 & SPIB & $\underline{6689}$ & $\begin{array}{l}\text { Spi-B transcription factor } \\
\text { (Spi-1/PU.1 related) }\end{array}$ & $\begin{array}{l}\text { RNA polymerase II } \\
\text { transcription factor } \\
\text { activity; } \\
\text { biological_process } \\
\text { unknown; cytoplasm; } \\
\text { molecular_function } \\
\text { unknown; nucleus; } \\
\text { regulation of } \\
\text { transcription from Pol } \\
\text { II promoter; } \\
\text { transcription factor } \\
\text { activity }\end{array}$ \\
\hline R98517 & 0.000726742 & $\frac{H I S T 1 H 1}{\underline{C}}$ & $\underline{3006}$ & histone 1, H1c & $\begin{array}{l}\text { DNA binding; } \\
\text { chromosome; } \\
\text { chromosome } \\
\text { organization and } \\
\text { biogenesis (sensu } \\
\text { Eukarya); } \\
\text { nucleosome; } \\
\text { nucleosome } \\
\text { assembly; nucleus }\end{array}$ \\
\hline H63763 & 0.000872181 & & & & \\
\hline H65775 & 0.000893253 & & & & \\
\hline
\end{tabular}




\begin{tabular}{|c|c|c|c|c|c|}
\hline AA213450 & 0.000934614 & & & & \\
\hline AA035066 & 0.001077269 & MGC4268 & $\underline{83607}$ & $\begin{array}{l}\text { hypothetical protein } \\
\text { MGC4268 }\end{array}$ & \\
\hline R47859 & 0.001191811 & NPR1 & 4881 & $\begin{array}{l}\text { natriuretic peptide receptor } \\
\text { A/guanylate cyclase } A \\
\text { (atrionatriuretic peptide } \\
\text { receptor } A \text { ) }\end{array}$ & $\begin{array}{l}\text { ATP binding; cGMP } \\
\text { biosynthesis; } \\
\text { guanylate cyclase } \\
\text { activity; integral to } \\
\text { membrane; } \\
\text { intracellular signaling } \\
\text { cascade; lyase } \\
\text { activity; peptide } \\
\text { receptor activity, G- } \\
\text { protein coupled; } \\
\text { protein amino acid } \\
\text { phosphorylation; } \\
\text { protein kinase activity; } \\
\text { receptor activity; } \\
\text { receptor guanylate } \\
\text { cyclase activity; } \\
\text { regulation of blood } \\
\text { pressure }\end{array}$ \\
\hline AA004845 & 0.001331715 & KIAA1529 & 57653 & KIAA1529 protein & \\
\hline AA002135 & 0.001365198 & $\underline{\mathrm{C} 2}$ & 717 & complement component 2 & $\begin{array}{l}\text { chymotrypsin activity; } \\
\text { classical-complement } \\
\text { pathway C3/C5 } \\
\text { convertase activity; } \\
\text { complement } \\
\text { activation, classical } \\
\text { pathway; complement } \\
\text { component C2 } \\
\text { complex; hydrolase } \\
\text { activity; proteolysis } \\
\text { and peptidolysis; } \\
\text { trypsin activity }\end{array}$ \\
\hline H64569 & 0.001442673 & & & & \\
\hline AA031859 & 0.001463558 & TIMM13 & 26517 & $\begin{array}{l}\text { translocase of inner } \\
\text { mitochondrial membrane } 13 \\
\text { homolog (yeast) }\end{array}$ & $\begin{array}{l}\text { hearing; mitochondrial } \\
\text { inner membrane pre- } \\
\text { sequence translocase } \\
\text { complex; } \\
\text { mitochondrial } \\
\text { translocation; } \\
\text { mitochondrion; protein } \\
\text { targeting; protein } \\
\text { translocase activity; } \\
\text { zinc ion binding }\end{array}$ \\
\hline W69432 & 0.001627691 & $\frac{\mathrm{MAPKAP}}{\mathrm{K} 2}$ & 9261 & $\begin{array}{l}\text { mitogen-activated protein } \\
\text { kinase-activated protein } \\
\text { kinase } 2\end{array}$ & $\begin{array}{l}\text { ATP binding; } \\
\text { MAPKKK cascade; } \\
\text { nucleus; protein } \\
\text { amino acid } \\
\text { phosphorylation; } \\
\text { protein } \\
\text { serine/threonine } \\
\text { kinase activity; signal }\end{array}$ \\
\hline
\end{tabular}




\begin{tabular}{|c|c|c|c|c|c|}
\hline & & & & & $\begin{array}{l}\text { transducer activity; } \\
\text { transferase activity }\end{array}$ \\
\hline AA135646 & 0.001668986 & hIAN6 & 155038 & $\begin{array}{l}\text { human immune associated } \\
\text { nucleotide } 6\end{array}$ & \\
\hline AA057286 & 0.002040228 & $\frac{\text { TA- }}{\text { WDRP }}$ & 134430 & $\begin{array}{l}\text { T-cell activation WD repeat } \\
\text { protein }\end{array}$ & $\begin{array}{l}\text { catalytic activity; } \\
\text { metabolism }\end{array}$ \\
\hline AA046245 & 0.00228188 & $\overline{\mathrm{OSF}-2}$ & $\underline{10631}$ & $\begin{array}{l}\text { osteoblast specific factor } 2 \\
\text { (fasciclin I-like) }\end{array}$ & $\begin{array}{l}\text { cell adhesion; cell } \\
\text { adhesion molecule } \\
\text { activity; extracellular } \\
\text { matrix; skeletal } \\
\text { development } \\
\end{array}$ \\
\hline AA010141 & 0.002547572 & $\frac{\text { SERPINH }}{1}$ & $\underline{871}$ & $\begin{array}{l}\text { serine (or cysteine) } \\
\text { proteinase inhibitor, clade } \mathrm{H} \\
\text { (heat shock protein 47), } \\
\text { member 1, (collagen binding } \\
\text { protein 1) }\end{array}$ & heat shock response \\
\hline AA031564 & 0.002660207 & $\begin{array}{l}\mathrm{LOC} 1134 \\
44\end{array}$ & 113444 & \begin{tabular}{|l} 
hypothetical protein \\
BC011880
\end{tabular} & \\
\hline N94432 & 0.002746068 & & & & \\
\hline N90527 & 0.00279075 & PIM1 & $\underline{5292}$ & pim-1 oncogene & $\begin{array}{l}\text { ATP binding; cAMP- } \\
\text { dependent protein } \\
\text { kinase activity; cell } \\
\text { growth and/or } \\
\text { maintenance; } \\
\text { cytoplasm; } \\
\text { development; protein } \\
\text { amino acid } \\
\text { phosphorylation; } \\
\text { protein kinase CK2 } \\
\text { activity; protein } \\
\text { serine/threonine } \\
\text { kinase activity; } \\
\text { transferase activity } \\
\end{array}$ \\
\hline AA046610 & 0.002804358 & & & & \\
\hline N24815 & 0.002848453 & UBA52 & $\underline{7311}$ & $\begin{array}{l}\text { ubiquitin A-52 residue } \\
\text { ribosomal protein fusion } \\
\text { product } 1\end{array}$ & $\begin{array}{l}\text { nucleus; protein } \\
\text { biosynthesis; protein } \\
\text { modification; } \\
\text { ribosome; structural } \\
\text { constituent of } \\
\text { ribosome }\end{array}$ \\
\hline N22392 & 0.002974381 & CLDN11 & $\underline{5010}$ & $\begin{array}{l}\text { claudin } 11 \text { (oligodendrocyte } \\
\text { transmembrane protein) }\end{array}$ & $\begin{array}{l}\text { integral to membrane; } \\
\text { structural molecule } \\
\text { activity; tight junction }\end{array}$ \\
\hline AA054115 & 0.002987791 & & & & \\
\hline R50905 & 0.003042081 & TUBB & $\underline{7280}$ & tubulin, beta polypeptide & $\begin{array}{l}\text { cytoskeleton; } \\
\text { structural constituent } \\
\text { of cytoskeleton }\end{array}$ \\
\hline N39407 & 0.003085277 & KIF21A & $\underline{55605}$ & kinesin family member $21 \mathrm{~A}$ & \\
\hline W60305 & 0.003247636 & & & & \\
\hline
\end{tabular}




\begin{tabular}{|c|c|c|c|c|c|}
\hline R75598 & 0.003519312 & NBL1 & $\underline{4681}$ & $\begin{array}{l}\text { neuroblastoma, suppression } \\
\text { of tumorigenicity } 1\end{array}$ & $\begin{array}{l}\text { negative regulation of } \\
\text { cell cycle }\end{array}$ \\
\hline H68885 & 0.003639707 & TSSC3 & $\underline{7262}$ & $\begin{array}{l}\text { tumor suppressing } \\
\text { subtransferable candidate } 3\end{array}$ & apoptosis; imprinting \\
\hline AA129727 & 0.00378033 & RAB5C & $\underline{5878}$ & $\begin{array}{l}\text { RAB5C, member RAS } \\
\text { oncogene family }\end{array}$ & $\begin{array}{l}\text { GTP binding; RAB } \\
\text { small monomeric } \\
\text { GTPase activity; } \\
\text { intracellular protein } \\
\text { transport; protein } \\
\text { transporter activity; } \\
\text { small GTPase } \\
\text { mediated signal } \\
\text { transduction }\end{array}$ \\
\hline AA151307 & 0.004494561 & GNB2 & $\underline{2783}$ & $\begin{array}{l}\text { guanine nucleotide binding } \\
\text { protein (G protein), beta } \\
\text { polypeptide } 2\end{array}$ & $\begin{array}{l}\text { G-protein coupled } \\
\text { receptor protein } \\
\text { signaling pathway; } \\
\text { heterotrimeric G- } \\
\text { protein GTPase } \\
\text { activity; heterotrimeric } \\
\text { G-protein complex; } \\
\text { signal transducer } \\
\text { activity; signal } \\
\text { transduction }\end{array}$ \\
\hline N48735 & 0.004677987 & & & & \\
\hline N29429 & 0.004746315 & CGI-57 & 27013 & hypothetical protein CGI-57 & \\
\hline W04610 & 0.004828823 & $\mathrm{H} 3 \mathrm{~F} 3 \mathrm{~A}$ & 3020 & H3 histone, family $3 \mathrm{~A}$ & \\
\hline W52156 & 0.005026981 & OXTR & $\underline{5021}$ & oxytocin receptor & $\begin{array}{l}\text { G-protein signaling, } \\
\text { coupled to IP3 second } \\
\text { messenger } \\
\text { (phospholipase C } \\
\text { activating); } \\
\text { endosome; integral to } \\
\text { plasma membrane; } \\
\text { lactation; muscle } \\
\text { contraction; oxytocin } \\
\text { receptor activity; } \\
\text { pregnancy; rhodopsin- } \\
\text { like receptor activity; } \\
\text { vasopressin receptor } \\
\text { activity }\end{array}$ \\
\hline AA204664 & 0.005257297 & SMC1L2 & $\underline{27127}$ & $\begin{array}{l}\text { SMC1 structural maintenance } \\
\text { of chromosomes 1-like } 2 \\
\text { (yeast) }\end{array}$ & $\begin{array}{l}\text { ATP binding; ATP- } \\
\text { binding cassette } \\
\text { (ABC) transporter } \\
\text { activity; cell cycle; } \\
\text { chromosome } \\
\text { segregation; kinesin } \\
\text { complex; meiosis; } \\
\text { membrane; nucleus; } \\
\text { transport }\end{array}$ \\
\hline N34901 & 0.005737532 & GALNT7 & $\underline{117248}$ & \multicolumn{2}{|c|}{$\begin{array}{l}\text { UDP-N-acetyl-alpha-D-galactosamine:polypeptide N } \\
\text { acetylgalactosaminyltransferase } 7\end{array}$} \\
\hline
\end{tabular}




\begin{tabular}{|l|l|l|l|l|l|}
\hline AA098865 & 0.005823667 & $\underline{B C L 2 L 10}$ & $\underline{10017}$ & $\begin{array}{l}\text { BCL2-like 10 (apoptosis } \\
\text { facilitator) }\end{array}$ & $\begin{array}{l}\text { anti-apoptosis; } \\
\text { apoptosis inhibitor } \\
\text { activity; caspase } \\
\text { activation; integral to } \\
\text { membrane; } \\
\text { membrane fraction; } \\
\text { mitochondrion; } \\
\text { oogenesis; protein } \\
\text { binding; } \\
\text { spermatogenesis }\end{array}$ \\
\hline
\end{tabular}


Appendix C. Genes Differentially Regulated by $15 \mathrm{~m} \mathrm{MPP}+$ and $15 \mathrm{~m}$ MPP+/SN50 in Combination.

\begin{tabular}{|c|c|c|c|c|c|}
\hline GENBANK & P VALUE & SYMBOL & $\begin{array}{l}\text { LOCUS } \\
\text { LINK }\end{array}$ & GENE NAME & GENE ONTOLOGY \\
\hline T87888 & $4.54 \mathrm{E}-10$ & KIAA1046 & $\underline{22867}$ & KIAA1046 protein & \\
\hline R94499 & 9.77E-10 & GNB5 & 10681 & \multicolumn{2}{|c|}{$\begin{array}{l}\text { guanine nucleotide binding protein (G } \\
\text { protein), beta } 5\end{array}$} \\
\hline H68885 & 9.58E-09 & TSSC3 & $\underline{7262}$ & \begin{tabular}{|l|} 
tumor \\
suppressing \\
subtransferable \\
candidate 3
\end{tabular} & apoptosis; imprinting \\
\hline R19119 & 1.01E-08 & & & & \\
\hline AA010141 & $1.23 \mathrm{E}-08$ & $\frac{\text { SERPINH }}{1}$ & $\underline{871}$ & \begin{tabular}{|l|} 
serine (or \\
cysteine) \\
proteinase \\
inhibitor, clade H \\
(heat shock \\
protein 47), \\
member 1, \\
(collagen binding \\
protein 1)
\end{tabular} & heat shock response \\
\hline H18190 & $2.29 \mathrm{E}-08$ & JAK1 & $\underline{3716}$ & $\begin{array}{l}\text { Janus kinase } 1 \text { (a } \\
\text { protein tyrosine } \\
\text { kinase) }\end{array}$ & $\begin{array}{l}\text { ATP binding; cytoskeleton; } \\
\text { intracellular signaling } \\
\text { cascade; protein amino } \\
\text { acid phosphorylation; } \\
\text { protein tyrosine kinase } \\
\text { activity; transferase activity }\end{array}$ \\
\hline H18298 & 4.04E-08 & & & & \\
\hline H84257 & $7.13 \mathrm{E}-08$ & & & & \\
\hline $\mathrm{H} 25578$ & 7.76E-08 & & & & \\
\hline R17538 & $2.15 \mathrm{E}-07$ & PABPC4 & 8761 & $\begin{array}{l}\text { poly(A) binding } \\
\text { protein, } \\
\text { cytoplasmic } 4 \\
\text { (inducible form) }\end{array}$ & $\begin{array}{l}\text { RNA binding; RNA } \\
\text { catabolism; RNA } \\
\text { processing; blood } \\
\text { coagulation; cytoplasm; } \\
\text { poly(A) binding; protein } \\
\text { biosynthesis; response to } \\
\text { pest/pathogen/parasite } \\
\end{array}$ \\
\hline H58631 & 2.48E-07 & & & & \\
\hline R41363 & $2.58 \mathrm{E}-07$ & & & & \\
\hline H65775 & 5.19E-07 & & & & \\
\hline R48610 & $6.16 \mathrm{E}-07$ & TTC7 & 57217 & tetratricopeptide re & peat domain 7 \\
\hline H18495 & 7.34E-07 & & & & \\
\hline H45355 & $2.47 \mathrm{E}-06$ & & & & \\
\hline W68050 & $2.54 \mathrm{E}-06$ & LGALS1 & 3956 & $\begin{array}{l}\text { lectin, } \\
\text { galactoside- } \\
\text { binding, soluble, }\end{array}$ & $\begin{array}{l}\text { apoptosis; heterophilic cell } \\
\text { adhesion; sugar binding }\end{array}$ \\
\hline
\end{tabular}




\begin{tabular}{|c|c|c|c|c|c|}
\hline & & & & 1 (galectin 1) & \\
\hline H68441 & $3.08 \mathrm{E}-06$ & FLJ14054 & 79614 & \multicolumn{2}{|c|}{ hypothetical protein FLJ14054 } \\
\hline $\mathrm{H} 29730$ & $3.44 \mathrm{E}-06$ & & & & \\
\hline R20373 & $3.78 \mathrm{E}-06$ & TMP21 & 10972 & $\begin{array}{l}\text { transmembrane } \\
\text { trafficking protein }\end{array}$ & $\begin{array}{l}\text { ER to Golgi transport; } \\
\text { Golgi apparatus; integral to } \\
\text { plasma membrane; } \\
\text { intracellular protein } \\
\text { transport; membrane } \\
\text { fraction; microsome; } \\
\text { protein carrier activity; } \\
\text { protein transporter activity }\end{array}$ \\
\hline $\mathrm{H} 09945$ & 8.07E-06 & & & & \\
\hline H19297 & 8.11E-06 & EDIL3 & 10085 & $\begin{array}{l}\text { EGF-like repeats } \\
\text { and discoidin I- } \\
\text { like domains } 3\end{array}$ & $\begin{array}{l}\text { calcium ion binding; cell } \\
\text { adhesion; cell adhesion } \\
\text { molecule activity; } \\
\text { development; integrin } \\
\text { binding }\end{array}$ \\
\hline $\mathrm{H} 23933$ & 8.56E-06 & & & & \\
\hline R47938 & 9.78E-06 & FLJ32096 & 148646 & \multicolumn{2}{|c|}{ hypothetical protein FLJ32096 } \\
\hline $\mathrm{H} 27352$ & 9.93E-06 & HRAS & $\underline{3265}$ & $\begin{array}{l}\text { v-Ha-ras Harvey } \\
\text { rat sarcoma viral } \\
\text { oncogene } \\
\text { homolog }\end{array}$ & $\begin{array}{l}\text { GTPase activity; cell } \\
\text { motility; cell shape and cell } \\
\text { size control; cell surface } \\
\text { receptor linked signal } \\
\text { transduction; chemotaxis; } \\
\text { cytoplasm; histogenesis } \\
\text { and organogenesis; } \\
\text { peripheral plasma } \\
\text { membrane protein; plasma } \\
\text { membrane; regulation of } \\
\text { cell cycle; signal } \\
\text { transduction }\end{array}$ \\
\hline R85191 & 9.96E-06 & FLJ31364 & 146956 & \multicolumn{2}{|c|}{ homolog of yeast EME1 endonuclease } \\
\hline R07186 & $1.18 \mathrm{E}-05$ & & & & \\
\hline H00498 & $1.20 \mathrm{E}-05$ & PPP2R3A & $\underline{5523}$ & \begin{tabular}{|l|} 
protein \\
phosphatase 2 \\
(formerly 2A), \\
regulatory subunit \\
B", alpha
\end{tabular} & $\begin{array}{l}\text { calcium ion binding; } \\
\text { protein phosphatase type } \\
2 A \text {, intrinsic regulator } \\
\text { activity }\end{array}$ \\
\hline H84293 & $1.58 \mathrm{E}-05$ & SLC12A5 & 57468 & \begin{tabular}{|l|} 
solute carrier \\
family 12, \\
(potassium- \\
chloride \\
transporter) \\
member 5
\end{tabular} & $\begin{array}{l}\text { amino acid transport; } \\
\text { amino acid-polyamine } \\
\text { transporter activity; cell ion } \\
\text { homeostasis; chloride } \\
\text { transport; integral to } \\
\text { membrane; ion transport; } \\
\text { potassium ion transport; } \\
\text { potassium:chloride } \\
\text { symporter activity; sodium } \\
\text { ion transport; symporter } \\
\text { activity; transporter activity }\end{array}$ \\
\hline N29429 & 1.77E-05 & CGI-57 & 27013 & hypothetical protei & in CGI-57 \\
\hline R26844 & 1.90E-05 & & & & \\
\hline
\end{tabular}




\begin{tabular}{|c|c|c|c|c|c|}
\hline $\mathrm{H} 47146$ & $2.57 \mathrm{E}-05$ & ERCC1 & $\underline{2067}$ & \begin{tabular}{|l|} 
excision repair \\
cross- \\
complementing \\
rodent repair \\
deficiency, \\
complementation \\
group 1 (includes \\
overlapping \\
antisense \\
sequence)
\end{tabular} & $\begin{array}{l}\text { DNA repair; } \\
\text { embryogenesis and } \\
\text { morphogenesis; } \\
\text { endodeoxyribonuclease } \\
\text { activity; nucleotide- } \\
\text { excision repair; nucleus }\end{array}$ \\
\hline $\mathrm{H} 26760$ & $4.12 \mathrm{E}-05$ & KIAA0375 & 9853 & \multicolumn{2}{|c|}{ KIAA0375 gene product } \\
\hline $\mathrm{H} 27334$ & 4.54E-05 & DDR1 & $\underline{780}$ & \begin{tabular}{|l|} 
discoidin domain \\
receptor family, \\
member 1
\end{tabular} & $\begin{array}{l}\text { ATP binding; cell } \\
\text { adhesion; integral to } \\
\text { plasma membrane; protein } \\
\text { amino acid } \\
\text { phosphorylation; receptor } \\
\text { activity; transferase } \\
\text { activity; transmembrane } \\
\text { receptor protein tyrosine } \\
\text { kinase activity; } \\
\text { transmembrane receptor } \\
\text { protein tyrosine kinase } \\
\text { signaling pathway }\end{array}$ \\
\hline T98139 & $5.04 \mathrm{E}-05$ & $\underline{\mathrm{HLA}-\mathrm{B}}$ & $\underline{3106}$ & \begin{tabular}{|l|} 
major \\
histocompatibility \\
complex, class I, \\
B \\
\\
\end{tabular} & $\begin{array}{l}\text { MHC class I receptor } \\
\text { activity; antigen } \\
\text { presentation, endogenous } \\
\text { antigen; antigen } \\
\text { processing, endogenous } \\
\text { antigen via MHC class I; } \\
\text { immune response; integral } \\
\text { to plasma membrane } \\
\end{array}$ \\
\hline R21970 & $5.86 \mathrm{E}-05$ & GTF2H2 & $\underline{2966}$ & \begin{tabular}{|l|} 
general \\
transcription \\
factor IIH, \\
polypeptide 2, \\
44kDa \\
\end{tabular} & $\begin{array}{l}\text { DNA repair; nucleus; } \\
\text { regulation of transcription, } \\
\text { DNA-dependent }\end{array}$ \\
\hline R50087 & 6.67E-05 & GREB1 & $\underline{9687}$ & GREB1 protein & \\
\hline H86672 & 7.16E-05 & & & & \\
\hline $\mathrm{H} 70974$ & 7.39E-05 & & & & \\
\hline H84008 & 8.89E-05 & & & & \\
\hline R44307 & 0.000103006 & PPP1R9B & $\underline{84687}$ & \begin{tabular}{|l|} 
protein \\
phosphatase 1, \\
regulatory subunit \\
9B, spinophilin \\
\end{tabular} & $\begin{array}{l}\text { intracellular signaling } \\
\text { cascade; membrane; } \\
\text { transport; transporter } \\
\text { activity }\end{array}$ \\
\hline $\mathrm{H} 83405$ & 0.000104317 & FGD1 & $\underline{2245}$ & \begin{tabular}{|l|} 
faciogenital \\
dysplasia \\
(Aarskog-Scott \\
syndrome)
\end{tabular} & $\begin{array}{l}\text { development; guanyl- } \\
\text { nucleotide exchange factor } \\
\text { activity; histogenesis and } \\
\text { organogenesis; signal } \\
\text { transduction; zinc ion } \\
\text { binding }\end{array}$ \\
\hline $\mathrm{H} 40607$ & 0.00010928 & & & & \\
\hline
\end{tabular}




\begin{tabular}{|c|c|c|c|c|c|}
\hline AA031859 & 0.000109417 & TIMM13 & $\underline{26517}$ & $\begin{array}{l}\text { translocase of } \\
\text { inner } \\
\text { mitochondrial } \\
\text { membrane 13 } \\
\text { homolog (yeast) }\end{array}$ & $\begin{array}{l}\text { hearing; mitochondrial } \\
\text { inner membrane pre- } \\
\text { sequence translocase } \\
\text { complex; mitochondrial } \\
\text { translocation; } \\
\text { mitochondrion; protein } \\
\text { targeting; protein } \\
\text { translocase activity; zinc } \\
\text { ion binding }\end{array}$ \\
\hline $\mathrm{H} 20790$ & 0.000123136 & & $\underline{348024}$ & \multirow{2}{*}{\multicolumn{2}{|c|}{$\begin{array}{l}\text { similar to TPIP alpha lipid phosphatase } \\
\text { hypothetical protein FLJ11753 }\end{array}$}} \\
\hline R72577 & 0.00017949 & FLJ11753 & 79712 & & \\
\hline H52741 & 0.000188793 & & & & \\
\hline R89056 & 0.000191859 & LAMP1 & $\underline{3916}$ & $\begin{array}{l}\text { lysosomal- } \\
\text { associated } \\
\text { membrane } \\
\text { protein } 1\end{array}$ & $\begin{array}{l}\text { integral to plasma } \\
\text { membrane; lysosome; } \\
\text { membrane fraction }\end{array}$ \\
\hline R22402 & 0.000194653 & & & & \\
\hline H02088 & 0.000194866 & RBAF600 & 23352 & \multicolumn{2}{|c|}{ retinoblastoma-associated factor 600} \\
\hline H82992 & 0.000210935 & PIGT & 51604 & \multicolumn{2}{|c|}{ phosphatidyl inositol glycan class T } \\
\hline H49225 & 0.00035608 & & & & \\
\hline H52939 & 0.000374234 & & & & \\
\hline R54918 & 0.000382138 & FLJ13912 & 64785 & \multicolumn{2}{|c|}{ hypothetical protein FLJ13912 } \\
\hline R28090 & 0.000402226 & KIAA1495 & 57631 & KIAA1495 protein & \\
\hline N45640 & 0.000411849 & $\mathrm{CH} 25 \mathrm{H}$ & $\underline{9023}$ & $\begin{array}{l}\text { cholesterol 25- } \\
\text { hydroxylase }\end{array}$ & $\begin{array}{l}\text { catalytic activity; lipid } \\
\text { metabolism; membrane } \\
\text { fraction; steroid } \\
\text { hydroxylase activity } \\
\end{array}$ \\
\hline $\mathrm{H} 27034$ & 0.000427779 & IGKC & $\underline{3514}$ & $\begin{array}{l}\text { immunoglobulin } \\
\text { kappa constant }\end{array}$ & $\begin{array}{l}\text { antigen binding; immune } \\
\text { response }\end{array}$ \\
\hline R23351 & 0.00045636 & & & & \\
\hline R88435 & 0.000463204 & DPP6 & 1804 & $\begin{array}{l}\text { dipeptidylpeptida } \\
\text { se } 6\end{array}$ & $\begin{array}{l}\text { catalytic activity; } \\
\text { dipeptidyl-peptidase IV } \\
\text { activity; dipeptidyl- } \\
\text { peptidase activity; integral } \\
\text { to membrane; proteolysis } \\
\text { and peptidolysis }\end{array}$ \\
\hline $\mathrm{H} 45746$ & 0.000474872 & & & & \\
\hline R85044 & 0.00049781 & SMPD1 & $\underline{6609}$ & \begin{tabular}{|l|} 
sphingomyelin \\
phosphodiesteras \\
e 1, acid \\
lysosomal (acid \\
sphingomyelinas \\
e)
\end{tabular} & $\begin{array}{l}\text { carbohydrate metabolism; } \\
\text { hydrolase activity, acting } \\
\text { on glycosyl bonds; } \\
\text { lysosome; neurogenesis; } \\
\text { signal transduction; } \\
\text { sphingomyelin } \\
\text { metabolism; } \\
\text { sphingomyelin } \\
\text { phosphodiesterase activity }\end{array}$ \\
\hline H63763 & 0.000624428 & & & & \\
\hline
\end{tabular}




\begin{tabular}{|c|c|c|c|c|c|}
\hline $\mathrm{H} 47026$ & 0.000674021 & MGAT3 & $\underline{4248}$ & $\begin{array}{l}\text { mannosyl (beta- } \\
1,4-)-g l y c o p r o t e i n \\
\text { beta-1,4-N- } \\
\text { acetylglucosamin } \\
\text { yltransferase }\end{array}$ & $\begin{array}{l}\text { Golgi apparatus; N-linked } \\
\text { glycosylation; beta-1,4- } \\
\text { mannosylglycoprotein } \\
\text { beta-1,4-N- } \\
\text { acetylglucosaminyltransfer } \\
\text { ase activity; integral to } \\
\text { membrane; transferase } \\
\text { activity, transferring } \\
\text { glycosyl groups }\end{array}$ \\
\hline N80976 & 0.000700273 & $\frac{\operatorname{LOC5} 5125}{2}$ & $\underline{51252}$ & \multicolumn{2}{|c|}{ hypothetical protein LOC51252 } \\
\hline R85150 & 0.000724255 & EPHB6 & 2051 & EphB6 & $\begin{array}{l}\text { ATP binding; ephrin } \\
\text { receptor activity; integral to } \\
\text { membrane; protein amino } \\
\text { acid phosphorylation; } \\
\text { protein tyrosine kinase } \\
\text { activity; receptor activity; } \\
\text { transmembrane receptor } \\
\text { protein tyrosine kinase } \\
\text { signaling pathway }\end{array}$ \\
\hline $\mathrm{H} 26552$ & 0.001011054 & MGC5395 & $\underline{79026}$ & $\begin{array}{l}\text { hypothetical } \\
\text { protein MGC5395 }\end{array}$ & $\begin{array}{l}\text { intracellular signaling } \\
\text { cascade }\end{array}$ \\
\hline $\mathrm{H} 43455$ & 0.001071695 & PP2447 & 80305 & \multicolumn{2}{|c|}{ hypothetical protein PP2447 } \\
\hline $\mathrm{H} 93450$ & 0.001256746 & ZNF347 & 84671 & $\begin{array}{l}\text { zinc finger protein } \\
347\end{array}$ & $\begin{array}{l}\text { DNA binding; nucleus; } \\
\text { regulation of transcription, } \\
\text { DNA-dependent }\end{array}$ \\
\hline $\mathrm{H} 71213$ & 0.001430042 & $\underline{F 2}$ & $\underline{2147}$ & $\begin{array}{l}\text { coagulation factor } \\
\text { II (thrombin) }\end{array}$ & $\begin{array}{l}\text { STAT protein nuclear } \\
\text { translocation; acute-phase } \\
\text { response; apoptosis; blood } \\
\text { coagulation; calcium ion } \\
\text { binding; caspase } \\
\text { activation; chymotrypsin } \\
\text { activity; development; } \\
\text { extracellular space; } \\
\text { hydrolase activity; } \\
\text { proteolysis and } \\
\text { peptidolysis; regulation of } \\
\text { cell cycle; response to } \\
\text { wounding; soluble fraction; } \\
\text { thrombin activity; trypsin } \\
\text { activity; tyrosine } \\
\text { phosphorylation of STAT } \\
\text { protein } \\
\end{array}$ \\
\hline N25523 & 0.001869222 & HSPE1 & 3336 & $\begin{array}{l}\text { heat shock } \\
10 \mathrm{kDa} \text { protein } 1 \\
\text { (chaperonin 10) }\end{array}$ & $\begin{array}{l}\text { co-chaperonin activity; } \\
\text { heat shock protein activity; } \\
\text { mitochondrion; protein } \\
\text { folding }\end{array}$ \\
\hline R49189 & 0.001912823 & SLC30A6 & $\underline{55676}$ & \multicolumn{2}{|c|}{$\begin{array}{l}\text { solute carrier family } 30 \text { (zinc transporter), } \\
\text { member } 6\end{array}$} \\
\hline H59454 & 0.002111161 & & & & \\
\hline R48615 & 0.002214369 & C14orf21 & 161424 & $\begin{array}{l}\text { chromosome } 14 \\
\text { open reading } \\
\text { frame } 21\end{array}$ & RNA binding \\
\hline
\end{tabular}




\begin{tabular}{|c|c|c|c|c|c|}
\hline H83025 & 0.002258751 & & & & \\
\hline H46133 & 0.002673406 & BAl2 & $\underline{576}$ & $\begin{array}{l}\text { brain-specific } \\
\text { angiogenesis } \\
\text { inhibitor } 2\end{array}$ & $\begin{array}{l}\text { G-protein coupled receptor } \\
\text { activity; integral to } \\
\text { membrane; neuropeptide } \\
\text { signaling pathway }\end{array}$ \\
\hline N39391 & 0.002748964 & $\frac{\text { MGC1479 }}{9}$ & $\underline{84296}$ & \multicolumn{2}{|c|}{ hypothetical protein MGC14799 } \\
\hline R47859 & 0.002825359 & NPR1 & 4881 & $\begin{array}{l}\text { natriuretic peptide } \\
\text { receptor } \\
\text { A/guanylate } \\
\text { cyclase A } \\
\text { (atrionatriuretic } \\
\text { peptide receptor } \\
\text { A) }\end{array}$ & $\begin{array}{l}\text { ATP binding; cGMP } \\
\text { biosynthesis; guanylate } \\
\text { cyclase activity; integral to } \\
\text { membrane; intracellular } \\
\text { signaling cascade; lyase } \\
\text { activity; peptide receptor } \\
\text { activity, G-protein coupled; } \\
\text { protein amino acid } \\
\text { phosphorylation; protein } \\
\text { kinase activity; receptor } \\
\text { activity; receptor guanylate } \\
\text { cyclase activity; regulation } \\
\text { of blood pressure }\end{array}$ \\
\hline H59405 & 0.002940249 & FLJ10298 & 54682 & \multicolumn{2}{|c|}{ hypothetical protein FLJ10298 } \\
\hline AA031950 & 0.003182394 & & & & \\
\hline R39421 & 0.003373338 & PIGM & $\underline{93183}$ & $\begin{array}{l}\text { phosphatidylinosit } \\
\text { ol glycan, class M }\end{array}$ & transferase activity \\
\hline T84788 & 0.003432787 & & & & \\
\hline R82834 & 0.00345738 & & & & \\
\hline $\mathrm{H} 20520$ & 0.003478042 & & & & \\
\hline $\mathrm{H} 69440$ & 0.003723647 & $\frac{\text { ANKRD1 }}{3}$ & $\underline{88455}$ & \multicolumn{2}{|c|}{ ankyrin repeat domain 13} \\
\hline H69011 & 0.003868629 & SKIL & $\underline{6498}$ & SKI-like & $\begin{array}{l}\text { cell differentiation; cell } \\
\text { growth and/or } \\
\text { maintenance; } \\
\text { molecular_function } \\
\text { unknown; nucleus } \\
\end{array}$ \\
\hline $\mathrm{H} 45972$ & 0.003905578 & & & & \\
\hline R90824 & 0.003908283 & TMEM10 & 93377 & $\begin{array}{l}\text { transmembrane } \\
\text { protein } 10\end{array}$ & integral to membrane \\
\hline H51160 & 0.004249526 & PPP2R1A & $\underline{5518}$ & $\begin{array}{l}\text { protein } \\
\text { phosphatase } 2 \\
\text { (formerly } 2 A), \\
\text { regulatory subunit } \\
\text { A (PR 65), alpha } \\
\text { isoform }\end{array}$ & $\begin{array}{l}\text { protein phosphatase type } \\
\text { 2A activity }\end{array}$ \\
\hline AA037284 & 0.004277598 & APRT & $\underline{353}$ & \begin{tabular}{|l|} 
adenine \\
phosphoribosyltra \\
nsferase
\end{tabular} & $\begin{array}{l}\text { adenine } \\
\text { phosphoribosyltransferase } \\
\text { activity; adenine salvage } \\
\text { pathway; nucleoside } \\
\text { metabolism; transferase } \\
\text { activity, transferring } \\
\text { glycosyl groups }\end{array}$ \\
\hline
\end{tabular}




\begin{tabular}{|l|l|l|l|l|l|}
\hline H04530 & 0.004311455 & ECHS1 & 1892 & $\begin{array}{l}\text { enoyl Coenzyme } \\
\text { A hydratase, } \\
\text { short chain, 1, } \\
\text { mitochondrial }\end{array}$ & $\begin{array}{l}\text { energy pathways; fatty } \\
\text { acid beta-oxidation; fatty } \\
\text { acid metabolism; long- } \\
\text { chain enoyl-CoA hydratase } \\
\text { activity; lyase activity; } \\
\text { mitochondrion; short-chain } \\
\text { enoyl-CoA hydratase } \\
\text { activity }\end{array}$ \\
\hline $\mathrm{H} 62770$ & 0.004355849 & & & & \\
\hline $\mathrm{T} 86338$ & 0.004449624 & & & & \\
\hline $\mathrm{R} 88711$ & 0.004475335 & & & & \\
\hline
\end{tabular}


Appendix D. Genes that change $+/-2$ fold in response to $90 \mathrm{~m} \mathrm{MPP}+$.

\begin{tabular}{|c|c|c|c|c|c|}
\hline GENBANK & $+/-L_{O} G_{2}$ & SYMBOL & $\begin{array}{l}\text { LOCUS } \\
\text { LINK }\end{array}$ & GENE NAME & GENE ONTOLOGY \\
\hline AA045373 & 1.441517459 & TCEAL1 & 9338 & \begin{tabular}{|l|} 
transcription \\
elongation factor $A$ \\
(SII)-like 1
\end{tabular} & $\begin{array}{l}\text { RNA polymerase II } \\
\text { transcription factor activity; } \\
\text { negative regulation of } \\
\text { transcription from Pol II } \\
\text { promoter; nucleus; } \\
\text { regulation of transcription, } \\
\text { DNA-dependent; } \\
\text { transcription factor activity; } \\
\text { translation elongation factor } \\
\text { activity }\end{array}$ \\
\hline H69656 & 1.403296938 & NARF & $\underline{26502}$ & $\begin{array}{l}\text { nuclear prelamin } \mathrm{A} \\
\text { recognition factor }\end{array}$ & $\begin{array}{l}\text { lamin binding; nuclear } \\
\text { lamina }\end{array}$ \\
\hline H68373 & 1.381707958 & TFCP2 & $\underline{7024}$ & $\begin{array}{l}\text { transcription factor } \\
\text { CP2 }\end{array}$ & $\begin{array}{l}\text { DNA binding; regulation of } \\
\text { transcription from Pol II } \\
\text { promoter; transcription } \\
\text { factor activity }\end{array}$ \\
\hline $\mathrm{H} 60376$ & 1.326456548 & & & & \\
\hline H66920 & 1.323418429 & & & & \\
\hline \begin{tabular}{|l}
$\mathrm{H} 62770$ \\
\end{tabular} & 1.313201378 & & & & \\
\hline H61030 & 1.291270823 & & & & \\
\hline H61972 & 1.278225142 & PIN4 & $\underline{5303}$ & $\begin{array}{l}\text { protein (peptidyl- } \\
\text { prolyl cis/trans } \\
\text { isomerase) NIMA- } \\
\text { interacting, } 4 \\
\text { (parvulin) }\end{array}$ & $\begin{array}{l}\text { FK506-sensitive peptidyl- } \\
\text { prolyl cis-trans isomerase; } \\
\text { cyclophilin; cyclophilin-type } \\
\text { peptidy-prolyl cis-trans } \\
\text { isomerase activity; } \\
\text { isomerase activity; } \\
\text { mitochondrial matrix; protein } \\
\text { folding }\end{array}$ \\
\hline N30939 & 1.269259316 & & & & \\
\hline H62766 & 1.26421627 & & & & \\
\hline H63794 & 1.263829666 & NFATC1 & $\underline{4772}$ & $\begin{array}{l}\text { nuclear factor of } \\
\text { activated T-cells, } \\
\text { cytoplasmic, } \\
\text { calcineurin- } \\
\text { dependent } 1\end{array}$ & $\begin{array}{l}\text { FK506 binding; cytoplasm; } \\
\text { nucleus; regulation of } \\
\text { transcription, DNA- } \\
\text { dependent; transcription } \\
\text { factor activity; transcription } \\
\text { from Pol II promoter }\end{array}$ \\
\hline $\mathrm{H} 48676$ & 1.263058048 & & & & \\
\hline H60458 & 1.252815748 & $\mathrm{ACOX} 2$ & $\underline{8309}$ & $\begin{array}{l}\text { acyl-Coenzyme A } \\
\text { oxidase } 2, \\
\text { branched chain }\end{array}$ & $\begin{array}{l}\text { acyl-CoA oxidase activity; } \\
\text { bile acid metabolism; } \\
\text { electron transport; fatty acid } \\
\text { beta-oxidation; fatty acid } \\
\text { metabolism; oxidoreductase } \\
\text { activity; peroxisome }\end{array}$ \\
\hline W86443 & 1.24926473 & & & & \\
\hline $\mathrm{H} 45355$ & 1.245053836 & & & & \\
\hline H09945 & 1.241279937 & & & & \\
\hline
\end{tabular}




\begin{tabular}{|c|c|c|c|c|c|}
\hline $\mathrm{H} 62473$ & 1.23886115 & TGFBR3 & $\overline{7049}$ & \begin{tabular}{|l|} 
transforming \\
growth factor, beta \\
receptor III \\
(betaglycan, \\
$300 \mathrm{kDa}$ ) \\
\end{tabular} & $\begin{array}{l}\text { TGFbeta receptor signaling } \\
\text { pathway; development; } \\
\text { glycosaminoglycan binding; } \\
\text { integral to membrane; } \\
\text { receptor activity; signal } \\
\text { transduction }\end{array}$ \\
\hline H84096 & 1.233985965 & & & & \\
\hline H70485 & 1.233118474 & MBNL3 & $\underline{5796}$ & $\begin{array}{l}\text { muscleblind-like } 3 \\
\text { (Drosophila) }\end{array}$ & $\begin{array}{l}\text { development; nucleic acid } \\
\text { binding; nucleus }\end{array}$ \\
\hline R96672 & 1.229567068 & CYP2D6 & 1565 & $\begin{array}{l}\text { Cytochrome P450, } \\
\text { family } 2 \text {, subfamily } \\
\text { D, polypeptide } 6\end{array}$ & cytochrome P450 activity \\
\hline $\mathrm{H} 60340$ & 1.218704746 & & & & \\
\hline H61974 & 1.213195891 & & & & \\
\hline H59062 & 1.20663091 & KIAA0602 & 23241 & KIAA0602 protein & \\
\hline W47000 & 1.205992418 & HAK & 115701 & heart alpha-kinase & \\
\hline AA045817 & 1.205511408 & MAGEA8 & $\underline{4107}$ & $\begin{array}{l}\text { melanoma antigen, } \\
\text { family A, } 8\end{array}$ & $\begin{array}{l}\text { biological_process } \\
\text { unknown; } \\
\text { cellular_component } \\
\text { unknown; } \\
\text { molecular_function unknown }\end{array}$ \\
\hline AA136884 & 1.204444126 & FLJ21924 & $\underline{79832}$ & $\begin{array}{l}\text { hypothetical protein } \\
\text { FLJ21924 }\end{array}$ & \\
\hline AA099373 & 1.204236905 & GYS1 & $\underline{2997}$ & $\begin{array}{l}\text { glycogen synthase } \\
1 \text { (muscle) }\end{array}$ & glycogen metabolism \\
\hline H51160 & 1.203703177 & PPP2R1A & $\underline{5518}$ & $\begin{array}{l}\text { protein } \\
\text { phosphatase } 2 \\
\text { (formerly 2A), } \\
\text { regulatory subunit } \\
\text { A (PR 65), alpha } \\
\text { isoform }\end{array}$ & $\begin{array}{l}\text { protein phosphatase type } 2 \mathrm{~A} \\
\text { activity }\end{array}$ \\
\hline $\mathrm{H} 82521$ & 1.195709551 & ATP6V0B & $\underline{533}$ & $\begin{array}{l}\text { ATPase, } \mathrm{H+} \\
\text { transporting, } \\
\text { lysosomal } 21 \mathrm{kDa} \text {, } \\
\text { V0 subunit c" }\end{array}$ & $\begin{array}{l}\text { ATP biosynthesis; hydrogen } \\
\text { ion transporter activity; } \\
\text { hydrogen-transporting two- } \\
\text { sector ATPase activity; } \\
\text { hydrolase activity; integral to } \\
\text { membrane; proton transport; } \\
\text { transporter activity }\end{array}$ \\
\hline AA029583 & 1.195391457 & TFF3 & $\underline{7033}$ & $\begin{array}{l}\text { trefoil factor } 3 \\
\text { (intestinal) }\end{array}$ & $\begin{array}{l}\text { defense response; } \\
\text { digestion; extracellular }\end{array}$ \\
\hline W86198 & 1.189144525 & KIAA0905 & 22872 & $\begin{array}{l}\text { yeast Sec31p } \\
\text { homolog }\end{array}$ & \\
\hline H83003 & 1.186074391 & IGSF1 & $\underline{3547}$ & $\begin{array}{l}\text { immunoglobulin } \\
\text { superfamily, } \\
\text { member } 1\end{array}$ & $\begin{array}{l}\text { cell adhesion; integral to } \\
\text { plasma membrane }\end{array}$ \\
\hline R85183 & 1.181843544 & C20orf98 & $\underline{80023}$ & $\begin{array}{l}\text { chromosome } 20 \\
\text { open reading frame } \\
98\end{array}$ & integral to membrane \\
\hline AA142989 & 1.173727565 & BMPER & $\underline{168667}$ & \begin{tabular}{|l|} 
likely ortholog of \\
mouse BMP- \\
binding endothelial \\
regulator precursor
\end{tabular} & $\begin{array}{l}\text { calcium ion binding; } \\
\text { extracellular }\end{array}$ \\
\hline
\end{tabular}




\begin{tabular}{|c|c|c|c|c|c|}
\hline & & & & protein & \\
\hline H59810 & 1.165111447 & $\underline{C L U}$ & 1191 & \begin{tabular}{|l|} 
clusterin \\
(complement lysis \\
inhibitor, SP-40,40, \\
sulfated \\
glycoprotein 2, \\
testosterone- \\
repressed prostate \\
message 2, \\
apolipoprotein J) \\
\end{tabular} & $\begin{array}{l}\text { apoptosis; cell death; } \\
\text { complement activation, } \\
\text { classical pathway; } \\
\text { fertilization (sensu } \\
\text { Animalia); lipid metabolism } \\
\end{array}$ \\
\hline H67584 & 1.163534475 & & & & \\
\hline R85191 & 1.15964466 & FLJ31364 & 146956 & $\begin{array}{l}\text { homolog of yeast } \\
\text { EME1 } \\
\text { endonuclease }\end{array}$ & \\
\hline W58177 & 1.158467769 & $\frac{\mathrm{HIST} 2 \mathrm{H} 2 \mathrm{~A}}{\underline{\mathrm{A}}}$ & 8337 & histone 2, H2aa & $\begin{array}{l}\text { DNA binding; chromosome; } \\
\text { chromosome organization } \\
\text { and biogenesis (sensu } \\
\text { Eukarya); nucleosome; } \\
\text { nucleosome assembly; } \\
\text { nucleus }\end{array}$ \\
\hline H62266 & 1.154316023 & & & & \\
\hline AA044141 & 1.151517188 & C20orf98 & $\underline{80023}$ & $\begin{array}{l}\text { chromosome } 20 \\
\text { open reading frame } \\
98\end{array}$ & integral to membrane \\
\hline H68441 & 1.149340598 & FLJ14054 & $\underline{79614}$ & $\begin{array}{l}\text { hypothetical protein } \\
\text { FLJ14054 }\end{array}$ & \\
\hline N45013 & 1.142586597 & & & & \\
\hline H65775 & 1.140757184 & & & & \\
\hline H18298 & 1.13931833 & & & & \\
\hline $\mathrm{H} 68440$ & 1.129174679 & PIP5K1B & $\underline{8395}$ & $\begin{array}{l}\text { phosphatidylinositol- } \\
\text { I, beta }\end{array}$ & -4-phosphate 5-kinase, type \\
\hline AA115377 & 1.129084061 & TMPO & $\underline{7112}$ & thymopoietin & $\begin{array}{l}\text { lamin binding; } \\
\text { lamin/chromatin binding; } \\
\text { nuclear membrane; nucleus }\end{array}$ \\
\hline R21970 & 1.128566868 & GTF2H2 & $\underline{2966}$ & \begin{tabular}{|l|} 
general \\
transcription factor \\
IIH, polypeptide 2, \\
$44 \mathrm{kDa}$
\end{tabular} & $\begin{array}{l}\text { DNA repair; nucleus; } \\
\text { regulation of transcription, } \\
\text { DNA-dependent }\end{array}$ \\
\hline $\mathrm{H} 29730$ & 1.127973609 & & & & \\
\hline $\mathrm{H} 68949$ & 1.121067809 & & & & \\
\hline AA059131 & 1.120131935 & BTBD1 & $\underline{53339}$ & $\begin{array}{l}\text { BTB (POZ) domain } \\
\text { containing } 1\end{array}$ & $\begin{array}{l}\text { biological_process } \\
\text { unknown; } \\
\text { cellular_component } \\
\text { unknown; protein binding }\end{array}$ \\
\hline $\mathrm{H} 60520$ & 1.117545032 & & & & \\
\hline H43455 & 1.114606867 & PP2447 & $\underline{80305}$ & $\begin{array}{l}\text { hypothetical protein } \\
\text { PP2447 }\end{array}$ & \\
\hline AA147503 & 1.113307029 & RRS1 & 23212 & \begin{tabular}{|l|} 
RRS1 ribosome \\
biogenesis \\
regulator homolog \\
(S. cerevisiae)
\end{tabular} & $\begin{array}{l}\text { nucleus; ribosome } \\
\text { biogenesis }\end{array}$ \\
\hline
\end{tabular}




\begin{tabular}{|c|c|c|c|c|c|}
\hline T51698 & 1.111278341 & & & & \\
\hline H18495 & 1.108995666 & & & & \\
\hline R89056 & 1.105715702 & LAMP1 & 3916 & $\begin{array}{l}\text { lysosomal- } \\
\text { associated } \\
\text { membrane protein } \\
1\end{array}$ & $\begin{array}{l}\text { integral to plasma } \\
\text { membrane; lysosome; } \\
\text { membrane fraction }\end{array}$ \\
\hline $\mathrm{H} 47026$ & 1.103738337 & MGAT3 & $\underline{4248}$ & \begin{tabular}{|l|} 
mannosyl (beta- \\
$1,4-)-$ glycoprotein \\
beta-1,4-N- \\
acetylglucosaminylt \\
ransferase
\end{tabular} & $\begin{array}{l}\text { Golgi apparatus; N-linked } \\
\text { glycosylation; beta-1,4- } \\
\text { mannosylglycoprotein beta- } \\
\text { 1,4-N- } \\
\text { acetylglucosaminyltransfera } \\
\text { se activity; integral to } \\
\text { membrane; transferase } \\
\text { activity, transferring glycosyl } \\
\text { groups }\end{array}$ \\
\hline AA037661 & 1.101101359 & C21orf70 & 85395 & $\begin{array}{l}\text { chromosome } 21 \\
\text { open reading frame } \\
70\end{array}$ & \\
\hline H68441 & 1.09871499 & FLJ14054 & $\underline{79614}$ & $\begin{array}{l}\text { hypothetical protein } \\
\text { FLJ14054 }\end{array}$ & \\
\hline R21373 & 1.098435204 & HMGN1 & 3150 & $\begin{array}{l}\text { high-mobility group } \\
\text { nucleosome } \\
\text { binding domain } 1\end{array}$ & $\begin{array}{l}\text { DNA binding; RNA } \\
\text { polymerase II transcription } \\
\text { factor activity; chromatin; } \\
\text { positive transcription } \\
\text { elongation factor activity }\end{array}$ \\
\hline AA151360 & 1.091171256 & $\begin{array}{l}\text { ARHGAP1 } \\
\underline{2}\end{array}$ & 94134 & $\begin{array}{l}\text { Rho GTPase } \\
\text { activating protein } \\
12\end{array}$ & \\
\hline AA054541 & 1.090113332 & & & & \\
\hline AA203677 & 1.087790185 & & & & \\
\hline R85333 & 1.087525971 & & & & \\
\hline AA047517 & 1.086114591 & VRK3 & 51231 & $\begin{array}{l}\text { vaccinia related } \\
\text { kinase } 3\end{array}$ & $\begin{array}{l}\text { ATP binding; protein amino } \\
\text { acid phosphorylation; } \\
\text { protein kinase activity; } \\
\text { transferase activity }\end{array}$ \\
\hline R12665 & 1.084961201 & & 197135 & $\begin{array}{l}\text { similar to RIKEN } \\
\text { cDNA 4930424G05 }\end{array}$ & \\
\hline N80432 & 1.084518406 & & & & \\
\hline W39129 & 1.081123099 & NUCB1 & $\underline{4924}$ & nucleobindin 1 & $\begin{array}{l}\text { DNA binding; Golgi } \\
\text { apparatus; calcium ion } \\
\text { binding; extracellular space }\end{array}$ \\
\hline N48003 & 1.079307263 & MGC9912 & 112487 & $\begin{array}{l}\text { similar to RIKEN } \\
\text { cDNA 4930578F06 } \\
\text { gene }\end{array}$ & $\begin{array}{l}\text { D-amino acid catabolism; } \\
\text { cytoplasm; hydrolase } \\
\text { activity, acting on ester } \\
\text { bonds }\end{array}$ \\
\hline H65231 & 1.077877352 & & & & \\
\hline H39058 & 1.07691367 & & & & \\
\hline H67094 & 1.075796998 & & & & \\
\hline AA149233 & 1.075759317 & PTGES & $\underline{9536}$ & $\begin{array}{l}\text { prostaglandin E } \\
\text { synthase }\end{array}$ & $\begin{array}{l}\text { antimicrobial humoral } \\
\text { response (sensu } \\
\text { Invertebrata); membrane } \\
\text { fraction; prostaglandin }\end{array}$ \\
\hline
\end{tabular}




\begin{tabular}{|c|c|c|c|c|c|}
\hline & & & & & $\begin{array}{l}\text { metabolism; signal } \\
\text { transduction }\end{array}$ \\
\hline AA098907 & 1.074777518 & ALAS1 & 211 & $\begin{array}{l}\text { aminolevulinate, } \\
\text { delta-, synthase } 1\end{array}$ & $\begin{array}{l}\text { 5-aminolevulinate synthase } \\
\text { activity; acyltransferase } \\
\text { activity; biosynthesis; heme } \\
\text { biosynthesis; mitochondrion; } \\
\text { transaminase activity; } \\
\text { transferase activity }\end{array}$ \\
\hline H93445 & 1.073357733 & & & & \\
\hline H38321 & 1.072843358 & FLJ14360 & $\underline{84861}$ & $\begin{array}{l}\text { hypothetical protein } \\
\text { FLJ14360 }\end{array}$ & protein binding \\
\hline $\mathrm{H} 48589$ & 1.068620209 & HSPA4 & $\underline{3308}$ & $\begin{array}{l}\text { heat shock } 70 \mathrm{kDa} \\
\text { protein } 4\end{array}$ & molecular_function \\
\hline H66835 & 1.068287191 & & & & \\
\hline H68976 & 1.062900057 & BXDC1 & $\underline{84154}$ & $\begin{array}{l}\text { brix domain } \\
\text { containing } 1\end{array}$ & nucleus \\
\hline AA058835 & 1.060918908 & & & & \\
\hline N52765 & 1.059040178 & CACNA1I & 8911 & $\begin{array}{l}\text { calcium channel, } \\
\text { voltage-dependent, } \\
\text { alpha } 1 \text { I subunit }\end{array}$ & $\begin{array}{l}\text { calcium ion binding; calcium } \\
\text { ion transport; cation } \\
\text { transport; integral to } \\
\text { membrane; low voltage- } \\
\text { gated calcium channel } \\
\text { activity; voltage-gated } \\
\text { calcium channel complex }\end{array}$ \\
\hline AA134752 & 1.055574306 & $\underline{\text { SLC30A5 }}$ & $\underline{64924}$ & $\begin{array}{l}\text { solute carrier family } \\
\text { member } 5\end{array}$ & 30 (zinc transporter) \\
\hline $\mathrm{H} 70468$ & 1.054468231 & & & & \\
\hline N94749 & 1.054030574 & & & & \\
\hline H68952 & 1.051992045 & ITGA1 & $\underline{3672}$ & integrin, alpha 1 & $\begin{array}{l}\text { cell adhesion receptor } \\
\text { activity; cell-matrix } \\
\text { adhesion; collagen binding; } \\
\text { integral to membrane; } \\
\text { integrin complex; integrin- } \\
\text { mediated signaling pathway; } \\
\text { magnesium ion binding; } \\
\text { receptor activity }\end{array}$ \\
\hline H68718 & 1.051870928 & ROCK1 & $\underline{6093}$ & $\begin{array}{l}\text { Rho-associated, } \\
\text { coiled-coil } \\
\text { containing protein } \\
\text { kinase } 1\end{array}$ & $\begin{array}{l}\text { ATP binding; Rho protein } \\
\text { signal transduction; actin } \\
\text { cytoskeleton organization } \\
\text { and biogenesis; intracellular; } \\
\text { intracellular signaling } \\
\text { cascade; protein amino acid } \\
\text { phosphorylation; protein } \\
\text { serine/threonine kinase } \\
\text { activity; protein tyrosine } \\
\text { kinase activity; transferase } \\
\text { activity }\end{array}$ \\
\hline H62909 & 1.049977564 & & & & \\
\hline R47938 & 1.049354437 & FLJ32096 & 148646 & $\begin{array}{l}\text { hypothetical protein } \\
\text { FLJ32096 }\end{array}$ & \\
\hline
\end{tabular}




\begin{tabular}{|c|c|c|c|c|c|}
\hline $\mathrm{H} 24891$ & 1.047426291 & & & & \\
\hline N40017 & 1.046865373 & MRPL24 & $\underline{79590}$ & $\begin{array}{l}\text { mitochondrial } \\
\text { ribosomal protein } \\
\text { L24 }\end{array}$ & $\begin{array}{l}\text { intracellular; protein } \\
\text { biosynthesis; ribosome; } \\
\text { structural constituent of } \\
\text { ribosome }\end{array}$ \\
\hline W55993 & 1.046467205 & FBN2 & $\underline{2201}$ & \begin{tabular}{|l} 
fibrillin 2 \\
(congenital \\
contractural \\
arachnodactyly)
\end{tabular} & $\begin{array}{l}\text { calcium ion binding; } \\
\text { embryogenesis and } \\
\text { morphogenesis; } \\
\text { extracellular matrix; } \\
\text { extracellular matrix } \\
\text { structural constituent; } \\
\text { histogenesis and } \\
\text { organogenesis } \\
\end{array}$ \\
\hline H09701 & 1.045444795 & & & & \\
\hline $\mathrm{H} 48578$ & 1.045334733 & & & & \\
\hline H69787 & 1.044928552 & & & & \\
\hline N27190 & 1.044272505 & $\underline{\mathrm{UCHL} 3}$ & $\underline{7347}$ & $\begin{array}{l}\text { ubiquitin carboxyl- } \\
\text { terminal esterase } \\
\text { L3 (ubiquitin } \\
\text { thiolesterase) }\end{array}$ & $\begin{array}{l}\text { cytoplasm; hydrolase } \\
\text { activity; ubiquitin C-terminal } \\
\text { hydrolase activity; ubiquitin- } \\
\text { dependent protein } \\
\text { catabolism }\end{array}$ \\
\hline H61036 & 1.043982636 & & & & \\
\hline AA099441 & 1.037648009 & NUCB1 & 4924 & nucleobindin 1 & $\begin{array}{l}\text { DNA binding; Golgi } \\
\text { apparatus; calcium ion } \\
\text { binding; extracellular space }\end{array}$ \\
\hline R87198 & 1.037149735 & TUBB5 & 10382 & tubulin, beta, 5 & $\begin{array}{l}\text { cytoskeleton; structural } \\
\text { constituent of cytoskeleton }\end{array}$ \\
\hline N78467 & 1.035593133 & PWP1 & 11137 & $\begin{array}{l}\text { nuclear } \\
\text { phosphoprotein } \\
\text { similar to S. } \\
\text { cerevisiae PWP1 }\end{array}$ & nucleus; transcription \\
\hline R90824 & 1.034623848 & TMEM10 & 93377 & $\begin{array}{l}\text { transmembrane } \\
\text { protein } 10\end{array}$ & integral to membrane \\
\hline AA099636 & 1.03442035 & KIAA1039 & $\underline{23108}$ & KIAA1039 protein & \\
\hline R31364 & 1.034277696 & $\frac{\mathrm{LOC} 28337}{7}$ & $\underline{283377}$ & $\begin{array}{l}\text { hypothetical protein } \\
\text { LOC283377 }\end{array}$ & \\
\hline $\mathrm{H} 27034$ & 1.033434891 & IGKC & $\underline{3514}$ & $\begin{array}{l}\text { immunoglobulin } \\
\text { kappa constant }\end{array}$ & $\begin{array}{l}\text { antigen binding; immune } \\
\text { response }\end{array}$ \\
\hline W86640 & 1.032117754 & RIPK1 & 8737 & $\begin{array}{l}\text { receptor } \\
\text { (TNFRSF)- } \\
\text { interacting serine- } \\
\text { threonine kinase } 1\end{array}$ & $\begin{array}{l}\text { ATP binding; apoptosis; } \\
\text { cAMP-dependent protein } \\
\text { kinase activity; protein } \\
\text { amino acid phosphorylation; } \\
\text { protein kinase CK2 activity; } \\
\text { protein serine/threonine } \\
\text { kinase activity; protein } \\
\text { tyrosine kinase activity; } \\
\text { signal transduction; } \\
\text { transferase activity }\end{array}$ \\
\hline R48311 & 1.030542722 & FLJ38608 & 132228 & $\begin{array}{l}\text { hypothetical protein } \\
\text { FLJ38608 }\end{array}$ & \\
\hline
\end{tabular}




\begin{tabular}{|c|c|c|c|c|c|}
\hline $\mathrm{H} 62477$ & 1.029641741 & CD36 & $\frac{948}{9}$ & $\begin{array}{l}\text { CD36 antigen } \\
\text { (collagen type I } \\
\text { receptor, } \\
\text { thrombospondin } \\
\text { receptor) }\end{array}$ & $\begin{array}{l}\text { blood coagulation; cell } \\
\text { adhesion; cell adhesion } \\
\text { molecule activity; fatty acid } \\
\text { metabolism; integral to } \\
\text { plasma membrane; } \\
\text { membrane fraction; receptor } \\
\text { activity; transport } \\
\end{array}$ \\
\hline H94763 & 1.028965825 & SH3GLB1 & $\underline{51100}$ & $\begin{array}{l}\text { SH3-domain } \\
\text { GRB2-like } \\
\text { endophilin B1 }\end{array}$ & \\
\hline H52253 & 1.027982817 & $\underline{\mathrm{IGHG}}$ & 3502 & $\begin{array}{l}\text { immunoglobulin } \\
\text { heavy constant } \\
\text { gamma } 3 \text { (G3m } \\
\text { marker) } \\
\end{array}$ & $\begin{array}{l}\text { antigen binding; immune } \\
\text { response; membrane } \\
\text { fraction }\end{array}$ \\
\hline W70111 & 1.025583613 & ADH5 & 128 & $\begin{array}{l}\text { alcohol } \\
\text { dehydrogenase } 5 \\
\text { (class III), chi } \\
\text { polypeptide }\end{array}$ & \begin{tabular}{|l|} 
alcohol dehydrogenase \\
activity, iron-dependent; \\
alcohol dehydrogenase \\
activity, metal ion- \\
independent; alcohol \\
dehydrogenase activity, \\
zinc-dependent; alcohol \\
metabolism; electron \\
transporter activity; ethanol \\
oxidation; fatty acid binding; \\
formaldehyde \\
dehydrogenase (glutathione) \\
activity; oxidoreductase \\
activity; zinc ion binding
\end{tabular} \\
\hline H27352 & 1.024405897 & HRAS & $\underline{3265}$ & $\begin{array}{l}\text { v-Ha-ras Harvey } \\
\text { rat sarcoma viral } \\
\text { oncogene homolog }\end{array}$ & $\begin{array}{l}\text { GTPase activity; cell motility; } \\
\text { cell shape and cell size } \\
\text { control; cell surface receptor } \\
\text { linked signal transduction; } \\
\text { chemotaxis; cytoplasm; } \\
\text { histogenesis and } \\
\text { organogenesis; peripheral } \\
\text { plasma membrane protein; } \\
\text { plasma membrane; } \\
\text { regulation of cell cycle; } \\
\text { signal transduction } \\
\end{array}$ \\
\hline AA033714 & 1.022700441 & FLJ14260 & $\underline{80095}$ & $\begin{array}{l}\text { hypothetical protein } \\
\text { FLJ14260 }\end{array}$ & $\begin{array}{l}\text { DNA binding; } \\
\text { metallopeptidase activity; } \\
\text { nucleus; proteolysis and } \\
\text { peptidolysis; regulation of } \\
\text { transcription, DNA- } \\
\text { dependent; zinc ion binding } \\
\end{array}$ \\
\hline AA029775 & 1.021800833 & FUS1 & $\underline{11334}$ & $\begin{array}{l}\text { lung cancer } \\
\text { candidate }\end{array}$ & $\begin{array}{l}\text { cell proliferation; cell-cell } \\
\text { signaling; negative } \\
\text { regulation of cell cycle }\end{array}$ \\
\hline AA152304 & 1.021453662 & ARF3 & $\underline{377}$ & $\begin{array}{l}\text { ADP-ribosylation } \\
\text { factor } 3\end{array}$ & $\begin{array}{l}\text { Golgi apparatus; intracellular } \\
\text { protein transport; } \\
\text { nonselective vesicle } \\
\text { assembly; protein } \\
\text { transporter activity; small }\end{array}$ \\
\hline
\end{tabular}




\begin{tabular}{|c|c|c|c|c|c|}
\hline & & & & & $\begin{array}{l}\text { GTPase mediated signal } \\
\text { transduction; small } \\
\text { monomeric GTPase activity }\end{array}$ \\
\hline R07186 & 1.019988898 & & & & \\
\hline AA148416 & 1.019647544 & SERAC1 & $\underline{84947}$ & $\begin{array}{l}\text { serine active site } \\
\text { containing } 1\end{array}$ & catalytic activity \\
\hline R27269 & 1.019572266 & & & & \\
\hline H56304 & 1.019388908 & ENTPD1 & 953 & \begin{tabular}{|l|} 
ectonucleoside \\
triphosphate \\
diphosphohydrolas \\
e 1 \\
\\
\\
\end{tabular} & $\begin{array}{l}\text { antimicrobial humoral } \\
\text { response (sensu } \\
\text { Invertebrata); apyrase } \\
\text { activity; blood coagulation; } \\
\text { cell adhesion; cell-cell } \\
\text { signaling; hydrolase activity; } \\
\text { integral to plasma } \\
\text { membrane; magnesium ion } \\
\text { binding; membrane fraction }\end{array}$ \\
\hline R90757 & 1.018109648 & RPH3A & $\underline{22895}$ & $\begin{array}{l}\text { likely ortholog of } \\
\text { mouse rabphilin } 3 \mathrm{~A}\end{array}$ & $\begin{array}{l}\text { intracellular protein } \\
\text { transport; membrane; } \\
\text { protein transporter activity; } \\
\text { synaptic junction; synaptic } \\
\text { vesicle; zinc ion binding }\end{array}$ \\
\hline R41363 & 1.017230382 & & & & \\
\hline T51972 & 1.017109657 & SEMA4C & $\underline{54910}$ & $\begin{array}{l}\text { sema domain, } \\
\text { immunoglobulin } \\
\text { domain (Ig), } \\
\text { transmembrane } \\
\text { domain (TM) and } \\
\text { short cytoplasmic } \\
\text { domain, } \\
\text { (semaphorin) 4C }\end{array}$ & $\begin{array}{l}\text { development; membrane; } \\
\text { receptor activity }\end{array}$ \\
\hline AA058632 & 1.015551086 & KIF1B & $\underline{23095}$ & $\begin{array}{l}\text { kinesin family } \\
\text { member } 1 \mathrm{~B}\end{array}$ & \\
\hline AA035437 & 1.01352019 & & & & \\
\hline H86672 & 1.012957874 & & & & \\
\hline $\mathrm{H} 60542$ & 1.011644443 & & & & \\
\hline N89894 & 1.010068232 & & & & \\
\hline AA057466 & 1.008653468 & & & & \\
\hline $\mathrm{H} 47518$ & 1.008141366 & & & & \\
\hline W47003 & 1.008005073 & HIF1A & 3091 & $\begin{array}{l}\text { hypoxia-inducible fa } \\
\text { helix-loop-helix trans }\end{array}$ & $\begin{array}{l}\text { actor 1, alpha subunit (basic } \\
\text { scription factor) }\end{array}$ \\
\hline R01799 & 1.00741527 & & & & \\
\hline N49763 & 1.007208746 & WTAP & 9589 & $\begin{array}{l}\text { Wilms tumor } 1 \\
\text { associated protein }\end{array}$ & \\
\hline AA009926 & 1.006656423 & & & & \\
\hline H68528 & 1.003205293 & FLJ32499 & 124637 & $\begin{array}{l}\text { hypothetical protein } \\
\text { FLJ32499 }\end{array}$ & \\
\hline H62045 & 1.00061111 & $\frac{\mathrm{LOC} 11758}{4}$ & 117584 & fring & \\
\hline H10658 & 1.000551286 & & & & \\
\hline
\end{tabular}




\begin{tabular}{|c|c|c|c|c|c|}
\hline AA059148 & 1.000473754 & KIAA1199 & 57214 & KIAA1199 protein & \\
\hline AA057126 & 1.000325845 & KIAA1416 & 55636 & KIAA1416 protein & \\
\hline N91341 & -1.00337743 & & & & \\
\hline T62916 & -1.00877983 & RPS29 & $\underline{6235}$ & $\begin{array}{l}\text { ribosomal protein } \\
\text { S29 }\end{array}$ & $\begin{array}{l}\text { RNA binding; cytosolic small } \\
\text { ribosomal subunit (sensu } \\
\text { Eukarya); intracellular; } \\
\text { protein biosynthesis; } \\
\text { ribosome; structural } \\
\text { constituent of ribosome; zinc } \\
\text { ion binding }\end{array}$ \\
\hline N32235 & -1.01486015 & IGFBP4 & $\underline{3487}$ & $\begin{array}{l}\text { insulin-like growth } \\
\text { factor binding } \\
\text { protein } 4\end{array}$ & $\begin{array}{l}\text { DNA metabolism; cell } \\
\text { proliferation; extracellular; } \\
\text { insulin-like growth factor } \\
\text { binding; regulation of cell } \\
\text { growth; signal transduction; } \\
\text { skeletal development }\end{array}$ \\
\hline N39407 & -1.017239042 & KIF21A & $\underline{55605}$ & $\begin{array}{l}\text { kinesin family } \\
\text { member } 21 \mathrm{~A}\end{array}$ & \\
\hline N29429 & -1.023442466 & CGI-57 & 27013 & $\begin{array}{l}\text { hypothetical protein } \\
\text { CGI-57 }\end{array}$ & \\
\hline N91501 & -1.052425513 & & & & \\
\hline W32438 & -1.107245642 & CRABP2 & 1382 & $\begin{array}{l}\text { cellular retinoic } \\
\text { acid binding protein } \\
2\end{array}$ & $\begin{array}{l}\text { epidermal differentiation; } \\
\text { lipid binding; regulation of } \\
\text { transcription, DNA- } \\
\text { dependent; retinoid binding; } \\
\text { signal transduction; } \\
\text { transport; transporter activity }\end{array}$ \\
\hline
\end{tabular}


Appendix E. Genes That Change $+/-2$ fold at both $15 \mathrm{~m}$ and $90 \mathrm{~m}$ of MPP+

treatment.

\begin{tabular}{|c|c|c|c|}
\hline GENBANK & SYMBOL & LOCUSLINK & GENE NAME \\
\hline AA059148 & KIAA1199 & 57214 & KIAA1199 protein \\
\hline AA057126 & KIAA1416 & 55636 & KIAA1416 protein \\
\hline H59810 & $\underline{\mathrm{CLU}}$ & 1191 & $\begin{array}{l}\text { clusterin (complement lysis inhibitor, SP- } \\
40,40, \text { sulfated glycoprotein } 2 \text {, testosterone- } \\
\text { repressed prostate message } 2, \\
\text { apolipoprotein J) }\end{array}$ \\
\hline R96672 & CYP2D6 & 1565 & $\begin{array}{l}\text { cytochrome P450, family } 2 \text {, subfamily D, } \\
\text { polypeptide } 6\end{array}$ \\
\hline R21970 & GTF2H2 & $\underline{2966}$ & $\begin{array}{l}\text { general transcription factor } \mathrm{IIH} \text {, polypeptide } \\
2,44 \mathrm{kDa}\end{array}$ \\
\hline W58177 & HIST2H2AA & 8337 & histone 2, H2aa \\
\hline R85191 & FLJ31364 & 146956 & homolog of yeast EME1 endonuclease \\
\hline N29429 & CGI-57 & 27013 & hypothetical protein CGI-57 \\
\hline H68441 & FLJ14054 & 79614 & hypothetical protein FLJ14054 \\
\hline H38321 & FLJ14360 & 84861 & hypothetical protein FLJ14360 \\
\hline R47938 & FLJ32096 & 148646 & hypothetical protein FLJ32096 \\
\hline H43455 & PP2447 & $\underline{80305}$ & hypothetical protein PP2447 \\
\hline H52253 & IGHG3 & $\underline{3502}$ & $\begin{array}{l}\text { immunoglobulin heavy constant gamma } 3 \\
\text { (G3m marker) }\end{array}$ \\
\hline $\mathrm{H} 27034$ & IGKC & $\underline{3514}$ & immunoglobulin kappa constant \\
\hline H83003 & IGSF1 & 3547 & immunoglobulin superfamily, member 1 \\
\hline AA058632 & KIF1B & 23095 & kinesin family member 1B \\
\hline N39407 & KIF21A & 55605 & kinesin family member 21A \\
\hline R90757 & RPH3A & 22895 & likely ortholog of mouse rabphilin $3 \mathrm{~A}$ \\
\hline R89056 & LAMP1 & $\underline{3916}$ & lysosomal-associated membrane protein 1 \\
\hline $\mathrm{H} 47026$ & MGAT3 & $\underline{4248}$ & $\begin{array}{l}\text { mannosyl (beta-1,4-)-glycoprotein beta-1,4- } \\
\mathrm{N} \text {-acetylglucosaminyltransferase }\end{array}$ \\
\hline N40017 & MRPL24 & 79590 & mitochondrial ribosomal protein L24 \\
\hline N78467 & PWP1 & 11137 & $\begin{array}{l}\text { nuclear phosphoprotein similar to S. } \\
\text { cerevisiae PWP1 }\end{array}$ \\
\hline H69656 & NARF & $\underline{26502}$ & nuclear prelamin A recognition factor \\
\hline H51160 & PPP2R1A & $\underline{5518}$ & $\begin{array}{l}\text { protein phosphatase } 2 \text { (formerly } 2 A) \text {, } \\
\text { regulatory subunit } A(P R 65) \text {, alpha isoform }\end{array}$ \\
\hline AA045373 & TCEAL1 & $\underline{9338}$ & transcription elongation factor $\mathrm{A}$ (SII)-like 1 \\
\hline R90824 & TMEM10 & 93377 & transmembrane protein 10 \\
\hline $\mathrm{H} 27352$ & $\underline{\text { HRAS }}$ & $\underline{3265}$ & $\begin{array}{l}\text { v-Ha-ras Harvey rat sarcoma viral oncogene } \\
\text { homolog }\end{array}$ \\
\hline \multicolumn{4}{|l|}{$\mathrm{H} 09945$} \\
\hline \multicolumn{4}{|l|}{ H18298 } \\
\hline H18495 & & & \\
\hline
\end{tabular}




\begin{tabular}{|l|l|l|l|}
\hline$H 29730$ & & & \\
\hline$H 45355$ & & & \\
\hline$H 62766$ & & & \\
\hline$H 62770$ & & & \\
\hline$H 65775$ & & & \\
\hline$H 86672$ & & & \\
\hline N45013 & & & \\
\hline R07186 & & & \\
\hline R41363 & & & \\
\hline R85333 & & & \\
\hline
\end{tabular}


Appendix F. Genes significantly different between 15m MPP+ \& 15m MPP+ with PTIO.

\begin{tabular}{|c|c|c|c|c|c|}
\hline GENBANK & PVALUE & SYMBOL & $\begin{array}{c}\text { LOCUS } \\
\text { LINK }\end{array}$ & $\begin{array}{l}\text { GENE } \\
\text { NAME }\end{array}$ & GENE ONTOLOGY \\
\hline $\mathrm{H} 01987$ & $8.26 \mathrm{E}-10$ & MLL3 & $\underline{58508}$ & \multicolumn{2}{|c|}{ myeloid/lymphoid or mixed-lineage leukemia3 } \\
\hline H84325 & $1.88 \mathrm{E}-09$ & PBX3 & $\underline{5090}$ & $\begin{array}{l}\text { pre-B-cell } \\
\text { leukemia } \\
\text { transcriptio } \\
\text { n factor } 3\end{array}$ & $\begin{array}{l}\text { DNA binding; anterior compartment } \\
\text { specification; oncogenesis; posterior } \\
\text { compartment specification }\end{array}$ \\
\hline R37089 & $2.11 \mathrm{E}-09$ & PABPC1 & 26986 & $\begin{array}{l}\text { poly }(\mathrm{A}) \\
\text { binding } \\
\text { protein, } \\
\text { cytoplasmi } \\
\text { c } 1\end{array}$ & $\begin{array}{l}\text { RNA binding; cytoplasm; mRNA } \\
\text { polyadenylation; poly }(\mathrm{A}) \text { binding }\end{array}$ \\
\hline R09196 & $2.50 \mathrm{E}-09$ & & & & \\
\hline H71562 & $3.80 \mathrm{E}-09$ & \begin{tabular}{|l} 
LOC2213 \\
03
\end{tabular} & $\underline{221303}$ & $\begin{array}{l}\text { hypothetic } \\
\text { al protein } \\
\text { LOC22130 } \\
3\end{array}$ & \\
\hline R26954 & $5.64 \mathrm{E}-08$ & $\underline{\text { CTSD }}$ & $\underline{1509}$ & $\begin{array}{l}\text { cathepsin } \\
D \\
\text { (lysosomal } \\
\text { aspartyl } \\
\text { protease) } \\
\end{array}$ & $\begin{array}{l}\text { cathepsin D activity; hydrolase activity; } \\
\text { lysosome; pepsin A activity; } \\
\text { proteolysis and peptidolysis }\end{array}$ \\
\hline R07461 & 7.99E-08 & & & & \\
\hline R01950 & 8.77E-08 & & & & \\
\hline H80104 & 1.20E-07 & & & & \\
\hline AA047309 & $1.30 \mathrm{E}-07$ & LAMC2 & $\underline{3918}$ & 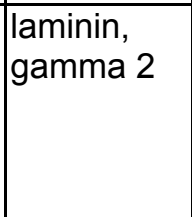 & $\begin{array}{l}\text { basement membrane; cell adhesion; } \\
\text { cell adhesion molecule activity; } \\
\text { epidermal differentiation; heparin } \\
\text { binding; kinesin complex; laminin-5; } \\
\text { structural molecule activity }\end{array}$ \\
\hline R66080 & $2.65 \mathrm{E}-07$ & KIAA1530 & $\underline{57654}$ & $\begin{array}{l}\text { KIAA1530 } \\
\text { protein }\end{array}$ & \\
\hline H09869 & $3.24 \mathrm{E}-07$ & GNAI2 & $\underline{2771}$ & $\begin{array}{l}\text { guanine } \\
\text { nucleotide } \\
\text { binding } \\
\text { protein (G } \\
\text { protein), } \\
\text { alpha } \\
\text { inhibiting } \\
\text { activity } \\
\text { polypeptid } \\
\text { e } 2\end{array}$ & $\begin{array}{l}\text { G-protein coupled receptor protein } \\
\text { signaling pathway; GTP binding; } \\
\text { heterotrimeric G-protein GTPase } \\
\text { activity; negative regulation of } \\
\text { adenylate cyclase activity; response to } \\
\text { nutrients; signal transducer activity; } \\
\text { signal transduction }\end{array}$ \\
\hline W32999 & $3.75 \mathrm{E}-07$ & RPS26 & 6231 & $\begin{array}{l}\text { ribosomal } \\
\text { protein } \\
\text { S26 }\end{array}$ & $\begin{array}{l}\text { RNA binding; cytosolic small ribosomal } \\
\text { subunit (sensu Eukarya); intracellular; } \\
\text { protein biosynthesis; ribosome; } \\
\text { structural constituent of ribosome }\end{array}$ \\
\hline
\end{tabular}




\begin{tabular}{|c|c|c|c|c|c|}
\hline R99604 & 4.53E-07 & & & & \\
\hline W19744 & 8.06E-07 & & & & \\
\hline T96291 & 8.09E-07 & & & & \\
\hline AA136363 & $9.79 \mathrm{E}-07$ & & & & \\
\hline N47077 & 1.27E-06 & FLJ38973 & $\underline{205327}$ & $\begin{array}{l}\text { hypothetic } \\
\text { al protein } \\
\text { FLJ38973 }\end{array}$ & \\
\hline R55491 & 1.30E-06 & & & & \\
\hline N92911 & $1.62 \mathrm{E}-06$ & DJ473B4 & $\underline{56180}$ & $\begin{array}{l}\text { hypothetic } \\
\text { al protein } \\
\text { dJ473B4 } \\
\end{array}$ & structural molecule activity \\
\hline R07186 & 2.21E-06 & & & & \\
\hline AA037284 & 2.26E-06 & APRT & $\underline{353}$ & $\begin{array}{l}\text { adenine } \\
\text { phosphorib } \\
\text { Osyltransfe } \\
\text { rase }\end{array}$ & $\begin{array}{l}\text { adenine phosphoribosyltransferase } \\
\text { activity; adenine salvage pathway; } \\
\text { nucleoside metabolism; transferase } \\
\text { activity, transferring glycosyl groups }\end{array}$ \\
\hline H83488 & $2.48 \mathrm{E}-06$ & & & & \\
\hline R72441 & $3.24 \mathrm{E}-06$ & KIAA1223 & $\underline{57182}$ & $\begin{array}{l}\text { KIAA1223 } \\
\text { protein }\end{array}$ & \\
\hline H59405 & $3.27 \mathrm{E}-06$ & FLJ10298 & $\underline{54682}$ & $\begin{array}{l}\text { hypothetic } \\
\text { al protein } \\
\text { FLJ10298 }\end{array}$ & \\
\hline AA031913 & 3.34E-06 & $\frac{\text { LOC 1238 }}{\underline{03}}$ & $\underline{123803}$ & $\begin{array}{l}\text { N-terminal } \\
\text { Asn } \\
\text { amidase }\end{array}$ & \\
\hline H51834 & 3.35E-06 & TTC1 & $\underline{7265}$ & \begin{tabular}{|l|} 
tetratricope \\
ptide \\
repeat \\
domain 1 \\
\end{tabular} & $\begin{array}{l}\text { chaperone activity; protein binding; } \\
\text { protein folding }\end{array}$ \\
\hline N64198 & 3.62E-06 & & & & \\
\hline R91005 & 5.68E-06 & & & & \\
\hline T86338 & 5.85E-06 & & & & \\
\hline R87345 & 5.98E-06 & MGC2656 & $\underline{79414}$ & $\begin{array}{l}\text { hypothetic } \\
\text { al protein } \\
\text { MGC2656 }\end{array}$ & \\
\hline R68921 & 6.03E-06 & XPO1 & $\underline{7514}$ & \begin{tabular}{|l} 
exportin 1 \\
(CRM1 \\
homolog, \\
yeast)
\end{tabular} & $\begin{array}{l}\text { cytoplasm; nuclear pore; nucleoplasm; } \\
\text { protein transporter activity; protein- } \\
\text { nucleus import, docking }\end{array}$ \\
\hline AA058661 & $6.29 \mathrm{E}-06$ & & & & \\
\hline R81337 & 6.57E-06 & $\frac{\text { LOC5115 }}{9}$ & $\underline{51159}$ & $\begin{array}{l}\text { colon } \\
\text { carcinoma } \\
\text { related } \\
\text { protein } \\
\end{array}$ & \\
\hline R39516 & 7.79E-06 & $\frac{\text { DKFZP76 }}{1 \text { 1N09121 }}$ & $\underline{57183}$ & \begin{tabular}{|l} 
hypothetic \\
al protein \\
DKFZp761 \\
N09121 \\
\end{tabular} & \\
\hline R08412 & 8.01E-06 & & & & \\
\hline H83464 & 8.49E-06 & & & & \\
\hline
\end{tabular}




\begin{tabular}{|c|c|c|c|c|c|}
\hline T85114 & $9.33 \mathrm{E}-06$ & DEF6 & $\underline{50619}$ & $\begin{array}{l}\text { differentially } \\
\text { (mouse) }\end{array}$ & y expressed in FDCP 6 homolog \\
\hline R99892 & $9.58 \mathrm{E}-06$ & & & & \\
\hline H06382 & $1.01 \mathrm{E}-05$ & & & & \\
\hline R76163 & 1.15E-05 & $Z Y X$ & 7791 & zyxin & $\begin{array}{l}\text { cell adhesion; cell adhesion molecule } \\
\text { activity; cell-cell signaling; focal } \\
\text { adhesion; integral to plasma } \\
\text { membrane; plasma membrane; signal } \\
\text { transduction }\end{array}$ \\
\hline N33577 & $1.23 \mathrm{E}-05$ & & $\underline{348396}$ & $\begin{array}{l}\text { similar to } \\
\text { hypothetic } \\
\text { al protein } \\
\text { FLJ20489 }\end{array}$ & \\
\hline W53003 & 1.51E-05 & RNASEL & 6041 & $\begin{array}{l}\text { ribonuclea } \\
\text { se L }\left(2 ', 5^{\prime}-\right. \\
\text { oligoisoade } \\
\text { nylate } \\
\text { synthetase } \\
- \\
\text { dependent }) \\
\end{array}$ & $\begin{array}{l}\text { ATP binding; RNA binding; } \\
\text { cellular_component unknown; } \\
\text { endoribonuclease activity; hydrolase } \\
\text { activity; protein amino acid } \\
\text { phosphorylation; protein kinase activity }\end{array}$ \\
\hline R10993 & 1.53E-05 & & & & \\
\hline H83987 & $1.57 \mathrm{E}-05$ & & & & \\
\hline AA121937 & 1.72E-05 & & & & \\
\hline R18850 & 1.74E-05 & TTYH1 & $\underline{57348}$ & $\begin{array}{l}\text { tweety } \\
\text { homolog } 1 \\
\text { (Drosophil } \\
\text { a) }\end{array}$ & $\begin{array}{l}\text { integral to membrane; iron ion } \\
\text { transport; iron ion transporter activity }\end{array}$ \\
\hline N90246 & 1.75E-05 & EPHA1 & $\underline{2041}$ & EphA1 & $\begin{array}{l}\text { ATP binding; ephrin receptor activity; } \\
\text { integral to plasma membrane; protein } \\
\text { amino acid phosphorylation; receptor } \\
\text { activity; signal transduction; } \\
\text { transferase activity; transmembrane } \\
\text { receptor protein tyrosine kinase } \\
\text { signaling pathway }\end{array}$ \\
\hline H47346 & 1.86E-05 & $\underline{\mathrm{KMO}}$ & 8564 & $\begin{array}{l}\text { kynurenine } \\
3- \\
\text { monooxyg } \\
\text { enase } \\
\text { (kynurenin } \\
\text { e 3- } \\
\text { hydroxylas } \\
\text { e) }\end{array}$ & $\begin{array}{l}\text { aromatic compound metabolism; } \\
\text { electron transport; electron transporter } \\
\text { activity; kynurenine 3-monooxygenase } \\
\text { activity }\end{array}$ \\
\hline H87118 & 1.98E-05 & FUT4 & 2526 & $\begin{array}{l}\text { fucosyltran } \\
\text { sferase } 4 \\
\text { (alpha } \\
(1,3) \\
\text { fucosyltran } \\
\text { sferase, } \\
\text { myeloid- } \\
\text { specific) } \\
\end{array}$ & $\begin{array}{l}\text { Golgi apparatus; carbohydrate } \\
\text { metabolism; fucosyltransferase } \\
\text { activity; integral to membrane; } \\
\text { membrane fraction; protein amino acid } \\
\text { glycosylation; transferase activity, } \\
\text { transferring glycosyl groups }\end{array}$ \\
\hline T51698 & 1.99E-05 & & & & \\
\hline R26598 & $2.48 \mathrm{E}-05$ & & & & \\
\hline
\end{tabular}




\begin{tabular}{|c|c|c|c|c|c|}
\hline T72012 & $2.87 \mathrm{E}-05$ & SFTPA2 & $\underline{6436}$ & $\begin{array}{l}\text { surfactant, } \\
\text { pulmonary- } \\
\text { associated } \\
\text { protein A2 }\end{array}$ & $\begin{array}{l}\text { extracellular space; heterophilic cell } \\
\text { adhesion; lipid transporter activity; } \\
\text { respiratory gaseous exchange; sugar } \\
\text { binding; surfactant activity }\end{array}$ \\
\hline H50015 & $3.10 \mathrm{E}-05$ & & & & \\
\hline AA047259 & $3.11 \mathrm{E}-05$ & EBAF & $\underline{7044}$ & $\begin{array}{l}\text { endometria } \\
\text { I bleeding } \\
\text { associated } \\
\text { factor (left- } \\
\text { right } \\
\text { determinati } \\
\text { on, factor } \\
\text { A; } \\
\text { transformin } \\
\text { g growth } \\
\text { factor beta } \\
\text { superfamil } \\
\text { y) } \\
\end{array}$ & $\begin{array}{l}\text { TGFbeta receptor signaling pathway; } \\
\text { cell growth; cell-cell signaling; } \\
\text { development; oocyte axis } \\
\text { determination; transforming growth } \\
\text { factor-beta receptor binding }\end{array}$ \\
\hline R15444 & $3.13 \mathrm{E}-05$ & $\begin{array}{l}\text { LOC3482 } \\
\underline{35}\end{array}$ & $\underline{348235}$ & $\begin{array}{l}\text { hypothetic } \\
\text { al protein } \\
\text { LOC34823 } \\
5\end{array}$ & \\
\hline $\mathrm{H} 49830$ & 3.18E-05 & & & & \\
\hline AA027831 & 3.53E-05 & & & & \\
\hline R40412 & 3.55E-05 & CTNND2 & 1501 & $\begin{array}{l}\text { catenin } \\
\text { (cadherin- } \\
\text { associated } \\
\text { protein), } \\
\text { delta 2 } \\
\text { (neural } \\
\text { plakophilin- } \\
\text { related } \\
\text { arm-repeat } \\
\text { protein) } \\
\end{array}$ & $\begin{array}{l}\text { cell adhesion; cell adhesion molecule } \\
\text { activity; cytoskeleton; development; } \\
\text { kinesin complex; neuronal cell } \\
\text { adhesion; structural molecule activity } \\
\end{array}$ \\
\hline AA045130 & $3.62 \mathrm{E}-05$ & FLJ10761 & $\underline{55224}$ & $\begin{array}{l}\text { hypothetic } \\
\text { al protein } \\
\text { FLJ10761 }\end{array}$ & $\begin{array}{l}\text { biological_process unknown; } \\
\text { cellular_component unknown; choline } \\
\text { kinase activity; transferase activity }\end{array}$ \\
\hline $\mathrm{H} 03447$ & 3.67E-05 & & & & \\
\hline T78085 & 3.77E-05 & $\begin{array}{l}\text { LOC2000 } \\
\underline{08}\end{array}$ & 200008 & $\begin{array}{l}\text { hypothetic } \\
\text { al protein } \\
\text { LOC20000 } \\
8\end{array}$ & $\begin{array}{l}\text { electron transport; oxidoreductase } \\
\text { activity }\end{array}$ \\
\hline H21648 & 3.98E-05 & & & & \\
\hline AA203189 & $4.03 E-05$ & TSP50 & 29122 & $\begin{array}{l}\text { testes- } \\
\text { specific } \\
\text { protease } \\
50\end{array}$ & $\begin{array}{l}\text { chymotrypsin activity; peptidase } \\
\text { activity; proteolysis and peptidolysis; } \\
\text { trypsin activity }\end{array}$ \\
\hline H45241 & $4.15 \mathrm{E}-05$ & RPL41 & $\underline{6171}$ & $\begin{array}{l}\text { ribosomal } \\
\text { protein L41 }\end{array}$ & $\begin{array}{l}\text { RNA binding; cytosolic large ribosomal } \\
\text { subunit (sensu Eukarya); protein } \\
\text { biosynthesis; structural constituent of } \\
\text { ribosome }\end{array}$ \\
\hline N34437 & 4.19E-05 & CAS1 & 64921 & O- & \\
\hline
\end{tabular}




\begin{tabular}{|c|c|c|c|c|c|}
\hline & & & & $\begin{array}{l}\text { acetyltrans } \\
\text { ferase }\end{array}$ & \\
\hline T87319 & $4.25 \mathrm{E}-05$ & C6orf56 & 9749 & $\begin{array}{l}\text { chromoso } \\
\text { me } 6 \text { open } \\
\text { reading } \\
\text { frame } 56\end{array}$ & \\
\hline R14112 & 4.27E-05 & $\underline{\text { CYP1A1 }}$ & 1543 & $\begin{array}{l}\text { cytochrom } \\
\text { e P450, } \\
\text { family } 1, \\
\text { subfamily } \\
\text { A, } \\
\text { polypeptid } \\
\text { e } 1\end{array}$ & $\begin{array}{l}\text { cytochrome P450 activity; electron } \\
\text { transport; endoplasmic reticulum; } \\
\text { membrane; microsome; } \\
\text { monooxygenase activity; } \\
\text { oxidoreductase activity, acting on } \\
\text { paired donors, with incorporation or } \\
\text { reduction of molecular oxygen, } \\
\text { reduced flavin or flavoprotein as one } \\
\text { donor, and incorporation of one atom } \\
\text { of oxygen }\end{array}$ \\
\hline R12622 & 4.29E-05 & FTSJ2 & $\underline{29960}$ & \begin{tabular}{|l|} 
FtsJ \\
homolog 2 \\
(E. coli)
\end{tabular} & $\begin{array}{l}\text { methyltransferase activity; nucleus; } \\
\text { rRNA processing; transferase activity }\end{array}$ \\
\hline AA099441 & 4.65E-05 & NUCB1 & $\underline{4924}$ & $\begin{array}{l}\text { nucleobindi } \\
\text { n } 1\end{array}$ & $\begin{array}{l}\text { DNA binding; Golgi apparatus; calcium } \\
\text { ion binding; extracellular space }\end{array}$ \\
\hline R52315 & 4.65E-05 & OCA2 & $\underline{4948}$ & \begin{tabular}{|l|} 
oculocutan \\
eous \\
albinism II \\
(pink-eye \\
dilution \\
homolog, \\
mouse) \\
\end{tabular} & $\begin{array}{l}\text { L-tyrosine transporter activity; } \\
\text { cytoplasm; eye pigment biosynthesis; } \\
\text { eye pigment precursor transporter } \\
\text { activity; integral to membrane; } \\
\text { transporter activity }\end{array}$ \\
\hline R26526 & 4.67E-05 & BNC & $\underline{646}$ & basonuclin & $\begin{array}{l}\text { epidermal differentiation; nucleus; } \\
\text { positive regulation of cell proliferation; } \\
\text { regulation of transcription, DNA- } \\
\text { dependent; transcription factor activity }\end{array}$ \\
\hline AA004424 & 4.74E-05 & & & & \\
\hline R07137 & $4.81 \mathrm{E}-05$ & $\mathrm{HIC2}$ & $\underline{23119}$ & $\begin{array}{l}\text { hypermeth } \\
\text { ylated in } \\
\text { cancer } 2\end{array}$ & $\begin{array}{l}\text { DNA binding; negative regulation of } \\
\text { transcription, DNA-dependent; } \\
\text { nucleus; protein C-terminus binding }\end{array}$ \\
\hline H68587 & 4.89E-05 & & $\underline{340833}$ & $\begin{array}{l}\mathrm{LOC} 34083 \\
3\end{array}$ & \\
\hline R00907 & 4.91E-05 & PLEKHG1 & 57480 & $\begin{array}{l}\text { pleckstrin he } \\
\text { (with RhoGe }\end{array}$ & $\begin{array}{l}\text { omology domain containing, family } G \\
\text { ef domain) member } 1\end{array}$ \\
\hline T80564 & 5.11E-05 & NMNAT2 & 23057 & nicotinamide & e nucleotide adenylyltransferase 2 \\
\hline AA042800 & $5.17 \mathrm{E}-05$ & & & & \\
\hline $\mathrm{H} 48570$ & 5.37E-05 & & & & \\
\hline H39927 & 5.71E-05 & & & & \\
\hline R18927 & 5.76E-05 & & & & \\
\hline T86133 & 5.95E-05 & & & & \\
\hline
\end{tabular}




\begin{tabular}{|c|c|c|c|c|c|}
\hline $\mathrm{H} 46116$ & $5.96 \mathrm{E}-05$ & GNG13 & $\underline{51764}$ & \begin{tabular}{|l|} 
guanine \\
nucleotide \\
binding \\
protein (G \\
protein), \\
gamma 13 \\
\end{tabular} & $\begin{array}{l}\text { G-protein coupled receptor protein } \\
\text { signaling pathway; heterotrimeric G- } \\
\text { protein GTPase activity; heterotrimeric } \\
\text { G-protein complex; signal transducer } \\
\text { activity; signal transduction }\end{array}$ \\
\hline $\mathrm{H} 12333$ & 6.00E-05 & TFPI2 & $\underline{7980}$ & $\begin{array}{l}\text { tissue } \\
\text { factor } \\
\text { pathway } \\
\text { inhibitor } 2\end{array}$ & $\begin{array}{l}\text { blood coagulation; extracellular matrix; } \\
\text { extracellular matrix structural } \\
\text { constituent; serine protease inhibitor } \\
\text { activity }\end{array}$ \\
\hline R24299 & 6.03E-05 & $\frac{\text { Rab11- }}{\text { FIP3 }}$ & $\underline{9727}$ & $\begin{array}{l}\text { KIAA0665 } \\
\text { gene } \\
\text { product }\end{array}$ & $\begin{array}{l}\text { Rab interactor activity; calcium ion } \\
\text { binding }\end{array}$ \\
\hline $\mathrm{H} 10851$ & 6.08E-05 & & & & \\
\hline W52537 & $6.17 \mathrm{E}-05$ & PSMA2 & $\underline{5683}$ & $\begin{array}{l}\text { proteasom } \\
\text { e } \\
\text { (prosome, } \\
\text { macropain) } \\
\text { subunit, } \\
\text { alpha type, } \\
2 \\
\end{array}$ & $\begin{array}{l}\text { 26S proteasome; cytosol; } \\
\text { endopeptidase activity; proteasome } \\
\text { core complex (sensu Eukarya); } \\
\text { proteasome endopeptidase activity; } \\
\text { ubiquitin-dependent protein catabolism }\end{array}$ \\
\hline R24727 & $6.17 \mathrm{E}-05$ & PTDSR & $\underline{23210}$ & \begin{tabular}{|l|} 
phosphatid \\
ylserine \\
receptor
\end{tabular} & \\
\hline $\mathrm{H} 24375$ & $6.26 \mathrm{E}-05$ & & & & \\
\hline R56097 & $6.47 \mathrm{E}-05$ & $\underline{P 29}$ & $\underline{25949}$ & $\begin{array}{l}\text { GCIP- } \\
\text { interacting } \\
\text { protein p29 }\end{array}$ & $\begin{array}{l}\text { biological_process unknown; nucleus; } \\
\text { protein binding }\end{array}$ \\
\hline W32180 & 6.49E-05 & & & & \\
\hline R62213 & 7.78E-05 & & & & \\
\hline T99071 & 7.94E-05 & & & & \\
\hline R37498 & 8.01E-05 & RALB & $\underline{5899}$ & $\begin{array}{l}\text { V-ral } \\
\text { simian } \\
\text { leukemia } \\
\text { viral } \\
\text { oncogene } \\
\text { homolog B } \\
\text { (ras } \\
\text { related; } \\
\text { GTP } \\
\text { binding } \\
\text { protein) }\end{array}$ & $\begin{array}{l}\text { GTP binding; RAS small monomeric } \\
\text { GTPase activity; signal transduction; } \\
\text { small GTPase mediated signal } \\
\text { transduction }\end{array}$ \\
\hline H98856 & $8.45 E-05$ & TCF12 & $\underline{6938}$ & $\begin{array}{l}\text { transcriptio } \\
\text { n factor } 12 \\
\text { (HTF4, } \\
\text { helix-loop- } \\
\text { helix } \\
\text { transcriptio } \\
\text { n factors 4) } \\
\end{array}$ & $\begin{array}{l}\text { DNA binding; RNA polymerase II } \\
\text { transcription factor activity; } \\
\text { development; immune response; } \\
\text { muscle development; nucleus; } \\
\text { regulation of transcription from Pol II } \\
\text { promoter }\end{array}$ \\
\hline $\mathrm{H} 26552$ & 8.54E-05 & MGC5395 & $\underline{79026}$ & $\begin{array}{l}\text { hypothetic } \\
\text { al protein } \\
\text { MGC5395 }\end{array}$ & intracellular signaling cascade \\
\hline
\end{tabular}




\begin{tabular}{|c|c|c|c|c|c|}
\hline W31358 & 8.63E-05 & & & & \\
\hline R49740 & $8.75 \mathrm{E}-05$ & FBX021 & $\underline{23014}$ & $\begin{array}{l}\text { F-box only } \\
\text { protein } 21\end{array}$ & \\
\hline H81214 & 8.99E-05 & & & & \\
\hline H68203 & $9.18 \mathrm{E}-05$ & & & & \\
\hline R20373 & 9.30E-05 & TMP21 & 10972 & $\begin{array}{l}\text { transmemb } \\
\text { rane } \\
\text { trafficking } \\
\text { protein }\end{array}$ & $\begin{array}{l}\text { ER to Golgi transport; Golgi apparatus; } \\
\text { integral to plasma membrane; } \\
\text { intracellular protein transport; } \\
\text { membrane fraction; microsome; } \\
\text { protein carrier activity; protein } \\
\text { transporter activity }\end{array}$ \\
\hline AA033795 & 9.32E-05 & & & & \\
\hline W47002 & $9.56 \mathrm{E}-05$ & NME2 & $\underline{4831}$ & $\begin{array}{l}\text { non- } \\
\text { metastatic } \\
\text { cells 2, } \\
\text { protein } \\
\text { (NM23B) } \\
\text { expressed } \\
\text { in }\end{array}$ & $\begin{array}{l}\text { ATP binding; CTP biosynthesis; GTP } \\
\text { biosynthesis; UTP biosynthesis; } \\
\text { kinase activity; negative regulation of } \\
\text { cell cycle; negative regulation of cell } \\
\text { proliferation; nucleoside triphosphate } \\
\text { biosynthesis; nucleoside-diphosphate } \\
\text { kinase activity; nucleus; regulation of } \\
\text { transcription, DNA-dependent; } \\
\text { transcription factor activity; transferase } \\
\text { activity }\end{array}$ \\
\hline W91885 & 9.85E-05 & & & & \\
\hline W68050 & 9.91E-05 & LGALS1 & $\underline{3956}$ & $\begin{array}{l}\text { lectin, } \\
\text { galactoside } \\
\text {-binding, } \\
\text { soluble, 1 } \\
\text { (galectin 1) }\end{array}$ & $\begin{array}{l}\text { apoptosis; heterophilic cell adhesion; } \\
\text { sugar binding }\end{array}$ \\
\hline H06803 & $\begin{array}{r}0.000102 \\
328 \\
\end{array}$ & & & & \\
\hline H73928 & $\begin{array}{r}0.000104 \\
464 \\
\end{array}$ & SEC61B & 10952 & $\begin{array}{l}\text { protein } \\
\text { translocati } \\
\text { on } \\
\text { complex } \\
\text { beta } \\
\end{array}$ & $\begin{array}{l}\text { endoplasmic reticulum; integral to } \\
\text { membrane; nonselective vesicle } \\
\text { transport; protein targeting; protein } \\
\text { translocase activity }\end{array}$ \\
\hline H10123 & $\begin{array}{r}0.000106 \\
775 \\
\end{array}$ & $\underline{\text { SHREW1 }}$ & $\underline{55966}$ & \begin{tabular}{|l|} 
transmemb \\
rane \\
protein \\
SHREW1 \\
\end{tabular} & metabolism; oxidoreductase activity \\
\hline R13021 & \begin{tabular}{|r|}
0.000108 \\
34
\end{tabular} & FLJ10751 & 55222 & $\begin{array}{l}\text { hypothetic } \\
\text { al protein } \\
\text { FLJ10751 }\end{array}$ & \\
\hline H86049 & $\begin{array}{r}0.000112 \\
712 \\
\end{array}$ & & & & \\
\hline W38655 & $\begin{array}{r}0.000113 \\
189\end{array}$ & GJA1 & 2697 & $\begin{array}{l}\text { gap } \\
\text { junction } \\
\text { protein, } \\
\text { alpha 1, } \\
43 k D a \\
\text { (connexin } \\
43 \text { ) } \\
\end{array}$ & $\begin{array}{l}\text { cell-cell signaling; connexon channel } \\
\text { activity; connexon complex; hearing; } \\
\text { heart development; integral to plasma } \\
\text { membrane; ion transporter activity; } \\
\text { muscle contraction; transport }\end{array}$ \\
\hline R41480 & 0.000116 & EFCBP1 & 64168 & EF hand & calcium ion binding \\
\hline
\end{tabular}




\begin{tabular}{|c|c|c|c|c|c|}
\hline & 081 & & & \begin{tabular}{|l} 
calcium \\
binding \\
protein 1
\end{tabular} & \\
\hline T75124 & $\begin{array}{r}0.000124 \\
039\end{array}$ & CGI-67 & $\underline{51104}$ & $\begin{array}{l}\text { CGI-67 } \\
\text { protein }\end{array}$ & \\
\hline R62986 & $\begin{array}{r}0.000129 \\
157\end{array}$ & & & & \\
\hline AA039600 & $\begin{array}{r}0.000129 \\
217\end{array}$ & FGFR4 & $\underline{2264}$ & $\begin{array}{l}\text { fibroblast } \\
\text { growth } \\
\text { factor } \\
\text { receptor } 4\end{array}$ & $\begin{array}{l}\text { ATP binding; FGF receptor signaling } \\
\text { pathway; fibroblast growth factor } \\
\text { receptor activity; integral to plasma } \\
\text { membrane; protein amino acid } \\
\text { phosphorylation; protein-tyrosine } \\
\text { kinase activity; receptor activity; } \\
\text { transferase activity }\end{array}$ \\
\hline H02012 & \begin{tabular}{|r|}
0.000129 \\
857
\end{tabular} & & & & \\
\hline R55379 & \begin{tabular}{|r|}
0.000130 \\
606
\end{tabular} & TNFSF12 & 8742 & $\begin{array}{l}\text { tumor } \\
\text { necrosis } \\
\text { factor } \\
\text { (ligand) } \\
\text { superfamil } \\
\text { y, member } \\
12 \\
\end{array}$ & $\begin{array}{l}\text { angiogenesis; immune response; } \\
\text { induction of apoptosis; integral to } \\
\text { plasma membrane; signal } \\
\text { transduction; tumor necrosis factor } \\
\text { receptor binding }\end{array}$ \\
\hline R68004 & \begin{tabular}{|r|}
0.000134 \\
931
\end{tabular} & PCBP2 & $\underline{5094}$ & \begin{tabular}{|l} 
poly $(\mathrm{rC})$ \\
binding \\
protein 2
\end{tabular} & $\begin{array}{l}\text { DNA binding; RNA binding; cytoplasm; } \\
\text { mRNA metabolism; nucleus; } \\
\text { ribonucleoprotein complex }\end{array}$ \\
\hline R36127 & $\begin{array}{r}0.000137 \\
083\end{array}$ & FLNB & $\underline{2317}$ & $\begin{array}{l}\text { filamin } B \\
\text { beta (actin } \\
\text { binding } \\
\text { protein } \\
\text { 278) }\end{array}$ & $\begin{array}{l}\text { actin binding; actin cytoskeleton; actin } \\
\text { cytoskeleton organization and } \\
\text { biogenesis; cytoskeletal anchoring; } \\
\text { integral to plasma membrane; } \\
\text { membrane associated actin binding; } \\
\text { signal transduction }\end{array}$ \\
\hline H67193 & \begin{tabular}{|r|}
0.000143 \\
814 \\
\end{tabular} & EIF2S3 & 1968 & $\begin{array}{l}\text { eukaryotic } \\
\text { translation } \\
\text { initiation } \\
\text { factor } 2 \\
\text { subunit } 3 \\
\text { gamma, } \\
52 \mathrm{kDa} \\
\end{array}$ & $\begin{array}{l}\text { GTPase activity; cytosolic small } \\
\text { ribosomal subunit (sensu Eukarya); } \\
\text { eukaryotic translation initiation factor } 2 \\
\text { complex; translation elongation factor } \\
\text { activity; translation initiation factor } \\
\text { activity; translational elongation }\end{array}$ \\
\hline R22402 & $\begin{array}{r}0.000145 \\
207 \\
\end{array}$ & & & & \\
\hline AA040852 & $\begin{array}{r}0.000146 \\
241\end{array}$ & KIAA1321 & 57532 & $\begin{array}{l}\text { KIAA1321 } \\
\text { protein }\end{array}$ & \\
\hline R38712 & \begin{tabular}{|r|}
0.000152 \\
754 \\
\end{tabular} & & & & \\
\hline N30228 & \begin{tabular}{|r|}
0.000155 \\
195 \\
\end{tabular} & KIAA1432 & $\underline{57589}$ & $\begin{array}{l}\text { KIAA1432 } \\
\text { protein }\end{array}$ & \\
\hline R83199 & $\begin{array}{r}0.000155 \\
413 \\
\end{array}$ & & & & \\
\hline
\end{tabular}




\begin{tabular}{|c|c|c|c|c|c|}
\hline $\mathrm{H} 68718$ & \begin{tabular}{|r|}
0.000158 \\
676 \\
\end{tabular} & ROCK1 & $\underline{6093}$ & $\begin{array}{l}\text { Rho- } \\
\text { associated, } \\
\text { coiled-coil } \\
\text { containing } \\
\text { protein } \\
\text { kinase 1 }\end{array}$ & $\begin{array}{l}\text { ATP binding; Rho protein signal } \\
\text { transduction; actin cytoskeleton } \\
\text { organization and biogenesis; } \\
\text { intracellular; intracellular signaling } \\
\text { cascade; protein amino acid } \\
\text { phosphorylation; protein } \\
\text { serine/threonine kinase activity; } \\
\text { protein-tyrosine kinase activity; } \\
\text { transferase activity }\end{array}$ \\
\hline H02328 & $\begin{array}{r}0.000163 \\
034\end{array}$ & $\underline{\mathrm{SLC} 2 \mathrm{~A} 1}$ & $\underline{6513}$ & $\begin{array}{l}\text { solute } \\
\text { carrier } \\
\text { family } 2 \\
\text { (facilitated } \\
\text { glucose } \\
\text { transporter } \\
\text { ), member } \\
1\end{array}$ & $\begin{array}{l}\text { carbohydrate transport; glucose } \\
\text { transport; glucose transporter activity; } \\
\text { integral to membrane; membrane } \\
\text { fraction; sugar porter activity; } \\
\text { transporter activity }\end{array}$ \\
\hline R43191 & \begin{tabular}{|r|}
0.000163 \\
622 \\
\end{tabular} & & & & \\
\hline R85093 & $\begin{array}{r}0.000165 \\
446 \\
\end{array}$ & & & & \\
\hline H20379 & $\begin{array}{r}0.000166 \\
734\end{array}$ & WDR22 & $\underline{8816}$ & $\begin{array}{l}\text { WD repeat } \\
\text { domain } 22\end{array}$ & oncogenesis \\
\hline T65261 & $\begin{array}{r}0.000168 \\
202 \\
\end{array}$ & & & & \\
\hline $\mathrm{H} 05660$ & \begin{tabular}{|r|}
0.000168 \\
597
\end{tabular} & C18orf1 & $\underline{753}$ & $\begin{array}{l}\text { chromoso } \\
\text { me } 18 \\
\text { open } \\
\text { reading } \\
\text { frame } 1 \\
\end{array}$ & $\begin{array}{l}\text { biological_process unknown; integral } \\
\text { to membrane; molecular_function } \\
\text { unknown }\end{array}$ \\
\hline R51354 & $\begin{array}{r}0.000171 \\
049 \\
\end{array}$ & $\begin{array}{l}\text { CNTNAP } \\
\underline{2}\end{array}$ & 26047 & $\begin{array}{l}\text { contactin } \\
\text { associated } \\
\text { protein-like } \\
2 \\
\end{array}$ & \\
\hline AA147654 & \begin{tabular}{|r|}
0.000171 \\
844
\end{tabular} & & & & \\
\hline N26917 & $\begin{array}{r}0.000171 \\
923\end{array}$ & & & & \\
\hline R45094 & $\begin{array}{r}0.000173 \\
118 \\
\end{array}$ & ME3 & $\underline{10873}$ & $\begin{array}{l}\text { malic } \\
\text { enzyme 3, } \\
\text { NADP }(+)- \\
\text { dependent, } \\
\text { mitochondr } \\
\text { ial }\end{array}$ & $\begin{array}{l}\text { electron transporter activity; malate } \\
\text { dehydrogenase (oxaloacetate- } \\
\text { decarboxylating) (NADP) activity; } \\
\text { malate metabolism; mitochondrion; } \\
\text { oxidoreductase activity; pyruvate } \\
\text { metabolism }\end{array}$ \\
\hline $\mathrm{H} 43658$ & \begin{tabular}{|r|}
0.000174 \\
592 \\
\end{tabular} & TRIM47 & $\underline{91107}$ & \begin{tabular}{|l} 
tripartite \\
motif- \\
containing \\
47
\end{tabular} & intracellular; zinc ion binding \\
\hline R81715 & \begin{tabular}{|r|}
0.000175 \\
054 \\
\end{tabular} & ATF4 & $\underline{468}$ & $\begin{array}{l}\text { activating } \\
\text { transcriptio } \\
\text { n factor } 4 \\
\text { (tax- }\end{array}$ & $\begin{array}{l}\text { DNA binding; RNA polymerase II } \\
\text { transcription factor activity; nucleus; } \\
\text { regulation of transcription, DNA- } \\
\text { dependent }\end{array}$ \\
\hline
\end{tabular}




\begin{tabular}{|c|c|c|c|c|c|}
\hline & & & & $\begin{array}{l}\text { responsive } \\
\text { enhancer } \\
\text { element } \\
\text { B67) }\end{array}$ & \\
\hline AA069448 & $\begin{array}{r}0.000177 \\
002 \\
\end{array}$ & & & & \\
\hline N95545 & $\begin{array}{r}0.000178 \\
172 \\
\end{array}$ & $\underline{\mathrm{IL} 11}$ & 3589 & $\begin{array}{l}\text { interleukin } \\
11\end{array}$ & $\begin{array}{l}\text { B-cell differentiation; adipocyte } \\
\text { differentiation; cell proliferation; cell- } \\
\text { cell signaling; cytokine activity; } \\
\text { extracellular; interleukin-11 receptor } \\
\text { binding; megakaryocyte differentiation; } \\
\text { platelet activation; positive regulation } \\
\text { of cell proliferation }\end{array}$ \\
\hline R51914 & \begin{tabular}{|r|}
0.000180 \\
31 \\
\end{tabular} & CGI-87 & $\underline{51112}$ & $\begin{array}{l}\text { CGI-87 } \\
\text { protein }\end{array}$ & \\
\hline H10439 & $\begin{array}{r}0.000188 \\
608 \\
\end{array}$ & & $\underline{338598}$ & $\begin{array}{l}\text { similar to } \\
\text { hypothetic } \\
\text { al protein } \\
\text { MGC5560 }\end{array}$ & \\
\hline R64301 & \begin{tabular}{|r|}
0.000189 \\
177
\end{tabular} & & & & \\
\hline H38881 & $\begin{array}{r}0.000190 \\
477\end{array}$ & WHSC1 & $\underline{7468}$ & $\begin{array}{l}\text { Wolf- } \\
\text { Hirschhorn } \\
\text { syndrome } \\
\text { candidate } \\
1\end{array}$ & $\begin{array}{l}\text { embryogenesis and morphogenesis; } \\
\text { oncogenesis }\end{array}$ \\
\hline AA037091 & $\begin{array}{r}0.000195 \\
333 \\
\end{array}$ & TCEB1 & 6921 & $\begin{array}{l}\text { transcriptio } \\
\text { n } \\
\text { elongation } \\
\text { factor B } \\
\text { (SIII), } \\
\text { polypeptid } \\
\text { e } 1 \\
(15 \mathrm{kDa}, \\
\text { elongin C) } \\
\end{array}$ & $\begin{array}{l}\text { nucleus; protein binding; regulation of } \\
\text { transcription from Pol II promoter; } \\
\text { transcriptional elongation regulator } \\
\text { activity }\end{array}$ \\
\hline N39397 & $\begin{array}{r}0.000198 \\
774 \\
\end{array}$ & BACH & $\underline{11332}$ & $\begin{array}{l}\text { brain acyl- } \\
\text { CoA } \\
\text { hydrolase }\end{array}$ & $\begin{array}{l}\text { acyl-CoA binding; cytoplasm; } \\
\text { hydrolase activity; lipid metabolism; } \\
\text { palmitoyl-CoA hydrolase activity; } \\
\text { serine esterase activity }\end{array}$ \\
\hline AA203329 & $\begin{array}{r}0.000200 \\
052 \\
\end{array}$ & & & & \\
\hline T89417 & $\begin{array}{r}0.000205 \\
386 \\
\end{array}$ & & & & \\
\hline R20209 & $\begin{array}{r}0.000206 \\
661 \\
\end{array}$ & & & & \\
\hline R41888 & $\begin{array}{r}0.000209 \\
395 \\
\end{array}$ & & & & \\
\hline R18261 & $\begin{array}{r}0.000210 \\
355 \\
\end{array}$ & & & & \\
\hline H65945 & \begin{tabular}{|r|}
0.000214 \\
501 \\
\end{tabular} & & & & \\
\hline
\end{tabular}




\begin{tabular}{|c|c|c|c|c|c|}
\hline R20223 & $\begin{array}{r}0.000214 \\
646 \\
\end{array}$ & $\underline{\text { ARHGDIA }}$ & $\underline{396}$ & $\begin{array}{l}\text { Rho GDP } \\
\text { dissociatio } \\
n \text { inhibitor } \\
\text { (GDI) } \\
\text { alpha } \\
\end{array}$ & $\begin{array}{l}\text { GTPase activator activity; Rho GDP- } \\
\text { dissociation inhibitor activity; Rho } \\
\text { protein signal transduction; cytoplasm; } \\
\text { negative regulation of cell adhesion; } \\
\text { protein binding }\end{array}$ \\
\hline AA099373 & $\begin{array}{r}0.000217 \\
019\end{array}$ & $\underline{\text { GYS1 }}$ & 2997 & \begin{tabular}{|l|} 
glycogen \\
synthase 1 \\
(muscle)
\end{tabular} & glycogen metabolism \\
\hline $\mathrm{H} 48578$ & $\begin{array}{r}0.000217 \\
829\end{array}$ & & & & \\
\hline W17311 & $\begin{array}{r}0.000218 \\
848\end{array}$ & $\underline{\mathrm{SDHB}}$ & $\underline{6390}$ & \begin{tabular}{|l|} 
succinate \\
dehydroge \\
nase \\
complex, \\
subunit B, \\
iron sulfur \\
(Ip) \\
\end{tabular} & $\begin{array}{l}\text { aerobic respiration; electron transport; } \\
\text { electron transporter activity; } \\
\text { mitochondrion; oxidoreductase activity; } \\
\text { quinol:fumarate oxidoreductase } \\
\text { activity; succinate dehydrogenase } \\
\text { (ubiquinone) activity; tricarboxylic acid } \\
\text { cycle }\end{array}$ \\
\hline R34648 & $\begin{array}{r}0.000219 \\
625\end{array}$ & $\underline{\mathrm{CHL} 1}$ & 10752 & \begin{tabular}{|l|} 
cell \\
adhesion \\
molecule \\
with \\
homology \\
to L1CAM \\
(close \\
homolog of \\
L1) \\
\end{tabular} & $\begin{array}{l}\text { cell adhesion; integral to membrane; } \\
\text { signal transduction }\end{array}$ \\
\hline T83013 & $\begin{array}{r}0.000220 \\
38\end{array}$ & $\underline{H G D}$ & 3081 & $\begin{array}{l}\text { homogentis } \\
\text { oxidase) }\end{array}$ & ate 1,2-dioxygenase (homogentisate \\
\hline $\mathrm{H} 62473$ & $\begin{array}{r}0.000221 \\
357 \\
\end{array}$ & TGFBR3 & $\underline{7049}$ & $\begin{array}{l}\text { transformin } \\
\text { g growth } \\
\text { factor, beta } \\
\text { receptor III } \\
\text { (betaglyca } \\
\text { n, 300kDa) }\end{array}$ & $\begin{array}{l}\text { TGFbeta receptor signaling pathway; } \\
\text { development; glycosaminoglycan } \\
\text { binding; integral to membrane; } \\
\text { receptor activity; signal transduction }\end{array}$ \\
\hline $\mathrm{H} 46382$ & $\begin{array}{r}0.000222 \\
325\end{array}$ & PFTK1 & $\underline{5218}$ & \begin{tabular}{|l|} 
PFTAIRE \\
protein \\
kinase 1
\end{tabular} & $\begin{array}{l}\text { ATP binding; cAMP-dependent protein } \\
\text { kinase activity; cytoplasm; nucleus; } \\
\text { protein amino acid phosphorylation; } \\
\text { protein kinase CK2 activity; protein } \\
\text { serine/threonine kinase activity; } \\
\text { transferase activity }\end{array}$ \\
\hline N76007 & $\begin{array}{r}0.000222 \\
498 \\
\end{array}$ & & & & \\
\hline R31471 & $\begin{array}{r}0.000229 \\
077 \\
\end{array}$ & & & & \\
\hline AA034962 & $\begin{array}{r}0.000230 \\
532 \\
\end{array}$ & & & & \\
\hline R55936 & $\begin{array}{r}0.000230 \\
896 \\
\end{array}$ & & & & \\
\hline R00940 & $\begin{array}{r}0.000233 \\
118 \\
\end{array}$ & & & & \\
\hline R94499 & $\begin{array}{r}0.000233 \\
405\end{array}$ & GNB5 & 10681 & \multicolumn{2}{|c|}{$\begin{array}{l}\text { guanine nucleotide binding protein (G protein), } \\
\text { beta } 5\end{array}$} \\
\hline
\end{tabular}




\begin{tabular}{|c|c|c|c|c|c|}
\hline W51811 & \begin{tabular}{|r|}
0.000236 \\
308
\end{tabular} & WNT5A & 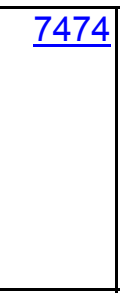 & $\begin{array}{l}\text { wingless- } \\
\text { type } \\
\text { MMTV } \\
\text { integration } \\
\text { site family, } \\
\text { member } \\
5 \mathrm{~A} \\
\end{array}$ & $\begin{array}{l}\text { cell-cell signaling; development; } \\
\text { embryogenesis and morphogenesis; } \\
\text { extracellular space; frizzled-2 signaling } \\
\text { pathway; receptor binding; signal } \\
\text { transduction; soluble fraction }\end{array}$ \\
\hline H77599 & $\begin{array}{r}0.000241 \\
297\end{array}$ & & & & \\
\hline W01319 & \begin{tabular}{|r|}
0.000249 \\
061
\end{tabular} & BHC80 & $\underline{51317}$ & $\begin{array}{l}\text { BRAF35/H } \\
\text { DAC2 } \\
\text { complex } \\
(80 \mathrm{kDa})\end{array}$ & \\
\hline AA007532 & $\begin{array}{r}0.000250 \\
771\end{array}$ & & & & \\
\hline T95166 & $\begin{array}{r}0.000251 \\
343\end{array}$ & & & & \\
\hline AA026351 & $\begin{array}{r}0.000251 \\
677 \\
\end{array}$ & & & & \\
\hline R43244 & $\begin{array}{r}0.000252 \\
089 \\
\end{array}$ & PPOX & $\underline{5498}$ & $\begin{array}{l}\text { protoporph } \\
\text { yrinogen } \\
\text { oxidase }\end{array}$ & $\begin{array}{l}\text { electron transport; electron transporter } \\
\text { activity; heme biosynthesis; } \\
\text { mitochondrion; oxidoreductase activity; } \\
\text { protoporphyrinogen oxidase activity }\end{array}$ \\
\hline R21107 & $\begin{array}{r}0.000252 \\
412 \\
\end{array}$ & & & & \\
\hline R25521 & \begin{tabular}{|r|}
0.000256 \\
979
\end{tabular} & NRCAM & $\underline{4897}$ & $\begin{array}{l}\text { neuronal } \\
\text { cell } \\
\text { adhesion } \\
\text { molecule }\end{array}$ & $\begin{array}{l}\text { cell adhesion; cell adhesion molecule } \\
\text { activity; integral to plasma membrane; } \\
\text { tumor suppressor }\end{array}$ \\
\hline H62909 & $\begin{array}{r}0.000260 \\
347\end{array}$ & & & & \\
\hline N23942 & $\begin{array}{r}0.000269 \\
889\end{array}$ & MEP50 & $\underline{79084}$ & $\begin{array}{l}\text { MEP50 } \\
\text { protein }\end{array}$ & \\
\hline H61379 & $\begin{array}{r}0.000271 \\
804 \\
\end{array}$ & & & & \\
\hline R83014 & $\begin{array}{r}0.000275 \\
307 \\
\end{array}$ & & & & \\
\hline $\mathrm{H} 12528$ & $\begin{array}{r}0.000276 \\
615 \\
\end{array}$ & ANXA5 & 308 & annexin A5 & $\begin{array}{l}\text { anticoagulant activity; blood } \\
\text { coagulation; calcium ion binding; } \\
\text { calcium-dependent phospholipid } \\
\text { binding; phospholipase inhibitor } \\
\text { activity }\end{array}$ \\
\hline R98591 & $\begin{array}{r}0.000277 \\
306\end{array}$ & & & & \\
\hline AA027815 & $\begin{array}{r}0.000280 \\
858 \\
\end{array}$ & KIAA1311 & $\underline{54439}$ & $\begin{array}{l}\text { KIAA1311 } \\
\text { protein }\end{array}$ & nucleic acid binding \\
\hline R24904 & \begin{tabular}{|r|}
0.000283 \\
965
\end{tabular} & PP1665 & $\underline{81544}$ & $\begin{array}{l}\text { hypothetic } \\
\text { al protein } \\
\text { PP1665 }\end{array}$ & \\
\hline R20383 & $\begin{array}{r}0.000284 \\
443 \\
\end{array}$ & & & & \\
\hline
\end{tabular}




\begin{tabular}{|c|c|c|c|c|c|}
\hline T92003 & \begin{tabular}{|r|}
0.000286 \\
696 \\
\end{tabular} & KIAA0342 & 9881 & $\begin{array}{l}\text { KIAA0342 } \\
\text { gene } \\
\text { product }\end{array}$ & $\begin{array}{l}\text { DNA binding; membrane; nucleus; } \\
\text { transport; transporter activity }\end{array}$ \\
\hline $\mathrm{H} 41889$ & $\begin{array}{r}0.000289 \\
679 \\
\end{array}$ & & & & \\
\hline R45684 & $\begin{array}{r}0.000291 \\
469 \\
\end{array}$ & & & & \\
\hline H05447 & $\begin{array}{r}0.000294 \\
637\end{array}$ & $\underline{\text { SPG7 }}$ & $\underline{6687}$ & $\begin{array}{l}\text { spastic } \\
\text { paraplegia } \\
7, \\
\text { paraplegin } \\
\text { (pure and } \\
\text { complicate } \\
\text { d } \\
\text { autosomal } \\
\text { recessive) }\end{array}$ & $\begin{array}{l}\text { ATP binding; cell adhesion; cell } \\
\text { adhesion molecule activity; chaperone } \\
\text { activity; extracellular matrix; integral to } \\
\text { membrane; metalloendopeptidase } \\
\text { activity; mitochondrion; neurogenesis; } \\
\text { nucleotide binding; proteolysis and } \\
\text { peptidolysis; signal transduction }\end{array}$ \\
\hline H93445 & $\begin{array}{r}0.000296 \\
991 \\
\end{array}$ & & & & \\
\hline R13346 & $\begin{array}{r}0.000304 \\
7\end{array}$ & ENT4 & $\underline{222962}$ & $\begin{array}{l}\text { equilibrativ } \\
\text { e } \\
\text { nucleoside } \\
\text { transporter } \\
4\end{array}$ & $\begin{array}{l}\text { membrane; nucleoside transporter } \\
\text { activity; transport }\end{array}$ \\
\hline AA035446 & $\begin{array}{r}0.000315 \\
977 \\
\end{array}$ & & & & \\
\hline $\mathrm{H} 77569$ & $\begin{array}{r}0.000318 \\
214\end{array}$ & & & & \\
\hline AA203184 & $\begin{array}{r}0.000320 \\
475\end{array}$ & & & & \\
\hline R40860 & $\begin{array}{r}0.000321 \\
443 \\
\end{array}$ & & & & \\
\hline AA203714 & $\begin{array}{r}0.000327 \\
318\end{array}$ & G3BP2 & 9908 & $\begin{array}{l}\text { Ras- } \\
\text { GTPase } \\
\text { activating } \\
\text { protein } \\
\text { SH3 } \\
\text { domain- } \\
\text { binding } \\
\text { protein 2 }\end{array}$ & $\begin{array}{l}\text { RAS protein signal transduction; RNA } \\
\text { binding; cytoplasm; cytoplasmic } \\
\text { sequestering of NF-kappaB; nucleus; } \\
\text { protein transporter activity; protein- } \\
\text { nucleus import; receptor signaling } \\
\text { complex scaffold activity; transport }\end{array}$ \\
\hline W47153 & $\begin{array}{r}0.000339 \\
402 \\
\end{array}$ & PTRF & $\underline{284119}$ & $\begin{array}{l}\text { polymeras } \\
\text { e I and } \\
\text { transcript } \\
\text { release } \\
\text { factor } \\
\end{array}$ & \\
\hline R98694 & $\begin{array}{r}0.000341 \\
697\end{array}$ & $\frac{\mathrm{LOC} 2860}{71}$ & $\underline{286071}$ & $\begin{array}{l}\text { hypothetic } \\
\text { al protein } \\
\text { LOC28607 } \\
1\end{array}$ & \\
\hline T70299 & $\begin{array}{r}0.000352 \\
597 \\
\end{array}$ & & & & \\
\hline
\end{tabular}




\begin{tabular}{|c|c|c|c|c|c|}
\hline AA131933 & $\begin{array}{r}0.000353 \\
436 \\
\end{array}$ & $\underline{\mathrm{ABP} 1}$ & $\underline{26}$ & $\begin{array}{l}\text { amiloride } \\
\text { binding } \\
\text { protein 1 } \\
\text { (amine } \\
\text { oxidase } \\
\text { (copper- } \\
\text { containing) }\end{array}$ & $\begin{array}{l}\text { amine oxidase (copper-containing) } \\
\text { activity; copper ion binding; drug } \\
\text { binding; heparin binding; metabolism; } \\
\text { oxidoreductase activity; peroxisome }\end{array}$ \\
\hline N22313 & $\begin{array}{r}0.000355 \\
08\end{array}$ & COL5A1 & 1289 & $\begin{array}{l}\text { collagen, } \\
\text { type V, } \\
\text { alpha } 1\end{array}$ & $\begin{array}{l}\text { cell adhesion; collagen; collagen type } \\
\text { V; extracellular matrix structural } \\
\text { constituent; heparin binding }\end{array}$ \\
\hline W32180 & $\begin{array}{r}0.000362 \\
284 \\
\end{array}$ & & & & \\
\hline N92610 & $\begin{array}{r}0.000363 \\
065\end{array}$ & ENPP5 & $\underline{59084}$ & $\begin{array}{l}\text { ectonucleo } \\
\text { tide } \\
\text { pyrophosp } \\
\text { hatase/pho } \\
\text { sphodieste } \\
\text { rase 5 } \\
\text { (putative } \\
\text { function) }\end{array}$ & $\begin{array}{l}\text { hydrolase activity; nucleotide } \\
\text { metabolism }\end{array}$ \\
\hline AA059277 & $\begin{array}{r}0.000363 \\
683 \\
\end{array}$ & & & & \\
\hline R92201 & $\begin{array}{r}0.000365 \\
314\end{array}$ & $\frac{\operatorname{LOC1311}}{\underline{18}}$ & 131118 & $\begin{array}{l}\text { similar to } \\
\text { RIKEN } \\
\text { cDNA } \\
1810055 D \\
05\end{array}$ & \\
\hline H86148 & $\begin{array}{r}0.000365 \\
708 \\
\end{array}$ & & & & \\
\hline R92085 & $\begin{array}{r}0.000367 \\
05\end{array}$ & & & & \\
\hline H93071 & $\begin{array}{r}0.000371 \\
869\end{array}$ & & & & \\
\hline H20128 & \begin{tabular}{|r|}
0.000376 \\
543 \\
\end{tabular} & & & & \\
\hline AA036798 & $\begin{array}{r}0.000380 \\
25\end{array}$ & $\frac{\mathrm{LOC} 2538}{27}$ & 253827 & $\begin{array}{l}\text { hypothetic } \\
\text { al protein } \\
\text { LOC25382 } \\
7\end{array}$ & \\
\hline R35932 & $\begin{array}{r}0.000380 \\
474\end{array}$ & & & & \\
\hline R89790 & $\begin{array}{r}0.000381 \\
932 \\
\end{array}$ & & & & \\
\hline AA058463 & \begin{tabular}{|r|}
0.000384 \\
372 \\
\end{tabular} & & & & \\
\hline R35706 & $\begin{array}{r}0.000386 \\
234\end{array}$ & GPM6A & $\underline{2823}$ & $\begin{array}{l}\text { glycoprotei } \\
\text { n M6A }\end{array}$ & integral to plasma membrane \\
\hline R01927 & $\begin{array}{r}0.000391 \\
302 \\
\end{array}$ & FST & 10468 & follistatin & $\begin{array}{l}\text { activin inhibitor activity; development; } \\
\text { extracellular; negative regulation of } \\
\text { follicle-stimulating hormone secretion }\end{array}$ \\
\hline
\end{tabular}




\begin{tabular}{|c|c|c|c|c|c|}
\hline H03532 & \begin{tabular}{|r|}
0.000391 \\
386 \\
\end{tabular} & DLG5 & $\underline{9231}$ & $\begin{array}{l}\text { discs, large } \\
\text { (Drosophil } \\
\text { a) homolog } \\
5\end{array}$ & $\begin{array}{l}\text { cell growth and/or maintenance; cell- } \\
\text { cell adhesion; intracellular signaling } \\
\text { cascade; plasma membrane; protein } \\
\text { binding; receptor signaling complex } \\
\text { scaffold activity; regulation of cell cycle }\end{array}$ \\
\hline R93705 & $\begin{array}{r}0.000393 \\
695 \\
\end{array}$ & & & & \\
\hline R00643 & \begin{tabular}{|r|}
0.000396 \\
004 \\
\end{tabular} & & & & \\
\hline R61879 & $\begin{array}{r}0.000396 \\
078 \\
\end{array}$ & & & & \\
\hline T80525 & \begin{tabular}{|r|}
0.000399 \\
471 \\
\end{tabular} & & & & \\
\hline $\mathrm{H} 17380$ & $\begin{array}{r}0.000401 \\
317\end{array}$ & & & & \\
\hline R93211 & $\begin{array}{r}0.000408 \\
894\end{array}$ & & & & \\
\hline R18947 & $\begin{array}{r}0.000417 \\
953 \\
\end{array}$ & & & & \\
\hline N49068 & $\begin{array}{r}0.000419 \\
155\end{array}$ & TMLHE & $\underline{55217}$ & \begin{tabular}{|l|} 
trimethyllys \\
ine \\
hydroxylas \\
e, epsilon \\
\end{tabular} & \\
\hline R13038 & \begin{tabular}{|r|}
0.000425 \\
346
\end{tabular} & $\begin{array}{l}\text { LOC1272 } \\
\underline{62}\end{array}$ & 127262 & $\begin{array}{l}\text { hypothetic } \\
\text { al protein } \\
\text { LOC12726 } \\
2\end{array}$ & \\
\hline R46521 & \begin{tabular}{|r|}
0.000429 \\
681 \\
\end{tabular} & TAGLN2 & $\underline{8407}$ & \begin{tabular}{|l} 
transgelin \\
2
\end{tabular} & muscle development \\
\hline H16828 & $\begin{array}{r}0.000446 \\
924 \\
\end{array}$ & ARPP-19 & $\underline{10776}$ & \begin{tabular}{l|} 
cyclic AMP \\
phosphopr \\
otein, 19 \\
kD
\end{tabular} & $\begin{array}{l}\text { cytoplasm; positive regulation of } \\
\text { gluconeogenesis; positive regulation of } \\
\text { glucose import; potassium channel } \\
\text { regulator activity; receptor binding }\end{array}$ \\
\hline $\mathrm{H} 77738$ & $\begin{array}{r}0.000460 \\
239\end{array}$ & & & & \\
\hline H84133 & \begin{tabular}{|r|}
0.000464 \\
071
\end{tabular} & FLJ36040 & $\underline{162963}$ & $\begin{array}{l}\text { hypothetic } \\
\text { al protein } \\
\text { FLJ36040 }\end{array}$ & $\begin{array}{l}\text { nucleic acid binding; nucleus; } \\
\text { regulation of transcription, DNA- } \\
\text { dependent }\end{array}$ \\
\hline AA033714 & \begin{tabular}{|r|}
0.000465 \\
544
\end{tabular} & FLJ14260 & $\underline{80095}$ & $\begin{array}{l}\text { hypothetic } \\
\text { al protein } \\
\text { FLJ14260 }\end{array}$ & $\begin{array}{l}\text { DNA binding; metallopeptidase } \\
\text { activity; nucleus; proteolysis and } \\
\text { peptidolysis; regulation of } \\
\text { transcription, DNA-dependent; zinc ion } \\
\text { binding }\end{array}$ \\
\hline N31736 & \begin{tabular}{|r|}
0.000470 \\
422 \\
\end{tabular} & TRIPIN & 151246 & tripin & \\
\hline T91013 & \begin{tabular}{|r|}
0.000473 \\
871 \\
\end{tabular} & & & & \\
\hline H74032 & $\begin{array}{r}0.000480 \\
42 \\
\end{array}$ & & & & \\
\hline
\end{tabular}




\begin{tabular}{|c|c|c|c|c|c|}
\hline H75766 & $\begin{array}{r}0.000480 \\
722 \\
\end{array}$ & $\underline{\text { CR1 }}$ & $\frac{1378}{13}$ & \begin{tabular}{|l|} 
compleme \\
nt \\
component \\
(3b/4b) \\
receptor 1, \\
including \\
Knops \\
blood \\
group \\
system
\end{tabular} & $\begin{array}{l}\text { complement activation; complement } \\
\text { component C3b receptor activity; } \\
\text { complement receptor activity; integral } \\
\text { to plasma membrane }\end{array}$ \\
\hline W31293 & $\begin{array}{r}0.000483 \\
427\end{array}$ & & & & \\
\hline H01085 & $\begin{array}{r}0.000483 \\
751\end{array}$ & & & & \\
\hline W47003 & $\begin{array}{r}0.000484 \\
083\end{array}$ & HIF1A & 3091 & \multicolumn{2}{|c|}{$\begin{array}{l}\text { hypoxia-inducible factor } 1 \text {, alpha subunit (basic } \\
\text { helix-loop-helix transcription factor) }\end{array}$} \\
\hline R43963 & $\begin{array}{r}0.000487 \\
097\end{array}$ & $\frac{\text { DKFZp76 }}{1 \mathrm{~A} 052}$ & $\underline{55593}$ & \begin{tabular}{|l|} 
hypothetic \\
al protein \\
DKFZp761 \\
A052
\end{tabular} & \\
\hline W85688 & $\begin{array}{r}0.000493 \\
237\end{array}$ & & & & \\
\hline R14617 & $\begin{array}{r}0.000494 \\
827\end{array}$ & EDRF1 & $\underline{26098}$ & \begin{tabular}{|l|} 
erythroid \\
differentiati \\
on-related \\
factor 1
\end{tabular} & \\
\hline N24178 & $\begin{array}{r}0.000512 \\
16 \\
\end{array}$ & & & & \\
\hline AA026902 & $\begin{array}{r}0.000521 \\
568\end{array}$ & FLJ11320 & $\underline{55343}$ & \begin{tabular}{|l|} 
GDP- \\
fucose \\
transporter \\
1
\end{tabular} & $\begin{array}{l}\text { Golgi apparatus; integral to } \\
\text { membrane; sugar porter activity; } \\
\text { transport }\end{array}$ \\
\hline T89772 & $\begin{array}{r}0.000528 \\
763 \\
\end{array}$ & KIAA0767 & 23151 & $\begin{array}{l}\text { KIAA0767 } \\
\text { protein }\end{array}$ & \\
\hline $\mathrm{H} 44375$ & $\begin{array}{r}0.000529 \\
467\end{array}$ & RFXANK & $\frac{8625}{6}$ & \begin{tabular}{l|} 
regulatory \\
factor X- \\
associated \\
ankyrin- \\
containing \\
protein
\end{tabular} & $\begin{array}{l}\text { humoral immune response; nucleus; } \\
\text { regulation of transcription, DNA- } \\
\text { dependent; transcription co-activator } \\
\text { activity; transcription factor activity; } \\
\text { transcription from Pol II promoter }\end{array}$ \\
\hline H14566 & $\begin{array}{r}0.000530 \\
146 \\
\end{array}$ & & & & \\
\hline R88098 & $\begin{array}{r}0.000530 \\
204\end{array}$ & MYO1C & 4641 & myosin IC & $\begin{array}{l}\text { ATP binding; actin binding; calmodulin } \\
\text { binding; motor activity; perception of } \\
\text { sound; unconventional myosin }\end{array}$ \\
\hline R35559 & $\begin{array}{r}0.000534 \\
118\end{array}$ & PGR1 & 93621 & \begin{tabular}{|l|} 
T-cell \\
activation \\
protein
\end{tabular} & \\
\hline R98556 & $\begin{array}{r}0.000552 \\
901\end{array}$ & $\underline{\text { CYB5 }}$ & $\underline{1528}$ & $\begin{array}{l}\text { cytochrom } \\
\text { e b-5 }\end{array}$ & $\begin{array}{l}\text { cytochrome-c oxidase activity; electron } \\
\text { transport; energy pathways; integral to } \\
\text { membrane; microsome; mitochondrion }\end{array}$ \\
\hline R39119 & 0.000556 & & & & \\
\hline
\end{tabular}




\begin{tabular}{|c|c|c|c|c|c|}
\hline & 986 & & & & \\
\hline R40156 & $\begin{array}{r}0.000560 \\
126\end{array}$ & ATSV & $\underline{547}$ & \begin{tabular}{|l} 
axonal \\
transport of \\
synaptic \\
vesicles
\end{tabular} & $\begin{array}{l}\text { ATP binding; anterograde axon cargo } \\
\text { transport; kinesin complex; } \\
\text { microtubule-based process; motor } \\
\text { activity }\end{array}$ \\
\hline T77530 & $\begin{array}{r}0.000568 \\
511 \\
\end{array}$ & & & & \\
\hline T95249 & $\begin{array}{r}0.000586 \\
757\end{array}$ & FMO3 & $\underline{2328}$ & $\begin{array}{l}\text { flavin } \\
\text { containing } \\
\text { monooxyg } \\
\text { enase 3 }\end{array}$ & $\begin{array}{l}\text { dimethylaniline monooxygenase }(\mathrm{N} \text { - } \\
\text { oxide-forming) activity; disulfide } \\
\text { oxidoreductase activity; electron } \\
\text { transport; integral to membrane; } \\
\text { microsome }\end{array}$ \\
\hline $\mathrm{H} 02590$ & $\begin{array}{r}0.000592 \\
926 \\
\end{array}$ & & & & \\
\hline R20809 & $\begin{array}{r}0.000599 \\
6\end{array}$ & RPLP1 & $\underline{6176}$ & $\begin{array}{l}\text { ribosomal } \\
\text { protein, } \\
\text { large, } \mathrm{P} 1\end{array}$ & $\begin{array}{l}\text { RNA binding; cytosolic large ribosomal } \\
\text { subunit (sensu Eukarya); intracellular; } \\
\text { ribosome; structural constituent of } \\
\text { ribosome; translational elongation }\end{array}$ \\
\hline $\mathrm{H} 06744$ & $\begin{array}{r}0.000600 \\
687 \\
\end{array}$ & & & & \\
\hline W85877 & $\begin{array}{r}0.000608 \\
409 \\
\end{array}$ & & & & \\
\hline N29108 & $\begin{array}{r}0.000611 \\
766 \\
\end{array}$ & & & & \\
\hline $\mathrm{H} 41330$ & $\begin{array}{r}0.000616 \\
443 \\
\end{array}$ & LRRC2 & $\underline{79442}$ & $\begin{array}{l}\text { leucine- } \\
\text { rich repeat- } \\
\text { containing } \\
2\end{array}$ & \\
\hline AA042920 & $\begin{array}{r}0.000622 \\
675\end{array}$ & $\underline{\text { GLI4 }}$ & $\underline{2738}$ & $\begin{array}{l}\text { GLI- } \\
\text { Kruppel } \\
\text { family } \\
\text { member } \\
\text { GLI4 }\end{array}$ & $\begin{array}{l}\text { DNA binding; biological_process } \\
\text { unknown; molecular_function } \\
\text { unknown; nucleus }\end{array}$ \\
\hline W01227 & $\begin{array}{r}0.000628 \\
914\end{array}$ & HDGF & $\underline{3068}$ & $\begin{array}{l}\text { hepatoma- } \\
\text { derived } \\
\text { growth } \\
\text { factor } \\
\text { (high- } \\
\text { mobility } \\
\text { group } \\
\text { protein 1- } \\
\text { like) } \\
\end{array}$ & $\begin{array}{l}\text { cell proliferation; cytoplasm; } \\
\text { extracellular space; growth factor } \\
\text { activity; heparin binding; signal } \\
\text { transduction }\end{array}$ \\
\hline R06054 & \begin{tabular}{|r|}
0.000629 \\
027
\end{tabular} & & & & \\
\hline R22945 & $\begin{array}{r}0.000630 \\
876 \\
\end{array}$ & $\underline{A L G 2}$ & $\underline{85365}$ & \multicolumn{2}{|c|}{$\begin{array}{l}\text { GDP-Man:Man(1)GIcNAc(2)-PP-dolichol } \\
\text { mannosyltransferase }\end{array}$} \\
\hline AA101859 & $\begin{array}{r}0.000631 \\
871\end{array}$ & ENSA & $\underline{2029}$ & $\begin{array}{l}\text { endosulfin } \\
\text { e alpha }\end{array}$ & $\begin{array}{l}\text { ion channel inhibitor activity; receptor } \\
\text { binding; response to nutrients; } \\
\text { transport }\end{array}$ \\
\hline
\end{tabular}




\begin{tabular}{|c|c|c|c|c|c|}
\hline R55497 & $\begin{array}{r}0.000644 \\
459 \\
\end{array}$ & \begin{tabular}{|l|} 
MGC1048 \\
$\underline{5}$
\end{tabular} & 112936 & $\begin{array}{l}\text { hypothetic } \\
\text { al protein } \\
\text { MGC1048 } \\
5\end{array}$ & $\begin{array}{l}\text { cytosol; intracellular protein transport; } \\
\text { protein binding; protein transporter } \\
\text { activity; retrograde (endosome to } \\
\text { Golgi) transport }\end{array}$ \\
\hline H50657 & $\begin{array}{r}0.000650 \\
395 \\
\end{array}$ & & & & \\
\hline H73931 & $\begin{array}{r}0.000654 \\
121\end{array}$ & & & & \\
\hline R08735 & $\begin{array}{r}0.000674 \\
376\end{array}$ & & & & \\
\hline N71628 & $\begin{array}{r}0.000686 \\
384\end{array}$ & SPIB & $\underline{6689}$ & $\begin{array}{l}\text { Spi-B } \\
\text { transcriptio } \\
\text { n factor } \\
\text { (Spi- } \\
\text { 1/PU.1 } \\
\text { related) }\end{array}$ & $\begin{array}{l}\text { RNA polymerase II transcription factor } \\
\text { activity; biological_process unknown; } \\
\text { cytoplasm; molecular_function } \\
\text { unknown; nucleus; regulation of } \\
\text { transcription from Pol II promoter; } \\
\text { transcription factor activity }\end{array}$ \\
\hline H95277 & $\begin{array}{r}0.000692 \\
001\end{array}$ & & $\underline{255065}$ & $\begin{array}{l}\mathrm{LOC} 25506 \\
5\end{array}$ & \\
\hline R25544 & $\begin{array}{r}0.000714 \\
957\end{array}$ & PCCB & $\underline{5096}$ & $\begin{array}{l}\text { propionyl } \\
\text { Coenzyme } \\
\text { A } \\
\text { carboxylas } \\
\text { e, beta } \\
\text { polypeptid } \\
\text { e }\end{array}$ & $\begin{array}{l}\text { fatty acid catabolism; mitochondrion; } \\
\text { propionyl-CoA carboxylase activity }\end{array}$ \\
\hline $\mathrm{N} / \mathrm{A} 1$ & $\begin{array}{r}0.000715 \\
432\end{array}$ & & & & \\
\hline W15154 & $\begin{array}{r}0.000717 \\
259\end{array}$ & FLJ11712 & $\underline{79621}$ & $\begin{array}{l}\text { hypothetic } \\
\text { al protein } \\
\text { FLJ11712 }\end{array}$ & \\
\hline R98517 & $\begin{array}{r}0.000726 \\
742 \\
\end{array}$ & $\frac{\mathrm{HIST} 1 \mathrm{H} 1}{\mathrm{C}}$ & $\underline{3006}$ & $\begin{array}{l}\text { histone 1, } \\
\text { H1c }\end{array}$ & $\begin{array}{l}\text { DNA binding; chromosome; } \\
\text { chromosome organization and } \\
\text { biogenesis (sensu Eukarya); } \\
\text { nucleosome; nucleosome assembly; } \\
\text { nucleus }\end{array}$ \\
\hline \begin{tabular}{|l|} 
R48249 \\
\end{tabular} & $\begin{array}{r}0.000729 \\
21\end{array}$ & & $\underline{91170}$ & $\begin{array}{l}\text { hypothetic } \\
\text { al gene } \\
\text { supported } \\
\text { by } \\
\text { AK002208 }\end{array}$ & \\
\hline N59242 & $\begin{array}{r}0.000732 \\
796\end{array}$ & $\mathrm{HH} 114$ & $\underline{84529}$ & $\begin{array}{l}\text { hypothetic } \\
\text { al protein } \\
\text { HH114 }\end{array}$ & \\
\hline R11233 & $\begin{array}{r}0.000734 \\
278\end{array}$ & P2RX5 & $\underline{5026}$ & $\begin{array}{l}\text { purinergic } \\
\text { receptor } \\
\text { P2X, } \\
\text { ligand- } \\
\text { gated ion } \\
\text { channel, } 5\end{array}$ & $\begin{array}{l}\text { ATP binding; ion channel activity; ion } \\
\text { transport; membrane; receptor activity }\end{array}$ \\
\hline R06542 & $\begin{array}{r}0.000735 \\
728 \\
\end{array}$ & & & & \\
\hline
\end{tabular}




\begin{tabular}{|c|c|c|c|c|c|}
\hline R48990 & \begin{tabular}{|r|}
0.000738 \\
059 \\
\end{tabular} & NUP155 & $\underline{9631}$ & $\begin{array}{l}\text { nucleopori } \\
\text { n } 155 \mathrm{kDa}\end{array}$ & $\begin{array}{l}\text { nuclear pore; nucleocytoplasmic } \\
\text { transport; nucleocytoplasmic } \\
\text { transporter activity; nucleus; transport; } \\
\text { transporter activity }\end{array}$ \\
\hline R25101 & $\begin{array}{r}0.000738 \\
646\end{array}$ & NR2F2 & $\underline{7026}$ & $\begin{array}{l}\text { nuclear } \\
\text { receptor } \\
\text { subfamily } \\
2, \text { group F, } \\
\text { member } 2\end{array}$ & $\begin{array}{l}\text { ligand-regulated transcription factor } \\
\text { activity; lipid metabolism; nucleus; } \\
\text { regulation of transcription from Pol II } \\
\text { promoter; signal transduction; steroid } \\
\text { hormone receptor activity; transcription } \\
\text { co-repressor activity; transcription } \\
\text { factor activity }\end{array}$ \\
\hline AA033764 & $\begin{array}{r}0.000750 \\
967\end{array}$ & & & & \\
\hline |T94800 & $\begin{array}{r}0.000755 \\
497\end{array}$ & & & & \\
\hline AA033635 & $\begin{array}{r}0.000762 \\
319\end{array}$ & & & & \\
\hline H39673 & $\begin{array}{r}0.000765 \\
384 \\
\end{array}$ & & $\underline{352246}$ & $\begin{array}{l}\text { LOC35224 } \\
6\end{array}$ & \\
\hline AA031347 & $\begin{array}{r}0.000765 \\
759 \\
\end{array}$ & & & & \\
\hline R14520 & $\begin{array}{r}0.000766 \\
904\end{array}$ & & & & \\
\hline R69584 & $\begin{array}{r}0.000776 \\
372 \\
\end{array}$ & & & & \\
\hline N54947 & $\begin{array}{r}0.000782 \\
204 \\
\end{array}$ & GOLGA2 & $\underline{2801}$ & $\begin{array}{l}\text { golgi } \\
\text { autoantige } \\
\text { n, golgin } \\
\text { subfamily } \\
\text { a, } 2 \\
\end{array}$ & Golgi apparatus \\
\hline R66251 & $\begin{array}{r}0.000783 \\
046 \\
\end{array}$ & & & & \\
\hline H37798 & $\begin{array}{r}0.000785 \\
652 \\
\end{array}$ & & & & \\
\hline R08249 & $\begin{array}{r}0.000798 \\
521\end{array}$ & $\underline{\mathrm{KIAA0317}}$ & $\underline{9870}$ & $\begin{array}{l}\text { KIAA0317 } \\
\text { gene } \\
\text { product }\end{array}$ & $\begin{array}{l}\text { intracellular; ubiquitin cycle; ubiquitin- } \\
\text { protein ligase activity }\end{array}$ \\
\hline H84657 & $\begin{array}{r}0.000810 \\
932 \\
\end{array}$ & GRWD & $\underline{83743}$ & $\begin{array}{l}\text { glutamate } \\
\text { rich WD } \\
\text { repeat } \\
\text { protein } \\
\text { GRWD } \\
\end{array}$ & \\
\hline W68275 & $\begin{array}{r}0.000827 \\
038\end{array}$ & $\frac{\text { CDC42EP }}{2}$ & $\underline{10435}$ & \multicolumn{2}{|c|}{ CDC42 effector protein (Rho GTPase binding) 2} \\
\hline R43233 & $\begin{array}{r}0.000832 \\
554 \\
\end{array}$ & & $\underline{349228}$ & $\begin{array}{l}\text { hypothetic } \\
\text { al gene } \\
\text { supported } \\
\text { by } \\
\text { AJ420560 }\end{array}$ & \\
\hline T70278 & $\begin{array}{r}0.000837 \\
11 \\
\end{array}$ & & & & \\
\hline
\end{tabular}




\begin{tabular}{|c|c|c|c|c|c|}
\hline $\mathrm{H} 17081$ & \begin{tabular}{|r|}
0.000837 \\
798 \\
\end{tabular} & COG7 & 91949 & $\begin{array}{l}\text { component } \\
\text { of } \\
\text { oligomeric } \\
\text { golgi } \\
\text { complex } 7\end{array}$ & $\begin{array}{l}\text { Golgi apparatus; intracellular protein } \\
\text { transport; membrane; protein } \\
\text { transporter activity }\end{array}$ \\
\hline W24394 & $\begin{array}{r}0.000840 \\
149 \\
\end{array}$ & A2M & & \begin{tabular}{|l|} 
alpha-2- \\
macroglob \\
ulin
\end{tabular} & $\begin{array}{l}\text { intracellular protein transport; protein } \\
\text { carrier activity; serine protease } \\
\text { inhibitor activity; wide-spectrum } \\
\text { protease inhibitor activity }\end{array}$ \\
\hline N32293 & $\begin{array}{r}0.000841 \\
745 \\
\end{array}$ & SEC24B & 10427 & $\begin{array}{l}\text { SEC24 } \\
\text { related } \\
\text { gene } \\
\text { family, } \\
\text { member B } \\
\text { (S. } \\
\text { cerevisiae) }\end{array}$ & $\begin{array}{l}\text { COPII vesicle; Golgi apparatus; } \\
\text { endoplasmic reticulum; intracellular } \\
\text { protein transport; membrane; protein } \\
\text { transporter activity; secretory vesicle; } \\
\text { vesicle-mediated transport }\end{array}$ \\
\hline R56055 & $\begin{array}{r}0.000856 \\
06\end{array}$ & RIC3 & $\underline{79608}$ & \begin{tabular}{|l|} 
RIC3 \\
protein
\end{tabular} & \\
\hline H49369 & \begin{tabular}{|r|}
0.000860 \\
187
\end{tabular} & RAB3IL1 & $\underline{5866}$ & $\begin{array}{l}\text { RAB3A } \\
\text { interacting } \\
\text { protein } \\
\text { (rabin3)- } \\
\text { like 1 }\end{array}$ & \\
\hline W47177 & $\begin{array}{r}0.000861 \\
608 \\
\end{array}$ & GCN5L1 & 2647 & $\begin{array}{l}\text { GCN5 } \\
\text { general } \\
\text { control of } \\
\text { amino-acid } \\
\text { synthesis } \\
\text { 5-like 1 } \\
\text { (yeast) }\end{array}$ & $\begin{array}{l}\text { biological_process unknown; } \\
\text { cellular_component unknown; } \\
\text { molecular_function unknown }\end{array}$ \\
\hline H63763 & $\begin{array}{r}0.000872 \\
181\end{array}$ & & & & \\
\hline R07997 & $\begin{array}{r}0.000876 \\
518\end{array}$ & & & & \\
\hline $\mathrm{H} 65775$ & $\begin{array}{r}0.000893 \\
253 \\
\end{array}$ & & & & \\
\hline R19554 & $\begin{array}{r}0.000910 \\
211\end{array}$ & & & & \\
\hline N91376 & $\begin{array}{r}0.000919 \\
833 \\
\end{array}$ & KIAA0247 & $\underline{9766}$ & $\begin{array}{l}\text { KIAA0247 } \\
\text { gene } \\
\text { product }\end{array}$ & integral to membrane \\
\hline AA031465 & $\begin{array}{r}0.000923 \\
723 \\
\end{array}$ & GEFT & 115557 & $\begin{array}{l}\text { RAC/CDC } \\
42 \\
\text { exchange } \\
\text { factor }\end{array}$ & \\
\hline AA213450 & $\begin{array}{r}0.000934 \\
614 \\
\end{array}$ & & & & \\
\hline AA128013 & $\begin{array}{r}0.000940 \\
491\end{array}$ & & & & \\
\hline $\mathrm{H} 40682$ & $\begin{array}{r}0.000941 \\
948\end{array}$ & & & & \\
\hline R24850 & 0.000942 & HSPA6 & 3310 & heat shock & ATP binding; heat shock protein \\
\hline
\end{tabular}




\begin{tabular}{|c|c|c|c|c|c|}
\hline & 491 & & & \begin{tabular}{|l|}
$70 \mathrm{kDa}$ \\
protein 6 \\
$($ HSP70B')
\end{tabular} & activity \\
\hline N25927 & $\begin{array}{r}0.000950 \\
965\end{array}$ & & & & \\
\hline R18453 & $\begin{array}{r}0.000960 \\
994\end{array}$ & & & & \\
\hline AA054541 & $\begin{array}{r}0.000965 \\
547\end{array}$ & & & & \\
\hline N52765 & $\begin{array}{r}0.000966 \\
351\end{array}$ & CACNA1I & $\underline{8911}$ & \begin{tabular}{|l|} 
calcium \\
channel, \\
voltage- \\
dependent, \\
alpha 1l \\
subunit
\end{tabular} & $\begin{array}{l}\text { calcium ion binding; calcium ion } \\
\text { transport; cation transport; integral to } \\
\text { membrane; low voltage-gated calcium } \\
\text { channel activity; voltage-gated calcium } \\
\text { channel complex }\end{array}$ \\
\hline H18581 & $\begin{array}{r}0.000968 \\
418\end{array}$ & CD164 & $\underline{8763}$ & \begin{tabular}{|l|} 
CD164 \\
antigen, \\
sialomucin
\end{tabular} & $\begin{array}{l}\text { cell adhesion; cell adhesion molecule } \\
\text { activity; development; immune } \\
\text { response; integral to membrane; } \\
\text { integral to plasma membrane; } \\
\text { membrane fraction; negative } \\
\text { regulation of cell proliferation; plasma } \\
\text { membrane; signal transduction; } \\
\text { soluble fraction }\end{array}$ \\
\hline T79481 & $\begin{array}{r}0.000972 \\
193 \\
\end{array}$ & ANK1 & $\underline{286}$ & \begin{tabular}{|l|} 
ankyrin 1, \\
erythrocyti \\
c
\end{tabular} & $\begin{array}{l}\text { actin cytoskeleton; plasma membrane; } \\
\text { structural constituent of cytoskeleton }\end{array}$ \\
\hline N25754 & $\begin{array}{r}0.000991 \\
313 \\
\end{array}$ & $\underline{\mathrm{LOC} 2833}$ & 283378 & \begin{tabular}{|l|} 
hypothetic \\
al protein \\
LOC28337 \\
8
\end{tabular} & \\
\hline W03627 & $\begin{array}{r}0.000992 \\
082\end{array}$ & DELGEF & 26297 & \multicolumn{2}{|c|}{$\begin{array}{l}\text { deafness locus associated putative guanine } \\
\text { nucleotide exchange factor }\end{array}$} \\
\hline H57509 & $\begin{array}{r}0.000992 \\
872\end{array}$ & & & & \\
\hline N31362 & $\begin{array}{r}0.000995 \\
169\end{array}$ & NF1 & $\underline{4763}$ & \begin{tabular}{|l|} 
neurofibro \\
min 1 \\
(neurofibro \\
matosis, \\
von \\
Recklingha \\
usen \\
disease, \\
Watson \\
disease) \\
\end{tabular} & $\begin{array}{l}\text { GTPase activator activity; RAS protein } \\
\text { signal transduction; Ras GTPase } \\
\text { activator activity; cell growth and/or } \\
\text { maintenance; cytoplasm; enzyme } \\
\text { inhibitor activity; negative regulation of } \\
\text { cell proliferation; tumor suppressor } \\
\end{array}$ \\
\hline $\mathrm{H} 23343$ & $\begin{array}{r}0.000999 \\
695 \\
\end{array}$ & & & & \\
\hline AA001336 & $\begin{array}{r}0.001000 \\
548 \\
\end{array}$ & & & & \\
\hline N77560 & $\begin{array}{r}0.001011 \\
142 \\
\end{array}$ & & & & \\
\hline
\end{tabular}




\begin{tabular}{|c|c|c|c|c|c|}
\hline W02503 & $\begin{array}{r}0.001017 \\
699 \\
\end{array}$ & $\frac{\operatorname{SLC25A1}}{0}$ & $\frac{1468}{14}$ & \begin{tabular}{|l|} 
solute \\
carrier \\
family 25 \\
(mitochond \\
rial carrier; \\
dicarboxyla \\
te \\
transporter \\
, member \\
10
\end{tabular} & $\begin{array}{l}\text { binding; dicarboxylic acid transport; } \\
\text { dicarboxylic acid transporter activity; } \\
\text { gluconeogenesis; integral to } \\
\text { membrane; mitochondrial inner } \\
\text { membrane; mitochondrial transport; } \\
\text { mitochondrion; transport }\end{array}$ \\
\hline AA029583 & $\begin{array}{r}0.001020 \\
959 \\
\end{array}$ & TFF3 & $\underline{7033}$ & \begin{tabular}{|l|} 
trefoil \\
factor 3 \\
(intestinal) \\
\end{tabular} & $\begin{array}{l}\text { defense response; digestion; } \\
\text { extracellular }\end{array}$ \\
\hline R68695 & $\begin{array}{r}0.001026 \\
928 \\
\end{array}$ & & & & \\
\hline W47411 & $\begin{array}{r}0.001027 \\
216\end{array}$ & THY1 & $\underline{7070}$ & $\begin{array}{l}\text { Thy-1 cell } \\
\text { surface } \\
\text { antigen }\end{array}$ & integral to plasma membrane \\
\hline H69318 & $\begin{array}{r}0.001039 \\
787\end{array}$ & ADAM10 & $\underline{102}$ & \begin{tabular}{|l|} 
a \\
disintegrin \\
and \\
metalloprot \\
einase \\
domain 10 \\
\end{tabular} & $\begin{array}{l}\text { cell-cell signaling; hydrolase activity; } \\
\text { integral to plasma membrane; } \\
\text { metalloendopeptidase activity; } \\
\text { proteolysis and peptidolysis; zinc ion } \\
\text { binding }\end{array}$ \\
\hline H59247 & $\begin{array}{r}0.001041 \\
911 \\
\end{array}$ & & & & \\
\hline H61751 & $\begin{array}{r}0.001043 \\
209\end{array}$ & $\underline{\text { SLC5A2 }}$ & $\underline{6524}$ & \begin{tabular}{|l|} 
solute \\
carrier \\
family 5 \\
(sodium/gl \\
ucose \\
cotransport \\
er), \\
member 2 \\
\end{tabular} & $\begin{array}{l}\text { carbohydrate metabolism; integral to } \\
\text { membrane; low-affinity } \\
\text { glucose:sodium symporter activity; } \\
\text { sodium ion transport; sugar porter } \\
\text { activity; symporter activity; transport; } \\
\text { transporter activity }\end{array}$ \\
\hline W90745 & \begin{tabular}{|r|}
0.001045 \\
087 \\
\end{tabular} & & & & \\
\hline R99293 & $\begin{array}{r}0.001045 \\
597 \\
\end{array}$ & & & & \\
\hline H13102 & $\begin{array}{r}0.001050 \\
919 \\
\end{array}$ & & & & \\
\hline R88720 & \begin{tabular}{|r|}
0.001054 \\
557 \\
\end{tabular} & & & & \\
\hline R55829 & $\begin{array}{r}0.001056 \\
852 \\
\end{array}$ & EDN1 & $\underline{1906}$ & $\begin{array}{l}\text { endothelin } \\
1\end{array}$ & $\begin{array}{l}\text { cell-cell signaling; extracellular space; } \\
\text { pathogenesis; peptide hormone; } \\
\text { positive regulation of cell proliferation; } \\
\text { regulation of blood pressure; signal } \\
\text { transduction; soluble fraction; toxin } \\
\text { activity }\end{array}$ \\
\hline N25619 & $\begin{array}{r}0.001063 \\
803 \\
\end{array}$ & & & & \\
\hline AA035066 & $\begin{array}{r}0.001077 \\
269\end{array}$ & MGC4268 & $\underline{83607}$ & $\begin{array}{l}\text { hypothetic } \\
\text { al protein } \\
\text { MGC4268 }\end{array}$ & \\
\hline
\end{tabular}




\begin{tabular}{|c|c|c|c|c|c|}
\hline AA001147 & \begin{tabular}{|r|}
0.001079 \\
796 \\
\end{tabular} & GK001 & 57003 & $\begin{array}{l}\text { GK001 } \\
\text { protein }\end{array}$ & \\
\hline R26320 & \begin{tabular}{|r|}
0.001090 \\
599 \\
\end{tabular} & FLNB & $\underline{2317}$ & $\begin{array}{l}\text { filamin B, } \\
\text { beta (actin } \\
\text { binding } \\
\text { protein } \\
278 \text { ) }\end{array}$ & $\begin{array}{l}\text { actin binding; actin cytoskeleton; actin } \\
\text { cytoskeleton organization and } \\
\text { biogenesis; cytoskeletal anchoring; } \\
\text { integral to plasma membrane; } \\
\text { membrane associated actin binding; } \\
\text { signal transduction }\end{array}$ \\
\hline N24163 & $\begin{array}{r}0.001097 \\
639 \\
\end{array}$ & & & & \\
\hline AA136271 & $\begin{array}{r}0.001098 \\
48\end{array}$ & & & & \\
\hline T74280 & $\begin{array}{r}0.001106 \\
704 \\
\end{array}$ & & & & \\
\hline N64618 & \begin{tabular}{|r|}
0.001111 \\
281 \\
\end{tabular} & BUB3 & $\underline{9184}$ & \begin{tabular}{|l|} 
BUB3 \\
budding \\
uninhibited \\
by \\
benzimidaz \\
oles 3 \\
homolog \\
(yeast)
\end{tabular} & $\begin{array}{l}\text { cell proliferation; kinetochore; mitosis; } \\
\text { mitotic spindle checkpoint; nucleus }\end{array}$ \\
\hline R21092 & $\begin{array}{r}0.001117 \\
965\end{array}$ & CA1 & $\underline{759}$ & \begin{tabular}{l|} 
carbonic \\
anhydrase \\
I
\end{tabular} & $\begin{array}{l}\text { carbonate dehydratase activity; } \\
\text { cytoplasm; lyase activity; one-carbon } \\
\text { compound metabolism; zinc ion } \\
\text { binding }\end{array}$ \\
\hline R23778 & $\begin{array}{r}0.001161 \\
765\end{array}$ & C7 & $\underline{730}$ & \begin{tabular}{|l|} 
compleme \\
nt \\
component \\
7 \\
\\
\end{tabular} & $\begin{array}{l}\text { complement activation, alternative } \\
\text { pathway; complement activation, } \\
\text { classical pathway; complement } \\
\text { activity; cytolysis; immune response; } \\
\text { integral to membrane; membrane } \\
\text { attack complex; response to } \\
\text { pathogenic bacteria }\end{array}$ \\
\hline AA142989 & $\begin{array}{r}0.001176 \\
611 \\
\end{array}$ & BMPER & $\underline{168667}$ & \begin{tabular}{|l|} 
likely \\
ortholog of \\
mouse \\
BMP- \\
binding \\
endothelial \\
regulator \\
precursor \\
protein
\end{tabular} & calcium ion binding; extracellular \\
\hline AA045611 & $\begin{array}{r}0.001181 \\
504\end{array}$ & FLJ20280 & $\underline{54876}$ & \begin{tabular}{|l|} 
hypothetic \\
al protein \\
FLJ20280 \\
\end{tabular} & \\
\hline T87794 & $\begin{array}{r}0.001190 \\
228 \\
\end{array}$ & & & & \\
\hline $\mathrm{H} 08517$ & $\begin{array}{r}0.001191 \\
761\end{array}$ & & & & \\
\hline
\end{tabular}




\begin{tabular}{|c|c|c|c|c|c|}
\hline R47859 & \begin{tabular}{|r|}
0.001191 \\
811 \\
\end{tabular} & NPR1 & 4881 & $\begin{array}{l}\text { natriuretic } \\
\text { peptide } \\
\text { receptor } \\
\text { A/guanylat } \\
\text { e cyclase } \\
\text { A } \\
\text { (atrionatriu } \\
\text { retic } \\
\text { peptide } \\
\text { receptor A) }\end{array}$ & $\begin{array}{l}\text { ATP binding; cGMP biosynthesis; } \\
\text { guanylate cyclase activity; integral to } \\
\text { membrane; intracellular signaling } \\
\text { cascade; lyase activity; peptide } \\
\text { receptor activity, G-protein coupled; } \\
\text { protein amino acid phosphorylation; } \\
\text { protein kinase activity; receptor } \\
\text { activity; receptor guanylate cyclase } \\
\text { activity; regulation of blood pressure }\end{array}$ \\
\hline R09247 & \begin{tabular}{|r|}
0.001197 \\
335
\end{tabular} & & & & \\
\hline N34765 & $\begin{array}{r}0.001201 \\
456\end{array}$ & & & & \\
\hline W42881 & \begin{tabular}{|r|}
0.001203 \\
458 \\
\end{tabular} & ERdi5 & $\underline{54431}$ & $\begin{array}{l}\text { ER- } \\
\text { resident } \\
\text { protein } \\
\text { ERdj5 }\end{array}$ & \\
\hline R16934 & \begin{tabular}{|r|}
0.001203 \\
671 \\
\end{tabular} & $\begin{array}{l}\text { TNFSF13 } \\
\underline{B}\end{array}$ & 10673 & $\begin{array}{l}\text { tumor } \\
\text { necrosis } \\
\text { factor } \\
\text { (ligand) } \\
\text { superfamil } \\
y, \text { member } \\
13 b \\
\end{array}$ & $\begin{array}{l}\text { cell proliferation; immune response; } \\
\text { integral to plasma membrane; positive } \\
\text { regulation of cell proliferation; signal } \\
\text { transduction; soluble fraction; tumor } \\
\text { necrosis factor receptor binding }\end{array}$ \\
\hline AA044940 & $\begin{array}{r}0.001204 \\
383 \\
\end{array}$ & & & & \\
\hline R56037 & $\begin{array}{r}0.001231 \\
967\end{array}$ & & & & \\
\hline AA040160 & $\begin{array}{r}0.001246 \\
704 \\
\end{array}$ & $\frac{\mid \mathrm{LOC9271}}{\underline{5}}$ & 92715 & \begin{tabular}{|l|} 
hypothetic \\
al protein \\
BC017335
\end{tabular} & \\
\hline R83852 & $\begin{array}{r}0.001251 \\
331\end{array}$ & & & & \\
\hline R13550 & \begin{tabular}{|r|}
0.001254 \\
784 \\
\end{tabular} & & & & \\
\hline $\mathrm{H} 26570$ & $\begin{array}{r}0.001277 \\
725\end{array}$ & AKT2 & $\underline{208}$ & $\begin{array}{l}\text { v-akt } \\
\text { murine } \\
\text { thymoma } \\
\text { viral } \\
\text { oncogene } \\
\text { homolog } 2 \\
\end{array}$ & $\begin{array}{l}\text { ATP binding; protein amino acid } \\
\text { phosphorylation; protein } \\
\text { serine/threonine kinase activity; } \\
\text { transferase activity }\end{array}$ \\
\hline N63396 & $\begin{array}{r}0.001314 \\
843 \\
\end{array}$ & & & & \\
\hline H51271 & \begin{tabular}{|r|}
0.001320 \\
774 \\
\end{tabular} & & & & \\
\hline AA004845 & $\begin{array}{r}0.001331 \\
715\end{array}$ & KIAA1529 & $\underline{57653}$ & $\begin{array}{l}\text { KIAA1529 } \\
\text { protein }\end{array}$ & \\
\hline N/A1 & $\begin{array}{r}0.001335 \\
105\end{array}$ & & & & \\
\hline W07648 & $\begin{array}{r}0.001344 \\
377\end{array}$ & & & & \\
\hline T66873 & 0.001346 & & & & \\
\hline
\end{tabular}




\begin{tabular}{|c|c|c|c|c|c|}
\hline & 468 & & & & \\
\hline R14326 & $\begin{array}{r}0.001349 \\
613 \\
\end{array}$ & HERC1 & $\underline{8925}$ & \begin{tabular}{|l|} 
hect \\
(homologo \\
us to the \\
E6-AP \\
(UBE3A) \\
carboxyl \\
terminus) \\
domain \\
and RCC1 \\
(CHC1)- \\
like domain \\
(RLD) 1 \\
\end{tabular} & \begin{tabular}{|l} 
ARF guanyl-nucleotide exchange \\
factor activity; Golgi apparatus; \\
catalytic activity; nonselective vesicle \\
transport; ubiquitin cycle; ubiquitin- \\
protein ligase activity
\end{tabular} \\
\hline H98668 & $\begin{array}{r}0.001354 \\
685 \\
\end{array}$ & & & & \\
\hline AA002135 & $\begin{array}{r}0.001365 \\
198\end{array}$ & $\underline{\mathrm{C} 2}$ & $\underline{717}$ & \begin{tabular}{|l|} 
compleme \\
nt \\
component \\
2 \\
\\
\end{tabular} & $\begin{array}{l}\text { chymotrypsin activity; classical- } \\
\text { complement-pathway C3/C5 } \\
\text { convertase activity; complement } \\
\text { activation, classical pathway; } \\
\text { complement component C2 complex; } \\
\text { hydrolase activity; proteolysis and } \\
\text { peptidolysis; trypsin activity }\end{array}$ \\
\hline R12665 & $\begin{array}{r}0.001368 \\
855\end{array}$ & & $\underline{197135}$ & \begin{tabular}{|l|} 
similar to \\
RIKEN \\
cDNA \\
$4930424 G$ \\
05 \\
\end{tabular} & \\
\hline N38953 & $\begin{array}{r}0.001373 \\
325 \\
\end{array}$ & & & & \\
\hline N90841 & $\begin{array}{r}0.001376 \\
131 \\
\end{array}$ & CDKN1C & 1028 & \begin{tabular}{|l|} 
cyclin- \\
dependent \\
kinase \\
inhibitor 1C \\
(p57, Kip2) \\
\end{tabular} & $\begin{array}{l}\text { G1 phase of mitotic cell cycle; cell } \\
\text { cycle; cell cycle arrest; cyclin- } \\
\text { dependent protein kinase inhibitor } \\
\text { activity; negative regulation of cell } \\
\text { cycle; negative regulation of cell } \\
\text { proliferation; nucleus; regulation of } \\
\text { CDK activity }\end{array}$ \\
\hline R42536 & $\begin{array}{r}0.001378 \\
95\end{array}$ & DACH & 1602 & \begin{tabular}{|l|} 
dachshund \\
homolog \\
(Drosophil \\
a) \\
\end{tabular} & $\begin{array}{l}\text { eye morphogenesis (sensu } \\
\text { Drosophila) }\end{array}$ \\
\hline R34537 & \begin{tabular}{|r|}
0.001381 \\
264 \\
\end{tabular} & & & & \\
\hline R43469 & $\begin{array}{r}0.001381 \\
555 \\
\end{array}$ & EPHB3 & 2049 & EphB3 & $\begin{array}{l}\text { ATP binding; ephrin receptor activity; } \\
\text { integral to plasma membrane; protein } \\
\text { amino acid phosphorylation; receptor } \\
\text { activity; signal transduction; } \\
\text { transferase activity; transmembrane } \\
\text { receptor protein tyrosine kinase } \\
\text { signaling pathway }\end{array}$ \\
\hline T78084 & $\begin{array}{r}0.001391 \\
293 \\
\end{array}$ & & & & \\
\hline T86217 & $\begin{array}{r}0.001431 \\
738 \\
\end{array}$ & & & & \\
\hline
\end{tabular}




\begin{tabular}{|c|c|c|c|c|c|}
\hline R09469 & $\begin{array}{r}0.001432 \\
031\end{array}$ & & & & \\
\hline H67999 & $\begin{array}{r}0.001432 \\
285\end{array}$ & CYP3A7 & $\underline{1551}$ & $\begin{array}{l}\text { cytochrom } \\
\text { e P450, } \\
\text { family 3, } \\
\text { subfamily } \\
\text { A, } \\
\text { polypeptid } \\
\text { e } 7\end{array}$ & cytochrome P450 activity \\
\hline T95687 & $\begin{array}{r}0.001435 \\
574 \\
\end{array}$ & $\frac{\text { RAP1GD }}{\text { S1 }}$ & $\underline{5910}$ & $\begin{array}{l}\text { RAP1, } \\
\text { GTP-GDP } \\
\text { dissociatio } \\
n \\
\text { stimulator } \\
1\end{array}$ & $\begin{array}{l}\text { GTPase activator activity; } \\
\text { biological_process unknown; } \\
\text { cellular_component unknown }\end{array}$ \\
\hline H64569 & $\begin{array}{r}0.001442 \\
673 \\
\end{array}$ & & & & \\
\hline R09844 & \begin{tabular}{|r|}
0.001443 \\
567 \\
\end{tabular} & & & & \\
\hline N49914 & $\begin{array}{r}0.001454 \\
344\end{array}$ & KIAA0423 & $\underline{23116}$ & $\begin{array}{l}\text { KIAA0423 } \\
\text { protein }\end{array}$ & $\begin{array}{l}\text { binding; mitochondrial inner } \\
\text { membrane; transport }\end{array}$ \\
\hline W80729 & \begin{tabular}{|r|}
0.001458 \\
212
\end{tabular} & SMUG1 & 23583 & \begin{tabular}{|l|} 
single- \\
strand \\
selective \\
monofuncti \\
onal uracil \\
DNA \\
glycosylas \\
e
\end{tabular} & $\begin{array}{l}\text { DNA repair; single-stranded DNA } \\
\text { binding; uracil DNA N-glycosylase } \\
\text { activity }\end{array}$ \\
\hline R06074 & $\begin{array}{r}0.001462 \\
305\end{array}$ & & & & \\
\hline AA031859 & $\begin{array}{r}0.001463 \\
558 \\
\end{array}$ & TIMM13 & $\underline{26517}$ & \begin{tabular}{|l|} 
translocas \\
e of inner \\
mitochondr \\
ial \\
membrane \\
13 \\
homolog \\
(yeast)
\end{tabular} & $\begin{array}{l}\text { hearing; mitochondrial inner } \\
\text { membrane pre-sequence translocase } \\
\text { complex; mitochondrial translocation; } \\
\text { mitochondrion; protein targeting; } \\
\text { protein translocase activity; zinc ion } \\
\text { binding }\end{array}$ \\
\hline AA034109 & \begin{tabular}{|r|}
0.001468 \\
252
\end{tabular} & MINK & $\underline{50488}$ & $\begin{array}{l}\text { misshapen } \\
\text { /NIK- } \\
\text { related } \\
\text { kinase } \\
\\
\end{array}$ & $\begin{array}{l}\text { ATP binding; cAMP-dependent protein } \\
\text { kinase activity; development; protein } \\
\text { amino acid phosphorylation; protein } \\
\text { kinase CK2 activity; protein kinase } \\
\text { cascade; protein serine/threonine } \\
\text { kinase activity; response to stress; } \\
\text { small GTPase regulatory/interacting } \\
\text { protein activity; transferase activity }\end{array}$ \\
\hline R34114 & $\begin{array}{r}0.001469 \\
248 \\
\end{array}$ & & & & \\
\hline R23880 & $\begin{array}{r}0.001488 \\
865\end{array}$ & & 340730 & $\begin{array}{l}\text { LOC34073 } \\
0\end{array}$ & \\
\hline
\end{tabular}




\begin{tabular}{|c|c|c|c|c|c|}
\hline R09962 & $\begin{array}{r}0.001493 \\
614 \\
\end{array}$ & PMS2L6 & $\underline{5384}$ & \begin{tabular}{|l|} 
postmeiotic \\
segregatio \\
n \\
increased \\
2 -like 6 \\
\end{tabular} & $\begin{array}{l}\text { damaged DNA binding; mismatch } \\
\text { repair; nucleus }\end{array}$ \\
\hline H82747 & $\begin{array}{r}0.001495 \\
072 \\
\end{array}$ & & & & \\
\hline R20140 & $\begin{array}{r}0.001495 \\
222\end{array}$ & MFN1 & 55669 & mitofusin 1 & \\
\hline T84619 & $\begin{array}{r}0.001499 \\
292 \\
\end{array}$ & & & & \\
\hline T95148 & $\begin{array}{r}0.001503 \\
153 \\
\end{array}$ & & & & \\
\hline R18138 & \begin{tabular}{|r|}
0.001512 \\
918 \\
\end{tabular} & & & & \\
\hline H01884 & $\begin{array}{r}0.001532 \\
617 \\
\end{array}$ & & & & \\
\hline W90154 & $\begin{array}{r}0.001537 \\
062\end{array}$ & $\frac{\text { DKFZP43 }}{4 \mathrm{~B} 168}$ & $\underline{25896}$ & \begin{tabular}{|l|} 
DKFZP434 \\
B168 \\
protein
\end{tabular} & \\
\hline R06564 & $\begin{array}{r}0.001538 \\
843 \\
\end{array}$ & GALE & 2582 & $\begin{array}{l}\text { galactose- } \\
4- \\
\text { epimerase, } \\
\text { UDP- }\end{array}$ & $\begin{array}{l}\text { UDP-glucose 4-epimerase activity; } \\
\text { carbohydrate metabolism; galactose } \\
\text { metabolism; isomerase activity; } \\
\text { nucleotide-sugar metabolism }\end{array}$ \\
\hline N54603 & $\begin{array}{r}0.001548 \\
733 \\
\end{array}$ & & & & \\
\hline AA039986 & $\begin{array}{r}0.001567 \\
752 \\
\end{array}$ & FBLP-1 & $\underline{54751}$ & $\begin{array}{l}\text { filamin- } \\
\text { binding } \\
\text { LIM } \\
\text { protein-1 }\end{array}$ & \\
\hline N21636 & $\begin{array}{r}0.001573 \\
222 \\
\end{array}$ & PBP & $\underline{5037}$ & $\begin{array}{l}\text { prostatic } \\
\text { binding } \\
\text { protein }\end{array}$ & $\begin{array}{l}\text { ATP binding; lipid binding; } \\
\text { phosphatidylethanolamine binding; } \\
\text { serine protease inhibitor activity }\end{array}$ \\
\hline H08101 & $\begin{array}{r}0.001597 \\
637\end{array}$ & $\underline{\mathrm{GA}}$ & $\underline{27165}$ & \begin{tabular}{|l|} 
liver \\
mitochondr \\
ial \\
glutaminas \\
e
\end{tabular} & $\begin{array}{l}\text { amino acid metabolism; glutaminase } \\
\text { activity; glutamine metabolism; } \\
\text { hydrolase activity; mitochondrion }\end{array}$ \\
\hline N49325 & $\begin{array}{r}0.001617 \\
303\end{array}$ & KIAA0962 & 23341 & \begin{tabular}{|l|} 
KIAA0962 \\
protein
\end{tabular} & \\
\hline W69432 & $\begin{array}{r}0.001627 \\
691 \\
\end{array}$ & $\frac{\text { MAPKAP }}{\text { K2 }}$ & $\underline{9261}$ & $\begin{array}{l}\text { mitogen- } \\
\text { activated } \\
\text { protein } \\
\text { kinase- } \\
\text { activated } \\
\text { protein } \\
\text { kinase 2 } \\
\end{array}$ & $\begin{array}{l}\text { ATP binding; MAPKKK cascade; } \\
\text { nucleus; protein amino acid } \\
\text { phosphorylation; protein } \\
\text { serine/threonine kinase activity; signal } \\
\text { transducer activity; transferase activity }\end{array}$ \\
\hline AA031630 & $\begin{array}{r}0.001635 \\
462 \\
\end{array}$ & $\frac{\mathrm{LOC} 3400}{61}$ & $\underline{340061}$ & $\begin{array}{l}\text { hypothetic } \\
\text { al protein } \\
\text { LOC34006 } \\
1\end{array}$ & \\
\hline R97368 & 0.001637 & & & & \\
\hline
\end{tabular}




\begin{tabular}{|c|c|c|c|c|c|}
\hline & 009 & & & & \\
\hline H67706 & $\begin{array}{r}0.001639 \\
338 \\
\end{array}$ & & & & \\
\hline R36169 & $\begin{array}{r}0.001648 \\
31\end{array}$ & & & & \\
\hline AA135646 & $\begin{array}{r}0.001668 \\
986 \\
\end{array}$ & hIAN6 & $\underline{155038}$ & \begin{tabular}{|l} 
human \\
immune \\
associated \\
nucleotide \\
6
\end{tabular} & \\
\hline T79552 & $\begin{array}{r}0.001673 \\
764\end{array}$ & & & & \\
\hline T67217 & $\begin{array}{r}0.001676 \\
026 \\
\end{array}$ & MGC3207 & $\underline{84245}$ & $\begin{array}{l}\text { hypothetic } \\
\text { al protein } \\
\text { MGC3207 }\end{array}$ & \\
\hline R65751 & $\begin{array}{r}0.001696 \\
666 \\
\end{array}$ & $\underline{\text { SLC16A4 }}$ & 9122 & \begin{tabular}{|l|} 
olute \\
carrier \\
family 16 \\
(monocarb \\
oxylic acid \\
transporter \\
s), member \\
4
\end{tabular} & $\begin{array}{l}\text { integral to plasma membrane; } \\
\text { membrane fraction; monocarboxylic } \\
\text { acid transport; monocarboxylic acid } \\
\text { transporter activity }\end{array}$ \\
\hline AA033652 & $\begin{array}{r}0.001697 \\
509 \\
\end{array}$ & DDX54 & 79039 & \multicolumn{2}{|c|}{ DEAD (Asp-Glu-Ala-Asp) box polypeptide 54} \\
\hline R44335 & $\begin{array}{r}0.001706 \\
69 \\
\end{array}$ & & & & \\
\hline W03006 & $\begin{array}{r}0.001708 \\
944 \\
\end{array}$ & MARCKS & $\underline{4082}$ & \begin{tabular}{|l|} 
myristoylat \\
ed alanine- \\
rich protein \\
kinase C \\
substrate \\
\end{tabular} & $\begin{array}{l}\text { actin cross-linking activity; actin } \\
\text { cytoskeleton; calmodulin binding; cell } \\
\text { motility; plasma membrane }\end{array}$ \\
\hline R56247 & $\begin{array}{r}0.001710 \\
31 \\
\end{array}$ & RASD2 & $\underline{23551}$ & \begin{tabular}{|l|} 
RASD \\
family, \\
member 2 \\
\end{tabular} & $\begin{array}{l}\text { GTP binding; RAS small monomeric } \\
\text { GTPase activity; biological_process } \\
\text { unknown; cellular_component } \\
\text { unknown; molecular_function } \\
\text { unknown; small GTPase mediated } \\
\text { signal transduction }\end{array}$ \\
\hline R76088 & \begin{tabular}{|r|}
0.001718 \\
762 \\
\end{tabular} & $\underline{\mathrm{UCHL} 3}$ & $\underline{7347}$ & \begin{tabular}{|l|} 
ubiquitin \\
carboxyl- \\
terminal \\
esterase \\
L3 \\
(ubiquitin \\
thiolestera \\
se) \\
\end{tabular} & $\begin{array}{l}\text { cytoplasm; hydrolase activity; ubiquitin } \\
\text { thiolesterase activity; ubiquitin- } \\
\text { dependent protein catabolism }\end{array}$ \\
\hline T84202 & $\begin{array}{r}0.001725 \\
688 \\
\end{array}$ & TAPBP & $\underline{6892}$ & \begin{tabular}{|l} 
TAP \\
binding \\
protein \\
(tapasin)
\end{tabular} & $\begin{array}{l}\text { MHC-interacting protein; endoplasmic } \\
\text { reticulum; endoplasmic reticulum } \\
\text { membrane; immune response; integral } \\
\text { to membrane; peptide antigen } \\
\text { transporter activity; protein binding; } \\
\text { protein complex assembly }\end{array}$ \\
\hline
\end{tabular}




\begin{tabular}{|c|c|c|c|c|c|}
\hline N23779 & \begin{tabular}{|r|}
0.001736 \\
785 \\
\end{tabular} & CD151 & 977 & \begin{tabular}{|l} 
CD151 \\
antigen
\end{tabular} & $\begin{array}{l}\text { cell adhesion; integral to plasma } \\
\text { membrane; membrane fraction }\end{array}$ \\
\hline R28465 & $\begin{array}{r}0.001739 \\
327\end{array}$ & & $\underline{221922}$ & \multicolumn{2}{|c|}{$\begin{array}{l}\text { hypothetical gene supported by AL713633; } \\
\text { BC014395 }\end{array}$} \\
\hline R94942 & $\begin{array}{r}0.001743 \\
843\end{array}$ & FLJ20522 & $\underline{54965}$ & $\begin{array}{l}\text { hypothetic } \\
\text { al protein } \\
\text { FLJ20522 } \\
\end{array}$ & \\
\hline R17806 & $\begin{array}{r}0.001749 \\
212\end{array}$ & C14orf37 & 145407 & $\begin{array}{l}\text { chromoso } \\
\text { me } 14 \\
\text { open } \\
\text { reading } \\
\text { frame } 37\end{array}$ & \\
\hline T99284 & \begin{tabular}{|r|}
0.001774 \\
889
\end{tabular} & & 145608 & $\begin{array}{l}\mathrm{LOC} 14560 \\
8\end{array}$ & \\
\hline T97033 & $\begin{array}{r}0.001780 \\
573 \\
\end{array}$ & $\frac{\text { DKFZP43 }}{4 \mid 116}$ & 25962 & $\begin{array}{l}\text { DKFZP434 } \\
\text { I116 } \\
\text { protein }\end{array}$ & nucleic acid binding \\
\hline H98614 & $\begin{array}{r}0.001790 \\
742 \\
\end{array}$ & ZFHX1B & 9839 & $\begin{array}{l}\text { zinc finger } \\
\text { homeobox } \\
1 b\end{array}$ & $\begin{array}{l}\text { SMAD binding; negative regulation of } \\
\text { transcription; neurogenesis; nucleus; } \\
\text { phosphatase regulator activity; } \\
\text { regulation of transcription, DNA- } \\
\text { dependent; transcription factor activity; } \\
\text { transcriptional repressor activity }\end{array}$ \\
\hline R07557 & $\begin{array}{r}0.001802 \\
913 \\
\end{array}$ & RPLP0 & $\underline{6175}$ & $\begin{array}{l}\text { ribosomal } \\
\text { protein, } \\
\text { large, P0 }\end{array}$ & $\begin{array}{l}\text { RNA binding; cytosolic large ribosomal } \\
\text { subunit (sensu Eukarya); intracellular; } \\
\text { ribosome; structural constituent of } \\
\text { ribosome; translational elongation }\end{array}$ \\
\hline H59829 & $\begin{array}{r}0.001815 \\
986\end{array}$ & & & & \\
\hline T85888 & $\begin{array}{r}0.001824 \\
002 \\
\end{array}$ & $\frac{\text { DKFZP56 }}{40043}$ & $\underline{57037}$ & $\begin{array}{l}\text { hypothetic } \\
\text { al protein } \\
\text { DKFZp564 } \\
\text { O043 } \\
\end{array}$ & \\
\hline R80523 & \begin{tabular}{|r|}
0.001824 \\
324 \\
\end{tabular} & & & & \\
\hline T70331 & $\begin{array}{r}0.001825 \\
185 \\
\end{array}$ & EPAS1 & $\underline{2034}$ & $\begin{array}{l}\text { endothelial } \\
\text { PAS } \\
\text { domain } \\
\text { protein 1 }\end{array}$ & $\begin{array}{l}\text { RNA polymerase II transcription factor } \\
\text { activity, enhancer binding; } \\
\text { angiogenesis; development; nucleus; } \\
\text { regulation of transcription, DNA- } \\
\text { dependent; signal transducer activity; } \\
\text { signal transduction; transcription co-- } \\
\text { activator activity; transcription from Pol } \\
\text { II promoter }\end{array}$ \\
\hline H77595 & $\begin{array}{r}0.001827 \\
873 \\
\end{array}$ & & & & \\
\hline R69535 & $\begin{array}{r}0.001829 \\
561\end{array}$ & $\underline{I G K C}$ & $\underline{3514}$ & $\begin{array}{l}\text { immunoglo } \\
\text { bulin } \\
\text { kappa } \\
\text { constant } \\
\end{array}$ & antigen binding; immune response \\
\hline R13273 & \begin{tabular}{|r|}
0.001843 \\
677 \\
\end{tabular} & & & & \\
\hline T72401 & 0.001845 & C8orf4 & 56892 & chromoso & \\
\hline
\end{tabular}




\begin{tabular}{|c|c|c|c|c|c|}
\hline & 907 & & & $\begin{array}{l}\text { me } 8 \text { open } \\
\text { reading } \\
\text { frame } 4\end{array}$ & \\
\hline H47114 & $\begin{array}{r}0.001847 \\
68 \\
\end{array}$ & & & & \\
\hline T99834 & $\begin{array}{r}0.001867 \\
049\end{array}$ & & & & \\
\hline H68599 & \begin{tabular}{|r|}
0.001869 \\
866
\end{tabular} & $\frac{\text { MGC1573 }}{7}$ & $\underline{85012}$ & $\begin{array}{l}\text { hypothetic } \\
\text { al protein } \\
\text { MGC1573 } \\
7\end{array}$ & \\
\hline N57396 & $\begin{array}{r}0.001890 \\
572 \\
\end{array}$ & $\begin{array}{l}\text { LOC1508 } \\
37\end{array}$ & 150837 & $\begin{array}{l}\text { hypothetic } \\
\text { al protein } \\
\text { LOC15083 } \\
7\end{array}$ & \\
\hline R23436 & $\begin{array}{r}0.001900 \\
3\end{array}$ & $\begin{array}{l}\text { PRKAR2 } \\
\underline{A}\end{array}$ & $\underline{5576}$ & $\begin{array}{l}\text { protein } \\
\text { kinase, } \\
\text { cAMP- } \\
\text { dependent, } \\
\text { regulatory, } \\
\text { type II, } \\
\text { alpha } \\
\end{array}$ & $\begin{array}{l}\text { cAMP-dependent protein kinase, } \\
\text { intrinsic regulator activity; cytoplasm; } \\
\text { intracellular signaling cascade; } \\
\text { membrane fraction; plasma membrane }\end{array}$ \\
\hline R21825 & $\begin{array}{r}0.001915 \\
345 \\
\end{array}$ & & & & \\
\hline AA114919 & \begin{tabular}{|r|}
0.001917 \\
815
\end{tabular} & NSEP1 & $\underline{4904}$ & $\begin{array}{l}\text { nuclease } \\
\text { sensitive } \\
\text { element } \\
\text { binding } \\
\text { protein } 1\end{array}$ & $\begin{array}{l}\text { DNA binding; double-stranded DNA } \\
\text { binding; nucleus; response to } \\
\text { pest/pathogen/parasite; single- } \\
\text { stranded DNA binding; transcription } \\
\text { factor activity; transcription from Pol II } \\
\text { promoter }\end{array}$ \\
\hline R02194 & $\begin{array}{r}0.001921 \\
302 \\
\end{array}$ & & & & \\
\hline N24943 & \begin{tabular}{|r|}
0.001935 \\
641 \\
\end{tabular} & FLJ13612 & 80303 & $\begin{array}{l}\text { likely } \\
\text { ortholog of } \\
\text { neuronally } \\
\text { expressed } \\
\text { calcium } \\
\text { binding } \\
\text { protein }\end{array}$ & calcium ion binding \\
\hline R87060 & \begin{tabular}{|r|}
0.001952 \\
501
\end{tabular} & GGCX & 2677 & $\begin{array}{l}\text { gamma- } \\
\text { glutamyl } \\
\text { carboxylas } \\
\text { e }\end{array}$ & $\begin{array}{l}\text { blood coagulation; gamma-glutamyl } \\
\text { carboxylase activity; integral to } \\
\text { membrane; ligase activity; membrane } \\
\text { fraction; protein modification }\end{array}$ \\
\hline AA045253 & \begin{tabular}{|r|}
0.001965 \\
951
\end{tabular} & & & & \\
\hline R00170 & $\begin{array}{r}0.001969 \\
316\end{array}$ & BFAR & 51283 & $\begin{array}{l}\text { bifunctional } \\
\text { apoptosis } \\
\text { regulator }\end{array}$ & $\begin{array}{l}\text { anti-apoptosis; apoptosis inhibitor } \\
\text { activity; integral to plasma membrane; } \\
\text { membrane fraction; structural } \\
\text { molecule activity }\end{array}$ \\
\hline R63553 & \begin{tabular}{|r|}
0.001971 \\
752 \\
\end{tabular} & ALEX1 & 51309 & $\begin{array}{l}\text { ALEX1 } \\
\text { protein }\end{array}$ & \\
\hline T82206 & $\begin{array}{r}0.001985 \\
116\end{array}$ & & & & \\
\hline
\end{tabular}




\begin{tabular}{|c|c|c|c|c|c|}
\hline $\mathrm{H} 17654$ & \begin{tabular}{|r|}
0.001985 \\
446 \\
\end{tabular} & & & & \\
\hline T65080 & $\begin{array}{r}0.001987 \\
878 \\
\end{array}$ & & & & \\
\hline H85905 & $\begin{array}{r}0.002010 \\
733 \\
\end{array}$ & & & & \\
\hline R80226 & $\begin{array}{r}0.002037 \\
069 \\
\end{array}$ & & & & \\
\hline AA057286 & $\begin{array}{r}0.002040 \\
228 \\
\end{array}$ & $\begin{array}{l}\text { TA- } \\
\text { WDRP }\end{array}$ & $\underline{134430}$ & $\begin{array}{l}\text { T-cell } \\
\text { activation } \\
\text { WD repeat } \\
\text { protein }\end{array}$ & catalytic activity; metabolism \\
\hline N59347 & $\begin{array}{r}0.002086 \\
366\end{array}$ & TARS & $\underline{6897}$ & $\begin{array}{l}\text { threonyl- } \\
\text { tRNA } \\
\text { synthetase }\end{array}$ & $\begin{array}{l}\text { ATP binding; cytoplasm; ligase } \\
\text { activity; soluble fraction; threonine- } \\
\text { tRNA ligase activity; threonyl-tRNA } \\
\text { aminoacylation }\end{array}$ \\
\hline R12879 & $\begin{array}{r}0.002091 \\
929 \\
\end{array}$ & KIAA1336 & $\underline{57539}$ & $\begin{array}{l}\text { KIAA1336 } \\
\text { protein }\end{array}$ & \\
\hline H42536 & $\begin{array}{r}0.002097 \\
603 \\
\end{array}$ & GPD1 & $\underline{2819}$ & $\begin{array}{l}\text { glycerol-3- } \\
\text { phosphate } \\
\text { dehydroge } \\
\text { nase } 1 \\
\text { (soluble) }\end{array}$ & $\begin{array}{l}\text { carbohydrate metabolism; glycerol-3- } \\
\text { phosphate dehydrogenase (NAD) } \\
\text { activity; glycerol-3-phosphate } \\
\text { dehydrogenase complex; glycerol-3- } \\
\text { phosphate metabolism; } \\
\text { oxidoreductase activity, acting on } \mathrm{CH} \text { - } \\
\text { OH group of donors }\end{array}$ \\
\hline R20019 & $\begin{array}{r}0.002118 \\
61 \\
\end{array}$ & & & & \\
\hline AA207094 & \begin{tabular}{|r|}
0.002132 \\
152 \\
\end{tabular} & & & & \\
\hline H84720 & $\begin{array}{r}0.002135 \\
212 \\
\end{array}$ & & & & \\
\hline N67634 & $\begin{array}{r}0.002150 \\
875 \\
\end{array}$ & $\frac{\mathrm{P} 1 \mathrm{P} 373 \mathrm{C}}{6}$ & $\underline{56053}$ & $\begin{array}{l}\text { hypothetic } \\
\text { al protein } \\
\text { P1 p373c6 }\end{array}$ & \\
\hline R36086 & $\begin{array}{r}0.002150 \\
941 \\
\end{array}$ & & & & \\
\hline N64399 & \begin{tabular}{|r|}
0.002151 \\
364 \\
\end{tabular} & OSBPL1A & $\underline{114876}$ & $\begin{array}{l}\text { oxysterol } \\
\text { binding } \\
\text { protein-like } \\
1 \mathrm{~A}\end{array}$ & $\begin{array}{l}\text { cholesterol metabolism; intracellular; } \\
\text { lipid transport; phospholipid binding; } \\
\text { steroid metabolism; vesicle-mediated } \\
\text { transport }\end{array}$ \\
\hline AA203254 & \begin{tabular}{|r|}
0.002155 \\
188
\end{tabular} & & & & \\
\hline N76908 & $\begin{array}{r}0.002175 \\
852\end{array}$ & & & & \\
\hline R31105 & $\begin{array}{r}0.002197 \\
489\end{array}$ & & & & \\
\hline T81437 & $\begin{array}{r}0.002224 \\
647 \\
\end{array}$ & & & & \\
\hline
\end{tabular}




\begin{tabular}{|c|c|c|c|c|c|}
\hline N75416 & \begin{tabular}{|r|}
0.002233 \\
007
\end{tabular} & GNA14 & 9630 & $\begin{array}{l}\text { guanine } \\
\text { nucleotide } \\
\text { binding } \\
\text { protein (G } \\
\text { protein), } \\
\text { alpha } 14\end{array}$ & $\begin{array}{l}\text { G-protein coupled receptor protein } \\
\text { signaling pathway; GTP binding; } \\
\text { heterotrimeric G-protein GTPase } \\
\text { activity; heterotrimeric G-protein } \\
\text { complex; plasma membrane; protein } \\
\text { amino acid ADP-ribosylation; signal } \\
\text { transducer activity; signal transduction }\end{array}$ \\
\hline T75185 & \begin{tabular}{|r|}
0.002242 \\
925
\end{tabular} & FLJ33761 & 125488 & $\begin{array}{l}\text { hypothetic } \\
\text { al protein } \\
\text { FLJ33761 }\end{array}$ & \\
\hline AA150729 & $\begin{array}{r}0.002252 \\
663 \\
\end{array}$ & NCOR2 & 9612 & \begin{tabular}{|l|} 
nuclear \\
receptor \\
co- \\
repressor 2 \\
\end{tabular} & \\
\hline AA203692 & \begin{tabular}{|r|}
0.002254 \\
753
\end{tabular} & & & & \\
\hline R15267 & $\begin{array}{r}0.002276 \\
617\end{array}$ & & & & \\
\hline AA046245 & \begin{tabular}{|r|}
0.002281 \\
88
\end{tabular} & OSF-2 & 10631 & \begin{tabular}{l|} 
osteoblast \\
specific \\
factor 2 \\
(fasciclin I- \\
like)
\end{tabular} & $\begin{array}{l}\text { cell adhesion; cell adhesion molecule } \\
\text { activity; extracellular matrix; skeletal } \\
\text { development }\end{array}$ \\
\hline R60906 & $\begin{array}{r}0.002297 \\
729 \\
\end{array}$ & $\frac{M G C 2438}{1}$ & 115939 & $\begin{array}{l}\text { hypothetic } \\
\text { al protein } \\
\text { MGC2438 } \\
1\end{array}$ & \\
\hline H59112 & $\begin{array}{r}0.002297 \\
904 \\
\end{array}$ & & & & \\
\hline N24066 & $\begin{array}{r}0.002299 \\
348 \\
\end{array}$ & CSDA & 8531 & \begin{tabular}{|l|} 
cold shock \\
domain \\
protein A
\end{tabular} & $\begin{array}{l}\text { RNA polymerase II transcription factor } \\
\text { activity; cytoplasm; double-stranded } \\
\text { DNA binding; negative regulation of } \\
\text { transcription from Pol II promoter; } \\
\text { perinuclear space; response to cold; } \\
\text { transcription co-repressor activity; } \\
\text { transcription factor activity }\end{array}$ \\
\hline H80679 & $\begin{array}{r}0.002311 \\
983 \\
\end{array}$ & & & & \\
\hline N78909 & $\begin{array}{r}0.002315 \\
35 \\
\end{array}$ & & & & \\
\hline N/A1 & $\begin{array}{r}0.002315 \\
428 \\
\end{array}$ & & & & \\
\hline W47145 & $\begin{array}{r}0.002340 \\
933 \\
\end{array}$ & EIF3S7 & 8664 & $\begin{array}{l}\text { eukaryotic } \\
\text { translation } \\
\text { initiation } \\
\text { factor } 3 \\
\text { subunit } 7 \\
\text { zeta, } \\
66 / 67 \mathrm{kDa} \\
\end{array}$ & $\begin{array}{l}\text { eukaryotic translation initiation factor } 3 \\
\text { complex; protein biosynthesis; } \\
\text { regulation of translational initiation; } \\
\text { translation initiation factor activity }\end{array}$ \\
\hline N62985 & $\begin{array}{r}0.002361 \\
781 \\
\end{array}$ & \begin{tabular}{|l}
$\underline{\mathrm{LOC} 9067}$ \\
$\underline{3}$
\end{tabular} & 90673 & \begin{tabular}{|l|} 
hypothetic \\
al protein \\
LOC90673
\end{tabular} & \\
\hline
\end{tabular}




\begin{tabular}{|c|c|c|c|c|c|}
\hline N42004 & $\begin{array}{r}0.002367 \\
601 \\
\end{array}$ & DPYSL3 & $\underline{1809}$ & $\begin{array}{l}\text { dihydropyri } \\
\text { midinase- } \\
\text { like } 3\end{array}$ & $\begin{array}{l}\text { dihydropyrimidinase activity; hydrolase } \\
\text { activity; neurogenesis; nucleobase, } \\
\text { nucleoside, nucleotide and nucleic } \\
\text { acid metabolism; signal transduction }\end{array}$ \\
\hline R65766 & $\begin{array}{r}0.002368 \\
414\end{array}$ & SEC22L1 & 9554 & $\begin{array}{l}\text { SEC22 } \\
\text { vesicle } \\
\text { trafficking } \\
\text { protein-like } \\
1 \text { (S. } \\
\text { cerevisiae) } \\
\end{array}$ & $\begin{array}{l}\text { ER to Golgi transport; endoplasmic } \\
\text { reticulum membrane }\end{array}$ \\
\hline R36114 & $\begin{array}{r}0.002399 \\
498\end{array}$ & $\underline{\text { FLJ33387 }}$ & 161145 & $\begin{array}{l}\text { hypothetic } \\
\text { al protein } \\
\text { FLJ33387 }\end{array}$ & \\
\hline H85811 & $\begin{array}{r}0.002403 \\
043\end{array}$ & HIPK2 & 28996 & $\begin{array}{l}\text { homeodom } \\
\text { ain } \\
\text { interacting } \\
\text { protein } \\
\text { kinase 2 } \\
\end{array}$ & $\begin{array}{l}\text { nucleus; protein kinase activity; } \\
\text { transcription co-repressor activity }\end{array}$ \\
\hline AA130140 & $\begin{array}{r}0.002403 \\
115 \\
\end{array}$ & & & & \\
\hline T93785 & $\begin{array}{r}0.002410 \\
919\end{array}$ & & & & \\
\hline H62006 & $\begin{array}{r}0.002432 \\
551 \\
\end{array}$ & $\underline{E V I 2 B}$ & 2124 & $\begin{array}{l}\text { ecotropic } \\
\text { viral } \\
\text { integration } \\
\text { site 2B } \\
\end{array}$ & $\begin{array}{l}\text { cell growth and/or maintenance; } \\
\text { integral to plasma membrane }\end{array}$ \\
\hline R25519 & $\begin{array}{r}0.002434 \\
119\end{array}$ & HPCAL4 & $\underline{51440}$ & $\begin{array}{l}\text { hippocalcin } \\
\text { like } 4\end{array}$ & $\begin{array}{l}\text { calcium ion binding; central nervous } \\
\text { system development }\end{array}$ \\
\hline H57272 & $\begin{array}{r}0.002474 \\
056\end{array}$ & & & & \\
\hline R09933 & $\begin{array}{r}0.002511 \\
822 \\
\end{array}$ & & & & \\
\hline W15390 & $\begin{array}{r}0.002545 \\
125\end{array}$ & BMPR1A & $\underline{657}$ & $\begin{array}{l}\text { bone } \\
\text { morphogen } \\
\text { etic protein } \\
\text { receptor, } \\
\text { type IA }\end{array}$ & $\begin{array}{l}\text { ATP binding; TGFbeta receptor } \\
\text { signaling pathway; cAMP-dependent } \\
\text { protein kinase activity; integral to } \\
\text { membrane; protein amino acid } \\
\text { phosphorylation; protein kinase CK2 } \\
\text { activity; protein serine/threonine } \\
\text { kinase activity; protein-tyrosine kinase } \\
\text { activity; receptor activity; transferase } \\
\text { activity; transforming growth factor- } \\
\text { beta receptor activity }\end{array}$ \\
\hline R16440 & $\begin{array}{r}0.002546 \\
399 \\
\end{array}$ & AD024 & $\underline{57405}$ & $\begin{array}{l}\text { AD024 } \\
\text { protein }\end{array}$ & \\
\hline AA010141 & $\begin{array}{r}0.002547 \\
572 \\
\end{array}$ & $\frac{\text { SERPINH }}{1}$ & $\underline{871}$ & $\begin{array}{l}\text { serine (or } \\
\text { cysteine) } \\
\text { proteinase } \\
\text { inhibitor, } \\
\text { clade H } \\
\text { (heat } \\
\text { shock } \\
\text { protein 47), }\end{array}$ & heat shock response \\
\hline
\end{tabular}




\begin{tabular}{|c|c|c|c|c|c|}
\hline & & & & $\begin{array}{l}\text { member 1, } \\
\text { (collagen } \\
\text { binding } \\
\text { protein 1) } \\
\end{array}$ & \\
\hline R88895 & $\begin{array}{r}0.002561 \\
656\end{array}$ & MANBAL & $\underline{63905}$ & $\begin{array}{l}\text { mannosida } \\
\text { se, beta A, } \\
\text { lysosomal- } \\
\text { like }\end{array}$ & integral to membrane \\
\hline H84599 & $\begin{array}{r}0.002580 \\
41 \\
\end{array}$ & & & & \\
\hline H06620 & $\begin{array}{r}0.002605 \\
411\end{array}$ & & & & \\
\hline H79410 & $\begin{array}{r}0.002607 \\
092\end{array}$ & & & & \\
\hline H19945 & $\begin{array}{r}0.002611 \\
532 \\
\end{array}$ & STX5A & $\underline{6811}$ & $\begin{array}{l}\text { syntaxin } \\
5 \mathrm{~A}\end{array}$ & nonselective vesicle targeting \\
\hline AA142924 & $\begin{array}{r}0.002612 \\
33\end{array}$ & $\underline{D F}$ & $\underline{1675}$ & $\begin{array}{l}\text { D } \\
\text { component } \\
\text { of } \\
\text { compleme } \\
\text { nt (adipsin) }\end{array}$ & $\begin{array}{l}\text { chymotrypsin activity; complement } \\
\text { activation, alternative pathway; } \\
\text { complement factor D activity; } \\
\text { hydrolase activity; proteolysis and } \\
\text { peptidolysis; trypsin activity }\end{array}$ \\
\hline W39594 & $\begin{array}{r}0.002627 \\
393\end{array}$ & NSEP1 & $\underline{4904}$ & $\begin{array}{l}\text { nuclease } \\
\text { sensitive } \\
\text { element } \\
\text { binding } \\
\text { protein } 1\end{array}$ & $\begin{array}{l}\text { DNA binding; double-stranded DNA } \\
\text { binding; nucleus; response to } \\
\text { pest/pathogen/parasite; single- } \\
\text { stranded DNA binding; transcription } \\
\text { factor activity; transcription from Pol II } \\
\text { promoter }\end{array}$ \\
\hline R10547 & $\begin{array}{r}0.002656 \\
444\end{array}$ & & & & \\
\hline H69845 & $\begin{array}{r}0.002659 \\
182 \\
\end{array}$ & & & & \\
\hline AA031564 & $\begin{array}{r}0.002660 \\
207\end{array}$ & $\frac{\operatorname{LOC1134}}{44}$ & 113444 & $\begin{array}{l}\text { hypothetic } \\
\text { al protein } \\
\text { BC011880 }\end{array}$ & \\
\hline AA152287 & $\begin{array}{r}0.002679 \\
818\end{array}$ & $\underline{\text { SLC35B2 }}$ & 347734 & $\begin{array}{l}\text { solute } \\
\text { carrier } \\
\text { family } 35 \\
\text { member } \\
\text { B2 } \\
\end{array}$ & $\begin{array}{l}\text { copper ion binding; electron transport; } \\
\text { electron transporter activity }\end{array}$ \\
\hline AA028109 & $\begin{array}{r}0.002693 \\
002 \\
\end{array}$ & RAB23 & $\underline{51715}$ & $\begin{array}{l}\text { RAB23, } \\
\text { member } \\
\text { RAS } \\
\text { oncogene } \\
\text { family }\end{array}$ & $\begin{array}{l}\text { GTP binding; RAB small monomeric } \\
\text { GTPase activity; intracellular protein } \\
\text { transport; protein transporter activity; } \\
\text { small GTPase mediated signal } \\
\text { transduction }\end{array}$ \\
\hline T91181 & $\begin{array}{r}0.002737 \\
341\end{array}$ & & & & \\
\hline
\end{tabular}




\begin{tabular}{|c|c|c|c|c|c|}
\hline AA054102 & $\begin{array}{r}0.002743 \\
091 \\
\end{array}$ & SOCS5 & $\underline{9655}$ & $\begin{array}{l}\text { suppressor } \\
\text { of cytokine } \\
\text { signaling } 5\end{array}$ & $\begin{array}{l}\text { JAK-STAT cascade; cytokine and } \\
\text { chemokine mediated signaling } \\
\text { pathway; cytoplasm; intracellular } \\
\text { signaling cascade; kinase inhibitor } \\
\text { activity; negative regulation of T-helper } \\
2 \text { cell differentiation; negative } \\
\text { regulation of signal transduction; } \\
\text { positive regulation of T-helper } 1 \text { cell } \\
\text { differentiation; protein binding; } \\
\text { regulation of cell growth }\end{array}$ \\
\hline N94432 & $\begin{array}{r}0.002746 \\
068 \\
\end{array}$ & & & & \\
\hline H28534 & $\begin{array}{r}0.002784 \\
444 \\
\end{array}$ & $\underline{A Q P 1}$ & $\underline{358}$ & \begin{tabular}{|l|} 
aquaporin \\
1 (channel- \\
forming \\
integral \\
protein, \\
$28 \mathrm{kDa}$ ) \\
\end{tabular} & $\begin{array}{l}\text { excretion; integral to plasma } \\
\text { membrane; transport; water transport; } \\
\text { water transporter activity }\end{array}$ \\
\hline N90527 & $\begin{array}{r}0.002790 \\
75 \\
\end{array}$ & PIM1 & $\underline{5292}$ & \begin{tabular}{|l|} 
pim-1 \\
oncogene
\end{tabular} & $\begin{array}{l}\text { ATP binding; cAMP-dependent protein } \\
\text { kinase activity; cell growth and/or } \\
\text { maintenance; cytoplasm; } \\
\text { development; protein amino acid } \\
\text { phosphorylation; protein kinase CK2 } \\
\text { activity; protein serine/threonine } \\
\text { kinase activity; transferase activity }\end{array}$ \\
\hline AA114872 & $\begin{array}{r}0.002801 \\
353 \\
\end{array}$ & $\underline{\mathrm{ACO} 1}$ & $\underline{48}$ & $\begin{array}{l}\text { aconitase } \\
1, \text { soluble }\end{array}$ & $\begin{array}{l}\text { RNA binding; aconitate hydratase } \\
\text { activity; cytoplasm; lyase activity; } \\
\text { metabolism; negative regulation of } \\
\text { translation; tricarboxylic acid cycle }\end{array}$ \\
\hline AA046610 & \begin{tabular}{|r|}
0.002804 \\
358 \\
\end{tabular} & & & & \\
\hline R88547 & $\begin{array}{r}0.002824 \\
047 \\
\end{array}$ & FLJ25530 & $\underline{220296}$ & \begin{tabular}{|l|} 
hypothetic \\
al protein \\
FLJ25530
\end{tabular} & \\
\hline N24815 & $\begin{array}{r}0.002848 \\
453 \\
\end{array}$ & UBA52 & 7311 & \begin{tabular}{|l|} 
ubiquitin A- \\
52 residue \\
ribosomal \\
protein \\
fusion \\
product 1 \\
\end{tabular} & $\begin{array}{l}\text { nucleus; protein biosynthesis; protein } \\
\text { modification; ribosome; structural } \\
\text { constituent of ribosome }\end{array}$ \\
\hline R52303 & $\begin{array}{r}0.002849 \\
583 \\
\end{array}$ & & & & \\
\hline N45514 & \begin{tabular}{|r|}
0.002870 \\
186 \\
\end{tabular} & NECL1 & $\underline{57863}$ & $\begin{array}{l}\text { nectin-like } \\
\text { protein } 1\end{array}$ & \\
\hline N75085 & \begin{tabular}{|r|}
0.002880 \\
082
\end{tabular} & OLR1 & $\underline{4973}$ & \begin{tabular}{|l|} 
oxidised \\
low density \\
lipoprotein \\
(lectin-like) \\
receptor 1 \\
\end{tabular} & $\begin{array}{l}\text { circulation; heterophilic cell adhesion; } \\
\text { integral to plasma membrane; } \\
\text { membrane fraction; proteolysis and } \\
\text { peptidolysis; receptor activity; sugar } \\
\text { binding }\end{array}$ \\
\hline H53599 & $\begin{array}{r}0.002899 \\
157 \\
\end{array}$ & & & & \\
\hline H51648 & 0.002907 & MGC1694 & 112479 & similar to & exonuclease activity; intracellular \\
\hline
\end{tabular}




\begin{tabular}{|c|c|c|c|c|c|}
\hline & 82 & & & $\begin{array}{l}\text { RIKEN } \\
\text { CDNA } \\
\text { 4933424N } \\
\text { 09 gene }\end{array}$ & \\
\hline N69468 & $\begin{array}{r}0.002945 \\
715 \\
\end{array}$ & & & & \\
\hline H82917 & $\begin{array}{r}0.002960 \\
524 \\
\end{array}$ & & & & \\
\hline R26716 & $\begin{array}{r}0.002964 \\
644 \\
\end{array}$ & ZBTB2 & $\underline{57621}$ & $\begin{array}{l}\text { zinc finger } \\
\text { and BTB } \\
\text { domain } \\
\text { containing } \\
2\end{array}$ & $\begin{array}{l}\text { DNA binding; nucleus; protein binding; } \\
\text { regulation of transcription, DNA- } \\
\text { dependent }\end{array}$ \\
\hline N22392 & $\begin{array}{r}0.002974 \\
381\end{array}$ & CLDN11 & $\underline{5010}$ & $\begin{array}{l}\text { claudin } 11 \\
\text { (oligodendr } \\
\text { ocyte } \\
\text { transmemb } \\
\text { rane } \\
\text { protein) } \\
\end{array}$ & $\begin{array}{l}\text { integral to membrane; structural } \\
\text { molecule activity; tight junction }\end{array}$ \\
\hline AA054115 & $\begin{array}{r}0.002987 \\
791 \\
\end{array}$ & & & & \\
\hline H88417 & \begin{tabular}{|r|}
0.002994 \\
186 \\
\end{tabular} & CGI-127 & $\underline{51646}$ & $\begin{array}{l}\text { yippee } \\
\text { protein }\end{array}$ & \\
\hline $\mathrm{H} 72512$ & $\begin{array}{r}0.003003 \\
414\end{array}$ & HSPC023 & 28974 & $\begin{array}{l}\text { HSPC023 } \\
\text { protein }\end{array}$ & \\
\hline T91277 & $\begin{array}{r}0.003025 \\
45 \\
\end{array}$ & & & & \\
\hline AA062622 & $\begin{array}{r}0.003033 \\
254 \\
\end{array}$ & $\frac{\text { PRKWNK }}{1}$ & $\underline{65125}$ & $\begin{array}{l}\text { protein } \\
\text { kinase, } \\
\text { lysine } \\
\text { deficient } 1\end{array}$ & \\
\hline W32940 & $\begin{array}{r}0.003035 \\
311 \\
\end{array}$ & FLJ32115 & 121506 & $\begin{array}{l}\text { hypothetic } \\
\text { al protein } \\
\text { FLJ32115 }\end{array}$ & $\begin{array}{l}\text { oxidoreductase activity, acting on } \\
\text { single donors with incorporation of } \\
\text { molecular oxygen, incorporation of two } \\
\text { atoms of oxygen }\end{array}$ \\
\hline R50905 & $\begin{array}{r}0.003042 \\
081\end{array}$ & TUBB & $\underline{7280}$ & $\begin{array}{l}\text { tubulin, } \\
\text { beta } \\
\text { polypeptid } \\
\text { e }\end{array}$ & $\begin{array}{l}\text { cytoskeleton; structural constituent of } \\
\text { cytoskeleton }\end{array}$ \\
\hline W47505 & $\begin{array}{r}0.003050 \\
809\end{array}$ & $\underline{\text { IGFBP5 }}$ & 3488 & $\begin{array}{l}\text { insulin-like } \\
\text { growth } \\
\text { factor } \\
\text { binding } \\
\text { protein 5 }\end{array}$ & $\begin{array}{l}\text { extracellular space; insulin-like growth } \\
\text { factor binding; regulation of cell } \\
\text { growth; signal transduction }\end{array}$ \\
\hline T84813 & $\begin{array}{r}0.003051 \\
414\end{array}$ & VDAC2 & 7417 & $\begin{array}{l}\text { voltage- } \\
\text { dependent } \\
\text { anion } \\
\text { channel } 2\end{array}$ & $\begin{array}{l}\text { anion transport; integral to membrane; } \\
\text { mitochondrial outer membrane; } \\
\text { mitochondrion; voltage-dependent } \\
\text { anion channel porin activity; voltage- } \\
\text { dependent ion-selective channel } \\
\text { activity }\end{array}$ \\
\hline AA054746 & $\begin{array}{r}0.003052 \\
362 \\
\end{array}$ & & & & \\
\hline
\end{tabular}




\begin{tabular}{|c|c|c|c|c|c|}
\hline W90748 & $\begin{array}{r}0.003061 \\
153 \\
\end{array}$ & & & & \\
\hline W90109 & $\begin{array}{r}0.003070 \\
945\end{array}$ & & & & \\
\hline N39407 & $\begin{array}{r}0.003085 \\
277\end{array}$ & KIF21A & $\underline{55605}$ & $\begin{array}{l}\text { kinesin } \\
\text { family } \\
\text { member } \\
21 \mathrm{~A}\end{array}$ & \\
\hline AA059211 & \begin{tabular}{|r|}
0.003121 \\
245
\end{tabular} & MAK & $\underline{4117}$ & $\begin{array}{l}\text { male germ } \\
\text { cell- } \\
\text { associated } \\
\text { kinase }\end{array}$ & $\begin{array}{l}\text { ATP binding; protein amino acid } \\
\text { phosphorylation; protein } \\
\text { serine/threonine kinase activity; } \\
\text { spermatogenesis; transferase activity }\end{array}$ \\
\hline AA059211 & $\begin{array}{r}0.003121 \\
245\end{array}$ & & 283963 & $\begin{array}{l}\text { hypothetic } \\
\text { al gene } \\
\text { supported } \\
\text { by } \\
\text { AK094432 }\end{array}$ & \\
\hline H81468 & $\begin{array}{r}0.003121 \\
494 \\
\end{array}$ & & & & \\
\hline T85314 & $\begin{array}{r}0.003146 \\
725 \\
\end{array}$ & & & & \\
\hline T90080 & $\begin{array}{r}0.003207 \\
004 \\
\end{array}$ & SPAG9 & $\underline{9043}$ & $\begin{array}{l}\text { sperm } \\
\text { associated } \\
\text { antigen } 9\end{array}$ & $\begin{array}{l}\text { integral to membrane; } \\
\text { spermatogenesis }\end{array}$ \\
\hline $\mathrm{H} 00627$ & $\begin{array}{r}0.003228 \\
67 \\
\end{array}$ & & & & \\
\hline H52273 & $\begin{array}{r}0.003234 \\
343 \\
\end{array}$ & & & & \\
\hline W60305 & $\begin{array}{r}0.003247 \\
636 \\
\end{array}$ & & & & \\
\hline T85676 & \begin{tabular}{|r|}
0.003274 \\
22
\end{tabular} & $\underline{\mathrm{VIL1}}$ & $\underline{7429}$ & villin 1 & $\begin{array}{l}\text { F-actin capping protein complex; actin } \\
\text { binding; actin bundling activity; actin } \\
\text { filament severing activity; protein } \\
\text { complex assembly }\end{array}$ \\
\hline N79080 & $\begin{array}{r}0.003276 \\
459 \\
\end{array}$ & PTMA & $\underline{5757}$ & $\begin{array}{l}\text { prothymosi } \\
\text { n, alpha } \\
\text { (gene } \\
\text { sequence } \\
\text { 28) }\end{array}$ & $\begin{array}{l}\text { development; nucleus; regulation of } \\
\text { cell cycle; transcription }\end{array}$ \\
\hline R80424 & $\begin{array}{r}0.003287 \\
749 \\
\end{array}$ & & & & \\
\hline R91375 & $\begin{array}{r}0.003321 \\
47\end{array}$ & & & & \\
\hline T92329 & $\begin{array}{r}0.003345 \\
347\end{array}$ & & & & \\
\hline N95805 & $\begin{array}{r}0.003352 \\
305 \\
\end{array}$ & KIAA1284 & $\underline{27152}$ & $\begin{array}{l}\text { KIAA1284 } \\
\text { protein }\end{array}$ & intracellular signaling cascade \\
\hline N77126 & $\begin{array}{r}0.003361 \\
642 \\
\end{array}$ & & & & \\
\hline W58007 & $\begin{array}{r}0.003380 \\
131 \\
\end{array}$ & & $\underline{339299}$ & $\begin{array}{l}\text { LOC33929 } \\
9\end{array}$ & \\
\hline
\end{tabular}




\begin{tabular}{|c|c|c|c|c|c|}
\hline N75083 & \begin{tabular}{|r|}
0.003398 \\
293 \\
\end{tabular} & $\underline{M M P 15}$ & $\underline{4324}$ & $\begin{array}{l}\text { matrix } \\
\text { metalloprot } \\
\text { einase 15 } \\
\text { (membran } \\
\text { e-inserted) }\end{array}$ & $\begin{array}{l}\text { enzyme activator activity; extracellular } \\
\text { matrix; hydrolase activity; integral to } \\
\text { plasma membrane; } \\
\text { metalloendopeptidase activity; protein } \\
\text { modification; proteolysis and } \\
\text { peptidolysis; zinc ion binding }\end{array}$ \\
\hline R87413 & $\begin{array}{r}0.003431 \\
986\end{array}$ & SEMA3B & $\underline{7869}$ & $\begin{array}{l}\text { sema } \\
\text { domain, } \\
\text { immunoglo } \\
\text { bulin } \\
\text { domain } \\
\text { (Ig), short } \\
\text { basic } \\
\text { domain, } \\
\text { secreted, } \\
\text { (semaphori } \\
\text { n) 3B }\end{array}$ & $\begin{array}{l}\text { axon guidance; cell-cell signaling; } \\
\text { endoplasmic reticulum }\end{array}$ \\
\hline AA031958 & \begin{tabular}{|r|}
0.003477 \\
242
\end{tabular} & & & & \\
\hline R75598 & \begin{tabular}{|r|}
0.003519 \\
312
\end{tabular} & $\underline{\mathrm{NBL1}}$ & $\underline{4681}$ & $\begin{array}{l}\text { neuroblast } \\
\text { oma, } \\
\text { suppressio } \\
\mathrm{n} \text { of } \\
\text { tumorigeni } \\
\text { city } 1\end{array}$ & negative regulation of cell cycle \\
\hline AA010093 & $\begin{array}{r}0.003548 \\
005 \\
\end{array}$ & & & & \\
\hline $\mathrm{H} 44869$ & $\begin{array}{r}0.003579 \\
353\end{array}$ & & & & \\
\hline W72400 & $\begin{array}{r}0.003625 \\
373\end{array}$ & C12orf2 & 11228 & $\begin{array}{l}\text { chromoso } \\
\text { me } 12 \\
\text { open } \\
\text { reading } \\
\text { frame } 2\end{array}$ & neuropeptide signaling pathway \\
\hline H68885 & $\begin{array}{r}0.003639 \\
707\end{array}$ & TSSC3 & $\underline{7262}$ & $\begin{array}{l}\text { tumor } \\
\text { suppressin } \\
\mathrm{g} \\
\text { subtransfer } \\
\text { able } \\
\text { candidate } \\
3 \\
\end{array}$ & apoptosis; imprinting \\
\hline R37412 & $\begin{array}{r}0.003648 \\
196\end{array}$ & GSTT1 & $\underline{2952}$ & $\begin{array}{l}\text { glutathione } \\
\text { S- } \\
\text { transferase } \\
\text { theta } 1\end{array}$ & $\begin{array}{l}\text { glutathione transferase activity; } \\
\text { response to stress; transferase activity }\end{array}$ \\
\hline R26131 & $\begin{array}{r}0.003661 \\
88\end{array}$ & C6orf37 & $\underline{55603}$ & $\begin{array}{l}\text { chromoso } \\
\text { me } 6 \text { open } \\
\text { reading } \\
\text { frame } 37\end{array}$ & \\
\hline R09692 & $\begin{array}{r}0.003663 \\
018\end{array}$ & & & & \\
\hline
\end{tabular}




\begin{tabular}{|c|c|c|c|c|c|}
\hline $\mathrm{H} 24259$ & \begin{tabular}{|r|}
0.003672 \\
244 \\
\end{tabular} & $\underline{\mathrm{KIAA} 1010}$ & 23268 & $\begin{array}{l}\text { KIAA1010 } \\
\text { protein }\end{array}$ & $\begin{array}{l}\text { endocytosis; guanyl-nucleotide } \\
\text { exchange factor activity; intracellular } \\
\text { signaling cascade }\end{array}$ \\
\hline R00688 & $\begin{array}{r}0.003689 \\
215\end{array}$ & & & & \\
\hline R22967 & $\begin{array}{r}0.003710 \\
451\end{array}$ & STAB1 & 23166 & stabilin 1 & \\
\hline N55283 & $\begin{array}{r}0.003739 \\
354 \\
\end{array}$ & KIAA0469 & $\underline{9903}$ & $\begin{array}{l}\text { KIAA0469 } \\
\text { gene } \\
\text { product }\end{array}$ & \\
\hline H99202 & \begin{tabular}{|r|}
0.003764 \\
722 \\
\end{tabular} & MGC4126 & $\underline{84859}$ & $\begin{array}{l}\text { hypothetic } \\
\text { al protein } \\
\text { MGC4126 }\end{array}$ & \\
\hline AA129727 & \begin{tabular}{|r|}
0.003780 \\
33
\end{tabular} & RAB5C & $\underline{5878}$ & $\begin{array}{l}\text { RAB5C, } \\
\text { member } \\
\text { RAS } \\
\text { oncogene } \\
\text { family }\end{array}$ & $\begin{array}{l}\text { GTP binding; RAB small monomeric } \\
\text { GTPase activity; intracellular protein } \\
\text { transport; protein transporter activity; } \\
\text { small GTPase mediated signal } \\
\text { transduction }\end{array}$ \\
\hline AA136708 & $\begin{array}{r}0.003781 \\
094 \\
\end{array}$ & & & & \\
\hline R86045 & $\begin{array}{r}0.003820 \\
446\end{array}$ & & & & \\
\hline R15278 & $\begin{array}{r}0.003834 \\
693 \\
\end{array}$ & EIF2S2 & 8894 & $\begin{array}{l}\text { eukaryotic } \\
\text { translation } \\
\text { initiation } \\
\text { factor 2, } \\
\text { subunit } 2 \\
\text { beta, } \\
38 \mathrm{kDa} \\
\end{array}$ & $\begin{array}{l}\text { RNA binding; eukaryotic translation } \\
\text { initiation factor } 2 \text { complex; ribosome; } \\
\text { translation initiation factor activity; } \\
\text { translational initiation }\end{array}$ \\
\hline H39156 & $\begin{array}{r}0.003865 \\
716\end{array}$ & MTMR6 & 9107 & $\begin{array}{l}\text { myotubular } \\
\text { in related } \\
\text { protein } 6\end{array}$ & $\begin{array}{l}\text { cellular_component unknown; } \\
\text { hydrolase activity; protein amino acid } \\
\text { dephosphorylation; protein } \\
\text { serine/threonine phosphatase activity; } \\
\text { protein tyrosine phosphatase activity }\end{array}$ \\
\hline H09744 & $\begin{array}{r}0.003883 \\
417 \\
\end{array}$ & & & & \\
\hline R46328 & $\begin{array}{r}0.003890 \\
309 \\
\end{array}$ & TNRC5 & 10695 & $\begin{array}{l}\text { trinucleotid } \\
\text { e repeat } \\
\text { containing } \\
5\end{array}$ & \\
\hline H63443 & $\begin{array}{r}0.003904 \\
024 \\
\end{array}$ & & & & \\
\hline W56823 & $\begin{array}{r}0.003927 \\
879\end{array}$ & FURIN & $\underline{5045}$ & $\begin{array}{l}\text { furin } \\
\text { (paired } \\
\text { basic } \\
\text { amino acid } \\
\text { cleaving } \\
\text { enzyme) } \\
\end{array}$ & $\begin{array}{l}\text { Golgi apparatus; cell-cell signaling; } \\
\text { furin activity; hydrolase activity; } \\
\text { integral to membrane; proteolysis and } \\
\text { peptidolysis; subtilase activity }\end{array}$ \\
\hline H66020 & $\begin{array}{r}0.003968 \\
552 \\
\end{array}$ & PIPOX & $\underline{51268}$ & $\begin{array}{l}\text { pipecolic } \\
\text { acid } \\
\text { oxidase }\end{array}$ & $\begin{array}{l}\text { oxidoreductase activity; peroxisome; } \\
\text { sarcosine oxidase activity; } \\
\text { tetrahydrofolate metabolism }\end{array}$ \\
\hline
\end{tabular}




\begin{tabular}{|c|c|c|c|c|c|}
\hline R13974 & $\begin{array}{r}0.003986 \\
489 \\
\end{array}$ & & & & \\
\hline H50984 & $\begin{array}{r}0.003986 \\
736 \\
\end{array}$ & & & & \\
\hline T86807 & $\begin{array}{r}0.003987 \\
072\end{array}$ & $\underline{\text { STK19 }}$ & $\underline{8859}$ & $\begin{array}{l}\text { serine/thre } \\
\text { onine } \\
\text { kinase } 19\end{array}$ & $\begin{array}{l}\text { ATP binding; cAMP-dependent protein } \\
\text { kinase activity; manganese ion } \\
\text { binding; nucleus; protein amino acid } \\
\text { phosphorylation; protein kinase CK2 } \\
\text { activity; protein serine/threonine } \\
\text { kinase activity; transferase activity }\end{array}$ \\
\hline T86807 & $\begin{array}{r}0.003987 \\
072 \\
\end{array}$ & & & & \\
\hline AA150837 & $\begin{array}{r}0.003991 \\
927 \\
\end{array}$ & & & & \\
\hline $\mathrm{H} 27730$ & $\begin{array}{r}0.004008 \\
584 \\
\end{array}$ & PPP2R1B & $\underline{5519}$ & $\begin{array}{l}\text { protein } \\
\text { phosphata } \\
\text { se } 2 \\
\text { (formerly } \\
2 A \text { ), } \\
\text { regulatory } \\
\text { subunit A } \\
\text { (PR 65), } \\
\text { beta } \\
\text { isoform } \\
\end{array}$ & $\begin{array}{l}\text { protein phosphatase type } 2 \mathrm{~A} \text {, intrinsic } \\
\text { regulator activity }\end{array}$ \\
\hline H88208 & $\begin{array}{r}0.004022 \\
824\end{array}$ & SUPV3L1 & $\underline{6832}$ & $\begin{array}{l}\text { suppressor } \\
\text { of var1, 3- } \\
\text { like } 1(\mathrm{~S} . \\
\text { cerevisiae) }\end{array}$ & $\begin{array}{l}\text { ATP binding; ATP dependent helicase } \\
\text { activity; RNA binding; hydrolase } \\
\text { activity; mitochondrion }\end{array}$ \\
\hline R42763 & $\begin{array}{r}0.004059 \\
641 \\
\end{array}$ & $\underline{\text { KIAA0319 }}$ & 9856 & \begin{tabular}{|l|} 
KIAA0319 \\
gene \\
product
\end{tabular} & \\
\hline H21697 & $\begin{array}{r}0.004102 \\
95\end{array}$ & TEGT & $\underline{7009}$ & \begin{tabular}{|l|} 
testis \\
enhanced \\
gene \\
transcript \\
(BAX \\
inhibitor 1) \\
\end{tabular} & $\begin{array}{l}\text { apoptosis; endoplasmic reticulum; } \\
\text { insoluble fraction; integral to plasma } \\
\text { membrane; nucleus }\end{array}$ \\
\hline N69188 & $\begin{array}{r}0.004119 \\
935 \\
\end{array}$ & & & & \\
\hline H65832 & $\begin{array}{r}0.004144 \\
741 \\
\end{array}$ & & & & \\
\hline AA029842 & $\begin{array}{r}0.004147 \\
955\end{array}$ & MTCP1 & $\underline{4515}$ & \begin{tabular}{|l|} 
mature T- \\
cell \\
proliferatio \\
n 1
\end{tabular} & $\begin{array}{l}\text { cell proliferation; oncogenesis; } \\
\text { regulation of cell cycle }\end{array}$ \\
\hline H01149 & $\begin{array}{r}0.004149 \\
361\end{array}$ & INPP5D & $\underline{3635}$ & $\begin{array}{l}\text { inositol } \\
\text { polyphosp } \\
\text { hate-5- } \\
\text { phosphata } \\
\text { se, } 145 \mathrm{kDa}\end{array}$ & $\begin{array}{l}\text { inositol-polyphosphate 5-phosphatase } \\
\text { activity; phosphate metabolism; signal } \\
\text { transduction }\end{array}$ \\
\hline
\end{tabular}




\begin{tabular}{|c|c|c|c|c|c|}
\hline AA128101 & $\begin{array}{r}0.004162 \\
313\end{array}$ & GM2A & $\underline{2760}$ & \begin{tabular}{|l|} 
GM2 \\
ganglioside \\
activator \\
protein
\end{tabular} & $\begin{array}{l}\text { glycolipid catabolism; } \\
\text { glycosphingolipid metabolism; } \\
\text { lysosome; sphingolipid activator } \\
\text { protein activity; sphingolipid } \\
\text { catabolism }\end{array}$ \\
\hline R97814 & $\begin{array}{r}0.004168 \\
698\end{array}$ & NACA & 4666 & \begin{tabular}{|l|} 
nascent- \\
polypeptid \\
e- \\
associated \\
complex \\
alpha \\
polypeptid \\
e
\end{tabular} & $\begin{array}{l}\text { nascent polypeptide association; } \\
\text { nascent polypeptide-associated } \\
\text { complex; protein biosynthesis }\end{array}$ \\
\hline AA015841 & $\begin{array}{r}0.004170 \\
424 \\
\end{array}$ & GNGT1 & $\underline{2792}$ & $\begin{array}{l}\text { guanine } \\
\text { nucleotide } \\
\text { binding } \\
\text { protein (G } \\
\text { protein), } \\
\text { gamma } \\
\text { transducin } \\
\text { g activity } \\
\text { polypeptid } \\
\text { e 1 }\end{array}$ & $\begin{array}{l}\text { G-protein coupled receptor protein } \\
\text { signaling pathway; heterotrimeric G- } \\
\text { protein GTPase activity; heterotrimeric } \\
\text { G-protein complex; signal transducer } \\
\text { activity; signal transduction }\end{array}$ \\
\hline H82982 & \begin{tabular}{|r|}
0.004178 \\
218
\end{tabular} & ZNF275 & 10838 & \begin{tabular}{|l|} 
zinc finger \\
protein 275
\end{tabular} & $\begin{array}{l}\text { DNA binding; nucleus; regulation of } \\
\text { transcription, DNA-dependent }\end{array}$ \\
\hline R59367 & $\begin{array}{r}0.004178 \\
499 \\
\end{array}$ & & & & \\
\hline R08181 & \begin{tabular}{|r|}
0.004234 \\
52 \\
\end{tabular} & & & & \\
\hline W03107 & $\begin{array}{r}0.004235 \\
497 \\
\end{array}$ & & & & \\
\hline R11934 & $\begin{array}{r}0.004247 \\
95\end{array}$ & & & & \\
\hline H95669 & $\begin{array}{r}0.004256 \\
235 \\
\end{array}$ & & & & \\
\hline \begin{tabular}{|l} 
N71659 \\
\end{tabular} & $\begin{array}{r}0.004258 \\
619 \\
\end{array}$ & & & & \\
\hline AA210692 & $\begin{array}{r}0.004283 \\
972\end{array}$ & KIAA0116 & 23016 & $\begin{array}{l}\text { KIAA0116 } \\
\text { protein }\end{array}$ & $\begin{array}{l}\text { 3'-5' exoribonuclease activity; RNA } \\
\text { binding; RNA catabolism; exonuclease } \\
\text { activity; exosome (RNase complex); } \\
\text { hydrolase activity; nucleus; rRNA } \\
\text { processing }\end{array}$ \\
\hline H75643 & $\begin{array}{r}0.004302 \\
059 \\
\end{array}$ & & & & \\
\hline R13675 & $\begin{array}{r}0.004310 \\
09\end{array}$ & PAK6 & 56924 & \begin{tabular}{|l|}
$\mathrm{p} 21(\mathrm{CDKN}$ \\
$1 \mathrm{~A})-$ \\
activated \\
kinase 6
\end{tabular} & $\begin{array}{l}\text { ATP binding; protein amino acid } \\
\text { phosphorylation; protein } \\
\text { serine/threonine kinase activity; } \\
\text { protein-tyrosine kinase activity; } \\
\text { transferase activity }\end{array}$ \\
\hline N22152 & $\begin{array}{r}0.004328 \\
295\end{array}$ & $\frac{\operatorname{LOC} 2557}{43}$ & $\underline{255743}$ & $\begin{array}{l}\text { hypothetic } \\
\text { al protein } \\
\text { LOC25574 }\end{array}$ & \\
\hline
\end{tabular}




\begin{tabular}{|c|c|c|c|c|c|}
\hline & & & & 3 & \\
\hline H78933 & \begin{tabular}{|r|}
0.004339 \\
196
\end{tabular} & UAP1 & $\underline{6675}$ & \begin{tabular}{|l|} 
UDP-N- \\
acteylgluco \\
samine \\
pyrophosp \\
horylase 1
\end{tabular} & $\begin{array}{l}\text { UDP-N-acetylglucosamine } \\
\text { biosynthesis; UDP-N- } \\
\text { acetylglucosamine diphosphorylase } \\
\text { activity; metabolism; transferase } \\
\text { activity }\end{array}$ \\
\hline N98333 & \begin{tabular}{|r|}
0.004367 \\
397
\end{tabular} & $\underline{\mathrm{RPL7}}$ & $\underline{6129}$ & \begin{tabular}{|l|} 
ribosomal \\
protein L7
\end{tabular} & $\begin{array}{l}\text { RNA binding; cytosolic large ribosomal } \\
\text { subunit (sensu Eukarya); intracellular; } \\
\text { protein biosynthesis; structural } \\
\text { constituent of ribosome; transcription } \\
\text { regulator activity }\end{array}$ \\
\hline H60498 & $\begin{array}{r}0.004370 \\
662 \\
\end{array}$ & & & & \\
\hline N24030 & $\begin{array}{r}0.004380 \\
518\end{array}$ & $\underline{\mathrm{IKBKG}}$ & 8517 & \begin{tabular}{|l|} 
inhibitor of \\
kappa light \\
polypeptid \\
e gene \\
enhancer \\
in B-cells, \\
kinase \\
gamma
\end{tabular} & $\begin{array}{l}\text { NIK-I-kappaB/NF-kappaB cascade; } \\
\text { immune response; induction of } \\
\text { apoptosis; kinesin complex; nucleus; } \\
\text { regulation of transcription, DNA- } \\
\text { dependent; signal transducer activity }\end{array}$ \\
\hline N22335 & \begin{tabular}{|r|}
0.004396 \\
797
\end{tabular} & $\frac{\text { LOC1295 }}{\underline{31}}$ & 129531 & \begin{tabular}{|l|} 
hypothetic \\
al protein \\
BC018453 \\
\end{tabular} & \\
\hline T70828 & $\begin{array}{r}0.004402 \\
95 \\
\end{array}$ & & & & \\
\hline $\mathrm{H} 28350$ & \begin{tabular}{|r|}
0.004422 \\
595 \\
\end{tabular} & & & & \\
\hline R84287 & \begin{tabular}{|r|}
0.004464 \\
223 \\
\end{tabular} & & & & \\
\hline H67348 & \begin{tabular}{|r|}
0.004474 \\
096
\end{tabular} & $\begin{array}{l}\text { DKFZp76 } \\
1 \mathrm{~B} 128 \\
\end{array}$ & 144348 & \begin{tabular}{|l|} 
hypothetic \\
al protein \\
DKFZp761 \\
B128 \\
\end{tabular} & nucleus \\
\hline W60936 & $\begin{array}{r}0.004492 \\
98\end{array}$ & TRIM8 & $\underline{81603}$ & $\begin{array}{l}\text { tripartite } \\
\text { motif- } \\
\text { containing } \\
8\end{array}$ & $\begin{array}{l}\text { biological_process unknown; } \\
\text { cellular_component unknown; kinesin } \\
\text { complex; molecular_function unknown; } \\
\text { nucleus; zinc ion binding }\end{array}$ \\
\hline AA151307 & \begin{tabular}{|r|}
0.004494 \\
561
\end{tabular} & GNB2 & $\underline{2783}$ & $\begin{array}{l}\text { guanine } \\
\text { nucleotide } \\
\text { binding } \\
\text { protein (G } \\
\text { protein), } \\
\text { beta } \\
\text { polypeptid } \\
\text { e 2 }\end{array}$ & $\begin{array}{l}\text { G-protein coupled receptor protein } \\
\text { signaling pathway; heterotrimeric G- } \\
\text { protein GTPase activity; heterotrimeric } \\
\text { G-protein complex; signal transducer } \\
\text { activity; signal transduction }\end{array}$ \\
\hline W03758 & $\begin{array}{r}0.004506 \\
816 \\
\end{array}$ & & & & \\
\hline W58007 & $\begin{array}{r}0.004509 \\
317 \\
\end{array}$ & & $\underline{339299}$ & $\begin{array}{l}\text { LOC33929 } \\
9\end{array}$ & \\
\hline
\end{tabular}




\begin{tabular}{|c|c|c|c|c|c|}
\hline AA149298 & $\begin{array}{r}0.004511 \\
158 \\
\end{array}$ & $\underline{\mathrm{DF}}$ & $\underline{1675}$ & \begin{tabular}{|l|} 
D \\
component \\
of \\
compleme \\
nt (adipsin)
\end{tabular} & $\begin{array}{l}\text { chymotrypsin activity; complement } \\
\text { activation, alternative pathway; } \\
\text { complement factor D activity; } \\
\text { hydrolase activity; proteolysis and } \\
\text { peptidolysis; trypsin activity }\end{array}$ \\
\hline AA021554 & $\begin{array}{r}0.004538 \\
873\end{array}$ & $\underline{N R L}$ & 4901 & $\begin{array}{l}\text { neural } \\
\text { retina } \\
\text { leucine } \\
\text { zipper }\end{array}$ & $\begin{array}{l}\text { DNA binding; nucleus; regulation of } \\
\text { rhodopsin gene activity; regulation of } \\
\text { transcription, DNA-dependent; specific } \\
\text { RNA polymerase II transcription factor } \\
\text { activity; transcription from Pol II } \\
\text { promoter; vision }\end{array}$ \\
\hline N/A1 & $\begin{array}{r}0.004554 \\
496 \\
\end{array}$ & & & & \\
\hline AA011554 & $\begin{array}{r}0.004565 \\
064\end{array}$ & & & & \\
\hline N52439 & $\begin{array}{r}0.004596 \\
897 \\
\end{array}$ & $\begin{array}{l}\text { KIDINS22 } \\
\underline{0}\end{array}$ & $\underline{57498}$ & \multicolumn{2}{|c|}{$\begin{array}{l}\text { likely homolog of rat kinase D-interacting } \\
\text { substance of } 220 \mathrm{kDa}\end{array}$} \\
\hline H45907 & $\begin{array}{r}0.004617 \\
352 \\
\end{array}$ & PKD1-like & 79932 & \begin{tabular}{|l|} 
polycystic \\
kidney \\
disease 1- \\
like
\end{tabular} & \\
\hline H73375 & $\begin{array}{r}0.004628 \\
302 \\
\end{array}$ & & & & \\
\hline H61842 & $\begin{array}{r}0.004643 \\
509 \\
\end{array}$ & & & & \\
\hline W86566 & $\begin{array}{r}0.004663 \\
212 \\
\end{array}$ & & & & \\
\hline T83293 & $\begin{array}{r}0.004663 \\
354 \\
\end{array}$ & & & & \\
\hline R70369 & $\begin{array}{r}0.004673 \\
353 \\
\end{array}$ & GPX4 & $\underline{2879}$ & \begin{tabular}{|l|} 
glutathione \\
peroxidase \\
4 \\
(phospholi \\
pid \\
hydroperox \\
idase)
\end{tabular} & $\begin{array}{l}\text { development; electron transporter } \\
\text { activity; glutathione peroxidase } \\
\text { activity; mitochondrion; oxidoreductase } \\
\text { activity; phospholipid metabolism; } \\
\text { response to oxidative stress }\end{array}$ \\
\hline W93335 & $\begin{array}{r}0.004675 \\
43\end{array}$ & & & & \\
\hline N48735 & $\begin{array}{r}0.004677 \\
987 \\
\end{array}$ & & & & \\
\hline R12985 & $\begin{array}{r}0.004679 \\
098\end{array}$ & $\underline{\text { SGNE1 }}$ & $\underline{6447}$ & \begin{tabular}{|l|} 
secretory \\
granule, \\
neuroendo \\
crine \\
protein 1 \\
(7B2 \\
protein) \\
\end{tabular} & $\begin{array}{l}\text { GTP binding; enzyme activator } \\
\text { activity; neuropeptide signaling } \\
\text { pathway; secretory vesicle }\end{array}$ \\
\hline R86861 & $\begin{array}{r}0.004694 \\
02\end{array}$ & RPIP8 & $\underline{10900}$ & \begin{tabular}{l|} 
RaP2 \\
interacting \\
protein 8
\end{tabular} & $\begin{array}{l}\text { small GTPase mediated signal } \\
\text { transduction; small GTPase } \\
\text { regulatory/interacting protein activity }\end{array}$ \\
\hline R89284 & $\begin{array}{r}0.004700 \\
856\end{array}$ & & & & \\
\hline
\end{tabular}




\begin{tabular}{|c|c|c|c|c|c|}
\hline R53914 & \begin{tabular}{|r|}
0.004722 \\
548 \\
\end{tabular} & HARC & $\underline{55664}$ & $\begin{array}{l}\text { Hsp90- } \\
\text { associating } \\
\text { relative of } \\
\text { Cdc37 }\end{array}$ & cytokinesis; regulation of cell cycle \\
\hline H54108 & $\begin{array}{r}0.004736 \\
708 \\
\end{array}$ & & & & \\
\hline N29429 & \begin{tabular}{|r|}
0.004746 \\
315
\end{tabular} & CGI-57 & 27013 & $\begin{array}{l}\text { hypothetic } \\
\text { al protein } \\
\text { CGI-57 }\end{array}$ & \\
\hline AA028961 & \begin{tabular}{|r|}
0.004756 \\
591
\end{tabular} & & & & \\
\hline H09429 & $\begin{array}{r}0.004758 \\
092\end{array}$ & & & & \\
\hline R97802 & $\begin{array}{r}0.004761 \\
377\end{array}$ & & & & \\
\hline R99685 & $\begin{array}{r}0.004818 \\
624\end{array}$ & & & & \\
\hline R96651 & \begin{tabular}{|r|}
0.004819 \\
469 \\
\end{tabular} & ATOX1 & 475 & $\begin{array}{l}\text { ATX1 } \\
\text { antioxidant } \\
\text { protein } 1 \\
\text { homolog } \\
\text { (yeast) }\end{array}$ & $\begin{array}{l}\text { chaperone activity; copper ion binding; } \\
\text { copper ion homeostasis; copper ion } \\
\text { transport; metal ion binding; metal ion } \\
\text { transport; response to oxidative stress }\end{array}$ \\
\hline W04610 & $\begin{array}{r}0.004828 \\
823 \\
\end{array}$ & $\mathrm{H} 3 \mathrm{~F} 3 \mathrm{~A}$ & $\underline{3020}$ & $\begin{array}{l}\text { H3 histone, } \\
\text { family } 3 \mathrm{~A}\end{array}$ & \\
\hline R13333 & \begin{tabular}{|r|}
0.004845 \\
099
\end{tabular} & \begin{tabular}{|l} 
LOC2834 \\
$\underline{45}$
\end{tabular} & $\underline{283445}$ & $\begin{array}{l}\text { hypothetic } \\
\text { al protein } \\
\text { LOC28344 } \\
5\end{array}$ & \\
\hline W15573 & \begin{tabular}{|r|}
0.004884 \\
151
\end{tabular} & FLJ33957 & 121551 & $\begin{array}{l}\text { hypothetic } \\
\text { al protein } \\
\text { FLJ33957 }\end{array}$ & protein binding \\
\hline AA128301 & \begin{tabular}{|r|}
0.004889 \\
001
\end{tabular} & & & & \\
\hline AA147589 & $\begin{array}{r}0.004889 \\
486\end{array}$ & & & & \\
\hline H67736 & $\begin{array}{r}0.004915 \\
864 \\
\end{array}$ & PPY2 & 23614 & $\begin{array}{l}\text { pancreatic } \\
\text { polypeptid } \\
\text { e } 2\end{array}$ & \\
\hline AA037107 & $\begin{array}{r}0.004945 \\
451 \\
\end{array}$ & TGFA & $\underline{7039}$ & $\begin{array}{l}\text { transformin } \\
\text { g growth } \\
\text { factor, } \\
\text { alpha }\end{array}$ & \\
\hline W23575 & $\begin{array}{r}0.004953 \\
754\end{array}$ & PFKP & 5214 & $\begin{array}{l}\text { phosphofru } \\
\text { ctokinase, } \\
\text { platelet }\end{array}$ & $\begin{array}{l}\text { 6-phosphofructokinase activity; 6- } \\
\text { phosphofructokinase complex; } \\
\text { glycolysis; kinase activity; magnesium } \\
\text { ion binding; transferase activity }\end{array}$ \\
\hline $\mathrm{H} 21773$ & $\begin{array}{r}0.004960 \\
414 \\
\end{array}$ & $\begin{array}{l}\text { LOC1457 } \\
58\end{array}$ & 145758 & $\begin{array}{l}\text { hypothetic } \\
\text { al protein } \\
\text { LOC14575 } \\
8\end{array}$ & \\
\hline R32813 & $\begin{array}{r}0.004961 \\
057 \\
\end{array}$ & MGC2668 & $\underline{81605}$ & $\begin{array}{l}\text { hypothetic } \\
\text { al protein }\end{array}$ & \\
\hline
\end{tabular}




\begin{tabular}{|c|c|c|c|c|c|}
\hline & & & & MGC2668 & \\
\hline H06538 & $\begin{array}{r}0.004987 \\
676 \\
\end{array}$ & $\underline{\mathrm{KCNK} 9}$ & $\underline{51305}$ & $\begin{array}{l}\text { potassium } \\
\text { channel, } \\
\text { subfamily } \\
\text { K, member } \\
9\end{array}$ & $\begin{array}{l}\text { integral to membrane; ion transport; } \\
\text { membrane fraction; potassium channel } \\
\text { activity; potassium ion transport; } \\
\text { voltage-gated ion channel activity }\end{array}$ \\
\hline W63762 & $\begin{array}{r}0.004989 \\
318 \\
\end{array}$ & COX15 & $\underline{1355}$ & \begin{tabular}{|l|} 
COX15 \\
homolog, \\
cytochrom \\
e c oxidase \\
assembly \\
protein \\
(yeast)
\end{tabular} & $\begin{array}{l}\text { cytochrome-c oxidase activity; electron } \\
\text { transporter activity; mitochondrion; } \\
\text { respiratory gaseous exchange }\end{array}$ \\
\hline N66115 & $\begin{array}{r}0.005016 \\
971\end{array}$ & & & & \\
\hline W52156 & $\begin{array}{r}0.005026 \\
981 \\
\end{array}$ & OXTR & $\underline{5021}$ & $\begin{array}{l}\text { oxytocin } \\
\text { receptor }\end{array}$ & $\begin{array}{l}\text { G-protein signaling, coupled to IP3 } \\
\text { second messenger (phospholipase C } \\
\text { activating); endosome; integral to } \\
\text { plasma membrane; lactation; muscle } \\
\text { contraction; oxytocin receptor activity; } \\
\text { pregnancy; rhodopsin-like receptor } \\
\text { activity; vasopressin receptor activity }\end{array}$ \\
\hline AA054715 & $\begin{array}{r}0.005032 \\
123\end{array}$ & & & & \\
\hline W38730 & $\begin{array}{r}0.005061 \\
733 \\
\end{array}$ & & & & \\
\hline R88987 & \begin{tabular}{|r|}
0.005075 \\
735 \\
\end{tabular} & TTR & $\underline{7276}$ & \begin{tabular}{|l|} 
transthyreti \\
n \\
(prealbumi \\
n, \\
amyloidosi \\
s type I)
\end{tabular} & $\begin{array}{l}\text { carrier activity; extracellular space; } \\
\text { retinol binding; steroid binding; thyroid } \\
\text { hormone generation; thyroid hormone } \\
\text { transporter activity; transport }\end{array}$ \\
\hline T66929 & $\begin{array}{r}0.005081 \\
981 \\
\end{array}$ & FLJ21603 & $\underline{79818}$ & $\begin{array}{l}\text { hypothetic } \\
\text { al protein } \\
\text { FLJ21603 }\end{array}$ & \\
\hline W05496 & $\begin{array}{r}0.005082 \\
158 \\
\end{array}$ & SEMA3F & $\frac{6405}{6}$ & \begin{tabular}{|l|} 
sema \\
domain, \\
immunoglo \\
bulin \\
domain \\
(lg), short \\
basic \\
domain, \\
secreted, \\
(semaphori \\
n) 3F
\end{tabular} & development; extracellular space \\
\hline H68528 & $\begin{array}{r}0.005143 \\
893\end{array}$ & FLJ32499 & 124637 & $\begin{array}{l}\text { hypothetic } \\
\text { al protein } \\
\text { FLJ32499 }\end{array}$ & \\
\hline R18381 & $\begin{array}{r}0.005146 \\
441\end{array}$ & & & & \\
\hline \begin{tabular}{|l|}
$H 62158$ \\
\end{tabular} & 0.005151 & & & & \\
\hline
\end{tabular}




\begin{tabular}{|c|c|c|c|c|c|}
\hline & $\begin{array}{r}847 \\
\end{array}$ & & & & \\
\hline H65385 & $\begin{array}{r}0.005214 \\
822\end{array}$ & & & & \\
\hline AA125808 & $\begin{array}{r}0.005222 \\
531\end{array}$ & CAPS & $\underline{828}$ & $\begin{array}{l}\text { calcyphosi } \\
\text { ne }\end{array}$ & $\begin{array}{l}\text { calcium ion binding; intracellular } \\
\text { signaling cascade }\end{array}$ \\
\hline H51675 & $\begin{array}{r}0.005230 \\
746 \\
\end{array}$ & & & & \\
\hline T80134 & $\begin{array}{r}0.005239 \\
732 \\
\end{array}$ & TBCD & $\underline{6904}$ & \begin{tabular}{|l|} 
tubulin- \\
specific \\
chaperone \\
d
\end{tabular} & $\begin{array}{l}\text { beta-tubulin folding; co-chaperonin } \\
\text { activity; cytosol; microtubule; protein } \\
\text { folding }\end{array}$ \\
\hline W69323 & $\begin{array}{r}0.005257 \\
25 \\
\end{array}$ & & & & \\
\hline AA204664 & \begin{tabular}{|r|}
0.005257 \\
297
\end{tabular} & SMC1L2 & $\underline{27127}$ & \begin{tabular}{|l} 
SMC1 \\
structural \\
maintenan \\
ce of \\
chromoso \\
mes 1-like \\
2 (yeast) \\
\end{tabular} & $\begin{array}{l}\text { ATP binding; ATP-binding cassette } \\
\text { (ABC) transporter activity; cell cycle; } \\
\text { chromosome segregation; kinesin } \\
\text { complex; meiosis; membrane; } \\
\text { nucleus; transport }\end{array}$ \\
\hline H73896 & $\begin{array}{r}0.005259 \\
852 \\
\end{array}$ & & & & \\
\hline R87739 & $\begin{array}{r}0.005263 \\
108 \\
\end{array}$ & & & & \\
\hline $\mathrm{H} 68720$ & $\begin{array}{r}0.005300 \\
974 \\
\end{array}$ & & & & \\
\hline AA203318 & $\begin{array}{r}0.005318 \\
056\end{array}$ & & & & \\
\hline R99774 & $\begin{array}{r}0.005318 \\
42 \\
\end{array}$ & NT5C2 & $\underline{22978}$ & \begin{tabular}{|l|}
$5 '-$ \\
nucleotidas \\
e, cytosolic \\
II
\end{tabular} & $\begin{array}{l}\text { IMP-GMP specific 5'-nucleotidase } \\
\text { activity; cytosol; hydrolase activity }\end{array}$ \\
\hline R19118 & $\begin{array}{r}0.005375 \\
804 \\
\end{array}$ & $\underline{\text { SDCBP }}$ & $\underline{6386}$ & $\begin{array}{l}\text { syndecan } \\
\text { binding } \\
\text { protein } \\
\text { (syntenin) }\end{array}$ & $\begin{array}{l}\text { actin modulating activity; adherens } \\
\text { junction; cytoskeletal adaptor activity; } \\
\text { cytoskeleton; endoplasmic reticulum; } \\
\text { interleukin-5 receptor binding; } \\
\text { interleukin-5 receptor complex; } \\
\text { intracellular signaling cascade; } \\
\text { membrane; neurexin binding; nucleus; } \\
\text { protein-membrane targeting; } \\
\text { regulation of synapse; substrate- } \\
\text { bound cell migration, cell extension; } \\
\text { syndecan binding }\end{array}$ \\
\hline N62188 & $\begin{array}{r}0.005378 \\
088 \\
\end{array}$ & & & & \\
\hline
\end{tabular}




\begin{tabular}{|c|c|c|c|c|c|}
\hline N62259 & \begin{tabular}{|r|}
0.005422 \\
106
\end{tabular} & DUSP1 & 1843 & $\begin{array}{l}\text { dual } \\
\text { specificity } \\
\text { phosphata } \\
\text { se } 1\end{array}$ & $\begin{array}{l}\text { CTD phosphatase activity; MAP } \\
\text { kinase phosphatase activity; calcium- } \\
\text { dependent protein serine/threonine } \\
\text { phosphatase activity; cell cycle; } \\
\text { hydrolase activity; magnesium- } \\
\text { dependent protein serine/threonine } \\
\text { phosphatase activity; myosin } \\
\text { phosphatase activity; non-membrane } \\
\text { spanning protein tyrosine phosphatase } \\
\text { activity; protein amino acid } \\
\text { dephosphorylation; protein } \\
\text { phosphatase type 2A activity; protein } \\
\text { phosphatase type 2B activity; protein } \\
\text { phosphatase type 2C activity; } \\
\text { response to oxidative stress } \\
\end{array}$ \\
\hline H48502 & \begin{tabular}{|r|}
0.005425 \\
544
\end{tabular} & $\frac{\text { LOC3394 }}{4 \underline{48}}$ & $\underline{339448}$ & $\begin{array}{l}\text { hypothetic } \\
\text { al protein } \\
\text { LOC33944 } \\
8 \\
\end{array}$ & \\
\hline H58461 & $\begin{array}{r}0.005467 \\
797\end{array}$ & & 339088 & $\begin{array}{l}\text { similar to } \\
\text { My016 } \\
\text { protein }\end{array}$ & \\
\hline AA009926 & $\begin{array}{r}0.005503 \\
977 \\
\end{array}$ & & & & \\
\hline R34574 & $\begin{array}{r}0.005535 \\
538 \\
\end{array}$ & & & & \\
\hline H85857 & $\begin{array}{r}0.005575 \\
674 \\
\end{array}$ & \begin{tabular}{|l}
$\underline{\mathrm{LOC} 2843}$ \\
$\underline{52}$
\end{tabular} & 284352 & $\begin{array}{l}\text { hypothetic } \\
\text { al protein } \\
\text { LOC28435 } \\
2\end{array}$ & \\
\hline R34347 & $\begin{array}{r}0.005611 \\
858 \\
\end{array}$ & KIAA0354 & 9925 & $\begin{array}{l}\text { KIAA0354 } \\
\text { gene } \\
\text { product }\end{array}$ & protein binding \\
\hline H46137 & \begin{tabular}{|r|}
0.005658 \\
161
\end{tabular} & NAPB & $\underline{63908}$ & $\begin{array}{l}\mathrm{N}- \\
\text { ethylmalei } \\
\text { mide- } \\
\text { sensitive } \\
\text { factor } \\
\text { attachment } \\
\text { protein, } \\
\text { beta } \\
\end{array}$ & $\begin{array}{l}\text { Golgi apparatus; endoplasmic } \\
\text { reticulum; intracellular protein } \\
\text { transport; intracellular transporter } \\
\text { activity; protein transporter activity }\end{array}$ \\
\hline T86459 & $\begin{array}{r}0.005660 \\
196 \\
\end{array}$ & & & & \\
\hline H70009 & $\begin{array}{r}0.005692 \\
46 \\
\end{array}$ & & & & \\
\hline N91458 & $\begin{array}{r}0.005694 \\
363 \\
\end{array}$ & $\begin{array}{l}\text { TA- } \\
\text { WDRP }\end{array}$ & $\underline{134430}$ & $\begin{array}{l}\text { T-cell } \\
\text { activation } \\
\text { WD repeat } \\
\text { protein } \\
\end{array}$ & catalytic activity; metabolism \\
\hline N30845 & $\begin{array}{r}0.005715 \\
041 \\
\end{array}$ & HPSE & $\underline{10855}$ & $\begin{array}{l}\text { heparanas } \\
\text { e }\end{array}$ & \begin{tabular}{|l|} 
beta-glucuronidase activity; \\
inflammatory response; invasive \\
growth; proteoglycan metabolism
\end{tabular} \\
\hline R65850 & 0.005722 & & & & \\
\hline
\end{tabular}




\begin{tabular}{|c|c|c|c|c|c|}
\hline & 801 & & & & \\
\hline N38966 & $\begin{array}{r}0.005736 \\
658 \\
\end{array}$ & & & & \\
\hline N34901 & $\begin{array}{r}0.005737 \\
532 \\
\end{array}$ & GALNT7 & $\underline{117248}$ & \multicolumn{2}{|c|}{$\begin{array}{l}\text { UDP-N-acetyl-alpha-D-galactosamine:polypeptide } \\
\mathrm{N} \text {-acetylgalactosaminyltransferase } 7\end{array}$} \\
\hline W70144 & $\begin{array}{r}0.005776 \\
662 \\
\end{array}$ & VDR & $\underline{7421}$ & \begin{tabular}{|l|} 
vitamin D \\
$(1,25-$ \\
dihydroxyvi \\
tamin D3) \\
receptor
\end{tabular} & $\begin{array}{l}\text { nucleus; regulation of transcription, } \\
\text { DNA-dependent; signal transduction; } \\
\text { steroid hormone receptor activity; } \\
\text { transcription factor activity; vitamin D3 } \\
\text { receptor activity }\end{array}$ \\
\hline H94763 & $\begin{array}{r}0.005782 \\
828\end{array}$ & $\underline{\mathrm{SH} 3 \mathrm{GLB} 1}$ & $\underline{51100}$ & \begin{tabular}{|l|} 
SH3- \\
domain \\
GRB2-like \\
endophilin \\
B1
\end{tabular} & \\
\hline AA132089 & $\begin{array}{r}0.005795 \\
396 \\
\end{array}$ & FLJ20522 & $\underline{54965}$ & $\begin{array}{l}\text { hypothetic } \\
\text { al protein } \\
\text { FLJ20522 }\end{array}$ & \\
\hline AA037207 & $\begin{array}{r}0.005804 \\
992\end{array}$ & MIRAB13 & $\underline{85377}$ & \begin{tabular}{|l|} 
molecule \\
interacting \\
with Rab13
\end{tabular} & $\begin{array}{l}\text { GTPase regulator activity; intracellular; } \\
\text { vesicle-mediated transport; zinc ion } \\
\text { binding }\end{array}$ \\
\hline AA098865 & $\begin{array}{r}0.005823 \\
667 \\
\end{array}$ & BCL2L10 & $\underline{10017}$ & $\begin{array}{l}\text { BCL2-like } \\
10 \\
\text { (apoptosis } \\
\text { facilitator) }\end{array}$ & $\begin{array}{l}\text { anti-apoptosis; apoptosis inhibitor } \\
\text { activity; caspase activation; female } \\
\text { gamete generation; integral to } \\
\text { membrane; membrane fraction; } \\
\text { mitochondrion; protein binding; } \\
\text { spermatogenesis }\end{array}$ \\
\hline AA211819 & $\begin{array}{r}0.005825 \\
701 \\
\end{array}$ & MGC3130 & $\underline{78995}$ & $\begin{array}{l}\text { hypothetic } \\
\text { al protein } \\
\text { MGC3130 }\end{array}$ & \\
\hline R83247 & $\begin{array}{r}0.005844 \\
822 \\
\end{array}$ & GLB1 & $\underline{2720}$ & $\begin{array}{l}\text { galactosida } \\
\text { se, beta } 1\end{array}$ & beta-galactosidase activity; lysosome \\
\hline T95864 & $\begin{array}{r}0.005905 \\
684\end{array}$ & & & & \\
\hline R10875 & $\begin{array}{r}0.005906 \\
909\end{array}$ & HSD17B1 & $\underline{3292}$ & \begin{tabular}{|l|} 
hydroxyste \\
roid $(17-$ \\
beta) \\
dehydroge \\
nase 1
\end{tabular} & $\begin{array}{l}\text { catalytic activity; cytoplasm; estrogen } \\
\text { metabolism; steroid biosynthesis }\end{array}$ \\
\hline R80475 & $\begin{array}{r}0.005911 \\
476\end{array}$ & FGFR1 & $\underline{2260}$ & \begin{tabular}{|l|} 
fibroblast \\
growth \\
factor \\
receptor 1 \\
(fms- \\
related \\
tyrosine \\
kinase 2, \\
Pfeiffer \\
syndrome) \\
\end{tabular} & $\begin{array}{l}\text { FGF receptor signaling pathway; } \\
\text { MAPKKK cascade; fibroblast growth } \\
\text { factor receptor activity; integral to } \\
\text { plasma membrane; oncogenesis; } \\
\text { skeletal development }\end{array}$ \\
\hline $\mathrm{H} 81331$ & $\begin{array}{r}0.005935 \\
414 \\
\end{array}$ & & & & \\
\hline
\end{tabular}




\begin{tabular}{|c|c|c|c|c|c|}
\hline R54877 & $\begin{array}{r}0.005962 \\
813 \\
\end{array}$ & FLJ10415 & $\underline{55139}$ & $\begin{array}{l}\text { hypothetic } \\
\text { al protein } \\
\text { FLJ10415 }\end{array}$ & \\
\hline R14363 & \begin{tabular}{|r|}
0.005963 \\
375
\end{tabular} & HDAC5 & 10014 & $\begin{array}{l}\text { histone } \\
\text { deacetylas } \\
\text { e } 5\end{array}$ & $\begin{array}{l}\text { chromatin modeling; chromatin } \\
\text { silencing; cytoplasm; histone } \\
\text { deacetylase activity; nucleus; } \\
\text { regulation of transcription, DNA- } \\
\text { dependent }\end{array}$ \\
\hline AA203710 & $\begin{array}{r}0.005978 \\
656 \\
\end{array}$ & XPO7 & 23039 & exportin 7 & \\
\hline H69803 & $\begin{array}{r}0.006018 \\
395 \\
\end{array}$ & & & & \\
\hline N76529 & 0.006024 & & & & \\
\hline N41764 & \begin{tabular}{|r|}
0.006029 \\
704
\end{tabular} & DLG7 & 9787 & $\begin{array}{l}\text { discs, large } \\
\text { homolog } 7 \\
\text { (Drosophil } \\
\text { a) }\end{array}$ & $\begin{array}{l}\text { biological_process unknown; cell-cell } \\
\text { signaling; cellular_component } \\
\text { unknown; molecular_function unknown }\end{array}$ \\
\hline
\end{tabular}


Appendix G. Genes significantly different after 15m MPP+ treatment +/- SN50.

\begin{tabular}{|c|c|c|c|c|c|}
\hline GENBANK & PVALUE & SYMBOL & $\begin{array}{l}\text { LOCUS } \\
\text { LINK }\end{array}$ & $\begin{array}{l}\text { GENE } \\
\text { NAME }\end{array}$ & GENE ONTOLOGY \\
\hline T87888 & $4.54 \mathrm{E}-10$ & KIAA1046 & $\underline{22867}$ & $\begin{array}{l}\text { KIAA1046 } \\
\text { protein }\end{array}$ & \\
\hline R94499 & $9.77 \mathrm{E}-10$ & GNB5 & 10681 & \multicolumn{2}{|c|}{ guanine nucleotide binding protein (G protein), beta } \\
\hline R63498 & 4.09E-09 & FLJ23563 & 79993 & $\begin{array}{l}\text { hypothetic } \\
\text { al protein } \\
\text { FLJ23563 }\end{array}$ & integral to membrane \\
\hline H68885 & 9.58E-09 & TSSC3 & $\underline{7262}$ & \begin{tabular}{|l|} 
tumor \\
suppressin \\
$\mathrm{g}$ \\
subtransfer \\
able \\
candidate \\
3 \\
\end{tabular} & apoptosis; imprinting \\
\hline R19119 & 1.01E-08 & & & & \\
\hline AA010141 & $1.23 \mathrm{E}-08$ & $\frac{\text { SERPINH }}{1}$ & 871 & \begin{tabular}{|l|} 
serine (or \\
cysteine) \\
proteinase \\
inhibitor, \\
clade H \\
(heat \\
shock \\
protein 47), \\
member 1, \\
(collagen \\
binding \\
protein 1) \\
\end{tabular} & heat shock response \\
\hline H18190 & $2.29 \mathrm{E}-08$ & JAK1 & $\underline{3716}$ & \begin{tabular}{|l|} 
Janus \\
kinase 1 (a \\
protein \\
tyrosine \\
kinase)
\end{tabular} & $\begin{array}{l}\text { ATP binding; cytoskeleton; intracellular } \\
\text { signaling cascade; protein amino acid } \\
\text { phosphorylation; protein-tyrosine kinase } \\
\text { activity; transferase activity }\end{array}$ \\
\hline H53827 & 2.39E-08 & & & & \\
\hline \begin{tabular}{|l|} 
R55491 \\
\end{tabular} & $3.73 \mathrm{E}-08$ & & & & \\
\hline H18298 & 4.04E-08 & & & & \\
\hline W44529 & $4.92 \mathrm{E}-08$ & MMP2 & $\underline{4313}$ & \begin{tabular}{|l|} 
matrix \\
metalloprot \\
einase 2 \\
(gelatinase \\
A, $72 \mathrm{kDa}$ \\
gelatinase, \\
$72 \mathrm{kDa}$ type \\
IV \\
collagenas \\
e)
\end{tabular} & $\begin{array}{l}\text { calcium ion binding; collagen } \\
\text { catabolism; extracellular matrix; } \\
\text { extracellular space; gelatinase A } \\
\text { activity; hydrolase activity; zinc ion } \\
\text { binding }\end{array}$ \\
\hline T79540 & $6.25 \mathrm{E}-08$ & & $\underline{253992}$ & \begin{tabular}{|l|l|l} 
\\
2
\end{tabular} & \\
\hline H84257 & 7.13E-08 & & & & \\
\hline
\end{tabular}




\begin{tabular}{|c|c|c|c|c|c|}
\hline $\mathrm{H} 25578$ & 7.76E-08 & & & & \\
\hline H10796 & $8.25 \mathrm{E}-08$ & SMN1 & $\underline{6606}$ & $\begin{array}{l}\text { survival of } \\
\text { motor } \\
\text { neuron 1, } \\
\text { telomeric } \\
\end{array}$ & \\
\hline T83013 & 8.72E-08 & $\underline{\mathrm{HGD}}$ & $\underline{3081}$ & \multicolumn{2}{|c|}{$\begin{array}{l}\text { homogentisate 1,2-dioxygenase (homogentisate } \\
\text { oxidase) }\end{array}$} \\
\hline T93785 & $1.32 \mathrm{E}-07$ & & & & \\
\hline R15267 & $1.34 \mathrm{E}-07$ & & & & \\
\hline $\mathrm{H} 63676$ & $1.52 \mathrm{E}-07$ & OPA1 & $\underline{4976}$ & \begin{tabular}{|l|} 
optic \\
atrophy 1 \\
(autosomal \\
dominant)
\end{tabular} & $\begin{array}{l}\text { GTP binding; mitochondrion; motor } \\
\text { activity; vision }\end{array}$ \\
\hline R20145 & 1.56E-07 & & & & \\
\hline R62213 & 1.57E-07 & & & & \\
\hline R17538 & $2.15 \mathrm{E}-07$ & PABPC4 & $\underline{8761}$ & $\begin{array}{l}\text { poly(A) } \\
\text { binding } \\
\text { protein, } \\
\text { cytoplasmi } \\
\text { c 4 } \\
\text { (inducible } \\
\text { form) } \\
\end{array}$ & $\begin{array}{l}\text { RNA binding; RNA catabolism; RNA } \\
\text { processing; blood coagulation; } \\
\text { cytoplasm; poly }(\mathrm{A}) \text { binding; protein } \\
\text { biosynthesis; response to } \\
\text { pest/pathogen/parasite }\end{array}$ \\
\hline H59552 & $2.28 \mathrm{E}-07$ & & & & \\
\hline H58631 & $2.48 \mathrm{E}-07$ & & & & \\
\hline R41363 & $2.58 \mathrm{E}-07$ & & & & \\
\hline T87535 & $2.68 \mathrm{E}-07$ & & & & \\
\hline R26954 & $3.09 E-07$ & CTSD & $\underline{1509}$ & $\begin{array}{l}\text { cathepsin } \\
\text { D } \\
\text { (lysosomal } \\
\text { aspartyl } \\
\text { protease) }\end{array}$ & $\begin{array}{l}\text { cathepsin D activity; hydrolase activity; } \\
\text { lysosome; pepsin A activity; proteolysis } \\
\text { and peptidolysis }\end{array}$ \\
\hline AA018742 & $3.66 \mathrm{E}-07$ & $\underline{I S L 1}$ & $\underline{3670}$ & \begin{tabular}{|l|} 
ISL1 \\
transcriptio \\
n factor, \\
LIM/homeo \\
domain, \\
(islet-1) \\
\end{tabular} & $\begin{array}{l}\text { RNA polymerase II transcription factor } \\
\text { activity; development; energy } \\
\text { pathways; nucleus; regulation of } \\
\text { transcription, DNA-dependent; } \\
\text { transcription factor activity }\end{array}$ \\
\hline H50657 & $3.78 \mathrm{E}-07$ & & & & \\
\hline H59765 & 4.77E-07 & C14orf68 & $\underline{283600}$ & $\begin{array}{l}\text { chromoso } \\
\text { me } 14 \\
\text { open } \\
\text { reading } \\
\text { frame } 68 \\
\end{array}$ & \\
\hline H72049 & 4.84E-07 & & & & \\
\hline $\mathrm{H} 47518$ & 4.96E-07 & & & & \\
\hline H65775 & 5.19E-07 & & & & \\
\hline R26325 & 5.74E-07 & & & & \\
\hline R48610 & $6.16 \mathrm{E}-07$ & TTC7 & $\underline{57217}$ & \begin{tabular}{|l|} 
tetratricope \\
ptide \\
repeat \\
domain 7 \\
\end{tabular} & \\
\hline
\end{tabular}




\begin{tabular}{|c|c|c|c|c|c|}
\hline $\mathrm{H} 18495$ & 7.34E-07 & & & & \\
\hline H82521 & 9.90E-07 & ATP6V0B & $\underline{533}$ & $\begin{array}{l}\text { ATPase, } \\
\mathrm{H}+ \\
\text { transportin } \\
\mathrm{g}, \\
\text { lysosomal } \\
21 \mathrm{kDa}, \mathrm{V} 0 \\
\text { subunit c" }\end{array}$ & $\begin{array}{l}\text { ATP biosynthesis; hydrogen ion } \\
\text { transporter activity; hydrogen- } \\
\text { transporting two-sector ATPase activity; } \\
\text { hydrolase activity; integral to } \\
\text { membrane; proton transport; } \\
\text { transporter activity }\end{array}$ \\
\hline R60838 & $1.46 \mathrm{E}-06$ & & & & \\
\hline T79552 & $1.74 \mathrm{E}-06$ & & & & \\
\hline \begin{tabular}{|l|} 
R20019 \\
\end{tabular} & $2.33 \mathrm{E}-06$ & & & & \\
\hline $\mathrm{H} 45355$ & $2.47 \mathrm{E}-06$ & & & & \\
\hline W68050 & $2.54 \mathrm{E}-06$ & LGALS1 & $\underline{3956}$ & $\begin{array}{l}\text { lectin, } \\
\text { galactoside } \\
\text {-binding, } \\
\text { soluble, 1 } \\
\text { (galectin 1) } \\
\end{array}$ & $\begin{array}{l}\text { apoptosis; heterophilic cell adhesion; } \\
\text { sugar binding }\end{array}$ \\
\hline R34114 & $2.81 \mathrm{E}-06$ & & & & \\
\hline H68441 & $3.08 \mathrm{E}-06$ & FLJ14054 & $\underline{79614}$ & $\begin{array}{l}\text { hypothetic } \\
\text { al protein } \\
\text { FLJ14054 }\end{array}$ & \\
\hline $\mathrm{H} 02590$ & $3.20 \mathrm{E}-06$ & & & & \\
\hline R00907 & $3.23 \mathrm{E}-06$ & PLEKHG1 & $\underline{57480}$ & $\begin{array}{l}\text { pleckstrin hc } \\
\text { (with RhoGe }\end{array}$ & $\begin{array}{l}\text { omology domain containing, family } G \\
\text { ef domain) member } 1\end{array}$ \\
\hline $\mathrm{H} 29730$ & $3.44 \mathrm{E}-06$ & & & & \\
\hline \begin{tabular}{|l}
$\mathrm{H} 71504$ \\
\end{tabular} & $3.44 \mathrm{E}-06$ & & & & \\
\hline H68976 & $3.47 \mathrm{E}-06$ & BXDC1 & $\underline{84154}$ & $\begin{array}{l}\text { brix } \\
\text { domain } \\
\text { containing } \\
1\end{array}$ & nucleus \\
\hline $\mathrm{H} 48570$ & $3.65 \mathrm{E}-06$ & & & & \\
\hline R54090 & $3.66 \mathrm{E}-06$ & & & & \\
\hline R20373 & $3.78 \mathrm{E}-06$ & TMP21 & 10972 & \begin{tabular}{|l|} 
transmemb \\
rane \\
trafficking \\
protein
\end{tabular} & $\begin{array}{l}\text { ER to Golgi transport; Golgi apparatus; } \\
\text { integral to plasma membrane; } \\
\text { intracellular protein transport; } \\
\text { membrane fraction; microsome; protein } \\
\text { carrier activity; protein transporter } \\
\text { activity }\end{array}$ \\
\hline R19859 & 3.94E-06 & $\frac{\mathrm{MGC} 2187}{4}$ & 93624 & $\begin{array}{l}\text { hypothetic } \\
\text { al protein } \\
\text { MGC2187 } \\
4\end{array}$ & \\
\hline H59135 & $4.80 \mathrm{E}-06$ & SPP2 & $\underline{6694}$ & $\begin{array}{l}\text { secreted } \\
\text { phosphopr } \\
\text { otein } 2 \\
24 \mathrm{kDa} \\
\end{array}$ & $\begin{array}{l}\text { endopeptidase inhibitor activity; } \\
\text { extracellular space; skeletal } \\
\text { development }\end{array}$ \\
\hline H73186 & $5.21 \mathrm{E}-06$ & & & & \\
\hline R51610 & $5.22 \mathrm{E}-06$ & & & & \\
\hline $\mathrm{H} 48282$ & 5.97E-06 & & & & \\
\hline $\mathrm{H} 60821$ & $6.08 \mathrm{E}-06$ & & & & \\
\hline W37870 & $6.13 \mathrm{E}-06$ & & & & \\
\hline
\end{tabular}




\begin{tabular}{|c|c|c|c|c|c|}
\hline $\mathrm{H} 09945$ & 8.07E-06 & & & & \\
\hline H19297 & 8.11E-06 & EDIL3 & 10085 & $\begin{array}{l}\text { EGF-like } \\
\text { repeats } \\
\text { and } \\
\text { discoidin I- } \\
\text { like } \\
\text { domains } 3 \\
\end{array}$ & $\begin{array}{l}\text { calcium ion binding; cell adhesion; cell } \\
\text { adhesion molecule activity; } \\
\text { development; integrin binding }\end{array}$ \\
\hline H86498 & $8.39 \mathrm{E}-06$ & & & & \\
\hline H23933 & $8.56 \mathrm{E}-06$ & & & & \\
\hline H48578 & 9.68E-06 & & & & \\
\hline AA150238 & $9.78 \mathrm{E}-06$ & & & & \\
\hline R47938 & 9.78E-06 & FLJ32096 & 148646 & $\begin{array}{l}\text { hypothetic } \\
\text { al protein } \\
\text { FLJ32096 }\end{array}$ & \\
\hline $\mathrm{H} 27352$ & 9.93E-06 & HRAS & 3265 & $\begin{array}{l}\text { v-Ha-ras } \\
\text { Harvey rat } \\
\text { sarcoma } \\
\text { viral } \\
\text { oncogene } \\
\text { homolog }\end{array}$ & $\begin{array}{l}\text { GTPase activity; cell motility; cell shape } \\
\text { and cell size control; cell surface } \\
\text { receptor linked signal transduction; } \\
\text { chemotaxis; cytoplasm; histogenesis } \\
\text { and organogenesis; peripheral plasma } \\
\text { membrane protein; plasma membrane; } \\
\text { regulation of cell cycle; signal } \\
\text { transduction }\end{array}$ \\
\hline R85191 & 9.96E-06 & FLJ31364 & 146956 & $\begin{array}{l}\text { homolog of } \\
\text { yeast } \\
\text { EME1 } \\
\text { endonucle } \\
\text { ase }\end{array}$ & \\
\hline R22957 & 1.07E-05 & pp9099 & $\underline{80301}$ & $\begin{array}{l}\mathrm{PH} \\
\text { domain- } \\
\text { containing } \\
\text { protein } \\
\end{array}$ & \\
\hline R77347 & 1.07E-05 & PPIG & 9360 & $\begin{array}{l}\text { peptidyl- } \\
\text { prolyl } \\
\text { isomerase } \\
\text { G } \\
\text { (cyclophilin } \\
\text { G) }\end{array}$ & $\begin{array}{l}\text { FK506-sensitive peptidyl-prolyl cis-trans } \\
\text { isomerase; cyclophilin; cyclophilin-type } \\
\text { peptidyl-prolyl cis-trans isomerase } \\
\text { activity; isomerase activity; mRNA } \\
\text { splicing; nucleoplasm; pre-mRNA } \\
\text { splicing factor activity; protein folding }\end{array}$ \\
\hline H03917 & 1.14E-05 & INSR & $\underline{3643}$ & $\begin{array}{l}\text { insulin } \\
\text { receptor }\end{array}$ & $\begin{array}{l}\text { ATP binding; carbohydrate metabolism; } \\
\text { cell growth and/or maintenance; } \\
\text { development; energy pathways; } \\
\text { epidermal growth factor receptor } \\
\text { activity; integral to plasma membrane; } \\
\text { protein amino acid phosphorylation; } \\
\text { receptor activity; receptor signaling } \\
\text { protein tyrosine kinase activity; signal } \\
\text { transduction; transferase activity; } \\
\text { transmembrane receptor protein } \\
\text { tyrosine kinase signaling pathway; } \\
\text { transmembrane receptor protein } \\
\text { tyrosine kinase signaling protein activity }\end{array}$ \\
\hline R07186 & 1.18E-05 & & & & \\
\hline
\end{tabular}




\begin{tabular}{|c|c|c|c|c|c|}
\hline $\mathrm{H} 00498$ & $1.20 \mathrm{E}-05$ & PPP2R3A & 5523 & $\begin{array}{l}\text { protein } \\
\text { phosphata } \\
\text { se } 2 \\
\text { (formerly } \\
2 A \text { ), } \\
\text { regulatory } \\
\text { subunit B", } \\
\text { alpha }\end{array}$ & $\begin{array}{l}\text { calcium ion binding; protein } \\
\text { phosphatase type } 2 A \text {, intrinsic regulator } \\
\text { activity }\end{array}$ \\
\hline R36086 & 1.38E-05 & & & & \\
\hline R50698 & 1.41E-05 & $\begin{array}{l}\text { MGC2316 } \\
\underline{6}\end{array}$ & $\underline{221504}$ & \begin{tabular}{|l|} 
hypothetic \\
al protein \\
MGC2316 \\
6
\end{tabular} & protein binding \\
\hline R81839 & 1.52E-05 & TXK & $\underline{7294}$ & $\begin{array}{l}\text { TXK } \\
\text { tyrosine } \\
\text { kinase }\end{array}$ & $\begin{array}{l}\text { ATP binding; cytoplasm; intracellular } \\
\text { signaling cascade; non-membrane } \\
\text { spanning protein tyrosine kinase } \\
\text { activity; protein amino acid } \\
\text { phosphorylation; transferase activity }\end{array}$ \\
\hline H12681 & 1.53E-05 & FCGR1A & 2209 & \begin{tabular}{|l|} 
Fc \\
fragment of \\
lgG, high \\
affinity la, \\
receptor \\
for (CD64)
\end{tabular} & $\begin{array}{l}\text { immune response; integral to plasma } \\
\text { membrane; phagocytosis, engulfment; } \\
\text { receptor signaling protein activity; } \\
\text { signal transduction }\end{array}$ \\
\hline H99202 & 1.54E-05 & MGC4126 & 84859 & $\begin{array}{l}\text { hypothetic } \\
\text { al protein } \\
\text { MGC4126 }\end{array}$ & \\
\hline H84293 & 1.58E-05 & $\underline{\text { SLC12A5 }}$ & 57468 & \begin{tabular}{|l|} 
solute \\
carrier \\
family 12, \\
(potassium \\
-chloride \\
transporter \\
) member \\
5
\end{tabular} & $\begin{array}{l}\text { amino acid transport; amino acid- } \\
\text { polyamine transporter activity; cell ion } \\
\text { homeostasis; chloride transport; } \\
\text { integral to membrane; ion transport; } \\
\text { potassium ion transport; } \\
\text { potassium:chloride symporter activity; } \\
\text { sodium ion transport; symporter activity; } \\
\text { transporter activity }\end{array}$ \\
\hline $\mathrm{H} 45241$ & 1.62E-05 & RPL41 & $\underline{6171}$ & $\begin{array}{l}\text { ribosomal } \\
\text { protein L41 }\end{array}$ & $\begin{array}{l}\text { RNA binding; cytosolic large ribosomal } \\
\text { subunit (sensu Eukarya); protein } \\
\text { biosynthesis; structural constituent of } \\
\text { ribosome }\end{array}$ \\
\hline R68131 & 1.68E-05 & SLC31A2 & 1318 & \begin{tabular}{|l|} 
solute \\
carrier \\
family 31 \\
(copper \\
transporter \\
s), member \\
2
\end{tabular} & $\begin{array}{l}\text { copper ion transport; copper ion } \\
\text { transporter activity; integral to plasma } \\
\text { membrane; transport }\end{array}$ \\
\hline N29429 & 1.77E-05 & CGI-57 & 27013 & $\begin{array}{l}\text { hypothetic } \\
\text { al protein } \\
\text { CGI-57 }\end{array}$ & \\
\hline R48603 & 1.78E-05 & AGS3 & 26086 & $\begin{array}{l}\text { activator of } \\
\text { G-protein } \\
\text { signaling } 3\end{array}$ & \\
\hline
\end{tabular}




\begin{tabular}{|c|c|c|c|c|c|}
\hline R41584 & $1.80 \mathrm{E}-05$ & KIAA0194 & $\underline{22993}$ & $\begin{array}{l}\text { KIAA0194 } \\
\text { protein }\end{array}$ & $\begin{array}{l}\text { DNA binding; nucleus; regulation of } \\
\text { transcription, DNA-dependent }\end{array}$ \\
\hline R26844 & 1.90E-05 & & & & \\
\hline H09314 & 1.90E-05 & PPP2CA & $\underline{5515}$ & $\begin{array}{l}\text { protein } \\
\text { phosphata } \\
\text { se } 2 \\
\text { (formerly } \\
2 A \text { ), } \\
\text { catalytic } \\
\text { subunit, } \\
\text { alpha } \\
\text { isoform }\end{array}$ & $\begin{array}{l}\text { CTD phosphatase activity; calcium- } \\
\text { dependent protein serine/threonine } \\
\text { phosphatase activity; hydrolase activity; } \\
\text { magnesium-dependent protein } \\
\text { serine/threonine phosphatase activity; } \\
\text { manganese ion binding; myosin } \\
\text { phosphatase activity; protein amino } \\
\text { acid dephosphorylation; protein } \\
\text { phosphatase type 2A complex; protein } \\
\text { phosphatase type 2A, intrinsic catalyst } \\
\text { activity; protein phosphatase type 2B } \\
\text { activity; protein phosphatase type 2C } \\
\text { activity; regulation of cell cycle }\end{array}$ \\
\hline R60546 & 1.96E-05 & CD63 & 967 & \begin{tabular}{|l} 
CD63 \\
antigen \\
(melanoma \\
1 antigen) \\
\end{tabular} & $\begin{array}{l}\text { integral to plasma membrane; } \\
\text { lysosomal membrane }\end{array}$ \\
\hline T65132 & $2.13 \mathrm{E}-05$ & SSTR1 & 6751 & $\begin{array}{l}\text { somatostat } \\
\text { in receptor } \\
1\end{array}$ & $\begin{array}{l}\text { G-protein signaling, coupled to cyclic } \\
\text { nucleotide second messenger; cell-cell } \\
\text { signaling; digestion; integral to plasma } \\
\text { membrane; negative regulation of cell } \\
\text { proliferation; response to nutrients; } \\
\text { rhodopsin-like receptor activity; } \\
\text { somatostatin receptor activity }\end{array}$ \\
\hline R22946 & $2.20 \mathrm{E}-05$ & & & & \\
\hline H73170 & $2.24 \mathrm{E}-05$ & & & & \\
\hline H09088 & $2.44 \mathrm{E}-05$ & & & & \\
\hline $\mathrm{H} 84096$ & $2.45 \mathrm{E}-05$ & & & & \\
\hline H47146 & $2.57 \mathrm{E}-05$ & ERCC1 & 2067 & $\begin{array}{l}\text { excision } \\
\text { repair } \\
\text { cross- } \\
\text { compleme } \\
\text { nting } \\
\text { rodent } \\
\text { repair } \\
\text { deficiency, } \\
\text { compleme } \\
\text { ntation } \\
\text { group 1 } \\
\text { (includes } \\
\text { overlappin } \\
\text { g } \\
\text { antisense } \\
\text { sequence) } \\
\end{array}$ & $\begin{array}{l}\text { DNA repair; embryogenesis and } \\
\text { morphogenesis; } \\
\text { endodeoxyribonuclease activity; } \\
\text { nucleotide-excision repair; nucleus }\end{array}$ \\
\hline R77382 & $2.68 \mathrm{E}-05$ & FLJ10276 & $\underline{55108}$ & $\begin{array}{l}\text { hypothetic } \\
\text { al protein } \\
\text { FLJ10276 }\end{array}$ & \\
\hline
\end{tabular}




\begin{tabular}{|c|c|c|c|c|c|}
\hline $\mathrm{H} 62447$ & 2.69E-05 & MASP1 & 5648 & $\begin{array}{l}\text { mannan- } \\
\text { binding } \\
\text { lectin } \\
\text { serine } \\
\text { protease } 1 \\
\text { (C4/C2 } \\
\text { activating } \\
\text { component } \\
\text { of Ra- } \\
\text { reactive } \\
\text { factor) }\end{array}$ & $\begin{array}{l}\text { complement activation; serine-type } \\
\text { endopeptidase activity }\end{array}$ \\
\hline R87060 & $3.10 \mathrm{E}-05$ & GGCX & $\underline{2677}$ & $\begin{array}{l}\text { gamma- } \\
\text { glutamyl } \\
\text { carboxylas } \\
\text { e }\end{array}$ & $\begin{array}{l}\text { blood coagulation; gamma-glutamyl } \\
\text { carboxylase activity; integral to } \\
\text { membrane; ligase activity; membrane } \\
\text { fraction; protein modification }\end{array}$ \\
\hline N23779 & $3.13 \mathrm{E}-05$ & CD151 & 977 & $\begin{array}{l}\text { CD151 } \\
\text { antigen }\end{array}$ & $\begin{array}{l}\text { cell adhesion; integral to plasma } \\
\text { membrane; membrane fraction }\end{array}$ \\
\hline AA035641 & $3.36 \mathrm{E}-05$ & IGFBP2 & $\underline{3485}$ & $\begin{array}{l}\text { insulin-like } \\
\text { growth } \\
\text { factor } \\
\text { binding } \\
\text { protein 2, } \\
36 \mathrm{kDa} \\
\end{array}$ & $\begin{array}{l}\text { extracellular space; insulin-like growth } \\
\text { factor binding; regulation of cell growth }\end{array}$ \\
\hline H14586 & $3.46 \mathrm{E}-05$ & PRPS1 & $\underline{5631}$ & $\begin{array}{l}\text { phosphorib } \\
\text { osyl } \\
\text { pyrophosp } \\
\text { hate } \\
\text { synthetase } \\
1\end{array}$ & $\begin{array}{l}\text { kinase activity; lipoate-protein ligase B } \\
\text { activity; magnesium ion binding; } \\
\text { neurogenesis; nucleoside metabolism; } \\
\text { nucleotide biosynthesis; purine base } \\
\text { metabolism; ribonucleoside } \\
\text { monophosphate biosynthesis; ribose- } \\
\text { phosphate diphosphokinase activity; } \\
\text { transferase activity }\end{array}$ \\
\hline H02379 & $3.66 \mathrm{E}-05$ & & & & \\
\hline H57136 & $3.77 \mathrm{E}-05$ & FXYD1 & $\underline{5348}$ & \begin{tabular}{|l|} 
FXYD \\
domain \\
containing \\
ion \\
transport \\
regulator 1 \\
(phosphole \\
mman) \\
\end{tabular} & $\begin{array}{l}\text { chloride channel activity; chloride } \\
\text { transport; integral to plasma } \\
\text { membrane; ion channel activity; ion } \\
\text { transport; muscle contraction } \\
\end{array}$ \\
\hline T95699 & $3.89 \mathrm{E}-05$ & C110RF4 & $\underline{56834}$ & \begin{tabular}{l|} 
chromoso \\
me 11 \\
hypothetic \\
al protein \\
ORF4 \\
\end{tabular} & \\
\hline R76214 & 3.89E-05 & $\underline{\mathrm{PCDH} 16}$ & $\underline{8642}$ & $\begin{array}{l}\text { protocadhe } \\
\text { rin } 16 \\
\text { dachsous- } \\
\text { like } \\
\text { (Drosophil } \\
\text { a) }\end{array}$ & $\begin{array}{l}\text { calcium ion binding; calcium-dependent } \\
\text { cell-cell adhesion; cell adhesion; cell } \\
\text { adhesion molecule activity; homophilic } \\
\text { cell adhesion; integral to membrane }\end{array}$ \\
\hline T86042 & 4.07E-05 & & & & \\
\hline
\end{tabular}




\begin{tabular}{|c|c|c|c|c|c|}
\hline R14364 & $4.08 \mathrm{E}-05$ & PPM1E & $\underline{22843}$ & \multicolumn{2}{|c|}{ protein phosphatase 1E (PP2C domain containing) } \\
\hline $\mathrm{H} 26760$ & $4.12 \mathrm{E}-05$ & KIAA0375 & $\underline{9853}$ & $\begin{array}{l}\text { KIAA0375 } \\
\text { gene } \\
\text { product }\end{array}$ & \\
\hline $\mathrm{H} 10327$ & $4.21 \mathrm{E}-05$ & RAP1B & $\underline{5908}$ & $\begin{array}{l}\text { RAP1B, } \\
\text { member of } \\
\text { RAS } \\
\text { oncogene } \\
\text { family }\end{array}$ & $\begin{array}{l}\text { GTP binding; RAS small monomeric } \\
\text { GTPase activity; membrane; small } \\
\text { GTPase mediated signal transduction }\end{array}$ \\
\hline AA133962 & $4.48 \mathrm{E}-05$ & & & & \\
\hline R73991 & $4.48 \mathrm{E}-05$ & KIAA1160 & $\underline{57461}$ & $\begin{array}{l}\text { KIAA1160 } \\
\text { protein }\end{array}$ & \\
\hline $\mathrm{H} 27334$ & $4.54 \mathrm{E}-05$ & DDR1 & $\underline{780}$ & $\begin{array}{l}\text { discoidin } \\
\text { domain } \\
\text { receptor } \\
\text { family, } \\
\text { member } 1\end{array}$ & $\begin{array}{l}\text { ATP binding; cell adhesion; integral to } \\
\text { plasma membrane; protein amino acid } \\
\text { phosphorylation; receptor activity; } \\
\text { transferase activity; transmembrane } \\
\text { receptor protein tyrosine kinase activity; } \\
\text { transmembrane receptor protein } \\
\text { tyrosine kinase signaling pathway }\end{array}$ \\
\hline N/A1 & 4.56E-05 & & & & \\
\hline $\mathrm{H} 25699$ & $4.78 \mathrm{E}-05$ & $\underline{\mathrm{OIP} 2}$ & $\underline{11340}$ & $\begin{array}{l}\text { Opa- } \\
\text { interacting } \\
\text { protein } 2\end{array}$ & $\begin{array}{l}\text { 3'-5' exoribonuclease activity; RNA } \\
\text { binding; biological_process unknown; } \\
\text { cellular_component unknown; } \\
\text { exonuclease activity; exosome (RNase } \\
\text { complex); hydrolase activity; } \\
\text { molecular_function unknown; nucleus; } \\
\text { rRNA processing }\end{array}$ \\
\hline $\mathrm{H} 77390$ & $4.89 \mathrm{E}-05$ & GOLGA1 & $\underline{2800}$ & \begin{tabular}{|l|} 
golgi \\
autoantige \\
n, golgin \\
subfamily \\
a, 1
\end{tabular} & \\
\hline R19064 & $4.95 \mathrm{E}-05$ & $\frac{\operatorname{LOC5127}}{\underline{5}}$ & $\underline{51275}$ & $\begin{array}{l}\text { apoptosis- } \\
\text { related } \\
\text { protein } \\
\text { PNAS-1 }\end{array}$ & \\
\hline R53020 & 4.95E-05 & & & & \\
\hline T98139 & $5.04 \mathrm{E}-05$ & $\underline{\mathrm{HLA}-\mathrm{B}}$ & $\underline{3106}$ & $\begin{array}{l}\text { major } \\
\text { histocomp } \\
\text { atibility } \\
\text { complex, } \\
\text { class I, B }\end{array}$ & $\begin{array}{l}\text { MHC class I receptor activity; antigen } \\
\text { presentation, endogenous antigen; } \\
\text { antigen processing, endogenous } \\
\text { antigen via MHC class I; immune } \\
\text { response; integral to plasma membrane }\end{array}$ \\
\hline $\mathrm{H} 62424$ & 5.04E-05 & & & & \\
\hline H58461 & $5.44 \mathrm{E}-05$ & & 339088 & $\begin{array}{l}\text { similar to } \\
\text { My016 } \\
\text { protein }\end{array}$ & \\
\hline
\end{tabular}




\begin{tabular}{|c|c|c|c|c|c|}
\hline W72707 & $5.65 E-05$ & PRDX6 & $\underline{9588}$ & $\begin{array}{l}\text { peroxiredo } \\
\text { xin } 6\end{array}$ & $\begin{array}{l}\text { antioxidant activity; cytosol; hydrolase } \\
\text { activity; lipid catabolism; lysosome; } \\
\text { non-selenium glutathione peroxidase } \\
\text { activity; oxidoreductase activity; } \\
\text { phospholipase A2 activity; phospholipid } \\
\text { catabolism; response to oxidative } \\
\text { stress }\end{array}$ \\
\hline T50388 & $5.74 \mathrm{E}-05$ & & & & \\
\hline AA046291 & 5.79E-05 & & & & \\
\hline R21970 & $5.86 \mathrm{E}-05$ & GTF2H2 & $\underline{2966}$ & \begin{tabular}{|l|} 
general \\
transcriptio \\
n factor \\
IIH, \\
polypeptid \\
e 2, 44kDa
\end{tabular} & $\begin{array}{l}\text { DNA repair; nucleus; regulation of } \\
\text { transcription, DNA-dependent }\end{array}$ \\
\hline $\mathrm{H} 60460$ & 6.01E-05 & DCL-1 & 9936 & $\begin{array}{l}\text { type I } \\
\text { transmemb } \\
\text { rane C- } \\
\text { type lectin } \\
\text { receptor } \\
\text { DCL-1 }\end{array}$ & $\begin{array}{l}\text { heterophilic cell adhesion; sugar } \\
\text { binding }\end{array}$ \\
\hline N21532 & 6.47E-05 & & & & \\
\hline R50087 & 6.67E-05 & GREB1 & 9687 & $\begin{array}{l}\text { GREB1 } \\
\text { protein }\end{array}$ & \\
\hline T83091 & $7.10 \mathrm{E}-05$ & & & & \\
\hline H86672 & $7.16 \mathrm{E}-05$ & & & & \\
\hline $\mathrm{H} 70974$ & 7.39E-05 & & & & \\
\hline H47346 & $7.80 \mathrm{E}-05$ & KMO & 8564 & $\begin{array}{l}\text { kynurenine } \\
\text { 3- } \\
\text { monooxyg } \\
\text { enase } \\
\text { (kynurenin } \\
\text { e 3- } \\
\text { hydroxylas } \\
\text { e) }\end{array}$ & $\begin{array}{l}\text { aromatic compound metabolism; } \\
\text { electron transport; electron transporter } \\
\text { activity; kynurenine 3-monooxygenase } \\
\text { activity }\end{array}$ \\
\hline R40597 & $7.95 \mathrm{E}-05$ & WARS2 & 10352 & $\begin{array}{l}\text { tryptophan } \\
\text { yl tRNA } \\
\text { synthetase } \\
2 \\
\text { (mitochond } \\
\text { rial) } \\
\end{array}$ & $\begin{array}{l}\text { ATP binding; ligase activity; } \\
\text { mitochondrion; soluble fraction; } \\
\text { tryptophan-tRNA ligase activity; } \\
\text { tryptophanyl-tRNA aminoacylation }\end{array}$ \\
\hline $\mathrm{H} 64609$ & 8.04E-05 & AHR & 196 & $\begin{array}{l}\text { aryl } \\
\text { hydrocarbo } \\
\text { n receptor }\end{array}$ & $\begin{array}{l}\text { apoptosis; cell cycle; ligand-dependent } \\
\text { nuclear receptor activity; nucleus; } \\
\text { regulation of transcription, DNA- } \\
\text { dependent; response to stress; } \\
\text { response to xenobiotic stimulus; signal } \\
\text { transduction; transcription factor } \\
\text { activity; transcription from Pol II } \\
\text { promoter }\end{array}$ \\
\hline AA031465 & 8.06E-05 & GEFT & 115557 & $\begin{array}{l}\text { RAC/CDC } \\
42 \\
\text { exchange }\end{array}$ & \\
\hline
\end{tabular}




\begin{tabular}{|c|c|c|c|c|c|}
\hline & & & & factor & \\
\hline T83168 & $8.41 \mathrm{E}-05$ & & & & \\
\hline R76162 & 8.68E-05 & KRT23 & 25984 & \begin{tabular}{|l|} 
keratin 23 \\
(histone \\
deacetylas \\
e \\
inducible) \\
\end{tabular} & \\
\hline H84008 & $8.89 \mathrm{E}-05$ & & & & \\
\hline AA130221 & 9.40E-05 & DSC3 & $\underline{1825}$ & \begin{tabular}{|l|} 
desmocolli \\
n 3 \\
\\
\end{tabular} & $\begin{array}{l}\text { calcium ion binding; cell adhesion; cell } \\
\text { adhesion molecule activity; } \\
\text { cytoskeleton; homophilic cell adhesion; } \\
\text { integral to membrane; intercellular } \\
\text { junction; membrane fraction; plasma } \\
\text { membrane }\end{array}$ \\
\hline R18841 & $\begin{array}{r}0.000101 \\
747\end{array}$ & HNT & $\underline{50863}$ & neurotrimin & $\begin{array}{l}\text { cell adhesion; cell adhesion molecule } \\
\text { activity; integral to plasma membrane; } \\
\text { neuronal cell recognition }\end{array}$ \\
\hline AA142939 & $\begin{array}{r}0.000102 \\
144\end{array}$ & ATP8B2 & $\underline{57198}$ & $\begin{array}{l}\text { ATPase, } \\
\text { Class I, } \\
\text { type 8B, } \\
\text { member } 2\end{array}$ & $\begin{array}{l}\text { ATPase activity; cation transport; } \\
\text { hydrolase activity; integral to } \\
\text { membrane; magnesium ion binding; } \\
\text { metabolism; phospholipid-translocating } \\
\text { ATPase activity }\end{array}$ \\
\hline R44307 & $\begin{array}{r}0.000103 \\
006\end{array}$ & PPP1R9B & $\underline{84687}$ & \begin{tabular}{|l|} 
protein \\
phosphata \\
se 1, \\
regulatory \\
subunit 9B, \\
spinophilin \\
\end{tabular} & $\begin{array}{l}\text { intracellular signaling cascade; } \\
\text { membrane; transport; transporter } \\
\text { activity }\end{array}$ \\
\hline T85558 & $\begin{array}{r}0.000103 \\
649 \\
\end{array}$ & & & & \\
\hline H83405 & $\begin{array}{r}0.000104 \\
317\end{array}$ & FGD1 & $\underline{2245}$ & \begin{tabular}{|l|} 
faciogenital \\
dysplasia \\
(Aarskog- \\
Scott \\
syndrome)
\end{tabular} & $\begin{array}{l}\text { development; guanyl-nucleotide } \\
\text { exchange factor activity; histogenesis } \\
\text { and organogenesis; signal transduction; } \\
\text { zinc ion binding }\end{array}$ \\
\hline $\mathrm{H} 40607$ & $\begin{array}{r}0.000109 \\
28 \\
\end{array}$ & & & & \\
\hline AA031859 & $\begin{array}{r}0.000109 \\
417\end{array}$ & TIMM13 & $\underline{26517}$ & \begin{tabular}{|l|} 
translocas \\
e of inner \\
mitochondr \\
ial \\
membrane \\
13 \\
homolog \\
(yeast)
\end{tabular} & $\begin{array}{l}\text { hearing; mitochondrial inner membrane } \\
\text { pre-sequence translocase complex; } \\
\text { mitochondrial translocation; } \\
\text { mitochondrion; protein targeting; protein } \\
\text { translocase activity; zinc ion binding } \\
\end{array}$ \\
\hline R08080 & $\begin{array}{r}0.000110 \\
623 \\
\end{array}$ & & & & \\
\hline $\mathrm{H} 05011$ & $\begin{array}{r}0.000113 \\
12 \\
\end{array}$ & & & & \\
\hline H84657 & $\begin{array}{r}0.000118 \\
031\end{array}$ & GRWD & $\underline{83743}$ & $\begin{array}{l}\text { glutamate } \\
\text { rich WD } \\
\text { repeat }\end{array}$ & \\
\hline
\end{tabular}




\begin{tabular}{|c|c|c|c|c|c|}
\hline & & & & $\begin{array}{l}\text { protein } \\
\text { GRWD }\end{array}$ & \\
\hline R71723 & \begin{tabular}{|r|}
0.000120 \\
086
\end{tabular} & $\underline{\text { SLC4A2 }}$ & $\underline{6522}$ & \begin{tabular}{|l|} 
solute \\
carrier \\
family 4, \\
anion \\
exchanger, \\
member 2 \\
(erythrocyt \\
e \\
membrane \\
protein \\
band 3-like \\
1) \\
\end{tabular} & $\begin{array}{l}\text { anion transport; anion transporter } \\
\text { activity; antiporter activity; inorganic } \\
\text { anion exchanger activity; integral to } \\
\text { membrane; membrane fraction }\end{array}$ \\
\hline H20790 & $\begin{array}{r}0.000123 \\
136\end{array}$ & & $\underline{348024}$ & $\begin{array}{l}\text { similar to } \\
\text { TPIP alpha } \\
\text { lipid } \\
\text { phosphata } \\
\text { se }\end{array}$ & \\
\hline H13744 & $\begin{array}{r}0.000125 \\
595 \\
\end{array}$ & $\underline{A L D O A}$ & $\underline{226}$ & $\begin{array}{l}\text { aldolase A, } \\
\text { fructose- } \\
\text { bisphosph } \\
\text { ate }\end{array}$ & \\
\hline R00710 & $\begin{array}{r}0.000126 \\
96 \\
\end{array}$ & & & & \\
\hline H47539 & \begin{tabular}{|r|}
0.000133 \\
883 \\
\end{tabular} & & & & \\
\hline R15155 & $\begin{array}{r}0.000135 \\
7 \\
\end{array}$ & & & & \\
\hline H18199 & $\begin{array}{r}0.000135 \\
845 \\
\end{array}$ & & & & \\
\hline R52852 & \begin{tabular}{|r|}
0.000136 \\
721 \\
\end{tabular} & & & & \\
\hline R55009 & $\begin{array}{r}0.000136 \\
973 \\
\end{array}$ & GNAI2 & $\underline{2771}$ & $\begin{array}{l}\text { guanine } \\
\text { nucleotide } \\
\text { binding } \\
\text { protein (G } \\
\text { protein), } \\
\text { alpha } \\
\text { inhibiting } \\
\text { activity } \\
\text { polypeptid } \\
\text { e 2 }\end{array}$ & $\begin{array}{l}\text { G-protein coupled receptor protein } \\
\text { signaling pathway; GTP binding; } \\
\text { heterotrimeric G-protein GTPase } \\
\text { activity; negative regulation of } \\
\text { adenylate cyclase activity; response to } \\
\text { nutrients; signal transducer activity; } \\
\text { signal transduction }\end{array}$ \\
\hline R52023 & $\begin{array}{r}0.000137 \\
073 \\
\end{array}$ & & $\underline{340249}$ & $\begin{array}{l}\text { similar to } \\
\text { hypothetic } \\
\text { al protein } \\
\text { FLJ35882 }\end{array}$ & \\
\hline $\mathrm{H} 54430$ & $\begin{array}{r}0.000142 \\
236 \\
\end{array}$ & & & & \\
\hline H78668 & $\begin{array}{r}0.000145 \\
452 \\
\end{array}$ & & & & \\
\hline
\end{tabular}




\begin{tabular}{|c|c|c|c|c|c|}
\hline AA045053 & \begin{tabular}{|r|}
0.000148 \\
34 \\
\end{tabular} & PTN & $\underline{5764}$ & $\begin{array}{l}\text { pleiotrophi } \\
\text { n (heparin } \\
\text { binding } \\
\text { growth } \\
\text { factor 8, } \\
\text { neurite } \\
\text { growth- } \\
\text { promoting } \\
\text { factor 1) }\end{array}$ & $\begin{array}{l}\text { cell proliferation; cytokine activity; } \\
\text { extracellular space; growth factor } \\
\text { activity; heparin binding; neurogenesis; } \\
\text { positive regulation of cell proliferation; } \\
\text { protein phosphatase inhibitor activity; } \\
\text { regulation of cell cycle; transmembrane } \\
\text { receptor protein tyrosine phosphatase } \\
\text { signaling pathway }\end{array}$ \\
\hline H61972 & $\begin{array}{r}0.000149 \\
717 \\
\end{array}$ & PIN4 & $\underline{5303}$ & \begin{tabular}{|l|} 
protein \\
(peptidyl- \\
prolyl \\
cis/trans \\
isomerase) \\
NIMA- \\
interacting, \\
4 (parvulin) \\
\end{tabular} & $\begin{array}{l}\text { FK506-sensitive peptidyl-prolyl cis-trans } \\
\text { isomerase; cyclophilin; cyclophilin-type } \\
\text { peptidyl-prolyl cis-trans isomerase } \\
\text { activity; isomerase activity; } \\
\text { mitochondrial matrix; protein folding } \\
\end{array}$ \\
\hline H59568 & $\begin{array}{r}0.000154 \\
717 \\
\end{array}$ & WBP3 & 91010 & \begin{tabular}{|l|} 
WW \\
domain \\
binding \\
protein 3
\end{tabular} & \\
\hline H18504 & $\begin{array}{r}0.000177 \\
193\end{array}$ & $\begin{array}{l}\text { NEUROD } \\
\underline{6}\end{array}$ & $\underline{63974}$ & $\begin{array}{l}\text { neurogenic } \\
\text { differentiati } \\
\text { on } 6\end{array}$ & $\begin{array}{l}\text { DNA binding; nucleus; regulation of } \\
\text { transcription, DNA-dependent }\end{array}$ \\
\hline R72577 & \begin{tabular}{|r|}
0.000179 \\
49
\end{tabular} & FLJ11753 & $\underline{79712}$ & \begin{tabular}{|l|} 
hypothetic \\
al protein \\
FLJ11753 \\
\end{tabular} & \\
\hline N76562 & $\begin{array}{r}0.000181 \\
883 \\
\end{array}$ & FTH1 & $\underline{2495}$ & $\begin{array}{l}\text { ferritin, } \\
\text { heavy } \\
\text { polypeptid } \\
\text { e } 1\end{array}$ & $\begin{array}{l}\text { binding; cell proliferation; ferric iron } \\
\text { binding; ferritin complex; intracellular } \\
\text { iron ion storage; iron ion transport }\end{array}$ \\
\hline R76890 & $\begin{array}{r}0.000185 \\
627 \\
\end{array}$ & KIAA1340 & $\underline{57542}$ & $\begin{array}{l}\text { KIAA1340 } \\
\text { protein }\end{array}$ & \\
\hline R91797 & $\begin{array}{r}0.000186 \\
22\end{array}$ & & & & \\
\hline H52741 & $\begin{array}{r}0.000188 \\
793\end{array}$ & & & & \\
\hline H11855 & \begin{tabular}{|r|}
0.000191 \\
519
\end{tabular} & ELK1 & $\underline{2002}$ & $\begin{array}{l}\text { ELK1, } \\
\text { member of } \\
\text { ETS } \\
\text { oncogene } \\
\text { family }\end{array}$ & \\
\hline R89056 & $\begin{array}{r}0.000191 \\
859 \\
\end{array}$ & LAMP1 & $\underline{3916}$ & $\begin{array}{l}\text { lysosomal- } \\
\text { associated } \\
\text { membrane } \\
\text { protein } 1\end{array}$ & $\begin{array}{l}\text { integral to plasma membrane; } \\
\text { lysosome; membrane fraction }\end{array}$ \\
\hline N55283 & $\begin{array}{r}0.000192 \\
731 \\
\end{array}$ & KIAA0469 & 9903 & $\begin{array}{l}\text { KIAA0469 } \\
\text { gene } \\
\text { product }\end{array}$ & \\
\hline W47525 & $\begin{array}{r}0.000193 \\
929\end{array}$ & & & & \\
\hline R22402 & $\begin{array}{r}0.000194 \\
653 \\
\end{array}$ & & & & \\
\hline
\end{tabular}




\begin{tabular}{|c|c|c|c|c|c|}
\hline $\mathrm{H} 02088$ & $\begin{array}{r}0.000194 \\
866\end{array}$ & RBAF600 & $\underline{23352}$ & $\begin{array}{l}\text { retinoblast } \\
\text { oma- } \\
\text { associated } \\
\text { factor } 600\end{array}$ & \\
\hline N34169 & $\begin{array}{r}0.000199 \\
375\end{array}$ & $\underline{N F 2}$ & $\underline{4771}$ & $\begin{array}{l}\text { neurofibro } \\
\text { min } 2 \\
\text { (bilateral } \\
\text { acoustic } \\
\text { neuroma) }\end{array}$ & $\begin{array}{l}\text { cytoskeleton; hearing; negative } \\
\text { regulation of cell cycle; negative } \\
\text { regulation of cell proliferation; plasma } \\
\text { membrane; structural molecule activity }\end{array}$ \\
\hline R09418 & $\begin{array}{r}0.000202 \\
042 \\
\end{array}$ & & & & \\
\hline H82992 & $\begin{array}{r}0.000210 \\
935\end{array}$ & PIGT & $\underline{51604}$ & $\begin{array}{l}\text { phosphatid } \\
\text { yl inositol } \\
\text { glycan } \\
\text { class T }\end{array}$ & \\
\hline H09429 & $\begin{array}{r}0.000214 \\
377 \\
\end{array}$ & & & & \\
\hline H60520 & $\begin{array}{r}0.000218 \\
429 \\
\end{array}$ & & & & \\
\hline R83017 & $\begin{array}{r}0.000234 \\
786 \\
\end{array}$ & & & & \\
\hline R88894 & $\begin{array}{r}0.000237 \\
613\end{array}$ & & & & \\
\hline AA152287 & $\begin{array}{r}0.000250 \\
526\end{array}$ & SLC35B2 & 347734 & \begin{tabular}{|l} 
solute \\
carrier \\
family 35 \\
member \\
B2
\end{tabular} & $\begin{array}{l}\text { copper ion binding; electron transport; } \\
\text { electron transporter activity }\end{array}$ \\
\hline $\mathrm{H} 01640$ & $\begin{array}{r}0.000251 \\
997\end{array}$ & PSG1 & $\underline{5669}$ & $\begin{array}{l}\text { pregnancy } \\
\text { specific } \\
\text { beta-1- } \\
\text { glycoprotei } \\
\text { n } 1\end{array}$ & extracellular space; pregnancy \\
\hline W72400 & $\begin{array}{r}0.000253 \\
169\end{array}$ & C12orf2 & 11228 & \begin{tabular}{|l} 
chromoso \\
me 12 \\
open \\
reading \\
frame 2 \\
\end{tabular} & neuropeptide signaling pathway \\
\hline N33037 & $\begin{array}{r}0.000260 \\
259\end{array}$ & $\underline{\mathrm{ABT} 1}$ & $\underline{29777}$ & $\begin{array}{l}\text { activator of } \\
\text { basal } \\
\text { transcriptio } \\
\text { n } 1\end{array}$ & $\begin{array}{l}\text { general RNA polymerase II } \\
\text { transcription factor activity; nucleus; } \\
\text { transcription co-activator activity; } \\
\text { transcription from Pol II promoter }\end{array}$ \\
\hline N95559 & $\begin{array}{r}0.000262 \\
254\end{array}$ & C20orf21 & $\underline{54915}$ & $\begin{array}{l}\text { chromoso } \\
\text { me } 20 \\
\text { open } \\
\text { reading } \\
\text { frame } 21\end{array}$ & \\
\hline R33026 & $\begin{array}{r}0.000269 \\
822\end{array}$ & & & & \\
\hline R61223 & $\begin{array}{r}0.000269 \\
985 \\
\end{array}$ & OACT1 & 154141 & \multicolumn{2}{|c|}{$\begin{array}{l}\text { O-acyltransferase (membrane bound) domain } \\
\text { containing } 1\end{array}$} \\
\hline R25152 & 0.000274 & & & & \\
\hline
\end{tabular}




\begin{tabular}{|c|c|c|c|c|c|}
\hline & 442 & & & & \\
\hline H61842 & $\begin{array}{r}0.000275 \\
864\end{array}$ & & & & \\
\hline W95842 & $\begin{array}{r}0.000278 \\
085\end{array}$ & MARCKS & $\underline{4082}$ & $\begin{array}{l}\text { myristoylat } \\
\text { ed alanine- } \\
\text { rich protein } \\
\text { kinase C } \\
\text { substrate }\end{array}$ & $\begin{array}{l}\text { actin cross-linking activity; actin } \\
\text { cytoskeleton; calmodulin binding; cell } \\
\text { motility; plasma membrane }\end{array}$ \\
\hline N49727 & $\begin{array}{r}0.000280 \\
013\end{array}$ & GDF11 & $\underline{10220}$ & $\begin{array}{l}\text { growth } \\
\text { differentiati } \\
\text { on factor } \\
11\end{array}$ & $\begin{array}{l}\text { cellular_component unknown; cytokine } \\
\text { activity; growth factor activity; } \\
\text { mesoderm development; neurogenesis; } \\
\text { skeletal development }\end{array}$ \\
\hline H78479 & $\begin{array}{r}0.000293 \\
917\end{array}$ & TXNL2 & $\underline{10539}$ & $\begin{array}{l}\text { thioredoxin } \\
\text {-like } 2\end{array}$ & $\begin{array}{l}\text { electron transport; electron transporter } \\
\text { activity }\end{array}$ \\
\hline H85095 & $\begin{array}{r}0.000296 \\
065\end{array}$ & POLR2J2 & 246721 & \begin{tabular}{|l|} 
DNA \\
directed \\
RNA \\
polymeras \\
e II \\
polypeptid \\
e J-related \\
gene \\
\end{tabular} & $\begin{array}{l}\text { DNA binding; DNA-directed RNA } \\
\text { polymerase activity; transcription }\end{array}$ \\
\hline $\mathrm{H} 16042$ & $\begin{array}{r}0.000297 \\
025\end{array}$ & & & & \\
\hline R84724 & $\begin{array}{r}0.000300 \\
636\end{array}$ & $\mathrm{ABCA3}$ & $\underline{21}$ & $\begin{array}{l}\text { ATP- } \\
\text { binding } \\
\text { cassette, } \\
\text { sub-family } \\
\text { A (ABC1), } \\
\text { member } 3\end{array}$ & $\begin{array}{l}\text { ATP binding; ATP-binding cassette } \\
\text { (ABC) transporter activity; drug } \\
\text { resistance; integral to membrane; } \\
\text { membrane fraction; nucleotide binding; } \\
\text { transport; transporter activity }\end{array}$ \\
\hline N33492 & $\begin{array}{r}0.000304 \\
628\end{array}$ & $\underline{\text { STHM }}$ & $\underline{10610}$ & $\begin{array}{l}\text { sialyltransf } \\
\text { erase }\end{array}$ & $\begin{array}{l}\text { Golgi apparatus; integral to membrane; } \\
\text { protein amino acid glycosylation; } \\
\text { sialyltransferase activity; transferase } \\
\text { activity, transferring glycosyl groups }\end{array}$ \\
\hline H18220 & $\begin{array}{r}0.000310 \\
781 \\
\end{array}$ & & & & \\
\hline W55993 & $\begin{array}{r}0.000310 \\
94\end{array}$ & FBN2 & $\underline{2201}$ & \begin{tabular}{|l|} 
fibrillin 2 \\
(congenital \\
contractura \\
I \\
arachnoda \\
ctyly)
\end{tabular} & $\begin{array}{l}\text { calcium ion binding; embryogenesis } \\
\text { and morphogenesis; extracellular } \\
\text { matrix; extracellular matrix structural } \\
\text { constituent; histogenesis and } \\
\text { organogenesis }\end{array}$ \\
\hline R14286 & $\begin{array}{r}0.000312 \\
815 \\
\end{array}$ & & & & \\
\hline R27036 & $\begin{array}{r}0.000316 \\
911\end{array}$ & & & & \\
\hline $\mathrm{H} 49310$ & $\begin{array}{r}0.000319 \\
938 \\
\end{array}$ & $\underline{4}$ & 155382 & \multicolumn{2}{|c|}{ Williams Beuren syndrome chromosome region 24} \\
\hline T65549 & $\begin{array}{r}0.000323 \\
563\end{array}$ & PPP1R2 & $\underline{5504}$ & $\begin{array}{l}\text { protein } \\
\text { phosphata } \\
\text { se } 1, \\
\text { regulatory }\end{array}$ & $\begin{array}{l}\text { energy pathways; glycogen } \\
\text { metabolism; type } 1 \text { serine/threonine } \\
\text { specific protein phosphatase inhibitor } \\
\text { activity }\end{array}$ \\
\hline
\end{tabular}




\begin{tabular}{|c|c|c|c|c|c|}
\hline & & & & $\begin{array}{l}\text { (inhibitor) } \\
\text { subunit } 2\end{array}$ & \\
\hline N89592 & $\begin{array}{r}0.000325 \\
074 \\
\end{array}$ & TUBB2 & $\underline{10383}$ & $\begin{array}{l}\text { tubulin, } \\
\text { beta, } 2\end{array}$ & $\begin{array}{l}\text { GTP binding; MHC class I protein } \\
\text { binding; chaperone activity; } \\
\text { cytoskeleton; microtubule-based } \\
\text { movement; natural killer cell mediated } \\
\text { cytolysis; structural constituent of } \\
\text { cytoskeleton; tubulin }\end{array}$ \\
\hline R09905 & \begin{tabular}{|r|}
0.000325 \\
399
\end{tabular} & & & & \\
\hline AA099381 & $\begin{array}{r}0.000330 \\
749 \\
\end{array}$ & COX15 & $\underline{1355}$ & \begin{tabular}{|l|} 
COX15 \\
homolog, \\
cytochrom \\
e c oxidase \\
assembly \\
protein \\
(yeast)
\end{tabular} & $\begin{array}{l}\text { cytochrome-c oxidase activity; electron } \\
\text { transporter activity; mitochondrion; } \\
\text { respiratory gaseous exchange } \\
\\
\end{array}$ \\
\hline H54691 & $\begin{array}{r}0.000337 \\
05\end{array}$ & $\frac{\mathrm{ARHGEF}}{12}$ & 23365 & $\begin{array}{l}\text { Rho } \\
\text { guanine } \\
\text { nucleotide } \\
\text { exchange } \\
\text { factor } \\
\text { (GEF) } 12 \\
\end{array}$ & $\begin{array}{l}\text { intracellular signaling cascade; signal } \\
\text { transducer activity }\end{array}$ \\
\hline N92911 & $\begin{array}{r}0.000338 \\
021\end{array}$ & DJ473B4 & $\underline{56180}$ & $\begin{array}{l}\text { hypothetic } \\
\text { al protein } \\
\text { dJ473B4 }\end{array}$ & structural molecule activity \\
\hline R51898 & $\begin{array}{r}0.000342 \\
18\end{array}$ & & & & \\
\hline $\mathrm{H} 50471$ & $\begin{array}{r}0.000345 \\
409 \\
\end{array}$ & PDCD6 & $\underline{10016}$ & $\begin{array}{l}\text { programm } \\
\text { ed cell } \\
\text { death } 6\end{array}$ & $\begin{array}{l}\text { apoptosis; calcium ion binding; } \\
\text { induction of apoptosis by extracellular } \\
\text { signals }\end{array}$ \\
\hline R50932 & $\begin{array}{r}0.000346 \\
131 \\
\end{array}$ & D4ST-1 & 113189 & $\begin{array}{l}\text { dermatan- } \\
\text { 4- } \\
\text { sulfotransf } \\
\text { erase-1 }\end{array}$ & transferase activity \\
\hline H62898 & $\begin{array}{r}0.000346 \\
19 \\
\end{array}$ & & & & \\
\hline $\mathrm{H} 49225$ & $\begin{array}{r}0.000356 \\
08 \\
\end{array}$ & & & & \\
\hline H83857 & $\begin{array}{r}0.000362 \\
813 \\
\end{array}$ & & & & \\
\hline H52939 & \begin{tabular}{|r|}
0.000374 \\
234 \\
\end{tabular} & & & & \\
\hline R54918 & $\begin{array}{r}0.000382 \\
138 \\
\end{array}$ & FLJ13912 & $\underline{64785}$ & $\begin{array}{l}\text { hypothetic } \\
\text { al protein } \\
\text { FLJ13912 }\end{array}$ & \\
\hline R21825 & $\begin{array}{r}0.000387 \\
8 \\
\end{array}$ & & & & \\
\hline H59238 & $\begin{array}{r}0.000395 \\
119\end{array}$ & $\frac{\text { RARRES }}{2}$ & $\underline{5919}$ & $\begin{array}{l}\text { retinoic } \\
\text { acid } \\
\text { receptor } \\
\text { responder }\end{array}$ & $\begin{array}{l}\text { cellular_component unknown; } \\
\text { molecular_function unknown; retinoid } \\
\text { metabolism }\end{array}$ \\
\hline
\end{tabular}




\begin{tabular}{|c|c|c|c|c|c|}
\hline & & & & $\begin{array}{l}\text { (tazarotene } \\
\text { induced) } 2\end{array}$ & \\
\hline H50385 & $\begin{array}{r}0.000397 \\
598\end{array}$ & CSEN & $\underline{30818}$ & $\begin{array}{l}\text { calsenilin, } \\
\text { presenilin } \\
\text { binding } \\
\text { protein, EF } \\
\text { hand } \\
\text { transcriptio } \\
\text { n factor }\end{array}$ & $\begin{array}{l}\text { DNA binding; calcium ion binding; } \\
\text { regulation of transcription from Pol II } \\
\text { promoter; signal transduction; } \\
\text { transcription co-repressor activity }\end{array}$ \\
\hline R28090 & \begin{tabular}{|r|}
0.000402 \\
226 \\
\end{tabular} & KIAA1495 & $\underline{57631}$ & $\begin{array}{l}\text { KIAA1495 } \\
\text { protein }\end{array}$ & \\
\hline AA142924 & $\begin{array}{r}0.000405 \\
923\end{array}$ & DF & 1675 & $\begin{array}{l}\text { D } \\
\text { component } \\
\text { of } \\
\text { compleme } \\
\text { nt (adipsin) }\end{array}$ & $\begin{array}{l}\text { chymotrypsin activity; complement } \\
\text { activation, alternative pathway; } \\
\text { complement factor D activity; hydrolase } \\
\text { activity; proteolysis and peptidolysis; } \\
\text { trypsin activity }\end{array}$ \\
\hline AA098963 & $\begin{array}{r}0.000410 \\
221\end{array}$ & $\underline{\text { SLC1A5 }}$ & $\underline{6510}$ & $\begin{array}{l}\text { solute } \\
\text { carrier } \\
\text { family } 1 \\
\text { (neutral } \\
\text { amino acid } \\
\text { transporter } \\
\text { ), member } \\
5\end{array}$ & $\begin{array}{l}\text { dicarboxylic acid transport; integral to } \\
\text { plasma membrane; membrane fraction; } \\
\text { neutral amino acid transport; neutral } \\
\text { amino acid transporter activity; receptor } \\
\text { activity; } \\
\text { sodium:dicarboxylate/tricarboxylate } \\
\text { symporter activity; transport }\end{array}$ \\
\hline N45640 & $\begin{array}{r}0.000411 \\
849\end{array}$ & $\underline{\mathrm{CH} 25 \mathrm{H}}$ & $\underline{9023}$ & $\begin{array}{l}\text { cholesterol } \\
25- \\
\text { hydroxylas } \\
\text { e }\end{array}$ & $\begin{array}{l}\text { catalytic activity; lipid metabolism; } \\
\text { membrane fraction; steroid hydroxylase } \\
\text { activity }\end{array}$ \\
\hline R79518 & $\begin{array}{r}0.000419 \\
88\end{array}$ & MCAM & 4162 & $\begin{array}{l}\text { melanoma } \\
\text { cell } \\
\text { adhesion } \\
\text { molecule }\end{array}$ & $\begin{array}{l}\text { cell adhesion; cell adhesion molecule } \\
\text { activity; embryogenesis and } \\
\text { morphogenesis; integral to plasma } \\
\text { membrane }\end{array}$ \\
\hline $\mathrm{H} 65175$ & $\begin{array}{r}0.000426 \\
372 \\
\end{array}$ & SLC31A1 & 1317 & $\begin{array}{l}\text { solute } \\
\text { carrier } \\
\text { family } 31 \\
\text { (copper } \\
\text { transporter } \\
\text { s), member } \\
1\end{array}$ & $\begin{array}{l}\text { copper ion transport; copper ion } \\
\text { transporter activity; integral to plasma } \\
\text { membrane; transport }\end{array}$ \\
\hline $\mathrm{H} 27034$ & $\begin{array}{r}0.000427 \\
779\end{array}$ & IGKC & 3514 & $\begin{array}{l}\text { immunoglo } \\
\text { bulin } \\
\text { kappa } \\
\text { constant } \\
\end{array}$ & antigen binding; immune response \\
\hline R69282 & $\begin{array}{r}0.000445 \\
804\end{array}$ & RSN & $\underline{6249}$ & \begin{tabular}{|l|} 
restin \\
(Reed- \\
Steinberg \\
cell- \\
expressed \\
intermediat \\
e filament- \\
associated
\end{tabular} & $\begin{array}{l}\text { endosome; intermediate filament; } \\
\text { kinesin complex; microtubule binding; } \\
\text { microtubule cytoskeleton; microtubule- } \\
\text { based process; nonselective vesicle } \\
\text { transport; nucleic acid binding }\end{array}$ \\
\hline
\end{tabular}




\begin{tabular}{|c|c|c|c|c|c|}
\hline & & & & protein) & \\
\hline T96973 & $\begin{array}{r}0.000446 \\
949 \\
\end{array}$ & & & & \\
\hline $\mathrm{H} 00760$ & $\begin{array}{r}0.000450 \\
476 \\
\end{array}$ & & & & \\
\hline H84224 & \begin{tabular}{|r|}
0.000451 \\
897 \\
\end{tabular} & & & & \\
\hline R23351 & $\begin{array}{r}0.000456 \\
36 \\
\end{array}$ & & & & \\
\hline R88435 & $\begin{array}{r}0.000463 \\
204 \\
\end{array}$ & DPP6 & $\underline{1804}$ & $\begin{array}{l}\text { dipeptidylp } \\
\text { eptidase } 6\end{array}$ & $\begin{array}{l}\text { catalytic activity; dipeptidyl-peptidase IV } \\
\text { activity; dipeptidyl-peptidase activity; } \\
\text { integral to membrane; proteolysis and } \\
\text { peptidolysis }\end{array}$ \\
\hline $\mathrm{H} 45746$ & \begin{tabular}{|r|}
0.000474 \\
872 \\
\end{tabular} & & & & \\
\hline N64478 & $\begin{array}{r}0.000481 \\
03\end{array}$ & $\frac{\mathrm{HUMYZ8}}{2 \mathrm{H} 07}$ & 29792 & $\begin{array}{l}\text { hypothetic } \\
\text { al protein } \\
\text { HUMYZ82 } \\
\text { H07 }\end{array}$ & \\
\hline R60030 & $\begin{array}{r}0.000485 \\
024 \\
\end{array}$ & KIAA0972 & $\underline{22869}$ & $\begin{array}{l}\text { KIAA0972 } \\
\text { protein }\end{array}$ & $\begin{array}{l}\text { DNA binding; nucleus; regulation of } \\
\text { transcription, DNA-dependent }\end{array}$ \\
\hline W80519 & $\begin{array}{r}0.000485 \\
204 \\
\end{array}$ & $\underline{\underline{\text { SDBCAG }}}$ & $\underline{51614}$ & \multicolumn{2}{|c|}{ serologically defined breast cancer antigen 84} \\
\hline R08165 & \begin{tabular}{|r|}
0.000487 \\
527 \\
\end{tabular} & & & & \\
\hline R85044 & $\begin{array}{r}0.000497 \\
81\end{array}$ & $\underline{\text { SMPD1 }}$ & $\underline{6609}$ & $\begin{array}{l}\text { sphingomy } \\
\text { elin } \\
\text { phosphodi } \\
\text { esterase 1, } \\
\text { acid } \\
\text { lysosomal } \\
\text { (acid } \\
\text { sphingomy } \\
\text { elinase) } \\
\end{array}$ & $\begin{array}{l}\text { carbohydrate metabolism; hydrolase } \\
\text { activity, acting on glycosyl bonds; } \\
\text { lysosome; neurogenesis; signal } \\
\text { transduction; sphingomyelin } \\
\text { metabolism; sphingomyelin } \\
\text { phosphodiesterase activity } \\
\end{array}$ \\
\hline H16242 & $\begin{array}{r}0.000498 \\
382 \\
\end{array}$ & $\frac{\operatorname{SDCCAG}}{\underline{16}}$ & $\underline{10813}$ & $\begin{array}{l}\text { serologicall } \\
\text { y defined } \\
\text { colon } \\
\text { cancer } \\
\text { antigen } 16 \\
\end{array}$ & tumor antigen \\
\hline H59136 & $\begin{array}{r}0.000506 \\
347\end{array}$ & CYP39A1 & $\underline{51302}$ & $\begin{array}{l}\text { cytochrom } \\
\text { e P450, } \\
\text { family } 39, \\
\text { subfamily } \\
\text { A, } \\
\text { polypeptid } \\
\text { e } 1\end{array}$ & $\begin{array}{l}\text { bile acid biosynthesis; bile acid } \\
\text { catabolism; digestion; electron } \\
\text { transport; electron transporter activity; } \\
\text { endoplasmic reticulum; membrane; } \\
\text { microsome; monooxygenase activity; } \\
\text { oxysterol 7-alpha-hydroxylase activity }\end{array}$ \\
\hline R10571 & $\begin{array}{r}0.000528 \\
464 \\
\end{array}$ & & & & \\
\hline
\end{tabular}




\begin{tabular}{|c|c|c|c|c|c|}
\hline $\mathrm{H} 27559$ & $\begin{array}{r}0.000539 \\
128\end{array}$ & C16orf35 & 8131 & \begin{tabular}{|l|} 
chromoso \\
me 16 \\
open \\
reading \\
frame 35 \\
\end{tabular} & $\begin{array}{l}\text { biological_process unknown; } \\
\text { cellular_component unknown; } \\
\text { molecular_function unknown }\end{array}$ \\
\hline N62241 & $\begin{array}{r}0.000545 \\
189\end{array}$ & FLJ32029 & 283209 & $\begin{array}{l}\text { hypothetic } \\
\text { al protein } \\
\text { FLJ32029 }\end{array}$ & $\begin{array}{l}\text { carbohydrate metabolism; } \\
\text { intramolecular transferase activity, } \\
\text { phosphotransferases }\end{array}$ \\
\hline H14999 & $\begin{array}{r}0.000547 \\
702\end{array}$ & $\underline{\text { ARHGEF }}$ & $\underline{9459}$ & $\begin{array}{l}\text { Rac/Cdc42 } \\
\text { guanine } \\
\text { nucleotide } \\
\text { exchange } \\
\text { factor } \\
\text { (GEF) } 6\end{array}$ & $\begin{array}{l}\text { GTPase activator activity; JNK } \\
\text { cascade; Rho guanyl-nucleotide } \\
\text { exchange factor activity; Rho interactor } \\
\text { activity; apoptosis; intracellular }\end{array}$ \\
\hline H58957 & $\begin{array}{r}0.000561 \\
922\end{array}$ & MCL1 & $\underline{4170}$ & $\begin{array}{l}\text { myeloid } \\
\text { cell } \\
\text { leukemia } \\
\text { sequence } \\
1 \text { (BCL2- } \\
\text { related) }\end{array}$ & $\begin{array}{l}\text { apoptotic program; development; heat } \\
\text { shock response }\end{array}$ \\
\hline T81715 & $\begin{array}{r}0.000562 \\
093\end{array}$ & & & & \\
\hline N95586 & $\begin{array}{r}0.000564 \\
814\end{array}$ & TPD52 & $\underline{7163}$ & $\begin{array}{l}\text { tumor } \\
\text { protein } \\
\text { D52 }\end{array}$ & $\begin{array}{l}\text { embryogenesis and morphogenesis; } \\
\text { kinesin complex }\end{array}$ \\
\hline R06552 & \begin{tabular}{|r|}
0.000566 \\
932 \\
\end{tabular} & & & & \\
\hline T92003 & $\begin{array}{r}0.000589 \\
798\end{array}$ & KIAA0342 & 9881 & $\begin{array}{l}\text { KIAA0342 } \\
\text { gene } \\
\text { product }\end{array}$ & $\begin{array}{l}\text { DNA binding; membrane; nucleus; } \\
\text { transport; transporter activity }\end{array}$ \\
\hline H10658 & $\begin{array}{r}0.000592 \\
146\end{array}$ & & & & \\
\hline N/A1 & $\begin{array}{r}0.000611 \\
815\end{array}$ & & & & \\
\hline H63763 & $\begin{array}{r}0.000624 \\
428 \\
\end{array}$ & & & & \\
\hline AA059213 & $\begin{array}{r}0.000647 \\
758\end{array}$ & & & & \\
\hline W32895 & $\begin{array}{r}0.000661 \\
226 \\
\end{array}$ & JUN & $\underline{3725}$ & $\begin{array}{l}\text { v-jun } \\
\text { sarcoma } \\
\text { virus } 17 \\
\text { oncogene } \\
\text { homolog } \\
\text { (avian) }\end{array}$ & $\begin{array}{l}\text { RNA polymerase II transcription factor } \\
\text { activity; cell growth and/or } \\
\text { maintenance; nuclear chromosome; } \\
\text { regulation of transcription, DNA- } \\
\text { dependent; transcription factor activity }\end{array}$ \\
\hline $\mathrm{H} 47026$ & $\begin{array}{r}0.000674 \\
021\end{array}$ & MGAT3 & 4248 & $\begin{array}{l}\text { mannosyl } \\
\text { (beta-1,4-)- } \\
\text { glycoprotei } \\
\text { n beta-1,4- } \\
\mathrm{N}- \\
\text { acetylgluco } \\
\text { saminyltra } \\
\text { nsferase } \\
\end{array}$ & $\begin{array}{l}\text { Golgi apparatus; N-linked glycosylation; } \\
\text { beta-1,4-mannosylglycoprotein 4-beta- } \\
\text { N-acetylglucosaminyltransferase } \\
\text { activity; integral to membrane; } \\
\text { transferase activity, transferring } \\
\text { glycosyl groups }\end{array}$ \\
\hline T65291 & 0.000691 & DKFZp76 & 222865 & hypothetic & \\
\hline
\end{tabular}




\begin{tabular}{|c|c|c|c|c|c|}
\hline & 869 & 1 L1417 & & \begin{tabular}{|l|} 
al protein \\
DKFZp761 \\
L1417
\end{tabular} & \\
\hline N68416 & $\begin{array}{r}0.000695 \\
289\end{array}$ & & & & \\
\hline N80976 & $\begin{array}{r}0.000700 \\
273\end{array}$ & $\frac{\operatorname{LOC5} 5125}{2}$ & $\underline{51252}$ & $\begin{array}{l}\text { hypothetic } \\
\text { al protein } \\
\text { LOC51252 }\end{array}$ & \\
\hline R19153 & $\begin{array}{r}0.000705 \\
028\end{array}$ & RPS7 & $\underline{6201}$ & $\begin{array}{l}\text { ribosomal } \\
\text { protein S7 }\end{array}$ & $\begin{array}{l}\text { RNA binding; cytosolic small ribosomal } \\
\text { subunit (sensu Eukarya); intracellular; } \\
\text { protein biosynthesis; ribosome; } \\
\text { structural constituent of ribosome }\end{array}$ \\
\hline R99067 & \begin{tabular}{|r|}
0.000710 \\
473
\end{tabular} & & & & \\
\hline R85150 & $\begin{array}{r}0.000724 \\
255\end{array}$ & EPHB6 & 2051 & EphB6 & $\begin{array}{l}\text { ATP binding; ephrin receptor activity; } \\
\text { integral to membrane; protein amino } \\
\text { acid phosphorylation; protein-tyrosine } \\
\text { kinase activity; receptor activity; } \\
\text { transmembrane receptor protein } \\
\text { tyrosine kinase signaling pathway }\end{array}$ \\
\hline H79433 & $\begin{array}{r}0.000728 \\
587 \\
\end{array}$ & & & & \\
\hline $\mathrm{H} 71072$ & $\begin{array}{r}0.000730 \\
464 \\
\end{array}$ & & & & \\
\hline R87193 & $\begin{array}{r}0.000760 \\
245 \\
\end{array}$ & & & & \\
\hline H18883 & $\begin{array}{r}0.000763 \\
027\end{array}$ & & & & \\
\hline H50015 & $\begin{array}{r}0.000791 \\
72\end{array}$ & & & & \\
\hline R87345 & $\begin{array}{r}0.000803 \\
526 \\
\end{array}$ & MGC2656 & $\underline{79414}$ & $\begin{array}{l}\text { hypothetic } \\
\text { al protein } \\
\text { MGC2656 }\end{array}$ & \\
\hline R88587 & \begin{tabular}{|r|}
0.000839 \\
108
\end{tabular} & & & & \\
\hline W87783 & $\begin{array}{r}0.000839 \\
432 \\
\end{array}$ & REV1L & $\underline{51455}$ & $\begin{array}{l}\text { REV1-like } \\
\text { (yeast) }\end{array}$ & $\begin{array}{l}\text { DNA repair; intracellular; transferase } \\
\text { activity }\end{array}$ \\
\hline H14566 & $\begin{array}{r}0.000845 \\
72 \\
\end{array}$ & & & & \\
\hline $\mathrm{H} 46055$ & $\begin{array}{r}0.000864 \\
372 \\
\end{array}$ & KIAA0725 & 23259 & $\begin{array}{l}\text { KIAA0725 } \\
\text { protein }\end{array}$ & metal ion binding \\
\hline $\mathrm{H} 43816$ & $\begin{array}{r}0.000865 \\
02\end{array}$ & & & & \\
\hline N72553 & $\begin{array}{r}0.000867 \\
797\end{array}$ & CALM3 & 808 & $\begin{array}{l}\text { calmodulin } \\
3 \\
\text { (phosphory } \\
\text { lase } \\
\text { kinase, } \\
\text { delta) }\end{array}$ & calcium ion binding \\
\hline
\end{tabular}




\begin{tabular}{|c|c|c|c|c|c|}
\hline $\mathrm{H} 44725$ & \begin{tabular}{|r|}
0.000868 \\
955
\end{tabular} & PAWR & $\underline{5074}$ & $\begin{array}{l}\text { PRKC, } \\
\text { apoptosis, } \\
\text { WT1, } \\
\text { regulator }\end{array}$ & $\begin{array}{l}\text { apoptosis; cytoplasm; negative } \\
\text { regulation of cell proliferation; negative } \\
\text { regulation of transcription from Pol II } \\
\text { promoter; nucleus; transcription co- } \\
\text { repressor activity }\end{array}$ \\
\hline $\mathrm{H} 17327$ & $\begin{array}{r}0.000891 \\
169 \\
\end{array}$ & PM5 & $\underline{23420}$ & $\begin{array}{l}\text { pM5 } \\
\text { protein }\end{array}$ & \\
\hline AA059242 & $\begin{array}{r}0.000899 \\
662 \\
\end{array}$ & & & & \\
\hline $\mathrm{H} 24891$ & $\begin{array}{r}0.000908 \\
478\end{array}$ & & & & \\
\hline R07137 & $\begin{array}{r}0.000911 \\
983\end{array}$ & $\mathrm{HIC2}$ & $\underline{23119}$ & $\begin{array}{l}\text { hypermeth } \\
\text { ylated in } \\
\text { cancer } 2\end{array}$ & $\begin{array}{l}\text { DNA binding; negative regulation of } \\
\text { transcription, DNA-dependent; nucleus; } \\
\text { protein C-terminus binding }\end{array}$ \\
\hline T86313 & $\begin{array}{r}0.000914 \\
196\end{array}$ & MAOB & $\underline{4129}$ & $\begin{array}{l}\text { monoamin } \\
\text { e oxidase } \\
\text { B }\end{array}$ & $\begin{array}{l}\text { amine oxidase (flavin-containing) } \\
\text { activity; electron transport; electron } \\
\text { transporter activity; integral to } \\
\text { membrane; mitochondrial membrane; } \\
\text { oxidoreductase activity }\end{array}$ \\
\hline AA054137 & $\begin{array}{r}0.000916 \\
815 \\
\end{array}$ & & & & \\
\hline $\mathrm{H} 43746$ & $\begin{array}{r}0.000931 \\
318\end{array}$ & TRIP8 & $\underline{221037}$ & $\begin{array}{l}\text { thyroid } \\
\text { hormone } \\
\text { receptor } \\
\text { interactor } 8\end{array}$ & $\begin{array}{l}\text { intracellular; ligand-dependent thyroid } \\
\text { hormone receptor interactor activity; } \\
\text { regulation of transcription, DNA- } \\
\text { dependent }\end{array}$ \\
\hline $\mathrm{H} 41330$ & $\begin{array}{r}0.000937 \\
356\end{array}$ & LRRC2 & $\underline{79442}$ & \begin{tabular}{|l} 
leucine- \\
rich repeat- \\
containing \\
2 \\
\end{tabular} & \\
\hline H90964 & $\begin{array}{r}0.000973 \\
406\end{array}$ & STRN4 & $\underline{29888}$ & $\begin{array}{l}\text { striatin, } \\
\text { calmodulin } \\
\text { binding } \\
\text { protein } 4\end{array}$ & $\begin{array}{l}\text { calmodulin binding; cytoplasm; kinesin } \\
\text { complex; membrane fraction; signal } \\
\text { transduction; structural molecule } \\
\text { activity; synaptic transmission }\end{array}$ \\
\hline R24476 & $\begin{array}{r}0.000995 \\
792 \\
\end{array}$ & & & & \\
\hline $\mathrm{H} 28503$ & $\begin{array}{r}0.000996 \\
126\end{array}$ & & & & \\
\hline $\mathrm{H} 26552$ & $\begin{array}{r}0.001011 \\
054 \\
\end{array}$ & MGC5395 & $\underline{79026}$ & $\begin{array}{l}\text { hypothetic } \\
\text { al protein } \\
\text { MGC5395 }\end{array}$ & intracellular signaling cascade \\
\hline $\mathrm{H} 42894$ & $\begin{array}{r}0.001028 \\
578 \\
\end{array}$ & KIAA0420 & 9717 & $\begin{array}{l}\text { KIAA0420 } \\
\text { gene } \\
\text { product }\end{array}$ & $\begin{array}{l}\text { intracellular; transport; transporter } \\
\text { activity }\end{array}$ \\
\hline R23778 & $\begin{array}{r}0.001030 \\
233 \\
\end{array}$ & $\underline{\mathrm{C} 7}$ & $\underline{730}$ & $\begin{array}{l}\text { compleme } \\
\mathrm{nt} \\
\text { component } \\
7\end{array}$ & $\begin{array}{l}\text { complement activation, alternative } \\
\text { pathway; complement activation, } \\
\text { classical pathway; complement activity; } \\
\text { cytolysis; immune response; integral to } \\
\text { membrane; membrane attack complex; } \\
\text { response to pathogenic bacteria }\end{array}$ \\
\hline W80487 & $\begin{array}{r}0.001053 \\
456 \\
\end{array}$ & DC50 & $\underline{81892}$ & $\begin{array}{l}\text { hypothetic } \\
\text { al protein }\end{array}$ & nucleic acid binding \\
\hline
\end{tabular}




\begin{tabular}{|c|c|c|c|c|c|}
\hline & & & & DC50 & \\
\hline H61030 & $\begin{array}{r}0.001061 \\
303\end{array}$ & & & & \\
\hline H29610 & $\begin{array}{r}0.001065 \\
284\end{array}$ & & & & \\
\hline R27269 & $\begin{array}{r}0.001066 \\
377 \\
\end{array}$ & & & & \\
\hline H43455 & $\begin{array}{r}0.001071 \\
695 \\
\end{array}$ & PP2447 & 80305 & $\begin{array}{l}\text { hypothetic } \\
\text { al protein } \\
\text { PP2447 }\end{array}$ & \\
\hline T74007 & $\begin{array}{r}0.001072 \\
8\end{array}$ & NCSTN & 23385 & nicastrin & $\begin{array}{l}\text { integral to membrane; } \\
\text { molecular_function unknown; } \\
\text { proteolysis and peptidolysis }\end{array}$ \\
\hline H38593 & $\begin{array}{r}0.001081 \\
703\end{array}$ & & & & \\
\hline R92948 & $\begin{array}{r}0.001093 \\
574 \\
\end{array}$ & & & & \\
\hline AA149222 & $\begin{array}{r}0.001115 \\
667\end{array}$ & $\frac{M G C 1483}{6}$ & $\underline{92014}$ & $\begin{array}{l}\text { hypothetic } \\
\text { al protein } \\
\text { similar to } \\
\text { CG7943 }\end{array}$ & $\begin{array}{l}\text { binding; mitochondrial inner membrane; } \\
\text { transport }\end{array}$ \\
\hline N91376 & $\begin{array}{r}0.001119 \\
322\end{array}$ & $\underline{\mathrm{KIAA0247}}$ & $\underline{9766}$ & $\begin{array}{l}\text { KIAA0247 } \\
\text { gene } \\
\text { product }\end{array}$ & integral to membrane \\
\hline R13021 & $\begin{array}{r}0.001136 \\
897\end{array}$ & $\underline{F L J 10751}$ & 55222 & $\begin{array}{l}\text { hypothetic } \\
\text { al protein } \\
\text { FLJ10751 }\end{array}$ & \\
\hline T80602 & \begin{tabular}{|r|}
0.001139 \\
61
\end{tabular} & & & & \\
\hline R59528 & $\begin{array}{r}0.001213 \\
546 \\
\end{array}$ & & & & \\
\hline AA045734 & $\begin{array}{r}0.001227 \\
907\end{array}$ & BET1L & $\underline{51272}$ & $\begin{array}{l}\text { blocked earl } \\
\text { like }\end{array}$ & ly in transport 1 homolog (S. cerevisiae) \\
\hline T97903 & $\begin{array}{r}0.001241 \\
936\end{array}$ & & & & \\
\hline H93450 & $\begin{array}{r}0.001256 \\
746 \\
\end{array}$ & ZNF347 & 84671 & \begin{tabular}{|l|} 
zinc finger \\
protein 347
\end{tabular} & $\begin{array}{l}\text { DNA binding; nucleus; regulation of } \\
\text { transcription, DNA-dependent }\end{array}$ \\
\hline AA136161 & $\begin{array}{r}0.001288 \\
343 \\
\end{array}$ & & & & \\
\hline N33229 & $\begin{array}{r}0.001288 \\
878 \\
\end{array}$ & & & & \\
\hline AA026902 & $\begin{array}{r}0.001289 \\
046\end{array}$ & FLJ11320 & $\underline{55343}$ & \begin{tabular}{|l|} 
GDP- \\
fucose \\
transporter \\
1 \\
\end{tabular} & $\begin{array}{l}\text { Golgi apparatus; integral to membrane; } \\
\text { sugar porter activity; transport }\end{array}$ \\
\hline T84539 & $\begin{array}{r}0.001311 \\
256\end{array}$ & & & & \\
\hline R78049 & $\begin{array}{r}0.001327 \\
308\end{array}$ & $\underline{\text { SUMF2 }}$ & $\underline{25870}$ & $\begin{array}{l}\text { sulfatase } \\
\text { modifying } \\
\text { factor } 2\end{array}$ & \\
\hline N73749 & $\begin{array}{r}0.001342 \\
171\end{array}$ & & & & \\
\hline
\end{tabular}




\begin{tabular}{|c|c|c|c|c|c|}
\hline N21091 & \begin{tabular}{r|}
0.001342 \\
53 \\
\end{tabular} & $\underline{\text { CAV1 }}$ & 857 & $\begin{array}{l}\text { caveolin 1, } \\
\text { caveolae } \\
\text { protein, } \\
22 \mathrm{kDa} \\
\end{array}$ & $\begin{array}{l}\text { caveola; integral to plasma membrane; } \\
\text { structural molecule activity }\end{array}$ \\
\hline N29422 & $\begin{array}{r}0.001354 \\
1\end{array}$ & MMP2 & 4313 & $\begin{array}{l}\text { matrix } \\
\text { metalloprot } \\
\text { einase } 2 \\
\text { (gelatinase } \\
\text { A, 72kDa } \\
\text { gelatinase, } \\
72 \mathrm{kDa} \text { type } \\
\text { IV } \\
\text { collagenas } \\
\text { e) }\end{array}$ & $\begin{array}{l}\text { calcium ion binding; collagen } \\
\text { catabolism; extracellular matrix; } \\
\text { extracellular space; gelatinase A } \\
\text { activity; hydrolase activity; zinc ion } \\
\text { binding }\end{array}$ \\
\hline N94626 & $\begin{array}{r}0.001371 \\
637 \\
\end{array}$ & SNRPD2 & $\underline{6633}$ & $\begin{array}{l}\text { small } \\
\text { nuclear } \\
\text { ribonucleo } \\
\text { protein D2 } \\
\text { polypeptid } \\
\text { e } 16.5 \mathrm{kDa}\end{array}$ & $\begin{array}{l}\text { pre-mRNA splicing factor activity; small } \\
\text { nuclear ribonucleoprotein complex; } \\
\text { small nucleolar ribonucleoprotein } \\
\text { complex; spliceosome assembly; } \\
\text { spliceosome complex }\end{array}$ \\
\hline AA126875 & $\begin{array}{r}0.001387 \\
999\end{array}$ & FYCO1 & $\underline{79443}$ & $\begin{array}{l}\text { FYVE and } \\
\text { coiled-coil } \\
\text { domain } \\
\text { containing } \\
1 \\
\end{array}$ & zinc ion binding \\
\hline R99223 & $\begin{array}{r}0.001391 \\
131 \\
\end{array}$ & & & & \\
\hline $\mathrm{H} 04901$ & $\begin{array}{r}0.001422 \\
235\end{array}$ & $\underline{\mathrm{MKNK1}}$ & 8569 & $\begin{array}{l}\text { MAP } \\
\text { kinase- } \\
\text { interacting } \\
\text { serine/thre } \\
\text { onine } \\
\text { kinase 1 }\end{array}$ & $\begin{array}{l}\text { ATP binding; cAMP-dependent protein } \\
\text { kinase activity; protein amino acid } \\
\text { phosphorylation; protein kinase CK2 } \\
\text { activity; protein kinase cascade; protein } \\
\text { serine/threonine kinase activity; } \\
\text { regulation of protein biosynthesis; } \\
\text { regulation of translation; response to } \\
\text { stress; transferase activity }\end{array}$ \\
\hline R64592 & $\begin{array}{r}0.001428 \\
63 \\
\end{array}$ & & & & \\
\hline H71213 & $\begin{array}{r}0.001430 \\
042 \\
\end{array}$ & $\underline{F 2}$ & $\underline{2147}$ & $\begin{array}{l}\text { coagulatio } \\
\text { n factor II } \\
\text { (thrombin) }\end{array}$ & $\begin{array}{l}\text { STAT protein nuclear translocation; } \\
\text { acute-phase response; apoptosis; } \\
\text { blood coagulation; calcium ion binding; } \\
\text { caspase activation; chymotrypsin } \\
\text { activity; development; extracellular } \\
\text { space; hydrolase activity; proteolysis } \\
\text { and peptidolysis; regulation of cell } \\
\text { cycle; response to wounding; soluble } \\
\text { fraction; thrombin activity; trypsin } \\
\text { activity; tyrosine phosphorylation of } \\
\text { STAT protein }\end{array}$ \\
\hline R98591 & $\begin{array}{r}0.001441 \\
915 \\
\end{array}$ & & & & \\
\hline R11336 & $\begin{array}{r}0.001449 \\
014\end{array}$ & & & & \\
\hline
\end{tabular}




\begin{tabular}{|c|c|c|c|c|c|}
\hline $\mathrm{H} 11235$ & \begin{tabular}{r|}
0.001468 \\
172 \\
\end{tabular} & PEX11A & $\underline{8800}$ & \begin{tabular}{|l|} 
peroxisom \\
al \\
biogenesis \\
factor $11 \mathrm{~A}$
\end{tabular} & $\begin{array}{l}\text { integral to peroxisomal membrane; } \\
\text { peroxisome organization and } \\
\text { biogenesis; signal transduction }\end{array}$ \\
\hline R83560 & $\begin{array}{r}0.001491 \\
671\end{array}$ & & & & \\
\hline R14363 & $\begin{array}{r}0.001500 \\
08\end{array}$ & HDAC5 & $\underline{10014}$ & \begin{tabular}{|l|} 
histone \\
deacetylas \\
e 5
\end{tabular} & $\begin{array}{l}\text { chromatin modeling; chromatin } \\
\text { silencing; cytoplasm; histone } \\
\text { deacetylase activity; nucleus; regulation } \\
\text { of transcription, DNA-dependent }\end{array}$ \\
\hline W79028 & $\begin{array}{r}0.001511 \\
113 \\
\end{array}$ & RPE & $\underline{6120}$ & \begin{tabular}{|l|} 
ribulose-5- \\
phosphate- \\
$3-$ \\
epimerase \\
\end{tabular} & ribulose-phosphate 3-epimerase activity \\
\hline H65832 & $\begin{array}{r}0.001524 \\
908 \\
\end{array}$ & & & & \\
\hline $\mathrm{H} 93200$ & $\begin{array}{r}0.001527 \\
254\end{array}$ & & & & \\
\hline T96118 & $\begin{array}{r}0.001545 \\
223\end{array}$ & & & & \\
\hline W24831 & $\begin{array}{r}0.001553 \\
14\end{array}$ & DPT & $\underline{1805}$ & $\begin{array}{l}\text { dermatopo } \\
\text { ntin }\end{array}$ & $\begin{array}{l}\text { cell adhesion; cell adhesion molecule } \\
\text { activity; extracellular matrix; protein } \\
\text { binding }\end{array}$ \\
\hline R76832 & $\begin{array}{r}0.001558 \\
703\end{array}$ & ATP5J2 & $\underline{9551}$ & \begin{tabular}{|l|} 
ATP \\
synthase, \\
$\mathrm{H}+$ \\
transportin \\
$\mathrm{g}$, \\
mitochondr \\
ial F0 \\
complex, \\
subunit f, \\
isoform 2 \\
\end{tabular} & $\begin{array}{l}\text { ATP biosynthesis; hydrogen ion } \\
\text { transporter activity; hydrogen- } \\
\text { transporting two-sector ATPase activity; } \\
\text { mitochondrion; proton transport }\end{array}$ \\
\hline AA193482 & $\begin{array}{r}0.001568 \\
499 \\
\end{array}$ & FLJ12287 & $\underline{64218}$ & \begin{tabular}{|l|} 
hypothetic \\
al protein \\
FLJ12287 \\
similar to \\
semaphori \\
ns
\end{tabular} & $\begin{array}{l}\text { development; integral to membrane; } \\
\text { neurogenesis; receptor activity }\end{array}$ \\
\hline T53075 & $\begin{array}{r}0.001585 \\
488\end{array}$ & ADCY5 & 111 & $\begin{array}{l}\text { adenylate } \\
\text { cyclase } 5\end{array}$ & $\begin{array}{l}\text { cAMP biosynthesis; } \\
\text { calcium/calmodulin-responsive } \\
\text { adenylate cyclase activity; guanylate } \\
\text { cyclase activity; integral to membrane; } \\
\text { intracellular signaling cascade; lyase } \\
\text { activity; magnesium ion binding }\end{array}$ \\
\hline $\mathrm{H} 70392$ & $\begin{array}{r}0.001614 \\
073 \\
\end{array}$ & DDX52 & 11056 & DEAD (Asp- & -Glu-Ala-Asp) box polypeptide 52 \\
\hline R87198 & $\begin{array}{r}0.001623 \\
29\end{array}$ & TUBB5 & 10382 & $\begin{array}{l}\text { tubulin, } \\
\text { beta, } 5\end{array}$ & $\begin{array}{l}\text { cytoskeleton; structural constituent of } \\
\text { cytoskeleton }\end{array}$ \\
\hline AA046082 & $\begin{array}{r}0.001623 \\
832 \\
\end{array}$ & & & & \\
\hline
\end{tabular}




\begin{tabular}{|c|c|c|c|c|c|}
\hline N70099 & $\begin{array}{r}0.001626 \\
892 \\
\end{array}$ & OSBP2 & 23762 & \begin{tabular}{|l|} 
oxysterol \\
binding \\
protein 2
\end{tabular} & $\begin{array}{l}\text { lipid transport; membrane; steroid } \\
\text { metabolism }\end{array}$ \\
\hline R88980 & $\begin{array}{r}0.001651 \\
243 \\
\end{array}$ & $\frac{\mathrm{LOC} 3482}{62}$ & 348262 & $\begin{array}{l}\text { hypothetic } \\
\text { al protein } \\
\text { LOC34826 } \\
2\end{array}$ & \\
\hline N90792 & $\begin{array}{r}0.001664 \\
459\end{array}$ & & & & \\
\hline R98461 & $\begin{array}{r}0.001667 \\
167\end{array}$ & SMC5L1 & 23137 & \begin{tabular}{|l|} 
SMC5 \\
structural \\
maintenan \\
ce of \\
chromoso \\
mes 5-like \\
1 (yeast) \\
\end{tabular} & $\begin{array}{l}\text { ATP binding; chromosome segregation; } \\
\text { nucleus }\end{array}$ \\
\hline H48488 & $\begin{array}{r}0.001669 \\
186 \\
\end{array}$ & & & & \\
\hline R36523 & $\begin{array}{r}0.001690 \\
459\end{array}$ & NRP2 & $\underline{8828}$ & $\begin{array}{l}\text { neuropilin } \\
2\end{array}$ & $\begin{array}{l}\text { axon guidance; membrane fraction; } \\
\text { receptor activity; vascular endothelial } \\
\text { growth factor receptor activity }\end{array}$ \\
\hline W48584 & $\begin{array}{r}0.001721 \\
581 \\
\end{array}$ & P4HA2 & 8974 & \begin{tabular}{|l|} 
procollage \\
n-proline, \\
$2-$ \\
oxoglutarat \\
e 4- \\
dioxygenas \\
e (proline \\
4 - \\
hydroxylas \\
e), alpha \\
polypeptid \\
e II \\
\end{tabular} & $\begin{array}{l}\text { electron transporter activity; } \\
\text { endoplasmic reticulum; oxidoreductase } \\
\text { activity; oxidoreductase activity, acting } \\
\text { on paired donors, with incorporation or } \\
\text { reduction of molecular oxygen, 2- } \\
\text { oxoglutarate as one donor, and } \\
\text { incorporation of one atom each of } \\
\text { oxygen into both donors; } \\
\text { oxidoreductase activity, acting on single } \\
\text { donors with incorporation of molecular } \\
\text { oxygen, incorporation of two atoms of } \\
\text { oxygen; procollagen-proline 4- } \\
\text { dioxygenase activity; protein } \\
\text { metabolism }\end{array}$ \\
\hline W76572 & $\begin{array}{r}0.001725 \\
886\end{array}$ & NUDT1 & $\underline{4521}$ & \begin{tabular}{|l|} 
nudix \\
(nucleosid \\
e \\
diphosphat \\
e linked \\
moiety X)- \\
type motif \\
1 \\
\end{tabular} & $\begin{array}{l}\text { 8-oxo-7,8-dihydroguanine } \\
\text { triphosphatase activity; DNA repair; } \\
\text { GTPase activity; hydrolase activity; } \\
\text { response to oxidative stress }\end{array}$ \\
\hline T79650 & $\begin{array}{r}0.001781 \\
352 \\
\end{array}$ & $\underline{\text { ARVCF }}$ & 421 & \begin{tabular}{|l|} 
armadillo \\
repeat \\
gene \\
deletes in \\
velocardiof \\
acial \\
syndrome \\
\end{tabular} & $\begin{array}{l}\text { cell adhesion; cell adhesion molecule } \\
\text { activity; cytoskeleton; development; } \\
\text { intracellular; kinesin complex; structural } \\
\text { molecule activity }\end{array}$ \\
\hline AA114945 & \begin{tabular}{|r|}
0.001792 \\
828 \\
\end{tabular} & & & & \\
\hline T99576 & 0.001796 & & & & \\
\hline
\end{tabular}




\begin{tabular}{|c|c|c|c|c|c|}
\hline & 991 & & & & \\
\hline T96928 & $\begin{array}{r}0.001799 \\
53 \\
\end{array}$ & & & & \\
\hline T89571 & $\begin{array}{r}0.001813 \\
156 \\
\end{array}$ & & & & \\
\hline N30288 & $\begin{array}{r}0.001814 \\
543\end{array}$ & PFDN2 & $\underline{5202}$ & prefoldin 2 & $\begin{array}{l}\text { chaperone activity; prefoldin complex; } \\
\text { protein folding }\end{array}$ \\
\hline AA128562 & $\begin{array}{r}0.001816 \\
196\end{array}$ & & & & \\
\hline R73050 & $\begin{array}{r}0.001833 \\
621 \\
\end{array}$ & CNTFR & 1271 & $\begin{array}{l}\text { ciliary } \\
\text { neurotrophi } \\
\text { c factor } \\
\text { receptor }\end{array}$ & $\begin{array}{l}\text { GPI-anchored membrane-bound } \\
\text { receptor; ciliary neurotrophic factor } \\
\text { receptor activity; membrane; } \\
\text { neurogenesis; receptor activity; signal } \\
\text { transduction }\end{array}$ \\
\hline H69787 & $\begin{array}{r}0.001837 \\
022 \\
\end{array}$ & & & & \\
\hline N25523 & $\begin{array}{r}0.001869 \\
222 \\
\end{array}$ & HSPE1 & $\underline{3336}$ & $\begin{array}{l}\text { heat shock } \\
10 \mathrm{kDa} \\
\text { protein } 1 \\
\text { (chaperoni } \\
\mathrm{n} 10)\end{array}$ & $\begin{array}{l}\text { co-chaperonin activity; heat shock } \\
\text { protein activity; mitochondrion; protein } \\
\text { folding }\end{array}$ \\
\hline R86231 & \begin{tabular}{|r|}
0.001881 \\
771 \\
\end{tabular} & PC326 & $\underline{55827}$ & $\begin{array}{l}\text { PC326 } \\
\text { protein }\end{array}$ & \\
\hline N68871 & $\begin{array}{r}0.001908 \\
816 \\
\end{array}$ & & & & \\
\hline R49189 & $\begin{array}{r}0.001912 \\
823 \\
\end{array}$ & SLC30A6 & $\underline{55676}$ & \multicolumn{2}{|c|}{ solute carrier family 30 (zinc transporter), member 6} \\
\hline $\mathrm{H} 26200$ & $\begin{array}{r}0.001942 \\
936\end{array}$ & & $\underline{349268}$ & $\begin{array}{l}\text { similar to } \\
\text { hypothetic } \\
\text { al protein } \\
\text { LOC28628 } \\
6\end{array}$ & \\
\hline R26108 & $\begin{array}{r}0.001950 \\
87 \\
\end{array}$ & & & & \\
\hline R14326 & $\begin{array}{r}0.002010 \\
252 \\
\end{array}$ & HERC1 & $\underline{8925}$ & $\begin{array}{l}\text { hect } \\
\text { (homologo } \\
\text { us to the } \\
\text { E6-AP } \\
\text { (UBE3A) } \\
\text { carboxyl } \\
\text { terminus) } \\
\text { domain } \\
\text { and RCC1 } \\
\text { (CHC1)- } \\
\text { like domain } \\
\text { (RLD) } 1 \\
\end{array}$ & $\begin{array}{l}\text { ARF guanyl-nucleotide exchange factor } \\
\text { activity; Golgi apparatus; catalytic } \\
\text { activity; nonselective vesicle transport; } \\
\text { ubiquitin cycle; ubiquitin-protein ligase } \\
\text { activity }\end{array}$ \\
\hline AA034144 & $\begin{array}{r}0.002010 \\
937\end{array}$ & $\underline{\text { CD36 }}$ & $\underline{948}$ & \begin{tabular}{|l|} 
CD36 \\
antigen \\
(collagen \\
type I \\
receptor, \\
thrombosp
\end{tabular} & $\begin{array}{l}\text { blood coagulation; cell adhesion; cell } \\
\text { adhesion molecule activity; fatty acid } \\
\text { metabolism; integral to plasma } \\
\text { membrane; membrane fraction; } \\
\text { receptor activity; transport }\end{array}$ \\
\hline
\end{tabular}




\begin{tabular}{|c|c|c|c|c|c|}
\hline & & & & $\begin{array}{l}\text { ondin } \\
\text { receptor) }\end{array}$ & \\
\hline R88895 & $\begin{array}{r}0.002028 \\
496 \\
\end{array}$ & MANBAL & $\underline{63905}$ & $\begin{array}{l}\text { mannosida } \\
\text { se, beta } A, \\
\text { lysosomal- } \\
\text { like }\end{array}$ & integral to membrane \\
\hline H50436 & $\begin{array}{r}0.002063 \\
585 \\
\end{array}$ & ALDH6A1 & 4329 & $\begin{array}{l}\text { aldehyde } \\
\text { dehydroge } \\
\text { nase } 6 \\
\text { family, } \\
\text { member } \\
\text { A1 }\end{array}$ & $\begin{array}{l}\text { metabolism; methylmalonate- } \\
\text { semialdehyde dehydrogenase } \\
\text { (acylating) activity; mitochondrion; } \\
\text { oxidoreductase activity; pyrimidine } \\
\text { nucleotide metabolism; valine } \\
\text { metabolism }\end{array}$ \\
\hline T48772 & $\begin{array}{r}0.002074 \\
625 \\
\end{array}$ & RPL12 & $\underline{6136}$ & $\begin{array}{l}\text { ribosomal } \\
\text { protein L12 }\end{array}$ & $\begin{array}{l}\text { RNA binding; cytosolic large ribosomal } \\
\text { subunit (sensu Eukarya); intracellular; } \\
\text { protein biosynthesis; ribosome; } \\
\text { structural constituent of ribosome }\end{array}$ \\
\hline AA204653 & $\begin{array}{r}0.002107 \\
156 \\
\end{array}$ & & & & \\
\hline H59454 & \begin{tabular}{|r|}
0.002111 \\
161 \\
\end{tabular} & & & & \\
\hline T64848 & $\begin{array}{r}0.002141 \\
857 \\
\end{array}$ & PER3 & $\underline{8863}$ & $\begin{array}{l}\text { period } \\
\text { homolog } 3 \\
\text { (Drosophil } \\
\text { a) } \\
\end{array}$ & $\begin{array}{l}\text { nucleus; regulation of transcription, } \\
\text { DNA-dependent; rhythmic behavior; } \\
\text { signal transducer activity; signal } \\
\text { transduction }\end{array}$ \\
\hline H65366 & $\begin{array}{r}0.002145 \\
291\end{array}$ & & & & \\
\hline H80680 & \begin{tabular}{|r|}
0.002172 \\
772 \\
\end{tabular} & & & & \\
\hline R48615 & $\begin{array}{r}0.002214 \\
369\end{array}$ & C14orf21 & $\underline{161424}$ & $\begin{array}{l}\text { chromoso } \\
\text { me } 14 \\
\text { open } \\
\text { reading } \\
\text { frame } 21\end{array}$ & RNA binding \\
\hline H28534 & $\begin{array}{r}0.002243 \\
158 \\
\end{array}$ & AQP1 & $\underline{358}$ & $\begin{array}{l}\text { aquaporin } \\
1 \text { (channel- } \\
\text { forming } \\
\text { integral } \\
\text { protein, } \\
28 \mathrm{kDa} \text { ) }\end{array}$ & $\begin{array}{l}\text { excretion; integral to plasma } \\
\text { membrane; transport; water transport; } \\
\text { water transporter activity }\end{array}$ \\
\hline H83025 & \begin{tabular}{|r|}
0.002258 \\
751 \\
\end{tabular} & & & & \\
\hline R43017 & \begin{tabular}{|r|}
0.002265 \\
502 \\
\end{tabular} & & & & \\
\hline W47347 & \begin{tabular}{|r|}
0.002302 \\
25 \\
\end{tabular} & ABCF3 & $\underline{55324}$ & \multicolumn{2}{|c|}{$\begin{array}{l}\text { ATP-binding cassette, sub-family F (GCN20), } \\
\text { member } 3\end{array}$} \\
\hline
\end{tabular}




\begin{tabular}{|c|c|c|c|c|c|}
\hline N34930 & \begin{tabular}{|r|}
0.002336 \\
485 \\
\end{tabular} & DLL3 & $\underline{10683}$ & $\begin{array}{l}\text { delta-like } 3 \\
\text { (Drosophil } \\
\text { a) }\end{array}$ & $\begin{array}{l}\text { N signaling pathway; Notch binding; } \\
\text { calcium ion binding; cell differentiation; } \\
\text { cell fate determination; embryonic } \\
\text { development (sensu Mammalia); } \\
\text { integral to membrane; neurogenesis; } \\
\text { skeletal development }\end{array}$ \\
\hline $\mathrm{H} 14143$ & $\begin{array}{r}0.002349 \\
457\end{array}$ & ATP1A3 & $\frac{478}{4}$ & \begin{tabular}{|l|} 
ATPase, \\
Na+/K+ \\
transportin \\
g, alpha 3 \\
polypeptid \\
e
\end{tabular} & $\begin{array}{l}\text { ATP binding; hydrolase activity; integral } \\
\text { to membrane; magnesium ion binding; } \\
\text { metabolism; monovalent inorganic } \\
\text { cation transporter activity; potassium } \\
\text { ion transport; sodium ion transport; } \\
\text { sodium/potassium-exchanging ATPase } \\
\text { activity; sodium/potassium-exchanging } \\
\text { ATPase complex; transport }\end{array}$ \\
\hline AA028961 & $\begin{array}{r}0.002366 \\
334\end{array}$ & & & & \\
\hline R66188 & $\begin{array}{r}0.002371 \\
586\end{array}$ & & & & \\
\hline$H 21568$ & $\begin{array}{r}0.002379 \\
889\end{array}$ & CNNM3 & 26505 & cyclin M3 & \\
\hline N64492 & $\begin{array}{r}0.002388 \\
716 \\
\end{array}$ & & & & \\
\hline H63711 & $\begin{array}{r}0.002410 \\
236\end{array}$ & & & & \\
\hline R09962 & \begin{tabular}{|r|}
0.002421 \\
401 \\
\end{tabular} & PMS2L6 & $\underline{5384}$ & $\begin{array}{l}\text { postmeiotic } \\
\text { segregatio } \\
\text { n } \\
\text { increased } \\
\text { 2-like } 6 \\
\end{array}$ & $\begin{array}{l}\text { damaged DNA binding; mismatch } \\
\text { repair; nucleus }\end{array}$ \\
\hline R43540 & \begin{tabular}{|r|}
0.002429 \\
377
\end{tabular} & & & & \\
\hline AA004343 & \begin{tabular}{|r|}
0.002454 \\
559 \\
\end{tabular} & & & & \\
\hline W32324 & \begin{tabular}{|r|}
0.002505 \\
773
\end{tabular} & GPX1 & $\underline{2876}$ & $\begin{array}{l}\text { glutathione } \\
\text { peroxidase } \\
1\end{array}$ & $\begin{array}{l}\text { glutathione peroxidase activity; } \\
\text { oxidoreductase activity; response to } \\
\text { oxidative stress }\end{array}$ \\
\hline H38541 & \begin{tabular}{|r|}
0.002515 \\
044
\end{tabular} & CNOT3 & $\underline{4849}$ & \begin{tabular}{|l|} 
CCR4- \\
NOT \\
transcriptio \\
n complex \\
subunit 3 \\
\end{tabular} & \\
\hline W96066 & \begin{tabular}{|r|}
0.002515 \\
087
\end{tabular} & ACTC & $\frac{70}{7}$ & \begin{tabular}{|l} 
actin, \\
alpha, \\
cardiac \\
muscle
\end{tabular} & $\begin{array}{l}\text { actin filament; motor activity; muscle } \\
\text { contraction; muscle development; } \\
\text { regulation of heart rate; structural } \\
\text { constituent of cytoskeleton; structural } \\
\text { constituent of muscle }\end{array}$ \\
\hline N/A2 & $\begin{array}{r}0.002532 \\
621 \\
\end{array}$ & & & & \\
\hline R31317 & \begin{tabular}{|r|}
0.002555 \\
1 \\
\end{tabular} & & & & \\
\hline T79362 & 0.002635 & & & & \\
\hline
\end{tabular}




\begin{tabular}{|c|c|c|c|c|c|}
\hline & 534 & & & & \\
\hline T87194 & $\begin{array}{r}0.002640 \\
554\end{array}$ & & & & \\
\hline $\mathrm{H} 46579$ & $\begin{array}{r}0.002641 \\
187\end{array}$ & $\frac{\mathrm{LOC} 2846}{11}$ & $\underline{284611}$ & $\begin{array}{l}\text { hypothetic } \\
\text { al protein } \\
\text { LOC28461 } \\
1\end{array}$ & \\
\hline W57989 & $\begin{array}{r}0.002661 \\
555\end{array}$ & TRIM2 & 23321 & \begin{tabular}{|l|} 
tripartite \\
motif- \\
containing \\
2
\end{tabular} & $\begin{array}{l}\text { biological_process unknown; } \\
\text { cytoplasm; myosin binding; zinc ion } \\
\text { binding }\end{array}$ \\
\hline H46133 & $\begin{array}{r}0.002673 \\
406\end{array}$ & BAl2 & $\underline{576}$ & \begin{tabular}{|l|} 
brain- \\
specific \\
angiogene \\
sis inhibitor \\
2
\end{tabular} & $\begin{array}{l}\text { G-protein coupled receptor activity; } \\
\text { integral to membrane; neuropeptide } \\
\text { signaling pathway }\end{array}$ \\
\hline $\mathrm{H} 43625$ & $\begin{array}{r}0.002710 \\
629\end{array}$ & $\frac{\mathrm{LOC} 2200}{74}$ & 220074 & $\begin{array}{l}\text { Hypothetica } \\
\text { chromosom }\end{array}$ & $\begin{array}{l}\text { III } 55.1 \mathrm{kDa} \text { protein F09G8.5 in } \\
\text { e III }\end{array}$ \\
\hline N63546 & $\begin{array}{r}0.002712 \\
26\end{array}$ & & & & \\
\hline H39920 & $\begin{array}{r}0.002718 \\
032\end{array}$ & & & & \\
\hline H89087 & $\begin{array}{r}0.002721 \\
451\end{array}$ & RNPS1 & 10921 & \begin{tabular}{|l|} 
RNA \\
binding \\
protein S1, \\
serine-rich \\
domain
\end{tabular} & $\begin{array}{l}\text { RNA binding; RNA splicing; nucleus; } \\
\text { transcription }\end{array}$ \\
\hline N39391 & $\begin{array}{r}0.002748 \\
964\end{array}$ & $\underline{\mathrm{MGC1479}}$ & 84296 & $\begin{array}{l}\text { hypothetic } \\
\text { al protein } \\
\text { MGC1479 } \\
9\end{array}$ & \\
\hline W79479 & $\begin{array}{r}0.002751 \\
486\end{array}$ & AP2M1 & 1173 & \begin{tabular}{|l|} 
adaptor- \\
related \\
protein \\
complex 2, \\
mu 1 \\
subunit \\
\end{tabular} & $\begin{array}{l}\text { clathrin vesicle coat; coated pit; } \\
\text { intracellular protein transport; } \\
\text { nonselective vesicle transport; } \\
\text { secretory vesicle; transporter activity }\end{array}$ \\
\hline H47114 & $\begin{array}{r}0.002765 \\
108\end{array}$ & & & & \\
\hline N74558 & $\begin{array}{r}0.002774 \\
032\end{array}$ & & & & \\
\hline N62283 & $\begin{array}{r}0.002775 \\
021\end{array}$ & TRAM1 & 23471 & $\begin{array}{l}\text { translocati } \\
\text { on } \\
\text { associated } \\
\text { membrane } \\
\text { protein 1 }\end{array}$ & $\begin{array}{l}\text { cotranslational membrane targeting; } \\
\text { endoplasmic reticulum; endoplasmic } \\
\text { reticulum receptor activity; integral to } \\
\text { membrane; protein targeting }\end{array}$ \\
\hline R45627 & $\begin{array}{r}0.002782 \\
889\end{array}$ & & & & \\
\hline R18705 & $\begin{array}{r}0.002796 \\
542\end{array}$ & $\frac{D K F Z p 76}{1 C 169}$ & $\underline{65056}$ & vasculin & \\
\hline
\end{tabular}




\begin{tabular}{|c|c|c|c|c|c|}
\hline R47859 & \begin{tabular}{|r|}
0.002825 \\
359 \\
\end{tabular} & NPR1 & $\underline{4881}$ & $\begin{array}{l}\text { natriuretic } \\
\text { peptide } \\
\text { receptor } \\
\text { A/guanylat } \\
\text { e cyclase } \\
\text { A } \\
\text { (atrionatriu } \\
\text { retic } \\
\text { peptide } \\
\text { receptor A) }\end{array}$ & $\begin{array}{l}\text { ATP binding; cGMP biosynthesis; } \\
\text { guanylate cyclase activity; integral to } \\
\text { membrane; intracellular signaling } \\
\text { cascade; lyase activity; peptide } \\
\text { receptor activity, G-protein coupled; } \\
\text { protein amino acid phosphorylation; } \\
\text { protein kinase activity; receptor activity; } \\
\text { receptor guanylate cyclase activity; } \\
\text { regulation of blood pressure }\end{array}$ \\
\hline AA125889 & \begin{tabular}{|r|}
0.002828 \\
64 \\
\end{tabular} & PTMS & $\underline{5763}$ & $\begin{array}{l}\text { parathymo } \\
\text { sin }\end{array}$ & $\begin{array}{l}\text { DNA replication; cellular defense } \\
\text { response; development; nucleus; } \\
\text { regulation of cell cycle }\end{array}$ \\
\hline N64488 & \begin{tabular}{|r|}
0.002852 \\
346
\end{tabular} & PHTF1 & $\underline{10745}$ & \begin{tabular}{|l|} 
putative \\
homeodom \\
ain \\
transcriptio \\
n factor 1 \\
\end{tabular} & $\begin{array}{l}\text { nucleus; regulation of transcription, } \\
\text { DNA-dependent; transcription factor } \\
\text { activity }\end{array}$ \\
\hline T91335 & $\begin{array}{r}0.002881 \\
994\end{array}$ & LUC7L & $\underline{55692}$ & $\begin{array}{l}\text { LUC7-like } \\
\text { (S. } \\
\text { cerevisiae) }\end{array}$ & \\
\hline R83014 & \begin{tabular}{|r|}
0.002892 \\
845 \\
\end{tabular} & & & & \\
\hline H84604 & \begin{tabular}{|r|}
0.002928 \\
862 \\
\end{tabular} & $\begin{array}{l}\frac{\text { SLC21A1 }}{2} \\
\underline{2}\end{array}$ & 28231 & \begin{tabular}{|l|} 
solute \\
carrier \\
family 21 \\
(organic \\
anion \\
transporter \\
), member \\
12 \\
\end{tabular} & $\begin{array}{l}\text { integral to membrane; ion transport; } \\
\text { transporter activity }\end{array}$ \\
\hline AA115064 & $\begin{array}{r}0.002933 \\
016 \\
\end{array}$ & & & & \\
\hline H59405 & \begin{tabular}{|r|}
0.002940 \\
249
\end{tabular} & FLJ10298 & $\underline{54682}$ & \begin{tabular}{|l|} 
hypothetic \\
al protein \\
FLJ10298 \\
\end{tabular} & \\
\hline H61036 & $\begin{array}{r}0.002943 \\
42\end{array}$ & & & & \\
\hline AA034109 & $\begin{array}{r}0.003019 \\
47\end{array}$ & MINK & $\underline{50488}$ & $\begin{array}{l}\text { misshapen } \\
\text { /NIK- } \\
\text { related } \\
\text { kinase }\end{array}$ & $\begin{array}{l}\text { ATP binding; cAMP-dependent protein } \\
\text { kinase activity; development; protein } \\
\text { amino acid phosphorylation; protein } \\
\text { kinase CK2 activity; protein kinase } \\
\text { cascade; protein serine/threonine } \\
\text { kinase activity; response to stress; } \\
\text { small GTPase regulatory/interacting } \\
\text { protein activity; transferase activity }\end{array}$ \\
\hline \begin{tabular}{|l|} 
N74414 \\
\end{tabular} & $\begin{array}{r}0.003068 \\
48 \\
\end{array}$ & $\begin{array}{l}\text { SDCCAG } \\
\underline{3}\end{array}$ & 10807 & \begin{tabular}{|l|} 
serologicall \\
y defined \\
colon \\
cancer \\
antigen 3
\end{tabular} & tumor antigen \\
\hline W32375 & 0.003103 & & & & \\
\hline
\end{tabular}




\begin{tabular}{|c|c|c|c|c|c|}
\hline & 289 & & & & \\
\hline T87509 & $\begin{array}{r}0.003115 \\
178\end{array}$ & & & & \\
\hline N42162 & $\begin{array}{r}0.003128 \\
355 \\
\end{array}$ & DC2 & 58505 & $\begin{array}{l}\text { DC2 } \\
\text { protein }\end{array}$ & \\
\hline N72164 & $\begin{array}{r}0.003137 \\
146 \\
\end{array}$ & & & & \\
\hline $\mathrm{H} 10026$ & $\begin{array}{r}0.003145 \\
39 \\
\end{array}$ & ZNF193 & $\underline{7746}$ & $\begin{array}{l}\text { zinc finger } \\
\text { protein } 193\end{array}$ & $\begin{array}{l}\text { nucleus; protein binding; regulation of } \\
\text { transcription, DNA-dependent; } \\
\text { transcription factor activity }\end{array}$ \\
\hline T97703 & $\begin{array}{r}0.003156 \\
48\end{array}$ & & & & \\
\hline AA031950 & $\begin{array}{r}0.003182 \\
394\end{array}$ & & & & \\
\hline T92612 & \begin{tabular}{|r|}
0.003193 \\
663 \\
\end{tabular} & CEPT1 & 10390 & $\begin{array}{l}\text { choline/eth } \\
\text { anolamine } \\
\text { phosphotra } \\
\text { nsferase }\end{array}$ & $\begin{array}{l}\text { ethanolaminephosphotransferase } \\
\text { activity; integral to membrane; lipid } \\
\text { metabolism; phospholipid biosynthesis; } \\
\text { transferase activity }\end{array}$ \\
\hline T52361 & $\begin{array}{r}0.003197 \\
303 \\
\end{array}$ & $\frac{\text { DKFZP43 }}{4 \mathrm{P} 1750}$ & $\underline{26000}$ & \begin{tabular}{|l|} 
DKFZP434 \\
P1750 \\
protein
\end{tabular} & catalytic activity; metabolism \\
\hline T70299 & $\begin{array}{r}0.003226 \\
285\end{array}$ & & & & \\
\hline T86284 & $\begin{array}{r}0.003273 \\
129 \\
\end{array}$ & & & & \\
\hline $\mathrm{H} 45174$ & \begin{tabular}{|r|}
0.003288 \\
345
\end{tabular} & CLMN & $\underline{79789}$ & $\begin{array}{l}\text { calmin } \\
\text { (calponin- } \\
\text { like, } \\
\text { transmemb } \\
\text { rane) }\end{array}$ & \\
\hline R19599 & $\begin{array}{r}0.003351 \\
854\end{array}$ & & & & \\
\hline R39421 & $\begin{array}{r}0.003373 \\
338 \\
\end{array}$ & PIGM & $\underline{93183}$ & \begin{tabular}{|l|} 
phosphatid \\
ylinositol \\
glycan, \\
class M
\end{tabular} & transferase activity \\
\hline R89424 & $\begin{array}{r}0.003383 \\
03\end{array}$ & & & & \\
\hline AA028907 & \begin{tabular}{|r|}
0.003403 \\
016 \\
\end{tabular} & & 153603 & $\begin{array}{l}\text { LOC15360 } \\
3\end{array}$ & \\
\hline R07810 & $\begin{array}{r}0.003407 \\
136\end{array}$ & & & & \\
\hline T84788 & $\begin{array}{r}0.003432 \\
787 \\
\end{array}$ & & & & \\
\hline R82834 & $\begin{array}{r}0.003457 \\
38\end{array}$ & & & & \\
\hline H56035 & \begin{tabular}{|r|}
0.003463 \\
096
\end{tabular} & FLJ31842 & 148534 & $\begin{array}{l}\text { hypothetic } \\
\text { al protein } \\
\text { FLJ31842 }\end{array}$ & \\
\hline H20520 & $\begin{array}{r}0.003478 \\
042 \\
\end{array}$ & & & & \\
\hline AA210905 & 0.003491 & & & & \\
\hline
\end{tabular}




\begin{tabular}{|c|c|c|c|c|c|}
\hline & 026 & & & & \\
\hline R94248 & $\begin{array}{r}0.003493 \\
034\end{array}$ & & & & \\
\hline $\mathrm{H} 62020$ & $\begin{array}{r}0.003494 \\
706 \\
\end{array}$ & & & & \\
\hline N93517 & $\begin{array}{r}0.003504 \\
284 \\
\end{array}$ & & & & \\
\hline T77987 & $\begin{array}{r}0.003518 \\
125\end{array}$ & & $\underline{345466}$ & \multicolumn{2}{|c|}{ similar to 6-pyruvoyl-tetrahydropterin synthase } \\
\hline N42817 & $\begin{array}{r}0.003546 \\
932\end{array}$ & COX6C & $\underline{1345}$ & $\begin{array}{l}\text { cytochrom } \\
\text { e c oxidase } \\
\text { subunit VIc }\end{array}$ & $\begin{array}{l}\text { aa3-type cytochrome c oxidase; ba3- } \\
\text { type cytochrome c oxidase; caa3-type } \\
\text { cytochrome c oxidase; cbb3-type } \\
\text { cytochrome c oxidase; cytochrome-c } \\
\text { oxidase activity; electron transport; } \\
\text { energy pathways; inner membrane; } \\
\text { mitochondrion; oxidoreductase activity }\end{array}$ \\
\hline AA047157 & $\begin{array}{r}0.003561 \\
795\end{array}$ & KAl1 & 3732 & \begin{tabular}{|l|} 
kangai 1 \\
(suppressi \\
on of \\
tumorigeni \\
city 6, \\
prostate; \\
CD82 \\
antigen \\
(R2 \\
leukocyte \\
antigen, \\
antigen \\
detected \\
by \\
monoclona \\
I and \\
antibody \\
IA4))
\end{tabular} & integral to plasma membrane \\
\hline AA125808 & $\begin{array}{r}0.003584 \\
725 \\
\end{array}$ & CAPS & $\underline{828}$ & \begin{tabular}{|l|} 
calcyphosi \\
ne
\end{tabular} & $\begin{array}{l}\text { calcium ion binding; intracellular } \\
\text { signaling cascade }\end{array}$ \\
\hline $\mathrm{H} 40811$ & $\begin{array}{r}0.003658 \\
811\end{array}$ & & & & \\
\hline AA045369 & $\begin{array}{r}0.003691 \\
143 \\
\end{array}$ & & & & \\
\hline $\mathrm{H} 22064$ & $\begin{array}{r}0.003703 \\
929 \\
\end{array}$ & PHF12 & $\underline{57649}$ & $\begin{array}{l}\text { PHD finger } \\
\text { protein } 12\end{array}$ & $\begin{array}{l}\text { DNA binding; metabolism; } \\
\text { oxidoreductase activity; regulation of } \\
\text { transcription, DNA-dependent }\end{array}$ \\
\hline R17223 & $\begin{array}{r}0.003712 \\
105 \\
\end{array}$ & & & & \\
\hline $\mathrm{H} 77938$ & $\begin{array}{r}0.003713 \\
497\end{array}$ & ETF1 & $\underline{2107}$ & \begin{tabular}{|l|} 
eukaryotic \\
translation \\
termination \\
factor 1 \\
\end{tabular} & $\begin{array}{l}\text { RNA binding; cytoplasm; regulation of } \\
\text { translational termination; translation } \\
\text { release factor activity, codon specific }\end{array}$ \\
\hline $\mathrm{H} 69440$ & $\begin{array}{r}0.003723 \\
647 \\
\end{array}$ & ANKRD13 & 88455 & \begin{tabular}{|l|} 
ankyrin \\
repeat \\
domain 13
\end{tabular} & \\
\hline
\end{tabular}




\begin{tabular}{|c|c|c|c|c|c|}
\hline $\mathrm{H78795}$ & \begin{tabular}{|r|}
0.003736 \\
927
\end{tabular} & HAND2 & $\underline{9464}$ & \begin{tabular}{|l|} 
heart and \\
neural \\
crest \\
derivatives \\
expressed \\
2
\end{tabular} & $\begin{array}{l}\text { angiogenesis; development; heart } \\
\text { development; nucleus; regulation of } \\
\text { transcription, DNA-dependent; } \\
\text { transcription factor activity; transcription } \\
\text { from Pol II promoter }\end{array}$ \\
\hline N63947 & \begin{tabular}{|r|}
0.003746 \\
113 \\
\end{tabular} & FLJ21940 & $\underline{64848}$ & \begin{tabular}{|l|} 
hypothetic \\
al protein \\
FLJ21940
\end{tabular} & \\
\hline H08918 & $\begin{array}{r}0.003746 \\
482 \\
\end{array}$ & LMLN & 89782 & \multicolumn{2}{|c|}{ leishmanolysin-like (metallopeptidase M8 family) } \\
\hline R48041 & \begin{tabular}{|r|}
0.003759 \\
537
\end{tabular} & GAA & $\underline{2548}$ & \begin{tabular}{|l|} 
glucosidas \\
e, alpha; \\
acid \\
(Pompe \\
disease, \\
glycogen \\
storage \\
disease \\
type II)
\end{tabular} & $\begin{array}{l}\text { alpha-glucosidase activity; } \\
\text { carbohydrate metabolism; energy } \\
\text { pathways; glycogen catabolism; } \\
\text { hydrolase activity, hydrolyzing O- } \\
\text { glycosyl compounds; lysosome }\end{array}$ \\
\hline AA121519 & \begin{tabular}{|r|}
0.003763 \\
832 \\
\end{tabular} & PCSK7 & $\underline{9159}$ & $\begin{array}{l}\text { proprotein } \\
\text { convertase } \\
\text { subtilisin } / \mathrm{k} \\
\text { exin type } 7\end{array}$ & $\begin{array}{l}\text { integral to Golgi membrane; peptidase } \\
\text { activity; peptide hormone processing; } \\
\text { proteolysis and peptidolysis; subtilase } \\
\text { activity }\end{array}$ \\
\hline R30807 & $\begin{array}{r}0.003804 \\
148\end{array}$ & & & & \\
\hline R97635 & \begin{tabular}{|r|}
0.003807 \\
487 \\
\end{tabular} & & & & \\
\hline W33113 & \begin{tabular}{|r|}
0.003866 \\
57 \\
\end{tabular} & & & & \\
\hline H69011 & \begin{tabular}{|r|}
0.003868 \\
629
\end{tabular} & SKIL & $\underline{6498}$ & SKI-like & $\begin{array}{l}\text { cell differentiation; cell growth and/or } \\
\text { maintenance; molecular_function } \\
\text { unknown; nucleus }\end{array}$ \\
\hline T79962 & $\begin{array}{r}0.003893 \\
833\end{array}$ & & & & \\
\hline $\mathrm{H} 45972$ & $\begin{array}{r}0.003905 \\
578 \\
\end{array}$ & & & & \\
\hline R90824 & \begin{tabular}{|r|}
0.003908 \\
283
\end{tabular} & TMEM10 & 93377 & \begin{tabular}{|l|} 
transmemb \\
rane \\
protein 10
\end{tabular} & integral to membrane \\
\hline H99439 & $\begin{array}{r}0.003915 \\
936\end{array}$ & & & & \\
\hline AA210785 & $\begin{array}{r}0.003917 \\
258 \\
\end{array}$ & & & & \\
\hline
\end{tabular}




\begin{tabular}{|c|c|c|c|c|c|}
\hline W47101 & $\begin{array}{r}0.003926 \\
794 \\
\end{array}$ & $\underline{\mathrm{IL} 1 \mathrm{~B}}$ & 3553 & $\begin{array}{l}\text { interleukin } \\
1 \text {, beta }\end{array}$ & $\begin{array}{l}\text { antimicrobial humoral response (sensu } \\
\text { Invertebrata); apoptosis; cell } \\
\text { proliferation; cell-cell signaling; } \\
\text { extracellular space; immune response; } \\
\text { inflammatory response; interleukin-1 } \\
\text { receptor antagonist activity; negative } \\
\text { regulation of cell proliferation; } \\
\text { regulation of cell cycle; signal } \\
\text { transducer activity; signal transduction }\end{array}$ \\
\hline H53118 & \begin{tabular}{|r|}
0.003979 \\
598 \\
\end{tabular} & & & & \\
\hline R70072 & \begin{tabular}{|r|}
0.003987 \\
828
\end{tabular} & ELN & 2006 & $\begin{array}{l}\text { elastin } \\
\text { (supravalv } \\
\text { ular aortic } \\
\text { stenosis, } \\
\text { Williams- } \\
\text { Beuren } \\
\text { syndrome) } \\
\end{array}$ & $\begin{array}{l}\text { cell proliferation; cell shape and cell } \\
\text { size control; circulation; extracellular } \\
\text { matrix; extracellular matrix structural } \\
\text { constituent; extracellular space; } \\
\text { histogenesis and organogenesis; } \\
\text { respiratory gaseous exchange }\end{array}$ \\
\hline R24502 & $\begin{array}{r}0.004002 \\
844\end{array}$ & ADSSL1 & 122622 & $\begin{array}{l}\text { adenylosuc } \\
\text { cinate } \\
\text { synthase } \\
\text { like } 1\end{array}$ & $\begin{array}{l}\text { GTP binding; adenylosuccinate } \\
\text { synthase activity; ligase activity; purine } \\
\text { nucleotide biosynthesis }\end{array}$ \\
\hline H69845 & $\begin{array}{r}0.004013 \\
994 \\
\end{array}$ & & & & \\
\hline H67054 & $\begin{array}{r}0.004041 \\
873\end{array}$ & $\underline{\text { OLR1 }}$ & 4973 & $\begin{array}{l}\text { oxidised } \\
\text { low density } \\
\text { lipoprotein } \\
\text { (lectin-like) } \\
\text { receptor } 1 \\
\end{array}$ & $\begin{array}{l}\text { circulation; heterophilic cell adhesion; } \\
\text { integral to plasma membrane; } \\
\text { membrane fraction; proteolysis and } \\
\text { peptidolysis; receptor activity; sugar } \\
\text { binding }\end{array}$ \\
\hline W15268 & $\begin{array}{r}0.004087 \\
506\end{array}$ & ARHA & $\underline{387}$ & $\begin{array}{l}\text { ras } \\
\text { homolog } \\
\text { gene } \\
\text { family, } \\
\text { member A }\end{array}$ & $\begin{array}{l}\text { GTP binding; Rho protein signal } \\
\text { transduction; Rho small monomeric } \\
\text { GTPase activity; actin cytoskeleton } \\
\text { organization and biogenesis; cell } \\
\text { growth and/or maintenance; } \\
\text { cytoskeleton }\end{array}$ \\
\hline AA025089 & $\begin{array}{r}0.004101 \\
469 \\
\end{array}$ & VDAC1 & $\underline{7416}$ & $\begin{array}{l}\text { voltage- } \\
\text { dependent } \\
\text { anion } \\
\text { channel } 1\end{array}$ & $\begin{array}{l}\text { anion transport; apoptogenic } \\
\text { cytochrome c release channel activity; } \\
\text { apoptotic program; integral to } \\
\text { membrane; mitochondrial outer } \\
\text { membrane; mitochondrion; voltage- } \\
\text { dependent anion channel porin activity; } \\
\text { voltage-dependent ion-selective } \\
\text { channel activity }\end{array}$ \\
\hline R68198 & $\begin{array}{r}0.004130 \\
001\end{array}$ & & & & \\
\hline $\mathrm{H} 65231$ & \begin{tabular}{|r|}
0.004212 \\
748 \\
\end{tabular} & & & & \\
\hline H51160 & \begin{tabular}{|r|}
0.004249 \\
526
\end{tabular} & PPP2R1A & $\underline{5518}$ & $\begin{array}{l}\text { protein } \\
\text { phosphata } \\
\text { se } 2 \\
\text { (formerly } \\
\text { 2A) }\end{array}$ & protein phosphatase type $2 \mathrm{~A}$ activity \\
\hline
\end{tabular}




\begin{tabular}{|c|c|c|c|c|c|}
\hline & & & & \begin{tabular}{|l|} 
regulatory \\
subunit A \\
(PR 65) \\
alpha \\
isoform \\
\end{tabular} & \\
\hline AA037284 & $\begin{array}{r}0.004277 \\
598\end{array}$ & $\underline{\text { APRT }}$ & 353 & \begin{tabular}{|l|} 
adenine \\
phosphorib \\
Osyltransfe \\
rase
\end{tabular} & $\begin{array}{l}\text { adenine phosphoribosyltransferase } \\
\text { activity; adenine salvage pathway; } \\
\text { nucleoside metabolism; transferase } \\
\text { activity, transferring glycosyl groups }\end{array}$ \\
\hline H73751 & $\begin{array}{r}0.004281 \\
436\end{array}$ & MAP3K6 & $\frac{9064}{9}$ & $\begin{array}{l}\text { mitogen- } \\
\text { activated } \\
\text { protein } \\
\text { kinase } \\
\text { kinase } \\
\text { kinase } 6 \\
\end{array}$ & $\begin{array}{l}\text { MAP kinase kinase kinase activity; } \\
\text { activation of JUNK; signal transduction }\end{array}$ \\
\hline H85811 & $\begin{array}{r}0.004299 \\
58\end{array}$ & HIPK2 & $\underline{28996}$ & \begin{tabular}{|l|} 
homeodom \\
ain \\
interacting \\
protein \\
kinase 2
\end{tabular} & $\begin{array}{l}\text { nucleus; protein kinase activity; } \\
\text { transcription co-repressor activity }\end{array}$ \\
\hline H04530 & $\begin{array}{r}0.004311 \\
455 \\
\end{array}$ & ECHS1 & $\underline{1892}$ & \begin{tabular}{|l|} 
enoyl \\
Coenzyme \\
A \\
hydratase, \\
short \\
chain, 1, \\
mitochondr \\
ial \\
\end{tabular} & $\begin{array}{l}\text { energy pathways; fatty acid beta- } \\
\text { oxidation; fatty acid metabolism; long- } \\
\text { chain-enoyl-CoA hydratase activity; } \\
\text { lyase activity; mitochondrion }\end{array}$ \\
\hline N33550 & $\begin{array}{r}0.004329 \\
96 \\
\end{array}$ & & & & \\
\hline $\mathrm{H} 27097$ & $\begin{array}{r}0.004331 \\
377 \\
\end{array}$ & $\underline{40 \text { LOC3386 }}$ & $\underline{338645}$ & \begin{tabular}{|l|} 
hypothetic \\
al protein \\
LOC33864 \\
5
\end{tabular} & \\
\hline $\mathrm{H} 62770$ & $\begin{array}{r}0.004355 \\
849 \\
\end{array}$ & & & & \\
\hline AA099281 & $\begin{array}{r}0.004365 \\
11\end{array}$ & COL18A1 & $\underline{80781}$ & $\begin{array}{l}\text { collagen, } \\
\text { type XVIII, } \\
\text { alpha } 1\end{array}$ & $\begin{array}{l}\text { cell adhesion; cell adhesion molecule } \\
\text { activity; collagen; extracellular matrix } \\
\text { structural constituent; histogenesis and } \\
\text { organogenesis; negative regulation of } \\
\text { cell proliferation; vision }\end{array}$ \\
\hline AA134572 & $\begin{array}{r}0.004368 \\
155 \\
\end{array}$ & & & & \\
\hline AA034076 & \begin{tabular}{|r|}
0.004373 \\
584 \\
\end{tabular} & & & & \\
\hline R63205 & $\begin{array}{r}0.004380 \\
177 \\
\end{array}$ & & & & \\
\hline R83247 & \begin{tabular}{|r|}
0.004394 \\
632 \\
\end{tabular} & GLB1 & $\underline{2720}$ & \begin{tabular}{|l|} 
galactosida \\
se, beta 1
\end{tabular} & beta-galactosidase activity; lysosome \\
\hline $\mathrm{H} 08266$ & $\begin{array}{r}0.004422 \\
345 \\
\end{array}$ & $\underline{\mathrm{H} 2 \mathrm{AV}}$ & 94239 & \begin{tabular}{|l|} 
histone \\
H2A.F/Z \\
variant \\
\end{tabular} & \\
\hline
\end{tabular}




\begin{tabular}{|l|r|l|l|l|l|}
\hline T86338 & $\begin{array}{r}0.004449 \\
624\end{array}$ & & & \\
\hline R88711 & $\begin{array}{r}0.004475 \\
335\end{array}$ & & & & \\
\hline AA036800 & $\begin{array}{r}0.004489 \\
289\end{array}$ & $\underline{\text { IHPK3 }}$ & $\underline{117283}$ & $\begin{array}{l}\text { inositol } \\
\text { hexaphosp } \\
\text { hate } \\
\text { kinase 3 }\end{array}$ & \\
\hline H83488 & 0.004495 & & & & \\
& 27 & & & & \\
\hline H06830 & 0.004560 & & & & \\
& 081 & & & & \\
\hline AA053136 & 0.004589 & KIAA1982 & 170960 & & KIAA1982 \\
protein & \\
\hline
\end{tabular}


Appendix H. Genes significantly different after 90m MPP+ treatment +/- PTIO.

\begin{tabular}{|c|c|c|c|c|c|}
\hline GENBANK & PVALUE & SYMBOL & $\begin{array}{l}\text { LOCUS } \\
\text { LINK }\end{array}$ & $\begin{array}{l}\text { GENE } \\
\text { NAME }\end{array}$ & GENE ONTOLOGY \\
\hline R64526 & $4.25 \mathrm{E}-08$ & & & & \\
\hline R39393 & 4.84E-08 & & & & \\
\hline $\mathrm{H} 14810$ & $2.84 \mathrm{E}-07$ & & $\underline{158819}$ & $\begin{array}{l}\text { hypothet } \\
\text { AL11753 }\end{array}$ & ical gene supported by AK057191; \\
\hline R11718 & $3.29 \mathrm{E}-07$ & TCF4 & $\underline{6925}$ & $\begin{array}{l}\text { transcri } \\
\text { ption } \\
\text { factor } 4\end{array}$ & $\begin{array}{l}\text { DNA binding; RNA polymerase II } \\
\text { transcription factor activity; nucleus; } \\
\text { regulation of transcription from Pol II } \\
\text { promoter }\end{array}$ \\
\hline W58007 & $5.31 \mathrm{E}-07$ & & 339299 & $\begin{array}{l}\text { LOC339 } \\
299\end{array}$ & \\
\hline $\mathrm{H} 03447$ & $6.23 \mathrm{E}-07$ & & & & \\
\hline R98825 & $6.82 \mathrm{E}-07$ & $\underline{\underline{L O C} 2835}$ & $\underline{283596}$ & $\begin{array}{l}\text { hypothe } \\
\text { tical } \\
\text { protein } \\
\text { LOC283 } \\
596\end{array}$ & \\
\hline H84229 & $3.46 \mathrm{E}-06$ & & & & \\
\hline T83371 & 4.28E-06 & & & & \\
\hline N72164 & 7.33E-06 & & & & \\
\hline AA029936 & $8.50 \mathrm{E}-06$ & $\frac{\text { PRKAR1 }}{\text { B }}$ & $\underline{5575}$ & $\begin{array}{l}\text { protein } \\
\text { kinase, } \\
\text { cAMP- } \\
\text { depend } \\
\text { ent, } \\
\text { regulato } \\
\text { ry, type } \\
\text { I, beta } \\
\end{array}$ & $\begin{array}{l}\text { 3',5'-cAMP binding; cAMP-dependent } \\
\text { protein kinase complex; cAMP- } \\
\text { dependent protein kinase, intrinsic } \\
\text { regulator activity; protein amino acid } \\
\text { phosphorylation; signal transduction }\end{array}$ \\
\hline H84599 & 1.04E-05 & & & & \\
\hline $\mathrm{H} 12977$ & 1.17E-05 & PRKCG & $\underline{5582}$ & $\begin{array}{l}\text { protein } \\
\text { kinase } \\
\text { C, } \\
\text { gamma }\end{array}$ & $\begin{array}{l}\text { ATP binding; cAMP-dependent protein } \\
\text { kinase activity; calcium ion binding; } \\
\text { diacylglycerol binding; intracellular } \\
\text { signaling cascade; protein amino acid } \\
\text { phosphorylation; protein kinase C } \\
\text { activity; protein kinase CK2 activity; } \\
\text { protein-tyrosine kinase activity; } \\
\text { transferase activity }\end{array}$ \\
\hline R56037 & 1.20E-05 & & & & \\
\hline R28465 & $1.68 \mathrm{E}-05$ & & $\underline{221922}$ & \multicolumn{2}{|c|}{$\begin{array}{l}\text { hypothetical gene supported by AL713633; } \\
\text { BC014395 }\end{array}$} \\
\hline T83702 & 1.71E-05 & & & & \\
\hline T78466 & $1.73 \mathrm{E}-05$ & PSG5 & $\underline{5673}$ & $\begin{array}{l}\text { pregnan } \\
\text { cy } \\
\text { specific } \\
\text { beta-1- } \\
\text { glycopr }\end{array}$ & $\begin{array}{l}\text { extracellular space; plasma glycoprotein; } \\
\text { pregnancy }\end{array}$ \\
\hline
\end{tabular}




\begin{tabular}{|c|c|c|c|c|c|}
\hline & & & & otein 5 & \\
\hline AA026351 & $1.94 \mathrm{E}-05$ & & & & \\
\hline T84214 & 1.97E-05 & & & & \\
\hline $\mathrm{H} 12575$ & $2.18 \mathrm{E}-05$ & MGC8902 & $\underline{284565}$ & \begin{tabular}{|l|} 
hypothe \\
tical \\
protein \\
MGC89 \\
02
\end{tabular} & \\
\hline R43469 & 2.68E-05 & EPHB3 & $\underline{2049}$ & EphB3 & $\begin{array}{l}\text { ATP binding; ephrin receptor activity; } \\
\text { integral to plasma membrane; protein } \\
\text { amino acid phosphorylation; receptor } \\
\text { activity; signal transduction; transferase } \\
\text { activity; transmembrane receptor protein } \\
\text { tyrosine kinase signaling pathway }\end{array}$ \\
\hline $\mathrm{H} 20004$ & $2.75 \mathrm{E}-05$ & WDR8 & $\underline{49856}$ & $\begin{array}{l}\text { WD } \\
\text { repeat } \\
\text { domain } \\
8\end{array}$ & \\
\hline AA129918 & 2.95E-05 & FLJ10385 & $\underline{55135}$ & $\begin{array}{l}\text { hypothe } \\
\text { tical } \\
\text { protein } \\
\text { FLJ103 } \\
85\end{array}$ & \\
\hline AA046498 & 3.36E-05 & & $\underline{347868}$ & \begin{tabular}{|l|} 
similar \\
to \\
hypothe \\
tical \\
protein \\
BC0153 \\
53 \\
\end{tabular} & \\
\hline T87122 & $3.53 \mathrm{E}-05$ & & & & \\
\hline R26558 & 4.44E-05 & $\begin{array}{l}\text { SDCCAG } \\
10 \\
\end{array}$ & 10283 & serologic & cally defined colon cancer antigen 10 \\
\hline W01227 & 4.64E-05 & HDGF & $\underline{3068}$ & \begin{tabular}{|l|} 
hepato \\
ma- \\
derived \\
growth \\
factor \\
(high- \\
mobility \\
group \\
protein \\
1-like) \\
\end{tabular} & $\begin{array}{l}\text { cell proliferation; cytoplasm; extracellular } \\
\text { space; growth factor activity; heparin } \\
\text { binding; signal transduction }\end{array}$ \\
\hline R05508 & 4.97E-05 & HSPC163 & $\underline{29097}$ & \begin{tabular}{|l|} 
HSPC1 \\
63 \\
protein
\end{tabular} & \\
\hline AA039224 & 5.09E-05 & & & & \\
\hline R51914 & 5.34E-05 & CGI-87 & $\underline{51112}$ & $\begin{array}{l}\text { CGI-87 } \\
\text { protein }\end{array}$ & \\
\hline T66875 & $6.08 \mathrm{E}-05$ & & & & \\
\hline R42763 & 6.49E-05 & KIAA0319 & 9856 & KIAA03 & \\
\hline
\end{tabular}




\begin{tabular}{|c|c|c|c|c|c|}
\hline & & & & $\begin{array}{l}19 \text { gene } \\
\text { product }\end{array}$ & \\
\hline W32438 & $7.02 E-05$ & CRABP2 & 1382 & $\begin{array}{l}\text { cellular } \\
\text { retinoic } \\
\text { acid } \\
\text { binding } \\
\text { protein } \\
2\end{array}$ & $\begin{array}{l}\text { epidermal differentiation; lipid binding; } \\
\text { regulation of transcription, DNA- } \\
\text { dependent; retinoid binding; signal } \\
\text { transduction; transport; transporter } \\
\text { activity }\end{array}$ \\
\hline R06569 & $7.28 \mathrm{E}-05$ & & & & \\
\hline H62185 & $7.96 \mathrm{E}-05$ & \begin{tabular}{|l}
$\underline{\mathrm{LOC}} 5696$ \\
$\underline{5}$
\end{tabular} & $\underline{56965}$ & \multicolumn{2}{|c|}{ hypothetical protein from EUROIMAGE 1977056} \\
\hline T84202 & 8.84E-05 & TAPBP & $\underline{6892}$ & \begin{tabular}{|l|} 
TAP \\
binding \\
protein \\
(tapasin \\
)
\end{tabular} & $\begin{array}{l}\text { MHC-interacting protein; endoplasmic } \\
\text { reticulum; endoplasmic reticulum } \\
\text { membrane; immune response; integral } \\
\text { to membrane; peptide antigen } \\
\text { transporter activity; protein binding; } \\
\text { protein complex assembly }\end{array}$ \\
\hline R21465 & 9.03E-05 & MAPT & 4137 & $\begin{array}{l}\text { microtu } \\
\text { bule- } \\
\text { associat } \\
\text { ed } \\
\text { protein } \\
\text { tau }\end{array}$ & $\begin{array}{l}\text { apoptosis; cytosol; microtubule } \\
\text { associated complex; microtubule } \\
\text { cytoskeleton organization and } \\
\text { biogenesis; plasma membrane; } \\
\text { structural constituent of cytoskeleton }\end{array}$ \\
\hline H70162 & $\begin{array}{r}0.000108 \\
65\end{array}$ & & & & \\
\hline N64388 & $\begin{array}{r}0.000109 \\
27\end{array}$ & NR4A1 & 3164 & $\begin{array}{l}\text { nuclear } \\
\text { receptor } \\
\text { subfamil } \\
\text { y } 4, \\
\text { group } \\
\text { A, } \\
\text { member } \\
1\end{array}$ & $\begin{array}{l}\text { DNA binding; ligand-dependent nuclear } \\
\text { receptor activity; signal transduction }\end{array}$ \\
\hline H85859 & $\begin{array}{r}0.000122 \\
345\end{array}$ & CPR8 & 9236 & \begin{tabular}{|l|} 
cell \\
cycle \\
progres \\
sion 8 \\
protein \\
\end{tabular} & \\
\hline $\mathrm{H} 15158$ & $\begin{array}{r}0.000127 \\
648\end{array}$ & HSPC166 & $\underline{29099}$ & $\begin{array}{l}\text { HSPC1 } \\
66 \\
\text { protein }\end{array}$ & \\
\hline R46859 & $\begin{array}{r}0.000140 \\
203 \\
\end{array}$ & & & & \\
\hline R88987 & $\begin{array}{r}0.000166 \\
588\end{array}$ & TTR & $\underline{7276}$ & $\begin{array}{l}\text { transthy } \\
\text { retin } \\
\text { (prealbu } \\
\text { min, } \\
\text { amyloid } \\
\text { osis } \\
\text { type I) } \\
\end{array}$ & $\begin{array}{l}\text { carrier activity; extracellular space; } \\
\text { retinol binding; steroid binding; thyroid } \\
\text { hormone generation; thyroid hormone } \\
\text { transporter activity; transport }\end{array}$ \\
\hline H30513 & $\begin{array}{r}0.000168 \\
118 \\
\end{array}$ & & & & \\
\hline
\end{tabular}




\begin{tabular}{|c|c|c|c|c|c|}
\hline R32199 & \begin{tabular}{|r|}
0.000177 \\
648 \\
\end{tabular} & & & & \\
\hline R97023 & $\begin{array}{r}0.000204 \\
063 \\
\end{array}$ & & & & \\
\hline AA044052 & $\begin{array}{r}0.000214 \\
526 \\
\end{array}$ & & & & \\
\hline W16794 & $\begin{array}{r}0.000221 \\
025 \\
\end{array}$ & $\underline{B I V M}$ & $\underline{54841}$ & \multicolumn{2}{|c|}{$\begin{array}{l}\text { basic, immunoglobulin-like variable motif } \\
\text { containing }\end{array}$} \\
\hline H17218 & $\begin{array}{r}0.000223 \\
699 \\
\end{array}$ & $\underline{\text { CALM2 }}$ & 805 & \begin{tabular}{|l|} 
calmod \\
ulin 2 \\
(phosph \\
orylase \\
kinase, \\
delta) \\
\end{tabular} & $\begin{array}{l}\text { G-protein coupled receptor protein } \\
\text { signaling pathway; calcium ion binding; } \\
\text { cytoplasm; plasma membrane; protein } \\
\text { binding }\end{array}$ \\
\hline T84134 & $\begin{array}{r}0.000228 \\
472\end{array}$ & $\underline{\mathrm{HMBS}}$ & $\underline{3145}$ & $\begin{array}{l}\text { hydroxy } \\
\text { methylbi } \\
\text { lane } \\
\text { synthas } \\
\text { e }\end{array}$ & $\begin{array}{l}\text { heme biosynthesis; hydroxymethylbilane } \\
\text { synthase activity; lyase activity }\end{array}$ \\
\hline H87044 & $\begin{array}{r}0.000235 \\
822\end{array}$ & $\underline{\text { TIMM22 }}$ & $\underline{29928}$ & \begin{tabular}{|l|} 
transloc \\
ase of \\
inner \\
mitocho \\
ndrial \\
membra \\
ne 22 \\
homolo \\
g \\
(yeast) \\
\end{tabular} & $\begin{array}{l}\text { inner membrane; integral to membrane; } \\
\text { intracellular protein transport; } \\
\text { mitochondrial inner membrane pre- } \\
\text { sequence translocase complex; } \\
\text { mitochondrion; protein translocase } \\
\text { activity }\end{array}$ \\
\hline R14705 & $\begin{array}{r}0.000243 \\
616 \\
\end{array}$ & FBXL2 & 25827 & \begin{tabular}{|l|} 
F-box \\
and \\
leucine- \\
rich \\
repeat \\
protein \\
2 \\
\end{tabular} & $\begin{array}{l}\text { cytoplasm; protein binding; protein } \\
\text { modification; proteolysis and } \\
\text { peptidolysis; ubiquitin-protein ligase } \\
\text { activity }\end{array}$ \\
\hline AA031681 & $\begin{array}{r}0.000245 \\
545 \\
\end{array}$ & & & & \\
\hline W69443 & $\begin{array}{r}0.000249 \\
658 \\
\end{array}$ & $\underline{\mathrm{HMGN1}}$ & $\underline{3150}$ & \begin{tabular}{|l|} 
high- \\
mobility \\
group \\
nucleos \\
ome \\
binding \\
domain \\
1
\end{tabular} & $\begin{array}{l}\text { DNA binding; RNA polymerase II } \\
\text { transcription factor activity; chromatin; } \\
\text { positive transcription elongation factor } \\
\text { activity }\end{array}$ \\
\hline W32180 & $\begin{array}{r}0.000251 \\
562 \\
\end{array}$ & & & & \\
\hline R06754 & $\begin{array}{r}0.000254 \\
895 \\
\end{array}$ & & & & \\
\hline R66012 & $\begin{array}{r}0.000286 \\
351 \\
\end{array}$ & & & & \\
\hline R27906 & 0.000291 & & & & \\
\hline
\end{tabular}




\begin{tabular}{|c|c|c|c|c|c|}
\hline & 142 & & & & \\
\hline $\mathrm{H} 77950$ & $\begin{array}{r}0.000292 \\
038\end{array}$ & & & & \\
\hline R56046 & $\begin{array}{r}0.000313 \\
918\end{array}$ & GNAZ & 2781 & $\begin{array}{l}\text { guanine } \\
\text { nucleoti } \\
\text { de } \\
\text { binding } \\
\text { protein } \\
\text { (G } \\
\text { protein), } \\
\text { alpha z } \\
\text { polypep } \\
\text { tide }\end{array}$ & $\begin{array}{l}\text { G-protein coupled receptor protein } \\
\text { signaling pathway; GTP binding; } \\
\text { endoplasmic reticulum; heterotrimeric G- } \\
\text { protein GTPase activity; nuclear } \\
\text { membrane; plasma membrane; receptor } \\
\text { signaling protein activity; signal } \\
\text { transduction }\end{array}$ \\
\hline R81039 & $\begin{array}{r}0.000324 \\
399\end{array}$ & MFGE8 & $\underline{4240}$ & $\begin{array}{l}\text { milk fat } \\
\text { globule- } \\
\text { EGF } \\
\text { factor } 8 \\
\text { protein }\end{array}$ & $\begin{array}{l}\text { cell adhesion; cell adhesion molecule } \\
\text { activity; lipid particle; milk protein; } \\
\text { oncogenesis }\end{array}$ \\
\hline R53914 & $\begin{array}{r}0.000336 \\
246\end{array}$ & HARC & $\underline{55664}$ & $\begin{array}{l}\text { Hsp90- } \\
\text { associat } \\
\text { ing } \\
\text { relative } \\
\text { of } \\
\text { Cdc37 } \\
\end{array}$ & cytokinesis; regulation of cell cycle \\
\hline R27994 & $\begin{array}{r}0.000401 \\
721\end{array}$ & $\begin{array}{l}\text { LOC1624 } \\
27\end{array}$ & 162427 & $\begin{array}{l}\text { hypothe } \\
\text { tical } \\
\text { protein } \\
\text { LOC162 } \\
427 \\
\end{array}$ & \\
\hline H52061 & $\begin{array}{r}0.000413 \\
197\end{array}$ & FLJ22313 & $\underline{64224}$ & \begin{tabular}{|l} 
hypothe \\
tical \\
protein \\
FLJ223 \\
13
\end{tabular} & \\
\hline $\mathrm{H} 17731$ & $\begin{array}{r}0.000428 \\
912\end{array}$ & & & & \\
\hline H68952 & $\begin{array}{r}0.000434 \\
047\end{array}$ & ITGA1 & $\underline{3672}$ & $\begin{array}{l}\text { integrin, } \\
\text { alpha } 1\end{array}$ & $\begin{array}{l}\text { cell adhesion receptor activity; cell- } \\
\text { matrix adhesion; collagen binding; } \\
\text { integral to membrane; integrin complex; } \\
\text { integrin-mediated signaling pathway; } \\
\text { magnesium ion binding; receptor activity }\end{array}$ \\
\hline AA046698 & $\begin{array}{r}0.000436 \\
088\end{array}$ & KIAA1724 & $\underline{85465}$ & \begin{tabular}{|l} 
KIAA17 \\
24 \\
protein
\end{tabular} & phospholipid biosynthesis \\
\hline T96360 & $\begin{array}{r}0.000442 \\
922\end{array}$ & & & & \\
\hline H84325 & $\begin{array}{r}0.000463 \\
215\end{array}$ & PBX3 & $\underline{5090}$ & $\begin{array}{l}\text { pre-B- } \\
\text { cell } \\
\text { leukemi } \\
\text { a } \\
\text { transcri } \\
\text { ption } \\
\text { factor } 3\end{array}$ & $\begin{array}{l}\text { DNA binding; anterior compartment } \\
\text { specification; oncogenesis; posterior } \\
\text { compartment specification }\end{array}$ \\
\hline
\end{tabular}




\begin{tabular}{|c|c|c|c|c|c|}
\hline T95099 & \begin{tabular}{|r|}
0.000466 \\
639 \\
\end{tabular} & MT1F & $\underline{4494}$ & \begin{tabular}{|l} 
metallot \\
hionein \\
$1 \mathrm{~F}$ \\
(functio \\
nal) \\
\end{tabular} & $\begin{array}{l}\text { biological_process unknown; cadmium } \\
\text { ion binding; copper ion binding; } \\
\text { cytoplasm; metal ion binding; zinc ion } \\
\text { binding }\end{array}$ \\
\hline R26644 & $\begin{array}{r}0.000471 \\
842 \\
\end{array}$ & & & & \\
\hline AA074208 & $\begin{array}{r}0.000476 \\
658 \\
\end{array}$ & NELL2 & $\underline{4753}$ & $\begin{array}{l}\text { NEL- } \\
\text { like } 2 \\
\text { (chicken }\end{array}$ & $\begin{array}{l}\text { calcium ion binding; cell adhesion; } \\
\text { extracellular; structural molecule activity }\end{array}$ \\
\hline $\mathrm{H} 18471$ & $\begin{array}{r}0.000480 \\
051\end{array}$ & & & & \\
\hline H53033 & $\begin{array}{r}0.000483 \\
447 \\
\end{array}$ & NUMB & $\underline{8650}$ & $\begin{array}{l}\text { numb } \\
\text { homolo } \\
\text { g } \\
\text { (Drosop } \\
\text { hila) }\end{array}$ & integral to plasma membrane \\
\hline
\end{tabular}


Appendix I. Genes differentially regulated in the presence of both PTIO and SN50 with $15 \mathrm{~m}$ concurrent MPP+ exposure.

\begin{tabular}{|c|c|c|c|c|}
\hline GENBANK & SYMBOL & $\begin{array}{c}\text { LOCUS } \\
\text { LINK }\end{array}$ & GENE NAME & GENE ONTOLOGY \\
\hline AA010141 & SERPINH1 & 871 & $\begin{array}{l}\text { serine (or cysteine) } \\
\text { proteinase inhibitor, } \\
\text { clade H (heat shock } \\
\text { protein 47), member } \\
1, \text { (collagen binding } \\
\text { protein 1) }\end{array}$ & heat shock response \\
\hline AA026902 & FLJ11320 & $\underline{55343}$ & $\begin{array}{l}\text { GDP-fucose } \\
\text { transporter } 1\end{array}$ & $\begin{array}{l}\text { Golgi apparatus; integral to } \\
\text { membrane; sugar porter activity; } \\
\text { transport }\end{array}$ \\
\hline \multicolumn{5}{|l|}{ AA028961 } \\
\hline AA031465 & GEFT & 115557 & $\begin{array}{l}\text { RAC/CDC42 } \\
\text { exchange factor }\end{array}$ & \\
\hline AA031859 & TIMM13 & $\underline{26517}$ & $\begin{array}{l}\text { translocase of inner } \\
\text { mitochondrial } \\
\text { membrane } 13 \\
\text { homolog (yeast) }\end{array}$ & $\begin{array}{l}\text { hearing; mitochondrial inner } \\
\text { membrane pre-sequence } \\
\text { translocase complex; mitochondrial } \\
\text { translocation; mitochondrion; protein } \\
\text { targeting; protein translocase } \\
\text { activity; zinc ion binding }\end{array}$ \\
\hline AA034109 & MINK & $\underline{50488}$ & $\begin{array}{l}\text { misshapen/NIK- } \\
\text { related kinase }\end{array}$ & $\begin{array}{l}\text { ATP binding; cAMP-dependent } \\
\text { protein kinase activity; development; } \\
\text { protein amino acid phosphorylation; } \\
\text { protein kinase CK2 activity; protein } \\
\text { kinase cascade; protein } \\
\text { serine/threonine kinase activity; } \\
\text { response to stress; small GTPase } \\
\text { regulatory/interacting protein activity; } \\
\text { transferase activity }\end{array}$ \\
\hline AA037284 & APRT & $\underline{353}$ & $\begin{array}{l}\text { adenine } \\
\text { phosphoribosyltransf } \\
\text { erase }\end{array}$ & $\begin{array}{l}\text { adenine phosphoribosyltransferase } \\
\text { activity; adenine salvage pathway; } \\
\text { nucleoside metabolism; transferase } \\
\text { activity, transferring glycosyl groups }\end{array}$ \\
\hline AA125808 & CAPS & $\underline{828}$ & calcyphosine & $\begin{array}{l}\text { calcium ion binding; intracellular } \\
\text { signaling cascade }\end{array}$ \\
\hline AA142924 & DF & $\underline{1675}$ & $\begin{array}{l}\text { D component of } \\
\text { complement (adipsin) }\end{array}$ & $\begin{array}{l}\text { chymotrypsin activity; complement } \\
\text { activation, alternative pathway; } \\
\text { complement factor D activity; } \\
\text { hydrolase activity; proteolysis and } \\
\text { peptidolysis; trypsin activity }\end{array}$ \\
\hline AA152287 & SLC35B2 & 347734 & $\begin{array}{l}\text { solute carrier family } \\
35, \text { member B2 }\end{array}$ & $\begin{array}{l}\text { copper ion binding; electron } \\
\text { transport; electron transporter } \\
\text { activity }\end{array}$ \\
\hline \multicolumn{5}{|l|}{$\mathrm{H} 02590$} \\
\hline \multicolumn{5}{|l|}{\begin{tabular}{|l|}
$\mathrm{H} 09429$ \\
\end{tabular}} \\
\hline \multicolumn{5}{|l|}{$\mathrm{H} 14566$} \\
\hline $\mathrm{H} 26552$ & MGC5395 & 79026 & hypothetical protein & intracellular signaling cascade \\
\hline
\end{tabular}




\begin{tabular}{|c|c|c|c|c|}
\hline & & & MGC5395 & \\
\hline $\mathrm{H} 28534$ & AQP1 & $\underline{358}$ & $\begin{array}{l}\text { aquaporin } 1 \\
\text { (channel-forming } \\
\text { integral protein, } \\
28 \mathrm{kDa} \text { ) }\end{array}$ & $\begin{array}{l}\text { excretion; integral to plasma } \\
\text { membrane; transport; water } \\
\text { transport; water transporter activity }\end{array}$ \\
\hline H41330 & LRRC2 & $\underline{79442}$ & $\begin{array}{l}\text { leucine-rich repeat- } \\
\text { containing } 2\end{array}$ & \\
\hline $\mathrm{H} 45241$ & RPL41 & $\underline{6171}$ & $\begin{array}{l}\text { ribosomal protein } \\
\text { L41 }\end{array}$ & $\begin{array}{l}\text { RNA binding; cytosolic large } \\
\text { ribosomal subunit (sensu Eukarya); } \\
\text { protein biosynthesis; structural } \\
\text { constituent of ribosome }\end{array}$ \\
\hline \multicolumn{5}{|l|}{$\mathrm{H} 47114$} \\
\hline \multicolumn{5}{|l|}{$\mathrm{H} 48570$} \\
\hline \multicolumn{5}{|l|}{$\mathrm{H} 48578$} \\
\hline \multicolumn{5}{|l|}{$\mathrm{H} 50015$} \\
\hline \multicolumn{5}{|l|}{\begin{tabular}{|l|}
$\mathrm{H} 50657$ \\
\end{tabular}} \\
\hline H58461 & & $\underline{339088}$ & $\begin{array}{l}\text { similar to My016 } \\
\text { protein }\end{array}$ & \\
\hline H59405 & FLJ10298 & $\underline{54682}$ & $\begin{array}{l}\text { hypothetical protein } \\
\text { FLJ10298 }\end{array}$ & \\
\hline \multicolumn{5}{|l|}{ H61842 } \\
\hline \multicolumn{5}{|l|}{\begin{tabular}{|l|}
$\mathrm{H} 63763$ \\
\end{tabular}} \\
\hline \multicolumn{5}{|l|}{$\mathrm{H} 65775$} \\
\hline \multicolumn{5}{|l|}{\begin{tabular}{|l}
$\mathrm{H} 65832$ \\
\end{tabular}} \\
\hline H68885 & TSSC3 & $\underline{7262}$ & $\begin{array}{l}\text { tumor suppressing } \\
\text { subtransferable } \\
\text { candidate } 3\end{array}$ & apoptosis; imprinting \\
\hline \multicolumn{5}{|l|}{$\mathrm{H} 69845$} \\
\hline \multicolumn{5}{|l|}{ H83488 } \\
\hline H84657 & GRWD & $\underline{83743}$ & $\begin{array}{l}\text { glutamate rich WD } \\
\text { repeat protein } \\
\text { GRWD }\end{array}$ & \\
\hline H85811 & HIPK2 & $\underline{28996}$ & $\begin{array}{l}\text { homeodomain } \\
\text { interacting protein } \\
\text { kinase } 2\end{array}$ & $\begin{array}{l}\text { nucleus; protein kinase activity; } \\
\text { transcription co-repressor activity }\end{array}$ \\
\hline H99202 & MGC4126 & 84859 & $\begin{array}{l}\text { hypothetical protein } \\
\text { MGC4126 }\end{array}$ & \\
\hline \multicolumn{5}{|l|}{ N/A1 } \\
\hline N23779 & CD151 & 977 & CD151 antigen & $\begin{array}{l}\text { cell adhesion; integral to plasma } \\
\text { membrane; membrane fraction }\end{array}$ \\
\hline N29429 & CGI-57 & $\underline{27013}$ & $\begin{array}{l}\text { hypothetical protein } \\
\text { CGI-57 }\end{array}$ & \\
\hline N55283 & KIAA0469 & $\underline{9903}$ & $\begin{array}{l}\text { KIAA0469 gene } \\
\text { product }\end{array}$ & \\
\hline N91376 & KIAA0247 & $\underline{9766}$ & $\begin{array}{l}\text { KIAA0247 gene } \\
\text { product }\end{array}$ & integral to membrane \\
\hline N92911 & DJ473B4 & $\underline{56180}$ & $\begin{array}{l}\text { hypothetical protein } \\
\text { dJ473B4 }\end{array}$ & structural molecule activity \\
\hline R00907 & PLEKHG1 & $\underline{57480}$ & $\begin{array}{l}\text { pleckstrin homology } \\
\text { RhoGef domain) me }\end{array}$ & $\begin{array}{l}\text { omain containing, family } \mathrm{G} \text { (with } \\
\text { hber } 1\end{array}$ \\
\hline
\end{tabular}




\begin{tabular}{|c|c|c|c|c|}
\hline R07137 & HIC2 & 23119 & $\begin{array}{l}\text { hypermethylated in } \\
\text { cancer } 2\end{array}$ & $\begin{array}{l}\text { DNA binding; negative regulation of } \\
\text { transcription, DNA-dependent; } \\
\text { nucleus; protein C-terminus binding }\end{array}$ \\
\hline \multicolumn{5}{|l|}{ R07186 } \\
\hline R09962 & PMS2L6 & $\underline{5384}$ & $\begin{array}{l}\text { postmeiotic } \\
\text { segregation } \\
\text { increased 2-like } 6\end{array}$ & $\begin{array}{l}\text { damaged DNA binding; mismatch } \\
\text { repair; nucleus }\end{array}$ \\
\hline R13021 & FLJ10751 & $\underline{55222}$ & $\begin{array}{l}\text { hypothetical protein } \\
\text { FLJ10751 }\end{array}$ & \\
\hline R14326 & HERC1 & $\underline{8925}$ & $\begin{array}{l}\text { hect (homologous to } \\
\text { the E6-AP (UBE3A) } \\
\text { carboxyl terminus) } \\
\text { domain and RCC1 } \\
\text { (CHC1)-like domain } \\
\text { (RLD) } 1\end{array}$ & $\begin{array}{l}\text { ARF guanyl-nucleotide exchange } \\
\text { factor activity; Golgi apparatus; } \\
\text { catalytic activity; nonselective } \\
\text { vesicle transport; ubiquitin cycle; } \\
\text { ubiquitin-protein ligase activity }\end{array}$ \\
\hline R14363 & HDAC5 & $\underline{10014}$ & $\begin{array}{l}\text { histone deacetylase } \\
5\end{array}$ & $\begin{array}{l}\text { chromatin modeling; chromatin } \\
\text { silencing; cytoplasm; histone } \\
\text { deacetylase activity; nucleus; } \\
\text { regulation of transcription, DNA- } \\
\text { dependent }\end{array}$ \\
\hline \multicolumn{5}{|l|}{ R15267 } \\
\hline \multicolumn{5}{|l|}{ R20019 } \\
\hline R20373 & TMP21 & 10972 & $\begin{array}{l}\text { transmembrane } \\
\text { trafficking protein }\end{array}$ & $\begin{array}{l}\text { ER to Golgi transport; Golgi } \\
\text { apparatus; integral to plasma } \\
\text { membrane; intracellular protein } \\
\text { transport; membrane fraction; } \\
\text { microsome; protein carrier activity; } \\
\text { protein transporter activity }\end{array}$ \\
\hline \multicolumn{5}{|r|}{ (1) } \\
\hline \multicolumn{5}{|l|}{ R22402 } \\
\hline R23778 & $\underline{\mathrm{C} 7}$ & $\underline{730}$ & $\begin{array}{l}\text { complement } \\
\text { component } 7\end{array}$ & $\begin{array}{l}\text { complement activation, alternative } \\
\text { pathway; complement activation, } \\
\text { classical pathway; complement } \\
\text { activity; cytolysis; immune response; } \\
\text { integral to membrane; membrane } \\
\text { attack complex; response to } \\
\text { pathogenic bacteria }\end{array}$ \\
\hline R26954 & $\underline{\text { CTSD }}$ & 1509 & $\begin{array}{l}\text { cathepsin D } \\
\text { (lysosomal aspartyl } \\
\text { protease) }\end{array}$ & $\begin{array}{l}\text { cathepsin D activity; hydrolase } \\
\text { activity; Iysosome; pepsin A activity; } \\
\text { proteolysis and peptidolysis }\end{array}$ \\
\hline \multicolumn{5}{|l|}{ R34114 } \\
\hline \multicolumn{5}{|l|}{ R36086 } \\
\hline R47859 & NPR1 & $\underline{4881}$ & $\begin{array}{l}\text { natriuretic peptide } \\
\text { receptor A/guanylate } \\
\text { cyclase A } \\
\text { (atrionatriuretic } \\
\text { peptide receptor A) }\end{array}$ & $\begin{array}{l}\text { ATP binding; cGMP biosynthesis; } \\
\text { guanylate cyclase activity; integral to } \\
\text { membrane; intracellular signaling } \\
\text { cascade; lyase activity; peptide } \\
\text { receptor activity, G-protein coupled; } \\
\text { protein amino acid phosphorylation; } \\
\text { protein kinase activity; receptor } \\
\text { activity; receptor guanylate cyclase } \\
\text { activity; regulation of blood pressure }\end{array}$ \\
\hline
\end{tabular}




\begin{tabular}{|c|c|c|c|c|}
\hline \multicolumn{5}{|l|}{ R55491 } \\
\hline \multicolumn{5}{|l|}{ R62213 } \\
\hline \multicolumn{5}{|l|}{ R83014 } \\
\hline R83247 & GLB1 & $\underline{2720}$ & galactosidase, beta 1 & $\begin{array}{l}\text { beta-galactosidase activity; } \\
\text { lysosome }\end{array}$ \\
\hline R87060 & GGCX & $\underline{2677}$ & $\begin{array}{l}\text { gamma-glutamyl } \\
\text { carboxylase }\end{array}$ & $\begin{array}{l}\text { blood coagulation; gamma-glutamyl } \\
\text { carboxylase activity; integral to } \\
\text { membrane; ligase activity; } \\
\text { membrane fraction; protein } \\
\text { modification }\end{array}$ \\
\hline R87345 & MGC2656 & $\underline{79414}$ & $\begin{array}{l}\text { hypothetical protein } \\
\text { MGC2656 }\end{array}$ & \\
\hline R88895 & MANBAL & $\underline{63905}$ & $\begin{array}{l}\text { mannosidase, beta } \\
\text { A, lysosomal-like }\end{array}$ & integral to membrane \\
\hline R94499 & GNB5 & 10681 & \multicolumn{2}{|c|}{ guanine nucleotide binding protein ( $G$ protein), beta 5} \\
\hline \multicolumn{5}{|l|}{ R98591 } \\
\hline \multicolumn{5}{|l|}{ T70299 } \\
\hline \multicolumn{5}{|l|}{ T79552 } \\
\hline T83013 & $\underline{H G D}$ & $\underline{3081}$ & \multicolumn{2}{|c|}{ homogentisate 1,2-dioxygenase (homogentisate oxidase) } \\
\hline \multicolumn{5}{|l|}{ T86338 } \\
\hline T92003 & KIAA0342 & $\underline{9881}$ & $\begin{array}{l}\text { KIAA0342 gene } \\
\text { product }\end{array}$ & $\begin{array}{l}\text { DNA binding; membrane; nucleus; } \\
\text { transport; transporter activity }\end{array}$ \\
\hline \multicolumn{5}{|l|}{ T93785 } \\
\hline W68050 & LGALS1 & $\underline{3956}$ & $\begin{array}{l}\text { lectin, galactoside- } \\
\text { binding, soluble, } 1 \\
\text { (galectin 1) }\end{array}$ & $\begin{array}{l}\text { apoptosis; heterophilic cell adhesion; } \\
\text { sugar binding }\end{array}$ \\
\hline W72400 & C12orf2 & 11228 & $\begin{array}{l}\text { chromosome } 12 \\
\text { open reading frame } 2\end{array}$ & neuropeptide signaling pathway \\
\hline
\end{tabular}

